\title{
Determining seismic shear-velocity from ambient noise sources at regional and local scales
}

Francesco Civilini

\author{
A thesis \\ submitted to the Victoria University of Wellington \\ in fulfilment of the \\ requirements for the degree of \\ Doctor of Philosophy \\ in Geophysics.
}

Victoria University of Wellington 2018 




\section{Abstract}

We present three projects that use different bandwidths of the ambient noise spectrum to solve geophysical problems. Specifically, we use signals within the noise field to determine surface and shear wave velocities, image the shallow and deep crust, and monitor time-dependent deformation resulting from geothermal fluid injection and extraction.

Harrat Al-Madinah, a Cenozoic bimodal alkaline volcanic field in west-central Saudi Arabia, is imaged using shear-velocities obtained from natural ambient seismic noise. To our knowledge, this project is the first analysis of Saudi Arabia structure using ambient noise methods. Surface wave arrivals are extracted from a year's worth of stationpair cross-correlations, which are approximations of the empirical Green's function of the interstation path. We determine group and phase velocity surface wave dispersion maps with a 0.1 decimal degree resolution and resolve a zone of slow surface wave velocity south-east of the city of Medina, which is spatially correlated with the most recent historical eruption (the 1256 CE Medina eruption). Dispersion curves are calculated at each grid-point of the surface-wave velocity maps and inverted to obtain measurements of shear-velocity with depth. The 1D velocity models are then used to produce average shear-velocity models for the volcanic field. A shear-velocity increase ranging from 0.5 to $1.0 \mathrm{~km} / \mathrm{s}$, suggesting a layer interface, is detected at approximately $20 \mathrm{~km}$ depth and compared to P-wave measurement from a previous refraction study. We compute cross-section profiles by interpolating the inversions into a pseudo-3D model and resolve a zone of slow shear-velocity below the $1256 \mathrm{CE}$ eruption location. These areas are also spatially correlated with low values of Bouguer gravity. We hypothesize that the low shear-velocity and gravity measurements are caused by fluids and fractures created from prior volcanic eruptions.

We use the coda of cross-correlations extracted from ambient noise to determine shear-velocity changes at Rotokawa and Ngatamariki, two electricity producing geothermal fields located in the North Island of New Zealand. Stacks of cross correlations between stations prior to the onset of production are compared to cross correlations of moving stacks in time periods of well stimulation and the onset of electricity production using the Moving Window Cross Spectral technique. An increase between $0.05 \%$ to $0.1 \%$ of shear-velocity is detected at Rotokawa coinciding with an increase of 
injection. The shear-velocity subsequently decreases by approximately $0.1 \%$ when the rate of production surpasses the rate of injection. A similar amplitude shear-velocity increase is detected at Ngatamariki during the beginning of injection. After the initial increase, the shear-velocity at Ngatamariki fluctuates in response to differences in injection and production rates. A straight-ray pseudo-tomography analysis is conducted at the geothermal fields, which reveals that localized positive velocity changes are colocated with injection wells.

Lastly, we use ambient noise and active sources at the Ngatamariki geothermal field to determine the structure of the top 200 meters using the Refraction Microtremor technique. We deployed a linear 72-channel array of vertical geophones with ten meter spacing at two locations of the geothermal field and determine average 1D and 2D shear-velocity profiles. We were able to image depths between 57 to 93 meters for 2D profiles and up to 165 meters for 1D profiles. A shear-velocity anomaly was detected across one of the lines that coincided with the inferred location of a fault determined from nearby well logs. This suggests that the method can be used to cheaply and quickly constrain near-surface geology at geothermal fields, where ambient noise is abundant and typical reflection and refraction surveys require large inputs of energy and are hindered by attenuation and scattering in near-surface layers. 


\section{Contents}

$\begin{array}{ll}\text { Abstract } & \text { iii }\end{array}$

Acknowledgments $\quad$ ix

List of Figures $\quad x$

List of Tables $\quad$ xvi

1 Introduction 1

1.1 Motivation ......................... 1

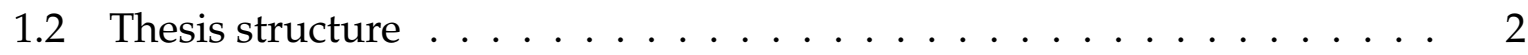

2 Theoretical framework $\quad 7$

2.1 Noise as a source . . . . . . . . . . . . . . . . 7

2.1.1 Green's functions and the stationary phase region . . . . . . . . 8

2.1.2 Station spacing and noise scaling . . . . . . . . . . . . . . 15

2.2 Computing the noise spectra . . . . . . . . . . . . 15

2.3 Computing the Green's function . . . . . . . . . . . . . . 18

3 Noise analysis and dispersion of the Harrat Al-Madinah volcanic field 23

3.1 Introduction . . . . . . . . . . . . . . . . . . . . 23

3.2 Regional geology and tectonics . . . . . . . . . . . . . . . 24

3.3 Local geology and prior geophysical studies . . . . . . . . . . . . 30

3.4 Site and instrument information . . . . . . . . . . . . 35

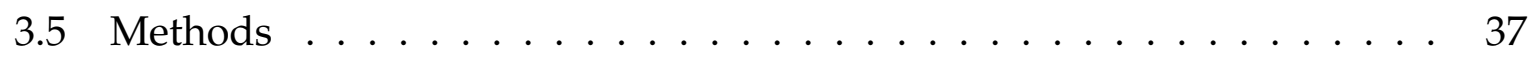

3.5 .1 Zero point noise . . . . . . . . . . . . . 38

3.5.2 Computing dispersion curves . . . . . . . . . . . . . . 39

3.5.3 Sensitivity Kernels . . . . . . . . . . . . . . . . . . . . . 45

3.5 .4 Tomographic inversion . . . . . . . . . . . . . . . 47

3.6 Results . . . . . . . . . . . . . . . . . . . . 50

3.6.1 Cross-correlation moveouts . . . . . . . . . . . . 50

3.6.2 Checkerboard tests ...................... 52 
3.6.3 Dispersion maps . . . . . . . . . . . . . . . . 60

3.6.4 Comparison with gravity measurements $\ldots \ldots \ldots \ldots 68$

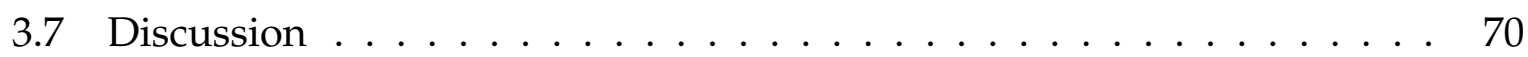

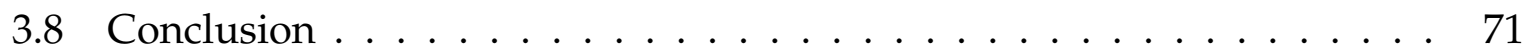

4 Shear-wave tomography of Harrat Al-Madinah 73

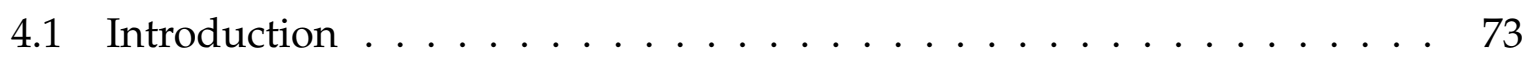

4.2 Methods . . . . . . . . . . . . . . . . . 73

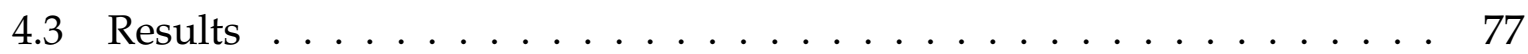

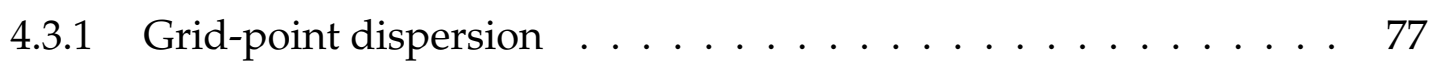

4.3 .2 1D Inversions . . . . . . . . . . . . . . . . . . . . . 81

4.3.3 Average Velocity Models . . . . . . . . . . . . . . . . . 84

4.3.4 Horizontal cross-sections of inverted S-wave velocities . . . . . 92

4.3.5 Pseudo-3D model and cross-sections . . . . . . . . . . . . . 97

4.4 Discussion . . . . . . . . . . . . . . . . . . . . . . . 106

4.5 Conclusion . . . . . . . . . . . . . . . . . . . . . . . . 110

5 Velocity variations at New Zealand geothermal fields 113

5.1 Introduction . . . . . . . . . . . . . . . . . . . . . . . . . . . 113

5.2 Velocity variations theory $\ldots \ldots \ldots \ldots \ldots \ldots$

5.3 Methods . . . . . . . . . . . . . . . . . . . . . . . . . . 119

5.3.1 Obtaining cross-correlations . . . . . . . . . . . . . . . . . 119

5.3 .2 Time-domain stability . . . . . . . . . . . . . . . . . . . 121

5.3.3 Frequency-domain stability . . . . . . . . . . . . . . . . 123

5.3.4 Computing velocity changes . . . . . . . . . . . . . 125

5.4 Rotokawa site information . . . . . . . . . . . . . . . . . . . . . . . 129

5.4.1 Rotokawa geologic information . . . . . . . . . . . . . . . . . . 129

5.4 .2 Rotokawa Instrumentation . . . . . . . . . . . . . . . . 132

5.5 Rotokawa results . . . . . . . . . . . . . . . . . . . 140

5.5.1 Rotokawa cross-correlations and moveouts . . . . . . . . . . 140

5.5 .2 Rotokawa stability . . . . . . . . . . . . . . . . . . . . 144

5.5.3 Sensor orientation issues at Rotokawa . . . . . . . . . . . . 150

5.5 .4 Rotokawa velocity changes . . . . . . . . . . . . . . 152

5.6 Ngatamariki site information . . . . . . . . . . . . . . . . 163

5.6 .1 Ngatamariki geology . . . . . . . . . . . . . . . . . . 163

5.6.2 Ngatamariki instrumentation . . . . . . . . . . . . . . . 167

5.7 Ngatamariki Results . . . . . . . . . . . . . . . . . . . . . . . 173

5.7.1 Ngatamariki cross-correlations and moveouts . . . . . . . . . 173

5.7 .2 Ngatamariki stability . . . . . . . . . . . . . . . . . . . . 173

5.7 .3 Ngatamariki velocity changes . . . . . . . . . . . . . . 178 
5.8 Discussion . . . . . . . . . . . . . . . . . . . . . . . . . 187

5.8.1 Cross-correlation stability, MWCS, and velocity changes . . . . 187

5.8.2 Time-dependent spatial relationships _ . . . . . . . . . . . . 190

5.8 .3 Mechanism for velocity changes _. . . . . . . . . . . . . 197

5.9 Conclusion . . . . . . . . . . . . . . . . . . . . . . . . . 201

6 Shear velocity structure inferred using Refraction Microtremor in the upper 200 meters at the Ngatamariki geothermal field, central North Island, New Zealand 205

6.1 Introduction . . . . . . . . . . . . . . . . . 206

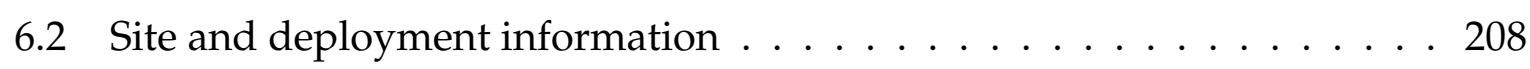

6.2 .1 Geology . . . . . . . . . . . . . . . . . . . 208

6.2 .2 Experimental setup . . . . . . . . . . . . . . . . . 208

6.3 ReMi method workflow and processing . . . . . . . . . . . 212

6.3 .1 Signal types . . . . . . . . . . . . . . . . . . 212

6.3.2 Characterizing noise sources of the geothermal field . . . . . . . 214

6.3.3 The slowness-frequency transform . . . . . . . . . . . 218

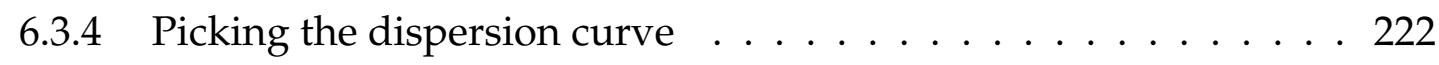

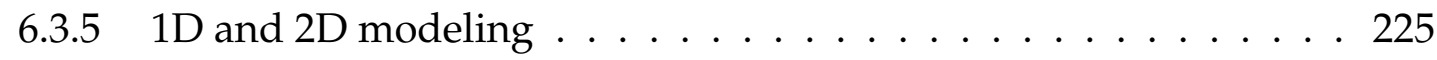

6.4 Results Line A . . . . . . . . . . . . . . . . . . . . . . . . . . . 229

6.5 Results Line B . . . . . . . . . . . . . . . . . . . . . 233

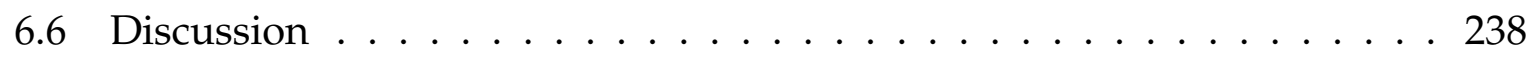

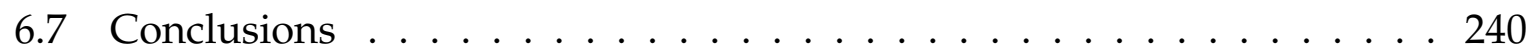

7 Summary of main results and opportunities for further research $\quad 243$

A Harrat Al-Madinah supplementary information $\quad 247$

A.1 Harrat Al-Madinah seismic stations . . . . . . . . . . . . . . . 247

A.2 Harrat Al-Madinah Station Noise . . . . . . . . . . . . . . . . 248

A.3 Four-month dispersion curves . . . . . . . . . . . . . . . . 252

B Velocity changes supplementary information 255

B.1 Rotokawa excluded pairs . . . . . . . . . . . . . . . 255

B.2 Ngatamariki excluded pairs . . . . . . . . . . . . . . 255

B.3 Rotokawa velocity changes reference stack $2 \ldots \ldots \ldots \ldots$

B.4 Noise at Rotokawa and Ngatamariki . . . . . . . . . . . . . . . . 264

C ReMi cultural noise supplementary information 277

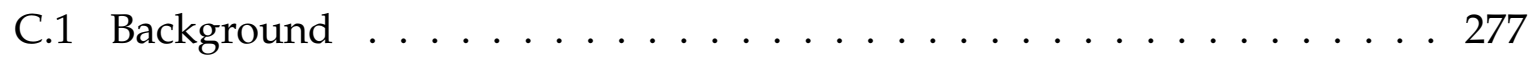

C.2 Methodology . . . . . . . . . . . . . . . . . . . . . 281

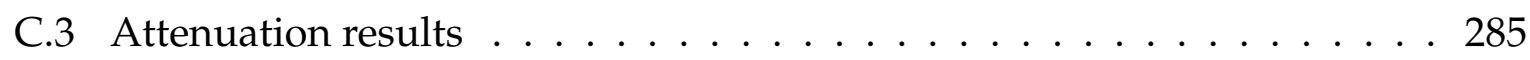


C.4 Application to microearthquakes . . . . . . . . . . . . . . . 287

C.5 Discussion and further work . . . . . . . . . . . . . . . . . . . . . . 290

C.6 Station Noise . . . . . . . . . . . . . . . . . . . . . . . . . . . . . 291 


\section{Acknowledgments}

First and foremost, I want to give a big thank you to Martha Savage and John Townend for their guidance over the last several years. None of this would have been possible without their dedication and support, and I am very grateful. This research was possible due to funding from the Victoria University Doctoral Scholarship and a generous travel grant from Mercury Energy, which sponsored conference attendance and overseas research opportunities that greatly improved this manuscript.

I had the opportunity to work at the US Geological Survey on the Saudi Arabia hazard project with Walter Mooney for several months, which was a great experience and research opportunity. I am grateful for his support, which resulted in a cooperative agreement between the US Geological Survey and Victoria University and funded six extra months for my thesis.

Within Victoria University, I'd also like to thank Adrian and Aleks for their coding suggestions and help diagnosing numerous computer problems, Aasha for her guidance at every stage of the ReMi project and on the paper, and Huw for help with the Paraview software during a crucial time. Conversations with Colin Wilson regarding the near-surface geology of the Taupo Volcanic Zone greatly improved the ReMi chapter. Probably every post-graduate student helped me at one point or other, and I'm especially thankful for the help from Steve and Chet for the geothermal fields and Katie for many discussions about ambient noise variations. And of course, a big hug to my office mates Laura-May, Rachel, and Jesse for their support and fun times throughout the last several years.

I'm very thankful for the invitation and supervision by Florent Brenguier and Gregor Hillers during my time at ISTerre in Grenoble. A big thanks to Johanes, Clara, and Vincenzo for making my time at Grenoble very enjoyable. Additional thanks to Michael Ritzwoller and Misha Barmin for their invitation to work at Boulder for a week and for their help on surface-wave tomography. Another thanks to Alex for his help during the Boulder trip and for showing me around Stanford. I am thankful for the many wonderful friends I have made during my time in Wellington, especially Nicola, Brit, and Sophie, who kept me sane during very trying times.

Lastly, I want to thank my sister and my parents for their constant support and always inspiring me to push ahead. "Presto e bene!" 


\section{List of Figures}

2.1 Noise distribution schematic . . . . . . . . . . . . . . . . . . 11

$2.21 \mathrm{D}$ and 2D Green's function example . . . . . . . . . . . . . . . . . 12

2.3 Coherence zone schematic . . . . . . . . . . . . . . . . . . . . . . 14

2.4 Coherence zone wavelength-distance geometry schematic . . . . . . . 14

2.5 Noise PDF example . . . . . . . . . . . . . . . . . . . . 17

2.6 Cross-correlation workflow . . . . . . . . . . . . . . . 18

2.7 Whitening order of operations . . . . . . . . . . . . . . . 20

2.8 Whitening order of operations filtered . . . . . . . . . . 20

3.1 Saudi Arabia seismic network map . . . . . . . . . . . . . . . . . . 24

3.2 Saudi Arabia geologic map . . . . . . . . . . . . . . 25

3.31985 refraction line . . . . . . . . . . . . . . . . 26

3.4 Saudi Arabia crustal thickness . . . . . . . . . . . . . . . . . . . . 27

3.5 Saudi Arabia harrats . . . . . . . . . . . . . . . . . . . . . . 28

3.6 Harrat Al-Madinah geologic boundaries . . . . . . . . . . . . . . 31

3.7 Medina eruption locations . . . . . . . . . . . . . . . 32

3.8 Harrat Al-Madinah surface geology . . . . . . . . . . . . . . 33

3.9 Harrat Al-Madinah eruption ages . . . . . . . . . . . . . . . . 34

3.10 Resistivity at $15 \mathrm{~km} \ldots \ldots \ldots \ldots \ldots$

3.11 Harrat Al-Madinah station map . . . . . . . . . . . . . . . 36

$3.12 \mathrm{~T} 120$ instrument response curves . . . . . . . . . . . . . . . 37

3.13 T120 instrument response removed example . . . . . . . . . . . . . 37

3.14 Zero point noise removal example . . . . . . . . . . . . . . . . 39

3.15 AFTAN procedure diagram . . . . . . . . . . . . . . . . . 40

3.16 AFTAN phase velocity selection example . . . . . . . . . . . . . . 42

3.17 AFTAN result example . . . . . . . . . . . . . . . . . . . . . . . . . . . . . . . . . . . . . . .

3.18 Al-Madinah sensitivity kernels . . . . . . . . . . . . . . . 46

3.19 Cone resolution example . . . . . . . . . . . . . . . . . . . . 49

3.20 Harrat Al-Madinah cross-correlations . . . . . . . . . . . . . . . . . 51

3.21 Al-Madinah checkerboard RR . . . . . . . . . . . . . . . . . 53

3.22 Al-Madinah checkerboard TT . . . . . . . . . . . . . . . . . . 54 
3.23 Al-Madinah checkerboard ZZ . . . . . . . . . . . . . . . . . . . 55

3.24 Map for additional stations used for the checkerboard test . . . . . . 57

3.25 Al-Madinah additional stations checkerboard test regional view . . . . 58

3.26 Al-Madinah additional stations checkerboard test local view . . . . . . 59

3.27 Al-Madinah RR phase velocity dispersion . . . . . . . . . . . . . . . 62

3.28 Al-Madinah TT phase velocity dispersion . . . . . . . . . . . . 63

3.29 Al-Madinah ZZ phase velocity dispersion . . . . . . . . . . . . 64

3.30 Al-Madinah RR group velocity dispersion . . . . . . . . . . . . 65

3.31 Al-Madinah TT group velocity dispersion . . . . . . . . . . . 66

3.32 Al-Madinah ZZ group velocity dispersion . . . . . . . . . . . 67

3.33 Harrat Al-Madinah dispersion with gravity measurements . . . . . . 69

4.1 Lat-lon point dispersion standard deviation . . . . . . . . . . 75

$4.21 \mathrm{D}$ shear velocity result example $\ldots \ldots \ldots \ldots \ldots \ldots$

4.3 Dispersion curve example $\ldots \ldots \ldots \ldots \ldots \ldots \ldots$

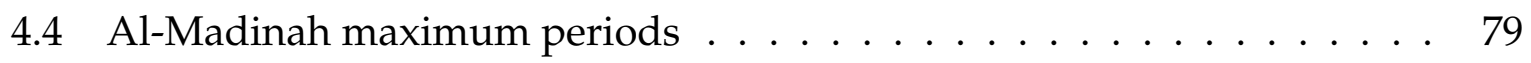

4.5 Grid-search results . . . . . . . . . . . . . . . . . . . 80

4.6 Shear-velocity inversion example for lat-lon point $24.4^{\circ}, 39.4^{\circ} \ldots \ldots 8$

4.7 Al-Madinah $10 \mathrm{~km} \mathrm{ZZ} \mathrm{shear-velocity} \mathrm{image} \mathrm{\ldots .} \mathrm{.} \mathrm{.} \mathrm{.} \mathrm{.} \mathrm{.} \mathrm{.} \mathrm{.} \mathrm{.} 84$

4.8 Al-Madinah average dispersion curves comparison . . . . . . . . . 85

4.9 Average dispersion for Harrat Al-Madinah . . . . . . . . . . . 86

4.10 Average shear-velocity models for Harrat Al-Madinah $\ldots \ldots \ldots$

4.11 Average shear-velocity models for the 1256 CE eruption area . . . . . . 89

4.12 Average shear-velocity models for the off-Harrat area . . . . . . . . 90

4.13 Harrat Al-Madinah average 1D shear-velocity models . . . . . . . . . 91

4.14 Al-Madinah shear-velocity from RR phase velocity . . . . . . . . . . . 92

4.15 Al-Madinah shear-velocity from TT phase velocity . . . . . . . . . . 93

4.16 Al-Madinah shear-velocity from ZZ phase velocity . . . . . . . . . . . 94

4.17 Al-Madinah shear-velocity from joint inversion phase velocity . . . . . 95

4.18 ParaView interpolated 3D model ZZ . . . . . . . . . . . . 97

4.19 ParaView $10 \mathrm{~km}$ horizontal cross-section ZZ . . . . . . . . . . . . 98

4.20 Al-Madinah cross-section locations . . . . . . . . . . . . . . . . . . 99

4.21 Al-Madinah cross-sections using RR phase velocities . . . . . . . . . 101

4.22 Al-Madinah cross-sections using TT phase velocities . . . . . . . . . 102

4.23 Al-Madinah cross-sections using ZZ phase velocities . . . . . . . 103

4.24 Al-Madinah cross-sections using the joint inversion . . . . . . . . 104

4.25 Data points for the ZZ phase velocity cross-section . . . . . . . . . . 105

4.26 Arabian Shield layers from Stern and Johnson [2010] . . . . . . . . 107

4.27 Saudi Arabia crustal model from refraction line . . . . . . . . . . . 108 
4.28 RR component calculated gravity . . . . . . . . . . . . . . . . . . . 109

4.29 Low-velocity mantle model . . . . . . . . . . . . . . . . . . . . 110

5.1 Rotokawa and Ngatamariki station map . . . . . . . . . . . . . . 115

5.2 NS07 winter-summer comparison . . . . . . . . . . . . . . . . 117

5.3 MWCS method schematic . . . . . . . . . . . . . . . . . . . . . . . 119

5.4 Ngatamariki station noise PDF example . . . . . . . . . . . . . . 120

5.5 NS08-NS09 daily ZZ cross-correlations . . . . . . . . . . . . . . . . 122

5.6 NS08-NS09 daily ZZ cross-correlations image . . . . . . . . . . . . . 122

5.7 NS08-NS09 one day magnitude squared coherence . . . . . . . . . . . . 124

5.8 NS08-NS09 combined magnitude squared coherence . . . . . . . . . . 124

5.9 MWCS workflow . . . . . . . . . . . . . . . . . . . . . . 125

5.10 RT01-RT02 MWCS DTT example . . . . . . . . . . . . . . . . 127

5.11 DVV example . . . . . . . . . . . . . . . . . . . . . 128

5.12 Rotokawa faults . . . . . . . . . . . . . . . . . . . . . . 130

5.13 Rotokawa geologic cross-section . . . . . . . . . . . . . . . . 131

5.14 Rotokawa 2009-2010 map . . . . . . . . . . . . . . . . . . . . . 134

5.15 Rotokawa 2009-2010 map production subset . . . . . . . . . . . . . 134

5.16 Rotokawa data availability $2009-2010$. . . . . . . . . . . . . . 136

5.17 Rotokawa data correction example . . . . . . . . . . . . . . . . 137

5.18 Injection flow at Rotokawa . . . . . . . . . . . . . . . . . . . . . 138

5.19 Production flow at Rotokawa . . . . . . . . . . . . . . . . . . . . 138

5.20 Rotokawa injection-production ratio . . . . . . . . . . . . . . . . . 139

5.21 RK radial correlations . . . . . . . . . . . . . . . . . . . . . . . . . 141

5.22 RK transverse correlations . . . . . . . . . . . . . . . . . . . . . 142

5.23 Rotokawa vertical correlations . . . . . . . . . . . . . . . . . 143

5.24 RT05-RT08 reference stack stability . . . . . . . . . . . . . . . . . 145

5.25 RT05-RT09 radial scatterers . . . . . . . . . . . . . . . . . . . . . . . 146

5.26 RT05-RT09 transverse scatterers . . . . . . . . . . . . . . . . . . . 147

5.27 RT05-RT09 vertical scatterers . . . . . . . . . . . . . . . . . . . . 148

5.28 Rotokawa bad data example . . . . . . . . . . . . . . . . . . . . 149

5.29 Rotokawa orientation issue . . . . . . . . . . . . . . . . . . . . 151

5.30 Rotokawa $\mathrm{M} 0$ and $\mathrm{M}$ comparison . . . . . . . . . . . . . . . . . 153

5.31 Rotokawa all field all components $d v v$. . . . . . . . . . . . . . . . . 154

5.32 Rotokawa all field diagonal components $\mathrm{dvv} \ldots \ldots \ldots \ldots$

5.33 Rotokawa injection-production subset pairs . . . . . . . . . . . . 156

5.34 Rotokawa injection subset all components $\mathrm{dvv}$. . . . . . . . . . . 157

5.35 Rotokawa injection subset diagonal components $\mathrm{dvv}$. . . . . . . . 158

5.36 Rotokawa production subset all components $d v v$. . . . . . . . . . . . 159 
5.37 Rotokawa production subset diagonal components dvv . . . . . . . . . 160

5.38 Rotokawa velocity step increase . . . . . . . . . . . . . . . . . 162

5.39 Ngatamariki surface geology . . . . . . . . . . . . . . . . . 165

5.40 Ngatamariki geologic cross-section . . . . . . . . . . . . . . . . 166

5.41 Ngatamariki station map . . . . . . . . . . . . . . . . . . 168

5.42 Ngatamariki data availability . . . . . . . . . . . . . . . . . . 169

5.43 Ngatamariki injection flow . . . . . . . . . . . . . . . . . . 170

5.44 Ngatamariki production flow . . . . . . . . . . . . . . . 171

5.45 Ngatamariki injection-production ratio . . . . . . . . . . . . . 172

5.46 Ngatamariki radial moveouts . . . . . . . . . . . . . . . . . . . . . . 174

5.47 Ngatamariki transverse moveouts . . . . . . . . . . . . . . . . 175

5.48 Ngatamariki vertical moveouts . . . . . . . . . . . . . . . . 176

5.49 Ngatamariki NS09 dead channel . . . . . . . . . . . . . . . . . . 177

5.50 Ngatamariki all field all components dvv . . . . . . . . . . . . . . 179

5.51 Ngatamariki all field diagonal components dvv . . . . . . . . . . . 180

5.52 Ngatamariki dvv subsets . . . . . . . . . . . . . . . . . . . . 181

5.53 Ngatamariki north subset all components dvv . . . . . . . . . . . . 182

5.54 Ngatamariki north subset diagonal components dvv . . . . . . . . . . . 183

5.55 Ngatamariki south subset all components dvv . . . . . . . . . . . . . 184

5.56 Ngatamariki south subset diagonal components dvv . . . . . . . . . . 185

5.57 Rotokawa 30 days MWCS animation . . . . . . . . . . . . . . . . . 192

5.58 Rotokawa 30 days MWCS lat-lon points . . . . . . . . . . . . . . . 193

5.59 Ngatamariki 30 days MWCS animation . . . . . . . . . . . . . . . 195

5.60 Ngatamariki 30 days MWCS lat-lon points . . . . . . . . . . . . . . . 196

5.61 Rotokawa rainfall comparison . . . . . . . . . . . . . . . . . . 198

5.62 Ngatamariki rainfall comparison . . . . . . . . . . . . . . . . . . 199

6.1 Ngatamariki ReMi line locations . . . . . . . . . . . . . . . . . . . . 210

6.2 ReMi schematic . . . . . . . . . . . . . . . . . . . . . 211

6.3 ReMi source types example . . . . . . . . . . . . . . . . . . 213

6.4 ReMi signal types Fourier transforms . . . . . . . . . . . . . . . . . 214

6.5 Rotokawa temporary deployment map . . . . . . . . . . . . . . . 216

6.6 Temporary Ngatamariki deployment weighted noise means . . . . . . . 217

6.7 ReMi background FFT comparisons . . . . . . . . . . . . . . . 218

6.8 Example slowness-frequency plots . . . . . . . . . . . . . . . 221

6.9 Accuracy of slowness measurements schematic . . . . . . . . . . . 223

6.10 ReMi rate of convergence . . . . . . . . . . . . . . . . . 223

6.11 ReMi line A 2D profile . . . . . . . . . . . . . . . . . . . 231

6.12 ReMi line A 1D profile . . . . . . . . . . . . . . . . . 232 
6.13 ReMi line B 2D profile . . . . . . . . . . . . . . . . . . 235

6.14 ReMi line B 1D profile . . . . . . . . . . . . . . . . . . 236

6.15 Line B three-layer and five-layer model model comparison . . . . . . . 237

A.1 PQLX PDF RHT01--RHT03 . . . . . . . . . . . . . . . . . . . . . 248

A.2 PQLX PDF RHT04-RHT07 . . . . . . . . . . . . . . . . . . . . . . . . 249

A.3 PQLX PDF RHT08--RHT11 and RHT13 . . . . . . . . . . . . . . . 250

A.4 PQLX PDF RHT14--RHT15 . . . . . . . . . . . . . . . . . . . . . . . 251

A.5 Grid-search dispersion January--April $2014 \ldots \ldots$. . . . . . . . . . 252

A.6 Grid-search dispersion February--May 2014 . . . . . . . . . . . . . . 252

A.7 Grid-search dispersion March--June 2014 . . . . . . . . . . . . . . . 252

A.8 Grid-search dispersion April--July 2014 . . . . . . . . . . . . . . 252

A.9 Grid-search dispersion May--August 2014 . . . . . . . . . . . . . . . 253

A.10 Grid-search dispersion June--September 2014 . . . . . . . . . . . . . 253

A.11 Grid-search dispersion July--October 2014 . . . . . . . . . . . . . . . 253

A.12 Grid-search dispersion August--November 2014 . . . . . . . . . . . 253

A.13 Grid-search dispersion September--December 2014 . . . . . . . . . . . . 254

B.1 Rotokawa Ref2 all field all components dvv . . . . . . . . . . . . 258

B.2 Rotokawa Ref2 all field diagonal components $d v v$. . . . . . . . . . 259

B.3 Rotokawa Ref2 injection subset all components dvv . . . . . . . . . . 260

B.4 Rotokawa Ref2 injection subset diagonal components dvv . . . . . . . 261

B.5 Rotokawa Ref2 production subset all components dvv . . . . . . . . . 262

B.6 Rotokawa Ref2 production subset diagonal components dvv . . . . . . 263

B.7 PQLX PDF RT01--RT03 summer . . . . . . . . . . . . . . . . . . . . . . 264

B.8 PQLX PDF RT05--RT08 summer . . . . . . . . . . . . . . . . . . . . . 265

B.9 PQLX PDF RT09--RT11 summer . . . . . . . . . . . . . . . . . . . . 266

B.10 PQLX PDF RT01--RT03 winter . . . . . . . . . . . . . . . . . . . . 267

B.11 PQLX PDF RT05--RT08 winter . . . . . . . . . . . . . . . . . . . . . . 268

B.12 PQLX PDF RT09--RT11 winter . . . . . . . . . . . . . . . . . . . . . . 269

B.13 PQLX PDF NM01--NM04 summer . . . . . . . . . . . . . . . . . 270

B.14 PQLX PDF NM05, NM07--NM09 summer . . . . . . . . . . . . . . . . 271

B.15 PQLX PDF NM10--NM11 summer . . . . . . . . . . . . . . . . . 272

B.16 PQLX PDF NM01--NM04 winter . . . . . . . . . . . . . . . . . . 273

B.17 PQLX PDF NM05, NM07--NM09 winter . . . . . . . . . . . . . . . 274

B.18 PQLX PDF NM10--NM11 winter . . . . . . . . . . . . . . . . . . 275

C.1 Rotokawa temporary deployment map with subsections . . . . . . . 278

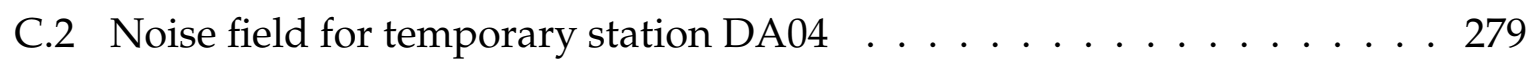

C.3 GS-11D instrument response curves . . . . . . . . . . . . . . 280 
C.4 Temporary deployment Area $1 \ldots \ldots$. . . . . . . . . . . . 282

C.5 Temporary deployment Area $2 \ldots \ldots \ldots \ldots . \ldots 283$

C.6 Temporary deployment Area $3 \ldots \ldots \ldots$

C.7 Decibel-amplitude conversion example . . . . . . . . . . . . 284

C.8 Area 1 EHE attenuation example . . . . . . . . . . . . . . . . 284

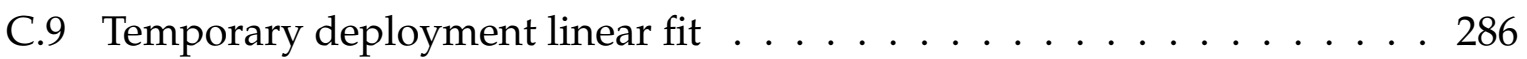

C.10 Attenuation earthquake example . . . . . . . . . . . . . . . 288

C.11 Microearthquake-noise comparison . . . . . . . . . . . . . . . . . 289

C.12 PQLX PDF DA01--DA03 . . . . . . . . . . . . . . . . . . . . . . . 291

C.13 PQLX PDF DA04--DA07 . . . . . . . . . . . . . . . . . . . . 292

C.14 PQLX PDF DB01--DB04 … . . . . . . . . . . . . . . . . . 293

C.15 PQLX PDF DB05--DB08 . . . . . . . . . . . . . . . . . . . . . . . 294

C.16 PQLX PDF DB09--DB11 . . . . . . . . . . . . . . . . . . . . . 295 


\section{List of Tables}

2.1 Station separation distance of datasets . . . . . . . . . . . 15

3.1 Harrat ages . . . . . . . . . . . . . . . . . . . . . . . . . . . 29

3.2 Saudi Arabia processing parameters . . . . . . . . . . . . 38

3.3 Parameters used for AFTAN analysis. . . . . . . . . . . . . . . . 43

4.1 Dinver parameters . . . . . . . . . . . . . . . . . 74

4.2 Values of Harrat Al-Madinah inversion . . . . . . . . . . . . . 83

4.3 Harrat Al-Madinah average shear velocities . . . . . . . . . . . . . . 87

4.4 Harrat Al-Madinah inversion depth velocity range . . . . . . . . . . . . 96

5.1 Rotokawa and Ngatamariki processing parameters . . . . . . . . . 120

5.2 MWCS velocity change parameters . . . . . . . . . . . . . . 128

5.3 Rotokawa original well depths . . . . . . . . . . . . . . . . . 132

5.4 Rotokawa well depths . . . . . . . . . . . . . . . . . . 133

5.5 Rotokawa velocity subset paths . . . . . . . . . . . . . . . . . 152

5.6 Rotokawa maximum positive velocity change . . . . . . . . . . . . . . 161

5.7 Rotokawa maximum negative velocity change . . . . . . . . . . . . 161

5.8 Ngatamariki 2012-2013 flow rates . . . . . . . . . . . . . . . . . . . . . . . . . . . . . . . . . . . . . . . . . . . .

5.9 Ngatamariki well depths . . . . . . . . . . . . . . . . . . . . 167

5.10 Ngatamariki velocity subset paths . . . . . . . . . . . . . . 178

5.11 Ngatamariki maximum positive velocity change . . . . . . . . . . . 186

5.12 Ngatamariki maximum negative velocity change . . . . . . . . . . 187

6.1 Angular coverage of slowness intervals . . . . . . . . . . . . . . . . 222

6.2 ReMi lines $p-f$ plot results . . . . . . . . . . . . . . . . . . . 225

6.3 ReMi lines manual inverse model interface depths . . . . . . . . . . . 226

6.4 ReMi lines manual inverse model layer velocities . . . . . . . . . . . . 227

6.5 ReMi lines automatic inverse models layer interface depths . . . . . . . 227

6.6 ReMi lines automatic inverse models layer velocities . . . . . . . . . . . 228

A.1 Harrat Rahat seismic stations . . . . . . . . . . . . . . . . . . 247 
B.1 Rotokawa reference stack 2 maximum positive velocity change . . . . . 257

B.2 Rotokawa reference stack 2 maximum negative velocity change . . . . 257

C.1 Temporary deployment station locations . . . . . . . . . . . . . . 281

C.2 Station source information . . . . . . . . . . . . . . . . . . 282

C.3 Temporary deployment attenuation results . . . . . . . . . . . . 285

C.4 Power station microearthquake attenuation . . . . . . . . . . . . . 287 


\section{Chapter 1}

\section{Introduction}

\subsection{Motivation}

Shear velocity models are useful to the scientific and engineering community to accurately locate earthquakes [Chiu et al., 1997], compute ground motion prediction equations [Castellaro et al., 2008, Bommer et al., 2010], and assess subsurface structure [Stein and Wysession, 2003]. Shear velocity changes have been used to observe inflation of volcano edifices prior to eruptions [Brenguier et al., 2008b], co-seismic deformation [Wegler and Sens-Schöndelfer, 2007], and even contraction of the surface of the moon from thermal heating [Sens-Schönfelder and Larose, 2008]. Typically, shear-velocity is determined through earthquake tomography or active source methods. Under ideal conditions, ambient noise seismology bypasses the greatest limitations of earthquake tomography (depth and spatial resolution dependent on earthquake location and occurrence (e.g. Ritzwoller and Levshin [1998]) and active source (cost of signal generation (e.g. Henrys et al. [2013]) and determines surface wave and shear velocities in the crust from repeatedly occurring background processes [Shapiro and Campillo, 2004].

The theoretical underpinning of this method was the discovery that cross-correlation of diffusive waves recorded at two receivers are approximately equivalent to the Green's function of the station path [Lobkis and Weaver, 2001]. Ambient noise was quickly embraced by the seismological community due to its ability to determine shear-velocity structure for environments where seismic activity is infrequent (e.g. Saygin and Kennet [2010], Overduin et al. [2015]). Within a few years of the first ambient noise tomography studies, it was discovered that signals within the coda of the cross-correlation corresponded to scatterers within the inter-station path [Sabra et al., 2005], and that changes of the scatters in time was analogous to changes in shear-velocity [Brenguier et al., 2008b].

The depth resolution of ambient noise methods depends primarily on the wavelength of the surface wave relative to the inter-station distance. This is a scalable 
system: shallow depths can be observed using high-frequency or cultural noise (> $1 \mathrm{~Hz})$ and short station spacing, while lower-frequency or natural noise $(<1 \mathrm{~Hz})$ can be used in conjunction with large station spacing to determine much deeper structure [Bonnefoy-Claudet et al., 2006]. The mathematics of these processes have been used for determining medium properties using earthquakes [Snieder, 2004], ocean waves [Shapiro and Campillo, 2004], acoustic waves [Lobkis and Weaver, 2001], and many other sources. Generally, a good rule of thumb for ambient noise tomography is that station spacing should be between one to five times the wavelength of the ambient noise signal to account for full depth sampling and minimizing the effects of smearing [Bensen et al., 2007]. This minimum wavelength criteria is not necessary for shearvelocity time-variations, as stable scatterers (whose variations correlate to changes in shear-velocity) can be generated at station distances of less than one wavelength [Brenguier et al., 2008b].

This thesis is a combination of three projects split into four chapters that use ambient noise to determine shear velocities at different depth scales: [1] A pseudo-3D inversion of the Harrat Al-Madinah volcanic field in Saudi Arabia using natural ambient noise tomography (Chapters 3 and 4), [2] shear-velocity variations at Rotokawa and Ngatamariki, two electricity-producing geothermal fields in the central North Island, New Zealand, (Chapter 5) and [3] a combined ambient noise and active source experiment at Ngatamariki to determine shear-velocity in the top 200 meters and constrain fault locations (Chapter 6).

Each chapter is presented as a stand-alone project with its own introduction and conclusion in a format suitable for publication. Due to the scope of the project, the Harrat Al-Madinah tomography project is split into two chapters. The combined ambient noise and source experiment was published in 2016 in an ambient noise special issue of SEG Interpretation [Civilini et al., 2016] ${ }^{1}$. Although the pronoun "we" is used throughout this manuscript to acknowledge the collaboration and feedback of my supervisors and secondary authors, all developed codes and presented results are original unless otherwise referenced. For the Civilini et al. [2016] journal article, secondary authors assisted with edits to the manuscript, data processing, and array deployment, but not with the writing of the publication.

\subsection{Thesis structure}

We begin with a general overview of the mathematics of ambient noise methods that provide a basis for most of the chapters. Additional theoretical information for the

\footnotetext{
${ }^{1}$ Civilini, F., Pancha, A., Savage, M.K., Sewell, S., and Townend, J., (2016). Shallow structure from cultural ambient noise at the Ngatamariki geothermal field using Refraction Microtremor (ReMi). SEG Interpretation, (4) 3, SJ87-SJ101, doi: 10.1190/INT-2015-0204.1
} 
projects is introduced at the beginning of each chapter as needed. The main body of this manuscript is composed of four chapters corresponding to each of the projects. We conclude with a chapter summarizing the key results of the manuscript and present suggestions for future research. Several appendices are included, which provide supplementary results and information that could not be included in the main chapters.

\section{Chapter 2 - Theoretical framework}

This chapter provides background on the theory of ambient seismic noise, specifically how surface waves can be extracted from a repeating background source. We discuss the stationary phase region of seismic stations, a geometrical area where ambient noise sources constructively interfere, and the effect of various source distributions on surface wave velocities. The background of the PQLX software [McNamara and Buland, 2004], which determines a seismic station's ambient noise field, is introduced. Lastly, we discuss the general workflow used to determine empirical Green's functions and the importance of a specific order of operations during time and frequency domain normalization.

\section{Chapter 3 - Noise analysis and dispersion of the Harrat Al-Madinah volcanic field, Saudi Arabia}

Chapter 3 describes the use of natural ambient noise signals to determine surface-wave maps beneath Harrat Al-Madinah, a volcanic structure underneath the city of Medina, Saudi Arabia. Harrat Al-Madinah is the northernmost extent of Harrat Rahat, the largest Cenozoic volcanic field in Saudi Arabia [Camp and Roobol, 1989]. This project uses the largest network aperture of the manuscript, with a maximum interstation distance of approximately $200 \mathrm{~km}$. After an introduction on the regional tectonics and the Harrat Rahat Seismic Network, we describe the methodology and parameters used to transform the cross-correlations described in theory in Chapter 2 to measurements of surface-wave dispersion. We asses the station coverage at Harrat Al-Madinah through checkerboard test and produce group and phase surface-wave velocity maps for radialradial, transverse-transverse, and vertical-vertical station-pair directional components.

\section{Chapter 4 - Shear-wave tomography of Harrat Al-Madinah}

Chapter 4 continues the work from Chapter 3 and determines shear-wave velocity values from the surface-wave dispersion maps. Each latitude-longitude point in the dispersion map grid is inverted to 1D shear-velocity with depth through use of the neighborhood algorithm [Sambridge, 1999, Wathelet, 2005, 2008], and the 1D profiles are interpolated into a pseudo-3D model [Ahrens et al., 2005]. We detect shear-velocity 
increases at approximately $20 \mathrm{~km}$ depth suggesting a layer interface. Cross-sections of the pseudo-3D model are then visually compared to Bouguer gravity measurements [Langenheim et al., 2016]. We find low shear-velocity values in the cross-sections that are co-located with measurements of low gravity and a previously inferred zone of magma intrusion [Abdelwahed et al., 2016].

\section{Chapter 5 - Velocity variations at New Zealand geothermal fields}

Chapter 5 uses variations within the coda of the empirical Green's functions to compute shear-velocity changes at Rotokawa and Ngatamariki, two geothermal fields located in the central North Island, New Zealand. Unlike tomographic studies (such as the one carried out in Chapter 3), which interpret the peak of a cross-correlation between station-pairs as a surface wave arrival, ambient noise time-variations use signals embedded in the coda of the cross-correlation, which are governed by changes in scattering properties of the wavefield [Brenguier et al., 2008b]. We analyze two timeperiods within the geothermal fields when velocity changes are expected: 2009-2010 Rotokawa, when injection and production was increased by a factor of four due to the start-up of a new power station after a decade of consistent electricity production, and 2012-2013 Ngatamariki, the beginning of injection and production at the geothermal field. After describing the methodology used to transform cross-correlations to measures of shear-velocity changes, we analyze the velocity changes using several instrument subsets. We find that changes in shear-velocity across the field appear to be driven at least in part by differences in production and injection rates and water table changes due to rainfall. Lastly, we create a pseudo-tomographic code to interpolate the velocity changes and determine spatial relationships across the field in time. Through this method, we detect an additional shear-velocity increase localized to the injection wells. We hypothesize that the responses are driven by mechanisms of rainfall, compaction and settling of the injection layer, or expansion and contraction of the formations above the injection layer.

\section{Chapter 6 - Refraction Microtremor at Ngatamariki}

The last project chapter is a combined ambient noise and active source Refraction Microtremor (ReMi) experiment. Two refraction lines of lengths of approximately 700 meters were deployed at the Ngatamariki geothermal field to determine subsurface shear velocities at depths shallower than 200 meters. We begin with an introduction of the ReMi method and compare it to other commonly used geotechnical engineering methods such as Spectral Analysis of Surface Waves and Multichannel Analysis of Surface Waves. 1D and 2D dispersion curves at each line are inverted to shear-velocity using the same neighborhood algorithm code utilized in Chapter 4 [Wathelet, 2005], 
compared to nearby well log geology, and used to constrain a fault location. Most of the chapter is taken directly from Civilini et al. [2016], but the theory [Section 6.3.3] and noise field [Section 6.3.2] sections have been expanded with additional information.

\section{Chapter 7 - Summary of main results and opportunities for further research}

We end this manuscript with a brief summary of the key findings for each project, implications for the field of ambient noise seismology, and future work that could be conducted to address the questions raised by this research.

\section{Appendix A - Harrat Al-Madinah supplementary information}

Appendix A includes the location of the Harrat Rahat seismic stations, the ambient noise field recorded throughout the year of data, and dispersion curves of the four month stacks used to compute the standard deviation of the inversion.

\section{Appendix B - Geothermal velocity changes supplementary informa- tion}

Appendix B contains the excluded pairs of each geothermal field for each component as well as velocity change results for Rotokawa using an additional reference stack. These results are described briefly in the discussion section of Chapter 5. Additionally, we display the ambient noise field at each station for both winter and summer seasons.

\section{Appendix C - ReMi cultural noise supplementary information}

Appendix C contains additional information and results from the ReMi experiment chapter. An array was installed at Rotokawa during the Ngatamariki ReMi deployment to characterize the typical noise present at the geothermal field. The objective was to determine the time and frequency domain characteristics and determine rates of attenuation. The time and frequency domain observations were added to Chapter 6, but the preliminary attenuation study was outside of the scope of that project. In this Appendix, we briefly discuss the amplitude decay recorded for each cultural noise source type and the potential applications of these investigations to seismic deployments. 


\section{Chapter 2}

\section{Theoretical framework}

In this chapter, we discuss the general theory of ambient noise methods, both within the context of the literature and the framework of my projects. The introduction section for each of the subsequent chapters will provide additional theoretical information as needed.

\subsection{Noise as a source}

Ambient noise methods use cross correlations of naturally occurring microseisms at two stations to construct an empirical Green's function of the station path [Shapiro et al., 2005]. A Green's function describes the response at a point from an applied force function [Shearer, 1999]. Within the context of this manuscript, an empirical Green's function can be idealized as the ground response resulting from a passing seismic wave. Lobkis and Weaver [2001] were the first to calculate a Green's function using correlations of dispersive waves from an acoustic diffusive source in a laboratory setting. A diffusive wave field in a solid is defined as an isotropic distribution of waves with uniformly distributed random phases [Egle, 1981], while dispersion refers to the property that wave speed depends on frequency.

It had long been known that coda waves of earthquakes are produced by the superposition of many backscattering waves during the interaction of primary waves with crustal heterogeneities [Aki, 1969]. Although this scattering was hypothesized to have a diffusive nature [Aki and Chouet, 1975], it was not until the studies of Shapiro et al. [2000] that this was observed in the seismic coda. This led to the extraction of Green's functions from coda waves [Campillo and Paul, 2003]. Shapiro and Campillo [2004] recognized that the random distribution of ambient noise taken over long time scales could be a considered a diffuse wavefield, and found good correlation between ambient noise calculated Rayleigh wave dispersion curves and established models.

Ambient noise refers to sources that continuously produce microseismicity. The 
noise field is primarily composed of surface waves, and is divided into two main categories: sources with frequencies greater than $1 \mathrm{~Hz}$ are referred to as cultural or human noise and sources with frequencies less than $1 \mathrm{~Hz}$ are natural noise [Bonnefoy-Claudet et al., 2006]. One of the first studies to analyze the frequency content and source of seismic noise fields was conducted by Lacoss et al. [1969] using power spectral density and beamforming, a technique that isolates the incident direction of a recorded signal. The study found both Rayleigh and Love waves present in natural ambient noise in two distinct microseism bands: below $0.15 \mathrm{~Hz}$ and between $0.2-0.3 \mathrm{~Hz}$. It is now known that below $0.3 \mathrm{~Hz}$, the microseism band is generally dominated by two signals of 7.5 and 15 second Rayleigh waves corresponding to the interaction of ocean waves [Friedrich et al., 1998, Shapiro et al., 2005]. The two period bands are usually referred to as the primary (12-18 seconds) and secondary (6-9 seconds) microseism bands [Friedrich et al., 1998]. The primary microseism is induced by the interaction of ocean waves with the shoreline, while the secondary microseism is generated by waves traveling in opposite directions due to reflection at the shoreline [Lacoss et al., 1969]. At frequencies less than $0.003 \mathrm{~Hz}$, the microseismicity is dominated by atmospheric disturbances (i.e. wind) [Tanimoto, 1999].

After the initial discovery of Shapiro and Campillo [2004], numerous papers were published about this phenomenon and its applications. Common applications for ambient noise include tomography [Shapiro et al., 2005, Brenguier et al., 2007, Harmon et al., 2007, Behr et al., 2010], volcano and geothermal monitoring [Jousset et al., 2010, Ohmi et al., 2008, Brenguier et al., 2007, Hillers et al., 2015], and observation of postseismic deformation [Brenguier et al., 2008a]. Others expanded the theory of noise correlations in an effort to understand how the results could be improved [Snieder, 2004, Asten, 2006, Wapenaar and Fokkema, 2006, Bensen et al., 2007].

\subsubsection{Green's functions and the stationary phase region}

We can determine the physical interpretation relating the cross correlation of a diffuse field to the Green's function by starting off with the 1D scalar wave equation [Stein and Wysession, 2003]:

$$
\frac{\partial^{2} u(x, t)}{\partial x^{2}}=\frac{1}{v^{2}} \frac{\partial^{2} u(x, t)}{\partial t^{2}}
$$

where $u$ is displacement and $v$ is velocity. A solution of the equation using $\cos (\omega t)$ is:

$$
u(x, t)=U(x, \omega) \cos (\omega t)
$$

where $\omega$ is angular velocity, $u$ is displacement, and $v$ is velocity. This replaces the scalar wave equation [Equation 2.1] as: 


$$
\frac{\partial^{2} U(x, \omega)}{\partial x^{2}}=\frac{-\omega^{2} U(x, \omega)}{v^{2}}
$$

The solution for $U(x, \omega)$ can be determined as:

$$
U(x, \omega)=\sin (\omega x / v)
$$

Here, $\omega$ can be replaced by $\omega_{n}$, corresponding to the eigenfrequencies of the system, the frequencies which constructively interfere and produce standing waves. The $n$ index represents the mode of the traveling wave, where the wave freely oscillates. With this we can also rewrite Equation 2.2:

$$
u(x, t)=U_{n}\left(x, \omega_{n}\right) \cos \left(\omega_{n} t\right)
$$

where $U_{n}\left(x, \omega_{n}\right)$ is the eigenfunction of the system, a function used to describe the displacement of standing wave at a certain eigenfrequency:

$$
U_{n}\left(x, \omega_{n}\right)=\sin \left(\omega_{n} x / v\right)
$$

A traveling wave can be represented as sum of eigenfunctions and eigenfrequencies weighed by complex factors $A_{n}$ [Stein and Wysession, 2003]:

$$
u(x, t)=\sum_{n=0}^{\infty} A_{n} U_{n}\left(x, \omega_{n}\right) \cos \left(\omega_{n} t\right)
$$

Equation 2.7 is very similar to the amplitude of a diffuse field $(\phi(x, t))$ in the modal form [Lobkis and Weaver, 2001, Shapiro and Campillo, 2004], where the total amplitude is the summation of the amplitudes produced by each eigenfrequency:

$$
\phi(x, t)=\mathbb{R} \sum_{n=1}^{\infty} a_{n} u_{n}(x) e^{i \omega_{n} t}
$$

where $a_{n}$ is the complex modal amplitude, $u_{n}$ and $\omega_{n}$ are eigenfunction and eigenfrequencies, and $\mathbb{R}$ denotes the real component. As the eigenfunctions are real and orthonormal, the impulse between modes $\left(\delta_{n m}\right)$ can be described as:

$$
\delta_{n m}=\int \rho u_{n} u_{m} d^{3} \mathbf{x}
$$

where $\rho$ is the modal density and $\mathbf{x}$ represents the dimensions of the region of interest. The modal amplitudes of the diffuse field are uncorrelated random variables, which are equivalent to the impulse between modes multiplied with $F\left(\omega_{n}\right)$, a smooth function related to the spectral energy density:

$$
\left\langle a_{n} a_{m}^{*}\right\rangle=\delta_{n m} F\left(\omega_{n}\right)
$$


The angular brackets \langle\rangle refer to the expectation value of the spectra, used when the incident waves are random variables, and equal to the time average of any sample function from the random process to which the random variable belongs [Cooper and McGillen, 1971, Snieder and Larose, 2013].

The cross correlation of a diffuse field at two points $x$ and $y$ can then be described as:

$$
\langle\phi(x, t) \phi(y, t+\tau)\rangle=\frac{1}{2} \mathbb{R} \sum_{n=1}^{\infty} F\left(\omega_{n}\right) u_{n}(x) u_{n}(y) e^{i \omega_{n} \tau} d \tau
$$

We will now relate Equation 2.11 to the Green's function by taking the integral $\int_{0}^{\tau}() d \tau:$

$$
\langle\phi(x, t) \phi(y, t+\tau)\rangle=\frac{1}{2} \mathbb{R} \sum_{n=1}^{\infty} F\left(\omega_{n}\right) u_{n}(x) u_{n}(y) \int_{0}^{\tau} e^{i \omega_{n} \tau} d \tau
$$

Using Euler's formula, we can evaluate Equation 2.12 as:

$$
\langle\phi(x, t) \phi(y, t+\tau)\rangle=\frac{1}{2} \mathbb{R} \sum_{n=1}^{\infty} F\left(\omega_{n}\right) u_{n}(x) u_{n}(y) \int_{0}^{\tau}\left(\cos \left(\omega_{n} \tau\right)+i \sin \left(\omega_{n} \tau\right)\right) d \tau
$$

As $F\left(\omega_{n}\right), u_{n}(x)$, and $u_{n}(y)$ are real, Equation 2.13 can be expressed as:

$$
\langle\phi(x, t) \phi(y, t+\tau)\rangle=\frac{1}{2} \sum_{n=1}^{\infty} F\left(\omega_{n}\right) u_{n}(x) u_{n}(y) \frac{\sin \left(\omega_{n} \tau\right)}{\omega_{n}}
$$

The above equation is only a factor $F\left(\omega_{n}\right) / 2$ different from the time derivative of the Green's function between points $x$ and $y\left(G_{x y}\right)$ and supports negative values of $\tau$ [Lobkis and Weaver, 2001]:

$$
G_{x y}=\sum_{n=1}^{\infty} u_{n}(x) u_{n}(y) \frac{\sin \left(\omega_{n} \tau\right)}{\omega_{n}}
$$

The retrieval of surface wave travel time measurements from ambient noise cross correlations uses the assumptions of a uniform noise distribution and equipartition of energy [Shapiro and Campillo, 2004]. Since these assumptions are not fully satisfied in practice, the validity of travel time measurements from ambient noise cross correlations can be ambiguous. Tsai [2009] used a ray-theoretical interpretation of the cross correlation of ambient noise to quantify the dependence of the velocity measurements on these approximations. He defined the cross correlation function $C_{x y}$ between two stations as a Gaussian pulse of amplitude $\rho$ and angular frequency $\omega$ integrated for a particular source distribution [Equation 2.16]:

$$
C_{x y}(\Delta t, \omega, W)=\int W\left(\Delta t_{d}\right) \rho(\Delta t, \omega) \cos \left[\omega\left(\Delta t-\Delta t_{d}\right)\right] d \Delta t_{d}
$$


where $\Delta t$ is the lag time, $\Delta t_{d}$ is the travel time difference between the pulses, and $W$ is a windowing function describing the pulse shape. Behr [2010] used this equation to illustrate how different source distributions would shape the resulting cross correlation function [Figure 2.1]. This figure shows that although a truly dispersed source results in a symmetrical cross correlation function, a single hemisphere of distributed noise should still be sufficient to obtain a valid measurement of surface wave travel time in the causal or acausal directions.
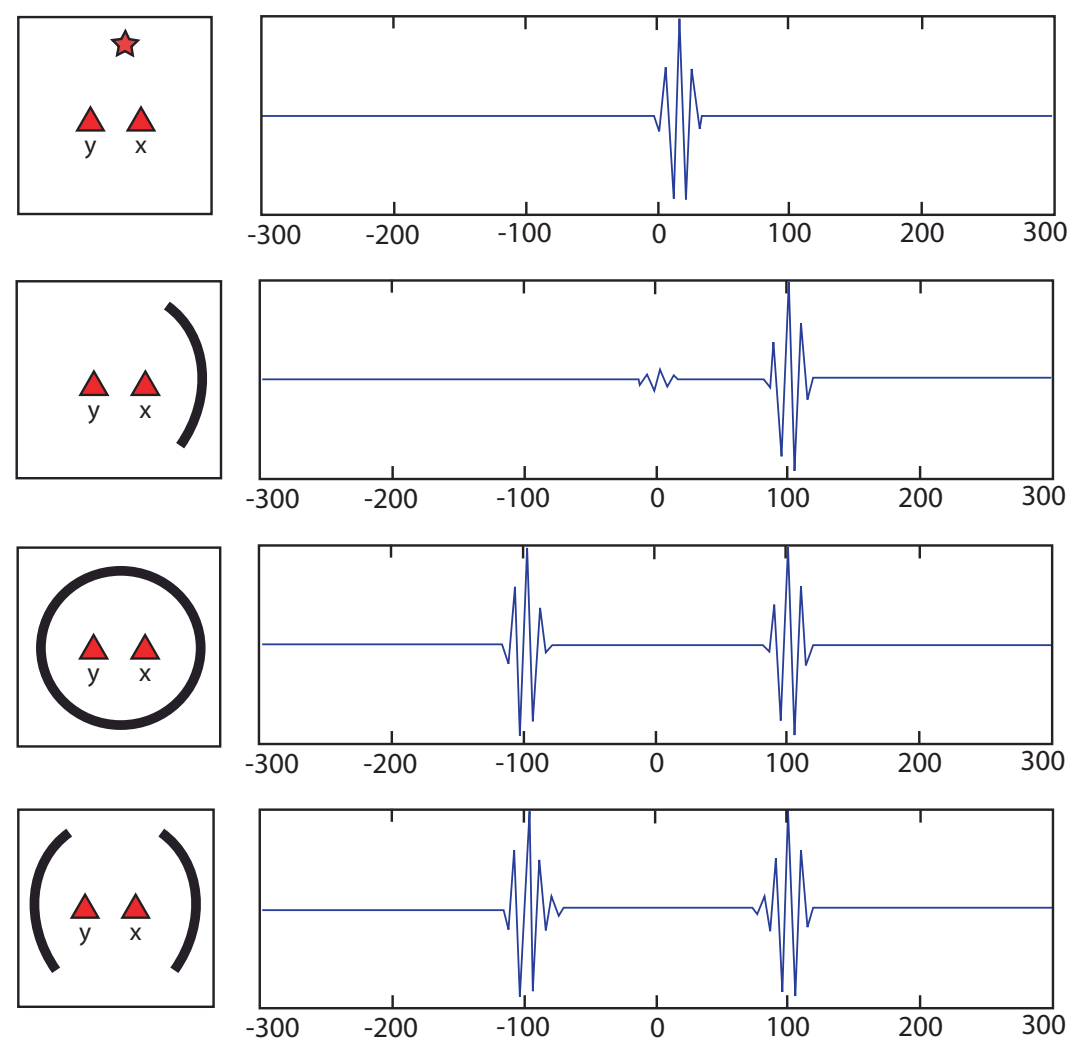

Figure 2.1: Distribution of noise sources and their resulting Gaussian-shaped pulse. Adapted from Behr [2010].

Although the cross correlation functions produced by ambient noise are analogous to the Green's function of the station path, they are actually the difference between the Green's function and its time reversed duplicate [Snieder et al., 2010]. We can relate the cross-correlation of ambient noise at two stations $A$ and $B$ to the Green's function in $1 \mathrm{D}$ and $2 \mathrm{D}$ in a homogeneous medium $\left(G_{1 D}(R, \omega)\right)$ using the work of Snieder and Larose [2013] [Figure 2.2].

In the 1D case, receiver $B$ is at position $x=0$ and receiver $A$ is at position $x=R$, where $R$ is the interstation distance [Figure 2.2a]. $S_{L}$ and $S_{R}$ are the spectrum of random waves incident from the left and right respectively. $k$ is the wavefield at frequency $\omega$. The total wavefield $(u(x))$ is:

$$
u(x)=S_{L} e^{i k x}+S_{R} e^{-i k x}
$$



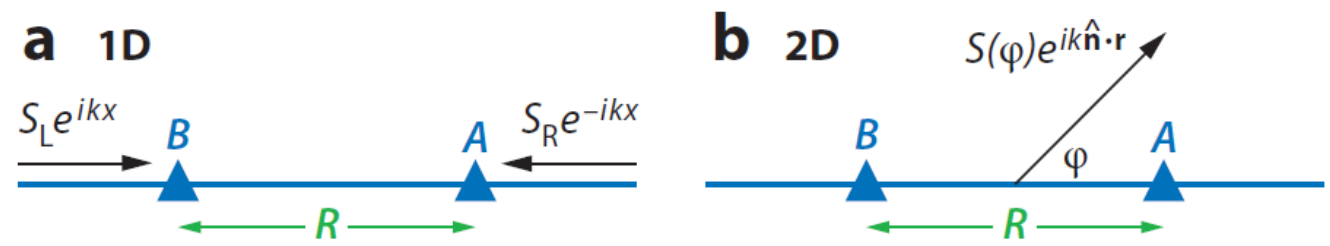

Figure 2.2: 1D [a] and 2D [b] schematic of the ambient noise wavefield at two receivers. Adapted from Snieder and Larose [2013].

The waves recorded at the two receivers are then:

$$
\begin{gathered}
u_{A}=S_{L} e^{i k x}+S_{R} e^{-i k x} \\
u_{A}=S_{L}+S_{R}
\end{gathered}
$$

Assuming that the noise wavefield incident from the left is uncorrelated with that incident from the right and that the power spectrum and has the same power spectrum, the following assumptions can be made [Snieder and Larose, 2013]:

$$
\begin{gathered}
\left\langle\left|S_{L}\right|^{2}\right\rangle=\left\langle\left|S_{R}\right|^{2}\right\rangle=\left\langle|S|^{2}\right\rangle \\
\left\langle S_{L} S_{R}^{*}\right\rangle=\left\langle S_{L}^{*} S_{R}\right\rangle=0
\end{gathered}
$$

The cross-correlation of the wavefields at $A$ and $B$ can then be resolved to:

$$
\left\langle u_{A} u_{B}^{*}\right\rangle=\left\langle|S|^{2}\right\rangle\left(e^{i k R}+e^{-i k R}\right)
$$

The Green's function in 1D homogeneous medium is defined as [Snieder and Larose, 2013]:

$$
G_{1 D}(r, \omega)=\frac{-i}{2 k} e^{i k R}
$$

We can then combine Equations 2.22 and 2.23 to obtain the cross-correlation of a diffuse field in terms of the 1D Green's function:

$$
\left\langle u_{A} u_{B}^{*}\right\rangle=2 i k\left\langle|S|^{2}\right\rangle\left[G_{1 D}(R, \omega)-G_{1 D}^{*}(R, \omega)\right]
$$

This derivation is based on the stationary phase assumption, where phases vary slowly and thus interfere constructively [Snieder and Larose, 2013]. The stationary phase assumption is more complicated in the $2 \mathrm{D}$ case, as the relative position of the two stations compared to the direction of the incoming wave can determine whether or not a signal will interfere constructively or destructively. Assume that the stations are located at $r_{B}=(0,0)$ and $r_{A}=(R, 0)$ [Figure 2.2b], and that the incoming plane waves have a random spectrum $S(\varphi)$ propagating in the direction $\hat{n}=(\cos \varphi, \sin \varphi)$, 
with $\varphi$ as the angle between the direction of propagation and the station path. The total wavefield recorded by the receivers is then [Snieder and Larose, 2013]:

$$
u_{A}=\int_{0}^{2 \pi} S(\varphi) e^{i k R \cos \varphi} d \varphi, \quad u_{B}=\int_{0}^{2 \pi} S\left(\varphi^{\prime}\right) d \varphi^{\prime}
$$

When the plane waves coming in from different directions are uncorrelated and each each has a power spectrum independent of the angle of incidence:

$$
\left\langle S(\varphi) S^{*}\left(\varphi^{\prime}\right)\right\rangle=|S|^{2} \delta\left(\varphi-\varphi^{\prime}\right)
$$

The average cross-correlation is given by:

$$
\left\langle u_{A} u_{B}^{*}\right\rangle=|S|^{2} \varphi \int_{0}^{2 \pi} e^{i k R \cos (\varphi)} d \varphi
$$

The cross-correlation in the frequency domain of these two wavefields can be correlated with the two dimensional Green's function $\left(G_{2 D}(R, \omega)\right)$ [Snieder and Larose, 2013]:

$$
\left\langle u_{A} u_{B}^{*}\right\rangle=4 i \pi|S|^{2}\left[G_{2 D}(R, \omega)-G_{2 D}^{*}(R, \omega)\right]
$$

The full derivation of Equation 2.28 from Equation 2.27 can be found in Appendix A of Snieder and Larose [2013].

In order for the ambient noise to constructively interfere between the station path, the source must lie in the stationary phase region of the two stations. Figure 2.3 displays the stationary phase region (grey shaded area) for two stations in two dimensions. If ambient source $S_{2}$, within the stationary phase region, varies slightly in position, the travel time difference $T_{B A}$ of the surface wave between the two stations remains unchanged. However, if source $S_{1}$ outside of the stationary phase region varies in position, the time between the stations will change accordingly, and the ambient signal will not stack coherently [Snieder and Larose, 2013].

This hyperbolic stationary phase region is known as the coherence zone of the two stations, and a source is defined to be in the coherence zone if it follows [Larose, 2006]:

$$
|S A-S B|<D-\frac{\lambda}{6}
$$

where $S A$ and $S B$ are station distances from the source to the stations, $D$ is the interstation distance, and $\lambda$ is the wavelength of the ambient noise signal. The coherence zone is also sometimes referred to in literature as the "end-fire lobe" [Sabra et al., 2005]. The coherence zone can be defined through the asymptote of the hyperbola $\theta_{0}$. This angle is based on on $\lambda$ and $D$, and changes the shape of the coherence zone depending on the values of $D$ and $\lambda$ [Equation 2.30, Figure 2.4]. 


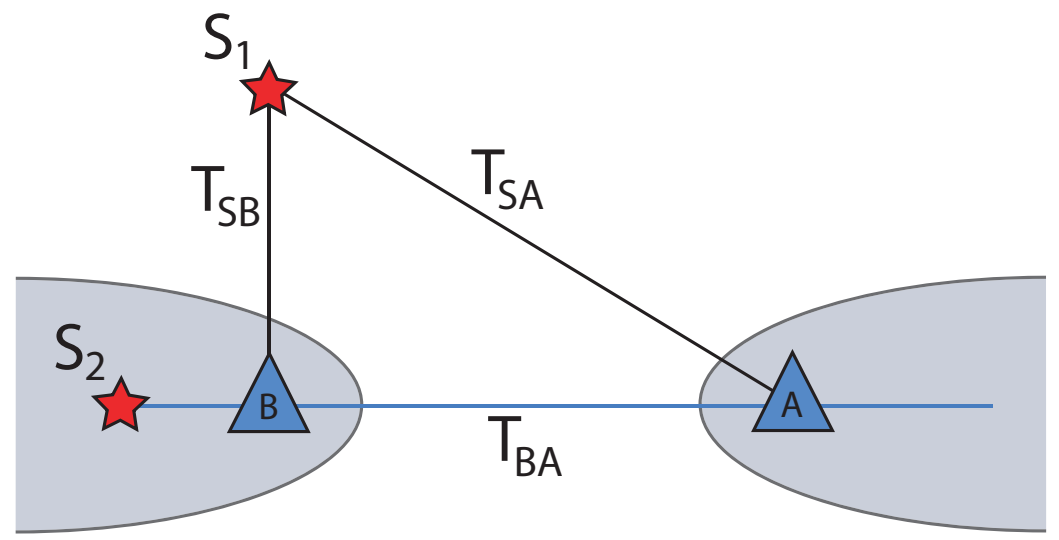

Figure 2.3: Schematic displaying sources inside and out the stationary phase region of a station pair, adapted from Snieder and Larose [2013]. Source $S_{2}$ varying in position within the stationary phase region will stack coherently, as $T_{B A}$ will remain mostly constant. Even small variations of source $S_{1}$ outside the stationary phase region will result in large changes to $T_{B A}$, and the source will not stack.

$$
\theta_{0}=\sqrt{\frac{\lambda}{2.2 D}}
$$

The stack of the cross-correlations of a split data record is analogous to the crosscorrelation of the entire data record [Goudard et al., 2008]. However, the smaller the coherence zone, the slower the convergence to the Green's function [Larose, 2006]. Crosscorrelation symmetry or lack thereof can be used in combination with station-pair azimuth to obtain an approximation of noise-field directionality [Figure 2.1]. However, methods like beamforming provide much more accurate results that are independent of the azimuth of the station pair path.
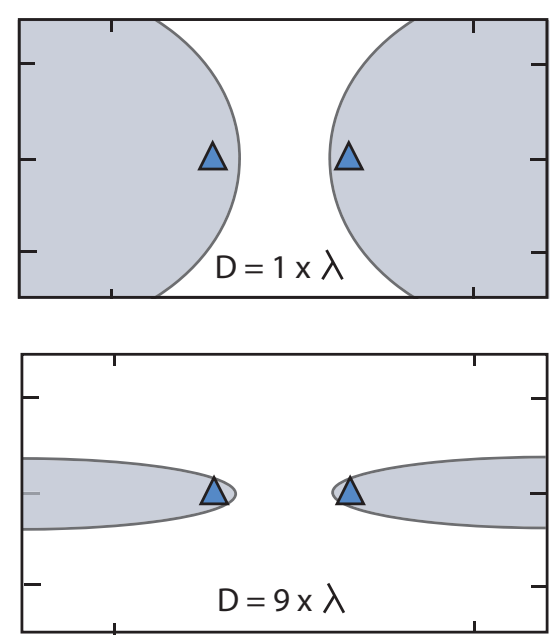

Figure 2.4: Geometry of coherence zones based on change of the ratio $\lambda / D$. Adapted from Larose [2006]. 


\subsubsection{Station spacing and noise scaling}

In general terms, ambient noise methods can scale effectively from small to large wavelengths if the station spacing matches the increase in scale. For tomography, which uses the ballistic arrivals of the surface waves, the station spacing must be far away enough to allow the full wavelength of the measured signal to be coherent from one station to the next. Within the context of ambient noise, ballistic waves refer to the cross-correlation peaks corresponding to phase and group velocity arrivals. Although some argue that the separation distance needs to be at least three times the wavelength of the signal [Tsai and Moschetti, 2010, Bensen et al., 2007], other research states that two wavelengths [Shapiro et al., 2005] or even one wavelength [Brenguier et al., 2007] is sufficient. Shear-velocity changes can be free from this wavelength restriction, as they can be computed from scatterers within the cross-correlation coda [Hillers et al., 2015] or ballistic arrivals [Brenguier et al., 2008b]. The approximate station separation for each of the datasets used in this manuscript is listed in Table 2.1.

\begin{tabular}{||l||l||}
\hline Dataset & Approximate Station Separation \\
\hline Harrat Rahat (Ch. 3 and 4) & $2-180 \mathrm{~km}$ \\
\hline Geothermal Fields (Ch. 5) & $1-15 \mathrm{~km}$ \\
\hline ReMi Lines (Ch. 6) & $10-720$ meters \\
\hline
\end{tabular}

Table 2.1: Approximate station separation distance for each of the datasets used in this manuscript.

\subsection{Computing the noise spectra}

As previously mentioned in Section 2.1, the noise field is generally divided into two bands: cultural ( $<1$ second) and natural ( $>1$ second) ambient noise [Bonnefoy-Claudet et al., 2006]. The strongest noise sources of the natural ambient noise band are the primary and secondary ocean waves, peaking at approximately 7 and 14 seconds (1.4 and $0.7 \mathrm{~Hz}$ ), and are the sources most commonly used for tomography [Friedrich et al., 1998, Shapiro et al., 2005]. The primary microseisms are generated by the interaction of waves on the coastline, while the secondary microseisms are generated by pressure on the seafloor from the interaction of ocean waves traveling in opposite directions [Lacoss et al., 1969]. The frequency distribution and amplitude of cultural noise can vary depending on the environment, but is most commonly produced by road traffic or machinery. This will be discussed more thoroughly in Chapter 6, where we analyze the effectiveness of cultural noise as a tomographic source.

The noise fields for each dataset were computed using the software PQLX, a suite 
of programs developed by the USGS to manage seismic data quality [McNamara and Boaz, 2011]. PQLX calculates probability density functions of power spectral densities (PSD) to map out the noise spectrum [McNamara and Buland, 2004]. To reduce variance in the PSD, each day of data is split into one hour segments with 50\% overlap, and each one hour segment is divided again into 13 segments with 75\% overlap [McNamara and Buland, 2004]. Each trace is then truncated to the nearest power of two, detrended, and cosine-tapered by $10 \%$. The traces are then converted to measures of PSD using the Tukey-Cooley method, which uses a finite range FFT to compute PSD values [Cooley and Tukey, 1965]. The PQLX developers chose this method to compute PSD due to its computational efficiency [McNamara and Buland, 2004], and is described in Equations 2.31-2.34. For a periodic time series $y(t)$, the finite-range Fourier transform is defined as:

$$
Y_{f}=\sum_{t=0}^{T_{r}} y_{t} e^{-i 2 \pi f t / T_{r}}
$$

where $T_{r}$ is the number of samples of the data segment and $f$ is frequency. Each point in frequency domain is represented by frequency $f_{k}$ :

$$
\begin{gathered}
f_{k}=\frac{k}{N \Delta t} \\
\{k=1,2, \ldots, N-1\}
\end{gathered}
$$

where $\Delta t$ is the sample interval, $N$ is the number of samples, and $k$ is the index of the Fourier coefficients. The total PSD estimate for each Fourier coefficient $\left(P_{k}\right)$ is then:

$$
P_{k}=\frac{2 \Delta t}{N}\left|Y_{k}\right|^{2}
$$

The segments are then multiplied by approximately 1.143 to account for the $10 \%$ cosine taper and the instrument response is removed [McNamara and Buland, 2004]. The amplitude is then converted to decibel with respect to acceleration [(meters $/$ second $\left.^{2}\right)^{2} / \mathrm{Hz}$ ] to facilitate comparison to the New High and New Low Noise Models of Peterson [1993], which represents world-wide expected upper and lower bounds of noise. The average power is then computed for each PSD for each octave with a 1/8 octave interval [McNamara and Buland, 2004], where an octave is a frequency band where the highest frequency is twice the lowest frequency. The results are then distributed into a histogram with 1-dB bins, and the probability density function (PDF) for the noise can be determined using:

$$
P\left(T_{c}\right)=\frac{N_{P T_{c}}}{N_{T_{c}}}
$$


where $P\left(T_{c}\right)$ is the PDF at central frequency $T_{c}, N_{P T_{c}}$ is the number of spectral estimates that fall into $P$, a 1-dB power bin, and $N_{T_{c}}$ is the total number of spectral estimates over all powers with a center period $T_{c}$.

The graphical output of the PQLX program is limited, so a Matlab code was written to adequately display the probability density functions. An example of noise plot is displayed in Figure 2.5. This example figure shows natural ambient noise for the east component of station AFFS of the Saudi Arabia network. The colorbar represents the probability distribution in percentages, and the New High and New Low Noise Models [Peterson, 1993] are marked as dashed lines. The solid black line is the mean weighed as a function of probability.

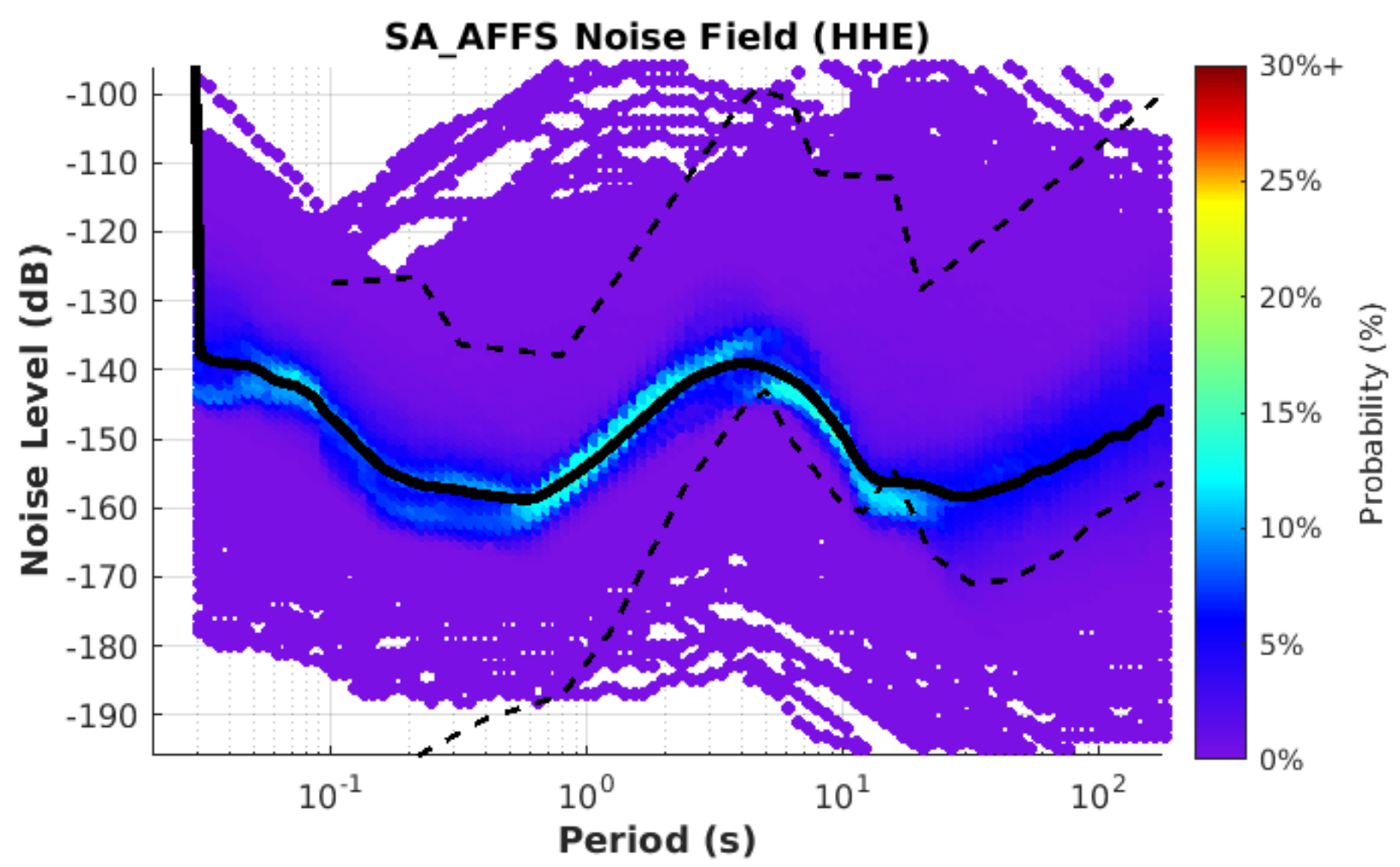

Figure 2.5: Probability density functions of the noise field at Saudi Arabia station AFFS, east component. Each function is computed using the program PQLX and the probability distribution is provided by the colorbar. The New High and New Low Noise Models [Peterson, 1993] are displayed as dashed lines.

Noise PDF plots for the stations of each network used in this manuscript are displayed in the Appendix. Various observations can be made from the noise distribution. For example, the Saudi Arabia stations [Appendix A.2], which are broadband instruments, have a much wider period band (approximately 0.05-200 s) than the short period Rotokawa and Ngamatariki stations (approximately 0.05-10 s) [Appendix B]. Additionally, the stations at the geothermal field consistently have a higher cultural noise peak than the other networks due to their close proximity to the power plant and 
pipes, which produce strong high $(>1 \mathrm{~Hz})$ frequency noise.

\subsection{Computing the Green's function}

In this section, we describe the methods used to compute cross-correlations for Saudi Arabia, Rotokawa, and Ngatamariki datasets. The data from the Refraction Microtremor experiment, due to its small inter-station spacing and observation of high frequency noise, had to be processed differently and will be described in detail in Chapter 6. Note that for the purposes of the projects described in this manuscript, the terms Green's function, Green's function difference [Snieder et al., 2010], and cross-correlation of ambient noise will be used interchangeably.

The robustness of ambient noise velocity calculations depends strongly on the accuracy of the extracted Green's functions. The signal-to-noise ratio of cross-correlated station pairs is a function of the pair azimuth with respect to the noise sources, the instrument response, and the station separation distance. Despite the uniqueness of each cross-correlation pair, several general processing steps can be used for most station configurations.

We followed methods similar to those outlined in [Bensen et al., 2007] to compute cross-correlations. A generalized version of the workflow is displayed in Figure 2.6. Instrument response removal (step 1) is a time-intensive procedure that is necessary before computing correlations between station pairs with different instrument types. A multi-core Python code was developed to remove instrument response for the Saudi Arabia dataset, the only project in this manuscript with varying instrument types [Section 3.4]. The software package MSNoise [Lecocq et al., 2014] was used to process and stack cross-correlation pairs (steps 3 through 7 in Figure 2.6). Specific parameters of the MSNoise steps will be discussed in each of the chapters as needed.

\section{Cross Correlation Workflow}

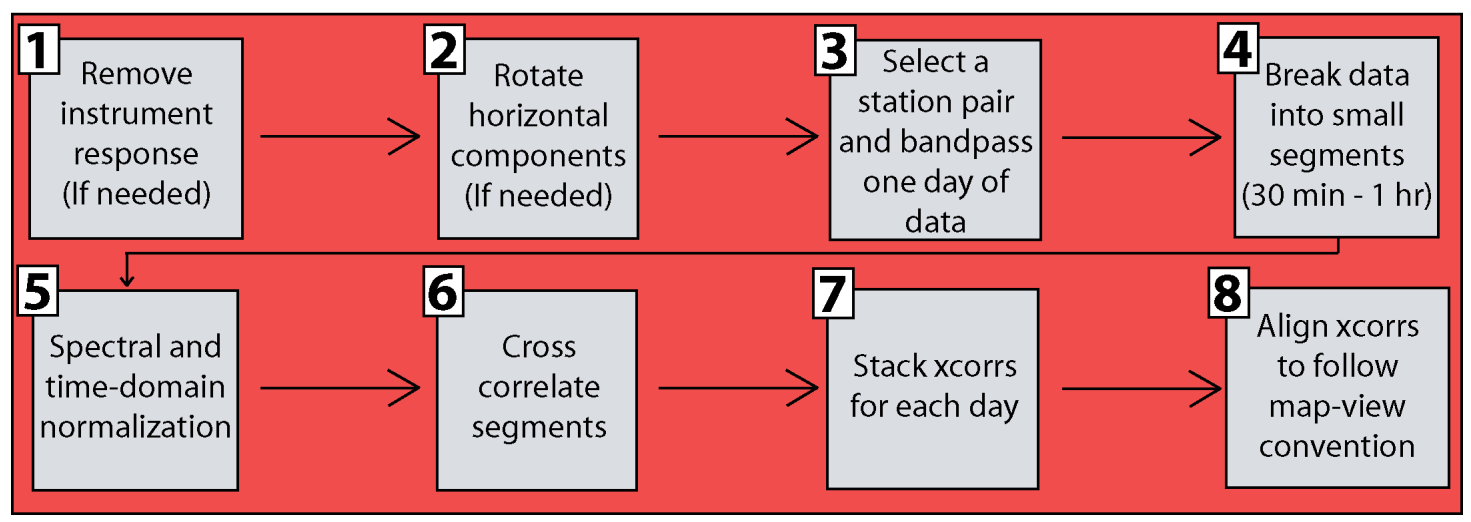

Figure 2.6: Displays the code workflow for calculating cross-correlations.

The last processing step is the alignment of the correlations to a map-view conven- 
tion (step 8). Generally, cross-correlations computed by MSNoise or other codes will be conducted in alphabetical order of the station pairs. As the causal and acausal sides of the cross-correlations represent the direction of noise in distinct geometrical areas on a map, they need to be made consistent for all pairs. We used a West to East as positive map-view convention for all our networks. In other words, the causal portion of the cross-correlation shows energy traveling West to East, while the acausal side shows energy traveling East to West. This is especially important for pairs where the noise field is asymmetric [Figure 2.1].

Two important details need to be considered during this procedure: [1] the effect of gaps on the cross-correlation and [2] order of normalization operations. As the cross-correlation of MSNoise occurs in the frequency domain [Lecocq et al., 2014], the Fourier transform of any gaps of data introduces higher harmonics in the Fourier domain due to the Gibbs Phenomenom. Given the small size of interval chosen for the cross-correlation and numerous stacking, this problem is largely minimized. However, when segments of data between cross-correlation pairs have a data gap at the same time, they result in a delta function at zero in the cross-correlation which overwhelms the rest of the signal. The cross-correlation code of MSNoise was rewritten to include a percentage threshold of zeros acceptable for each segment of data.

The most crucial step in the processing routine is the time and frequency domain normalization (step 5). These steps are necessary to remove the effect of earthquakes that might grossly dominate the cross-correlation stack. Due to the small size of the ambient noise signal, the amplitude of the data segments that is being cross correlated is negligible. Bensen et al. [2007] demonstrated that one bit normalization, where positive and negative data points are turned into values of positive and negative one, led to a cleaner resulting cross-correlation than clipping by a multiple of the Root Mean Square error (RMS).

We found that the default MSNoise routine, which carries out 1-Bit normalization prior to spectral whitening, to be extremely detrimental for sites with large amounts of cultural noise. Figure 2.7 displays the cross-correlations and Fourier transform between Rotokawa stations RT09 and RT12 for one month of data with two order of operations: 1-Bit normalization before spectral whitening [top] and whitening before 1-Bit normalization [bottom]. In both cases, the data was bandpass filtered between 0.01 and $8 \mathrm{~Hz}$ and whitened between 0.1 and $7 \mathrm{~Hz}$. The spectral amplitude below $2 \mathrm{~Hz}$, which contains the microseism signals used for ambient noise, is not observed when computing 1-bit normalization prior to spectral whitening [Figure 2.7 top]. This can be highlighted by filtering each signal between 0.1 to $1.0 \mathrm{~Hz}$ [Figure 2.8]. 

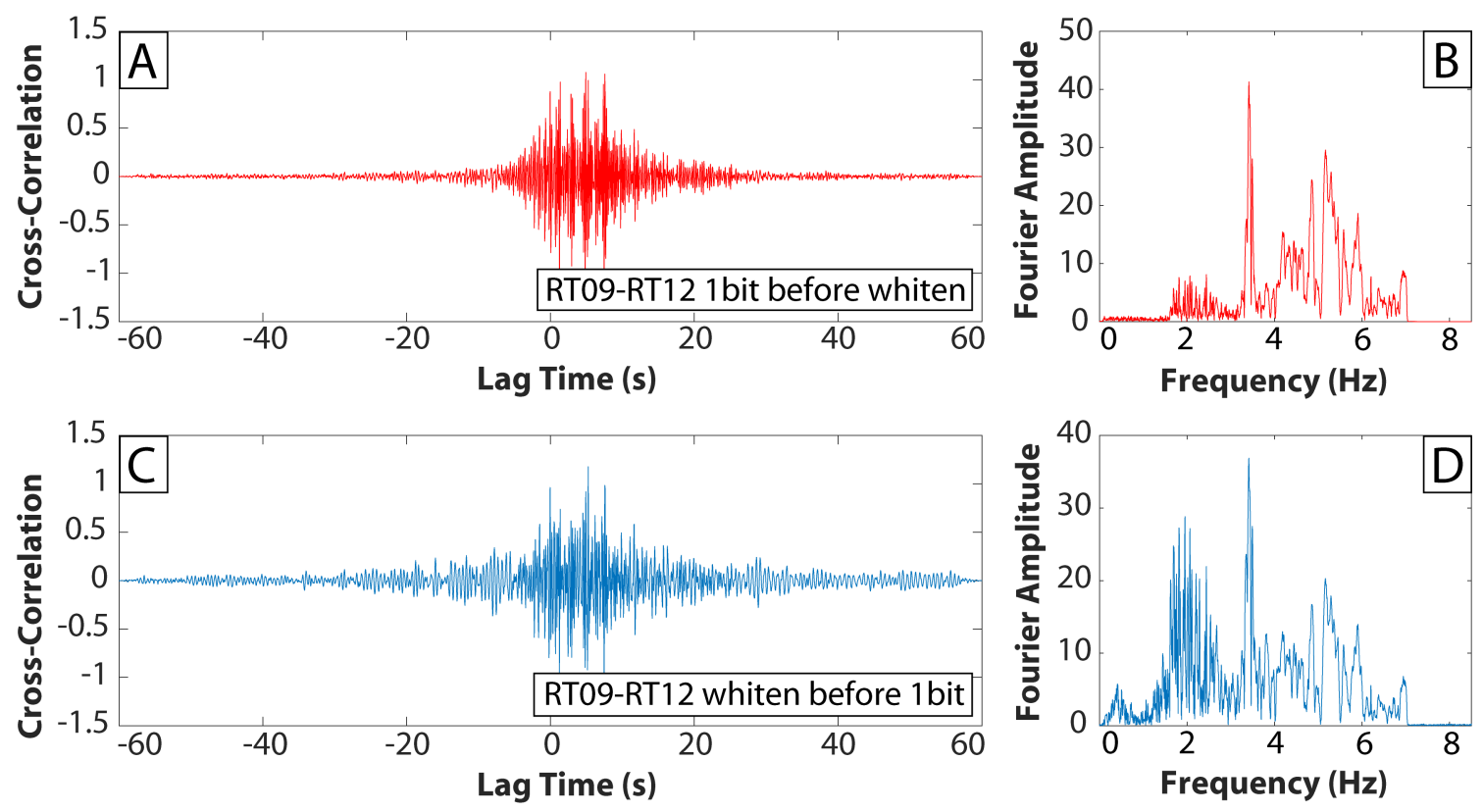

Figure 2.7: cross-correlations between two Rotokawa stations for one month of data. Data was bandpass filtered between 0.01 to $8 \mathrm{~Hz}$ and whitened between 0.1 to $7 \mathrm{~Hz}$. [Top] Time series and Fourier transform using 1-Bit normalization prior to whitening. [Bottom] Time series and Fourier transform using whitening prior to 1-Bit normalization.
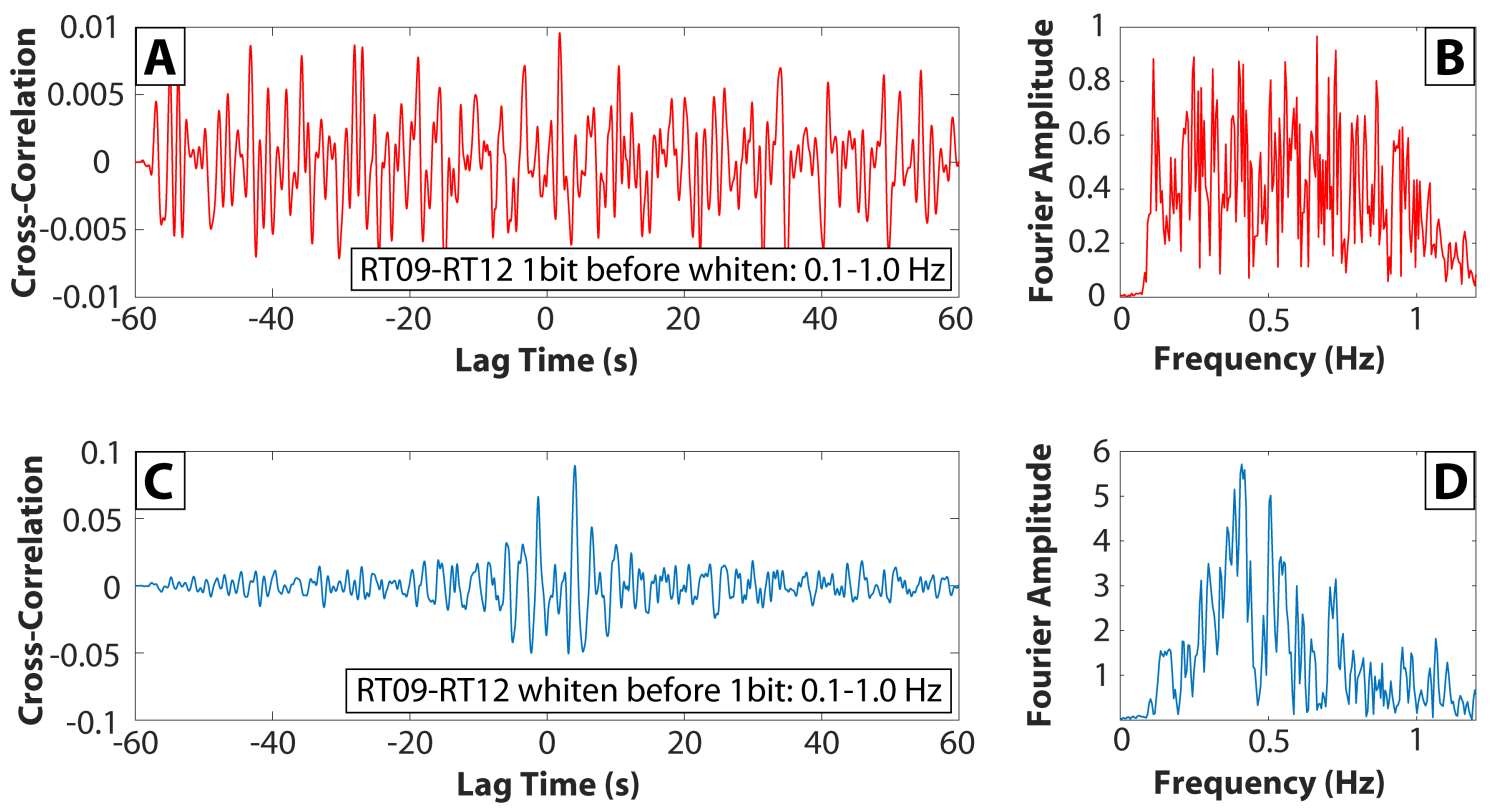

Figure 2.8: Correlations from Figure 2.7 after being filtered between 0.1 to $1.0 \mathrm{~Hz}$. 
As the amplitude of ambient noise signals is very small, the cross-correlation depends almost entirely on matching phases of the signal. By computing 1-bit normalization prior to whitening, a high frequency signal (such as that caused by cultural noise), can entirely dominate the spectrum over smaller signals of lower frequency.

The example provided in Figures 2.7 and 2.8 is meant to illustrate this principle, and is therefore displayed in a band that is much wider than would be common for ambient noise signals. However, the same principle applies even if the filter bands are much lower in frequency. This is especially important for computing velocity changes and dispersion curves, as both the Moving Window Cross Spectral (MWCS) [Section 5.2] and Automatic Frequency-Time Analysis (AFTAN) [Section 3.5.2] require the application of numerous thin filters to the cross-correlations. 


\section{Chapter 3}

\section{Noise analysis and dispersion of the Harrat Al-Madinah volcanic field}

\subsection{Introduction}

From April 18th to May 19th of 2009, an earthquake swarm consisting of over 4000 earthquakes $\mathrm{Mw}>4$ and seven events $\mathrm{M}>4$ occurred in Harrat Lunyarr, a volcanic zone located approximately $200 \mathrm{~km}$ northwest of the city of Medina in Saudi Arabia [Figure 3.1]. The swarm, which was the largest ever recorded in the region, had a peak magnitude of Mw 5.7, and was driven by the intrusion of an approximately $12 \mathrm{~km}$ long and $2.5 \mathrm{~m}$ thick dike [Baer and Hamiel, 2010, Pallister et al., 2010]. Due to this event, the Saudi Geological Survey began installing broadband surface stations with the objective to better characterize the hazard of the country. By the end of 2014, which is when the data for this project were collected, the Saudi Arabia Seismic Network consisted of 136 broadband stations.

This project is part of an overall effort by the United States Geological Survey and the Saudi Geological Survey to assess hazards within Saudi Arabia. One of the objectives of the hazard assessment is to develop velocity models for the country that can be used for earthquake relocation and determination of subsurface structure. Recently, teleseismic events recorded on the Saudi Seismic Network were used to develop upper mantle velocity structure [Yao et al., 2017]. The periodicity of ocean waves, the sources typically utilized in ambient noise tomography, are approximately seven and fourteen seconds [Bonnefoy-Claudet et al., 2006] and therefore can sample the crust.

In this chapter and the next, we assess the ability of ambient noise methods to resolve crustal structure by determining surface-wave dispersion maps [Chapter 3] and shear-velocities [Chapter 4] for a subset of stations around Harrat Al-Madinah, the volcanic zone encompassing the city of Medina. The methods used will soon be expanded to the complete set of 136 stations of the Saudi Arabia Seismic Network to 
produce shear-velocity models of the entire country.

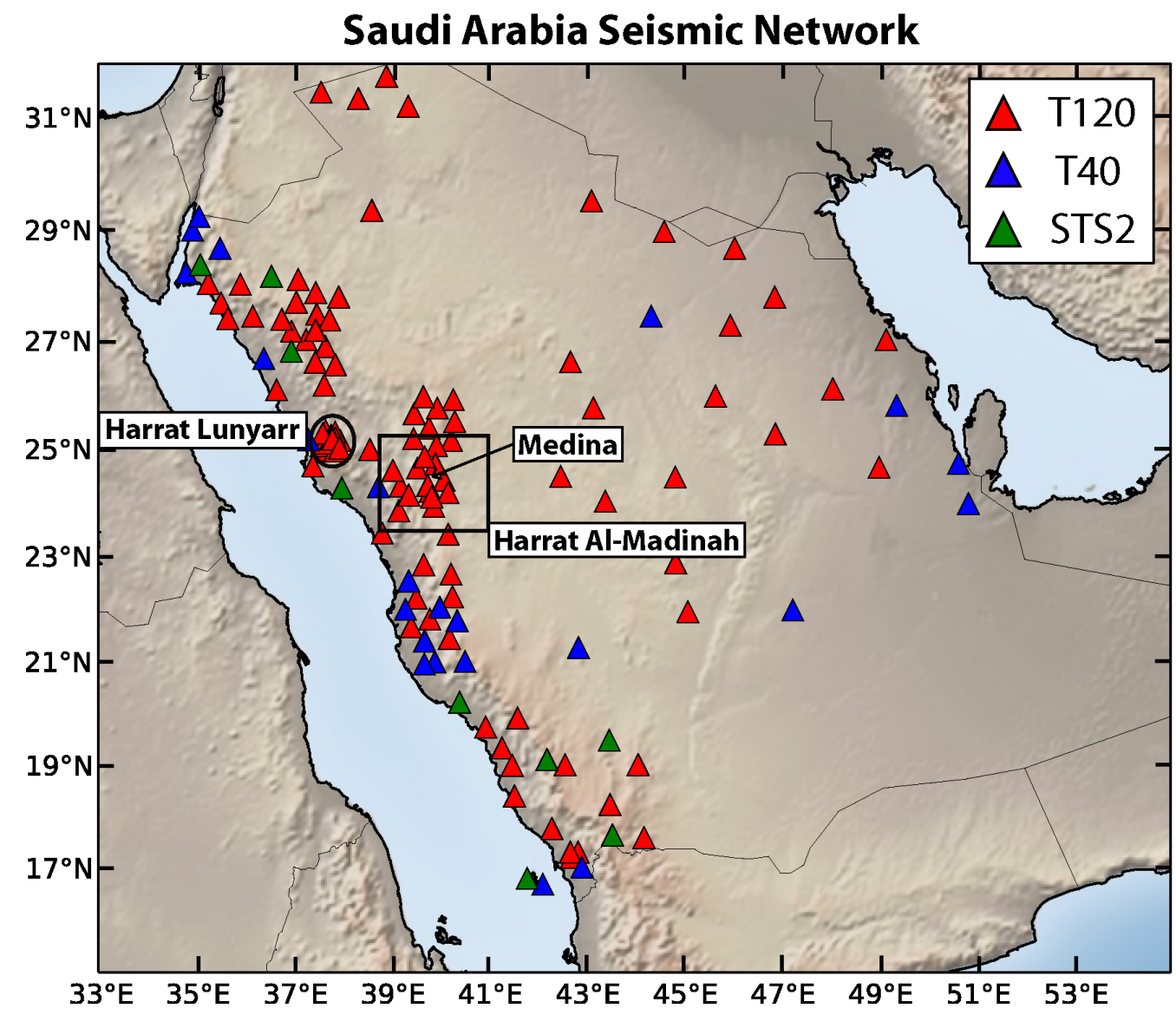

Figure 3.1: Map of the Saudi Arabia Seismic Network, which includes 106 Nanometrics Trillium T120, 9 Kinemetrics STS2, and 24 Nanometrics T40 tri-axial broadband seismometers. The round and rectangular shapes display the locations of the Lunayyir and Al-Madinah volcanic areas respectively.

\subsection{Regional geology and tectonics}

Harrat Al-Madinah is located on the Arabian Shield segment of the Arabian plate, a $\sim 25$ Ma old lithospheric plate created by rifting in the Red Sea and in the Gulf of Aden [Figure 3.2] [Stern and Johnson, 2010]. Since its separation from Africa, the Arabian Plate has rotated anticlockwise and drifted north at a rate of approximately 2-3 $\mathrm{cm} / \mathrm{yr}$ [Bird, 2002]. The Arabian Shield is a $\sim 725,000 \mathrm{~km}^{2}$ surface expression of Precambrian rocks that form the crystalline basement of the Arabian Plate [Johnson and Kattan, 2012]. The Arabian Shield is composed of six terrains, separated by four major ophiolite bearing suture zones [Tang et al., 2016]. Harrat Al-Madinah lies on the Hijaz terrane.

The mechanisms for exposing this large expanse of Precambrian rocks has been hypothesized to be the tilt of the Arabian plate towards the north and east due to 
uplift associated with the Red Sea and Gulf of Aden rifting and buoyancy differences within the Arabian lithosphere [Stern and Johnson, 2010, Johnson and Kattan, 2012]. Seismic activity is observed on all plate boundaries, but is especially frequent in the Zagros fold belt, the Dead Sea transform, and the Red Sea spreading axis [Johnson and Kattan, 2012] [Figure 3.2]. To the north and east of the Arabian Shield lies the Arabian Platform, which is primarily covered by Paleozoic, Mesozoic, and Cenozoic sedimentary rocks [Brown, 1972] [Figure 3.5].

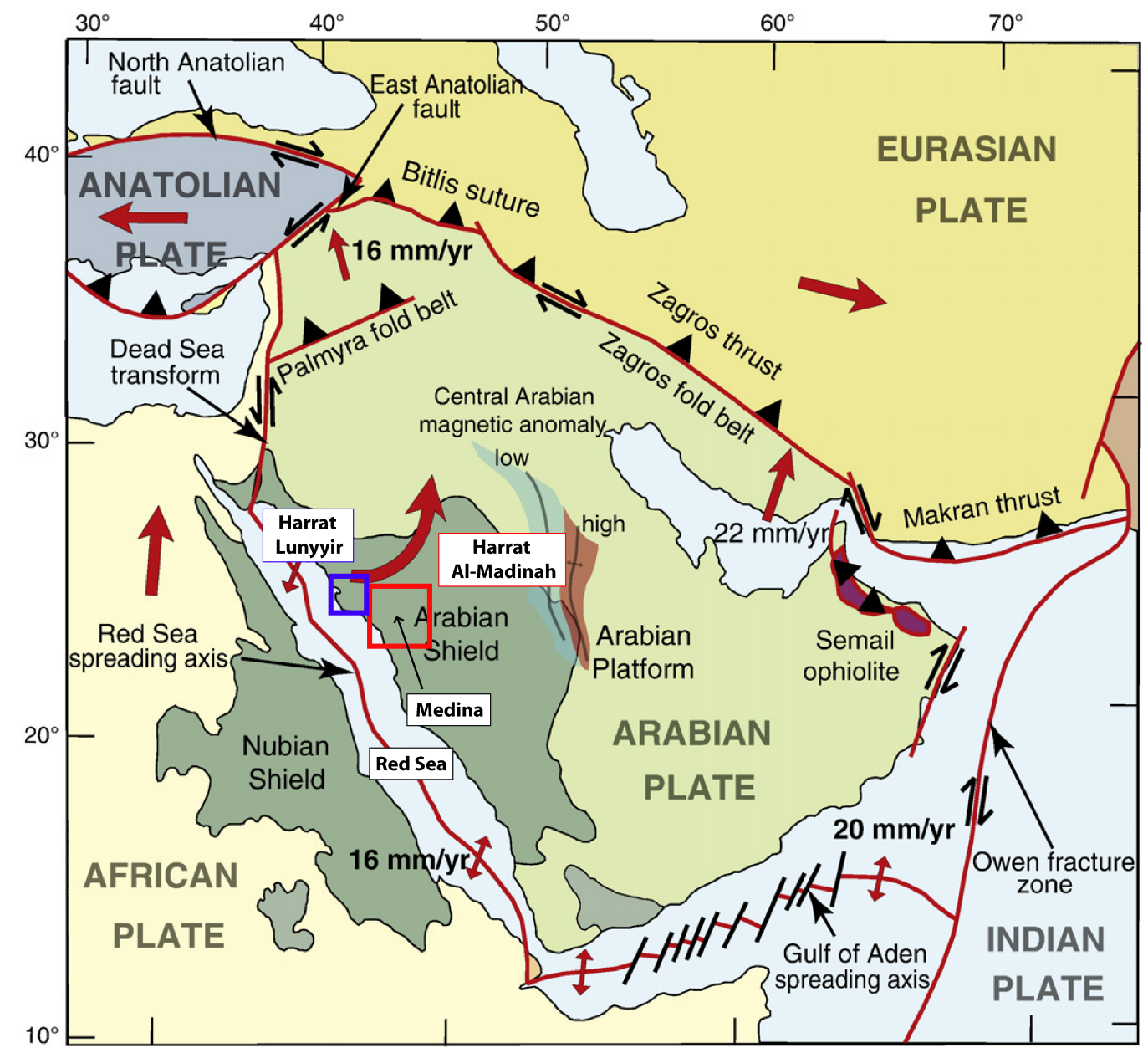

Figure 3.2: Map of Saudi Arabia. Includes plate boundaries, geologic features, and approximate plate convergent vectors [Bird, 2002]. Our study area (Harrat Al-Madinah) and Harrat Lunayyir (the location of the 2009 earthquake swarm) [Baer and Hamiel, 2010] are displayed as red and blue outlines respectively. Adapted from Stern and Johnson [2010].

Elevations across the country are varied, reaching over 3000 meters above sea level in the southwest edge near the Red Sea and the Gulf of Aden and down to sea level at the Arabian Gulf [Bosworth et al., 2005]. In 1985, a $1000 \mathrm{~km}$ long refraction profile was deployed east-west from Riyadh to Jizan to characterize the crustal structure to the mantle [Mooney et al., 1985] [Figures 3.5 and 3.3]. Using two-dimensional ray- 
tracing techniques, they found that the crust of the Arabian Shield is primarily composed of two layers approximately $20 \mathrm{~km}$ thick with average P-wave velocities of 6.3 and $7.0 \mathrm{~km} / \mathrm{s}$ [Mooney et al., 1985]. Although the refraction profile was located approximately $550 \mathrm{~km}$ south of Harrat Al-Madinah, a shot point location (SP4) was carried out near Bishah and Harrat Al-Buqum, another Cenozoic basaltic volcanic area [Figures 3.5 and 3.3].

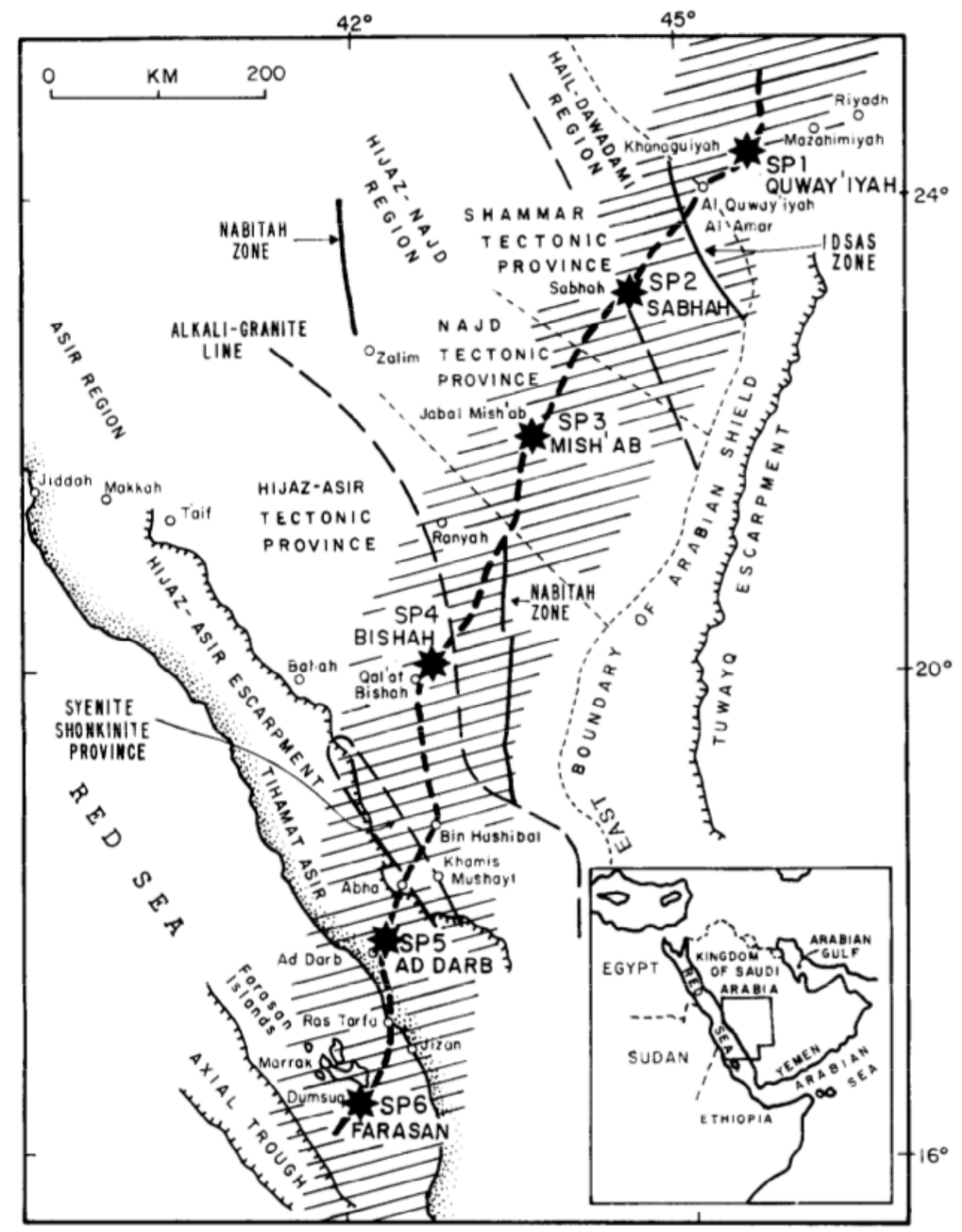

Figure 3.3: Location of the refraction line conducted by Mooney et al. [1985].

A currently unpublished study by Galvin et al. [2017] combined a total of 14 reflection/refraction and 29 receiver function studies to determine the crustal thickness across the Middle East. They found that the maximum and minimum crustal thicknesses were $65 \mathrm{~km}$ underneath the Zagros Mountains and $6 \mathrm{~km}$ in the Red Sea [Fig- 
ure 3.4].

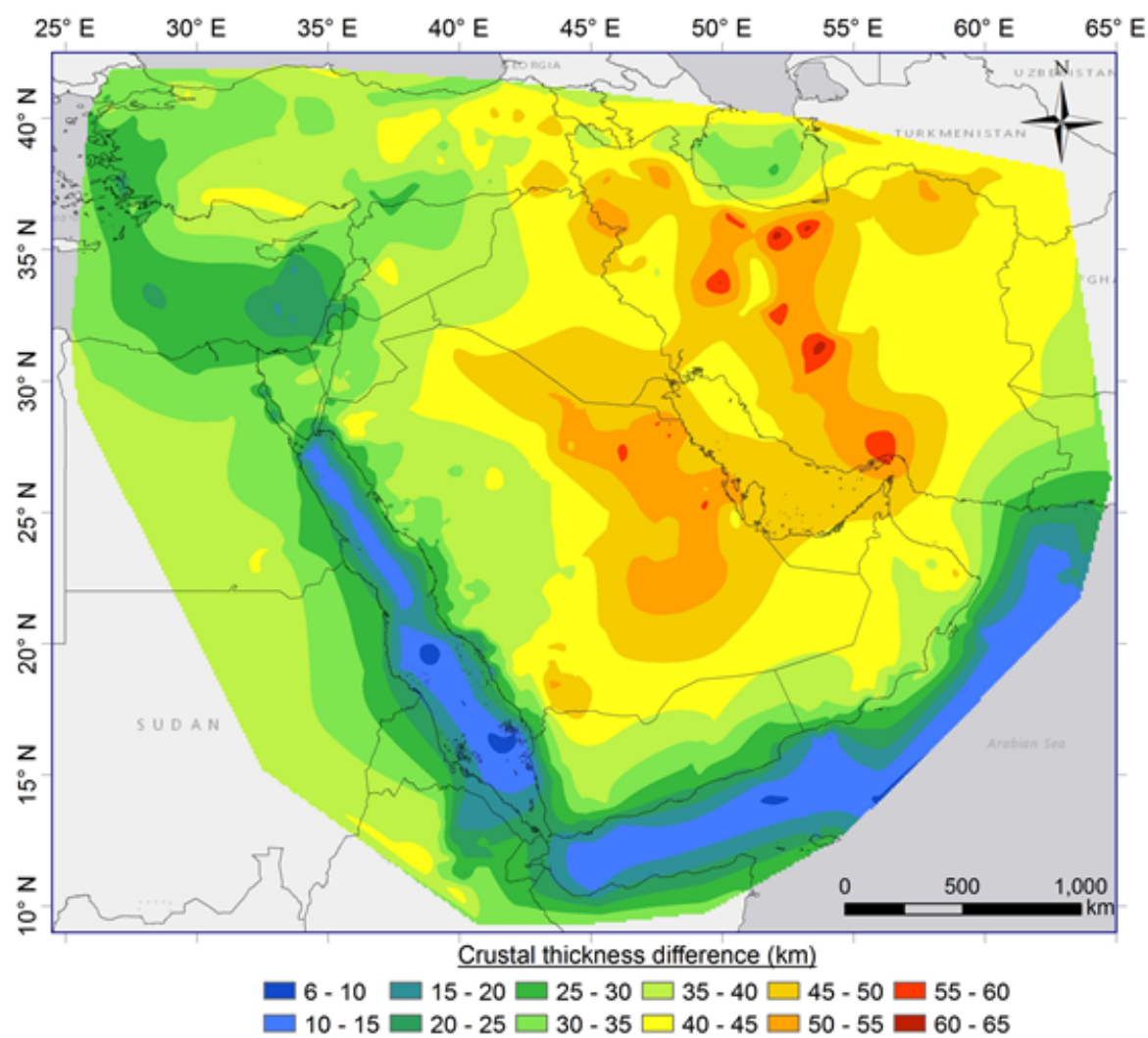

Figure 3.4: Crustal thickness of Saudi Arabia. Adapted from Galvin et al. [2017].

Of the entire size of the Arabian Shield, only $\sim 445,000 \mathrm{~km}^{2}$ is exposed Precambrian [Johnson and Kattan, 2012]. Interior parts of the shield are locally covered by Mesozoic and Cenozoic sedimentary rocks, Cenozoic alluvium and eolian sand, and Cenozoic basaltic lava (referred to as "harrat"). The word "Harrat" is based on the Arabic noun "harra" meaning "stony area, volcanic country, lava field" and is related to the adjective "harr" which means hot [Wehr, 1976]. Harrat Rahat is the largest Cenozoic volcanic field in Saudi Arabia, and its basalt is between $100 \mathrm{~m}$ and $400 \mathrm{~m}$ in thickness [Blank and Sadek, 1983, Camp and Roobol, 1989, Johnson and Kattan, 2012]. Our study area, Harrat Al-Madinah, is the northernmost portion of Harrat Rahat that includes the city of Medina (above latitude $24^{\circ} \mathrm{N}$ ) [Figure 3.5].

Although the volcanism in the western part of the Arabian Shield is related to the opening of the Red Sea at approximately 30 Ma [Camp and Roobol, 1992], its precise origin and mechanism is still unresolved [Tang et al., 2016]. The mechanics that brought the Red Sea to its present configuration are thought to have been a two step process: rifting was initialized passively, where plate-driven extensional stresses stretch and thin the lithosphere, then transitioned to active rifting, where uplift and lithospheric erosion is driven by hot mantle material [Camp and Roobol, 1992, Ebinger and Sleep, 1998]. Currently, the southern Red Sea (latitudes $<21^{\circ} \mathrm{N}$ ) is an area of active 


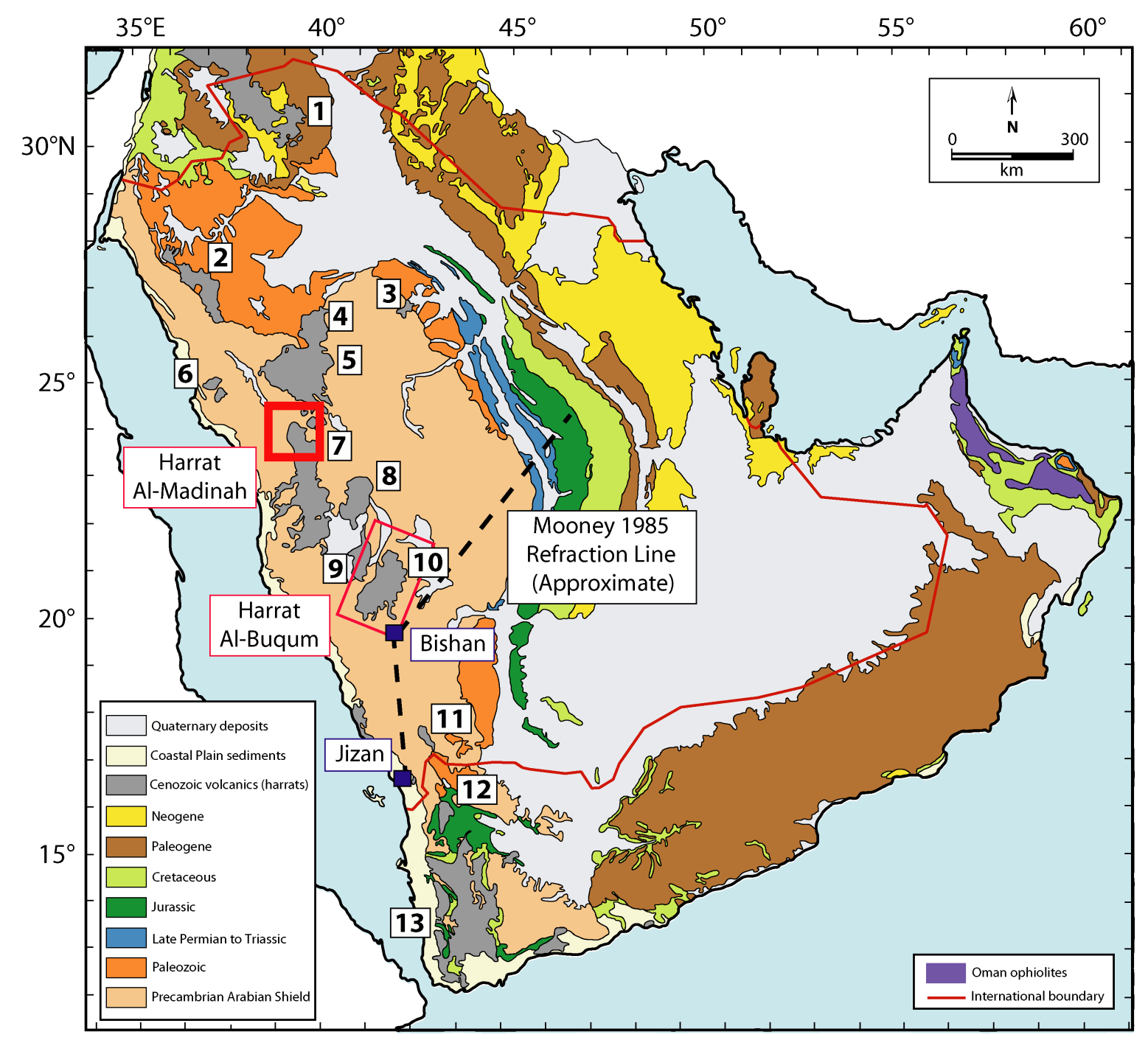

Figure 3.5: Geologic map of Saudi Arabia. The Cenozoic Harrats are displayed as a grey color. Harrat Al-Madinah (red outline) is the northernmost extent of Harrat Rahat. The approximate location of the refraction line from [Mooney et al., 1985] is displayed as a dashed line (please refer to Figure 3.3 for a more accurate depiction of the line). Adapted from Camp and Roobol [1992]. Numbers correspond to the harrats, and their names and lava ages can be found in Table 3.1.

seafloor spreading [Ebinger and Sleep, 1998], while the northern Red Sea (latitudes $>21^{\circ} \mathrm{N}$ ) is hypothesized to be a zone that is at the end of its continental stage and is transitioning into seafloor spreading [Cochran and Martinez, 1998]. The Harrats have two stages of volcanism which reflect the two stages of rifting: tholeiitic-to-transitional (30-20 Ma) and transitional-to-alkalic (12 Ma - recent) [Camp and Roobol, 1992]. Table 3.1 details the lava ages for the harrats displayed in Figure 3.5.

There are several hypotheses regarding the source of the Arabian Shield volcanism. Originally, it was hypothesized that the volcanism was due to a local mantle plume beneath the Arabian Shield [Camp and Roobol, 1992]. However, the absence of uni- 


\begin{tabular}{||l||l||}
\hline Harrat & Lava ages (Ma) \\
\hline 1. Shaam & $26-22,13-8,7<0.5$ \\
\hline 2. Uwayrid & $9-$ present \\
\hline 3. Hutaymah & 1.8 \\
\hline 4. Ithnayn & $3-$ present \\
\hline 5. Khaybar & $5-$ present \\
\hline 6. Lunayyir & $1.0-0.5$ \\
\hline 7. Rahat & $10-$ present \\
\hline 8. Kishb & $2-0.02$ \\
\hline 9. Hadn & $28-15$ \\
\hline 10. Al Buqum & $9-$ present \\
\hline 11. As Sirat & $30-25$ \\
\hline 12. Tihama Asir & $25-20$ \\
\hline 13. Yemen Traps & $30-20$ \\
\hline
\end{tabular}

Table 3.1: Lava ages for the Saudi Arabian harrats [Coleman, 1993, Johnson and Kattan, 2012]. Numbers refer to the boxes displayed in Figure 3.5.

directional volcanic migration and NNW-trending distribution of volcanic vents parallel to the Red Sea has suggested that volcanism in this region is related to periodic extensional episodes along the reactivated Red Sea fault system [Moufti et al., 2012a]. Chang et al. [2011] suggested that the volcanism may be due to lateral flow from the the Afar or Jordan hotspots. Recent work by Tang et al. [2016], utilizing teleseismic P-receiver functions and fundamental Rayleigh wave group velocities, observed high mantle lid temperatures and slow velocities on the north and south tips of the Arabian shield. They attributed these results to thermal conduction from the Afar and Jordan plumes, and hypothesized that the volcanism in the Arabian Shield is caused by small-scale adiabatic ascent of magma diapirs. This hypothesis was supported by Yao et al. [2017], who found a low velocity zone in the upper mantle beneath western Saudi Arabia using teleseismic Rayleigh phase velocities. 


\subsection{Local geology and prior geophysical studies}

Harrat Rahat is a Cenozoic bimodal alkaline volcanic field in Saudi Arabia extending over an area of approximately 20,000 $\mathrm{km}^{2}$, bounded by the cities of Jeddah and Mecca in the south and Medina to the north [Figure 3.5] [Camp and Roobol, 1989, Moufti et al., 2011, 2012a,b, Moufti and Németh, 2016]. Volcanism in Harrat Rahat began approximately $10 \mathrm{Ma}$ in the Neogene, with at least three pulses around 10, 5, and $2 \mathrm{Ma}$, and at least several pulses in the Quaternary from 1.9 Ma to present [Coleman, 1993, Moufti et al., 2012a]. Harrat Al-Madinah is the northern extent of Harrat Rahat located near the city of Medina [Figure 3.5, red square].

Harrat Rahat is composed of three main geological units: Shawahit basalt $(\sim 10-$ $2.5 \mathrm{Ma}$ ), Hammah basalt ( 2.5 Ma-1.7 Ma), and Madinah basalt ( 1.7 Ma to recent), reflecting an overall northward progression with age [Camp and Roobol, 1992, Moufti et al., 2012a]. Harrat Al-Madinah is differentiated from the rest of Harrat Rahat by a NNW-SSE volcanic chain that extends about $100 \mathrm{~km}$ to Medina, composed of more than 500 scoria cones between 30 to 200 meters high [Figure 3.11].

The most recent volcanic eruption in this area was the 1256 CE Al-Madinah eruption, a $2.25 \mathrm{~km}$ long fissure eruption that produced a $23 \mathrm{~km}$ long lava flow that stopped just $8 \mathrm{~km}$ east of Medina [Camp et al., 1987]. Figure 3.6 displays the visible contrast between the Madinah Basalt and the Precambrian rocks at Harrat Al-Madinah along with the seismic stations of the Harrat Rahat seismic network. Although certain areas of the city have been built over the flow, the vents for this eruption can still be observed on satellite imagery, located at latitude-longitude point $24.36^{\circ}, 39.77^{\circ}$ [Camp et al., 1987] [Figure 3.7]. In comparison to our network, the fissure location of the previously mentioned Al-Madinah 1256 CE eruption is approximately $4 \mathrm{~km}$ south of RHT14 [Figures 3.7 and 3.11]. 


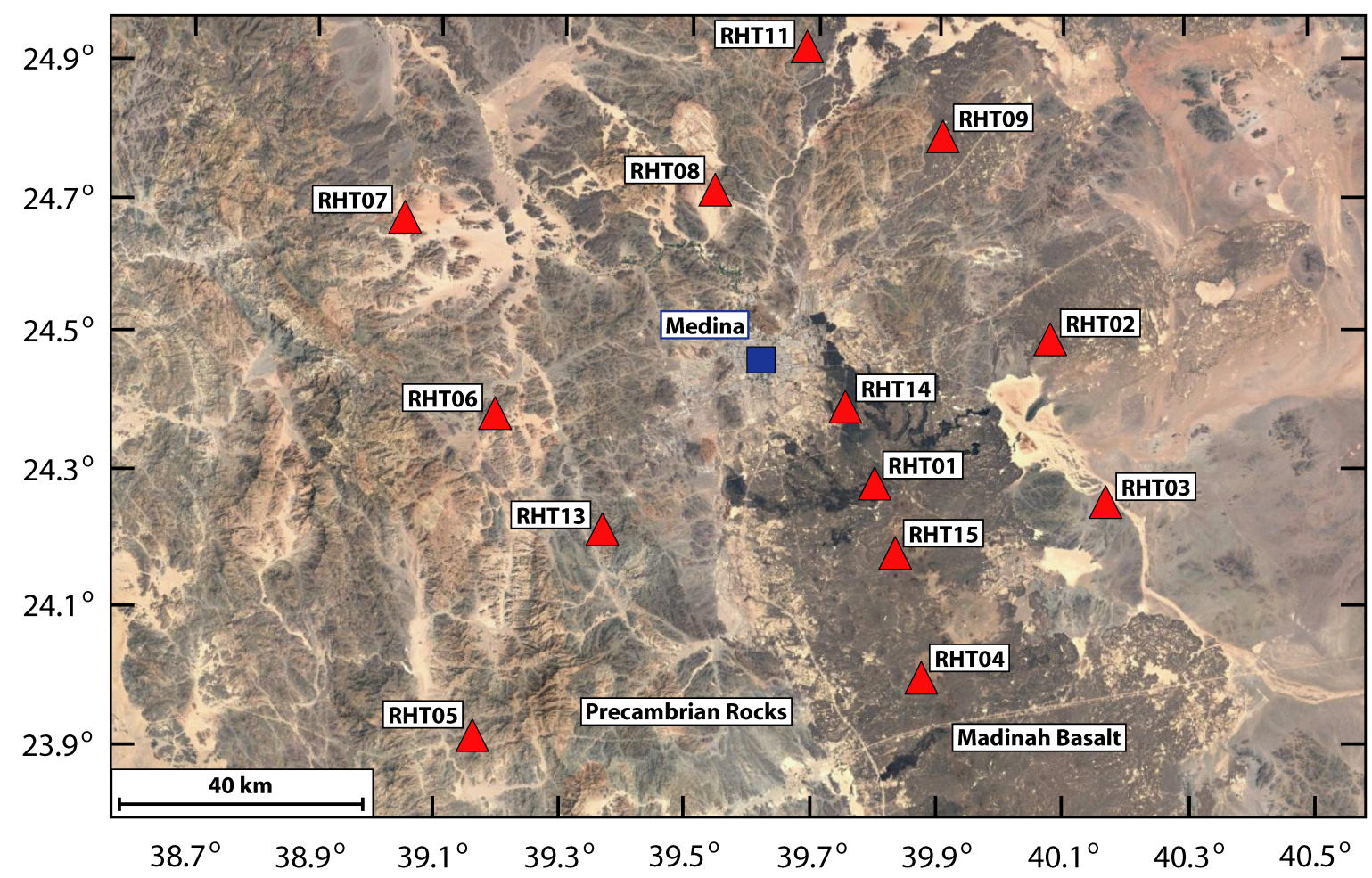

Figure 3.6: Satellite map of Harrat Al-Madinah displaying the contrast between the black Madinah Basalt and the light grey Precambrian rocks. Stations of the Harrat Rahat seismic network are shown as red triangles and the city of Medina as a blue square. Satellite image (c) CNES/AIRBUS [2017] obtained using the software Google Earth Pro. 


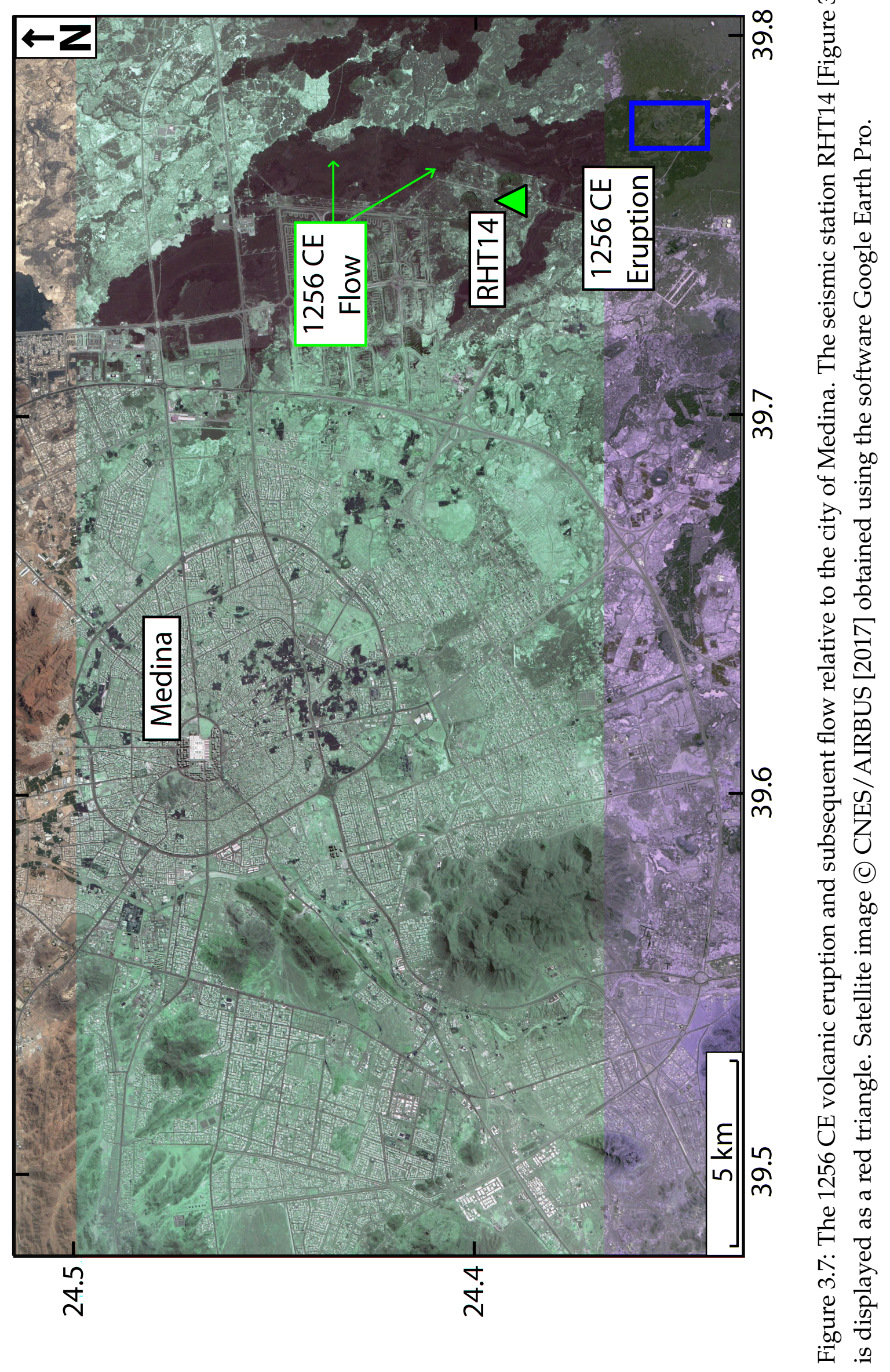


A geologic map of Harrat Al-Madinah is displayed in Figure 3.8 (adapted from Downs et al. [2017]) and contains several important features. The topography due to the volcanic chain can be observed parallel to RHT04, RHT15, RHT01, and RHT14, surrounded by lava flows of various ages [Figure 3.9]. The lava extent of the $1256 \mathrm{CE}$ eruption is displayed in red near RHT14, just east of the city of Medina (whose roads are displayed in black). Additionally, there are two flows from the late Pleistocene that extend westward from near RHT01 to south of the city of Medina.

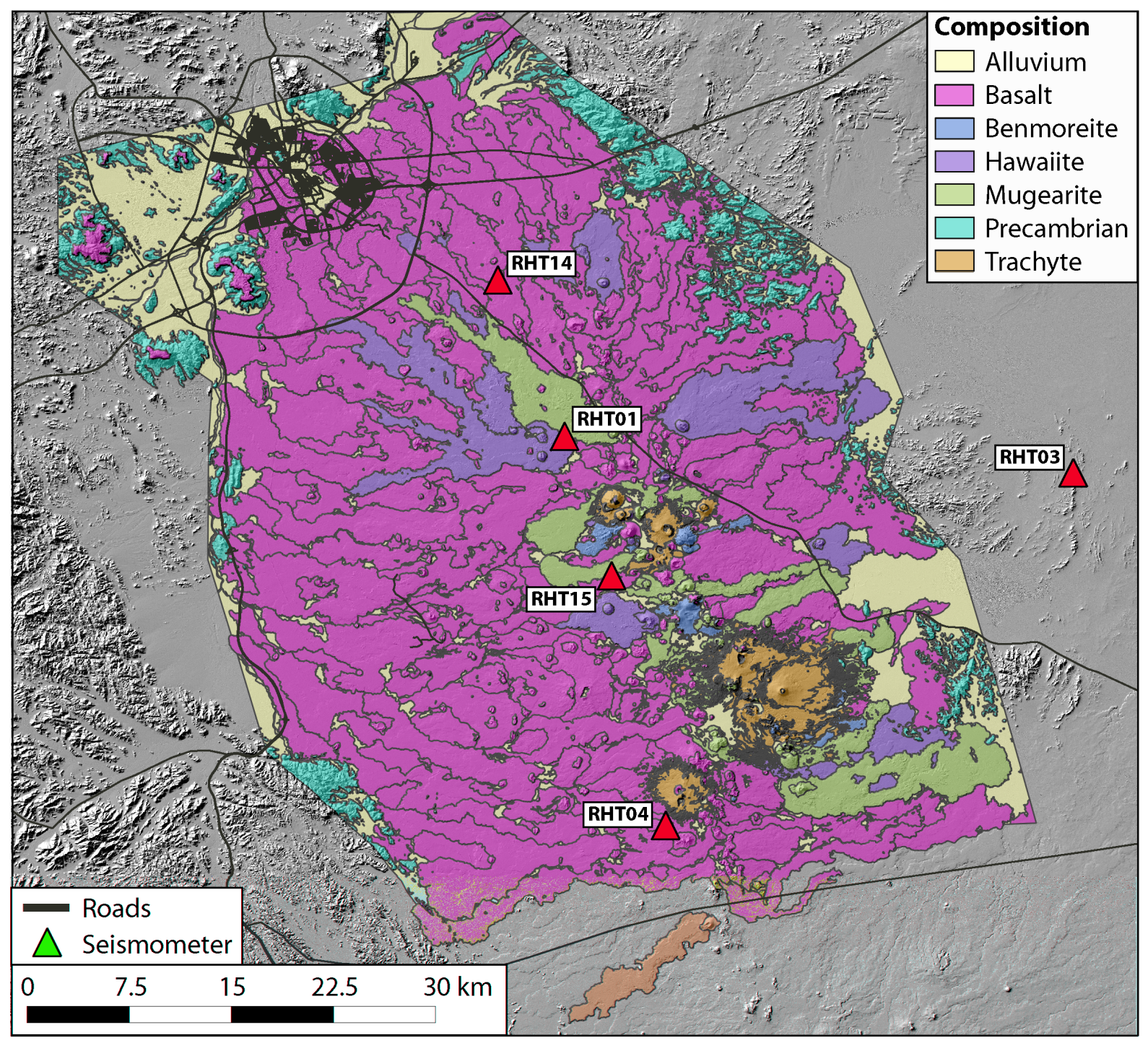

Figure 3.8: Geological map and surface topography of Harrat Al-Madinah. Adapted from Downs et al. [2017].

Several geophysical analyses have been done in this area, including: a surface-wave and P-wave receiver function joint inversion utilizing a portion of the Saudi Arabia Seismic Array [Tang et al., 2016], a gravity survey [Langenheim et al., 2016], 3D resistivity modeling [Bedrosian et al., 2017]. The resistivity survey found numerous conductors starting at approximately $15 \mathrm{~km}$ that are not solely co-located with the Harrat 


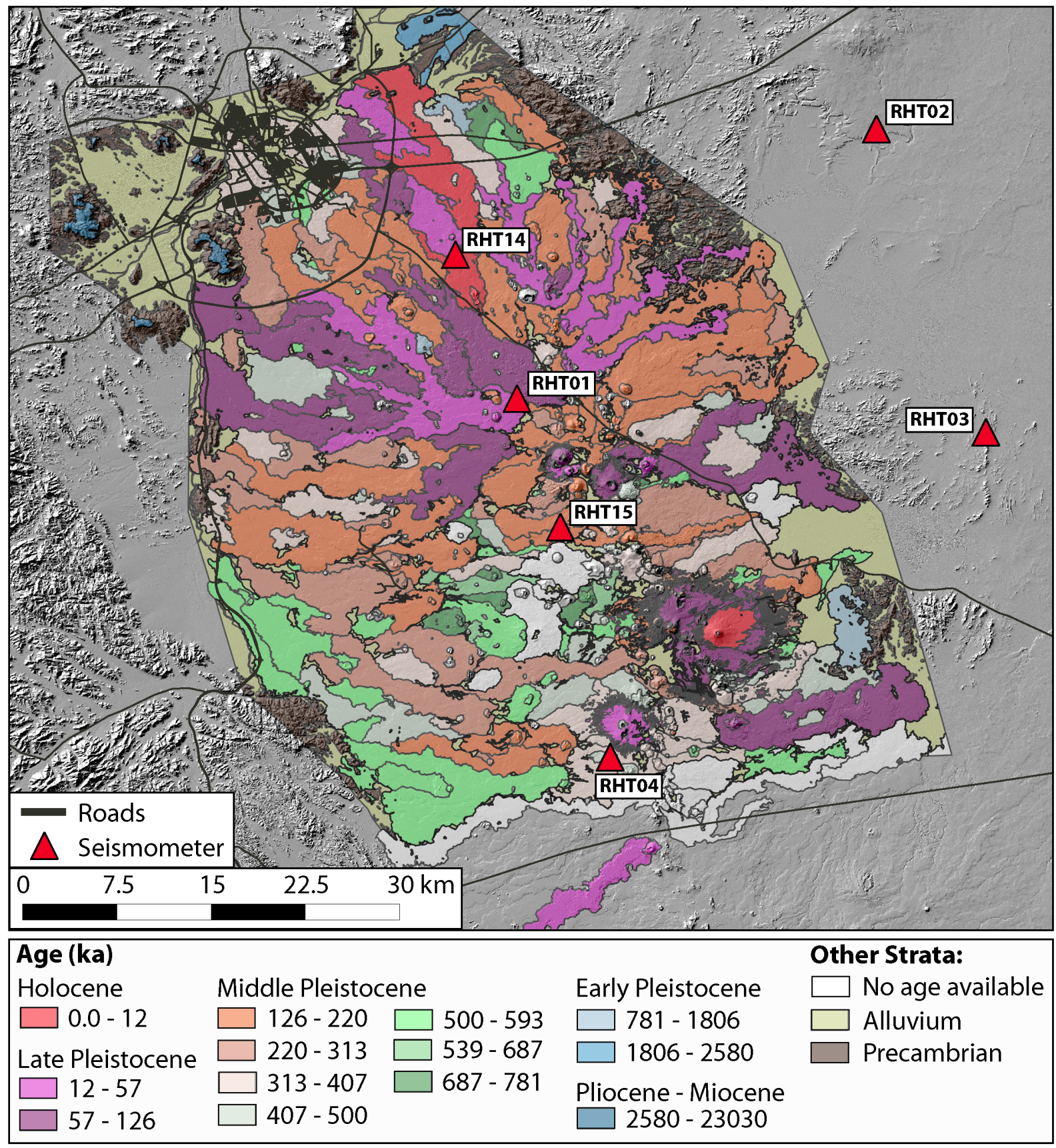

Figure 3.9: Eruption ages and surface topography of Harrat Al-Madinah. Adapted from Downs et al. [2017].

[Bedrosian et al., 2017] [Figure 3.10].

Recently, subsurface imaging was carried out at Harrat Al-Madinah by Abdelwahed et al. [2016] using local and teleseismic earthquakes recorded at a borehole array [Figure 3.11]. They found numerous high and low velocity anomalies in the shallow and deep parts of the Harrat, hypothesized to be a result of basaltic and trachytic eruptions [Abdelwahed et al., 2016]. Additionally, a low velocity anomaly was found at depths between 2 to $10 \mathrm{~km}$ beneath the $1256 \mathrm{CE}$ eruption [Abdelwahed et al., 2016]. 


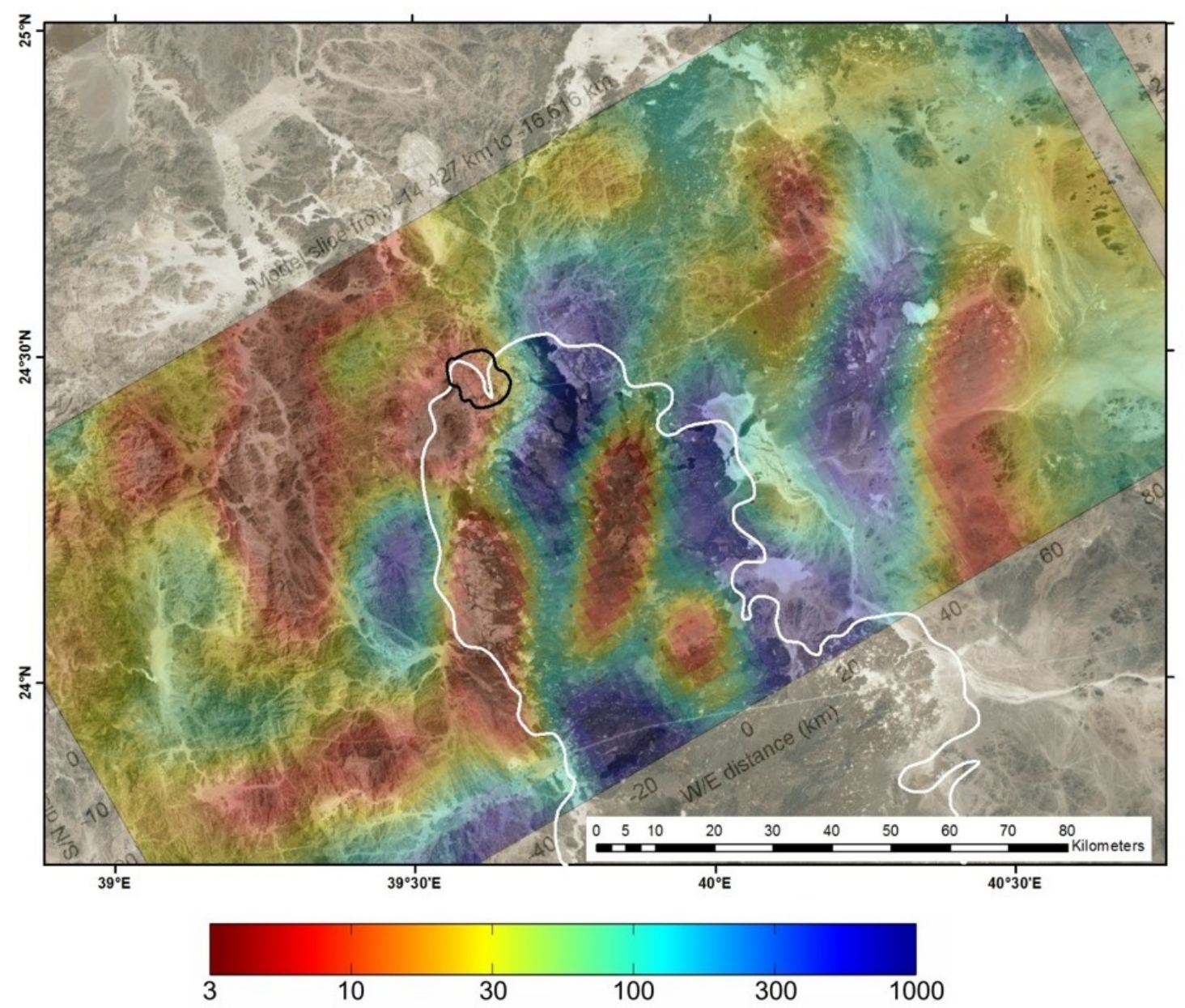

Figure 3.10: Resistivity (in $\Omega \mathrm{m}$ ) at $15 \mathrm{~km}$ for Harrat Al-Madinah. From Bedrosian et al. [2017].

\subsection{Site and instrument information}

The Harrat Rahat seismic array is a subset of the Saudi Arabia seismic network, and contains 14 Nanometrics Trillium T120 instruments across an array aperture of approximately 130 kilometers [Figure 3.11]. Although the subset of stations have the designation Harrat Rahat, they encompass only the Al-Madinah section of the Harrat. The T120s are broadband seismometers with a flat frequency response range of approximately 0.01-20 Hz [Streckeisen, 1995][Figure 3.12]. Instrument locations are listed in Table A.1 in Appendix A.1.

The instrument response must be removed from data in order to accurately compute cross-correlations between station pairs from different types of instruments. As all the instruments used in this subset are of the same type, this is not a necessary step. However, as these data are meant to be incorporated into the rest of the Saudi Arabian stations, instrument response was nevertheless removed. Instrument response 


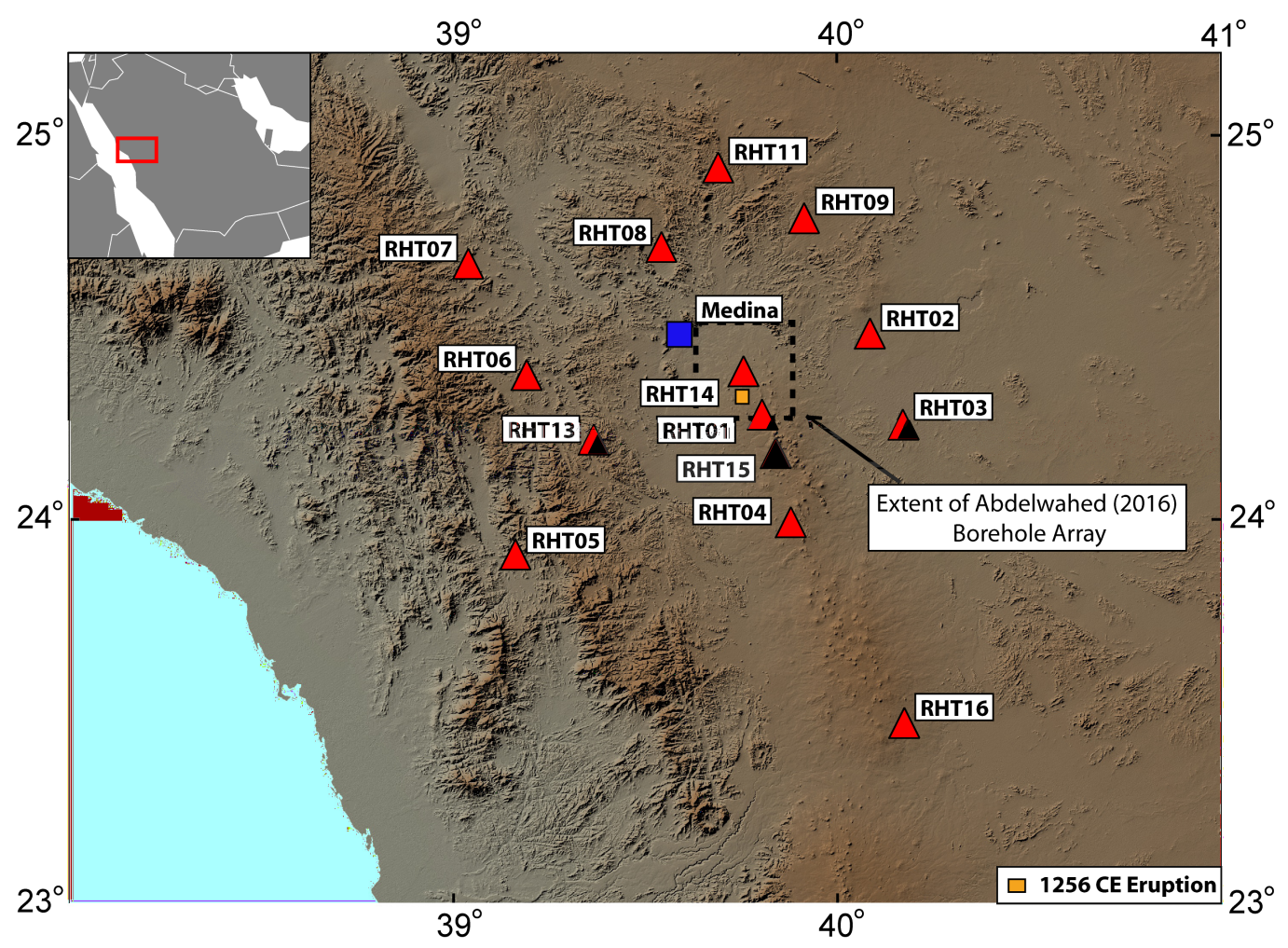

Figure 3.11: The Harrat Rahat seismic network (red triangles), composed of 14 T120 broadband seismometers. Velocity results from RHT16 were consistently much faster than other pairs, so the station was removed from the analysis. The city of Medina and the location of the $1256 \mathrm{CE}$ eruption are displayed as a blue and orange square respectively. The extent of the Abdelwahed et al. [2016] borehole array is displayed as a dotted black rectangle.

files were generated for each station using the IRIS program $r d s e e d$ [IRIS, 2012]. The instrument response was removed using the ObsPy function simulate [Beyreuther et al., 2010] within a multi-core Python wrapper code.

We analyzed the spectra of a teleseismic earthquake before and after instrument response removal to check whether or not the processing was successful. The M7.9 Aleutian Islands earthquake occurred on 2014-06-23 20:53:09 UTC $\left(51.849^{\circ} \mathrm{N}, 178.735^{\circ} \mathrm{E}\right)$ and was recorded across the entire Saudi Arabia array. The teleseismic signal was used to check that the instrument response was removed correctly. Figure 3.13 displays the earthquake trace at a T120 station (AFFS) and compares the Fourier spectra before and after instrument response removal. 

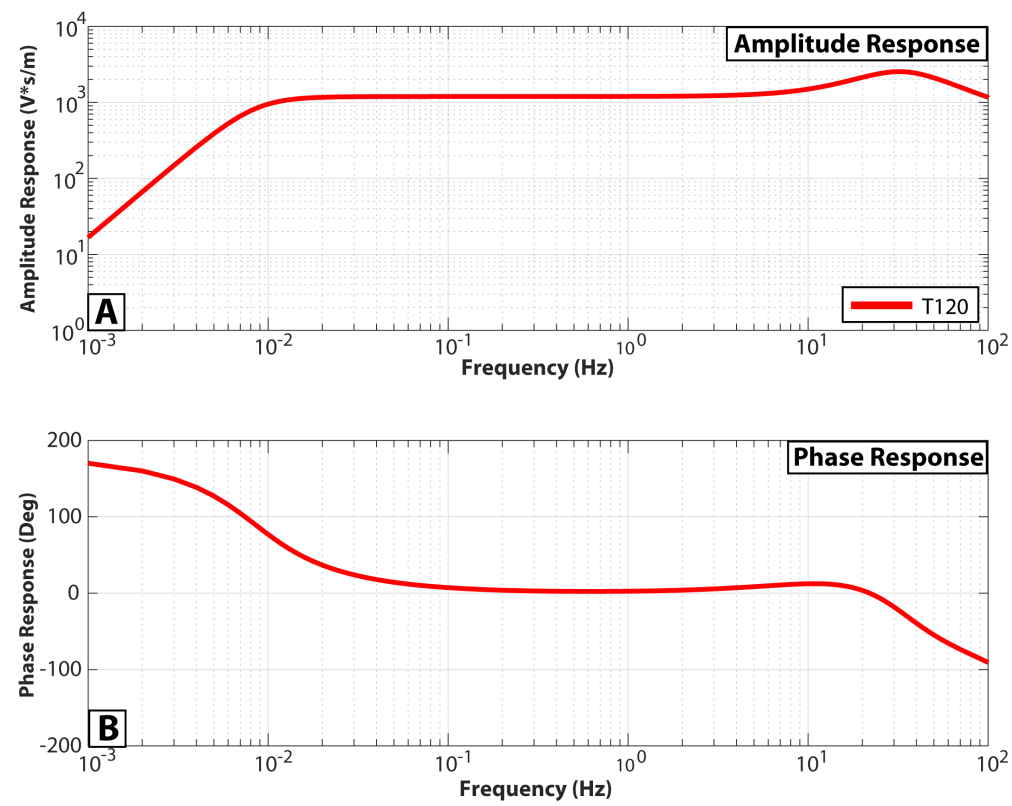

Figure 3.12: Amplitude [A] and phase [B] instrument response curves for the T120 broadband instruments [Streckeisen, 1995].
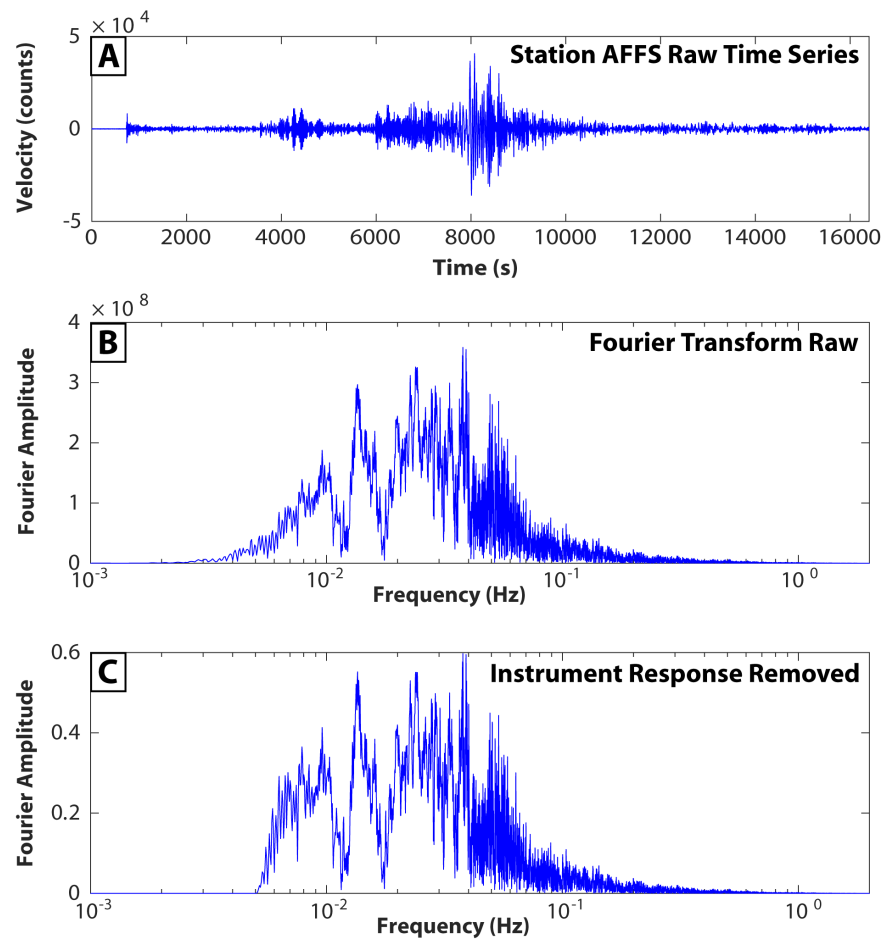

Figure 3.13: [A] Time series signal of the M7.9 Aleutian Islands earthquake. [B] Fourier transform of the teleseismic earthquake without instrument response removed. [C] Fourier transform of the teleseismic earthquake with instrument response removed.

\subsection{Methods}

In this section, we define the methods used to compute dispersion curves and maps from natural ambient noise. These steps require the computation of stable cross-correlations 
described in Chapter 2. The specific parameters used for cross-correlation computation are listed in Table 3.2. After computing cross-correlations, the processing steps are as follows: [1] Removal of zero-point noise from cross-correlation data, [2] determination of dispersion curves through the Frequency Time Analysis (FTAN) [Levshin et al., 1972, 1989], and [3] computation of surface wave dispersion maps [Barmin et al., 2001].

\begin{tabular}{||l||l||}
\hline Property & Value \\
\hline Analysis duration (s) & 86400 \\
\hline Cross-correlation sample-rate $(\mathrm{Hz})$ & 20 \\
\hline Correlation duration $(\mathrm{s})$ & 1800 \\
\hline Maximum lag-time $(\mathrm{s})$ & 700 \\
\hline Filtering $(\mathrm{Hz})$ & $0.005-1.1$ \\
\hline Whitening $(\mathrm{Hz})$ & $0.006-1.0$ \\
\hline Time normalization type (s) & One-bit \\
\hline
\end{tabular}

Table 3.2: Saudi Arabia processing parameters.

\subsubsection{Zero point noise}

Ambient noise cross-correlation results are sometimes negatively affected by a phenomena often called "zero-point noise" or a "near-zero time" signal [Landes̀ et al., 2010]. This phenomenon produces large amplitudes near zero lag time in the crosscorrelation, which results in faster velocities when determining dispersion curves. After computing daily cross-correlations for the stations pairs, we noticed that only some days were affected by zero-point noise, while other days were not affected. Zero-point noise can be caused by numerous factors, including teleseismic P-waves generated by distant storms [Gerstoft et al., 2008] and in deep oceans [Landes̀ et al., 2010] or body waves reflected by the Moho [Zhan et al., 2010, Poli et al., 2012].

Due to this, an algorithm was written to remove the days affected by this phenomena from the cumulative cross-correlation stack. We used an unrealistic $5 \mathrm{~km} / \mathrm{s}$ surface wave velocity threshold to determine a window of the cross-correlation lag-time where we don't expect surface wave arrivals. For example, if a station pair is $60 \mathrm{~km}$ apart, the zero-point detection lag time window would be +-12 seconds. If the maximum value in this segment is more than 4 times the RMS of the noise, the day is discarded from the cross-correlation stack. In our case, the noise part of the cross-correlation was chosen to be the data between 150 to 700 seconds lag-time, where we did not expect surface waves to occur. The effect of this procedure is illustrated in Figure 3.14. Additionally, some station pairs were affected by a low-frequency signal, which was removed prior to stacking. Regardless of the presence of zero-point noise, cross-correlations were 
discarded if the signal-to-noise ratio was less than 15.
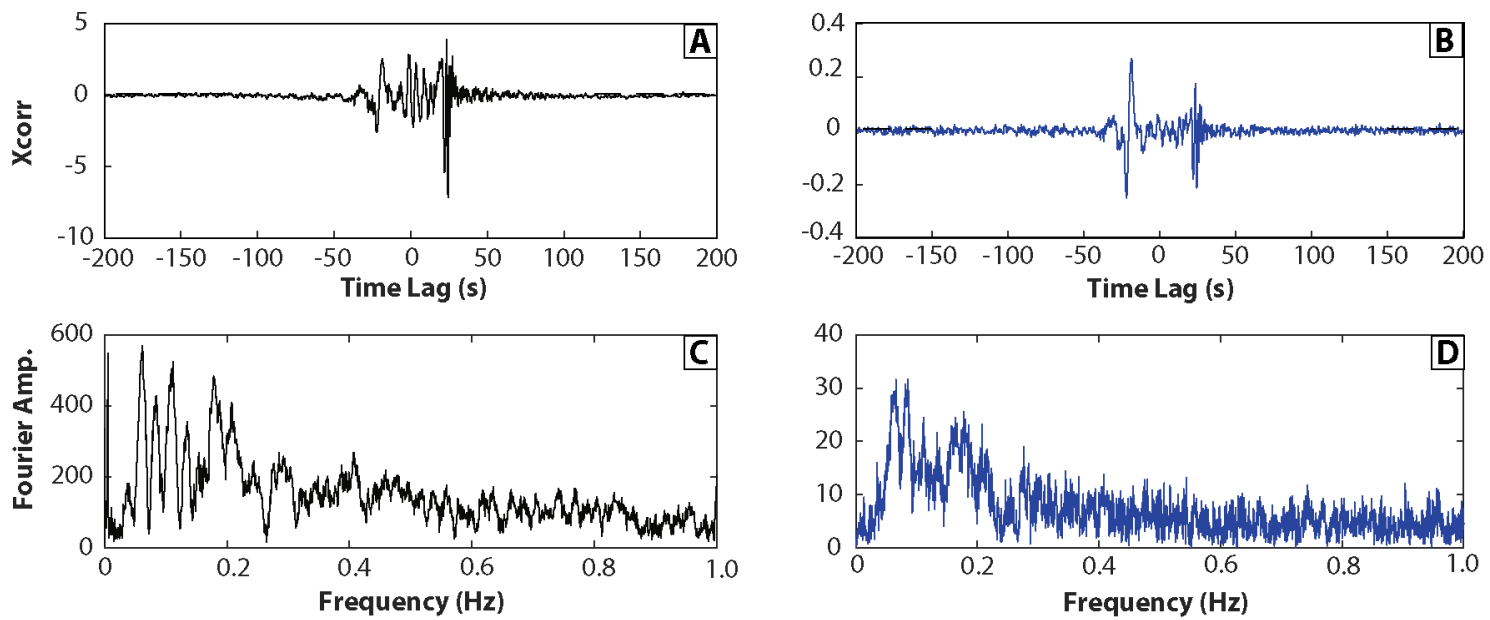

Figure 3.14: Zero point removal for station pair RHT01 and RHT06 vertical cross components. Time series [A, B] and Fourier transform [C, D] before [black] and after [blue] removing the zero-point noise and low-frequency signal spike.

\subsubsection{Computing dispersion curves}

Dispersion of surface waves is most commonly characterized as a function of velocity or slowness in the frequency domain. Frequency-Time Analysis (FTAN) [Levshin et al., 1972, 1989] is a method to extract dispersion curves from time series data. Essentially, it is a method that computes a dispersion curve (velocity according to frequency) through a moving Gaussian filter in the frequency domain. We define the real part of a Gaussian filter $H\left(\omega-\omega^{H}\right)$ around a central frequency $\omega^{H}$ [Levshin et al., 1989, Bensen et al., 2007]:

$$
H\left(\omega-\omega^{H}\right)=e^{-\alpha\left(\frac{\omega-\omega^{H}}{\omega^{H}}\right)^{2}}
$$

where $\alpha$ is the parameter controlling the width of the Gaussian filter and is dependent on the separation distance of the station pair [Levshin et al., 1989] and $H$ is the index of the central frequency. In the codes that we used for the FTAN computation, there was no way to explicitly set $\alpha$, but instead set a factor of the Gaussian width (ffact) [Table 3.3]. This will be discussed at the end of this section when we list all of the used parameters. Assuming a smoothly varying dispersion $\tau(\omega)$, the amplitude of a signal filtered by Equation 3.1 will cluster around the time $t$ :

$$
t=\tau\left(\omega^{H}\right)
$$

In other words, $\tau\left(\omega^{H}\right)$ is the intersection between the spectrum $K(\omega)$ and the Gaussian filter, and $t$ is the inverse Fourier transform (time-domain) equivalent of that sig- 
nal. The FTAN representation of the signal, or the representation of the signal for all filters $H\left(\omega-\omega^{H}\right)$, is then defined as the complex function:

$$
S\left(\omega^{H}, t\right)=\int_{\omega_{\min }}^{\omega_{\max }} H\left(\omega-\omega^{H}\right) K(\omega) e^{i \omega t} d \omega
$$

where $K(\omega)$ is the spectral amplitude of analytical signal and $\left|S\left(\omega^{H}, t\right)\right|$, the signal envelope for each filter, is the FTAN map most commonly used for visual representation. $\omega_{\min }$ and $\omega_{\max }$ are the minimum and maximum frequency boundaries, determined by the maximum and minimum period bound variables tmin and tmax [Table 3.3]. Within this region, we define $S_{m}$, a function obtained from $S\left(\omega^{H}, t\right)$ where $|S|$ has a maximum along $t$, and is defined as the dispersion curve $t\left(\omega^{H}\right)=\tau\left(\omega^{H}\right)$ [Levshin et al., 1989]. The FTAN map is manipulated by changing the range and width of the $H\left(\omega-\omega^{H}\right)$ filter, and it should calculate accurate values provided that the filter doesn't extend past the ends of the spectrum [Figure 3.15]. In other words, the FTAN method generates a dispersion curve through use of a moving Gaussian filter in the frequency domain.

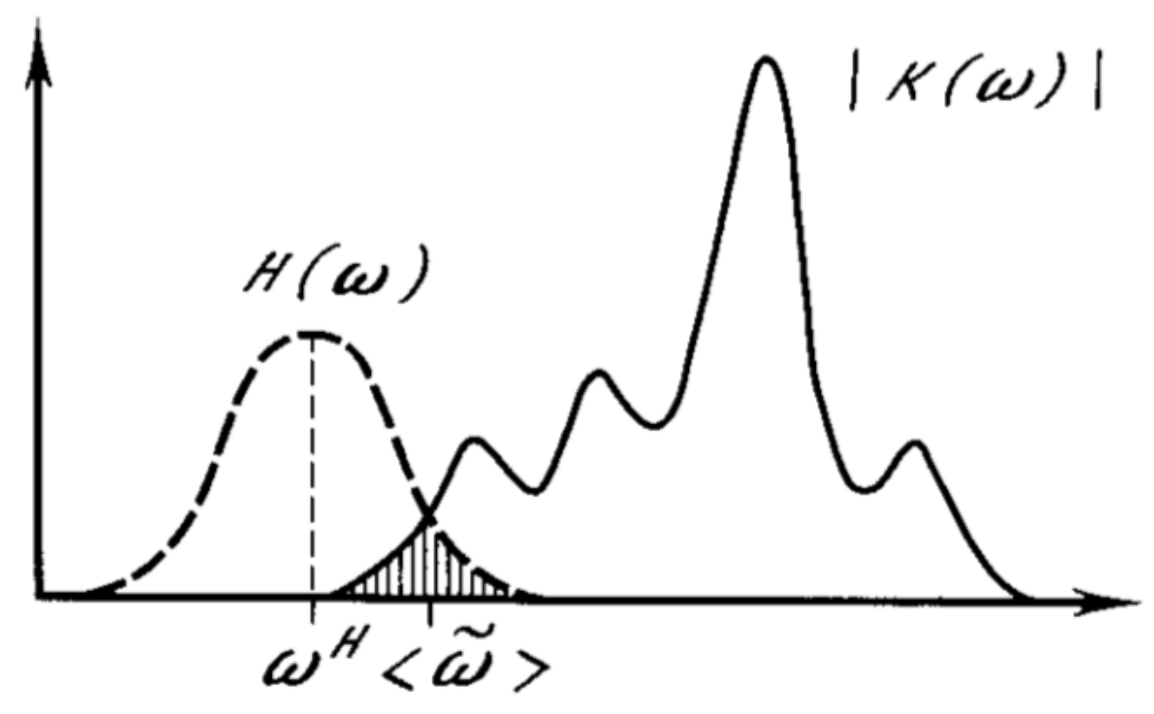

Figure 3.15: Diagram displaying a filter $H(\omega)$, symmetric around frequency $\omega^{H}$, the spectrum $K(\omega)$ of a seismic signal, and the resulting area of intersection in the frequency domain. This filter on the edge of the spectrum would introduce errors in the resulting FTAN plot. From [Levshin et al., 1989].

The group speed $U$ is determined from the peak of the of the envelope function:

$$
U\left(\omega^{H}\right)=r / \tau\left(\omega_{H}\right)
$$

where $r$ is the distance between the two stations.

Group velocity measurements can sometimes be improved through the use of a phase-matched filter. A phase-matched filter is defined as a linear filter whose phase is equal to that of an input signal [Herrin and Goforth, 1977]. In the case of FTAN, a 
phase matched filter is used to smooth the measured group speeds and avoid neighboring noise [Levshin and Ritzwoller, 2001]. The application of the phase-matched filter generally has three steps [Levshin and Ritzwoller, 2001, Bensen et al., 2007]: [1] the cross-correlation is passed through a phase-matched filter whose spectrum is defined by the originally calculated group velocity dispersion curve, [2] the peak of the "compressed" result is identified and isolated by a certain time bandwidth ${ }^{1}$, and [3] the resulting signal is inverted back through the original phase matched filter. The peak of the envelope function is calculated again to obtain a cleaner group velocity. The positive and negative sides of the cross-correlation were stacked to increase the signal-to-noise ratio.

We can relate group and phase velocity through the following equations [Stein and Wysession, 2003]:

$$
\begin{aligned}
U & =\frac{\delta \omega}{\delta k} \\
c & =\frac{\omega}{k} \\
s_{u} & =U^{-1} \\
s_{c} & =c^{-1}
\end{aligned}
$$

where $s_{c}$ and $s_{u}$ are phase and group slowness at frequency $\omega$ and $k$ is the wavenumber.

Phase velocity can be calculated by using the group envelope function and the phase of the analytical signal. For an instantaneous frequency $\omega$, the phase of the crosscorrelation signal $(\phi(t, \omega))$ is:

$$
\phi(t, \omega)=k \Delta-\omega t-\phi_{s}-\phi_{a}
$$

where $t$ is the travel time of the signal, $\Delta$ is the interstation distance, $k$ is the wavenumber, $\phi_{s}$ is the source phase and $\phi_{a}$ is the phase ambiguity, a correction based on the $2 \pi$ ambiguity of the phase spectrum [Snieder, 2004, Bensen et al., 2007]. We then evaluate the phase at the observed group arrival time $t_{u}=\frac{\Delta}{U}$ and $k=\omega s_{c}$ and evaluate Equation 3.9 for phase slowness:

$$
s_{c}=s_{u}+(\omega \Delta)^{-1}\left(\phi\left(t_{u}\right)+\phi_{s}+\phi_{a}\right)
$$

where a phase velocity value is calculated for every group velocity time. For ambient noise cross-correlations, the source phase term $\phi_{s}$ is approximated to zero as the deviation given an inhomegenous source distribution is minimal [Bensen et al., 2007, Lin et al., 2008].

\footnotetext{
${ }^{1}$ This is useful for earthquake tomography to cancel out pre or post-event noise. Due to our preprocessing and zero-point noise removal, there is no need to cut out data and we use the entire crosscorrelation record. However, this parameter could be very important for cross-correlations records with low signal-to-noise ratios.
} 
The determination of the $2 \pi$ ambiguity is more complex. The ambiguity is defined as $\phi_{a}=2 \pi N$, where $N=0, \pm 1, \pm 2, \ldots$. An additional $-\frac{\pi}{4}$ term arises from the stationary phase integration of ambient noise cross-correlations due the interference of a homogeneous source distribution [Snieder, 2004, Lin et al., 2008]. Equation 3.10 is thus expanded to:

$$
s_{c}=s_{u}+(\omega \Delta)^{-1}\left(\phi\left(t_{u}\right)+2 \pi N-\pi / 4\right)
$$

The $N$ term can be determined by phase velocity maps [Bensen et al., 2007]. A reference phase curve is compared to phase velocities calculated using different $N$ terms and the closest branch is selected [Figure 3.16] [Lin et al., 2008]. If a baseline Love speed isn't available, the value from the Rayleigh wave increase by $9 \%$ is sometimes used [Lin et al., 2008]. The $N$ term can be off by one for periods that are greater than 20 s or station-pair distances that are greater than $1800 \mathrm{~km}$, and a second correction using reference velocities from an initial network phase velocity map is sometimes used [Lin et al., 2008].

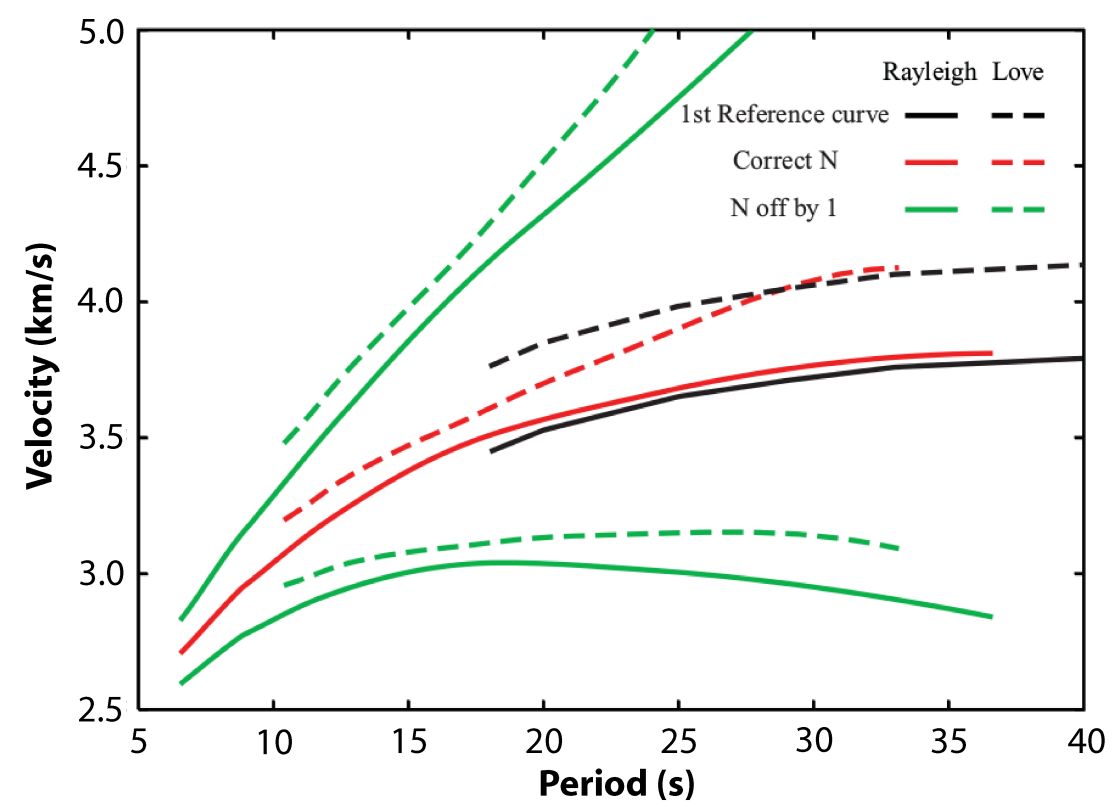

Figure 3.16: Comparison between a reference curve determined from phase velocity maps (black) and phase velocities from three different $\mathrm{N}$ integers (green and red). From Lin et al. [2008].

AFTAN is a set of Fortran subroutines and C functions developed by the University of Colorado Boulder to compute an automatic FTAN for the inversion of dispersion curves [AFTAN, 2013]. AFTAN follows the methodology described above, but does not automatically apply the $N$ term correction. The causal and acausal parts of the cross-correlation were stacked together to increase the signal-to-noise ratio prior to FTAN. Figure 3.17 displays the result of the AFTAN procedure for a station-pair 
RHT04-RHT07. We found that the regular FTAN [Figure 3.17A] and the phase matched filter FTAN [Figure 3.17C] produced approximately the same results in the period band of interest (less than 15 seconds period). As the phase matched filter sometimes excluded the 5 and 6 seconds period, we decided to use the regular FTAN for the dispersion maps and inversions. The full parameters used in the AFTAN procedure are listed in Table 3.3.

As previously mentioned, the $\alpha$ variable describing the width of the moving Gaussian filter cannot be set within the AFTAN codes. $\alpha$ is defined as follows:

$$
\alpha=\text { ffact } * 20 * \sqrt{\text { delta } / 1000}
$$

where ffact is a constant that multiplies the Gaussian and delta is the distance between the stations in kilometers [AFTAN, 2013]. The ffact variable should be written as one for distances greater than one wavelength of the ambient noise, but in any case, recent unpublished work suggests that values of $\alpha$ don't significantly change dispersion curve measurements [Personal comm. Ritzwoller 2016].

\begin{tabular}{||l||l||l||}
\hline Parameter & Description & Value [Units] \\
\hline piover4 & Factor for pi/4 phase shift & -1.0 \\
\hline vmin & Minimum group velocity value & 1.5 \\
\hline vmax & Maximum group velocity value & 5.0 \\
\hline tmin & Minimum period & 4.0 \\
\hline tmax & Maximum period & 30.0 \\
\hline tresh & $\begin{array}{l}\text { Threshold for second derivative of } \\
\text { group velocity }\end{array}$ & 10.0 \\
\hline ffact & Factor to width of FTAN filters & 1.0 \\
\hline taperl & $\begin{array}{l}\text { Factor for the left end seismogram ta- } \\
\text { pering }\end{array}$ & 1.0 \\
\hline snr & Minimum signal-to-noise ratio & 0.2 [Db] \\
\hline fmatch & Factor to compressed signal width & 1.0 \\
\hline
\end{tabular}

Table 3.3: Parameters used for AFTAN analysis. 

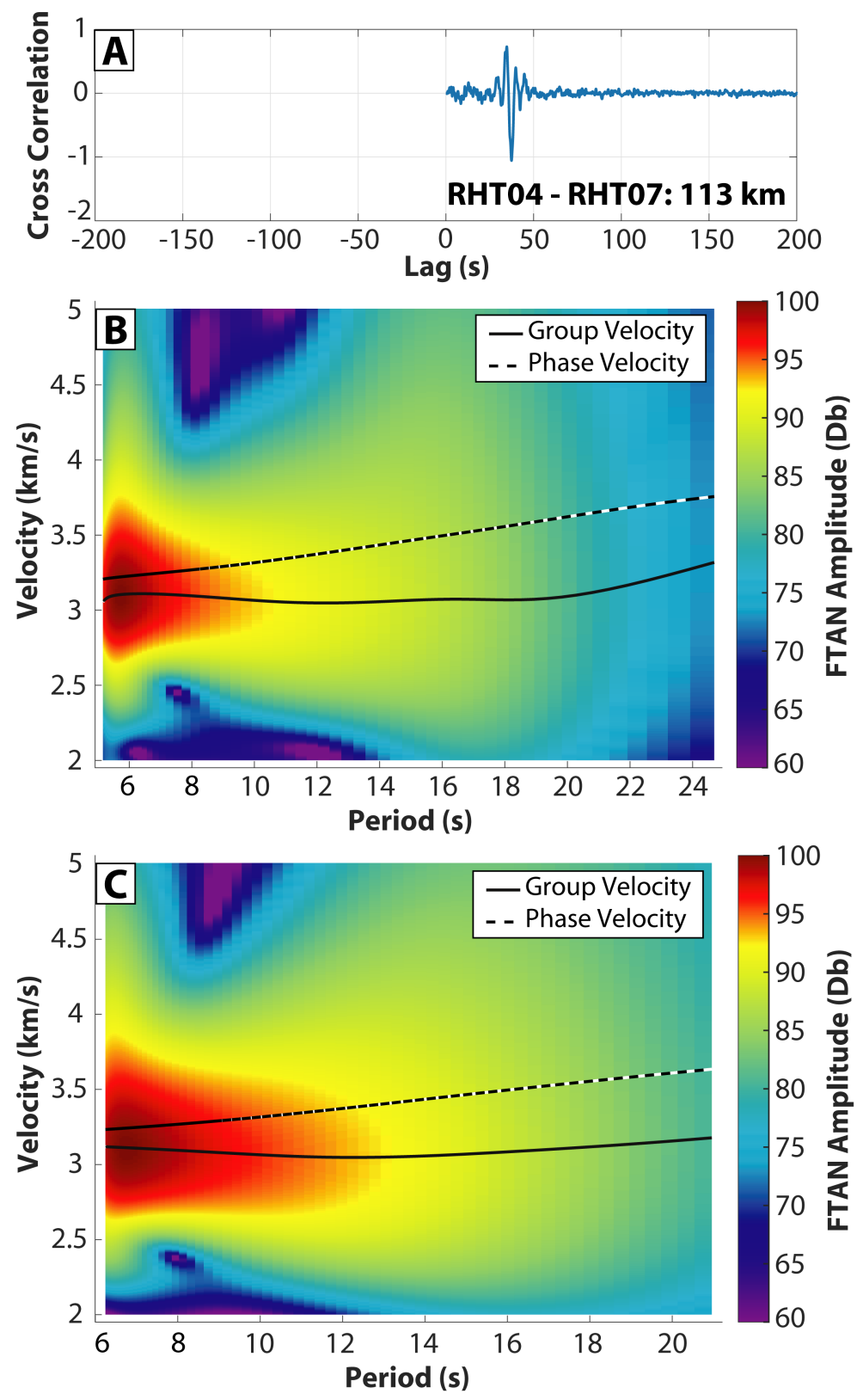

Figure 3.17: Effect of the phase-matched filter on obtained group velocity values. [A] Cross-correlation between Saudi Arabia stations RHT01 and RHT05. [B] FTAN and raw group velocity. [C] FTAN and cleaned group velocity after phase-matched filter. 


\subsubsection{Sensitivity Kernels}

The senskernel software package, developed by the University of Colorado Boulder, is a tool for determining group and phase velocity sensitivity kernels for a set of periods based on an input velocity model, and is available for free on the Center for Imaging the Earth's Interior (CIEI) “Research Products" webpage:

http:/ / ciei.colorado.edu/Products/senskernel-1.0.tgz

During inversions, a kernel $\left(G_{i}(r)\right)$ is used as an operator to correlate a change in an observable quantity $\left(\Delta d_{i}\right)$ to a change in a physical property $(\Delta m(r))$ [Stein and Wysession, 2003]:

$$
\Delta d_{i}=\int_{0}^{a} G_{i}(r) \Delta m(r) d r
$$

where $a$ is the distance along a ray path. Using the partial derivatives:

$$
\begin{gathered}
\frac{\delta c(T)}{\delta b}(r) \\
\frac{\delta U(T)}{\delta b}(r)
\end{gathered}
$$

where $c(T)$ and $U(T)$ are the phase and group velocity at a certain period and $b$ is the shear-velocity, we can describe the changes in phase and group velocities in response to shear velocity perturbation at each depth, where the resolved kernel peaks at the depth of highest resolution [Stein and Wysession, 2003].

To our knowledge, there currently aren't any publicly available P-wave or S-wave velocity models for Harrat Al-Madinah. However, certain areas of the $1000 \mathrm{~km}$ refraction survey conducted by Mooney et al. [1985] can be used to obtain an approximate value of depth resolution. We used the values of Shot Point 4 North-East, located at latitude-longitude point $20.09^{\circ}, 42.65^{\circ}$ [Mooney et al., 1985], to compute sensitivity kernels for Rayleigh and Love shear velocities [Figure 3.18]. We chose this particular shot-point as it was located near Harrat Al-Buqum, a location we expect to be similar in velocities to Harrat Al-Madinah. The shear-velocity was approximated to $60 \%$ of the recorded P-wave velocity [Stein and Wysession, 2003].

The maximum depth computed by the kernels is approximately $30 \mathrm{~km}$, with the largest peaks typically occurring within the top $15 \mathrm{~km}$ for periods of 4-14 seconds [Figure 3.18]. Assuming that this velocity model is relatively descriptive of the velocities of Harrat Al-Madinah, we can expect that the boundary between the first two crustal layers [Figure 3.18A] can be observed by our Rayleigh velocities at long periods [Figure 3.18B, C], while shallow structure can be determined by our Love velocities at short periods [Figure 3.18D, E]. 

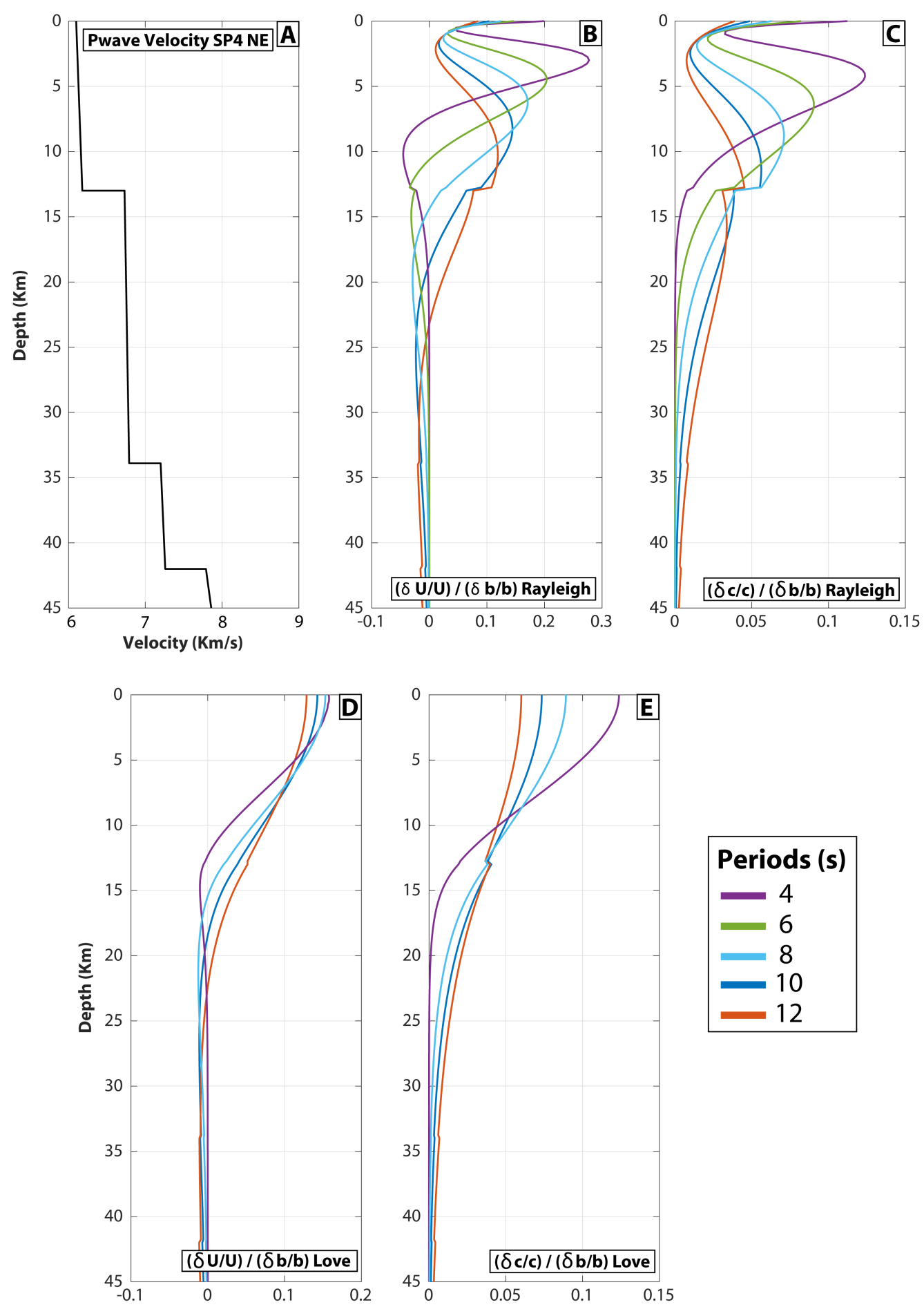

\section{Periods (s)}

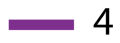

$-6$

8

10
-12

Figure 3.18: Sensitivity kernels computed for Shot Point 4 North-East from Mooney et al. [1985] (Their Figure 4). [A] Original P-wave model for Shot Point 4 North-East, [B] Rayleigh group velocity sensitivity kernel, [C] Rayleigh phase velocity sensitivity kernel, [D] Love group velocity sensitivity kernel, and [E] Love phase velocity sensitivity kernel. 


\subsubsection{Tomographic inversion}

We used the "nearly-linear" methods of Barmin et al. [2001] to estimate 2D dispersion maps for the Harrat Al-Madinah dispersion curves generated by AFTAN. In this section, we briefly discuss the theory of the code and the functions most relevant for the parameter selection use by our dataset. Refer to Barmin et al. [2001] for more detailed information.

The objective of the inversion method is to determine the vector function of the velocity perturbation $\mathbf{m}(\mathbf{r})$ :

$$
\mathbf{m}(\mathbf{r})=\left[m_{0}(\mathbf{r}), \ldots, m_{n}(\mathbf{r})\right]
$$

where $m$ is the velocity perturbation for an $n$ number of rays with surface position $\mathbf{r}=[\theta, \phi]$, where $\theta$ is the colatitude and $\phi$ the longitude. Assuming spherical geometry, we can define $\delta t$ as the travel time perturbation along a path $p$ of a ray:

$$
\begin{gathered}
\delta t=t-t_{0}=\int_{p} \frac{m}{c_{0}(\mathbf{r})} d s \\
m=\frac{c_{0}(\mathbf{r})-c(\mathbf{r})}{c(\mathbf{r})}
\end{gathered}
$$

where $c(\mathbf{r})$ is the velocity of the ray, $c_{0}(\mathbf{r})$ is the velocity of the ray for a $2 \mathrm{D}$ reference map, and $t_{0}$ is the origin time. We assume that the paths between $c_{0}(\mathbf{r})$ and $c(\mathbf{r})$ are the same. For an anisotropic solid, the $c(\mathbf{r})$ term splits into isotropic $\left(c_{I}\right)$ and anisotropic $\left(c_{A}\right)$ components:

$$
\begin{gathered}
c(\mathbf{r})=c_{I}(\mathbf{r})+c_{A}(\mathbf{r}) \\
c_{I}(\mathbf{r})=A_{0}(\mathbf{r}) \\
c_{A}(\mathbf{r})=A_{1}(\mathbf{r}) \cos (2 \psi)+A_{2}(\mathbf{r}) \sin (2 \psi)+A_{3}(\mathbf{r}) \cos (4 \psi)+A_{4}(\mathbf{r}) \sin (4 \psi)
\end{gathered}
$$

where $\psi$ is the local azimuth of the ray, $A_{0}$ is an isotropic coefficient, and $A_{1} \ldots A_{4}$ are anisotropic coefficients. This transforms Equation 3.18 into:

$$
m \approx \frac{c_{0}-c_{I}}{c_{I}}-\frac{c_{A}}{c_{I}^{2}} c_{0}
$$

From this, we define $d$ as the travel time residual using Equations 3.17 and 3.22:

$$
d=\delta t^{o b s}-t_{0}=\int_{p} \frac{m}{c_{0}} d s+\epsilon
$$


where $\epsilon$ is a combination of the model error and the observational error. The objective of the inversion is to use the travel time residuals $d$ to estimate the velocity perturbation $m$. For each path index $i$, we transform Equation 3.23 using a vector of linear functionals $G(m)$ :

$$
d_{i}=\delta t_{i}^{o b s}=G_{i}(m)+\epsilon
$$

The penalty function that is to be minimized is a summation of three terms:

$$
(G(m)-d)^{T} C^{-1}(G(m)-d)+\sum_{k=0}^{n} \alpha_{k}^{2}\left\|F_{k}(m)\right\|^{2}+\sum_{k=0}^{n} \beta^{2}\left\|H_{k}(m)\right\|^{2}
$$

where first function corresponds to the travel time fit with an input model, the second to spatial smoothing, and the last to the weight of the path density. The variables in the travel time time fit (first term) were discussed in Equations 3.17-3.24. The $C$ term is a convarience matrix of observational errors for each path $\epsilon_{i}$ [Equation 3.24].

The second term of the penalty function:

$$
\sum_{k=0}^{n} \alpha_{k}^{2}\left\|F_{k}(m)\right\|^{2}
$$

regulates the spatial smoothing of the function. The summation of index $k$ to $n$ adds the anisotropic components, but is not essential for our current discussion. We define the spatial smoothing condition $F_{k}$ and the smoothing kernel $S_{k}$ as:

$$
\begin{gathered}
F_{k}(m)=m_{k}(r)-\int_{S} S_{k}\left(r, r^{\prime}\right) m_{k}\left(r^{\prime}\right) d r^{\prime} \\
S_{k}\left(r, r^{\prime}\right)=K_{0 k} \exp \left(-\frac{\left|r-r^{\prime}\right|^{2}}{2 \sigma_{k}^{2}}\right)
\end{gathered}
$$

where $m_{k}$ is the velocity change for ray index $k$, where $n$ is the total number of rays. As can be surmised, the smoothing kernel in Equation 3.28 is a Gaussian smoothing filter, where $\sigma_{k}$ is the spatial smoothing width. In order to obtain meaningful results, $\alpha$ and $\sigma$ must be selected accordingly to the spacing of the dataset. In general terms, $\alpha$ represents the degree of amplitude smoothing, while $\sigma$ corresponds to the width of the Gaussian peak. We use the approximation outlined by Barmin et al. [2001], where $\sigma=2 l$, where $l$ is the separation distance between the nodes of the discrete grid, and $\alpha=4 \sigma$. The Harrat Rahat inversion was done with a node spacing of $0.1^{\circ}$ degrees (approximately $11 \mathrm{Km}$ at the equator), so $\sigma$ and $\alpha$ were set to 25 and 100 respectively.

The final term establishes the weight of the input model relative to the path density:

$$
\sum_{k=0}^{n} \beta^{2}\left\|H_{k}(m)\right\|^{2}
$$


where $H_{k}$ is the weighted norm of the model with weighing function $B$ that depends on path density $\rho(r)$ (isotropic) and azimuthal distribution $\chi(r)$ (anisotropic):

$$
H_{k}(m)=B(\rho(r), \chi(R)) m_{k}
$$

Generally, an appropriate dampening factor $B$ must be selected based on available ray paths. However, as we did not use a reference input model, this last term is not used in the penalty function.

In addition to producing dispersion maps, the Barmin et al. [2001] code automatically conducts a cone resolution analysis, which determines the minimum distance at which velocity anomalies can be resolved [Figure 3.19]. Based on the distribution of the resolution in our study area, we chose a maximum cone-resolution threshold of $35 \mathrm{~km}$ for all periods. In other words, any velocity measurement with a resolution cone-radius greater than $35 \mathrm{~km}$ is removed from the dispersion curve. The $35 \mathrm{~km}$ boundary is displayed as a single contour on every map presented in our dispersion results [Section 3.6.3]. Areas of resolution cone-radius greater than $50 \mathrm{~km}$ are not displayed in Figure 3.19 due to an exponential resolution decrease near the boundaries, which makes the resolution contours inside the field impossible to distinguish.

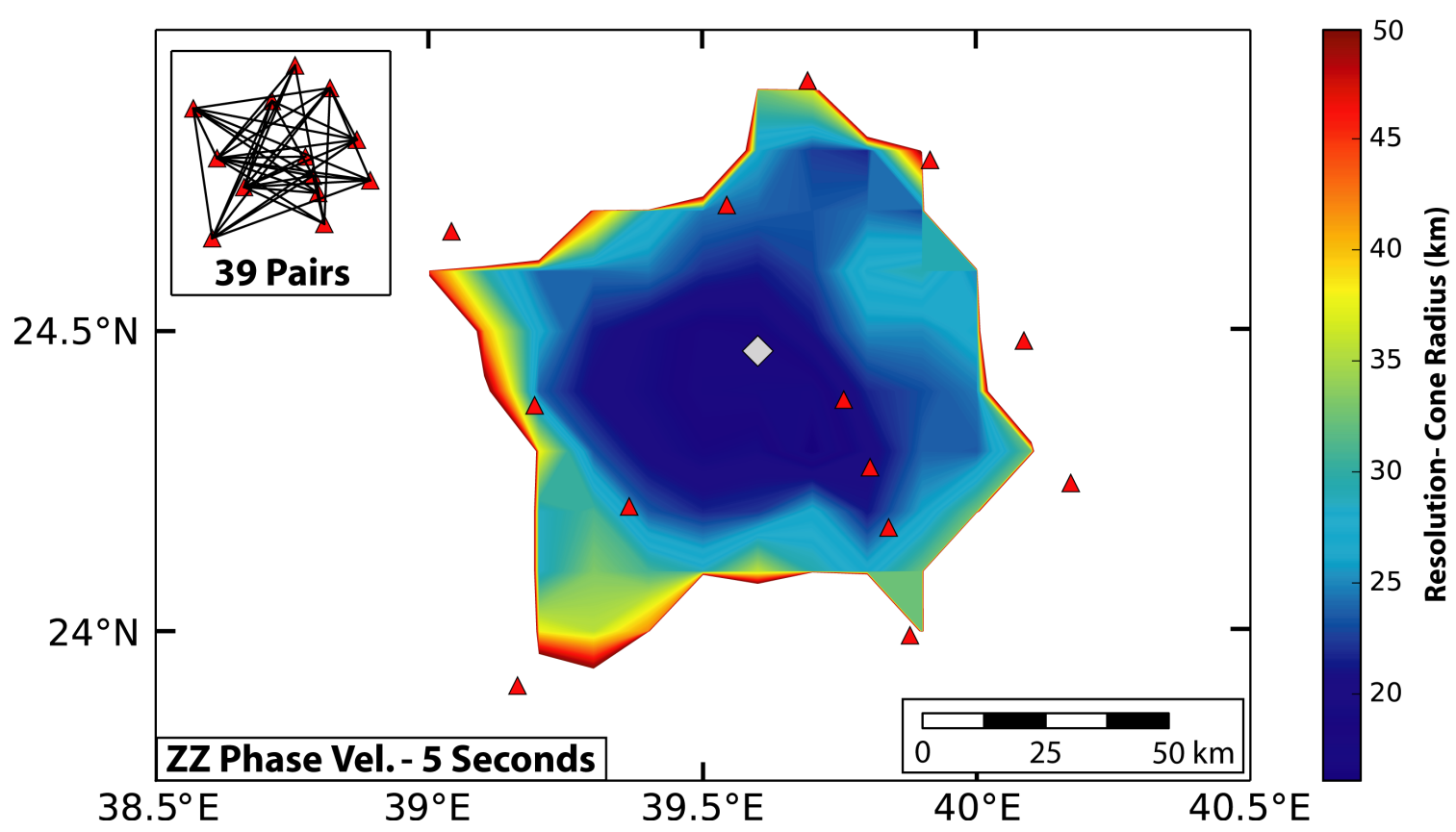

Figure 3.19: Cone-radius resolution plot for 5 second period for vertical-vertical (ZZ) phase velocities. The resolution exponentially decreases near the edges of the array where there are fewer cross-correlation pairs, so contours only up to $50 \mathrm{~km}$ are displayed here. The city of Medina is represented as a silver diamond. 


\subsection{Results}

\subsubsection{Cross-correlation moveouts}

Raw and filtered cross-correlations of station pairs were computed and stacked using the zero-point exclusion algorithm described in Section 3.5.1 [Figures 3.20]. The entire time period (one year) of data was stacked to compute these cross-correlations. Overlapping four month segments of cross-correlations were used to compute a standard deviation for the dispersion curves prior to shear-velocity inversion [Section 4.3.1].

A first order approximation of the group velocity was determined by measuring the lag time across the station distance for the filtered cross-correlation. We found that the radial-radial (RR), transverse-transverse (TT), and vertical-vertical (ZZ) components had group velocities of $3.0 \mathrm{~km} / \mathrm{s}, 3.4 \mathrm{~km} / \mathrm{s}$, and $3.1 \mathrm{~km} / \mathrm{s}$ respectively [Figure 3.20]. The higher velocity for the TT component suggests that the observed moveout corresponds to a Love wave, while the RR and ZZ components reflect the horizontal and vertical components of a Rayleigh wave. Note that only the cross-correlations that passed the signal-to-noise ratio threshold of 15 are displayed in Figure 3.20. 

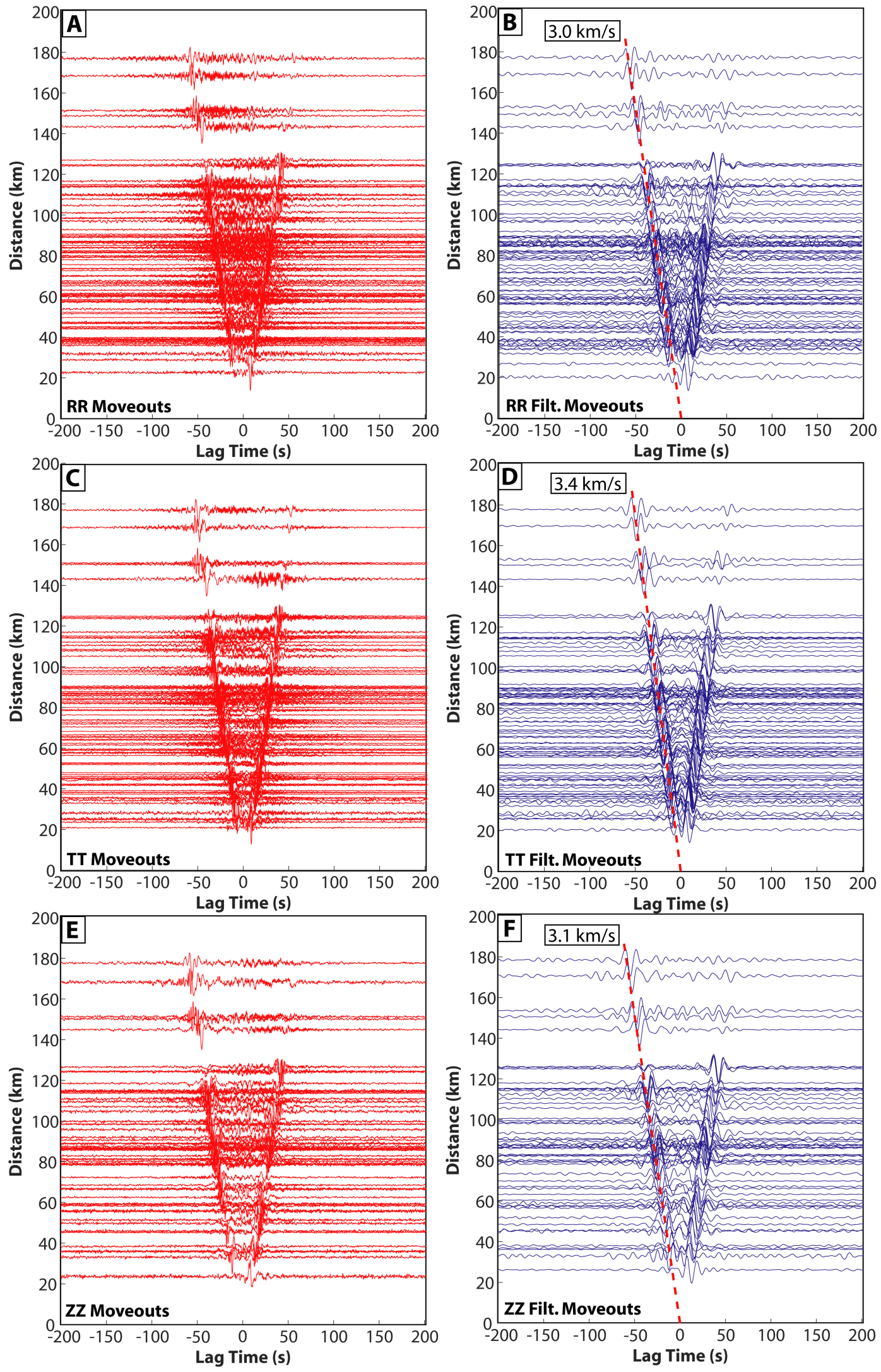

Figure 3.20: cross-correlation moveouts for Harrat Al-Madinah station pairs for unfiltered and $0.05-0.12 \mathrm{~Hz}$ filtered radial-radial (RR) [A, B], transverse-transverse (TT) [C, $\mathrm{D}]$, and vertical-vertical (ZZ) [E, F] components. 


\subsubsection{Checkerboard tests}

Checkerboard tests were conducted to asses the resolution of the tomographic results. In this method, station-pair velocities are computed across a synthetic geologic model, in this case a "checkerboard" of low and high velocities. The velocity values for these station pairs are inverted using the same method described in Section 3.5.4. The resulting dispersion map is then compared to the original synthetic geologic model. We chose checkerboards of sizes of 0.2-0.5 decimal degrees with alternating checkers of 3.0 and $3.5 \mathrm{~km} / \mathrm{s}$, which are approximately within the calculated phase velocity bounds of the tomographic results [Figures 3.27-3.29]. Velocities through the checkerboard were computed using Fatiando, an open-source Python library for geophysical inversions [Uieda et al., 2013]. Three figures of the checkerboard models are shown in Figures 3.21-3.23, comprised of the same number of station-pairs as the 5 second dispersion maps for each component. In order to better identify the differences in velocity values and boundaries, individual colorbars were chosen for each sub plot.

Although the total number of station pairs between the RR and TT checkerboard test is similar (67 and 65 pairs respectively), some of the pairs between the two datasets are different due to the signal-to-noise threshold (i.e., the same pair in different components can have different measures of SNR). Regardless, the two components produce identical results [Figures 3.21 and 3.22]. The checkerboard tests for the $\mathrm{ZZ}$ were able to reproduce virtually all boundaries observed for the RR and TT components with only minor differences to the velocity (less than $0.05 \mathrm{~km} / \mathrm{s}$ ) [Figure 3.23].

Although each checkerboard test suffers some degree of smearing, the images are less resolved for $0.2^{\circ}$ and $0.3^{\circ}$ tiles [Figures 3.21-3.23]. This matches up with the results of the cone-resolution test [Figure 3.19], which resolved a 15 to $30 \mathrm{~km}$ resolution for the field. However, the inverted velocities of the checkerboard test were very similar to the input model. The fastest velocity in the tomographic inversion with greatest difference from the input model [Figure 3.21B] is within $0.2 \mathrm{~km} / \mathrm{s}$ of the $3.5 \mathrm{~km} / \mathrm{s}$ input model, which represents approximately a $6 \%$ deviation. The slowest velocity with greatest difference from the slow velocity checkers [Figure $3.21 \mathrm{H}$ ] is within $0.4 \mathrm{~km} / \mathrm{s}$ of the 3.0 $\mathrm{km} / \mathrm{s}$ input model, which is a $13 \%$ deviation. 

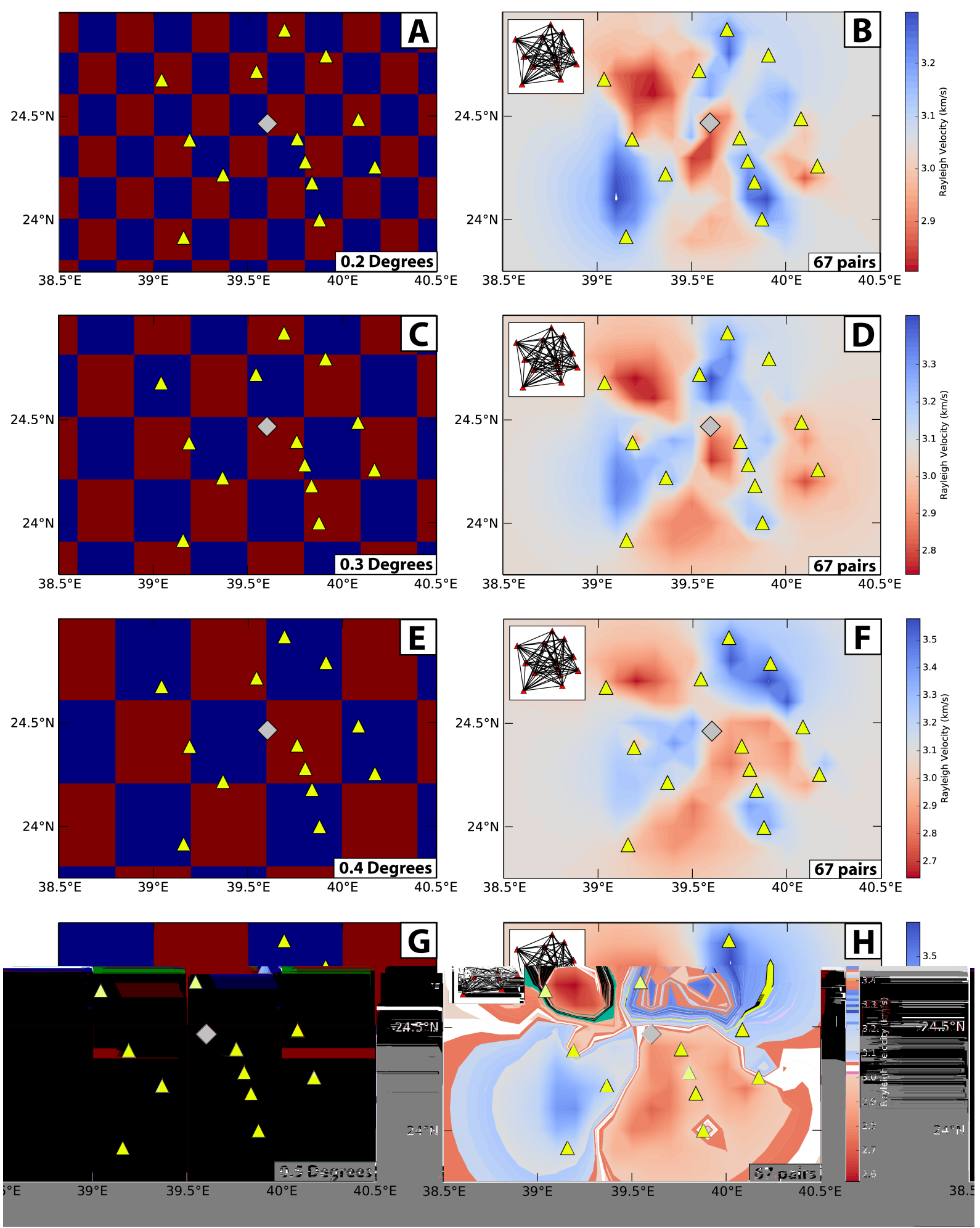

Figure 3.21: Checkerboard model and radial-radial (RR) tomography output for $0.2^{\circ}$ $[\mathrm{A}, \mathrm{B}], 0.3^{\circ}[\mathrm{C}, \mathrm{D}], 0.4^{\circ}[\mathrm{E}, \mathrm{F}]$, and $0.5^{\circ}$ tiles $[\mathrm{G}, \mathrm{H}]$. Checkerboard input model alternates between $3.0 \mathrm{~km} / \mathrm{s}$ (red) and $3.5 \mathrm{~km} / \mathrm{s}$ (blue) tiles. Harrat Rahat seismic stations and the city of Medina are displayed as yellow triangles and a silver diamond respectively. 

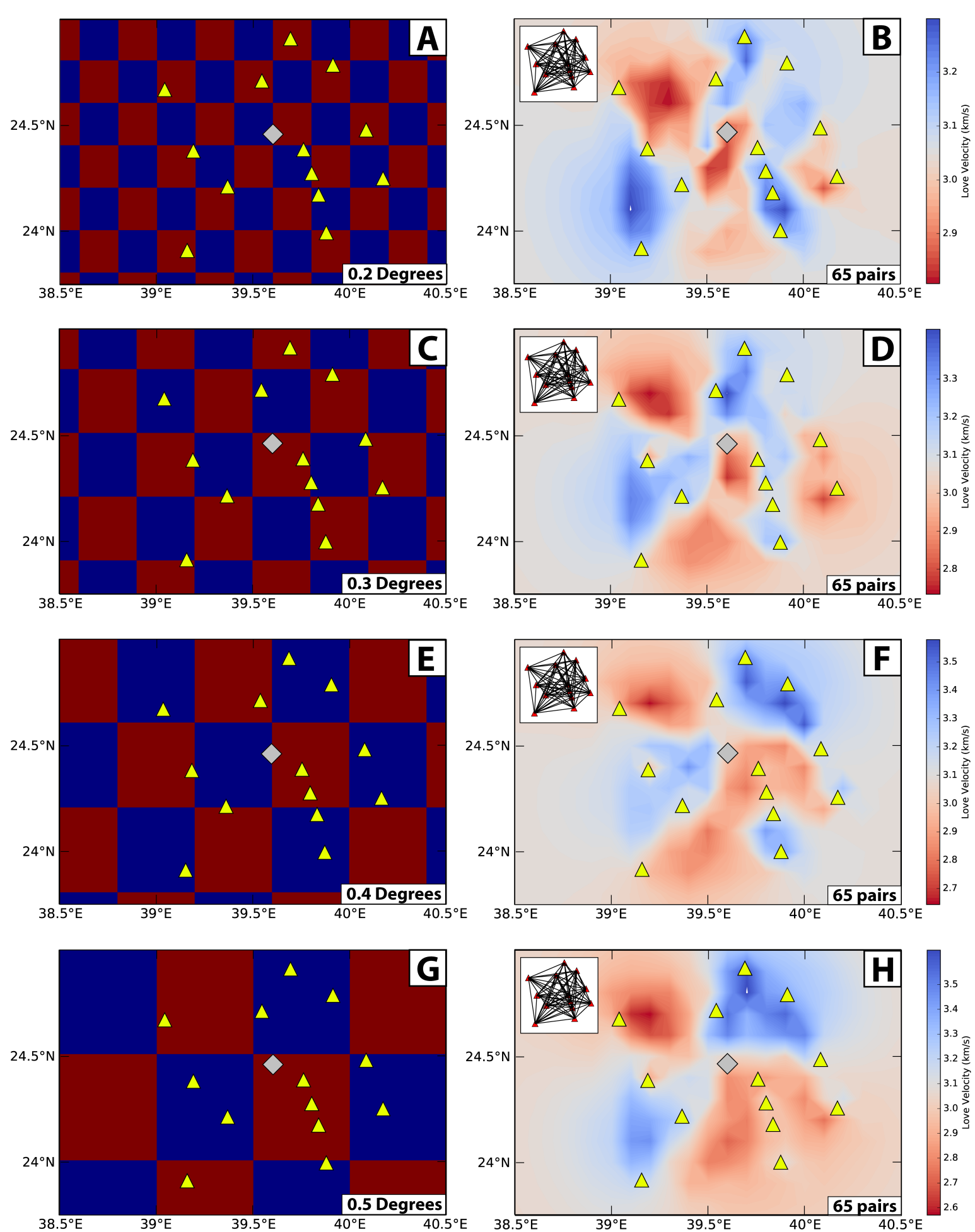

Figure 3.22: Checkerboard model and transverse-transverse (TT) tomography output for $0.2^{\circ}[\mathrm{A}, \mathrm{B}], 0.3^{\circ}[\mathrm{C}, \mathrm{D}], 0.4^{\circ}[\mathrm{E}, \mathrm{F}]$, and $0.5^{\circ}$ tiles $[\mathrm{G}, \mathrm{H}]$. 

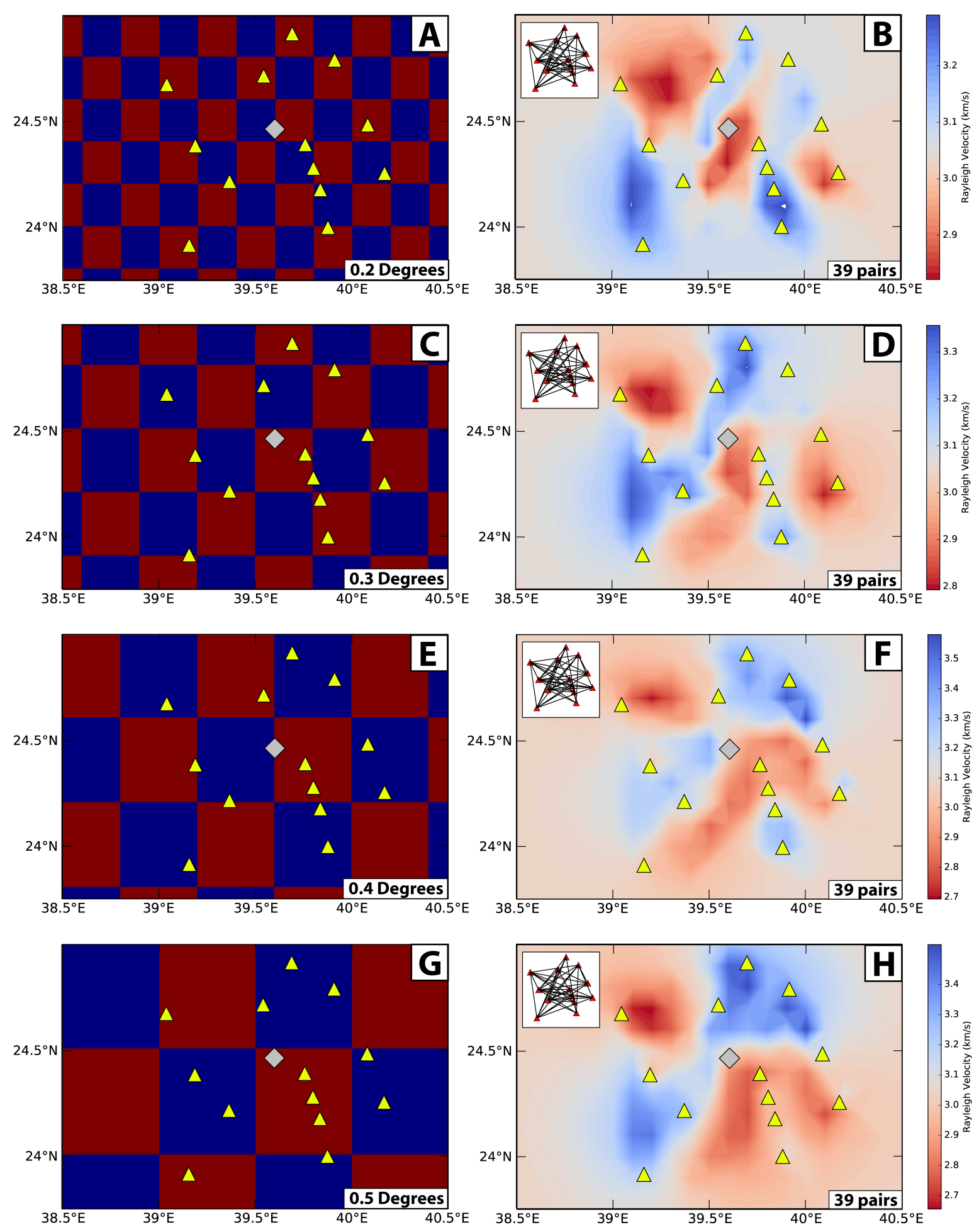

Figure 3.23: Checkerboard model and vertical-vertical (ZZ) tomography output for $0.2^{\circ}[\mathrm{A}, \mathrm{B}], 0.3^{\circ}[\mathrm{C}, \mathrm{D}], 0.4^{\circ}[\mathrm{E}, \mathrm{F}]$, and $0.5^{\circ}$ tiles $[\mathrm{G}, \mathrm{H}]$. 
Checkerboard tests can also be used in ambient noise studies to predict how the resolution may or may not improve when using additional stations. As previously described in the AFTAN theory section [Section 3.5.2], velocity retrieved from a crosscorrelation is an average across the entire station-path. The tomographic inversion is simply the discretization and averaging of path velocities into a grid [Section 3.5.4]. In other words, if station paths become larger, the accuracy at smaller scales may decrease.

We conducted more checkerboard tests using additional nearby stations of the Saudi Arabia Seismic network to see if the resolution of the measurements at Harrat AlMadinah would change [Figure 3.24]. Figure 3.25 displays the results of the checkerboard test at a regional scale and Figure 3.26 displays them at the local scale previously used for Figures 3.21-3.23. These checkerboard tests were conducted under the hypothetical that every available station-path was used in the inversion. We found that the new station configuration was unable to detect the smaller boundaries previously observed in our original Harrat Al-Madinah checkerboard results. This is most likely a result of the large inter-station paths averaging out smaller features, something that should be considered when doing local analysis of ambient noise results within largescale tomographic studies. 


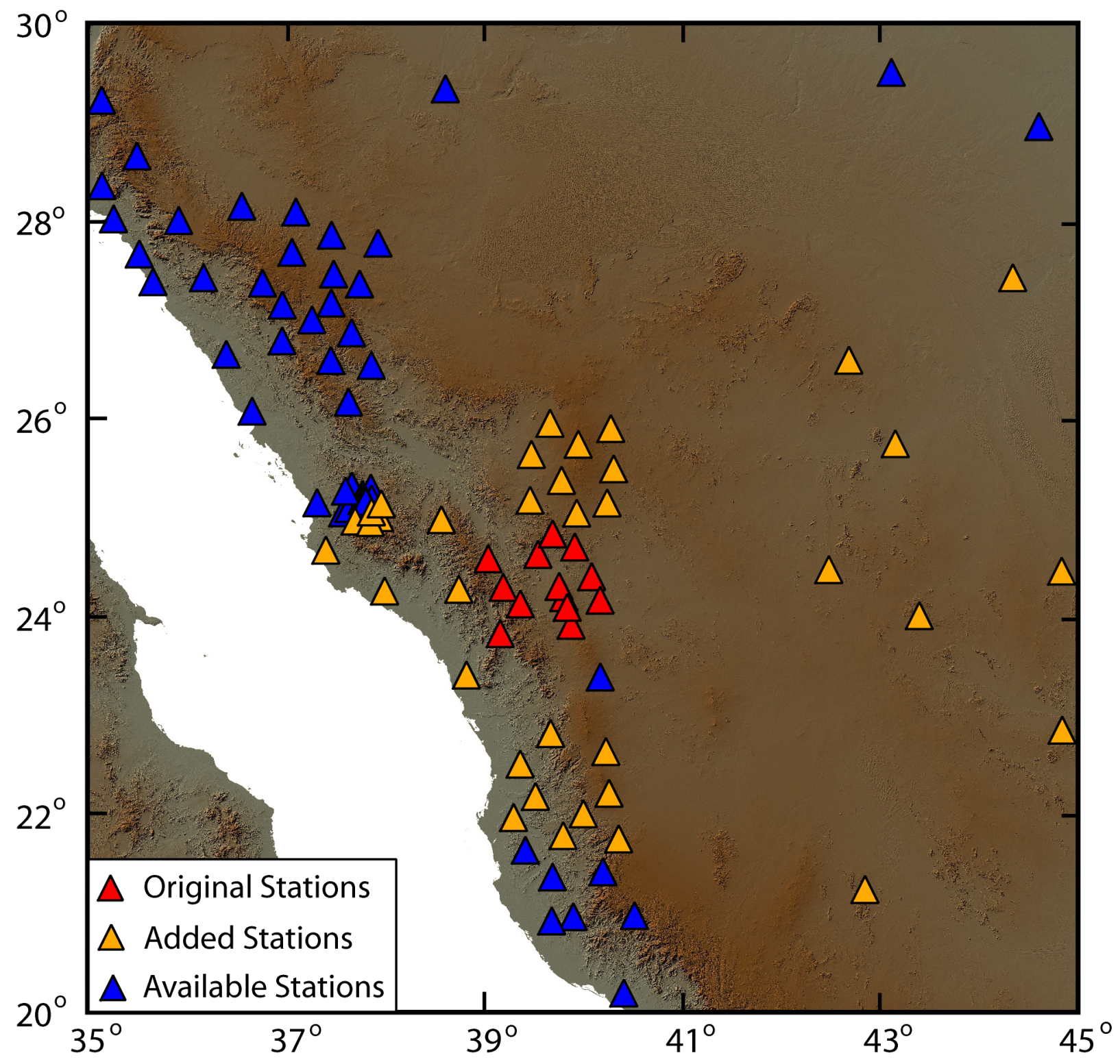

Figure 3.24: Map of the additional stations (orange triangles) used in the checkerboard tests of Figures 3.25 and 3.26 in conjunction with the original stations (red triangles) that were previously used in the checkerboard tests of Figures 3.21-3.23. The rest of the stations of the Saudi Seismic Network that were not used in our analysis are marked as blue triangles. 

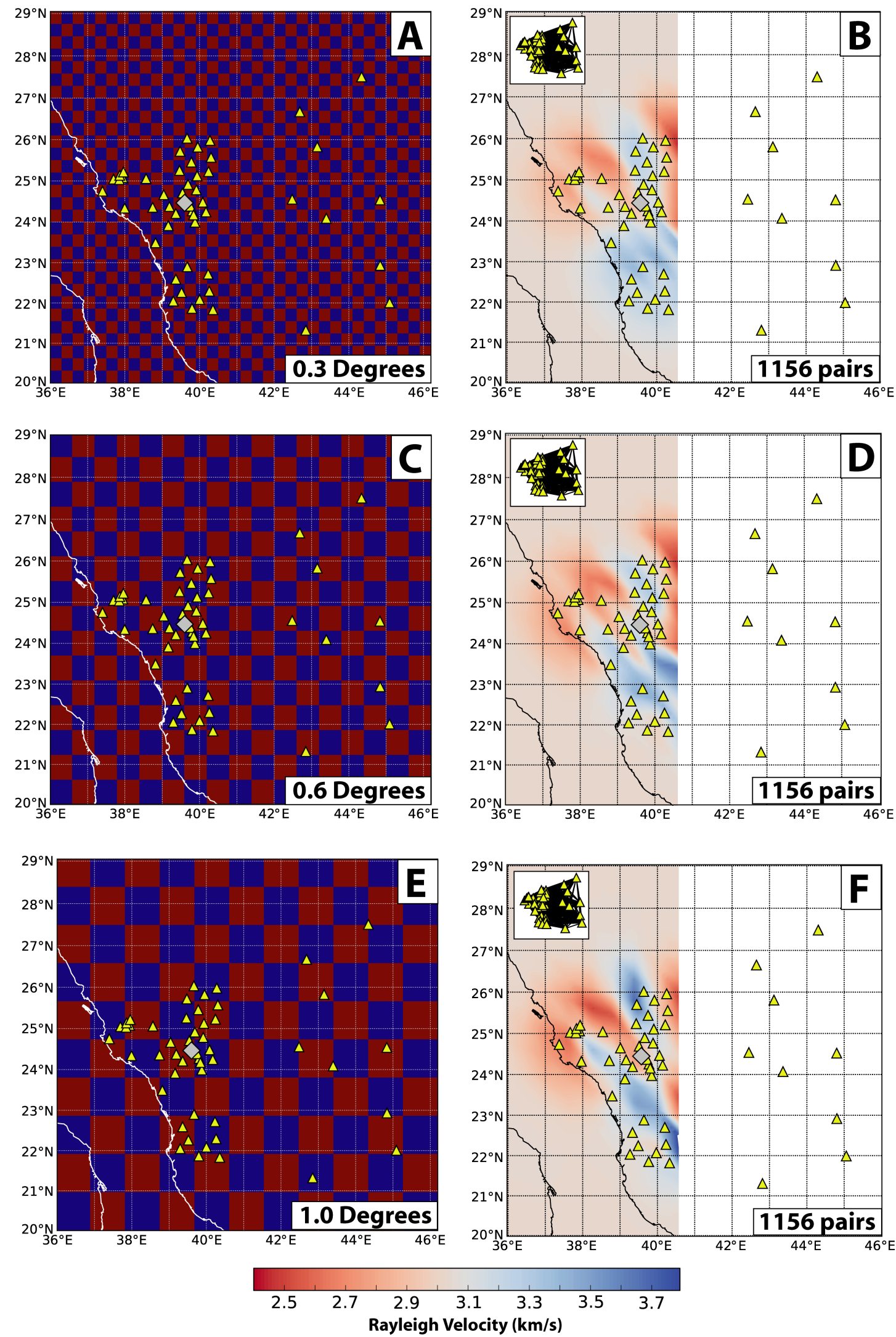

Figure 3.25: Checkerboard model and tomography output for $0.3^{\circ}[\mathrm{A}, \mathrm{B}], 0.6^{\circ}[\mathrm{C}, \mathrm{D}]$, and $1.0^{\circ}[\mathrm{E}, \mathrm{F}]$ tiles. 

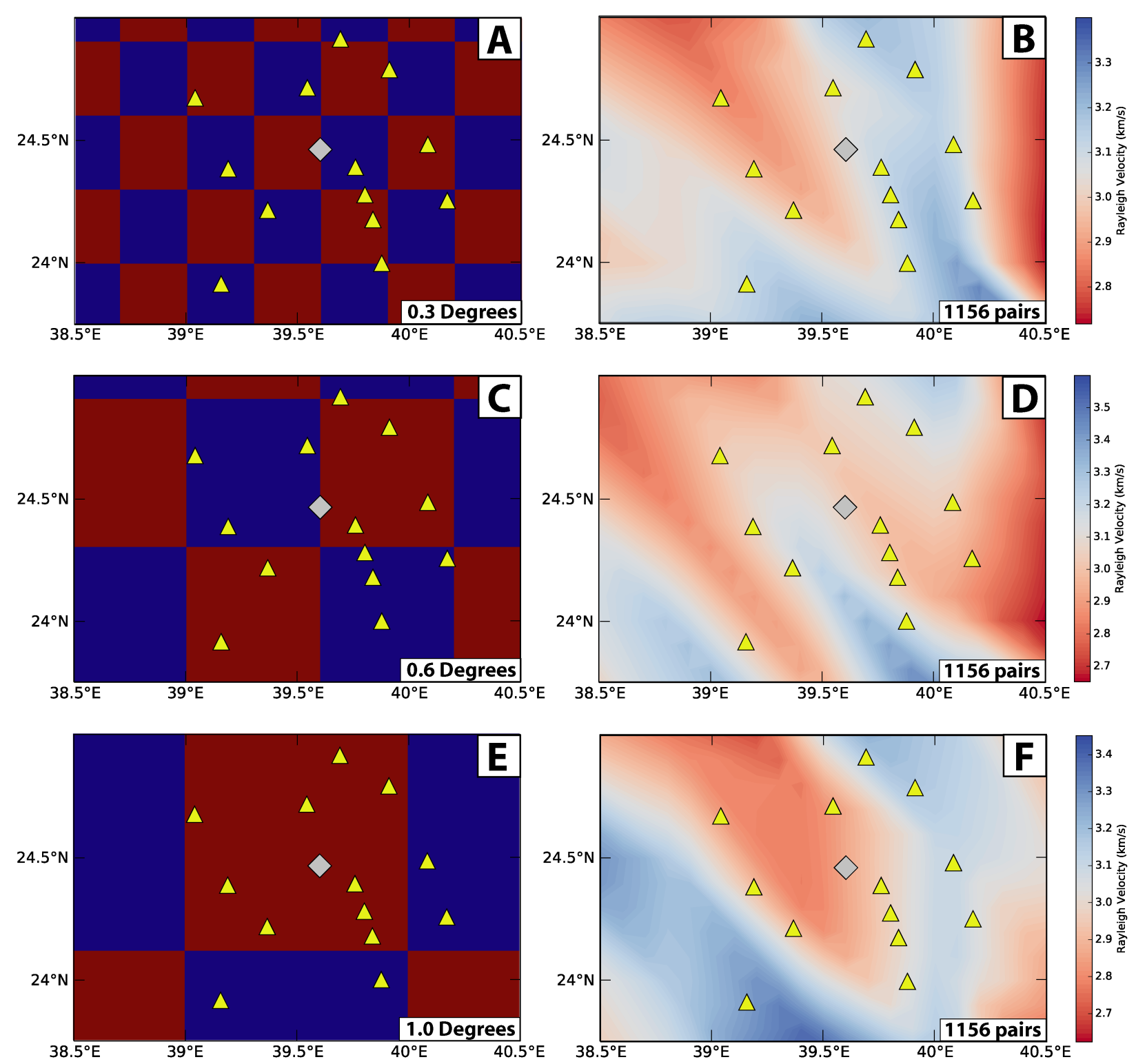

Figure 3.26: Checkerboard model and tomography output of Figure 3.25 for the Harrat Al-Madinah volcanic field using the additional stations described in Figure 3.24. The models use $0.3^{\circ}[\mathrm{A}, \mathrm{B}], 0.6^{\circ}[\mathrm{C}, \mathrm{D}]$, and $1.0^{\circ}[\mathrm{E}, \mathrm{F}]$ tiles alternating between $3.0 \mathrm{~km} / \mathrm{s}$ (red) and $3.5 \mathrm{~km} / \mathrm{s}$ (blue) velocities. 


\subsubsection{Dispersion maps}

Using the AFTAN procedure [Section 3.5.2] and the inversion methods of Barmin et al. [2001] [Section 3.5.4], we obtained dispersion maps of Harrat Al-Madinah for phase [Figures 3.27-3.29] and group velocities [Figures 3.30-3.32]. For each inversion, we display the used station paths in an inset on the top left of the frame and a gray contour corresponding to the $35 \mathrm{~km}$ cone-radius resolution [Figure 3.19]. RHT16, located at the bottom right of the array [Figure 3.11], had to be eliminated from the inversion due to consistent, unrealistically high surface wave velocities for all pairs. The location of the $1256 \mathrm{CE}$ eruption is labeled throughout the dispersion maps as a green box and will be shown as such throughout the rest of teh chapter.

There are areas of low velocity in both group and phase velocities that appear colocated with the $1256 \mathrm{CE}$ eruption [Figure 3.11]. These areas of low velocity are detected between 5 to 10 seconds in RR and ZZ phase velocities [Figures 3.27, 3.29], up to 9 seconds for TT phase velocities [Figure 3.28], and up to 13 seconds for all components of group velocities [Figures 3.30-3.32].

As may be expected, the contour representing the minimum resolution $(35 \mathrm{~km})$ changes for each period slice depending on the number of available station pairs. The cause of decreasing station paths is due to the 2 wavelength criteria previously established in Section 2.1.2. For example, the TT phase velocity contour greatly shrinks between 5 and 14 seconds as the number of pairs decreases from 65 to 13 [Figure 3.28A, F]. The contours provide the threshold for which latitude-longitude points will be used in the 1D shear-velocity inversion [Chapter 4]. For example, in Figure 3.28, points located in the south-east edge of the Harrat may only have dispersion curves reaching 8 or 9 second periods.

Phase velocities for RR and ZZ, displayed in Figures 3.27 and 3.29 using the same colorbar (approximately $2.9-3.5 \mathrm{~km} / \mathrm{s}$ ), are very consistent with one another, with distinctive low velocity zones detected south of Medina up to 10 seconds period. We found that ZZ group velocities [Figure 3.32] were approximately $0.2 \mathrm{~km} / \mathrm{s}$ higher than their RR counterparts [Figure 3.30], and could not be plotted using the same colorbar without losing contrast within certain tomographic slices. Additionally, the ZZ group velocities lacked the sharp boundaries observed in all other tomographic maps. Both the group and phase velocities for the TT component are approximately between 0.3 to $0.4 \mathrm{~km} / \mathrm{s}$ higher velocity than what is observed for the RR and $\mathrm{ZZ}$ components.

Similar low-velocity features can be observed between the TT and the RR and ZZ components of the phase velocity up to 10 seconds period. Boundaries between low and high velocity features were overall very consistent between phase and group velocities, especially in the RR component. RR phase and group velocities [Figures 3.27 and 3.30] imaged very similar low velocity features up to 10 seconds. The TT phase and 
group velocity plots [Figures 3.28 and 3.31] were similar up to 9 seconds period, but the group velocity plots were able to delineate features within the resolution threshold up to 14 seconds. Boundaries between low and high velocities for $\mathrm{ZZ}$ phase velocities [Figure 3.29] are more distinguishable than ZZ group velocities [Figure 3.32], but areas of low velocity south-east of Medina can still be observed for both up to 10 seconds period. 

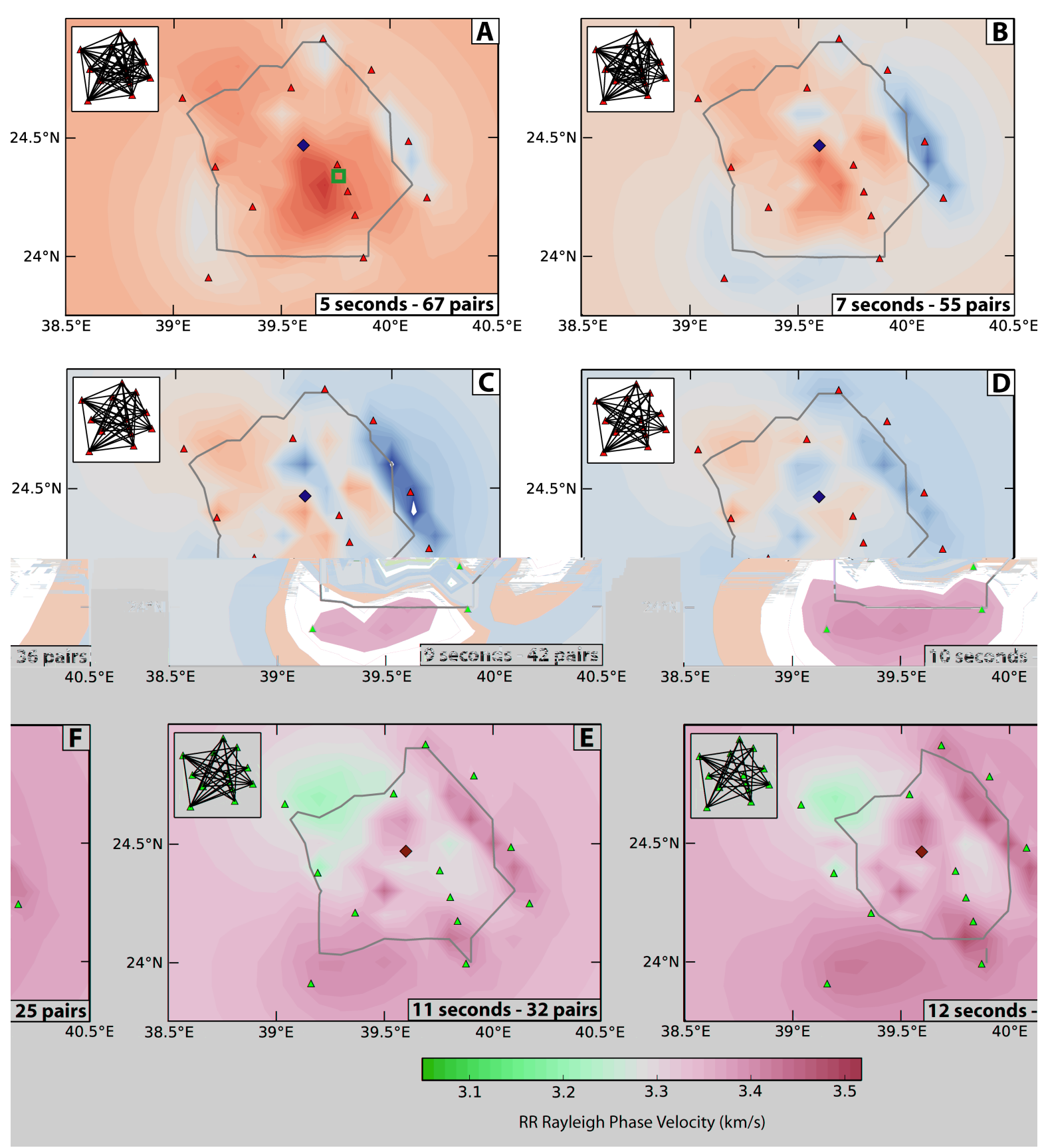

Figure 3.27: Dispersion maps for radial-radial (RR) phase velocities. The colorbar is scaled to the maximum and minimum values of all periods. The grey contour represents the $35 \mathrm{~km}$ resolution threshold of Figure 3.19. Harrat Rahat stations and the city of Medina are displayed as red triangles and a blue diamond, respectively. The location of the 1256 CE Medina eruption vents is displayed as a green box. Areas of white outside the resolution contour are anomalous values that were removed to prevent a skew to the color scaling. 

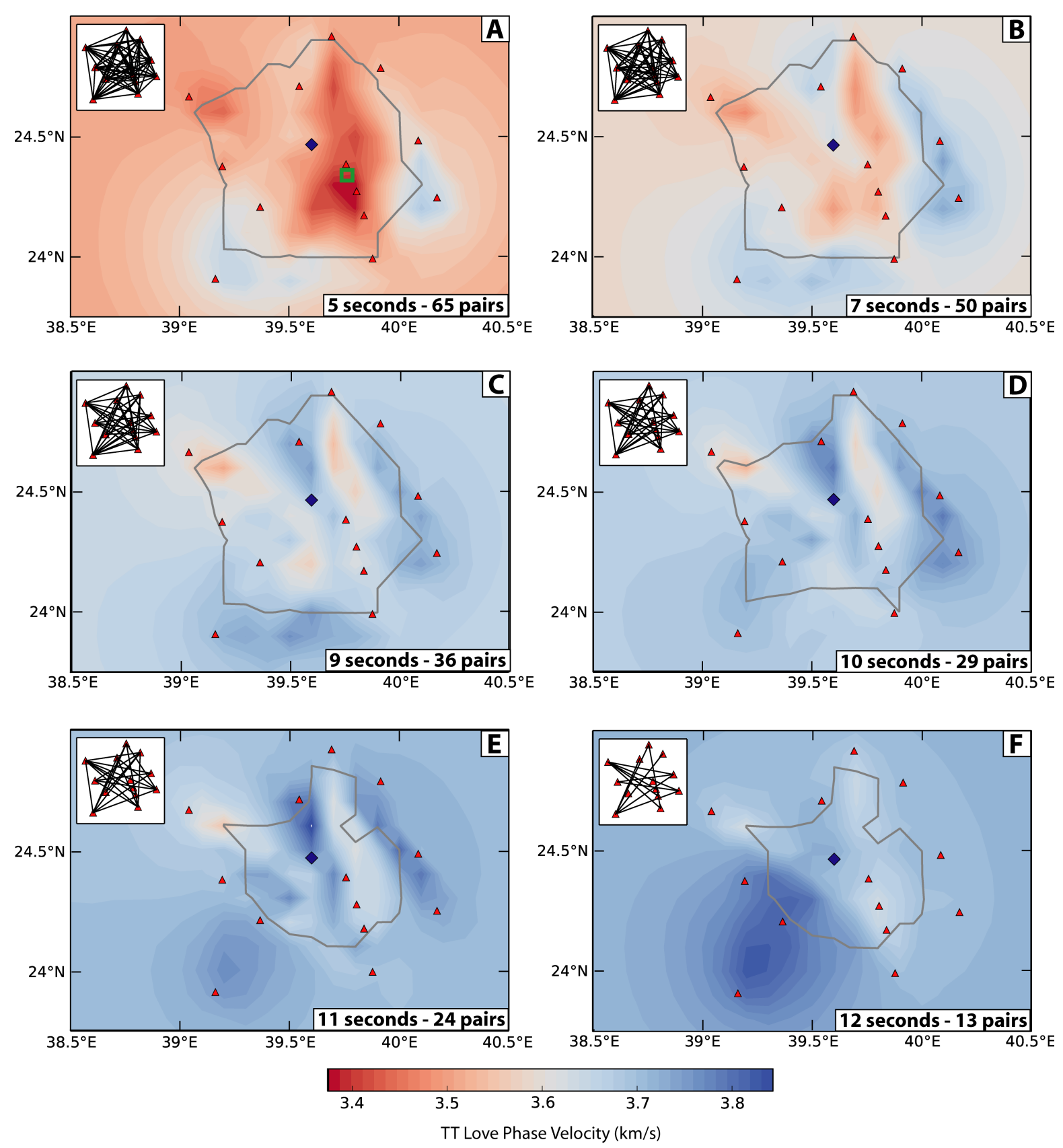

Figure 3.28: Dispersion maps for transverse-transverse (TT) phase velocities. Explanation same as Figure 3.27. 

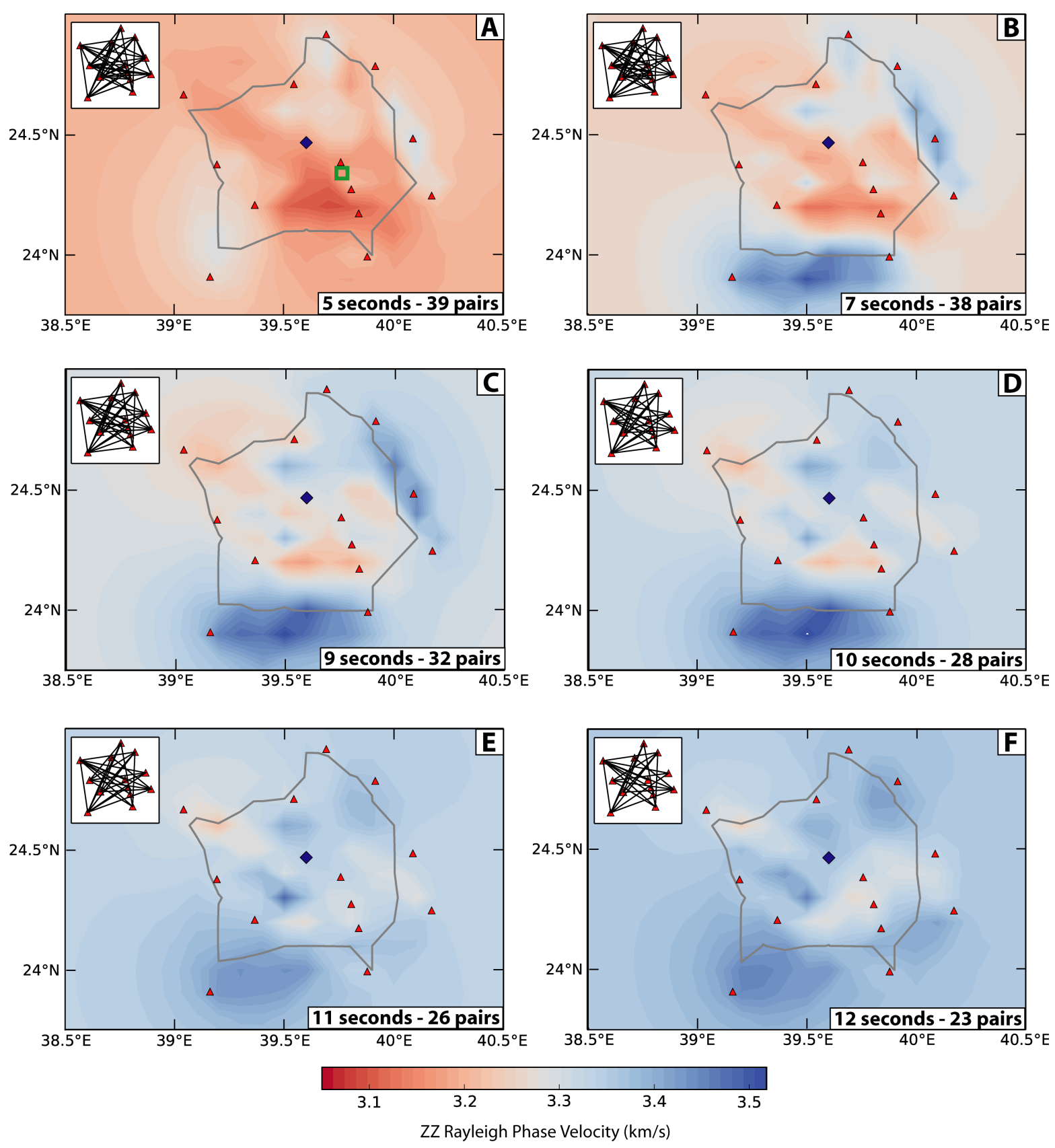

Figure 3.29: Dispersion maps for vertical-vertical (ZZ) phase velocities. Explanation same as Figure 3.27. 

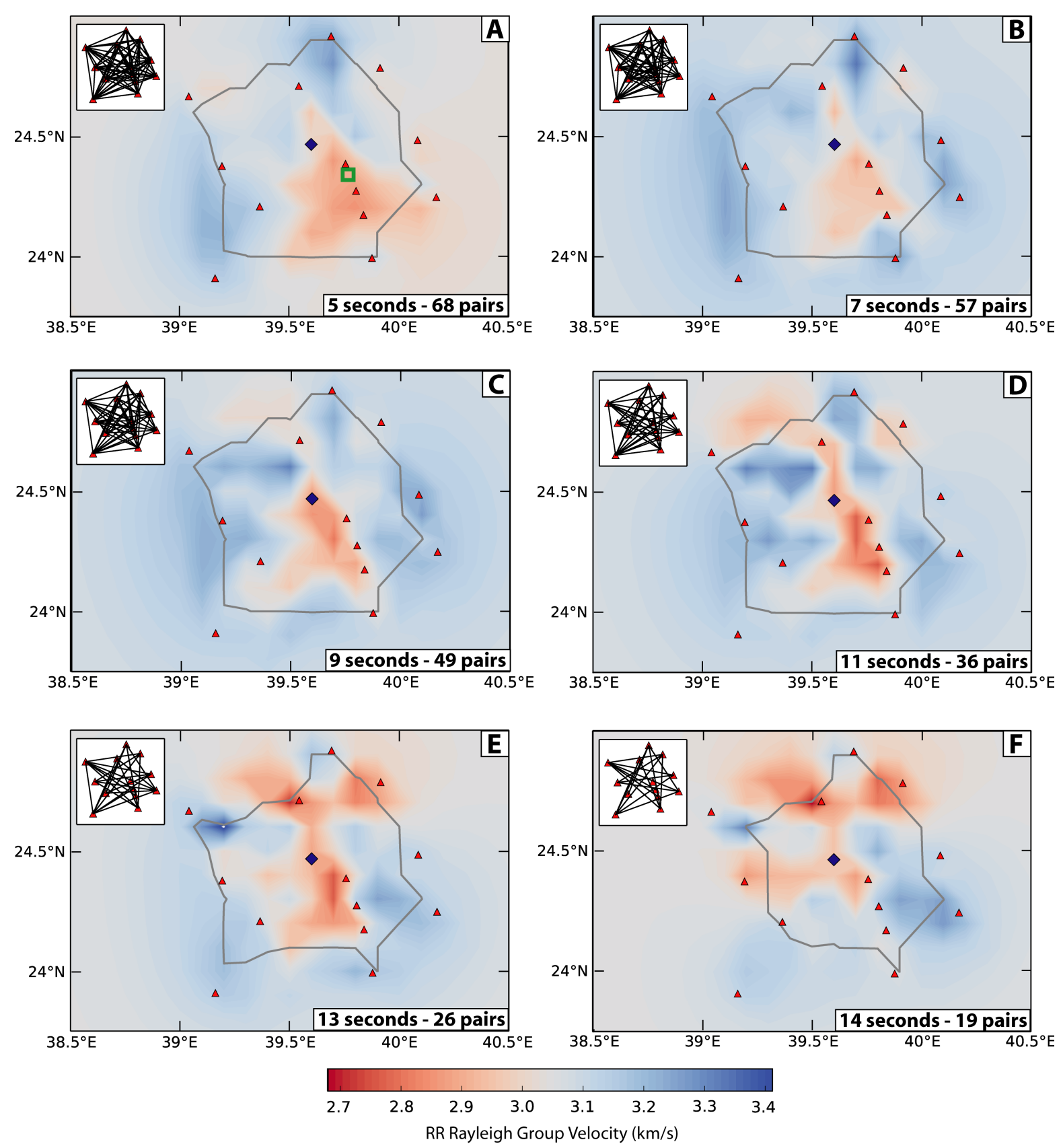

Figure 3.30: Dispersion maps for radial-radial (RR) group velocities. Explanation same as Figure 3.27. 

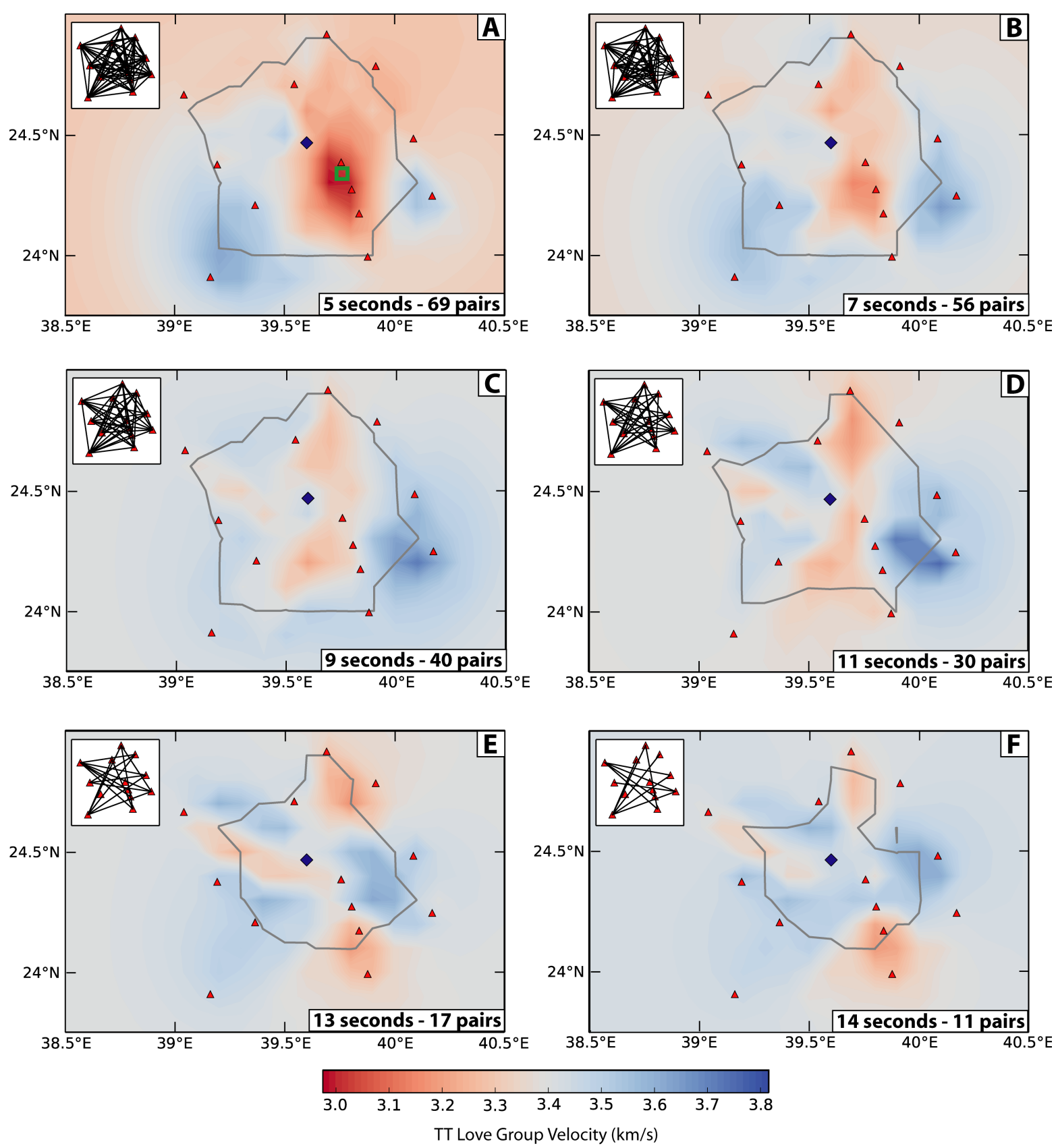

Figure 3.31: Dispersion maps for transverse-transverse (TT) group velocities. Explanation same as Figure 3.27. 

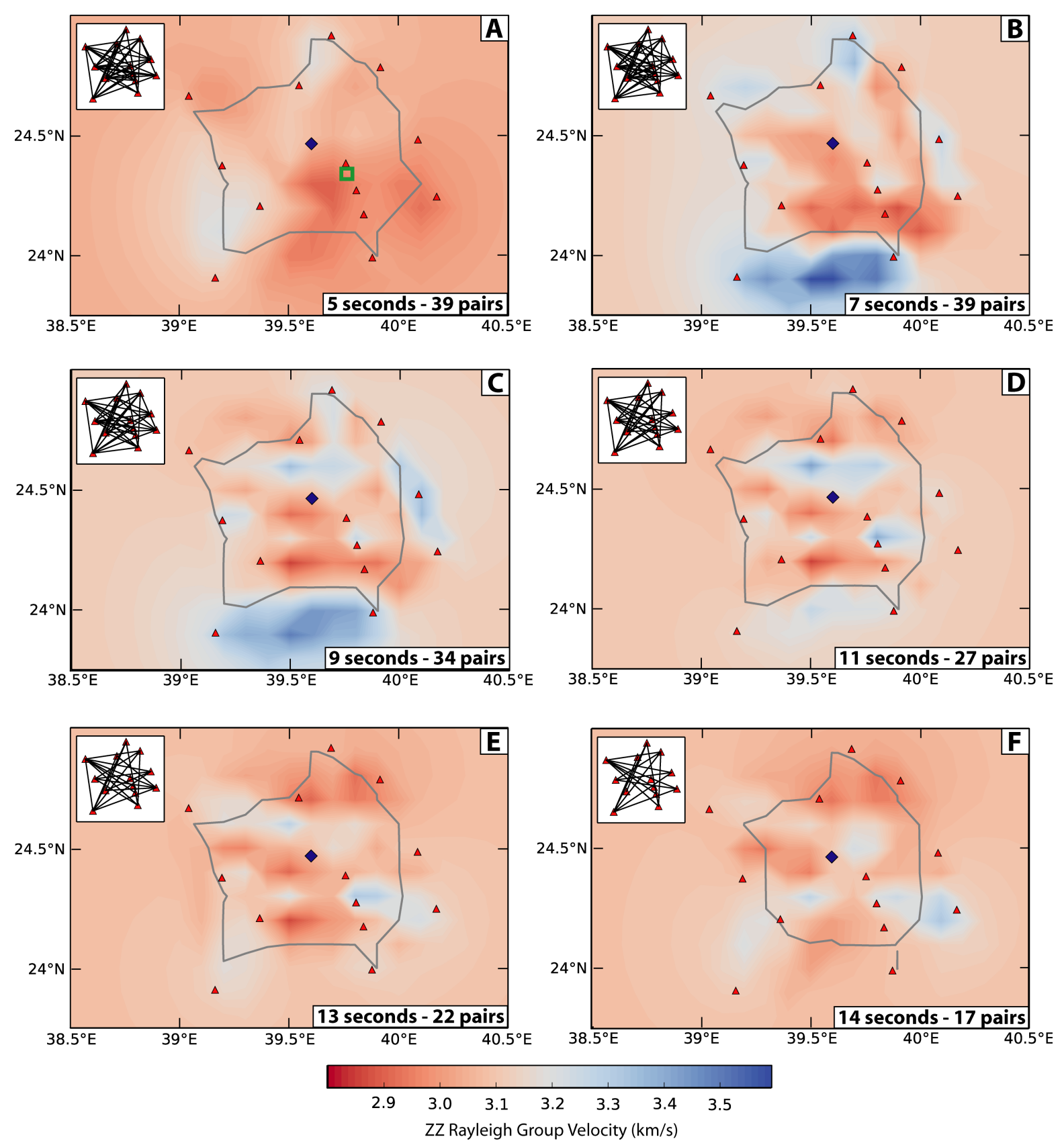

Figure 3.32: Dispersion maps for vertical-vertical (ZZ) group velocities. Explanation same as Figure 3.27. 


\subsubsection{Comparison with gravity measurements}

The US Geological Survey collected almost 300 gravity measurements in the Harrat AlMedinah area in November 2014 [Langenheim et al., 2016]. The measurements were processed to complete Bouguer gravity anomalies, which should reflect the density of the crust and changes in Moho depth [Turcotte and Schubert, 2002]. The chosen fixed point was the Madinah Technical College campus [Langenheim et al., 2016].

Figure 3.33 displays the gravity measurements on the 5 second RR, TT, and ZZ phase velocity dispersion maps. Unlike Figures 3.27-3.32, which were scaled using the same color bar to display the transition of low to high velocities for increasing periods, the dispersion maps of Figure 3.33 are scaled independently to highlight velocity contrasts. The gravity measurements trend high to low to high from west to east, and their minimum anomaly is co-located with the $1256 \mathrm{CE}$ historical eruption [Figure 3.33]. This corresponds to a high-low-high density sequence that may be observed as a fast-slow-fast surface wave velocity. Although a fast-slow-fast transition is observed in our data [Figure 3.33], it is quite difficult to compare the two, as the gravity values extend over the entirety of the slow velocity areas of the tomographic maps, and many of the gravity measurements lie outside the minimum resolution contour. However, the zone of minimum gravity is approximately co-located with the $1256 \mathrm{CE}$ eruption vent location, as shown by the areas of slow Rayleigh and Love phase velocity in the RR and TT components [Figure 3.33, green square]. Additionally, several low gravity anomaly values west of the city of Medina are collocated with a zone of low phase velocity for the $\mathrm{RR}$ and $\mathrm{ZZ}$ components. The low velocity zone may reflect sedimentary fill beneath the volcanic rocks, magma within the crust, density variations within the basement, or some combination of each [Langenheim et al., 2016]. 


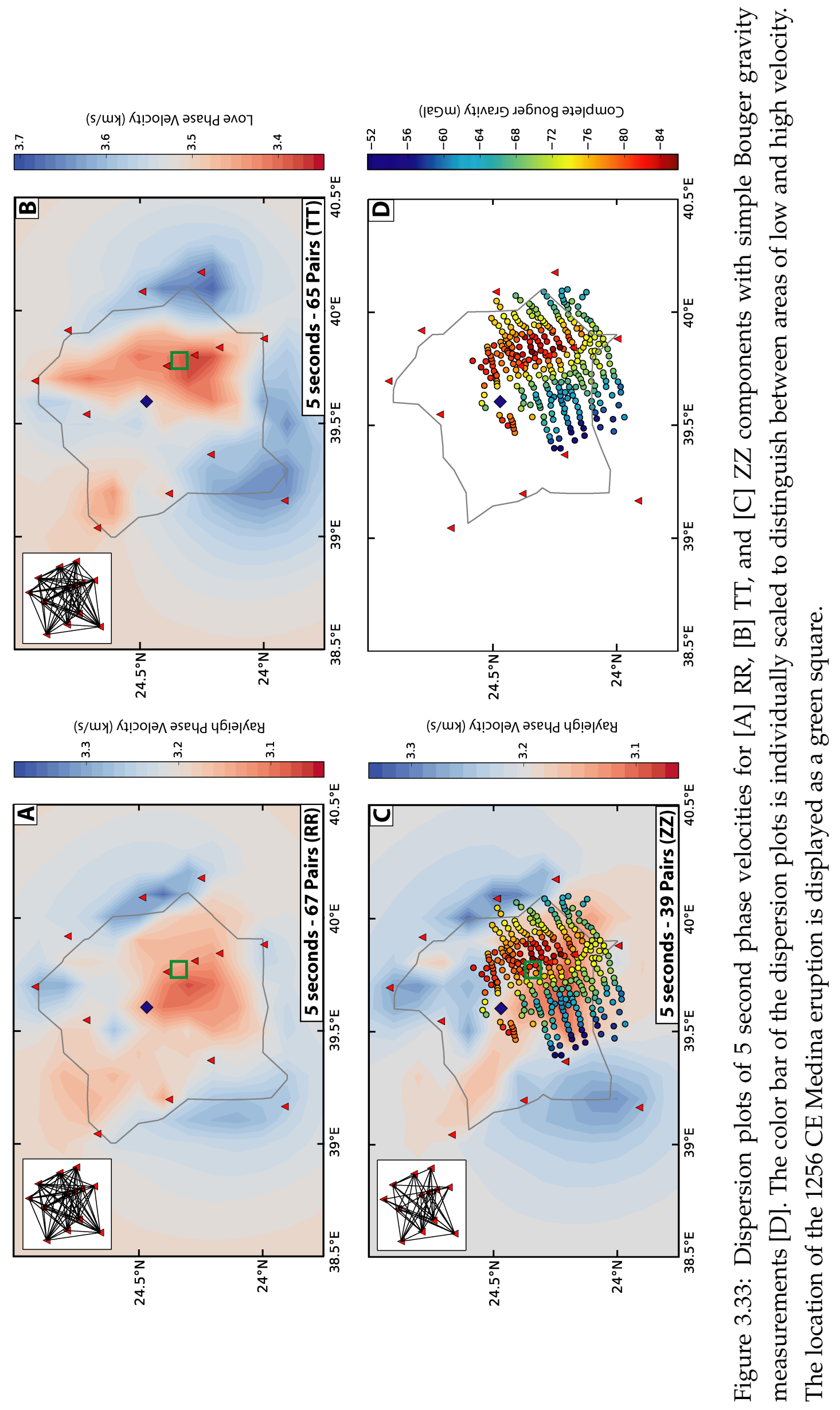




\subsection{Discussion}

The surface wave velocity maps displayed in Figures 3.27-3.32 are directly dependent on the quality of the AFTAN results. As described in Section 3.5.2, the AFTAN method utilizes a simple sliding filter using a Gaussian in the frequency domain [Levshin et al., 1989], so its accuracy is dependent on the signal-to-noise ratio of the crosscorrelations. The instruments used in this study were Trillium-120 broadband instruments, which have an effective frequency range of $0.01-100 \mathrm{~Hz}$ [Figure 3.12], and each station recorded consistent measurements of natural ambient noise at less than $1 \mathrm{~Hz}$ [Appendix A.2]. However, the signal-to-noise ratio for certain station-path correlations was negatively impacted due to the removal of zero-point noise [Figure 3.14]. As discussed in Section 3.5.1, this operation was absolutely necessary prior to the AFTAN, as unrealistically high measurements of shear-velocity would have been computed for long periods. In certain cases, zero-point noise was so prevalent that its removal brought down the cross-correlation below the signal-to-noise threshold. We found that the vertical-vertical (ZZ) component was more likely to be impacted by zero-point noise than the horizontals, and as a result, the tomographic maps of the $\mathrm{ZZ}$ component use less pairs (for example, Figures 3.27A and 3.29A). This suggests that the recorded zero-point noise may be due to teleseismic P-wave arrivals, as has been found in other studies [Landes̀ et al., 2010, Zhan et al., 2010].

Two resolution tests were conducted for the dispersion maps: a cone resolution test [Figure 3.19] [Barmin et al., 2001] and a checkerboard test [Figures 3.21-3.26]. Although the checkerboard resolution test computed between 20 to $30 \mathrm{~km}$ lateral resolution for most parts of the field, the checkerboard test was able to detect boundaries to much higher accuracy within the central parts of the field (e.g. Figure 3.21F). However, the resolution of the checkerboards greatly diminishes near the edges of the field for 0.2 and 0.3 decimal degree tiles.

All of the tomographic maps presented in Figures 3.27-3.32 resolved a zone of slow velocity south-east of the city of Medina which appears to extend across the Harrat [Figure 3.6] and the location of the 1256 CE Medina eruption (e.g. Figure 3.30). The velocity maps, transitioning across high-low-high velocities west to east, follow a similar trend to gravity anomalies in the region [Langenheim et al., 2016] [Figure 3.33]. Several of the dispersion maps contained additional areas of low velocity to the northwest (e.g. Figure 3.29A), which may be due to edge effects. However, a gravity low was detected west of the city of Medina [Figure 3.33D], which could be related to the low velocity area. 


\subsection{Conclusion}

In this chapter, we used ambient seismic noise to obtain the first surface-wave dispersion maps of the shallow crust beneath Harrat Al-Madinah, the northern extent of Harrat Rahat, the largest Cenozoic volcanic field in Saudi Arabia. The primary geologic features in our study area are basalts and related volcanic rocks of the Harrat located in the south-east of our array, which overlie Precambrian basement [Figure 3.6]. A year of data was recorded at the Harrat Rahat seismic array and used to determine crosscorrelations of repeating natural microseismic noise, which are analogous to empirical Green's functions of the station path. The cross-correlations were inverted to surface wave velocity maps, which resolved low-velocity areas co-located with the Madinah Basalt and the $1256 \mathrm{CE}$ Medina eruption. However, additional low-velocity areas were observed in some surface wave maps in the North-West of the array, which may be explained by lack of station coverage or resolution errors.

This project was part of an overall joint effort by the U.S. Geological Survey and the Saudi Geological Survey to characterize the hazard of the Saudi Arabian shield. This work is continued in Chapter 4, where we invert the dispersion curve at each grid-point of the surface-wave maps to obtain a pseudo-3D shear-velocity model of the Harrat. 


\section{Chapter 4}

\section{Shear-wave tomography of Harrat Al-Madinah}

\subsection{Introduction}

In this chapter, we expand from the surface-wave dispersion analysis conducted in Chapter 3 to determine shear-velocities for Harrat Al-Madinah. We obtain 1D inversions for each latitude-longitude grid-point in the volcanic field and use them to compute average velocity models, a pseudo-3D model, and cross-sections. This chapter will primarily focus on results and discussion, as the geology of the region and other background information were previously presented in Chapter 3.

\subsection{Methods}

Our methodology has three main steps: [1] The velocity measurement at each surfacewave map grid-point is combined for each period into a dispersion curve, [2] the gridpoint dispersion curve is inverted into 1D shear-velocity using the neighborhood algorithm [Sambridge, 1999], and [3] each 1D inversion is interpolated into a pseudo-3D model.

Dispersion maps are velocity measurements according to period, and can be thought of as an averaged horizontal cross-section through the earth. As observed in the surfacewave velocity maps [Section 3.6.3], resolution decreases with increasing period due to a lack of station-paths from the two-wavelength minimum threshold. Therefore, we only chose the periods at each grid-point that matched the $35 \mathrm{~km}$ minimum resolution discussed in Figure 3.19. In practical terms, it means that the dispersion curve at gridpoints in the center of the field (where there is most overlap of paths) may extend for longer periods than a dispersion curve for a grid-point at the edge of the field.

We used the Dinver software package to invert the dispersion curves to shear- 
velocity profiles at each latitude-longitude point for each component. We present four inversions for each grid point: radial-radial (RR), transverse-transverse (TT), verticalvertical (ZZ), and a joint inversion. Dinver is an open-source tool for geophysical research developed by the Geopsy project, a team of researchers of various European institutions first established during the SESAME European project in 2005 [Wathelet, 2005]. It incorporates the neighborhood algorithm of Sambridge [1999], which preferentially samples regions near values of good fit instead of utilizing the random distribution of Monte Carlo methods. This algorithm was expanded to include irregular limits (i.e., imposed geological boundaries) and developed into an open-source code by Wathelet [2008]. Each automatic inversion yields models based on minimizing the misfit values for each model. The layer parameters chosen for the inversion are listed in Table 4.1, and are based on the results of the refraction survey conducted by Mooney et al. [1985], previously discussed in Section 3.3. The inversion routine computed a total of 50,000 models for each latitude-longitude point.

\begin{tabular}{||l||l||l||}
\hline Layer & Velocity $\mathbf{( m / s )}$ & Interface Depth $\mathbf{( m )}$ \\
\hline Layer 1 & $150-3000$ & $150-3000$ \\
\hline Layer 2 & $1000-3800$ & $1000-6000$ \\
\hline Layer 3 & $3000-4000$ & $12000-30000$ \\
\hline Halfspace & $3500-4800$ & \\
\hline
\end{tabular}

Table 4.1: Parameters chosen for the Dinver inversion.

A standard deviation for each dispersion curve was determined using four-month stacked increments of cross-correlations [Figure 4.1]. Plots of the dispersion curves for each of the 4 month increments can be found in Appendix A.3. The values of standard deviation were used in the Dinver inversion routine to compute the model misfit $\left(M_{f i t}\right)$ [Wathelet, 2005]:

$$
M_{f i t}=\sqrt{\sum_{i}^{N} \frac{1}{N} \frac{\left(x_{i}-x_{i, s y n}\right)^{2}}{\sigma_{i}^{2}}}
$$

where $x_{i}$ and $x_{i, s y n}$ are observed and synthetic surface wave velocities respectively and $\sigma_{i}$ is the observational error for periods with index $i$ to $N$.

Figure 4.2 displays the total number of models produced for a sample inversion profile of latitude-longitude point $24.5^{\circ}, 39.5^{\circ}$. Each computed model has an associated misfit value representing the percentage difference from the starting dispersion curve. We computed a weighted mean of the inversion models [Figure 4.2 white line] using a misfit threshold of 0.1 (i.e., 10\% deviation from the starting dispersion curve). The models outside the misfit threshold are displayed as gray lines. The weighted mean of 

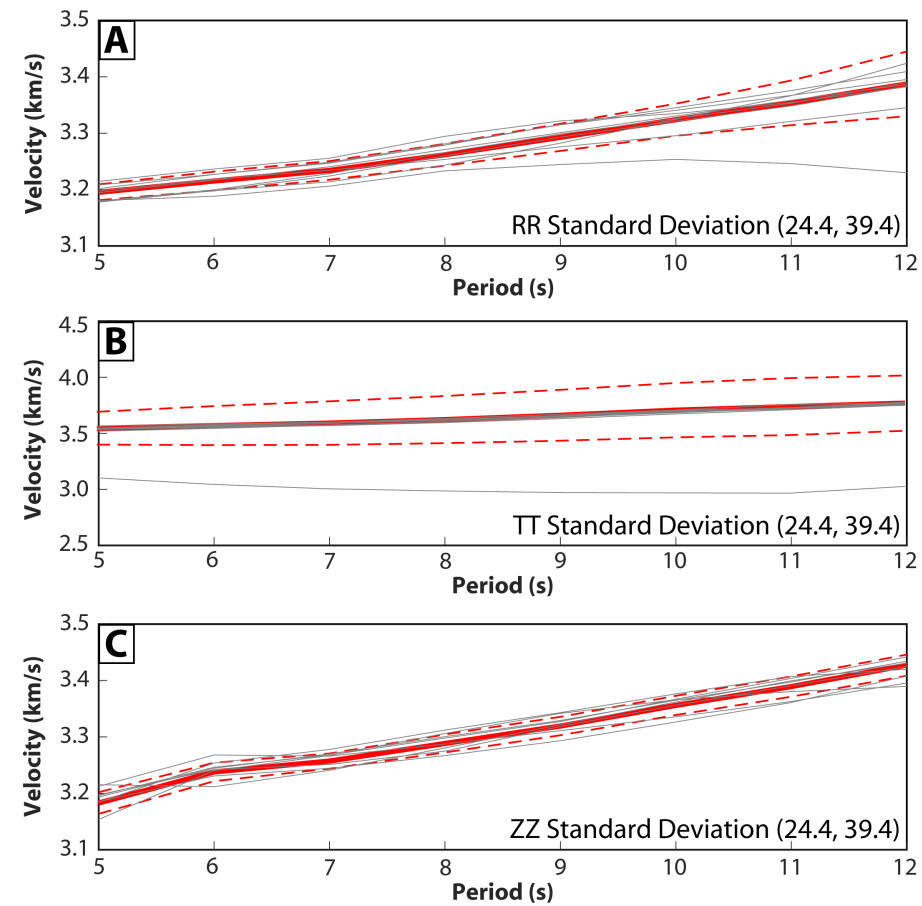

Figure 4.1: Standard deviation (red dotted line) for latitude-longitude point $24.4^{\circ}, 39.4^{\circ}$ using four-month increments. Each four-month dispersion curve is displayed in grey, and the full 12-month dispersion curve is represented by a red solid line.

the thousands of models is a way to assess the likelyhood of an inverse solution in a comprehensive manner.

Lastly, the weighted mean from each latitude-longitude point was interpolated into a pseudo-3D model using the software ParaView [Ahrens et al., 2005]. Paraview is an open-source package developed by Los Alamos National Laboratory and Kitware Inc. to both visualize and conduct analysis of large datasets, and can be downloaded free of charge [www.paraview.org]. We used ParaView to conduct 3D Deauleny interpolation [Delaunay, 1934] on the weighted mean values for each latitude-longitude depth, and then used their "slice" tool to obtain vertical and horizontal cross-sections of the model. 


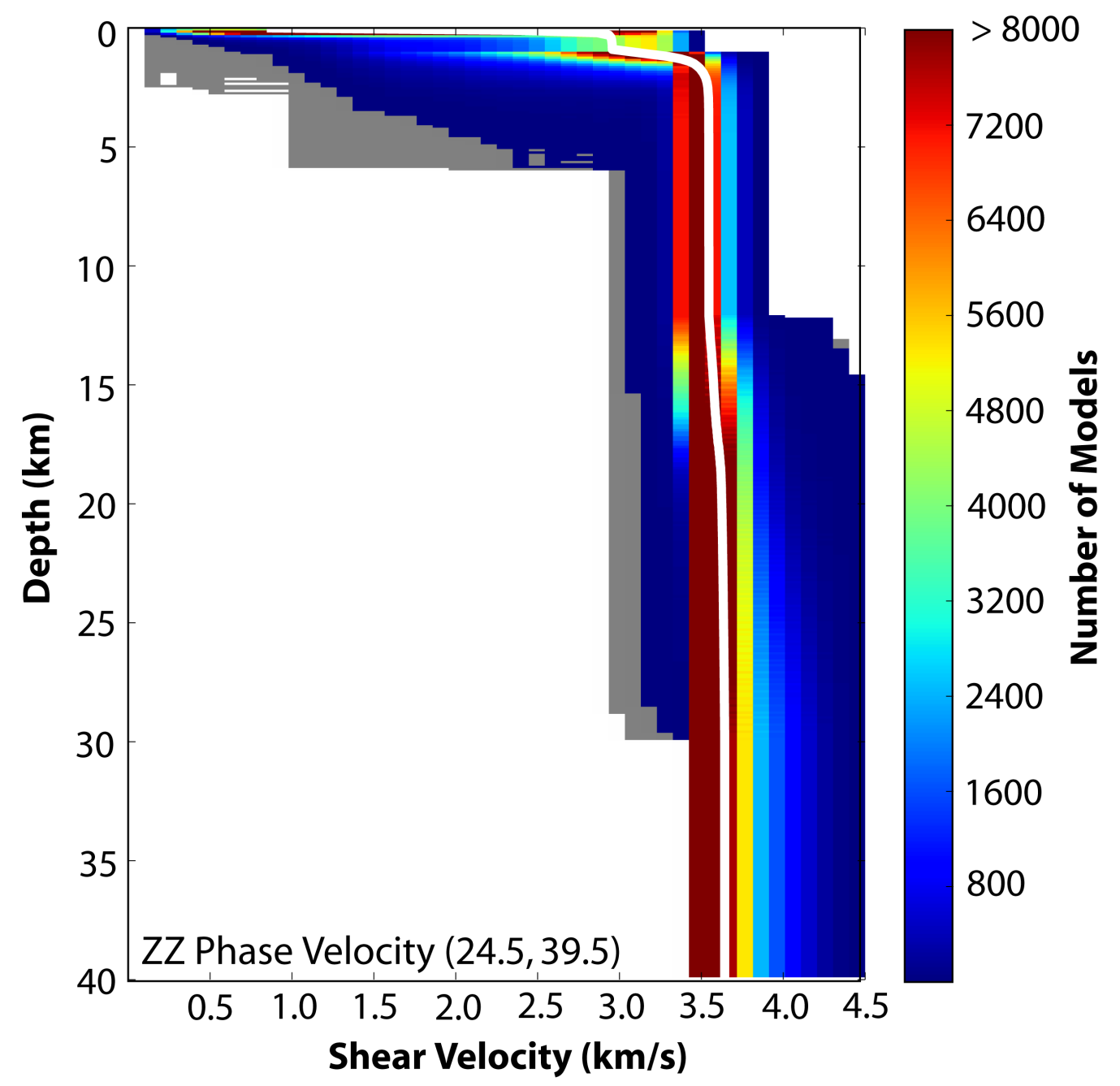

Figure 4.2: $1 \mathrm{D}$ shear-velocity model for latitude-longitude point $24.5^{\circ}, 39.5^{\circ}$. The color bar displays the number of models, and the weighted mean is represented as a white solid line. 


\subsection{Results}

\subsubsection{Grid-point dispersion}

Dispersion curves for each latitude-longitude grid-point were obtained from the surface wave maps [Figure 4.3]. Each point of the dispersion curve was tested against the $35 \mathrm{~km}$ cone-resolution threshold established in Figure 3.19. If the point was outside of the threshold, it was not used in the dispersion curve. Additionally, the dispersion curve at each point was required to contain at least four points. A total of 70,71, and 63 latitude-longitude points passed these criteria for the RR, TT, and ZZ components respectively. Figure 4.4 displays the maximum period following the threshold for each type of velocity. As may be expected, long periods cannot be observed near the edges of the field due to a shrinking in the resolution. An example of this can be observed in Figures 4.4D and 3.28. 

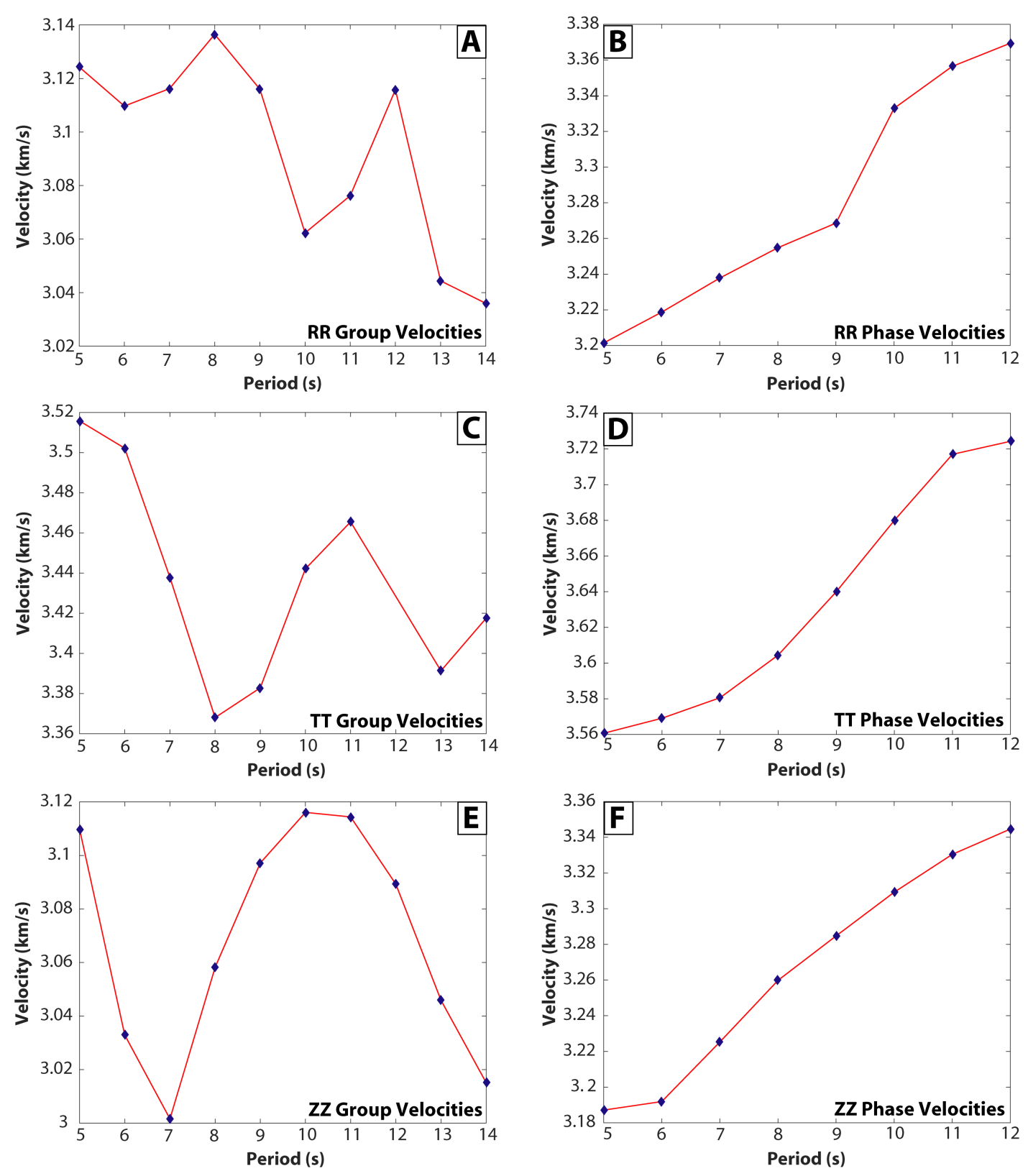

Figure 4.3: Dispersion curves for latitude-longitude point $24.5^{\circ}, 39.5^{\circ}$. Each point in the displayed curves passed the resolution threshold established in Figure 3.19. 

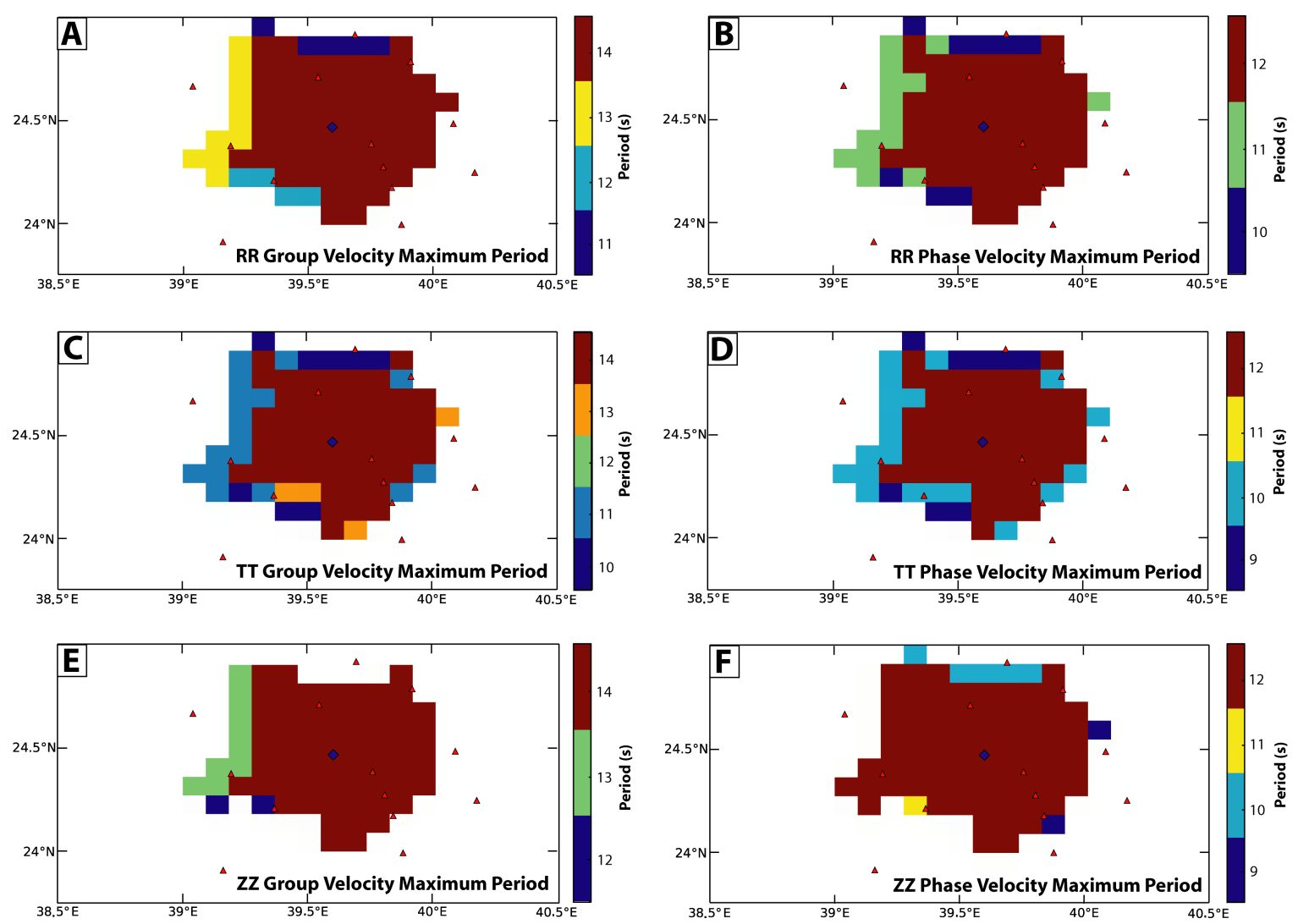

Figure 4.4: Maximum periods chosen for shear-velocity inversion for [A] RR group velocities, [B] RR phase velocities, [C] TT group velocities, [D] TT phase velocities, [E] $\mathrm{ZZ}$ group velocities and $[\mathrm{F}] \mathrm{ZZ}$ phase velocities. 
In our latitude-longitude point dispersion curves example, the group velocity dispersion curves oscillate between values of low and high velocity [Figure 4.3A, C, E] while the phase velocity consistently increase with period [Figure 4.3B, D, F]. This phenomenon is also observed in FTAN results of certain group-velocity station-pairs [Figure 3.17B is a mild example]. This is thought to be a result of a strong anisotropic source off the station path [Tsai, 2009, Behr, 2010], which would explain why only certain station-pairs experience this behavior. The oscillating nature of the group velocity makes it impossible to obtain realistic velocity models through inversions. Due to this, only phase velocities were used for our inversions. All the phase velocity dispersion curves used in the inversions are displayed in Figure 4.5.
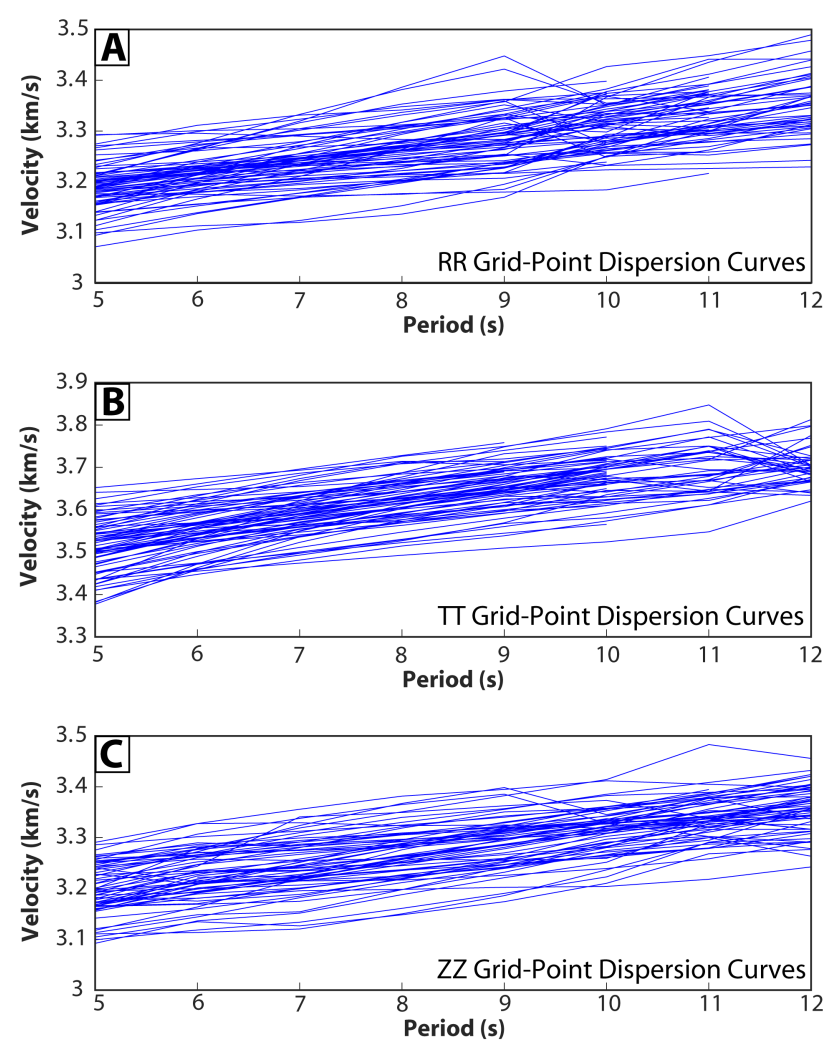

Figure 4.5: [A] RR, [B] TT, and [C] ZZ component latitude-longitude point phase dispersion curves resulting from the grid-search method of the dispersion maps of Figures 3.27-3.29. 


\subsubsection{D Inversions}

1D shear-velocity inversions were computed from RR, TT, and ZZ phase-velocity [Figure 4.3] using the Dinver software package [Sambridge, 1999, Wathelet, 2005, 2008] with the parameters of Table 4.1. Each inversion generated approximately 50,000 models. Models which were below a 10\% misfit threshold were not included in our weighted mean calculation.

Figure 4.6 displays the RR, TT, and ZZ inversions for latitude-longitude point $24.4^{\circ}$, $39.4^{\circ}$. In this figure, hot colors represent a large number of models, the white solid line is the weighted mean, and the gray areas represent models below the misfit threshold which are not included in the weighted mean. For each component, we found that the inversion was generally consistent in terms of boundaries and velocities [Figure 4.6]. 

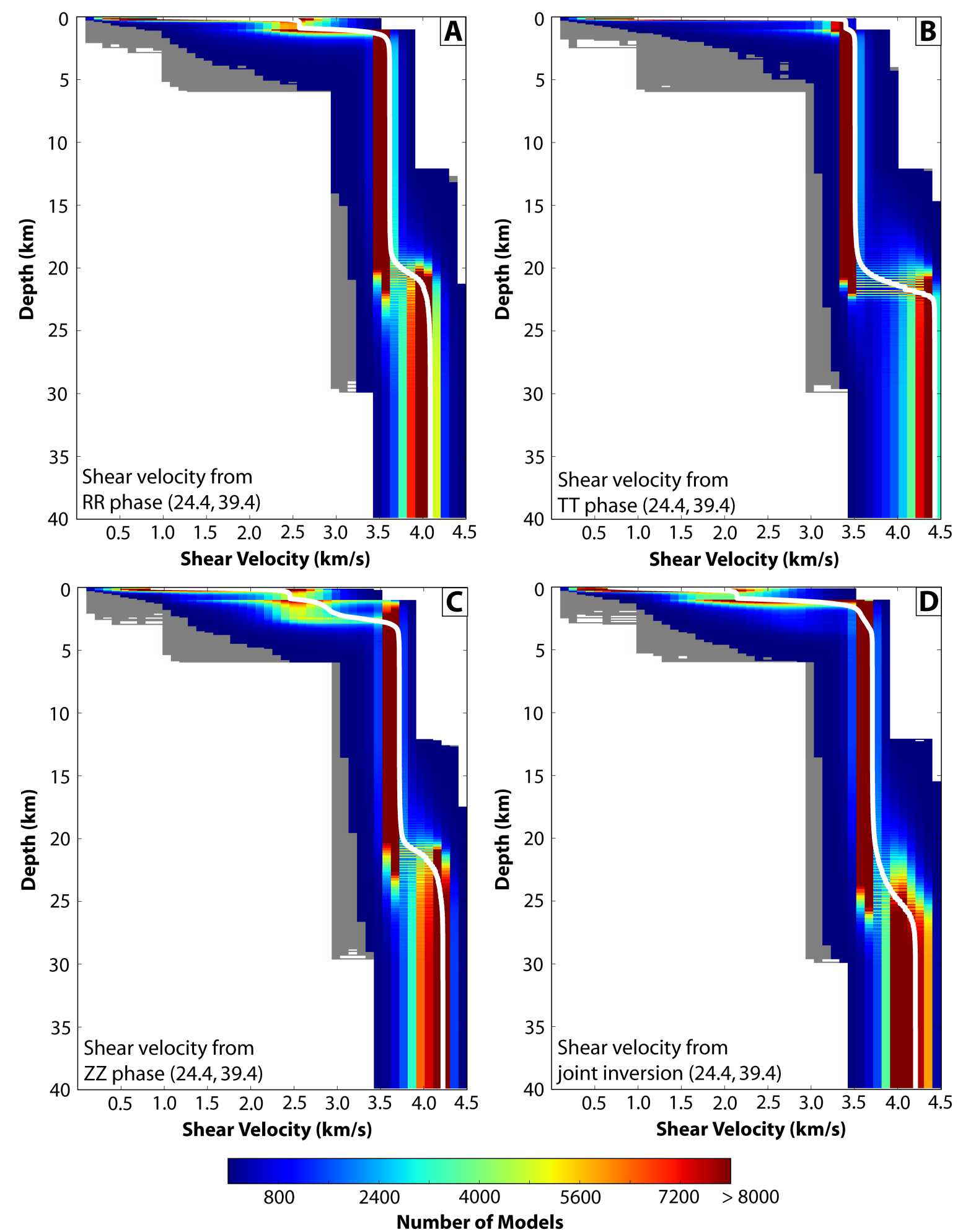

Figure 4.6: Shear-velocity inversions of latitude-longitude point $24.4^{\circ}, 39.4^{\circ}$ using [A] RR, [B] TT, [C] ZZ, and [D] joint inversion phase velocities. The color bar represents the number of models. The weighted mean is displayed as a solid white line. Grey lines represent models below the misfit threshold. 
In this particular example, a boundary in shear-velocity is observed in all components at approximately $20 \mathrm{~km}$ depth. The velocity increase through that boundary is approximately 470, 920, and $500 \mathrm{~m} / \mathrm{s}$ for RR, TT, and ZZ components respectively [Figure 4.6].

We conducted a broader analysis on the inversions to check whether or not this layer change is required. We took the difference in shear velocities between $30 \mathrm{~km}$ and $15 \mathrm{~km}$ to find the velocity increase at the boundary and computed the minimum, maximum, mean, and median values of that value for RR, TT, and $\mathrm{ZZ}$ components [Table 4.2]. Both the mean and median velocity increases were above $300 \mathrm{~m} / \mathrm{s}$ for all components, suggesting that a velocity increase indicative of a layer change is present in most 1D models. We found that the TT component, despite having a shallower resolution than the RR and ZZ [Figure 3.18], still resolved the $20 \mathrm{~km}$ layer interface for most of the latitude-longitude grid-points [Table 4.2].

\begin{tabular}{||l||l||l||l||}
\hline Component & Range $\mathbf{( m / s )}$ & Mean $\mathbf{( m / s )}$ & Median $\mathbf{( m / s )}$ \\
\hline Radial-radial (RR) & $18-945$ & 408 & 410 \\
\hline Transverse-transverse (TT) & $8-1097$ & 433 & 330 \\
\hline Vertical-vertical (ZZ) & $12-916$ & 376 & 321 \\
\hline
\end{tabular}

Table 4.2: Values of velocity change across boundary from 1D inversions. Velocity change was calculated from the difference between velocities at $30 \mathrm{~km}$ and $15 \mathrm{~km}$. 


\subsubsection{Average Velocity Models}

The combined result of $1 \mathrm{D}$ inversions are grids of $0.1^{\circ}$ decimal degree resolution for each depth [Figure 4.7]. $0.1^{\circ}$ degree spacing was used because that is the same gridspacing used to produce the surface wave tomography and the checkerboards. Additionally, although the checkerboard tests produce some smearing for $0.2^{\circ}$ and $0.3^{\circ}$ [Figures 3.21-3.23], the cone-radius resolution tests suggest that central areas of the Harrat have a resolution between 15 to $20 \mathrm{~km}$ [Figure 3.19]. Horizontal [Section 4.3.4] and vertical [Section 4.3.5] cross-sections can be determined from the interpolation of the grid-point 1D models. Prior to this, we present average velocity models for Harrat Al-Madinah, a zone surrounding the 1256 CE eruption, and an "off-Harrat" zone west of the city of Medina [Figure 4.7]. We define the off-Harrat as an area between longitudes $39.3-39.5^{\circ} \mathrm{E}$ and latitudes $24.4-24.6^{\circ} \mathrm{N}$ and the $1256 \mathrm{CE}$ eruption area between longitudes $39.7-39.9^{\circ} \mathrm{E}$ and latitudes $24.2-24.4^{\circ} \mathrm{N}$ [Figure 4.7, blue and red boundaries].

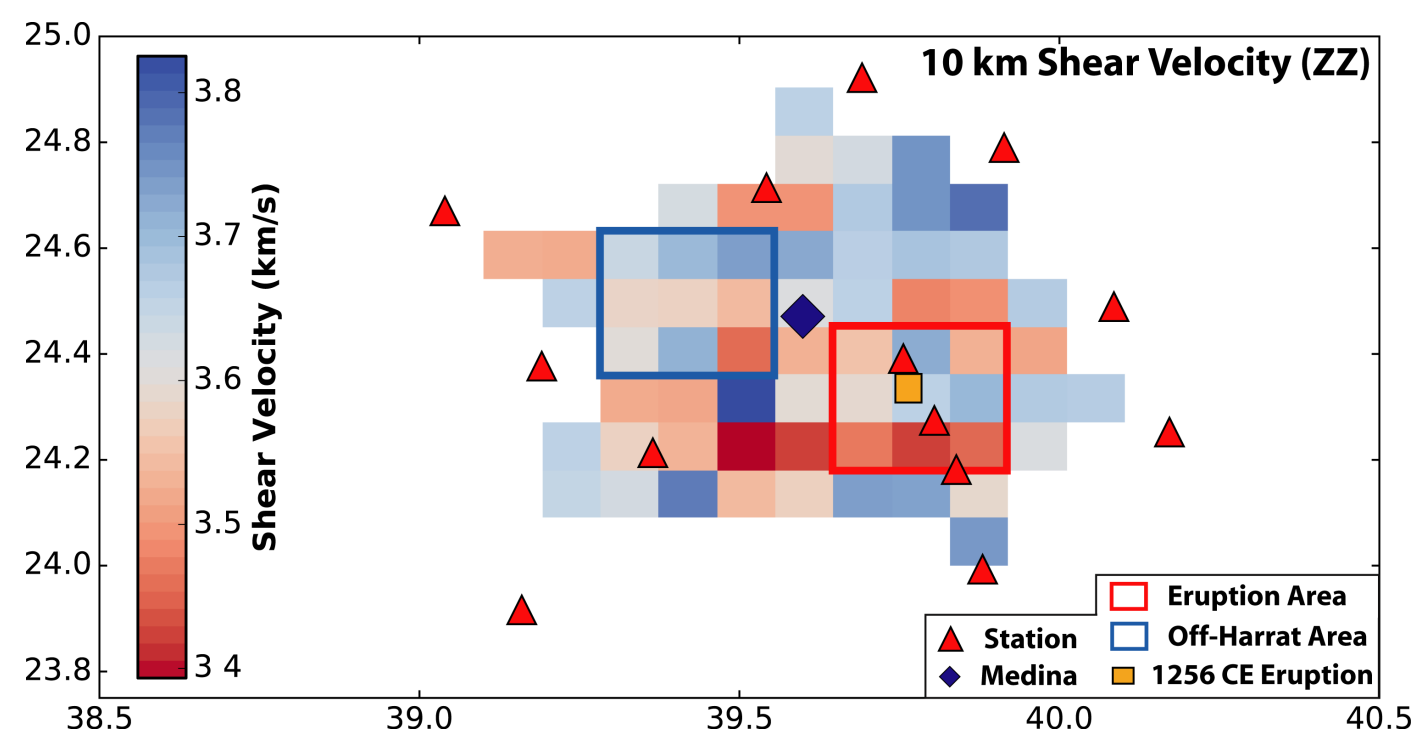

Figure 4.7: Shear-velocity $(\mathrm{km} / \mathrm{s})$ with depth obtained from vertical-vertical (ZZ) phase velocities. The Harrat Rahat seismic stations and the city of Medina are represented as red triangles and a blue diamond respectively. The location of the $1256 \mathrm{CE}$ eruption vent is marked as an orange square. The contoured version of this horizontal cross-section is displayed in Figure 4.16B.

Figures 4.9 and 4.8 display the average dispersion curves and standard deviation for Harrat Al-Madinah, the 1256 CE eruption area, and the off-Harrat area. Although the dispersion curves for the eruption area were slower than the dispersion curves for the entire Harrat at all periods, the curves for the off-Harrat area were mostly of slightly lower velocity than the average [Figure 4.8]. The average dispersion curves were then inverted to shear-velocity using the same methods and parameters as Section 4.3.2 [Figures 4.10-4.12]. 

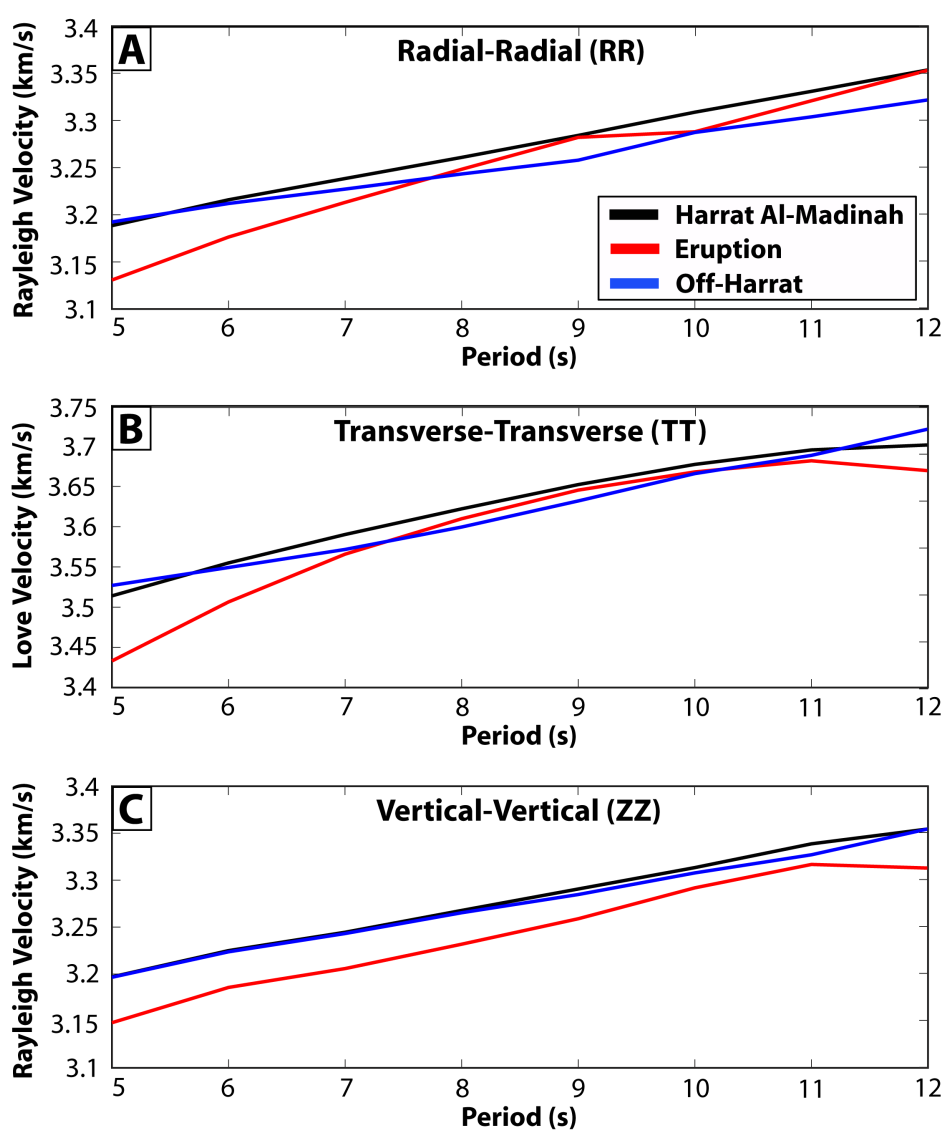

Figure 4.8: Average dispersion curves for Harrat Al-Madinah (black lines), the 1256 CE eruption area [B], and the off-Harrat area (blue) for components RR [A], TT [B], and ZZ [C]. Each individual dispersion curve is displayed in Figure 4.9. 


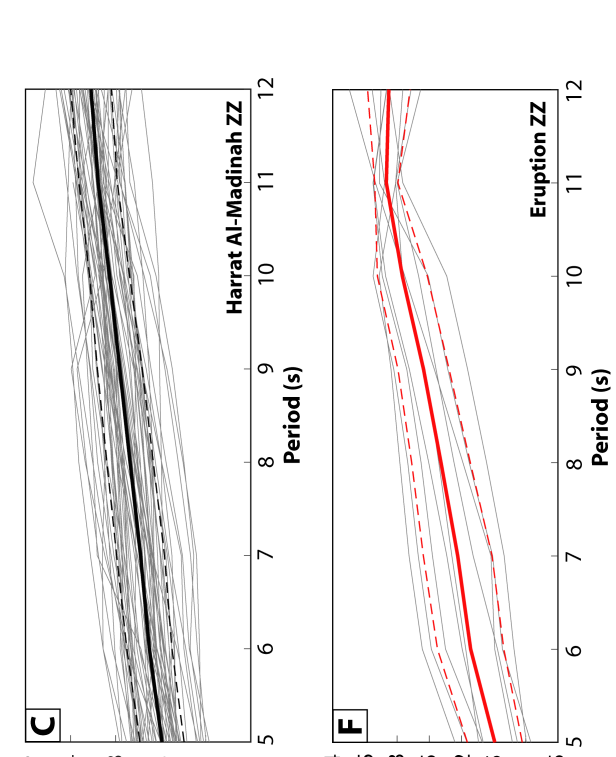

$\stackrel{n}{m} \stackrel{\forall}{m} \stackrel{m}{m} \stackrel{m}{m} \bar{m} m$

(s/uy) Кұ!วо|әА чб!әКеу

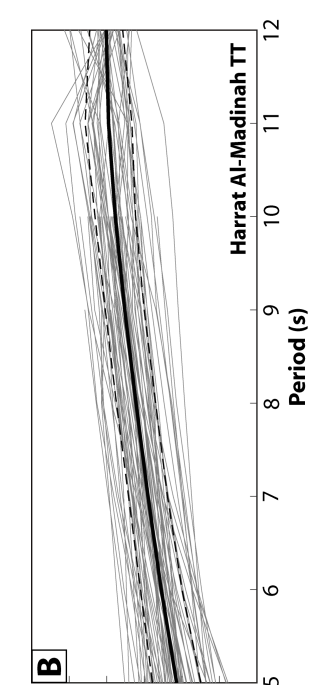

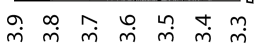
(s/யy) Кұ!วоə әло7

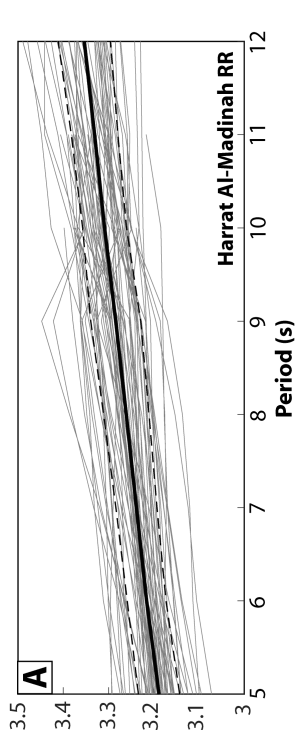

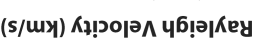

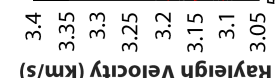

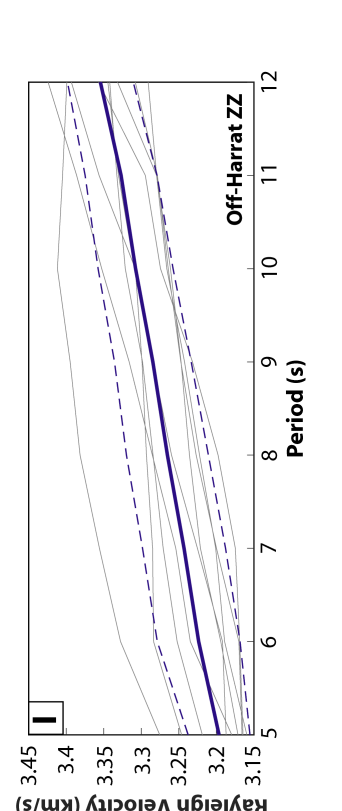

ฮี ప ప

.

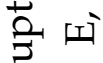

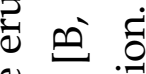

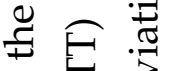

कृ

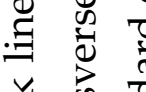

范 吾

可

ฮ

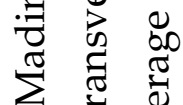

$\sum_{i}^{\infty}$

षंठ

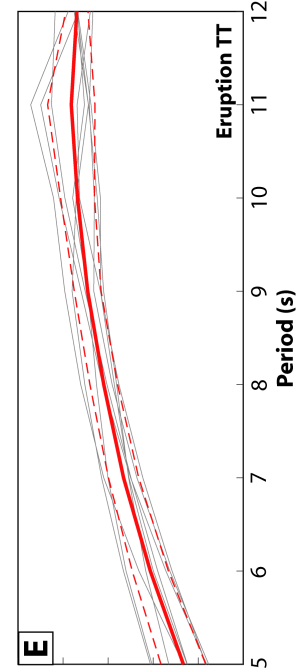

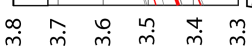

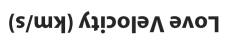
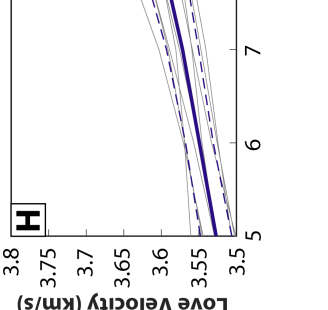

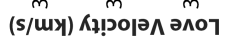
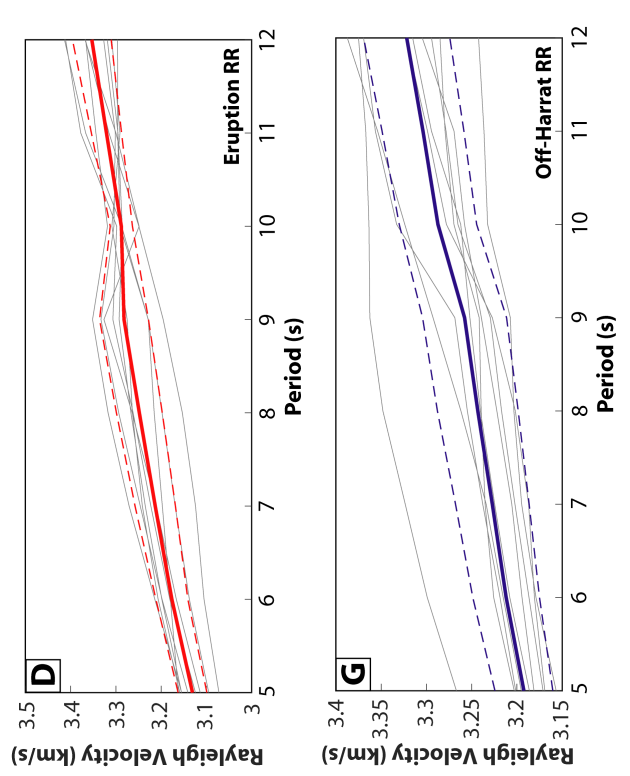

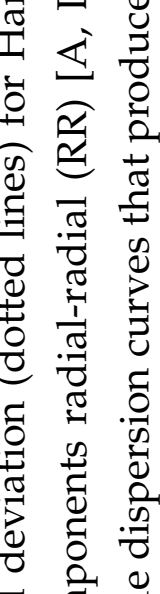

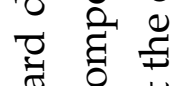

ฮृ

픙

के है

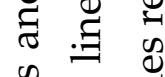

$\stackrel{0}{\Xi} . \Xi$

ప

๘

.유

氙

:

$\infty \begin{gathered}1 \\ 0\end{gathered}$

힝 0

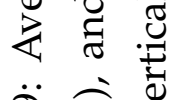

亏े

号 
The RR and $\mathrm{ZZ}$ components of all three areas displayed a velocity increase between 15-25 km depth, while the TT component only resolved the boundary in the off-Harrat area [Figures 4.10-4.13 B]. Additionally, the off-Harrat area [Figure 4.12] placed the boundary at approximately 5 to $10 \mathrm{~km}$ deeper depth than the eruption area [Figure 4.11]. As may be expected, the average model for Harrat Al-Madinah resolved the boundary at a depth in the middle of the two areas, between 20 to $25 \mathrm{~km}$ for the $\mathrm{RR}$ and TT components and between 13 to $20 \mathrm{~km}$ for TT [Figure 4.10]. The joint inversion models were similar to the RR and ZZ components for the entire Harrat and for the off-Harrat area [Figures 4.10D and 4.12D], but placed a lower depth boundary for the eruption area [Figure 4.11D]. Figure 4.13 displays the average 1D models for each of the three areas for all three components. Surprisingly, the off-Harrat region had a slower shear-velocity than the Harrat Al-Madinah average or the eruption region for the RR component [Figure 4.13A]. Additionally, the shear-velocity model for Harrat Al-Madinah was very similar to the eruption average. The velocities for each of the three layers is tabulated in Table 4.3.

\begin{tabular}{||l||l||ll||l||ll||}
\hline Subset & Component & $\begin{array}{l}\text { Layer } \mathbf{1} \\
\mathbf{( k m / s )}\end{array}$ & $\begin{array}{l}\text { Layer } \\
\mathbf{( k m / s )}\end{array}$ & $\begin{array}{l}\text { Layer } 3 \quad V_{s} \\
(\mathbf{k m} / \mathbf{s})\end{array}$ \\
\hline All Harrat & RR & 2.1 & 3.7 & 4.0 \\
\hline & TT & 2.7 & 3.6 & 3.9 \\
\hline & ZZ & 2.4 & 3.7 & 3.9 \\
\hline & Joint & 2.2 & 3.7 & 3.9 \\
\hline Eruption & RR & 1.6 & 3.7 & 4.0 \\
\hline & TT & 2.1 & 3.6 & 3.8 \\
\hline & ZZ & 2.5 & 3.6 & 3.8 \\
\hline & Joint & 1.7 & 3.7 & 4.1 \\
\hline Off Harrat & RR & 2.8 & 3.6 & 3.9 \\
\hline & TT & 3.2 & 3.5 & 4.3 \\
\hline & ZZ & 2.0 & 3.7 & 4.2 \\
\hline & Joint & 2.5 & 3.6 & 3.9 \\
\hline
\end{tabular}

Table 4.3: Layer shear-velocity for each component and joint inversion. 

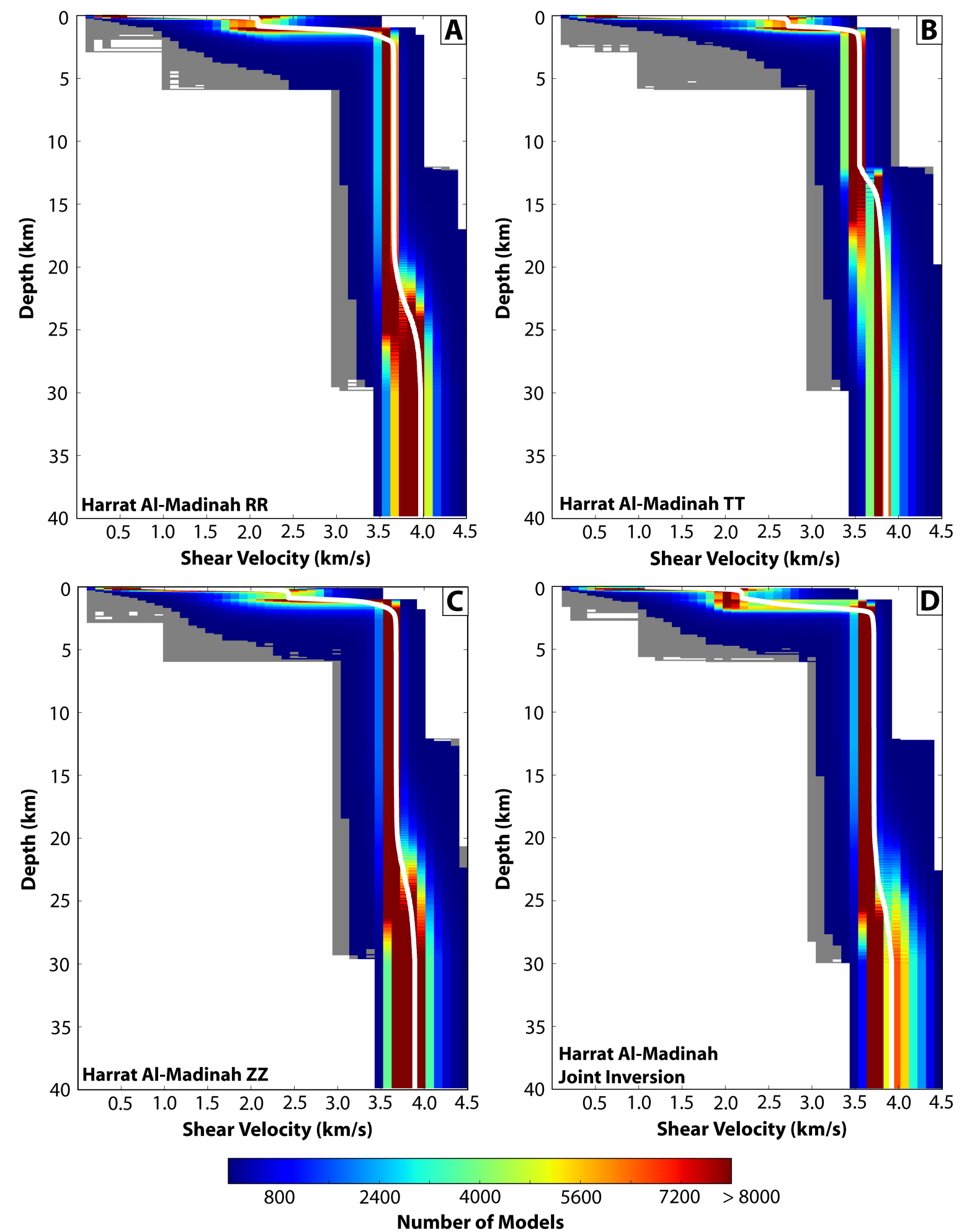

Figure 4.10: Average shear-velocity models for Harrat Al-Madinah using [A] RR, [B] $\mathrm{TT},[\mathrm{C}] \mathrm{ZZ}$, and [D] joint inversion phase velocities. The color bar represents the number of models and the weighted mean is displayed as a solid white line. 

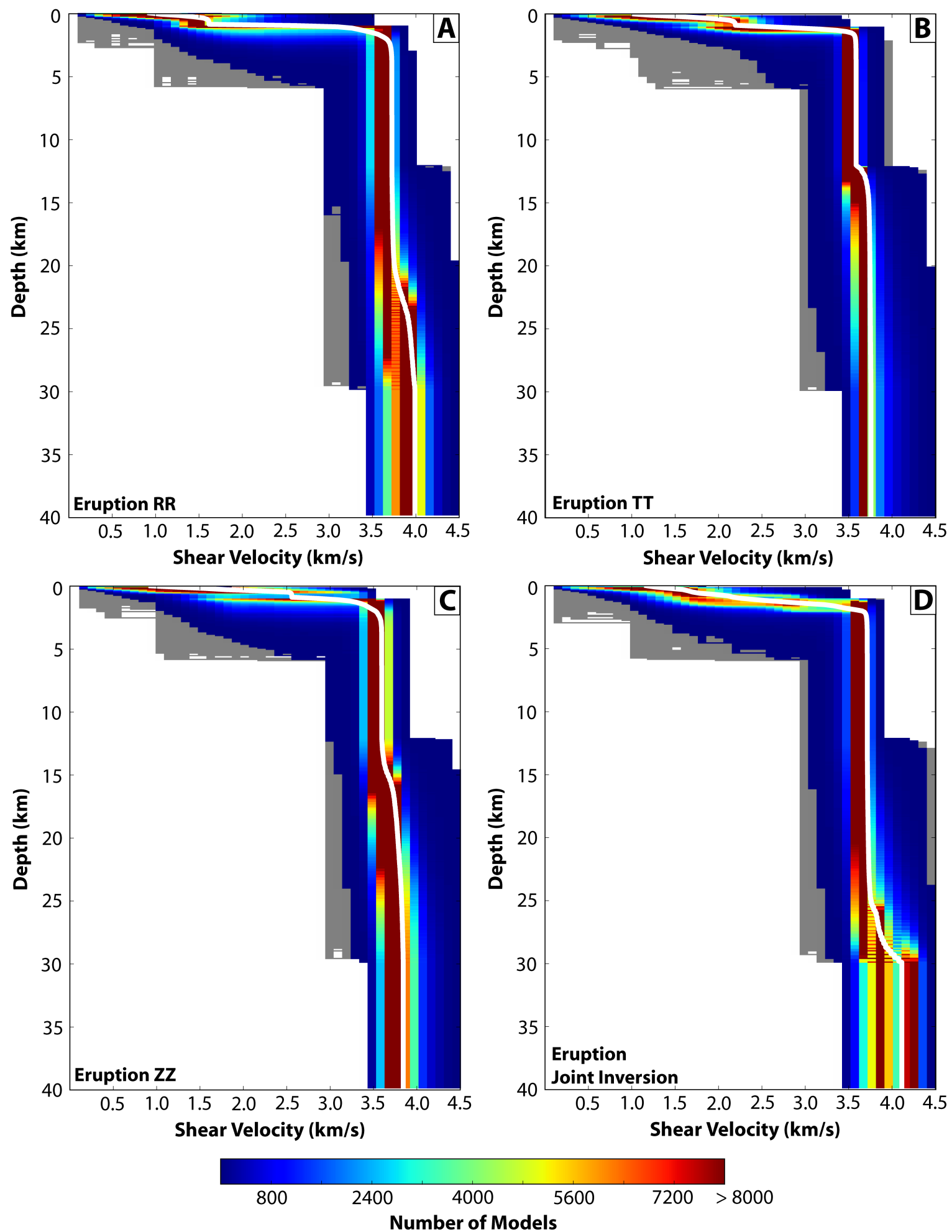

Figure 4.11: Average shear-velocity models for the $1256 \mathrm{CE}$ eruption area using [A] RR, [B] TT, [C] ZZ, and [D] joint inversion phase velocities. The color bar represents the number of models and the weighted mean is displayed as a solid white line. 

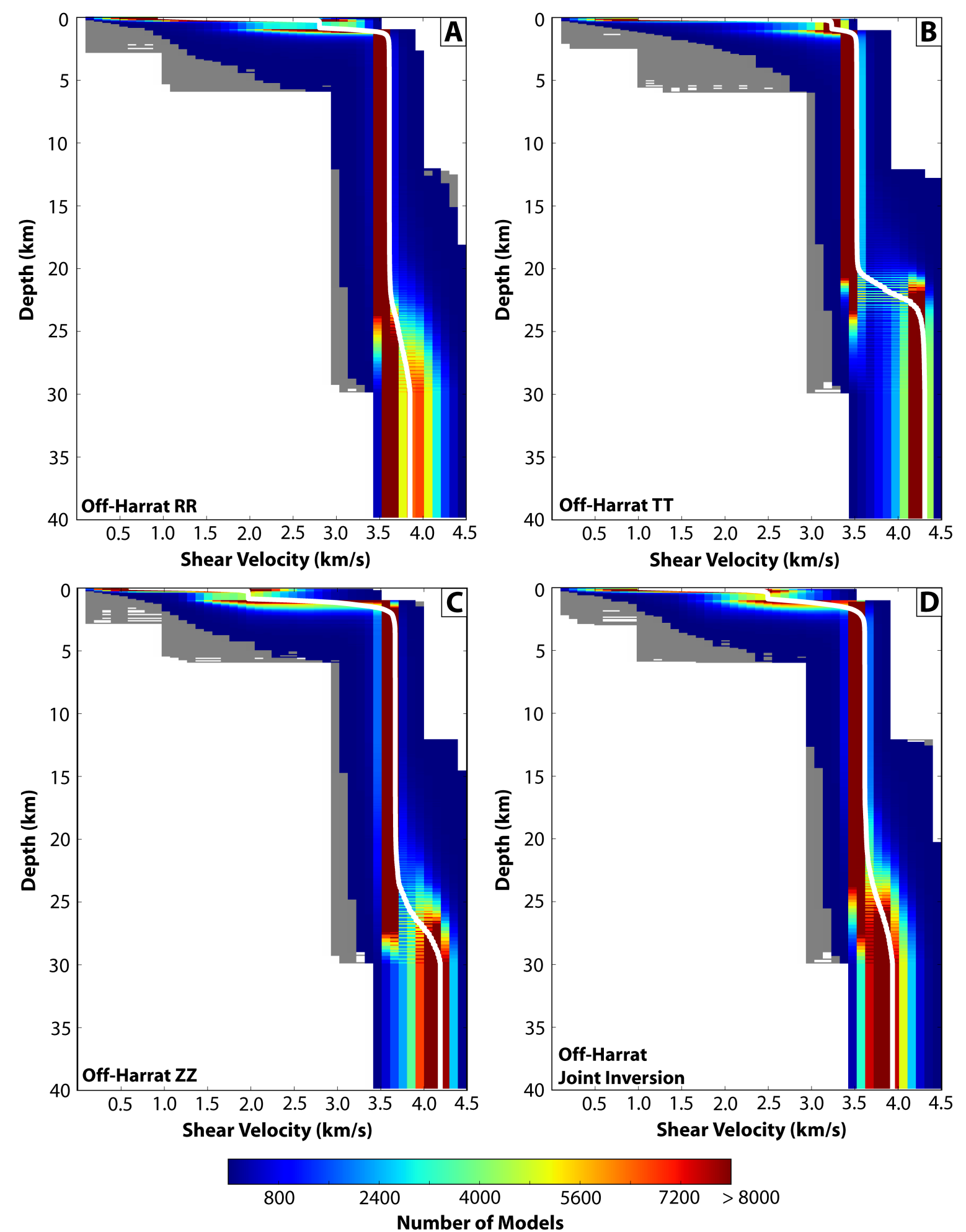

Figure 4.12: Average shear-velocity models for the off-Harrat area using [A] RR, [B] TT, $[C] \mathrm{ZZ}$, and [D] joint inversion phase velocities. The color bar represents the number of models and the weighted mean is displayed as a solid white line. 

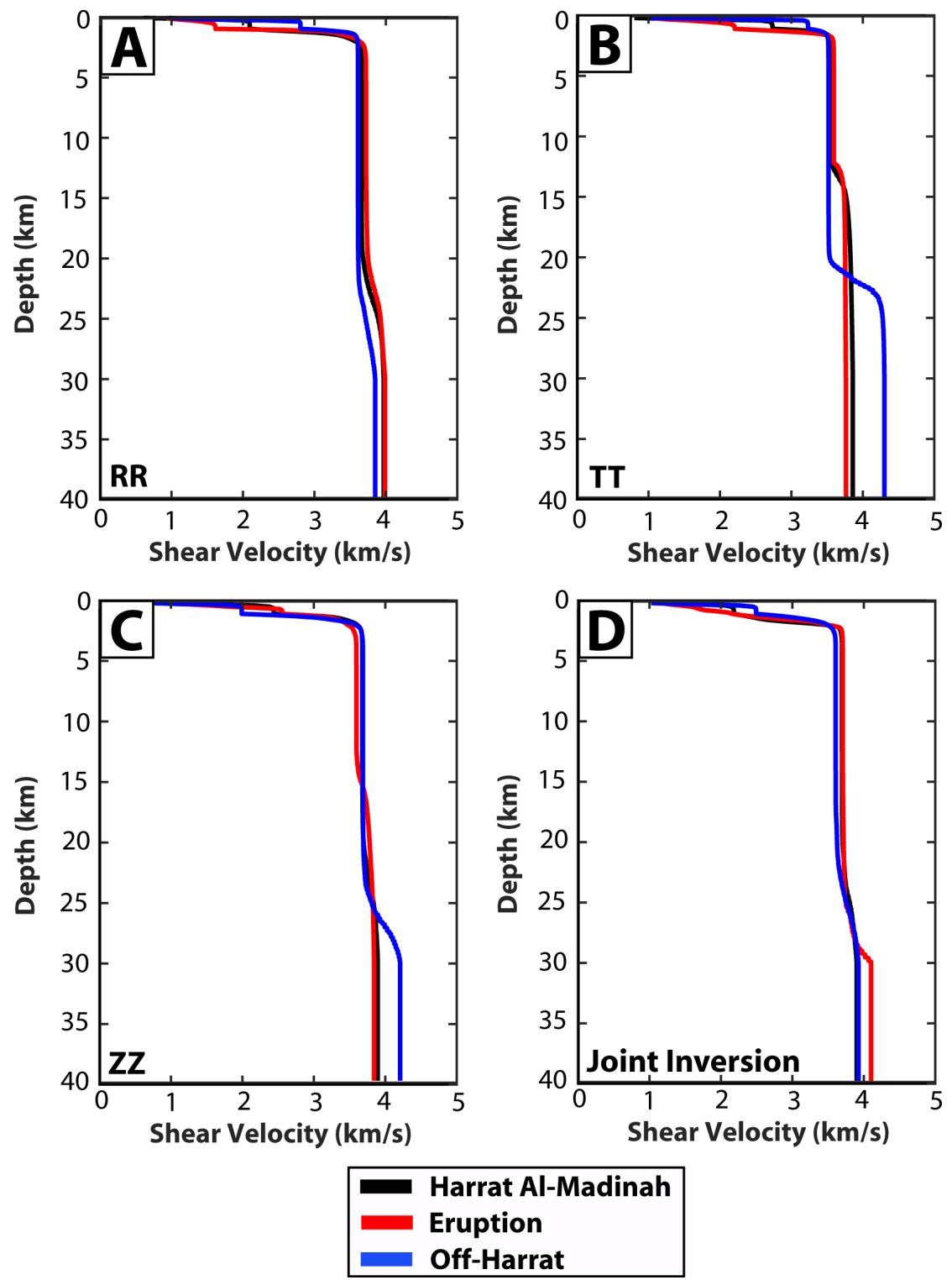

Figure 4.13: Average 1D shear-velocity models for Harrat Al-Madinah (black line), the 1256 CE eruption area (red line), and the off-Harrat area (blue line) for [A] RR, [B] TT, [C] ZZ, and [D] joint inversion phase velocities. 


\subsubsection{Horizontal cross-sections of inverted S-wave velocities}

Similar to the dispersion maps presented in Figures 3.27-3.32, the weighted means obtained by the 1D inversions can be analyzed as a function of depth. Velocity values from the 1D models for specific depths were interpolated to produce horizontal crosssections. The resolution of our tomography are cells of 0.1 by 0.1 decimal degrees, the same size as the dispersion maps [Figure 4.7]. Each horizontal cross-section is then contoured to smooth the data [Figures 4.14-4.16].
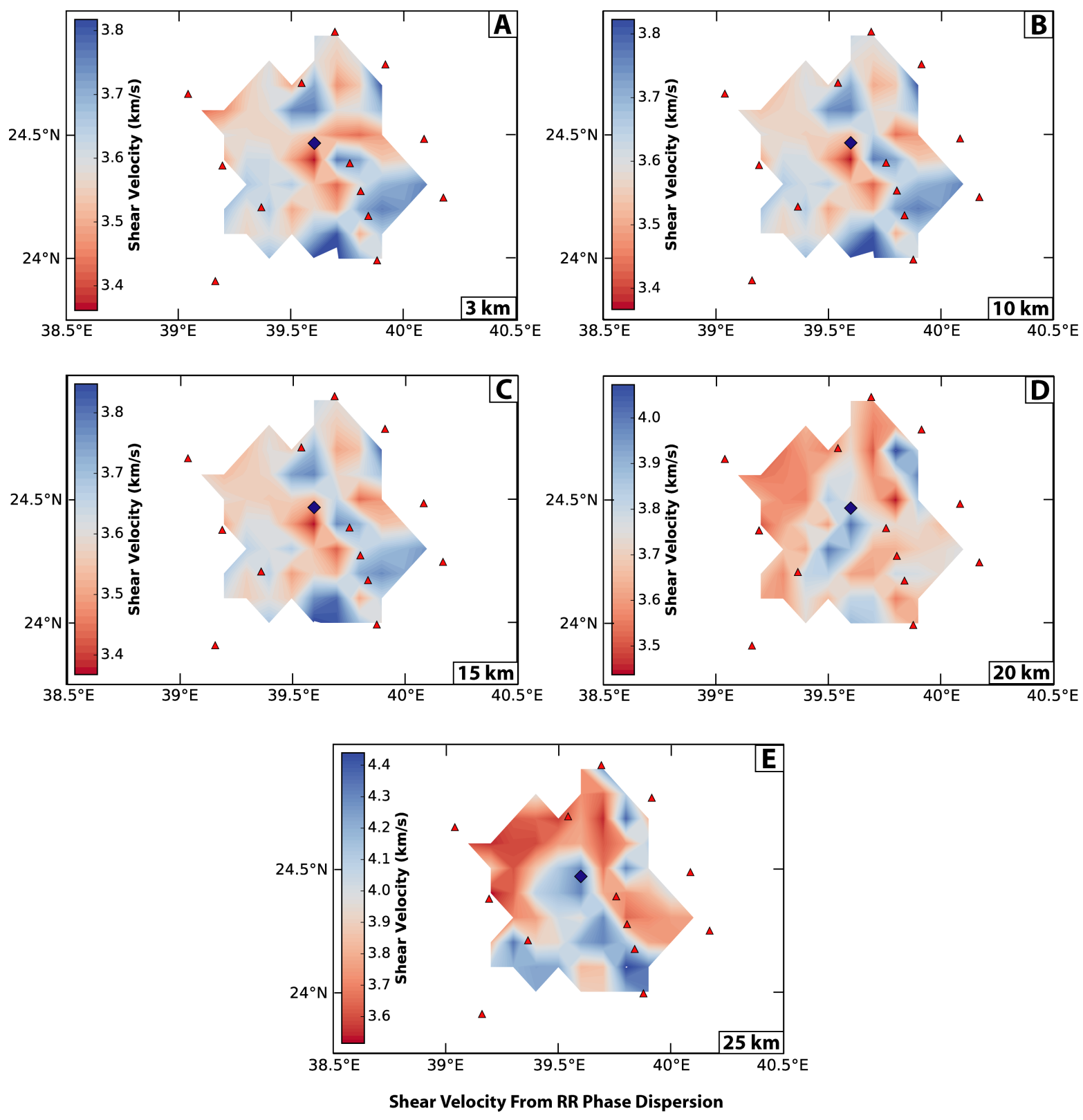

Figure 4.14: Shear-velocity $(\mathrm{km} / \mathrm{s})$ with depth obtained from radial-radial (RR) phase velocities. The Harrat Rahat seismic stations and the city of Medina are displayed as red triangles and a blue diamond respectively. 

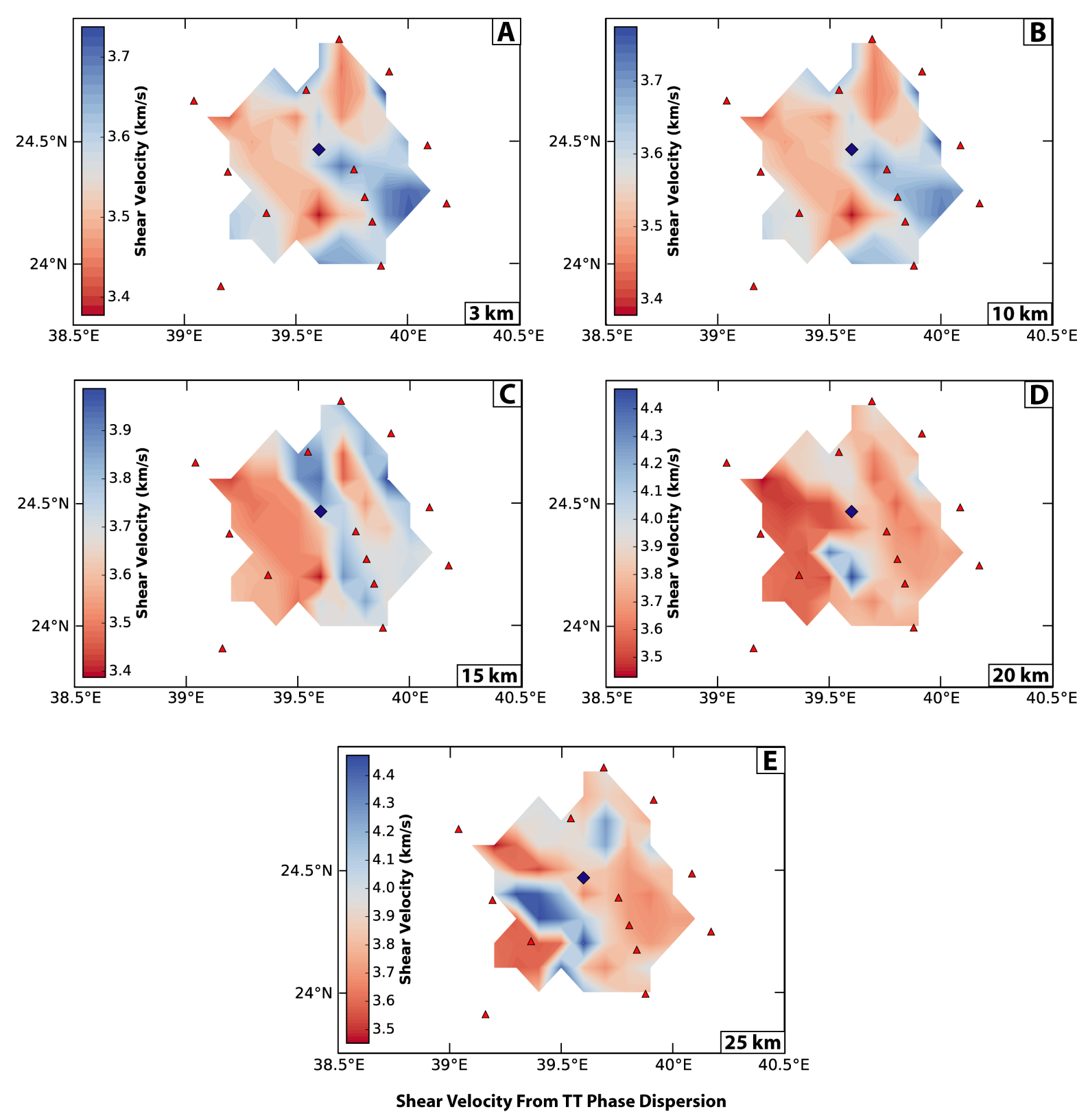

Figure 4.15: Shear-velocity $(\mathrm{km} / \mathrm{s})$ with depth obtained from transverse-transverse (TT) phase velocities. 

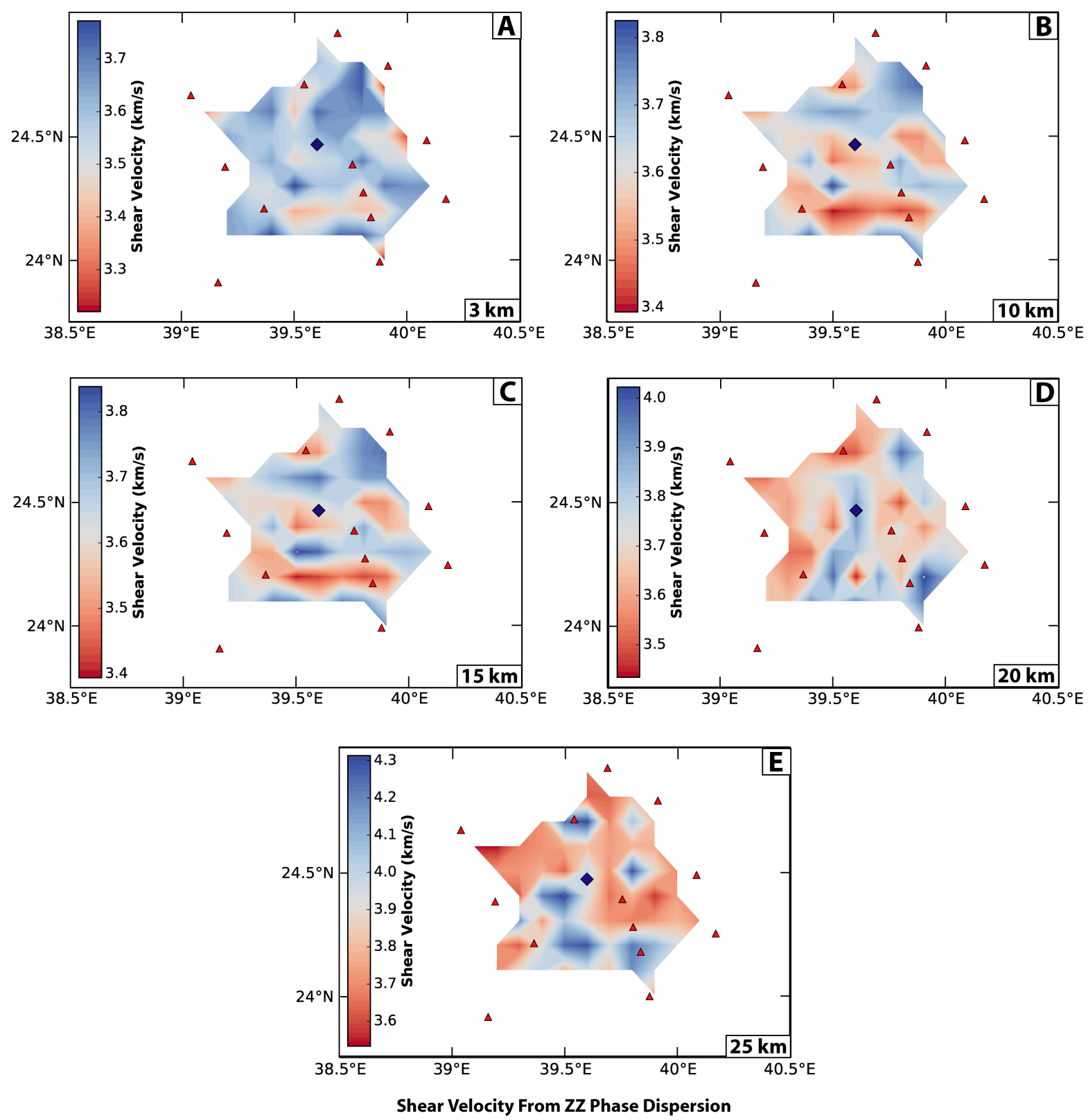

Figure 4.16: Shear-velocity $(\mathrm{km} / \mathrm{s})$ with depth obtained from vertical-vertical $(\mathrm{ZZ})$ phase velocities.

Each subplot in Figures 4.14-4.16 is individually scaled to accentuate the boundaries between low and high shear velocities. Low velocities near the $1256 \mathrm{CE}$ eruption area, located south of the city of Medina [Figure 3.11], are observed for shear velocities from the RR and TT components shallower than $20 \mathrm{~km}$ [Figure 4.14A-D, 4.15A-C] and for 10 and $15 \mathrm{~km}$ depths in the $\mathrm{ZZ}$ component [Figure 4.16]. This low-velocity area appears to change to east of the 1256 CE eruption vent for depths greater than $20 \mathrm{~km}$ for all components.

We check the robustness of the inversions by comparing the velocity boundaries and the overall high and low velocity features at the same depths. RR and ZZ shearvelocity are computed by inverting the dispersion produced by the horizontal and 

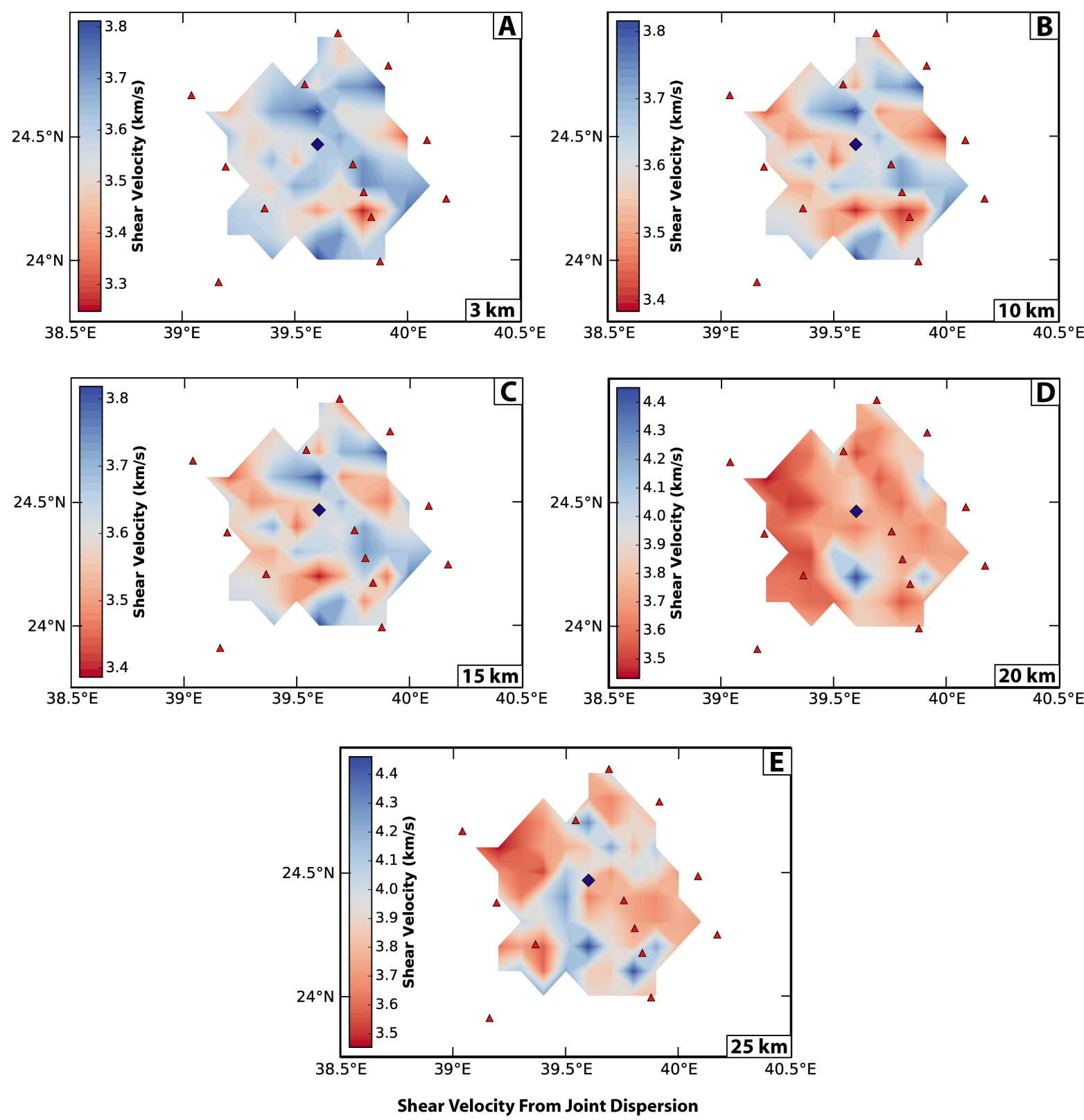

Figure 4.17: Shear-velocity $(\mathrm{km} / \mathrm{s})$ with depth obtained from joint inversion phase velocities.

vertical component of Rayleigh waves, while the TT shear velocities are inverted from Love wave dispersion. As we know from the sensitivity kernels [Figure 3.18] that the TT phase velocities sample shallower depths than the other two components, we expected that RR and ZZ tomographic results would be more similar to each other than with the TT. Instead, we found that the features of the RR and TT components were most similar to one another. Both the RR and TT shear-velocity inversions feature northwest-southeast trending low-velocity south of the city of Medina at depths shallower than $15 \mathrm{~km}$ [Figures $4.14 \mathrm{~A}-\mathrm{C}$ and $4.15 \mathrm{~A}-\mathrm{C}$ ] that are not as pronounced in the $\mathrm{ZZ}$ inversion [Figure 4.16A-C]. However, the slow velocity southeast of Medina at depths greater than $20 \mathrm{~km}$ is observed for all components [Figures $4.14-4.16 \mathrm{D}-\mathrm{F}$ ]. The maxi- 
mum and minimum values of shear-velocity were very consistent with depths, and are listed in Table 4.4 .

\begin{tabular}{||l||l||l||l||}
\hline Depth $\mathbf{( k m )}$ & RR Range $\mathbf{( k m} / \mathbf{s})$ & TT Range $\mathbf{( k m} / \mathbf{s})$ & ZZ Range $\mathbf{( k m} / \mathbf{s})$ \\
\hline 3 & $3.36-3.83$ & $3.38-3.74$ & $3.22-3.78$ \\
\hline 10 & $3.37-3.83$ & $3.38-3.77$ & $3.39-3.83$ \\
\hline 15 & $3.37-3.85$ & $3.39-4.00$ & $3.39-3.85$ \\
\hline 20 & $3.43-4.08$ & $3.43-4.48$ & $3.43-4.03$ \\
\hline 25 & $3.52-4.45$ & $3.45-4.48$ & $3.53-4.32$ \\
\hline 30 & $3.56-4.45$ & $3.69-4.48$ & $3.59-4.43$ \\
\hline
\end{tabular}

Table 4.4: Minimum and maximum inversion velocity with depth for RR, TT, and ZZ components.

The observed features in the shear-velocity inversions [Figures 4.14-4.16] correlate well with those of the phase velocity tomography maps [Figures 3.27-3.29]. The previously mentioned low velocity feature trending northwest-southeast below the city of Medina at depths $15 \mathrm{~km}$ and below [Figures 4.14A-C and 4.15A-C] is observed in the RR and TT tomographic maps below 10 seconds [Figures 3.27A-D and 3.28A-D]. At 10-12 seconds period for RR and TT dispersion maps, areas of low velocity are located near the northwest and southeast boundaries of the $35 \mathrm{~km}$ minimum resolution theshrold [Figures 3.27D-E and 3.28D-E], and are also observed in the shear-velocity at depths deeper than $20 \mathrm{~km}$ [Figures 4.14D-E and 4.15D-E].

The northwest-southeast low-velocity structure observed within the top 10 seconds of the ZZ phase velocity [Figure 3.29A-D] can be distinctly observed at the $10 \mathrm{~km}$ depth tomographic inversion [Figure 4.16B], but is not clear in the 3 and $15 \mathrm{~km}$ segments [Figure 4.16A, C]. However, a segment of that feature, a horizontal low-velocity strip at $24.2^{\circ}$ north extending from approximately $39.5^{\circ}-39.9^{\circ}$ east, can be observed at $15 \mathrm{~km}$ and shallower [Figure 4.16A-C]. 


\subsubsection{Pseudo-3D model and cross-sections}

The software ParaView [Ahrens et al., 2005], was used to interpolate the weighted means at each latitude-longitude point to create a pseudo-3D model of the field. To avoid drastic differences in scaling, the location of each 1D weighted mean model was converted to UTM. A downsample of the depth to one value per $1 \mathrm{~km}$ was required by the software to correctly interpolate the data [Figure 4.18]. In order to avoid drastic differences in color-scaling, the vertical cross-sections are plotted from $3 \mathrm{~km}$ downwards like the previously presented horizontal cross-sections. The results of the 3D interpolation can be compared by checking horizontal cross sections of the 3D model with the tomographic depth slices of Figures 4.14-4.16. We found that the 3D interpolation matched the features and velocity measurements of the tomographic slices [Figures 4.19 and $4.16 \mathrm{~B}$ ].

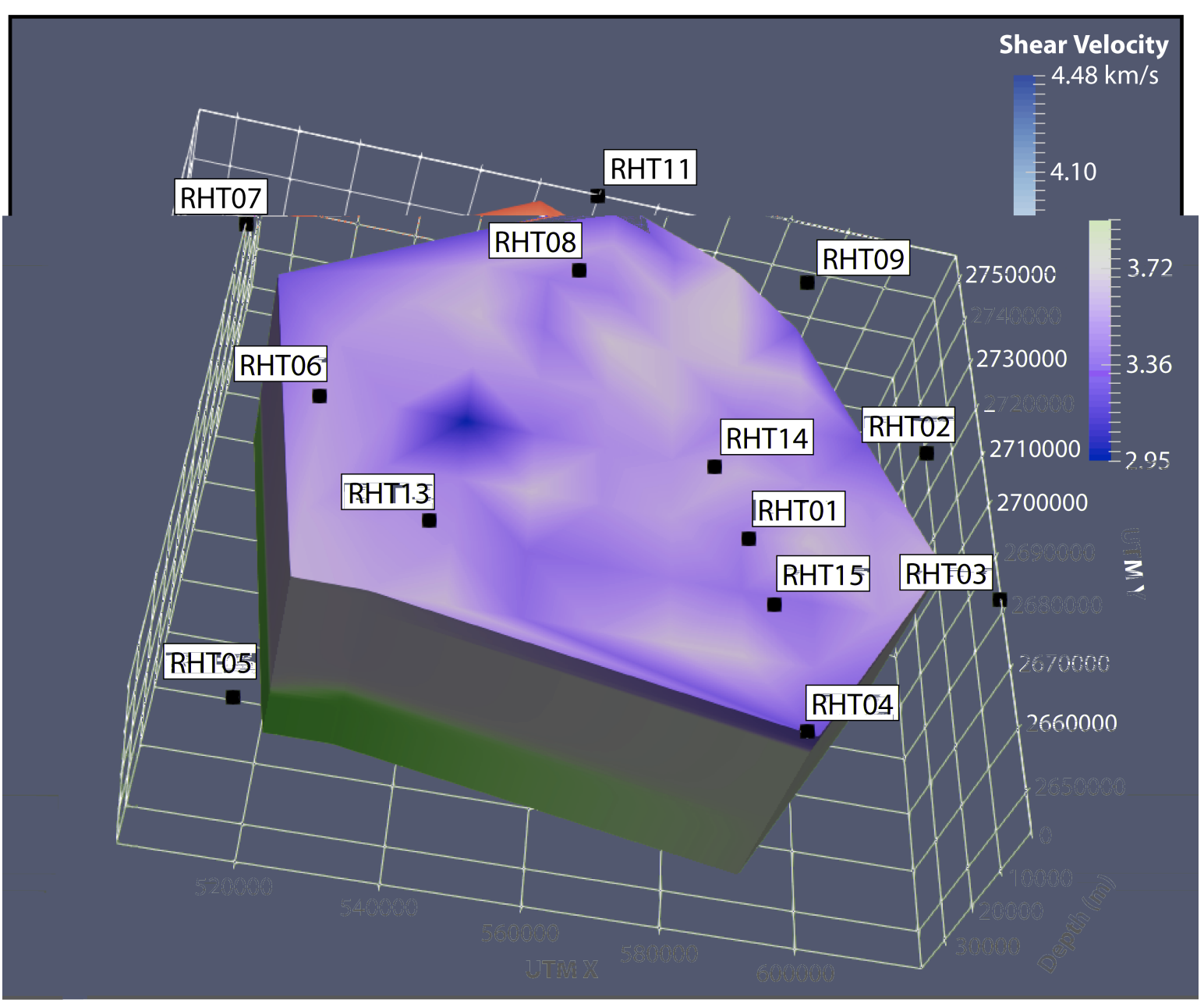

Figure 4.18: Shear-velocity model of the vertical-vertical (ZZ) 1D velocity models using 3D Delaunay interpolation [Delaunay, 1934] by the ParaView software package [Ahrens et al., 2005]. The color bar is scaled by the maximum and minimum shearvelocity values of the model. Black squares represent the Harrat Rahat seismic stations. 


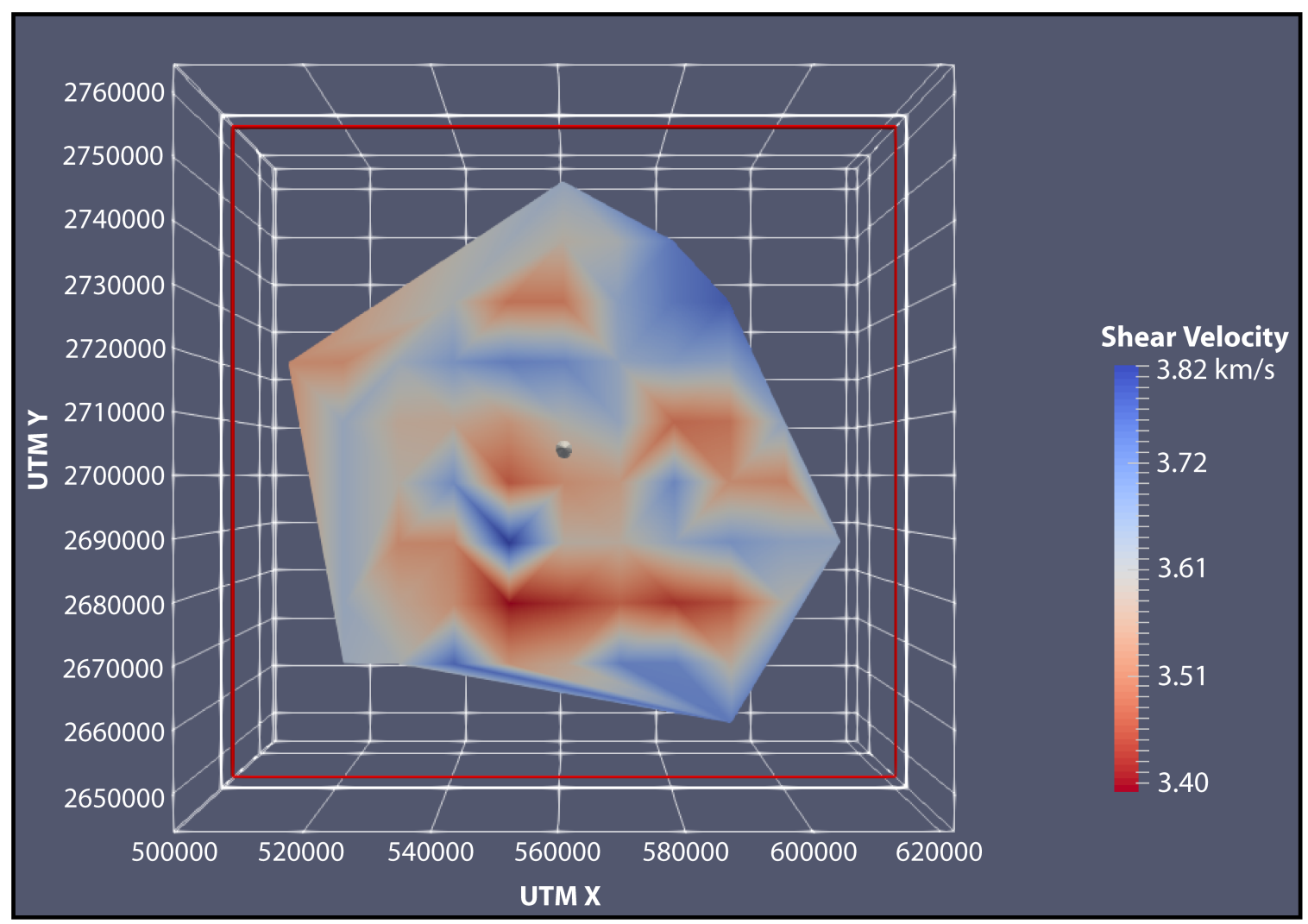

Figure 4.19: Shear-velocity for a horizontal cross-section at $10 \mathrm{~km}$ depth of the 3D interpolated ZZ shear-velocity model of Figure 4.18. The color bar is scaled by the maximum and minimum shear-velocity values of the cross-section. This cross-section is the $3 \mathrm{D}$ interpolated equivalent of the tomographic slice in Figure 4.16B. 
Two cross-sections of the 3D shear-velocity models [Figure 4.18] were chosen across the gravity deployment of Langenheim et al. [2016] [Figure 4.20]. Cross section a-a' traverses across the Harrat and the general trend of the gravity measurements, while cross-section $b-b^{\prime}$ follows the axis of the Harrat. The red, blue, and black lines correspond to the lateral boundaries of the RR, TT, and ZZ 3D model. Gravity profiles corresponding to the cross-section paths were extracted by interpolating the gravity data. Figures 4.21-4.23 display shear-velocity values of the cross section [B and D] alongside the interpolated complete Bouguer gravity anomaly value along the cross-section path [A and C]. Note that the extent of a-a' for ZZ is shorter than RR and TT [Figure 4.20]. Due to this, the distance values of the a-a' segment of the $\mathrm{ZZ}$ results [Figure 4.23A, B] were changed to line up with the those of the RR and TT velocities. The color map of the cross-sections is consistent between all components to facilitate comparisons.

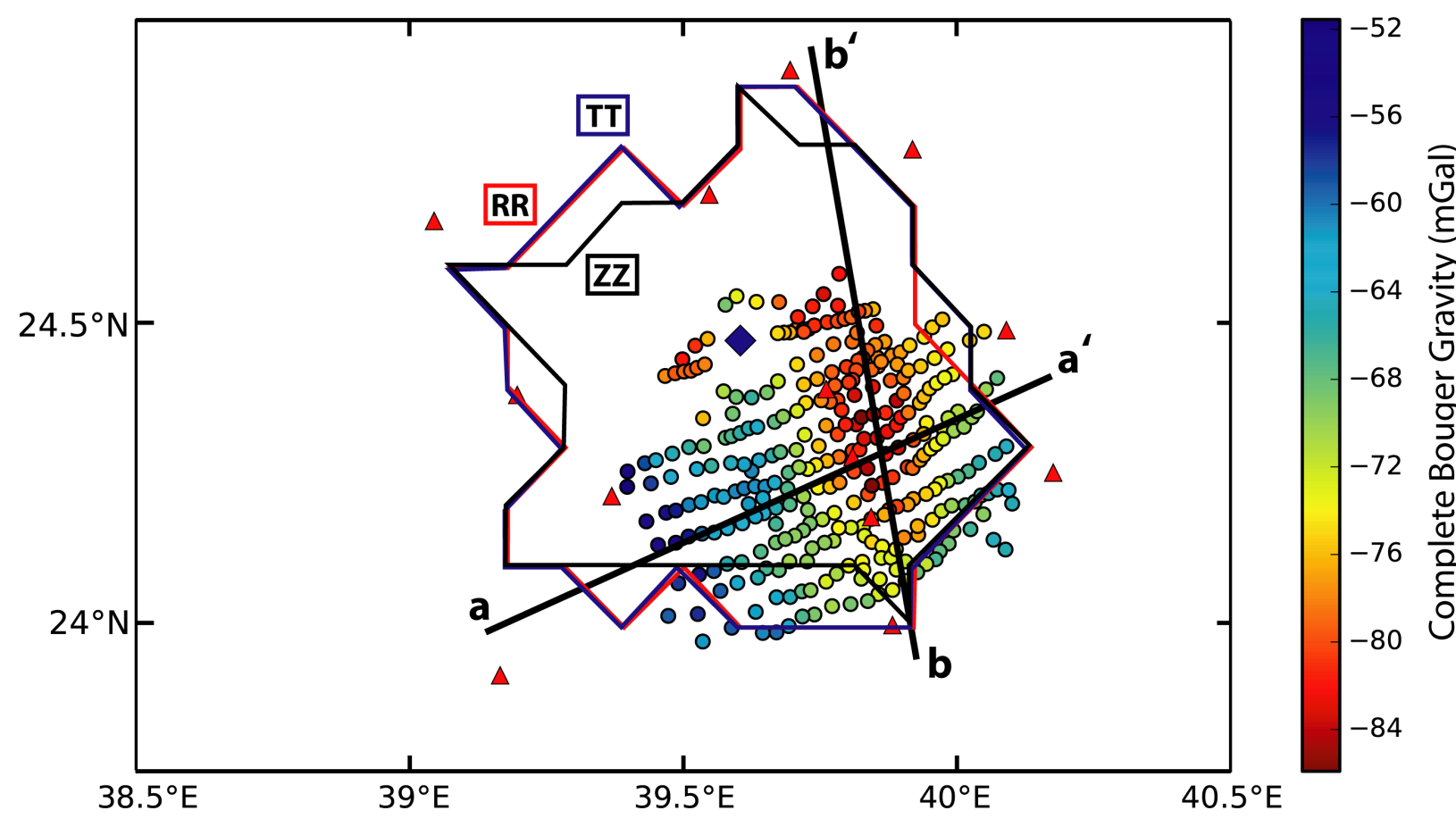

Figure 4.20: Cross-sections a-a' and b-b' used in Figures 4.21-4.23 alongside the extents of the RR (red line), TT (blue line), and ZZ (black line) 3D models interpolated by the Paraview software and the complete Bouguer gravity anomaly values of Langenheim et al. [2016].

We found that the cross-section shear-velocity values ranged between $3.2-4.5 \mathrm{~km} / \mathrm{s}$ and were similar to the Bouguer gravity variation. The boundary layer observed in the shear-velocity inversions [Figure 4.6 and Figures 4.10-4.13] was extremely consistent in both cross-sections at depths between 20 to $25 \mathrm{~km}$ for the radial-radial (RR) component [Figure 4.21]. In the a-a' cross section, we observed that western gravity low (located along 25 to $40 \mathrm{~km}$ in the profile), is matched by a slow-velocity below 25 $\mathrm{km}$ at approximately $30 \mathrm{~km}$ down the profile [Figure $4.21 \mathrm{~A}, \mathrm{~B}$ ]. The main gravity low, 
with values as low as approximately $-83 \mathrm{mGal}$, coincides with a wider and slower lowvelocity below $25 \mathrm{~km}$. East of $70 \mathrm{~km}$ along the profile, the gravity increases, as does the shear-velocity. Similar trends with the gravity peaks and troughs can be noticed in the b-b' profile [Figure 4.21C, D].

The cross-sections from the transverse-transverse (TT) component [Figure 4.22] correlate well with the gravity anomalies and what was observed in the RR component. However, in this data, the first $20 \mathrm{~km}$ in cross-section a-a' place a layer interface between $25-30 \mathrm{~km}$ instead of 20-25 km [Figure 4.21B]. However, a much more distinct area of low-velocity below $20 \mathrm{~km}$ is detected at $30 \mathrm{~km}$ corresponding with the western gravity low. An area of low velocity is still detected corresponding to the second trough, and begins approximately at $50 \mathrm{~km}$ down the profile. The b-b' velocity crosssection matches the gravity similarly to what was observed in the RR data, including the low-velocity between $65-75 \mathrm{~km}$, but has generally lower velocity than the RR component.

The cross-sections of vertical-vertical (ZZ) component [Figure 4.23] were very similar to the RR component. Here, the western gravity low in the a-a' cross section [Figure 4.23A] coincides with a low shear-velocity at approximately $22 \mathrm{~km}$ horizontal distance along the profile [Figure 4.23B]. A similar distance between is responses is observed in the eastern gravity low in a-a', as it appears to match a low shear-velocity area starting at approximately $58 \mathrm{~km}$ along the profile. The distance-lag between the gravity low and slow shear velocity in line $a-a^{\prime}$ is not present in the b-b' cross-section [Figure 4.23C, D]. Instead, we observe low-velocity below a depth of $20 \mathrm{~km}$ between 30-45 km and 65-75 km along the profile, similar to what was observed for the RR and TT components. The joint inversion cross-section displayed similar characteristics as the RR, TT, and ZZ components, and had shear-velocities that matched low and high gravity anomaly features.

Lastly, it's important to consider the possible error in sampling inherent in the crosssections derived from the 3D Delaunay interpolated model. Figure 4.25 displays the $\mathrm{ZZ}$ shear-velocity cross-section average velocity. The average velocity is not a very useful metric, but it allows us to describe the profile in terms of the number of samples along the profile. The figure suggests that the number of points at each depth of the cross-section should be sufficient to describe the shear-velocity change that we observe in Figures 4.21-4.23. 

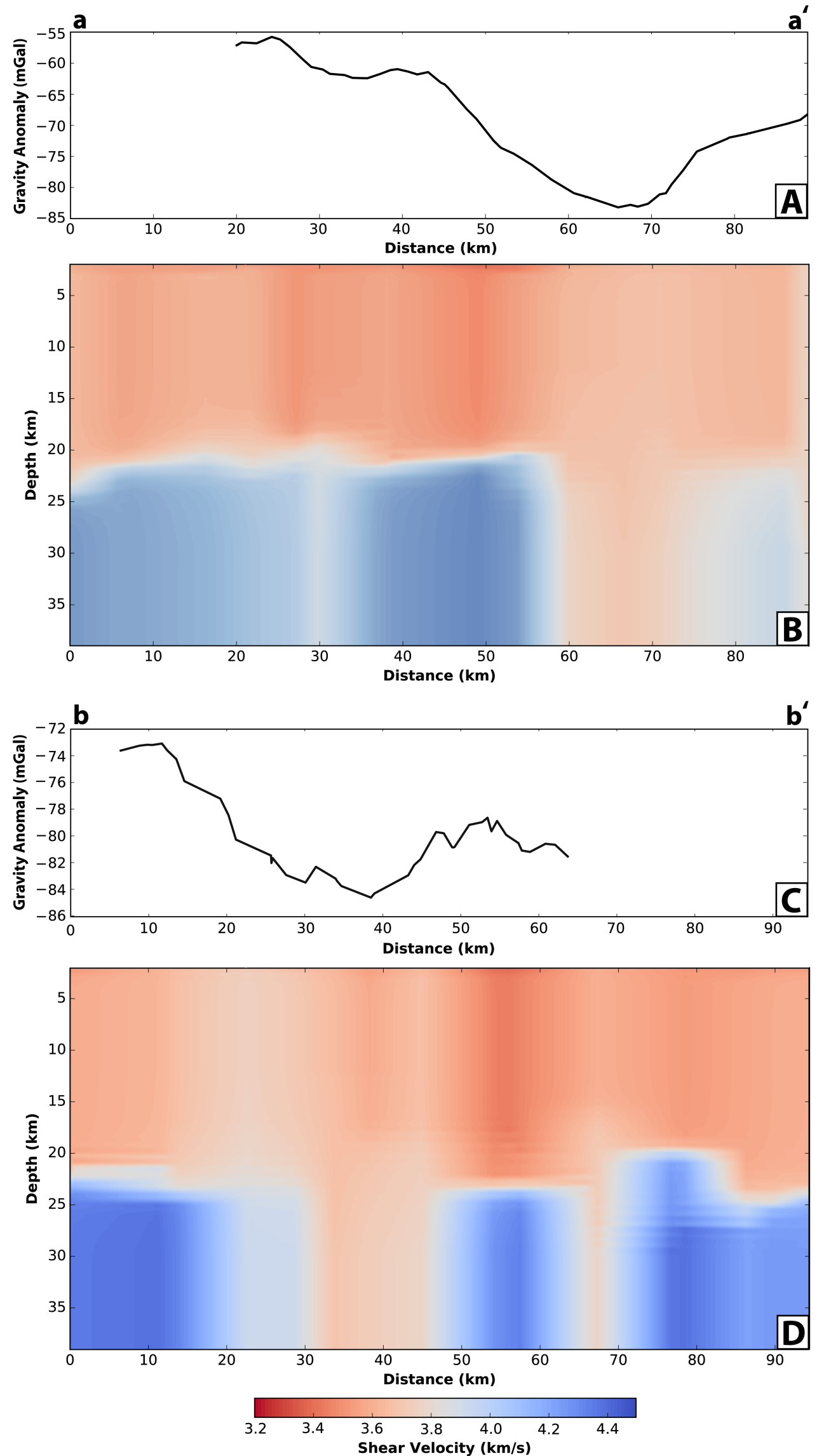

Figure 4.21: [A] Complete Bouguer gravity across a-a' [Figure 4.20], [b] shear-velocity across $a-a^{\prime},[C]$ complete Bouguer gravity across $b-b^{\prime}$, and [d] shear-velocity across b- $b^{\prime}$ using radial-radial (RR) phase velocities. 

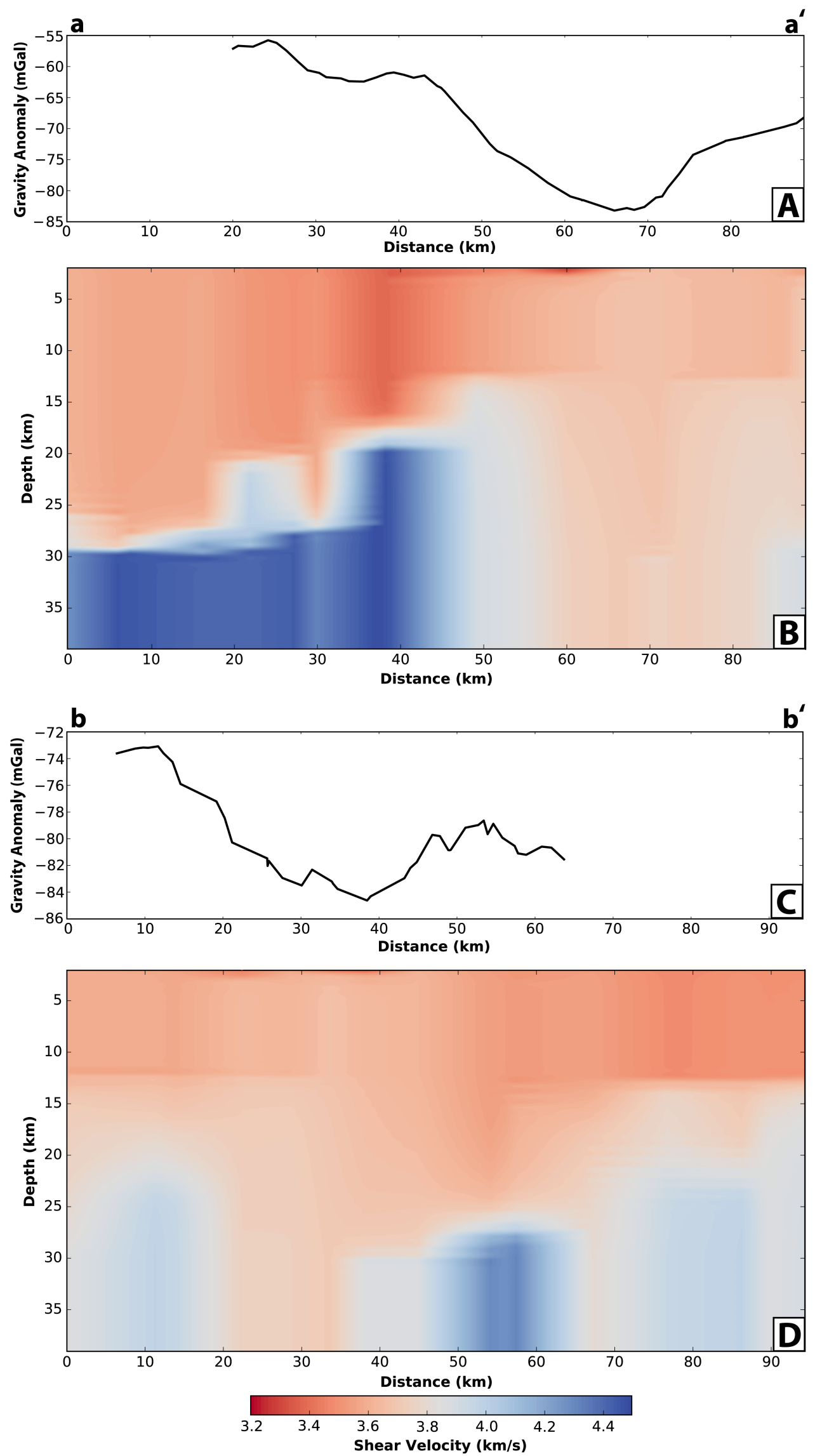

Figure 4.22: [A] Complete Bouguer gravity across a-a' [Figure 4.20], [b] shear-velocity across $a-a^{\prime},[C]$ complete Bouguer gravity across b-b', and [d] shear-velocity across b- $b^{\prime}$ using transverse-transverse (TT) phase velocities. 

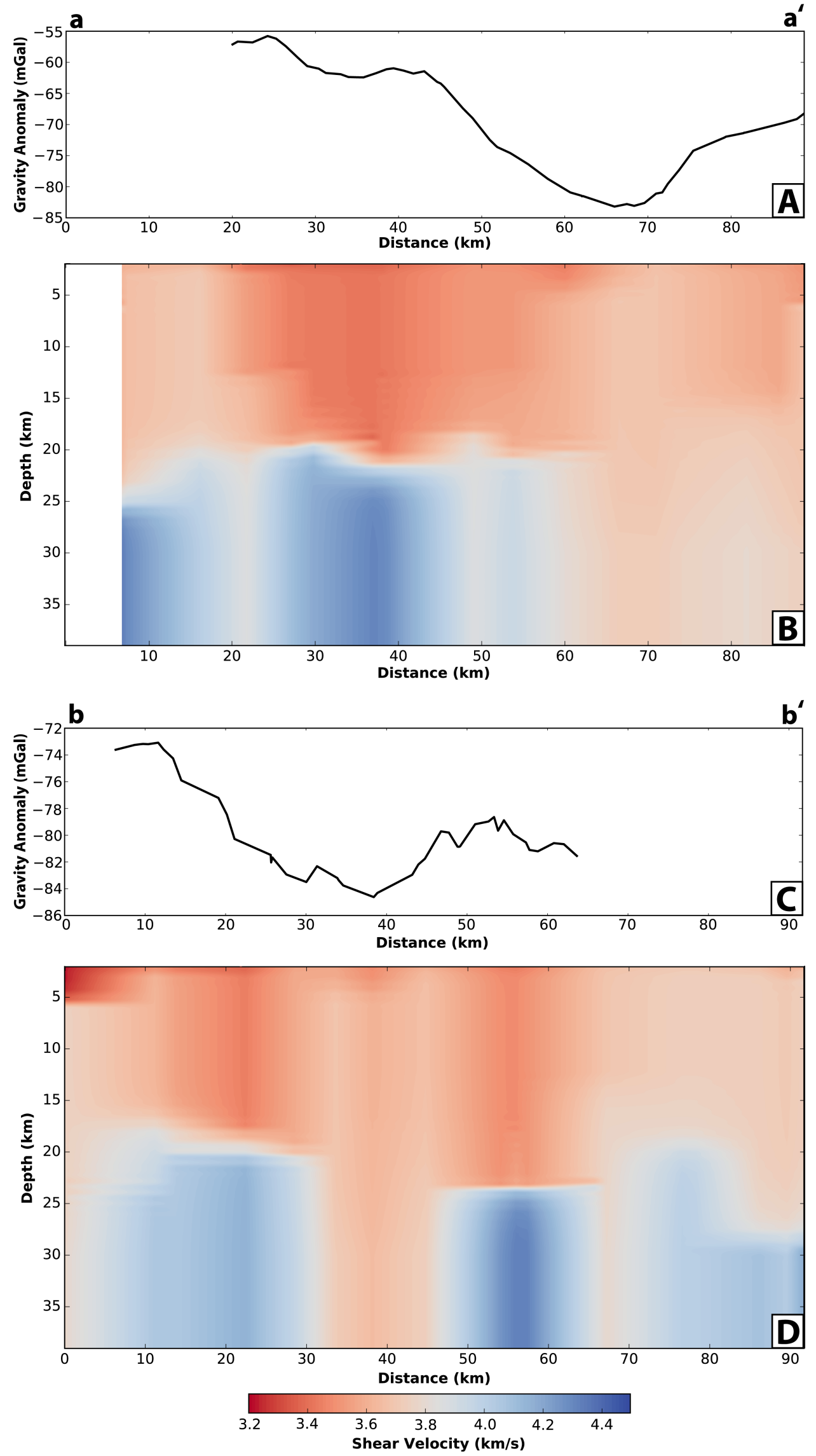

Figure 4.23: [A] Complete Bouguer gravity across a-a'[Figure 4.20], [b] shear-velocity across a-a', [C] complete Bouguer gravity across b-b', and [d] shear-velocity across b-b' using vertical-vertical (ZZ) phase velocities. 

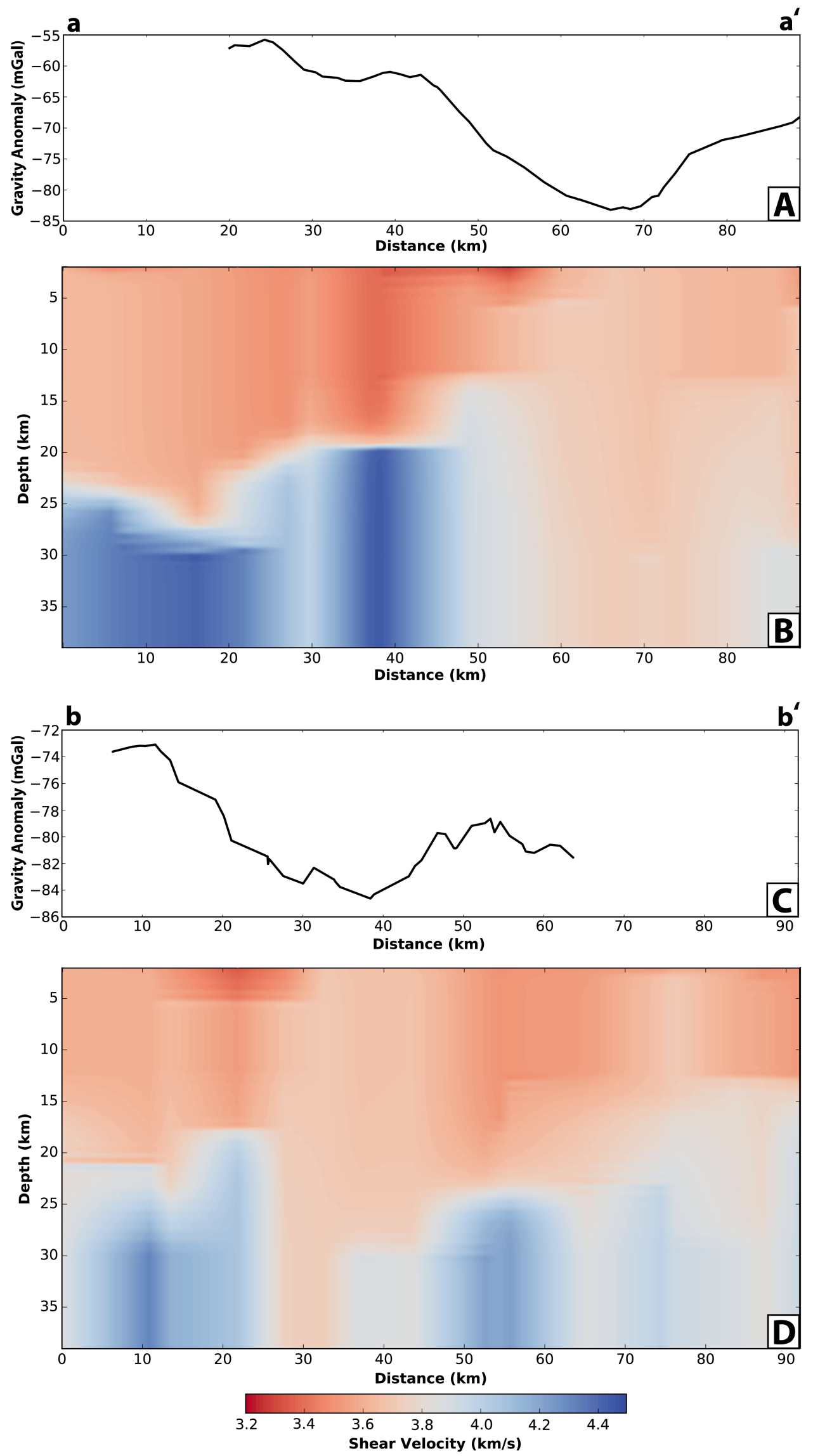

Figure 4.24: [A] Complete Bouguer gravity across a-a'[Figure 4.20], [b] shear-velocity across $a-a^{\prime},[C]$ complete Bouguer gravity across b- $b^{\prime}$, and [d] shear-velocity across b- $b^{\prime}$ using the joint inversion. 

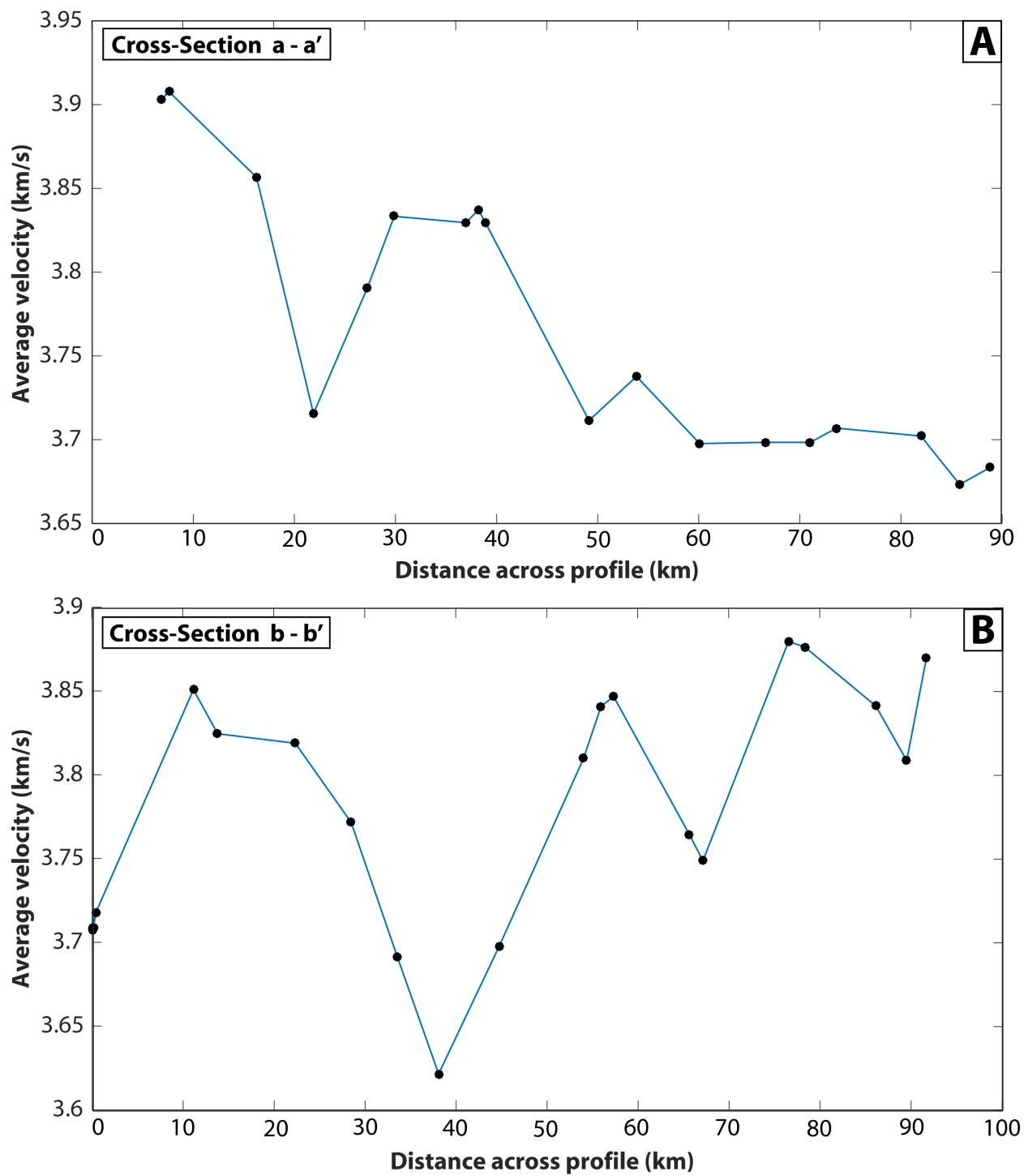

Figure 4.25: Displays the average shear-velocity of the a-a' [A] and b-b' [B] ZZ crosssections. The samples are displayed as black scatter points. 


\subsection{Discussion}

We computed dispersion curves for each latitude-longitude grid-point under the $35 \mathrm{~km}$ resolution threshold up to 12 seconds for phase velocities and 14 seconds for group velocities. Although certain station-paths, and in turn, several latitude-longitude points, could resolve periods deeper than 12 or 14 seconds [Figure 3.17], we decided to establish this period threshold to have consistency in the field [Figure 4.4] and increase stability in the inversion process and the 3D interpolation [Section 4.3.5].

Unfortunately, we found that the group velocities were too unstable to be inverted to shear-velocity [Figure 4.3, Section 4.3.1]. This has been previously observed in other ambient noise research as a result of a strong anisotropic source along the station path [Tsai, 2009, Behr, 2010]. However, our tomographic analysis does not show particular streaks in the results that might be indicative of this [Figures 3.30-3.31]. Another possible hypothesis is that certain station-paths are influenced by the Airy phase [M. Ritzwoller, Personal comm., 2016], a term used to describe a maximum or minimum point in a group velocity dispersion curve caused by energy from a range of periods arriving at the same time, although that typically only affects group velocities at periods between 50 and 240 seconds [Shearer, 1999].

The $1 \mathrm{D}$ inversions were overall very consistent with one another [Figures 4.6 and 4.13]. The RR and $\mathrm{ZZ}$ components and the TT component indicated a change in velocity at approximately $20 \mathrm{~km}$ and $14 \mathrm{~km}$ depth respectively [Figure 4.13], although the layer-change was consistent among all components for certain latitude-longitude points [Figure 4.6]. The 1D inversions were bounded by standard deviations using 4 month segments of data [Figure 4.1].

Average 1D velocity models were obtained for the entirety of the Harrat [Figure 4.10], a region encompassing the $1256 \mathrm{CE}$ eruption [Figure 4.11], and an area off the Harrat [Figure 4.12]. These average values are negatively affected by certain latitudelongitude points which were unable to detect the bottom layer (e.g. Figure 4.11B, C), which may be the result of a shortened dispersion curve bandwidth due to certain periods crossing the $35 \mathrm{~km}$ resolution threshold in the surface-wave maps [Figure 3.19].

The velocity values at each depth were interpolated across the field to obtain shearvelocity maps [Figures 4.14-4.16]. We decided to plot these results with relative scales in order to accentuate the boundaries between low and high shear velocities. Similar features to the dispersion maps can be observed, including the presence of a lowvelocity area south-east of the city of Medina. The boundaries generated by the coneresolution test [Figure 3.19, Figures 3.27-3.32 white outline] are not as sharp as they appear to be in the in the tomographic inversions [Figures 4.7, 4.14-4.16]. This observed sharpness is due to graphical representation in the Python contour function.

The shear-velocity cross-sections [Figures 4.21-4.23] have a velocity increase repre- 
sentative of a layer change at approximately $20 \mathrm{~km}$ depth. This interface depth matches the Conrad discontinuity [Wever, 1989], the boundary between a heterogeneous but broadly andesitic upper crust and the mafic lower crust in the Arabian Shield [Figure 4.26]. This change has been inferred from xenoliths [Stern and Johnson, 2010] and refraction surveys [Mooney et al., 1985]. The resistivity survey of Bedrosian et al. [2017] observed that conductors at $15 \mathrm{~km}$ are not limited to the Harrat area [Figure 3.10], which suggests that the low velocities in the lower crust underneath the 1256 CE eruption area are most likely not due to magma. Instead, we hypothesize that the low velocities in this area are caused by fractures and fluid from prior volcanic eruptions.

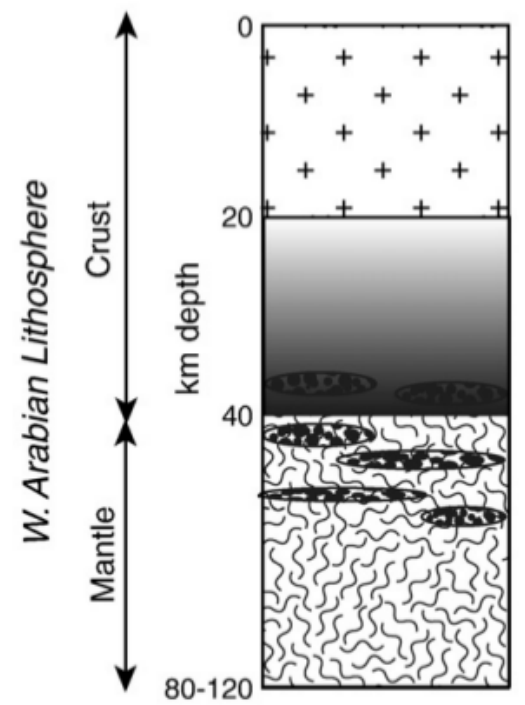

\author{
Upper Crust (heterogeneous \\ but broadly andesitic) \\ Conrad Discontinuity \\ Mafic Lower Crust (plagioclase-rich near \\ top, pyroxene-rich towards bottom)
}

Moho

Al-Augite Group Pyroxenites common

at top of mantle and base of crust

Cr-Diopside Group Spinel Peridotites

increasingly dominate with depth

Figure 4.26: Lithospheric structure of the Saudi Arabian Shield inferred from xenoliths. Figure from Stern and Johnson [2010].

We can use a VsVp ratio of 1.8 [Christensen, 1996] to obtain an estimate of what the P-wave velocity in the lower crust of the cross-sections. In the RR cross-section [Figure 4.21], if we split the lower crust at $55 \mathrm{~km}$ distance down the profile, the average shear-wave velocity of the $0-55 \mathrm{~km}$ (blue) block and the $55-88 \mathrm{~km}$ (red) block are 4.16 and $3.78 \mathrm{~km} / \mathrm{s}$, which correspond to P-wave velocities of $7.49 \mathrm{~km} / \mathrm{s}$ and 6.80 $\mathrm{km} / \mathrm{s}$ respectively. These values can then be compared with the velocities observed by the refraction line of Mooney et al. [1985] [Figure 4.27]. In Figure 4.27, the plagioclaserich and pyroxene-rich components of the lower crust [Figure 4.26] are represented by markedly different P-wave velocities ( $\sim 6.8$ and $7.8 \mathrm{~km} / \mathrm{s})$. Our $\sim 7.5 \mathrm{~km} / \mathrm{s}$ velocity may reflect a mix of those two layers. It is likely that the depth resolution of our maximum surface-wave period (12 seconds) is not enough to distinguish between the two areas across the entire field.

We used the empirical relationships of Christensen and Mooney [1995] to calculate density from the P-wave velocities, and then used the densities to compute an uncom- 


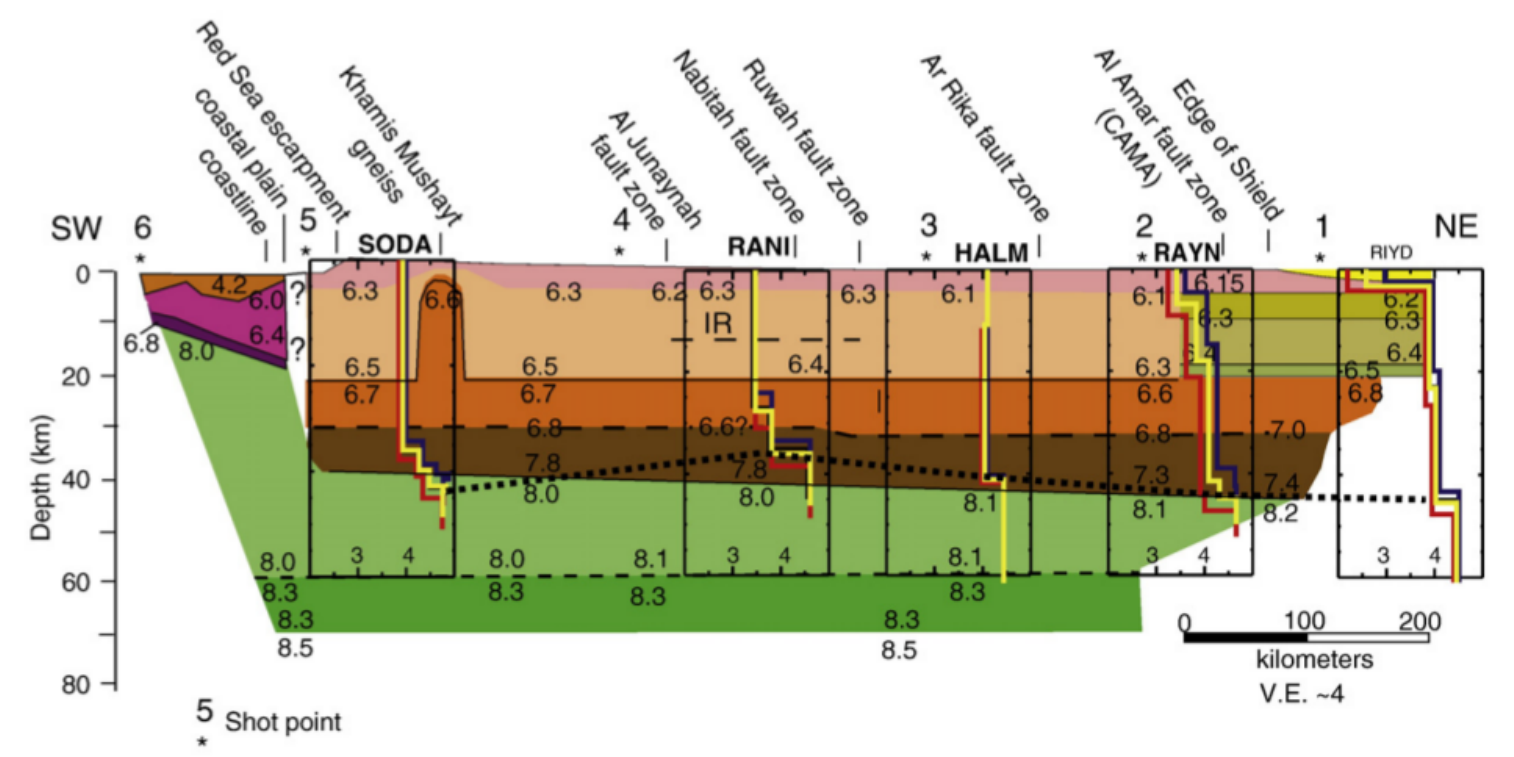

Figure 4.27: Crustal model interpreted from the Mooney et al. [1985] refraction line. Shear-velocity models from Sandvol et al. [1998] are overlain across the profile. Figure from Stern and Johnson [2010].

pensated gravity model for the RR cross-sections of Figure 4.21 [Figure 4.28]. From these relationships, the 7.49 and $6.80 \mathrm{~km} / \mathrm{s}$ P-wave velocities correspond to densities of 3.29 and $3.02 \mathrm{~g} / \mathrm{m}^{3}$ respectively. To simplify the gravity model, we split the crosssections into two density measurements: the red and blue segments of the cross-section were input as gravity measurements of zero and $+0.3 \mathrm{~g} / \mathrm{m}^{3}$ respectively. We utilized the gm-sys software [www.geosoft.com] to compute the expected gravity and compared the result with the observed values of Langenheim et al. [2016] [Figure 4.28]. The gravity calculations appear to reflect some of the long-wavelength measurements of the observed gravity, but their amplitude difference across the cross-section is much larger.

The high velocity values observed in the lower crust at Harrat Al-Madinah $(\mathrm{Vp}=$ $7.5 \mathrm{~km} / \mathrm{s}$ assuming a $\mathrm{VpVs}$ ratio of 1.8) are not a local high-velocity anomaly. Instead they reflect a mafic, pyroxene-rich segment of the lower crust that occurs throughout the Arabian Shield [Stern and Johnson, 2010]. Mooney et al. [1985] observed velocities ranging from 7.3 to $7.8 \mathrm{~km} / \mathrm{s}$ at the base of the lower crust and 6.6 to $6.9 \mathrm{~km} / \mathrm{s}$ in the top segment of the lower crust [Figure 4.27]. The two velocity differences correspond to compositional differences in the lower crust: a plagioclase-rich top layer and a pyroxene-rich bottom layer [Figure 4.26]. We could not differential these layers by doing a four-layer shear-velocity inversion, which suggests that our maximum 12 second phase velocity period may not have much resolution at this depth.

The measured velocities for the lower crust are within the range that might be expected for underplated crust in a rift system [Mooney et al., 1983, 1985, Catchings and Mooney, 1988, Stern et al., 2005, Stratford and Thybo, 2011]. Although volcanism in 

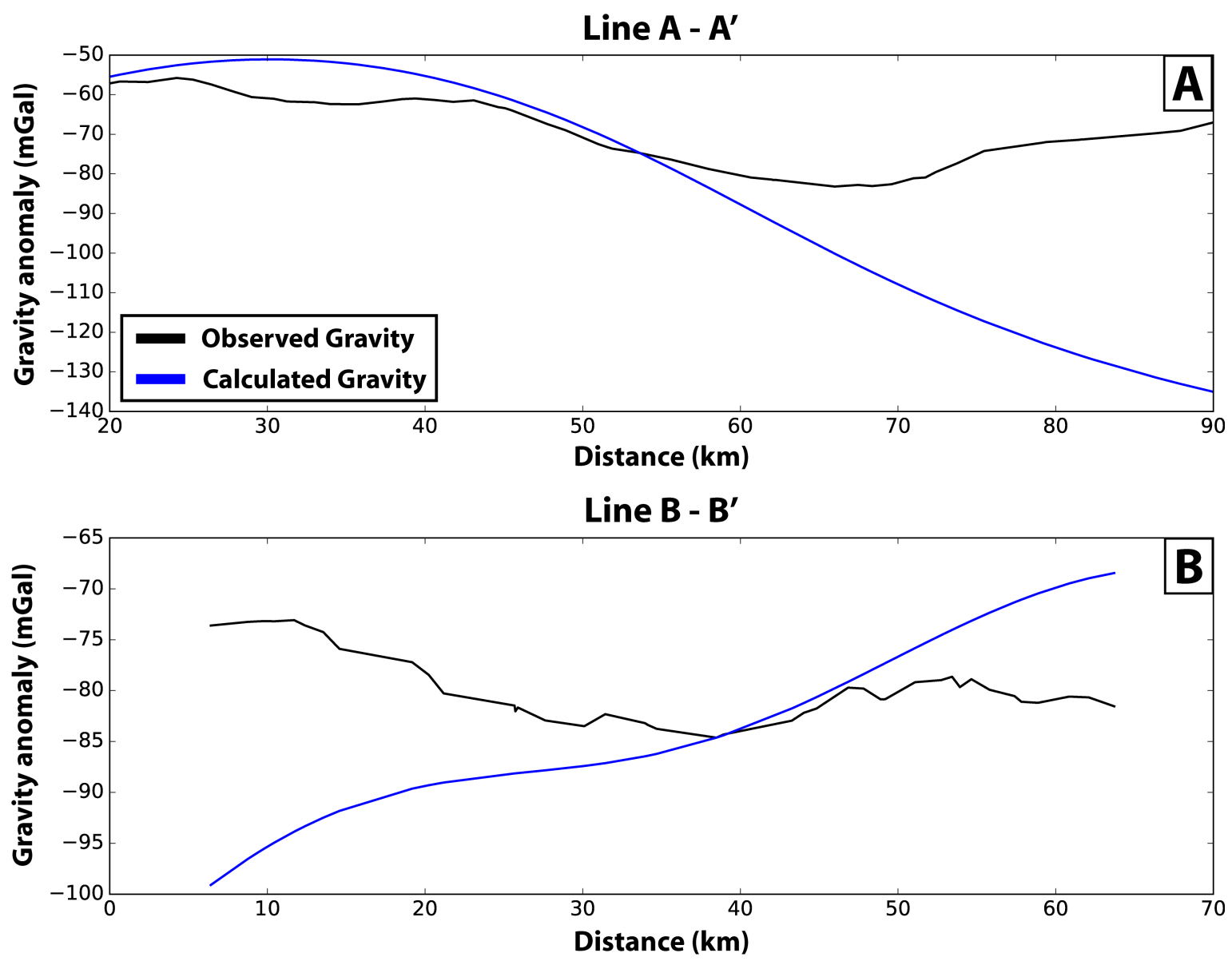

Figure 4.28: Comparisons between observed (black) and calculated (blue) gravity for the RR cross-sections [Figure 4.21] A-A' [A] and B-B' [B].

the Arabian Shield coincided with the opening of the Red Sea rift $32 \mathrm{Ma}$, the crustal layers of the Shield were instead formed in the Neoproterozoic from amalgamated volcanic arcs [Mooney et al., 1985, Stern and Johnson, 2010]. Although they may have similar crustal thicknesses, rift zones typically have high velocities at much shallower depths than what is observed in the Arabian Shield [Mooney et al., 1985] [Figure 4.21]. For example, the Mississippi Embayment has high velocities starting from approximately $7 \mathrm{~km}$ depth [Mooney et al., 1983, 1985]. Rifts commonly have a zone of anomalously low-density rocks called rift pillows in the mantle or lower crust that support the high-density layers [Mooney et al., 1983, Catchings and Mooney, 1988, Stratford and Thybo, 2011]. The dense, mafic lower crust of the Arabian Shield is supported by an anomalously low-velocity mantle lid produced by conduction heating from the Afar hot spot [Chang et al., 2011, Yao et al., 2017, Galvin et al., 2017] [Figure 4.29]. 


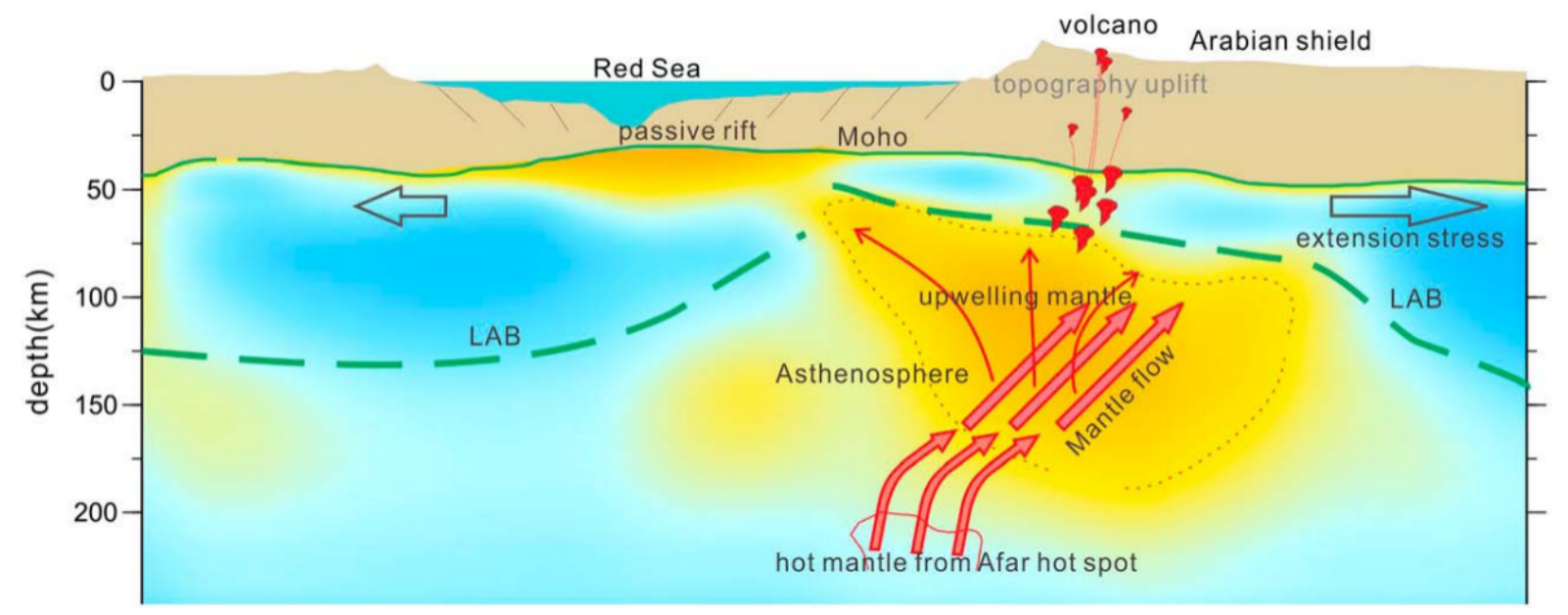

Figure 4.29: Illustration from Yao et al. [2017] showing the model for volcanism in the Western Arabian Shield. Mantle flow from the Afar hotspot produces volcanism in areas of crustal weakness.

\subsection{Conclusion}

In this chapter, we expanded the work conducted in Chapter 3 to determine shear velocities at Harrat Al-Madinah. 1D inversions were conducted for the phase velocities at each grid-point of the dispersion maps using a neighborhood algorithm code [Wathelet, 2008], and determined a velocity increase representative of a layer interface at approximately $20 \mathrm{~km}$ for $\mathrm{RR}, \mathrm{TT}$, and $\mathrm{ZZ}$ components and in the joint inversion [Table 4.3]. We hypothesize that this velocity increase corresponds to the boundary between the andesitic upper crust and the mafic lower crust [Mooney et al., 1985, Stern and Johnson, 2010] [Figures 4.21, 4.26, and 4.27]. Differences between the plagioclaserich and pyroxene-rich portions of the lower crust could not be resolved, most likely due to insufficient depth resolution of the maximum surface-wave period used in the inversion (12 second period). The dispersion curves for group velocities oscillate between high and low velocity values most likely due to a strong anisotropic source off the station path [Tsai, 2009, Behr, 2010] and could not be inverted.

Horizontal cross-sections were computed for all three components and joint inversions, and produced low shear-velocities South-East of the city of Medina for the top $10 \mathrm{~km}$. The 1D velocity models were interpolated into a pseudo-3D volume, and crosssections were taken across the field and compared to gravity measurements [Langenheim et al., 2016]. We found that zones of low shear-velocity below 10 kilometers were spatially co-located with decreases in gravity, suggesting that the same process manifesting in a low gravity anomaly also affects shear-velocity. All shear velocity crosssections were able to observe a velocity change associated with the transition from the andesitic upper crust and the mafic lower crust. High velocities observed in the lower 
crust are most likely due to a pyroxene-rich layer close to the mantle. Abdelwahed et al. [2016] found magma intrusions below Harrat Al-Madinah using P-wave tomography, which is mentioned by Langenheim et al. [2016] as a possible cause for the low gravity anomaly. However, conductive features in a resistivity survey were found both in and off the Harrat, suggesting that liquid magma probably is not present at these depths. We suggest that the observed slow shear velocities are caused by fractures and fluids produced by previous eruption events. 


\section{Chapter 5}

\section{Velocity variations at New Zealand geothermal fields}

\subsection{Introduction}

Ambient noise methods have been used in the geothermal sector to determine the depths at which magmatic processes occur, to distinguish between production and non-production areas, and to observe seismic velocity perturbations associated with fluid extraction [Jousset et al., 2010, Tibuleac and Eneva, 2011, Yang et al., 2011]. More recently, ambient noise methods were used to locate deformation induced by the stimulation of the 2006 Deep Heat Mining Project in Basel, Switzerland [Hillers et al., 2015]. In this chapter, we use ambient noise to obtain time-dependent measurements of shearvelocity to monitor deformation within the geothermal reservoirs of Rotokawa and Ngatamariki, two producing geothermal fields in the central North Island of New Zealand operated by Mercury Energy (formerly Mighty River Power).

Both fields are located in the central-east portion of the Taupo Volcanic Zone (TVZ), a region of rhyolitic and andesitic volcanism [Wilson et al., 1995, Chambefort et al., 2014]. The TVZ began forming approximately 2 Ma as a result of the subduction of the Pacific plate underneath the North Island [Wilson et al., 1995] and subsequent backarc extension [Rowland and Sibson, 2001]. Rifting along the TVZ ranges from less than 5 $\mathrm{mm} /$ year at the southern extent of the rifting (terminating approximately $40 \mathrm{~km}$ south of Lake Taupo) and between 13-19 mm/year at its northern extent (offshore in the Bay of Plenty) [Villamor and Berryman, 2006, 2010, McNamara et al., 2016]. Mercury Energy maintains seismic networks of $4.5 \mathrm{~Hz}$ short-period instruments at each of the fields whose primary purpose is to locate microseismicity [Figure 5.1].

We chose two time periods and regions to analyze for velocity changes: [1] 20092010 Rotokawa, which included the activation of the 140 MW “Nga Awa Purua” triple flash power plant that increased electricity production from 34 to 174 MW [Hernan- 
dez et al., 2015], and [2] 2012-2013 Ngatamariki, which contained the stimulation of three injection wells and the beginning of $82 \mathrm{MW}$ of electricity production [Clearwater et al., 2015]. These datasets represent an opportunity to observe velocity changes associated with increased injection (Rotokawa) and the onset of geothermal production (Ngatamariki). 


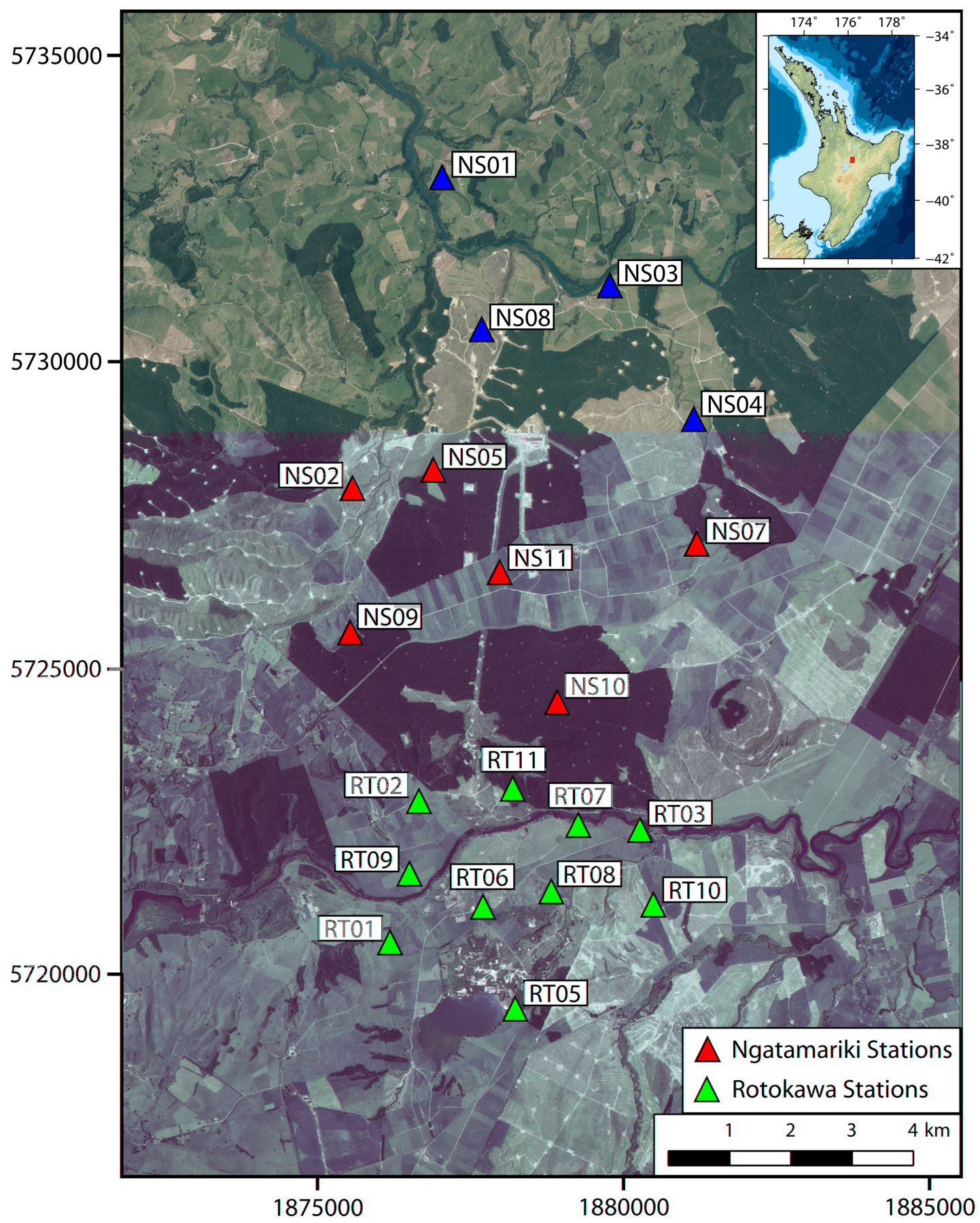

Figure 5.1: Map of the seismometers operated by Mercury Energy at Rotokawa (red triangles) and Ngatamariki (blue triangles). Note: The Rotokawa and Ngatamariki stations displayed are from 2009-2010 and 2012-2013 respectively, the time periods used in this study. Coordinates are in New Zealand Transverse Mercator 2000 coordinates (NZTM2000) 


\subsection{Velocity variations theory}

As discussed in detail in previous chapters, seismic records produced by a diffusive ambient noise source can be cross correlated to calculate an estimate of the empirical Green's function of the station path [Lobkis and Weaver, 2001, Shapiro and Campillo, 2004]. In Chapter 3, we explored how the group and phase velocities could be extracted from the amplitude and phase of "ballistic" arrivals, the large amplitude peak corresponding to a surface wave traveling between the stations. However, instead of using these arrivals, we now turn our attention to the cross-correlation coda, whose changes are governed by variations in the elastic or scattering properties of the wavefield [Brenguier et al., 2008b, Hillers et al., 2015].

This method has been applied to detect changes in velocity for a variety of deformation mechanisms, including: inflation of volcano edifices prior to eruptions [Brenguier et al., 2008b, Obermann et al., 2013], co-seismic deformation and recovery due to crustal stress or creation of new fractures [Wegler and Sens-Schöndelfer, 2007, Brenguier et al., 2008a, Wegler et al., 2009, Rivet et al., 2011], saturation of fractures due to increased groundwater level [Sens-Schönfelder and Wegler, 2006, Hillers et al., 2014], and thermal heating [Sens-Schönfelder and Larose, 2008].

There are two main methods used by the ambient noise community to obtain velocity changes from cross-correlations: the doublet or Moving Window Cross Spectral Technique [Brenguier et al., 2008b, Clarke et al., 2011] and the stretching method [Sens-Schönfelder and Wegler, 2006]. The Moving Window Cross Spectral technique (MWCS), initially developed by Ratdomopurbo and Poupinet [1995] for earthquake doublets and later applied to ambient noise, compares the cross-spectrum between two stacks of cross-correlations to measure a delay-time [Brenguier et al., 2008b, Clarke et al., 2011]. The stretching method instead computes a delay time between two traces by stretching and compressing a waveform until it optimizes its cross-correlation [SensSchönfelder and Wegler, 2006]. Each method has specific strengths and weaknesses. The MWCS method is extremely dependent on parametrization, specifically the window functions used to determine the time delay from the cross-spectrum [Clarke et al., 2011], while the stretching method measures delay from the time-domain [Sens-Schönfelder and Wegler, 2006]. However, a recent study has demonstrated that the stretching method can produce significant errors in its velocity measurements if the noise changes frequency content [Zhan et al., 2013]. This can prove a problem for studies using many months of natural ambient noise data, as ambient noise changes in amplitude and frequency during the summer and winter months [Zhan et al., 2013], a phenomenon that is also observed in our data [Figure 5.2, Appendix B.4]. Due to this, we chose to use the MWCS method for determining velocity changes.

The MWCS technique uses a reference and moving stack to compute delay times 

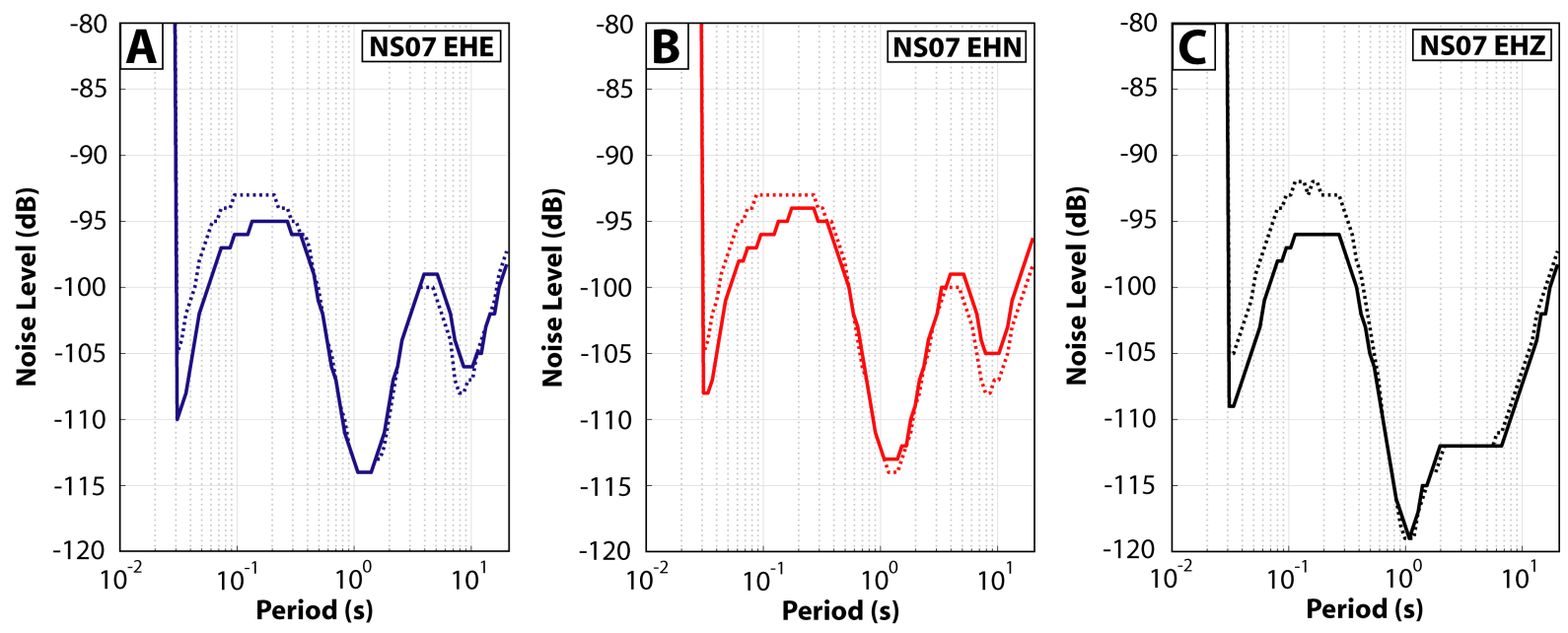

Figure 5.2: Weighted noise means of winter (solid line) and summer (dotted line) for Ngatamariki station NS07 east [A], north [B], and vertical [C] components.

within the cross-correlation coda. A reference stack represents a stable time period where velocity is assumed to be approximately constant, while a moving stack is taken during a time period where velocity changes are expected.

The first step in computing velocity changes is to calculate the cross-spectrum between the reference stack and the moving stack for each station pair. The method was first developed by Poupinet et al. [1984] to observe seismic velocity changes associated with large earthquakes using earthquake doublets (a time series before and after the earthquake). The cross-spectrum $(X(f))$ between a reference and moving stack is calculated by [Poupinet et al., 1984, Clarke et al., 2011]:

$$
X(f)=F_{r e f}(f) F_{m o v}^{*}(f)
$$

where $F_{\text {ref }}$ and $F_{\text {mov }}$ are the Fourier transforms at frequency $f$ of the reference and moving cross-correlations and the asterisk denotes the complex conjugate. Note that the equation for the cross-spectrum [Equation 5.1] is analogous to a Fourier transform of a cross-correlation between two time series. The slope of the phase of the cross spectrum $(\phi(f))$ contains information regarding the time-delay between the two time series $\delta t_{i}$, where $i$ represents the index of the moving window [Figure 5.3] [Poupinet et al., 1984].

For a frequency range of interest containing samples $j=1,2,3, \ldots, h$, we define the phase of the cross-spectrum $(\phi(f))$ as:

$$
\phi_{j}(f)=m f_{j}
$$

where the units of the phase are radians. The slope $m$ of the phase is related to the time delay $(\delta t)$ by:

$$
m=2 \pi \delta t_{i}
$$


where $i$ is the index of the moving window. The slope is determined by a weighted linear regression, where both the spectral amplitude and cross-coherence of the energy densities are used. The coherence between the two signals is an important parameter that directly effects the quality of the results. We will discuss its importance at length in Section 5.3.3. The cross-coherence $(C(\omega))$ is defined as:

$$
C(f)=\frac{|\overline{X(f)}|}{\sqrt{\overline{\left|F_{\text {ref }}(f)\right|^{2}} * \overline{\left|F_{\text {mov }}(f)\right|^{2}}}}
$$

where the overline represents sliding raised-cosine smoothing with width $0.1 \mathrm{~Hz}$. The weighting of each frequency sample $j$ is:

$$
w_{j}=\sqrt{\frac{C_{j}^{2}}{1-C_{j}^{2}} * \sqrt{\left|X_{j}\right|}}
$$

The weighted linear regression to determine the slope $m$ of the phase is:

$$
m=\frac{\sum_{j=1}^{h} w_{j} f_{j} \phi_{j}}{\sum_{j=1}^{h} w_{j} f_{j}^{2}}
$$

where $h$ is total number of samples in the frequency range.

The associated error is calculated by:

$$
e_{m}=\sqrt{\sum_{j}\left(\frac{\omega_{j} f_{j}}{\sum_{i} \omega_{i} f_{i}^{2}}\right)^{2} \sigma_{\phi}^{2}}
$$

where $\sigma_{p h i}^{2}$ is the squared misfit of the data to the modeled slope:

$$
\sigma_{\phi}^{2}=\frac{\sum_{j}\left(\phi_{j}-m v_{j}\right)^{2}}{h-1}
$$

A graphical representation of the weighted linear regression is displayed in Figure 5.3 [Clarke et al., 2011]. The MWCS time window of this example is between -18 to -12 seconds of the acausal part of the cross-correlation [Figure 5.3A], has approximately the same coherence throughout the window [Figure 5.3B], and the slope is well-fit throughout the frequency band of interest [Figure 5.3C].

The slope the phase is related to time delay by a factor of $2 \pi$ [Equation 5.3]. After time delay is computed for each moving window (index $i$ ), changes in velocity $(\delta v)$ can be obtained through [Ratdomopurbo and Poupinet, 1995]:

$$
\delta v / v=-\delta t / t
$$


a)

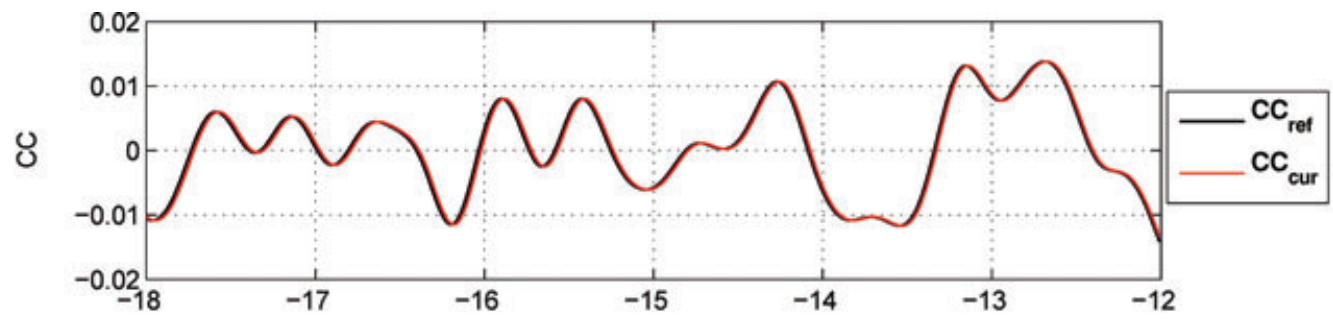

b)

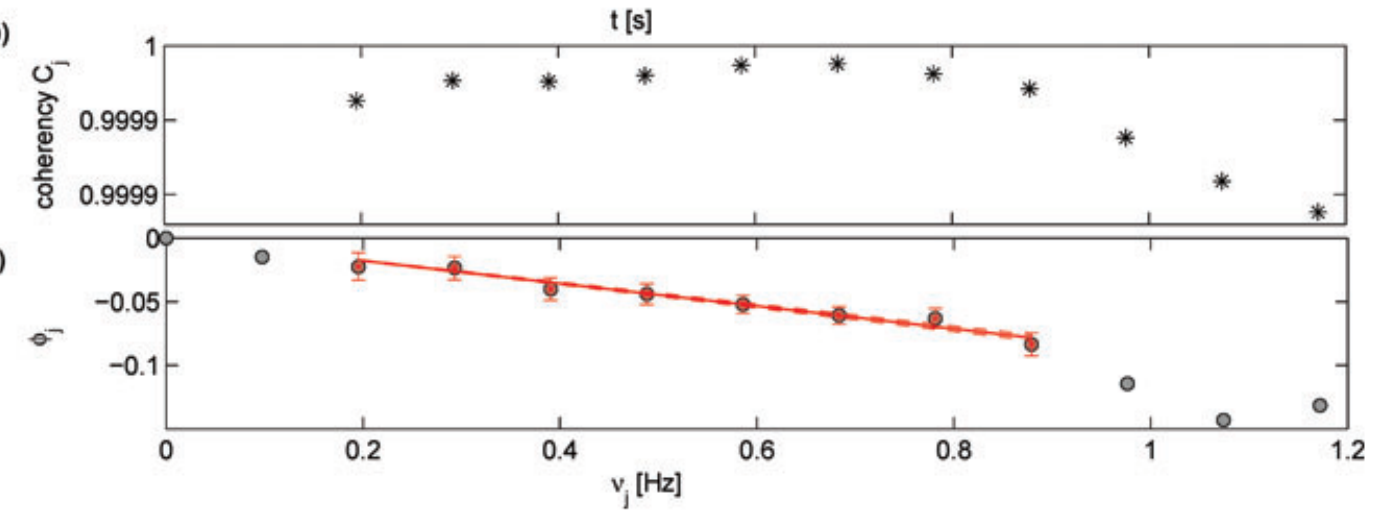

Figure 5.3: Example of the slope calculation of the MWCS method from Clarke et al. [2011]. [A] The cross-correlation traces for the reference $\left(C C_{r e f}\right)$ and moving $\left(C C_{\text {mov }}\right)$ stacks for the -18 to -12 seconds time window of the acausal side. [B] Coherence values computed using Equation 5.4 for the entire frequency spectrum. [C] Phase values $\left(\phi_{j}\right)$ for the time window (index $i$ ) and the corresponding slope $m$ for the frequency band of interest (Equation 5.6).

\subsection{Methods}

In this section, we describe the procedure used to compute velocity changes at the geothermal fields according to the theory of Section 5.2. The steps for determining velocity changes are as follows: [1] computation of daily empirical Green's function from natural ambient noise, [2] checking the stability of scatterers within the daily cross-correlations in the time and frequency domain, and [3] the parametrization of the MWCS technique.

\subsubsection{Obtaining cross-correlations}

As in the previous chapter, we begin with the computation of daily cross-correlations from natural ambient seismic noise. Our processing routine for determining crosscorrelations is similar to the one discussed in Chapter 3 for the tomography of Saudi Arabia, but has a few different parameters due to changes in network aperture and corner frequency of the instruments [Table 5.1]:

The noise field for each station at Rotokawa and Ngatamariki was determined using the USGS software PQLX [McNamara and Boaz, 2011] [Section 2.2]. One month 


\begin{tabular}{||l||l||}
\hline Property & Value \\
\hline Analysis duration (s) & 86400 \\
\hline Cross-correlation sample-rate $(\mathrm{Hz})$ & 20 \\
\hline Correlation duration $(\mathrm{s})$ & 900 \\
\hline Maximum lag-time $(\mathrm{s})$ & 60 \\
\hline Filtering $(\mathrm{Hz})$ & $0.01-1.1$ \\
\hline Whitening $(\mathrm{Hz})$ & $0.1-0.9$ \\
\hline Time normalization type (s) & One-bit \\
\hline
\end{tabular}

Table 5.1: Rotokawa and Ngatamariki processing parameters.

segments of both summer and winter were computed to assess the variability of the signals [Appendix B.4]. Due to the surrounding geothermal production, the data recorded at both networks includes high frequency cultural noise. All stations at Rotokawa and Ngatamariki contained high frequency noise, but its amplitude relative to the natural noise peak varied from station to station. An example of the noise field at one of the Ngatamariki sites is displayed in Figure 5.4.

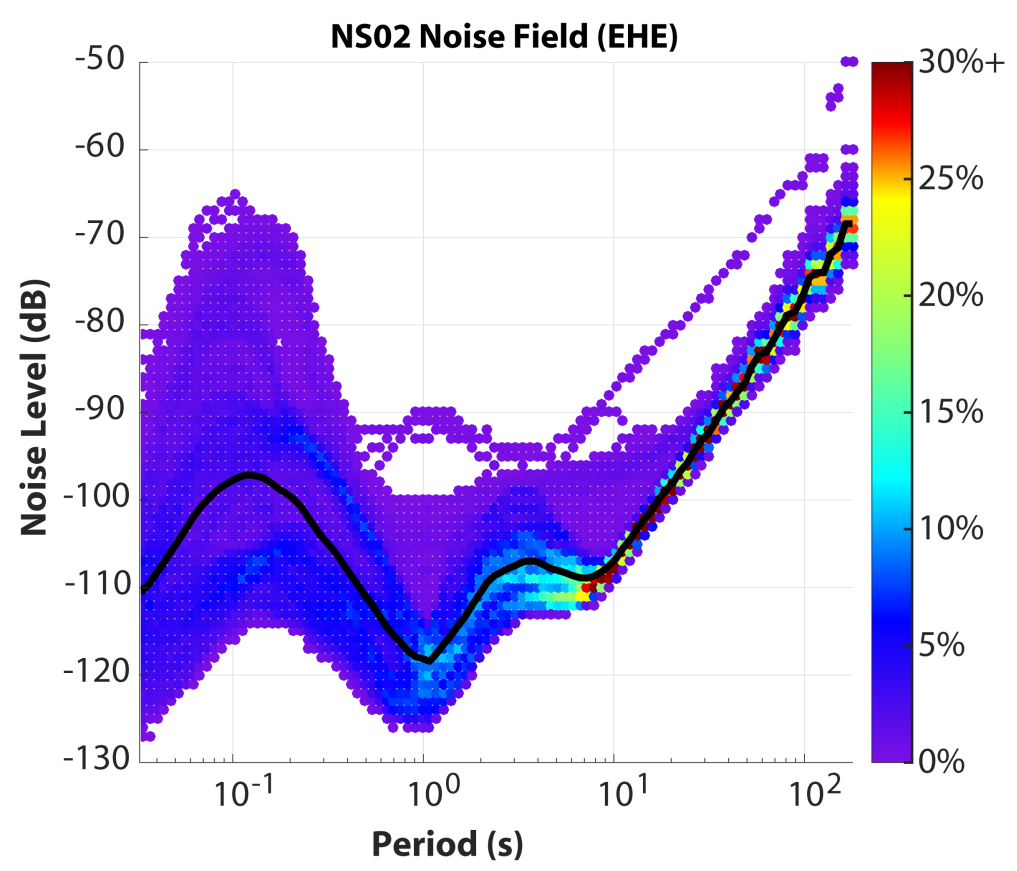

Figure 5.4: The noise field for the East component of the $4.5 \mathrm{~Hz}$ Ngatamariki station NS02. Both cultural (short period) and natural (long period) noise peaks can be observed. The weighted mean is displayed as a solid black line. The ramp in the long period is caused by the corner frequency of the instrument response.

Due to the high frequency noise, the data from each station is bandpass filtered between $0.9-10 \mathrm{~s}(0.1-1.1 \mathrm{~Hz})$. Unlike the broadband instruments of the Saudi Arabia study, the short period instruments are not reliable above 10 seconds, so there are no 
advantages to expanding the pass-band. Zero-point noise is not observed in the data, most likely due to the short inter-station distance.

Each segment is spectral whitened and one-bit (time-domain) normalized prior to cross-correlation and stacked for the entire day. Whitening prior to one-bit normalization and a $0.1-1.1 \mathrm{~Hz}$ filter were necessary to prevent the cultural noise from masking the natural ambient signal, as previously discussed in Section 2.3.

\subsubsection{Time-domain stability}

An understanding of the stability of cross-correlation pairs is essential prior to computing velocity changes. If cross-correlation peaks are not stable in time, they can be falsely interpreted as velocity changes. Common causes for cross-correlation instability include: instrument maintenance or hardware replacement, station outages, MWCS band-pass frequencies unaligned with the noise spectra, and incorrect MWCS time windows. In this section, we cover the cross-correlation stability in the time-domain. Frequency-domain stability using magnitude-squared coherence will be discussed in Section 5.3.3.

A simple but effective way to check time-domain stability is through visual inspection by plotting each daily correlation in time [Figure 5.5]. This was possible due to the small number of stations in our network. Noise peaks, whether they be ballistic arrivals or scatterers, should be observed throughout the whole record if they are to be used for velocity changes. From the graphical representation of the example pair in Figure 5.5, we can observe stable signals at approximately -5 and 5 seconds, as well as clear scatterers at approximately 10 and 25 seconds. These amplitude plots can become difficult to interpret as the number of days increases. Therefore, we use the Matlab function imagesc to convert the amplitude plots to an image, with high amplitudes marked as a light color and low amplitudes marked as a dark color [Figure 5.6]. 


\section{NZ_NS08 - NZ_NS09 (5.40 km) || ZZ || Xcorr: 2012-05-17 to 2012-10-01}

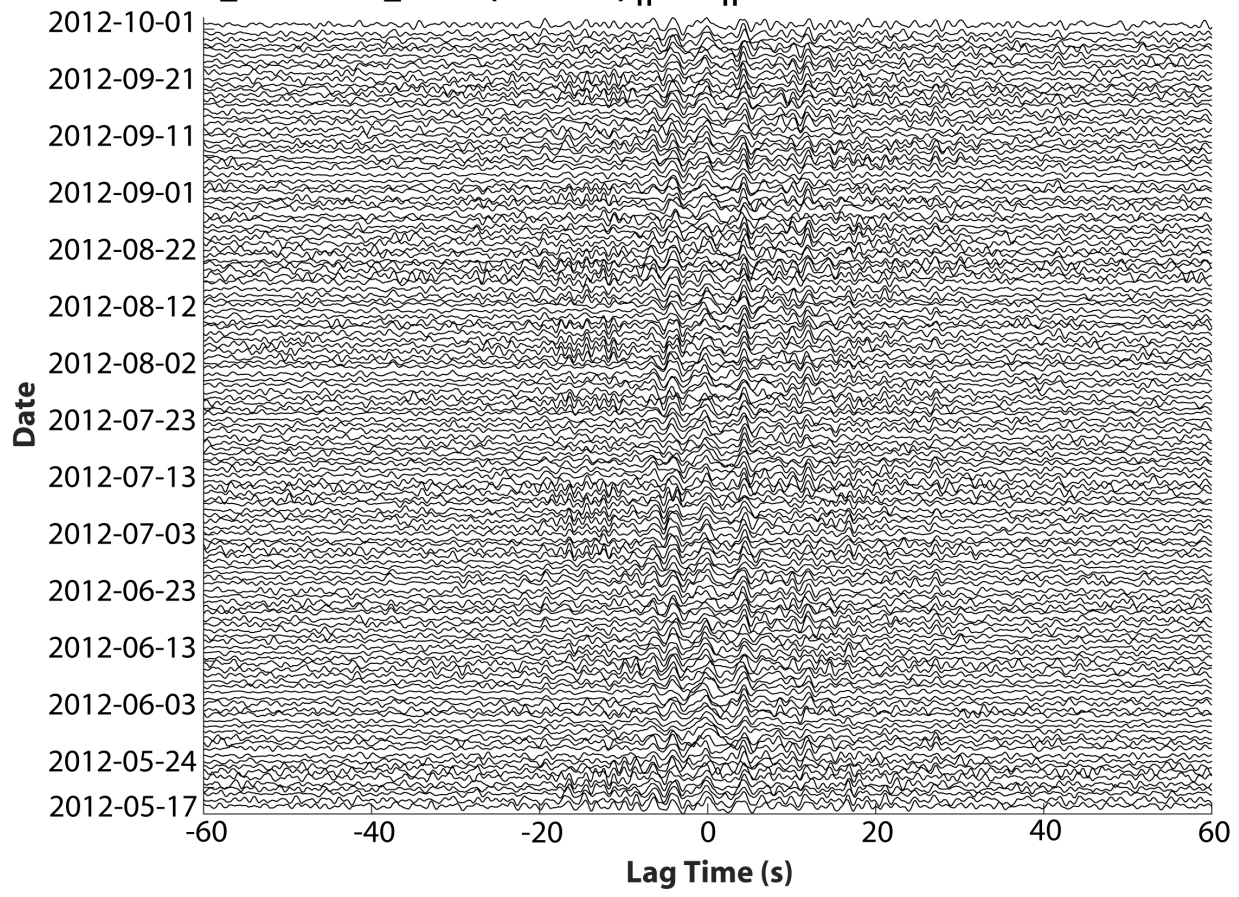

Figure 5.5: Daily vertical-vertical (ZZ) cross-correlations for Ngatamariki network station pair NS08-NS09 from 2012-05-17 to 2012-10-01 (140 days).

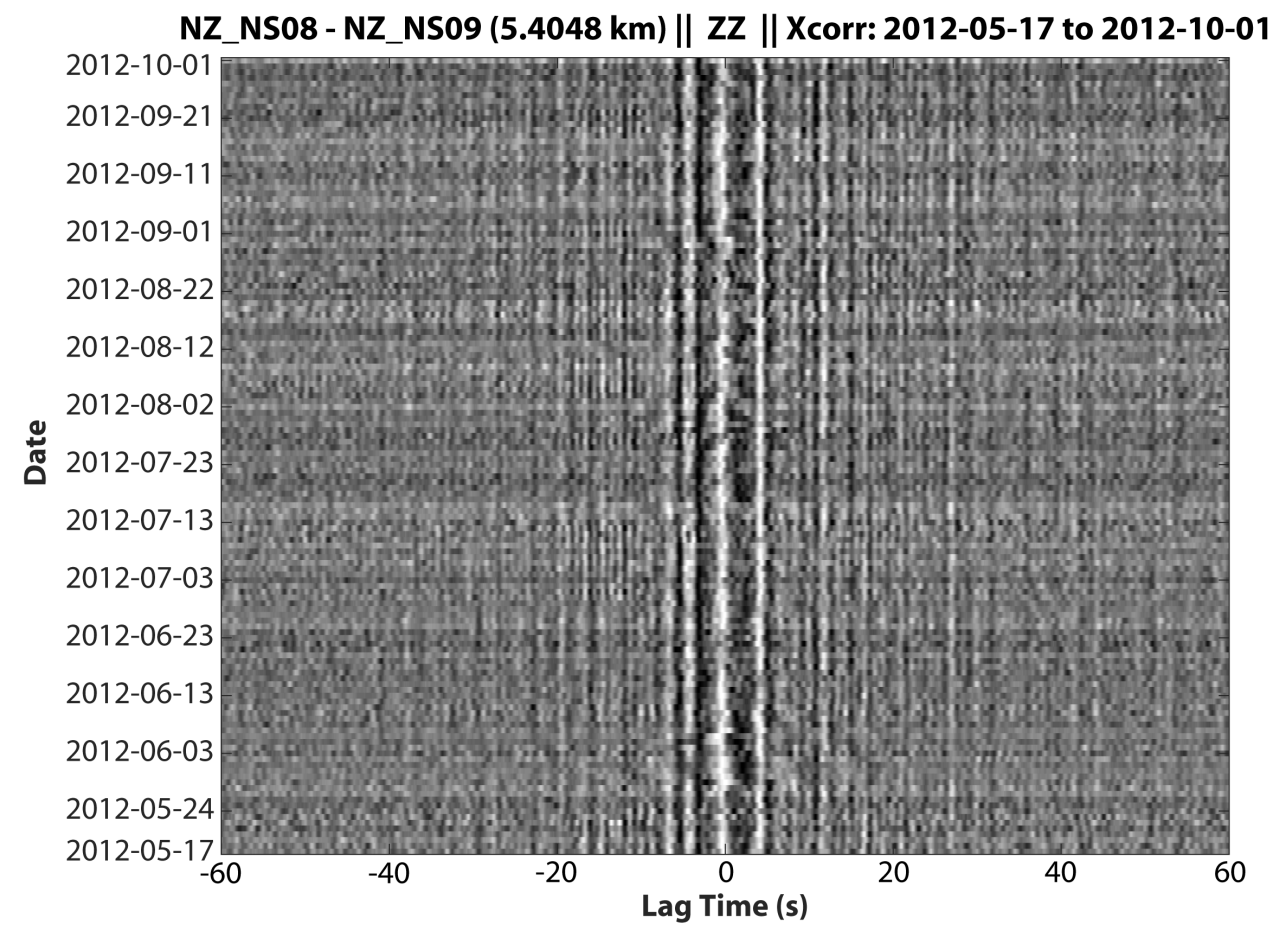

Figure 5.6: Image of daily vertical-vertical (ZZ) cross-correlations for station pair NS08NS09 (See Figure 5.5). Light colors represent areas of high amplitude and dark colors represent areas of low amplitude. 


\subsubsection{Frequency-domain stability}

Magnitude squared coherence measures the stability of scatterers in the frequency domain. The magnitude squared coherence $\left(C_{x y}\right)$ is a function between zero and one which gives a measure of correlation between two signals for each frequency $(\omega)$ [Smith, 2007]:

$$
C_{x y}(\omega)=\frac{\left|R_{x y}(\omega)\right|^{2}}{R_{x}(\omega) R_{y}(\omega)}
$$

where $R_{x}$ and $R_{y}$ are the Fourier transforms of signals $x$ and $y$ and $R_{x y}$ is their crossspectral density. In our case, the $x$ and $y$ variables correspond to the reference and moving stacks. The magnitude squared coherence was computed between each day of the moving stack and the reference stack [Figure 5.7]. Each daily coherence value [Figure 5.7E] is then combined to form an image using the Matlab routine imagesc [Figure 5.8]. For the example provided in Figure 5.8, a consistently high coherence value is observed at approximately 0.25 and $0.45 \mathrm{~Hz}$. Due to the pre-processing bandpass chosen in Section 5.3.1 (0.1-1.1 Hz)[Table 5.1], frequencies above $1.1 \mathrm{~Hz}$ are not coherent for any station-pair [Figure 5.8]. 

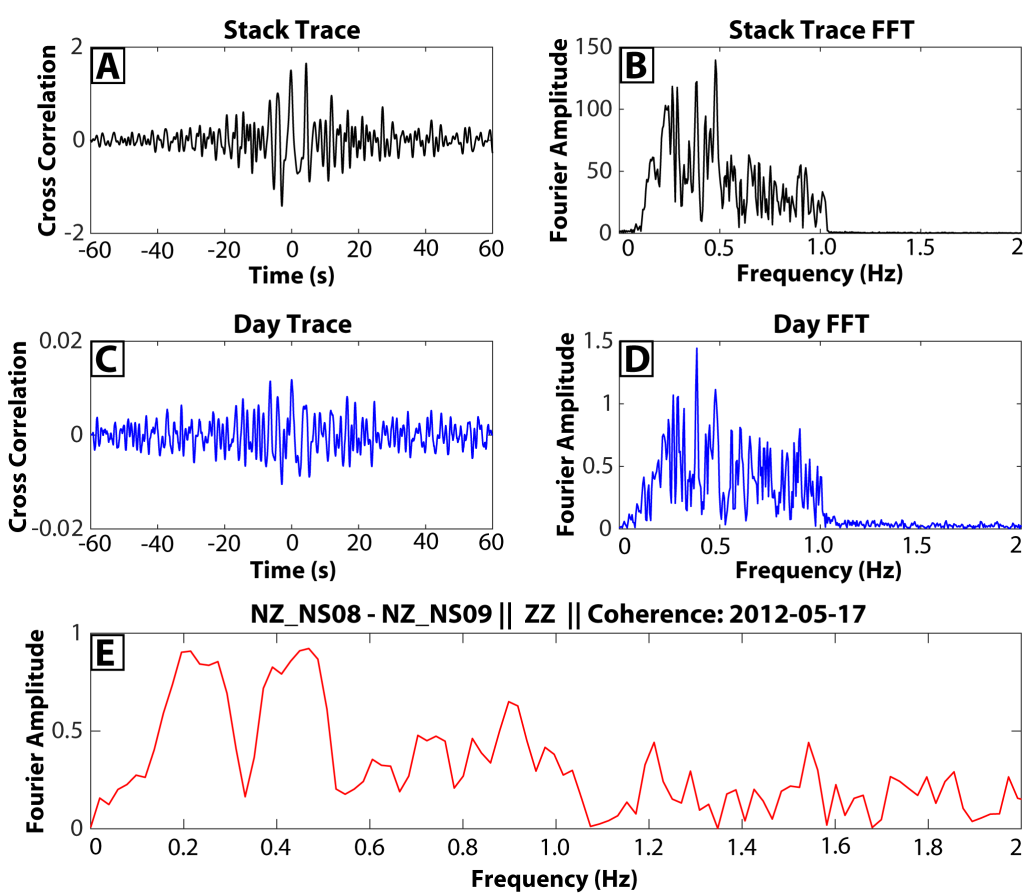

Figure 5.7: One day calculation of magnitude squared coherence (MSC) for station pair NS08-NS09 in the vertical-vertical (ZZ) component [E] using 140 day stack [A] and a one day trace $[\mathrm{C}]$ of cross-correlation. Comparisons between the Fourier transforms of the stack trace $[\mathrm{B}]$ and the one day of data [D] are used to verify the calculated MSC.

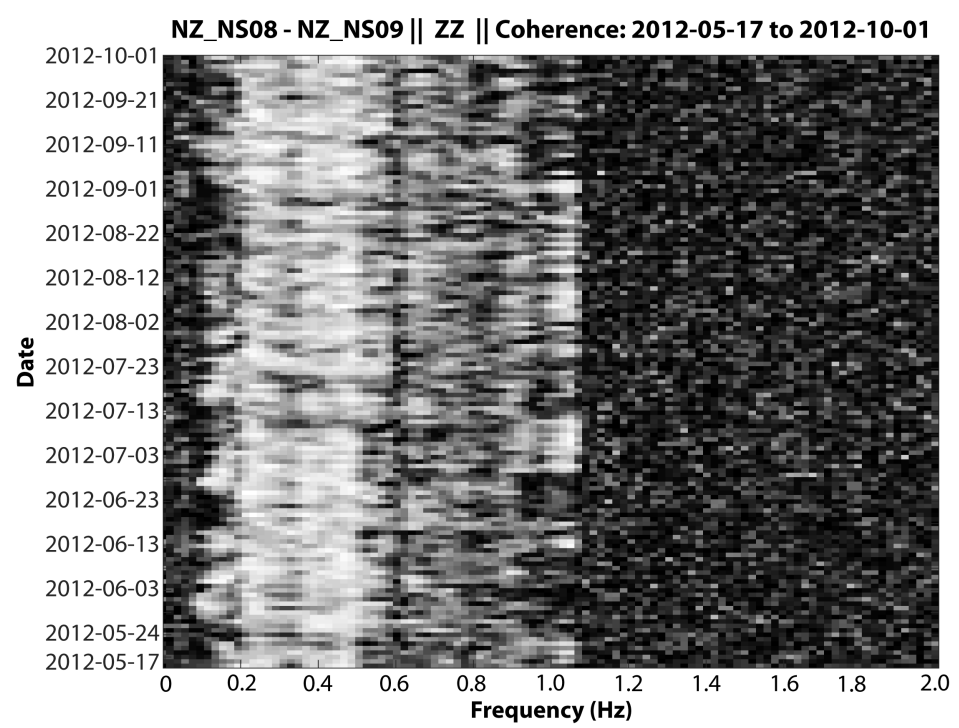

Figure 5.8: Daily coherence for station pair NS08-NS09 in the vertical-vertical (ZZ) component from 2012-05-17 to 2012-10-01. Light colors display areas of high coherence and dark colors are areas of low coherence. Signals beyond $1.1 \mathrm{~Hz}$ are not coherent due to the pre-processing bandpass chosen in Section 5.3.1. 


\subsubsection{Computing velocity changes}

Velocity changes were computed using the software MSNoise [Lecocq et al., 2014], based on the Moving-Window Cross Spectral method (MWCS) of Clarke et al. [2011] previously discussed in Section 5.2. Time-delays are computed incrementally across daily cross-correlation stacks of station-pairs using a time-window $T$ and overlap $L$ [Figure 5.9, Equations 5.2 and 5.3, Table 5.2]. It is important to remember that the time-delay is weighed by the coherence prior to the linear regression [Equation 5.5]. Additionally, the number of stacked days within the moving stack must be considered. One of the objectives when using the MWCS method is to use a moving stack whose length is appropriate for the time-scale of measured signal. For example, large moving stacks can average out short, transient velocity changes. We knew from the injection and production schedules (later discussed in Sections 5.4.2 and 5.6.2) that they operated on the scales of weeks and months, so we utilized 10, 20, 30, 40 and 50 day windows in the moving stacks for both of the fields.

\section{MWCS Workflow}

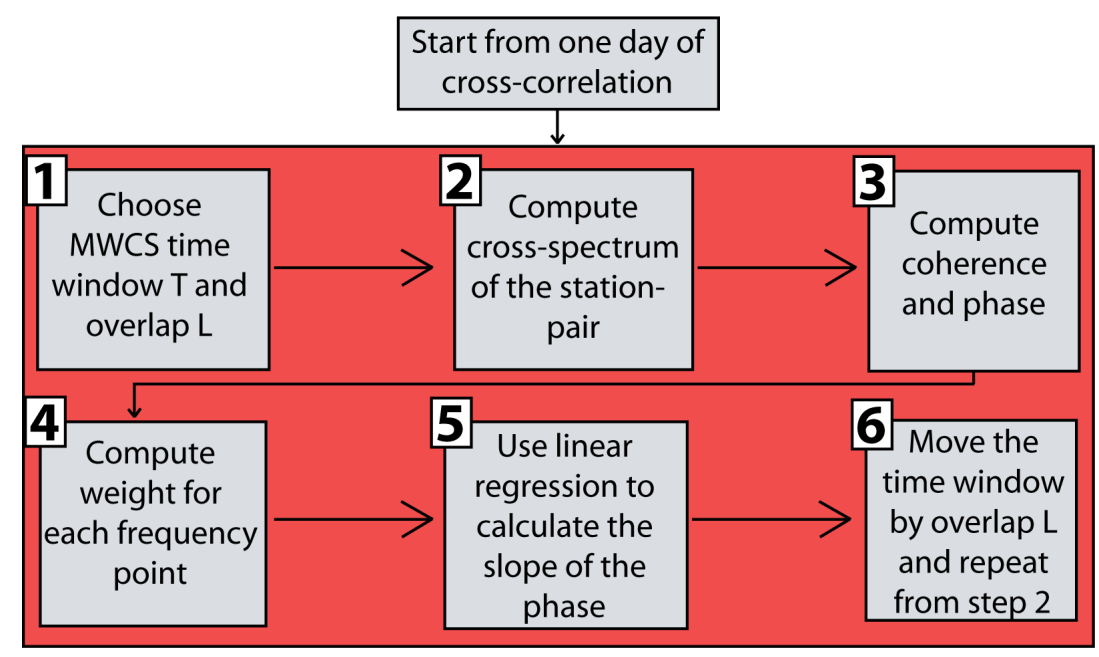

Figure 5.9: MWCS workflow starting from a daily cross-correlation, using the theory described in Section 5.2.

The overall time delay for a daily cross-correlation $(\delta t)$ is calculated using a weighted least-square regression. MSNoise calculates the WLS forced through the origin [Equation 5.11] and allowing a non-zero y-intercept (a) time-delay [Equation 5.12] [Lecocq et al., 2014]:

$$
\begin{gathered}
\delta t_{0}=m_{0} t \\
\delta t=a+m t
\end{gathered}
$$


The effect on data of the unrestricted linear regression and one forced through the origin will be re-addressed in Section 5.5.4, specifically in Figure 5.30. These slopes are calculated from both acausal (lag-time $t<0$ ) and causal (lag-time $t>0$ ) delay values.

Figure 5.10 displays the MWCS delay-time for a single day (2009-01-01) between Rotokawa station-pair RT01-RT02 for the RR component using a 10 second movingwindow and 2 second overlap. In addition to the time-window $T$ and overlap $L$ parameters, four additional thresholds (or delay-time parameters) are used to eliminate incorrect outlier values prior to the WLS computation: maximum lag-time, maximum time-delay, minimum coherence, and maximum error [Table 5.2]. The result from each station-pair is then averaged into a single measure of velocity change [Figure 5.11]. The calculated error (lightly shaded area) is the squared misfit of the data to the modeled slope [Clarke et al., 2011]. 


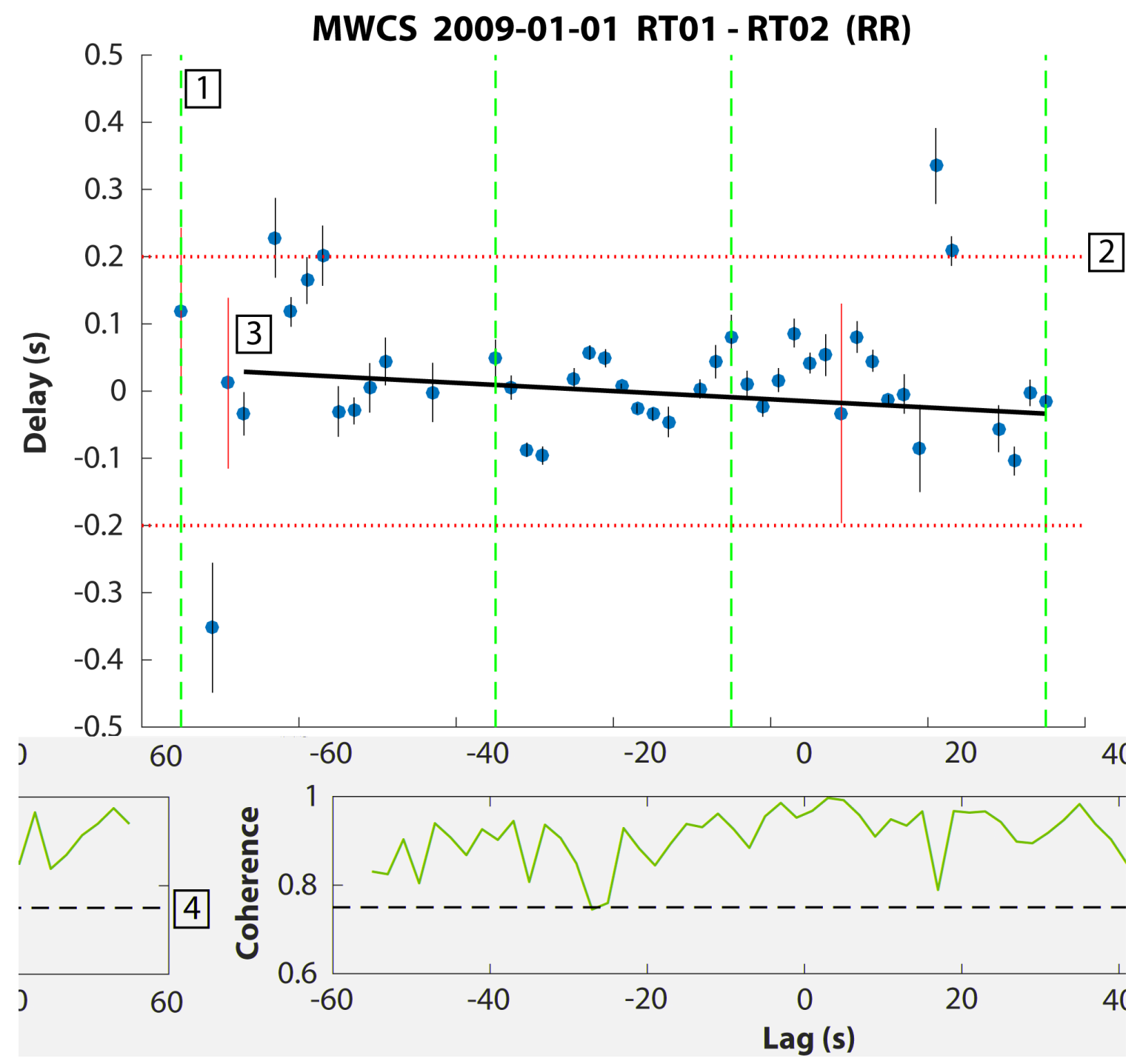

Figure 5.10: Time delay [top] and coherence [bottom] for a day of data for Rotokawa station-pair RT01-RT02. The delay-time parameters are as follows: [1] 15 to 55 seconds lag-time bandwidth (vertical green dotted line), [2] 0.2 seconds time-delay bandwidth (solid red horizontal line), [3] 0.1 seconds DTT error-threshold (red error bars), and [4] 0.75 coherence threshold (dotted black line). The weighted linear regression is displayed as a solid black line. 


\begin{tabular}{||l||l||l||}
\hline Property & Value & Definition \\
\hline [1] Lag-time bandwidth & $15-55$ seconds & $\begin{array}{l}\text { Lag-time in the causal and } \\
\text { acausal chosen for the WLS re- } \\
\text { gression. }\end{array}$ \\
\hline [2] Time-delay bandwidth & +-0.2 seconds & $\begin{array}{l}\text { Positive and negative time- } \\
\text { delay bounds used in the WLS } \\
\text { regression. }\end{array}$ \\
\hline [3] Error threshold & 0.1 seconds & $\begin{array}{l}\text { Maximum error for time-delay } \\
\text { values. }\end{array}$ \\
\hline [4] Coherence threshold & 0.75 & $\begin{array}{l}\text { Minimum coherence required } \\
\text { for time-delay values. }\end{array}$ \\
\hline
\end{tabular}

Table 5.2: Value and definition of the delay-time parameters applied after the MWCS calculation.
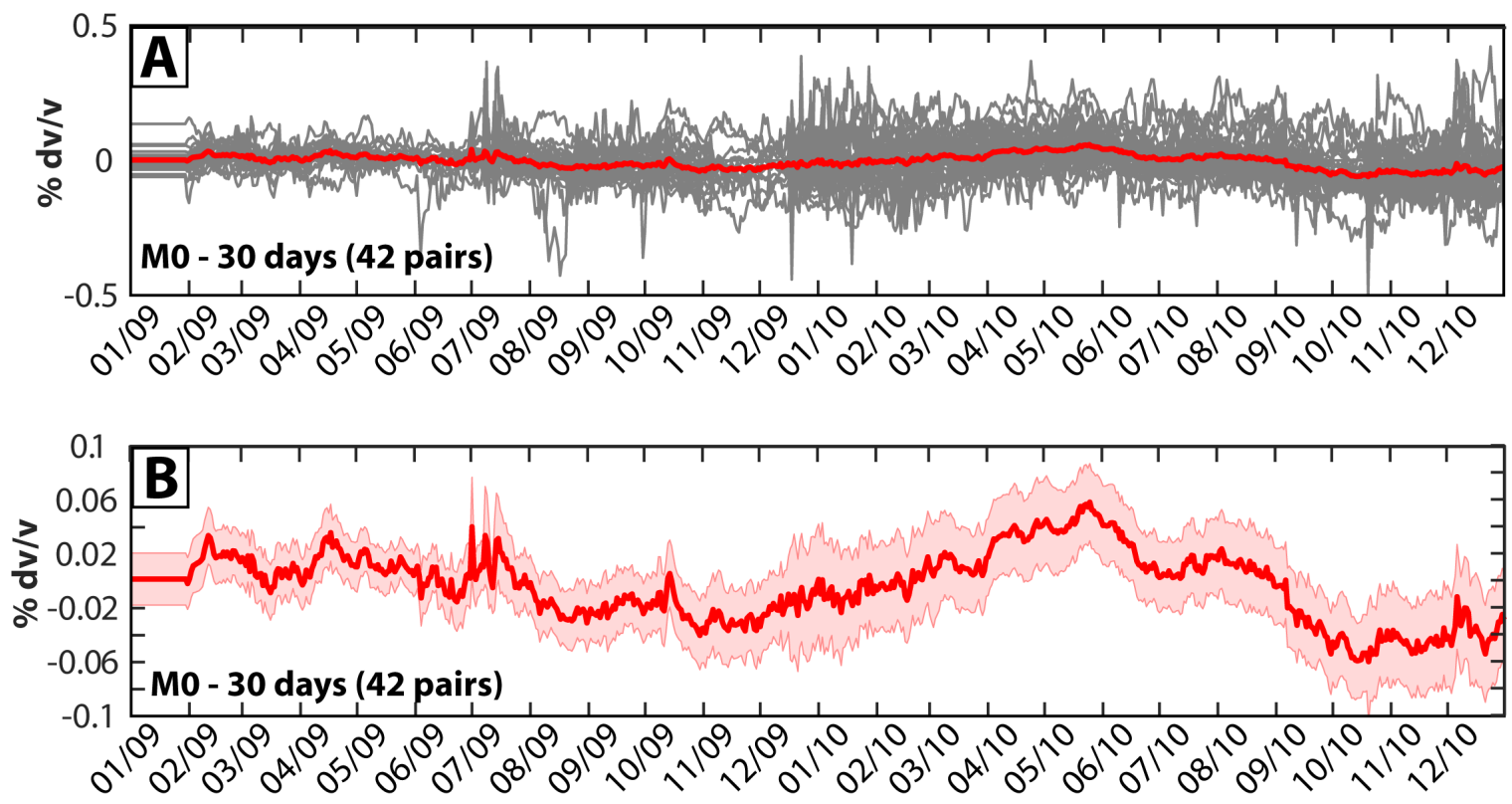

Figure 5.11: [A] Example displaying individual pairs of velocity changes (gray) and average velocity (red). [B] The same average velocity change as in [A] scaled between $+-0.1 \%$. 


\subsection{Rotokawa site information}

Rotokawa is a high-temperature $\left(>300^{\circ} \mathrm{C}\right.$ resource) [Henley and Middendorf, 1985] geothermal field located approximately $10 \mathrm{~km}$ north-east of Taupo that has been used for electricity production since 1997 [McNamara et al., 2016]. Our objective is to determine if velocity changes due to the increased injection and production during the activation of the Nga Awa Purua (NAP) power station in February of 2010 could be determined by ambient noise. Activation of NAP production increased electricity production at Rotokawa from $34 \mathrm{MW}$ to $172 \mathrm{MW}$ [McLoughlin et al., 2010]. After a few months of increased injection flow, production was increased at a rate surpassing injection, resulting in a pressure drawdown of up to 40 bar in the production area of the field [Hernandez et al., 2015].

\subsubsection{Rotokawa geologic information}

The geology of the Rotokawa geothermal field has been well-characterized due to continuing electricity production over the last several decades. The surface geology is dominated by thermal alteration including: hydrothermal eruption craters, a fumarole, rhyolite domes, and pumice alluvium [Milicich and Hunt, 2007, Rae, 2007, Rawlinson, 2011, McNamara et al., 2016].

The current understanding of subsurface geology at Rotokawa was primarily formulated from well cuttings of the 35 total wells (with depths ranging from 500 meters to $3.07 \mathrm{~km}$ ) drilled at Rotokawa from the start of development [Winick et al., 2011, McNamara et al., 2016]. This information is summarized in a geologic cross-section striking south-north across the geothermal field in Figures 5.12 and 5.13, adapted from Winick et al. [2011] and McNamara et al. [2016].

The basement at Rotokawa is composed of Late-Paleozoic to Late-Mesozoic greywacke sandstone, and was encountered in injection well RK04 (south of the Central Field Fault) at a depth of approximately $2.1 \mathrm{~km}$ [Rae, 2007, Wallis et al., 2013]. Rotokawa Andesite overlies the basement, extending to a maximum thickness of approximately 2.2 $\mathrm{km}$ in the north part of the field, above the Central Field Fault [Rae, 2007] (in our crosssection, the maximum thickness of the Rotokawa Andesite is approximately $1.75 \mathrm{~km}$ ). The Tahorakuri and Waikora Formations (volcaniclastic and sedimentary deposits each up to $250 \mathrm{~m}$ thick) and the Wairakei Ignimbrite (between 200-390 m thick) lie above the Rotokawa Andesite [Browne et al., 1992, Rae, 2007, McNamara et al., 2016]. Above the formations and the Wairakei Ingimbrite lies the volcano-sedimentary Waioria Formation, containing rhyolite lavas, breccias, and domes of the Haparangi Rhyolite Group, which range between 450-800 $\mathrm{m}$ in thickness (thickness increases to much shallower depths to the north of the field) [Rae, 2007, Bowyer and Holt, 2010, McNamara et al., 2016]. 


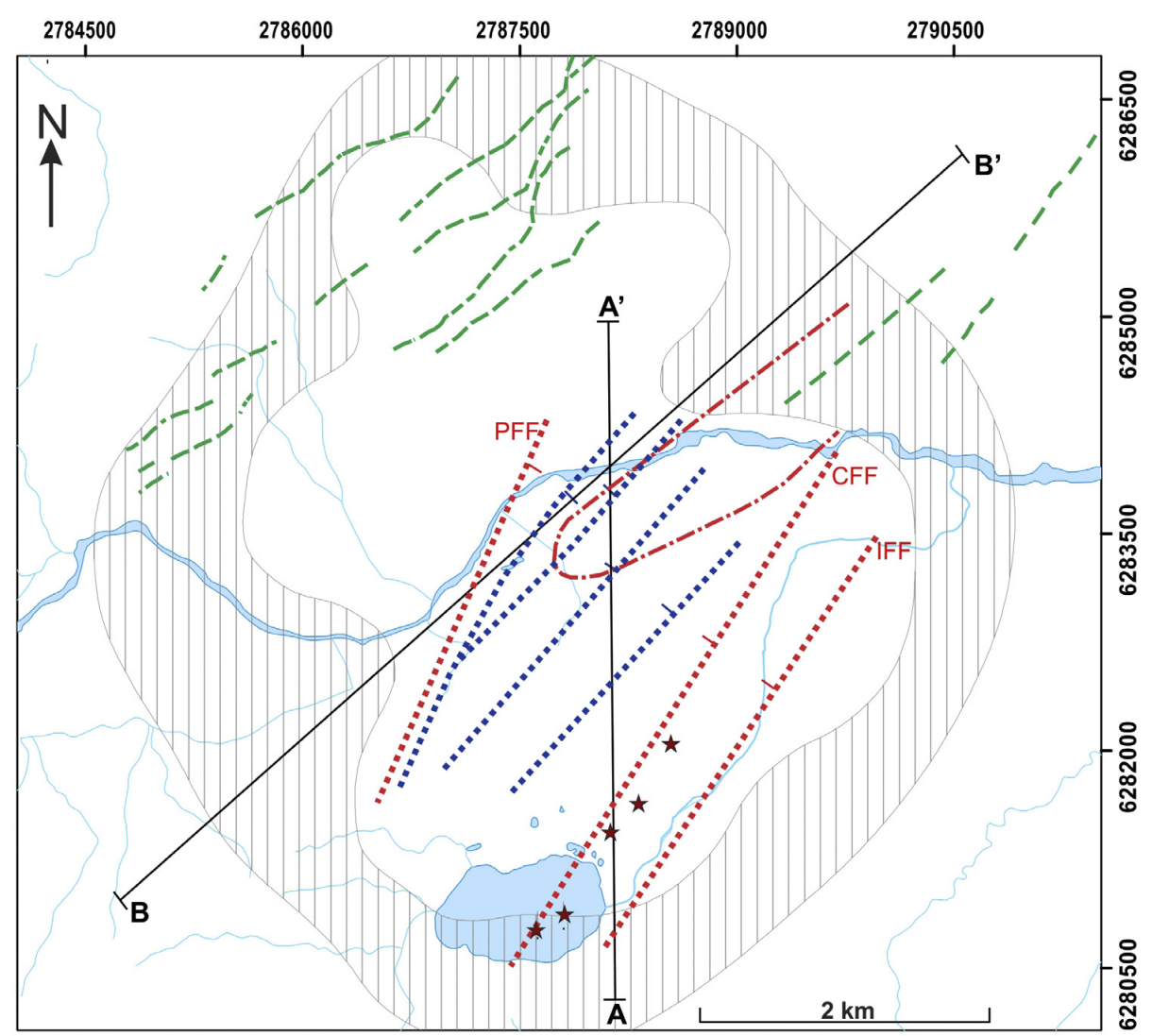

[ . . . . . Bower and Holt (2008) Modelled Faults

. . . . . . Wallis et al. (2013) Modelled Faults

- - GNS Science Active Fault Database, 2014

- - Wallis et al. (2013) Andesite Valley Outline

Hydrothermal Eruption Vents

Risk (2000) Resistivity Boundary

Figure 5.12: Displays the location of the geologic cross section of Figure 5.13 and the Production Field Fault (PFF), Central Field Fault (CFF), and Injection Field Fault (IFF). Adapted from McNamara et al. [2016].

There are three main aquifers at Rotokawa: [1] a shallow groundwater aquifer in the Oruanui Formation, contained by the impermeable Parariki Breccia and Huka Falls Formation below it, [2] an intermediate aquifer within the Waiora Formation and Haparangi Rhyolite, and [3] a deep aquifer and main geothermal reservoir hosted in the Rotokawa Andesite, Waikora and Tahorakuri Formations, and the basement greywacke [Winick et al., 2011, McNamara et al., 2016]. The shallow aquifer is characterized by a mixture of steam-heated chloride-sulphate waters and cold groundwater [Winick et al., 2011]. The deep reservoir is capped by a smectite-rich clay zone [Figure 5.13, dotted line] and characterized by $\mathrm{Cl}, \mathrm{B}, \mathrm{Li}, \mathrm{Cs}$ and non-condensable gases [Winick et al., 2011]. The intermediate aquifer is a mixture of fluids migrating down from the shallow aquifer and up from the deep aquifer, steam condensates, carbon dioxide and hydrogen sulfide gases, and heated, marginal groundwater [Winick et al., 2011, McNamara et al., 2016]. 


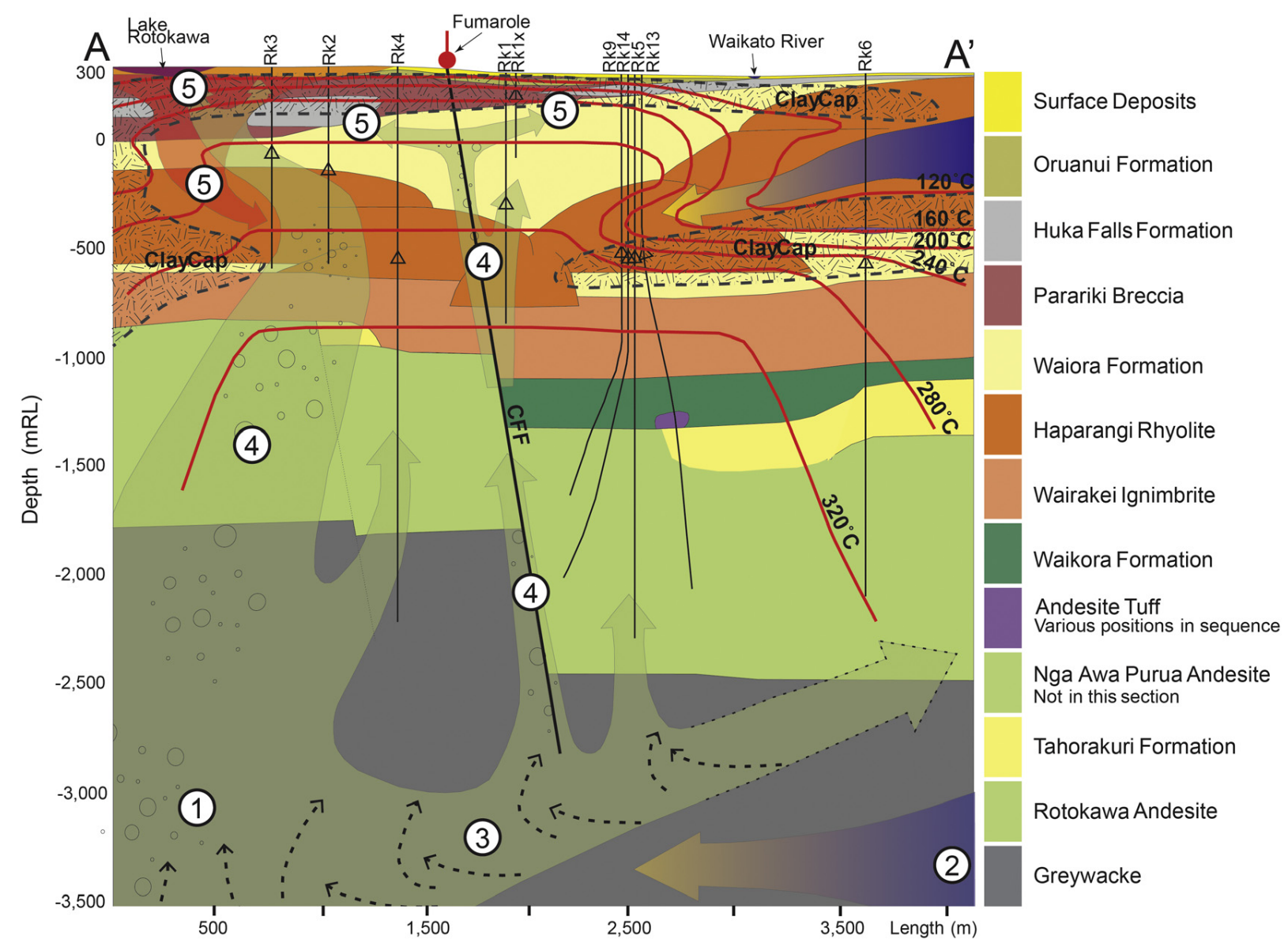

Figure 5.13: Rotokawa geologic cross section adapted from McNamara et al. [2016]. The location of the A- $\mathrm{A}^{\prime}$ profile is displayed in Figure 5.12. 


\subsubsection{Rotokawa Instrumentation}

From 1997 to 2003, Rotokawa produced 34 MW of electricity using a steam turbine and binary plant from injection and production at four wells: RK05 and RK09, two production wells situation in the deep aquifer for the Rotokawa Andesite, and RK11 and RK12, shallow injection wells located in the Haparangi Rhyolite (intermediate aquifer) [Table 5.3]. Due to fluid migration, two new injection wells (RK16 and RK18) with much deeper depths were drilled in 2003 [McNamara et al., 2016].

\begin{tabular}{||l||l||l||}
\hline Well & $\begin{array}{l}\text { Downhole } \\
\text { Depth (m) }\end{array}$ & Geology \\
\hline RK05 (Production) & 2647 & Rotokawa Andesite \\
\hline RK09 (Production) & 2045 & Rotokawa Andesite \\
\hline RK11 (Injection) & 697 & Haparangi Rhyolite \\
\hline RK12 (Injection) & 2647 & Haparangi Rhyolite \\
\hline RK16 (Injection) & 2647 & Greywacke \\
\hline RK18 (Injection) & 2647 & Rotokawa Andesite \\
\hline \hline
\end{tabular}

Table 5.3: Injection and production wells at Rotokawa from 1997 through 2010.

Additional wells (RK19-RK33) were drilled prior to the activation of the Nga Awa Purua power station. During the time period of our analysis (2009-2010), flow at Rotokawa was from a total of 5 injection and 9 production wells. Injection and production was exclusively in the deep aquifer, with injection occurring in the south-east portion of the field in the Greywacke bedrock and production occurring in the north-west portion of the field in the Rotokawa Andesite [Table 5.4, Figures 5.14 and 5.15].

We display the instrumentation within the injection and production areas of the field in Figures 5.14 and 5.15. Seismometers of the Rotokawa network are labeled as red triangles, while the injection and production wells (circles and squares respectively), are individually colored to match the subsequent flow-rate injection and production figures. During this time, the Rotokawa seismic network consisted of 10 Geospace seismometers with a corner frequency of $4.5 \mathrm{~Hz}$ [Figure 5.14]. The instrument response curves of the Geospace seismometers are displayed in Figure C.3 in Appendix C [Geo, 1992]. The maximum station distance is approximately $4.5 \mathrm{Km}$, which is over a magnitude smaller than the minimum inter-station distance at Harrat Al-Madinah in Chapter 3 . 


\begin{tabular}{||l||l||l||}
\hline Well & $\begin{array}{l}\text { Downhole } \\
\text { Depth } \mathbf{( m )}\end{array}$ & Geology \\
\hline RK20 (Injection) & 2850 & Greywacke \\
\hline RK21 (Injection) & 2659 & Greywacke \\
\hline RK22 (Injection) & 3100 & Greywacke \\
\hline RK23 (Injection) & 3147 & Greywacke \\
\hline RK24 (Injection) & 2992 & Greywacke \\
\hline RK05 (Production) & 2647 & Rotokawa Andesite \\
\hline RK14 (Production) & 2499 & Rotokawa Andesite \\
\hline RK17 (Production) & 1952 & Rotokawa Andesite \\
\hline RK25 (Production) & 2707 & Rotokawa Andesite \\
\hline RK26 (Production) & 2218 & Rotokawa Andesite \\
\hline RK27 (Production) & 2560 & Rotokawa Andesite \\
\hline RK28 (Production) & 2500 & Rotokawa Andesite \\
\hline RK29 (Production) & 2235 & Rotokawa Andesite \\
\hline RK30 (Production) & 2571 & Rotokawa Andesite \\
\hline \hline
\end{tabular}

Table 5.4: Injection and production wells at Rotokawa 2009-2010, their downhole depth, and geology at depth. 


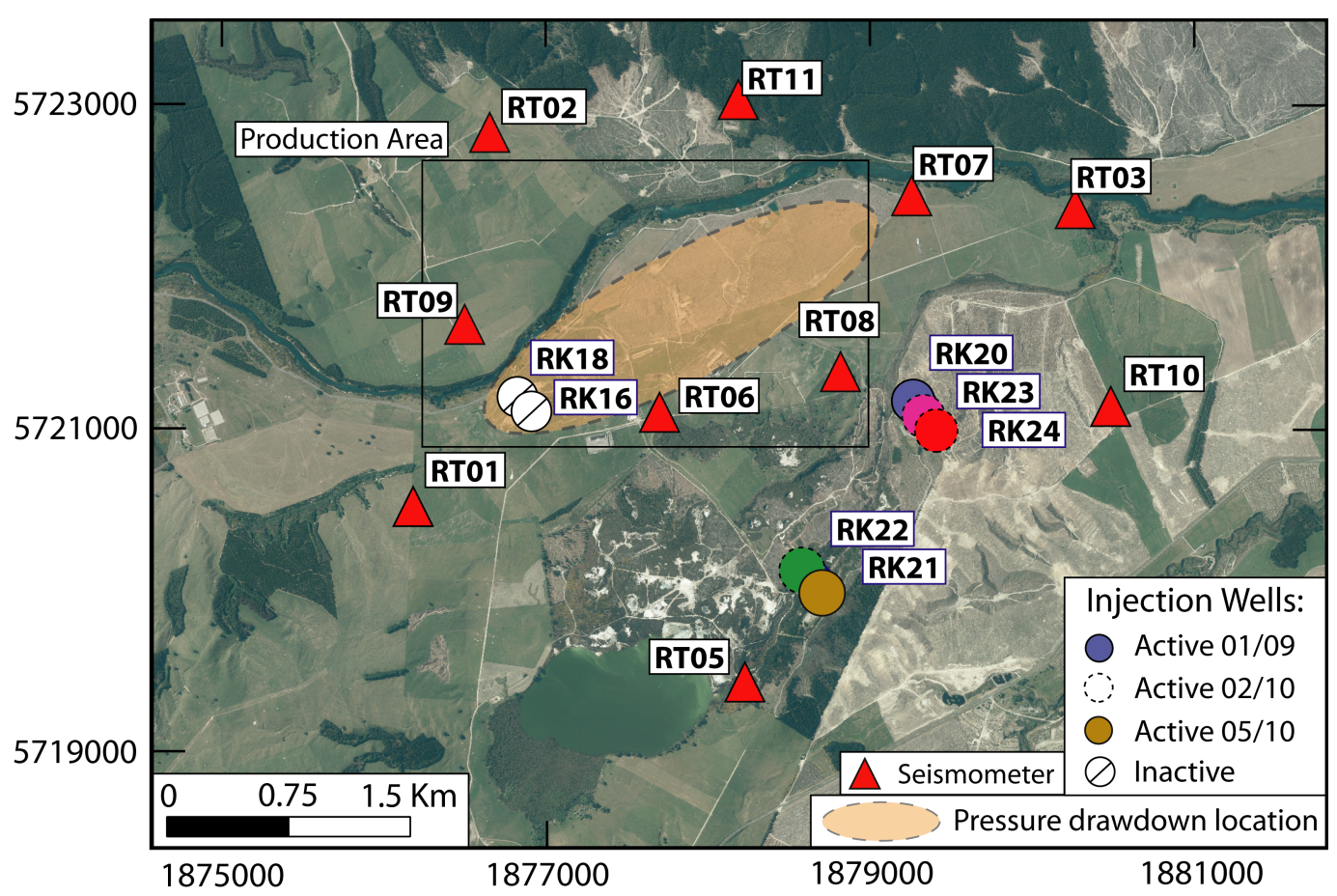

Figure 5.14: 2009-2010 Rotokawa station map. Displays the Rotokawa seismic network (red triangles), injection wells (circles), and a zone of pressure drawdown in the production area [Hernandez et al., 2015]. The production area is further detailed in Figure 5.15. Injection well colors are consistent with the flow rate color of Figure 5.18.

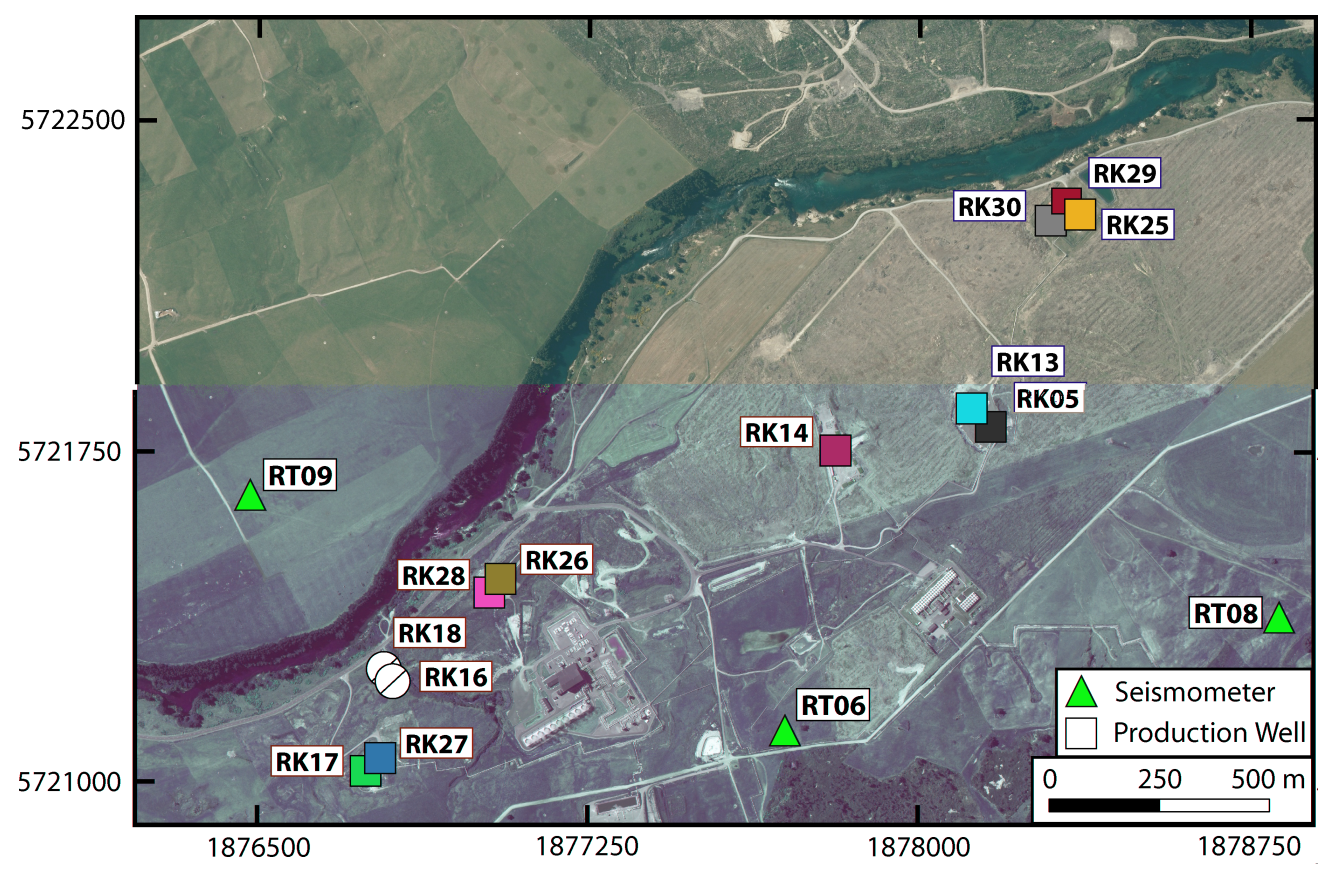

Figure 5.15: Production map subset from Figure 5.14. Production wells are squares. RK05 was not active during 2009-2010. Production well colors are consistent with the flow rate color of Figure 5.19. 
The data availability of the Rotokawa Seismic Network is displayed in Figure 5.16. Red bars display days where there is at least partial data. The times of initial Nga Awa Purua (NAP) injection and production are displayed as green and blue lines respectively. Due to the large amount of missing data, stations RT04 and RT03 were not used for analysis. As previously stated in Section 5.3.4, a reference stack of stable data is required to compute velocity changes. For the velocity change results displayed in this chapter [Section 5.5], we chose a reference stack from January 1st through October 1st 2009 [Figure 5.16, black rectangle]. This time period was chosen because it is before the onset of injection and includes both summer and winter seasons. An additional reference stack was used to compute velocity changes spanning from July 1st through October 1st 2009 [Figure 5.16, blue rectangle] due to uncertainties regarding the orientation of certain seismometers prior to July 2009. This second reference stack will be discussed in further detail in Section 5.5.2 and its results are located in Appendix B.3. 


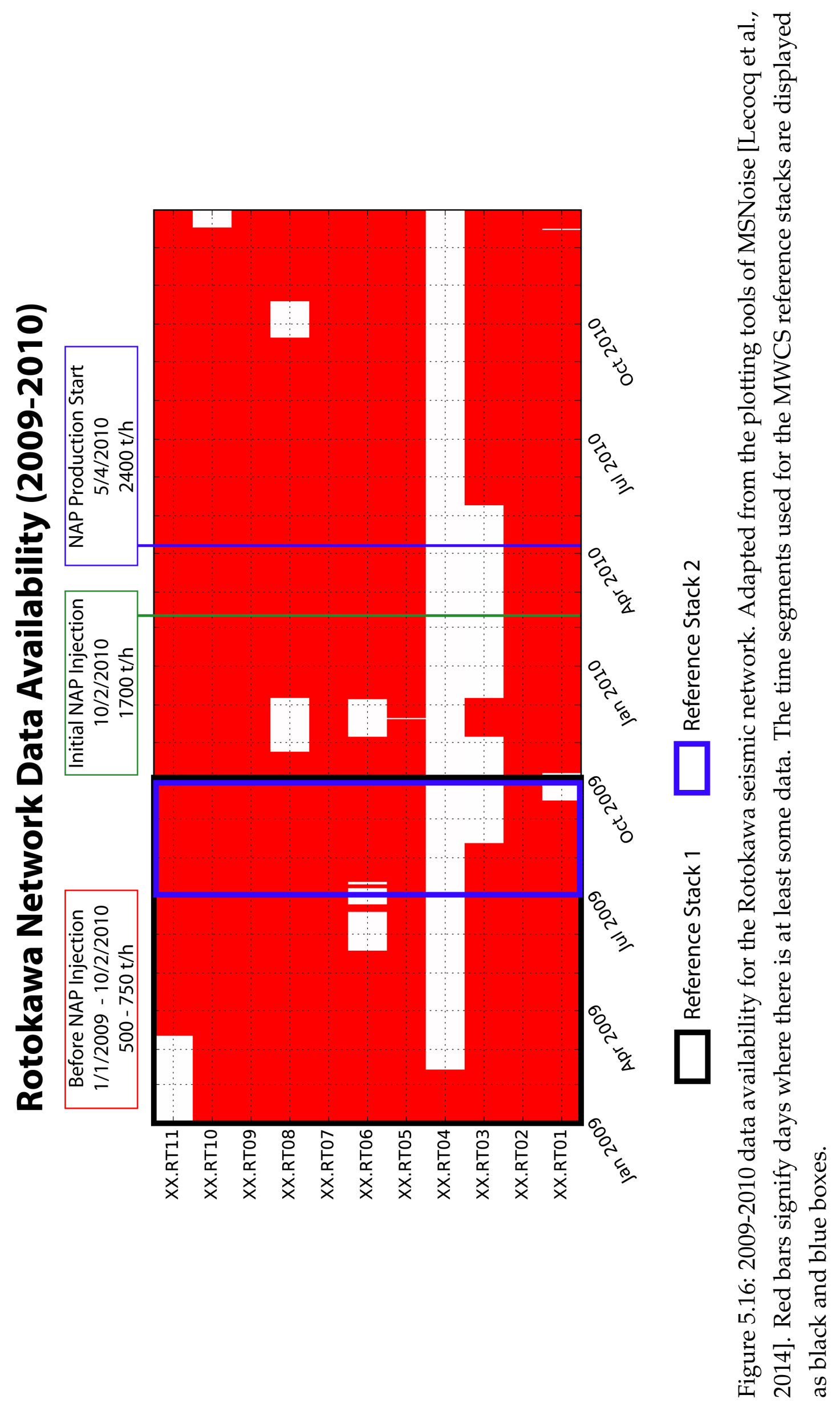


Although the MSNoise routine is able to detect gaps in data and fill them with a velocity change of zero, most station outages are accompanied with faulty data prior or following the outage. Time periods before and after station outages are not skipped by the software, so this problem is of significant concern since data contains many gaps [Figure 5.16]. Due to this, we wrote a code to remove velocity changes for ten days before and after time periods for which a station-pair experienced an outage outage. Figure 5.17 displays the effect of the code. In this example, we can observe that the corrected trace (red line) removes high-amplitude spikes before and after the station outages (gray line).

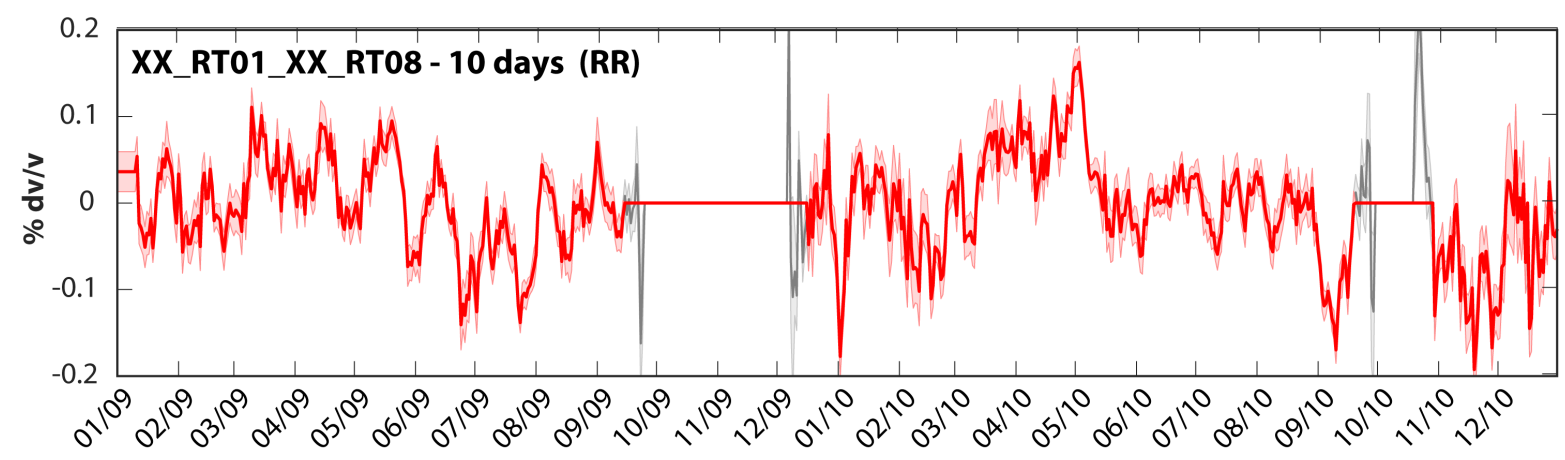

Figure 5.17: Corrected 10-day MWCS window velocity change result (red line) for station-pair RT01-RT08 radial-radial component. The original trace is displayed in gray.

Figures 5.18 and 5.19 display the flow rates for injection and production at Rotokawa. The color of each flow-rate corresponds to the injection or production well color displayed in Figures 5.14 and 5.15. A lag-time between the injection and production is observed, and injection in the south side of the field begins in May 2010 with RK21 [Figures 5.14 and 5.18, brown line].

Figure 5.20 displays the total injection and production $[\mathrm{A}]$, the total liquid volume in the system [B], and the injection-production ratio [C]. The total liquid volume is a measure relative to the start of the our data (i.e., at January 1st 2009 the total volume in the system is zero). As the flow rates between and injection and production can vary quickly, an 8-day smoothed curve is added to the injection-production ratio to facilitate comparisons. Prior to November 2009, the rate of injection at Rotokawa was equal to the rate of production, resulting in an injection-production ratio of approximately one. Between November 2009 and April 2010, additional injection began at the geothermal field due to the beginning of electricity production at the Nga Awa Purua power station. After this time period, production rates exceeded injection rates, leading to a decrease in total liquid volume and an injection-production ratio less than one [Figure 5.20C]. This over-production state is thought to be the mechanism which resulted in a pressure drawdown across the production area of the field [Hernandez 
et al., 2015].

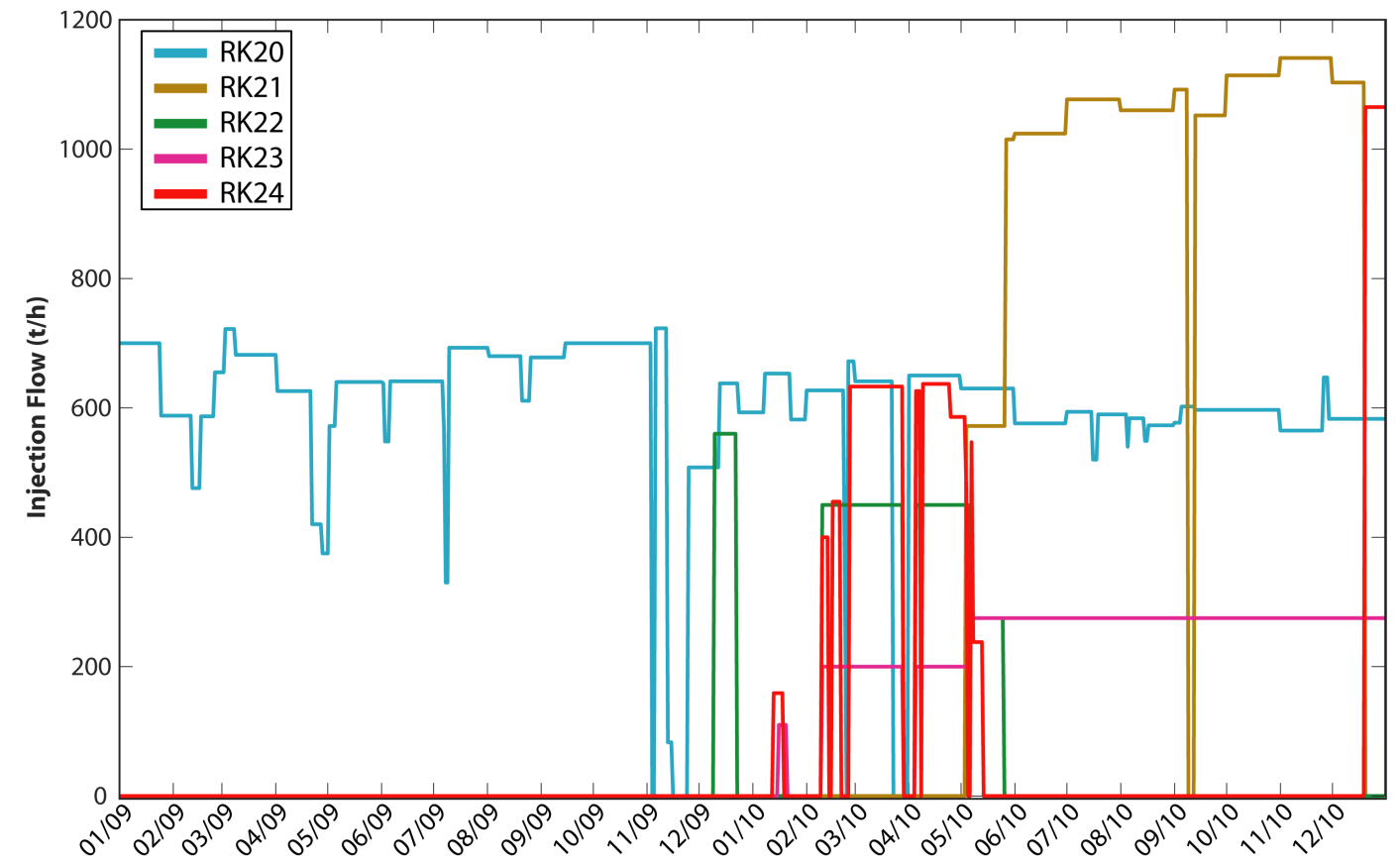

Figure 5.18: Rotokawa 2009-2010 injection flow in tons per hour. Line color matches injection well color in Figure 5.14.

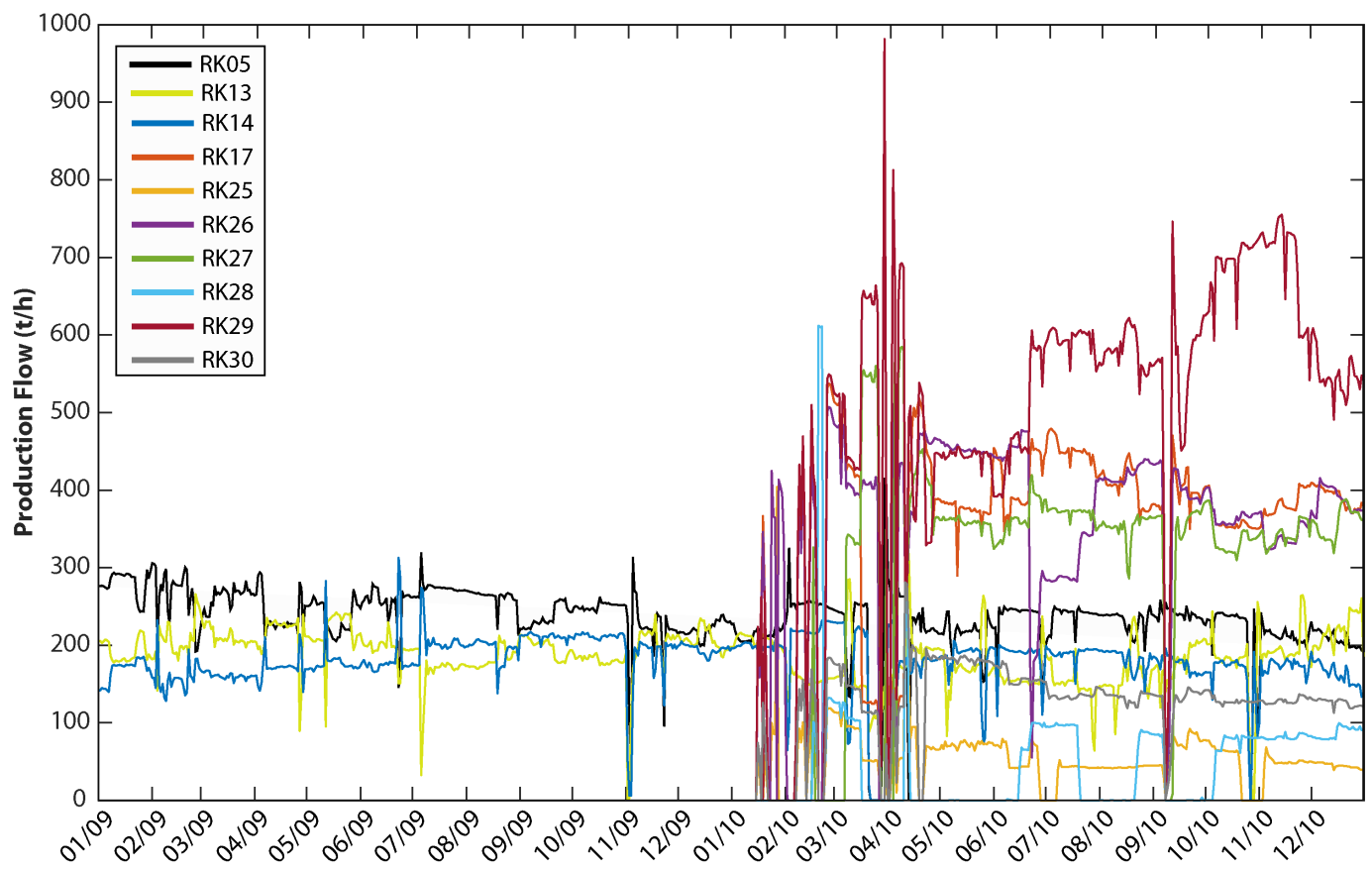

Figure 5.19: Rotokawa 2009-2010 production flow in tons per hour. Line color matches production well color in Figure 5.15. 

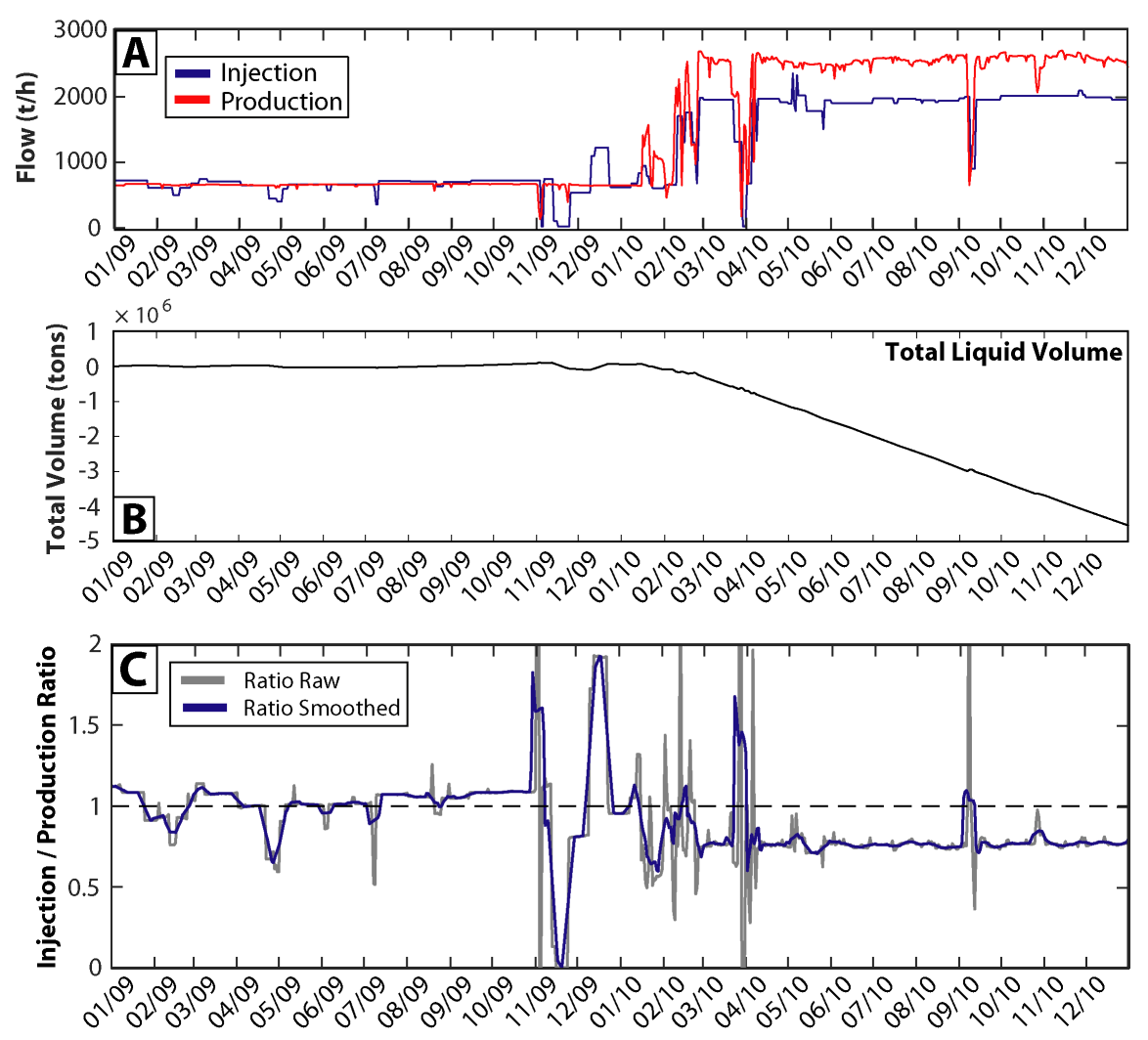

Figure 5.20: [A] Rotokawa total injection and production flow in tons per hour. [B] Total liquid volume computed from the flow rates relative to January 1st, 2009. [C] 8-day smoothed and raw overall injection-production ratios for Rotokawa 2009-2010. 


\subsection{Rotokawa results}

\subsubsection{Rotokawa cross-correlations and moveouts}

Cross-correlation moveouts are displayed for each of the 9 components of the Green's function tensor in Figures 5.21, 5.22, and 5.23. These moveouts are computed from all data, so they include both the reference and moving stack time periods. Each of the raw cross-correlations (red) are filtered between $0.1-0.4 \mathrm{~Hz}$ (blue) to facilitate the observation of scatterers. Ideally, cross-correlations used for velocity changes are characterized by a large amplitude peak in the ballistic wave range and distinctly separate, stable scatterers in the coda. We observe this in the diagonal components RR, TT, and ZZ. The $\mathrm{RZ}$ and ZR components display this as well, while the threshold between the ballistic wave and the cross-correlation coda is vague for the rest of the cross-components. This is not necessarily indicative of incorrect data as long as the scatterers are consistent, as we will discuss at length in the next section. We observe that the ballistic waves are generally symmetric and increase in time-lag with distance, which suggests that the network is recording a diffusive noise source. Additionally, we observe that scatterers are present in the cross-correlation coda for this stacked data, which is an essential check to conduct prior to continuing with the methodology. 

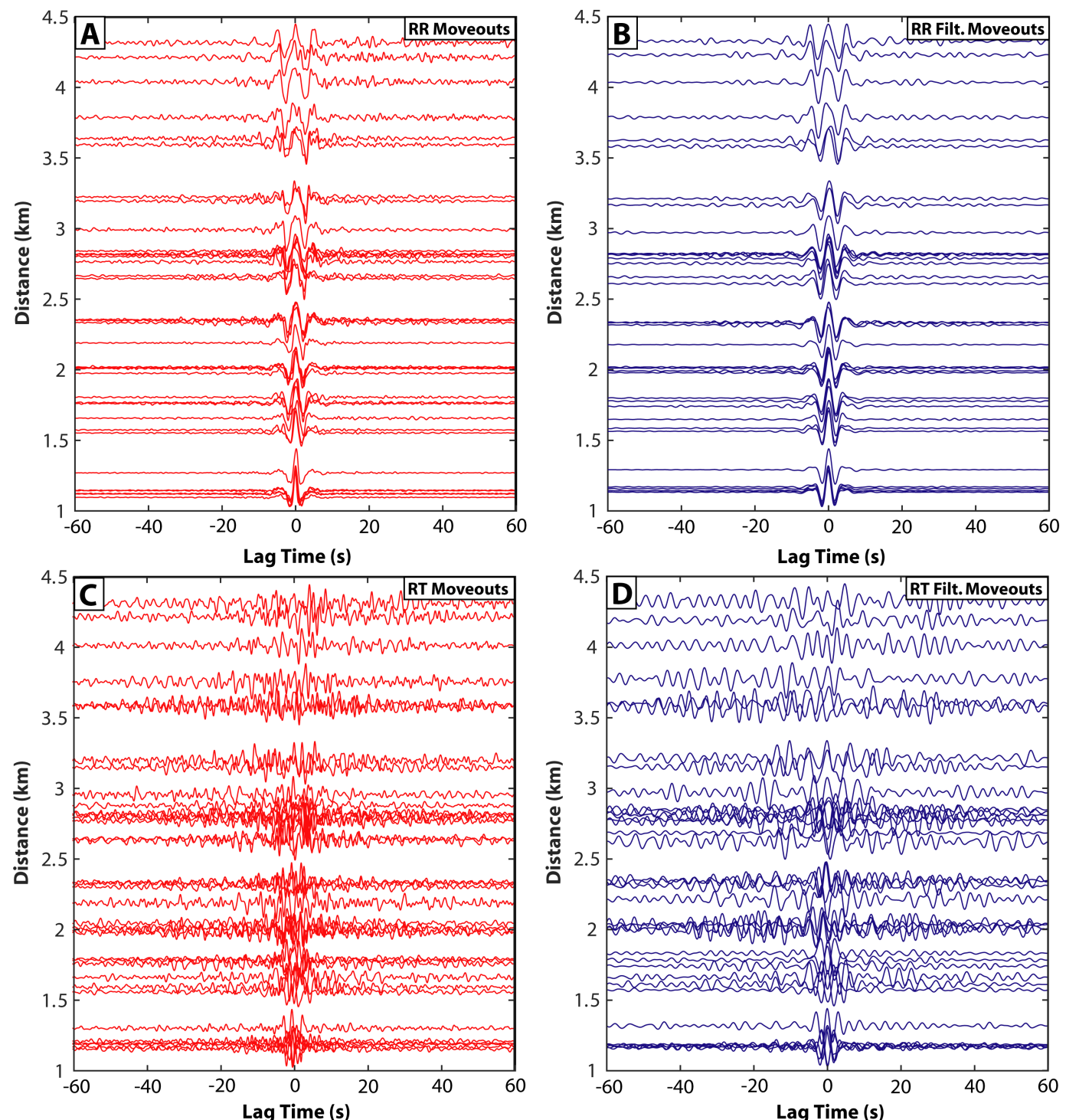

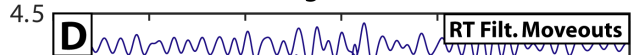

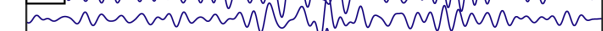
4 ammonsmy

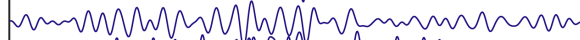

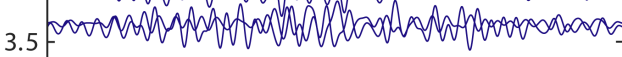
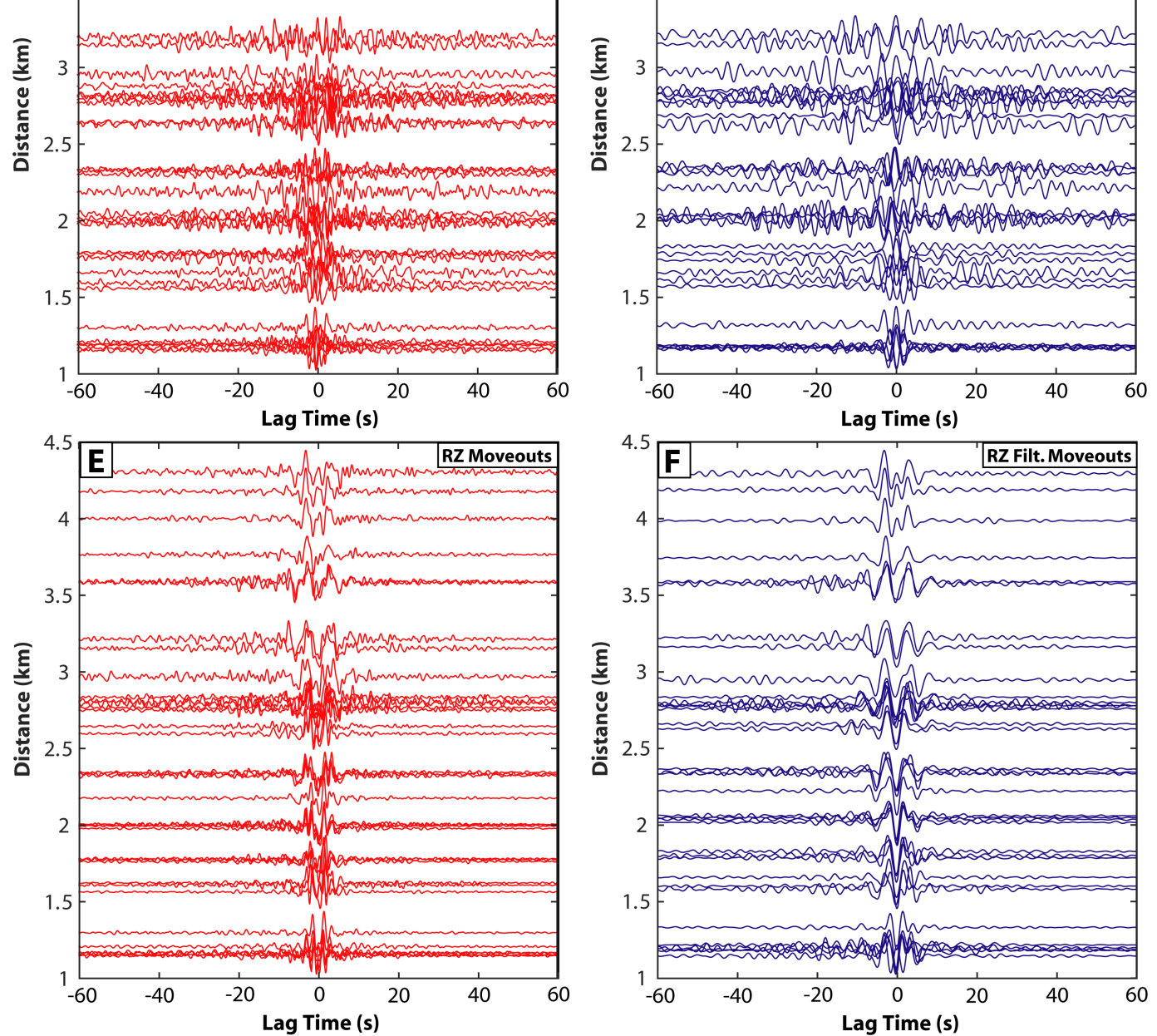

Figure 5.21: Rotokawa 2009-2010 radial cross-correlation moveouts. The red data in $[\mathrm{A}],[\mathrm{C}]$, and $[\mathrm{E}]$ is raw while the blue data $[\mathrm{B}],[\mathrm{D}]$, and $[\mathrm{F}]$ is filtered between $0.1-0.4$ $\mathrm{Hz}$. 

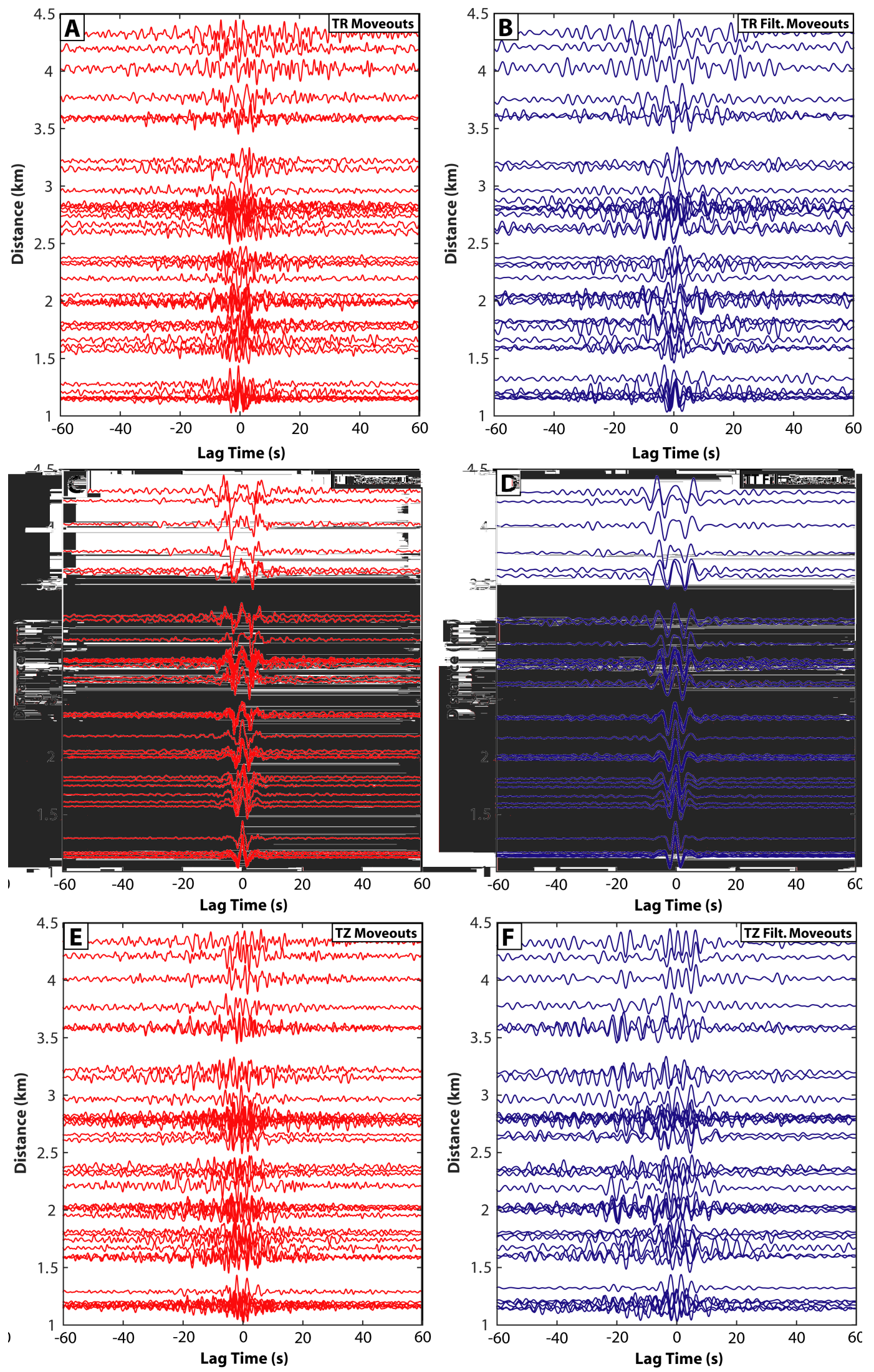

Figure 5.22: Rotokawa 2009-2010 transverse cross-correlation moveouts. 

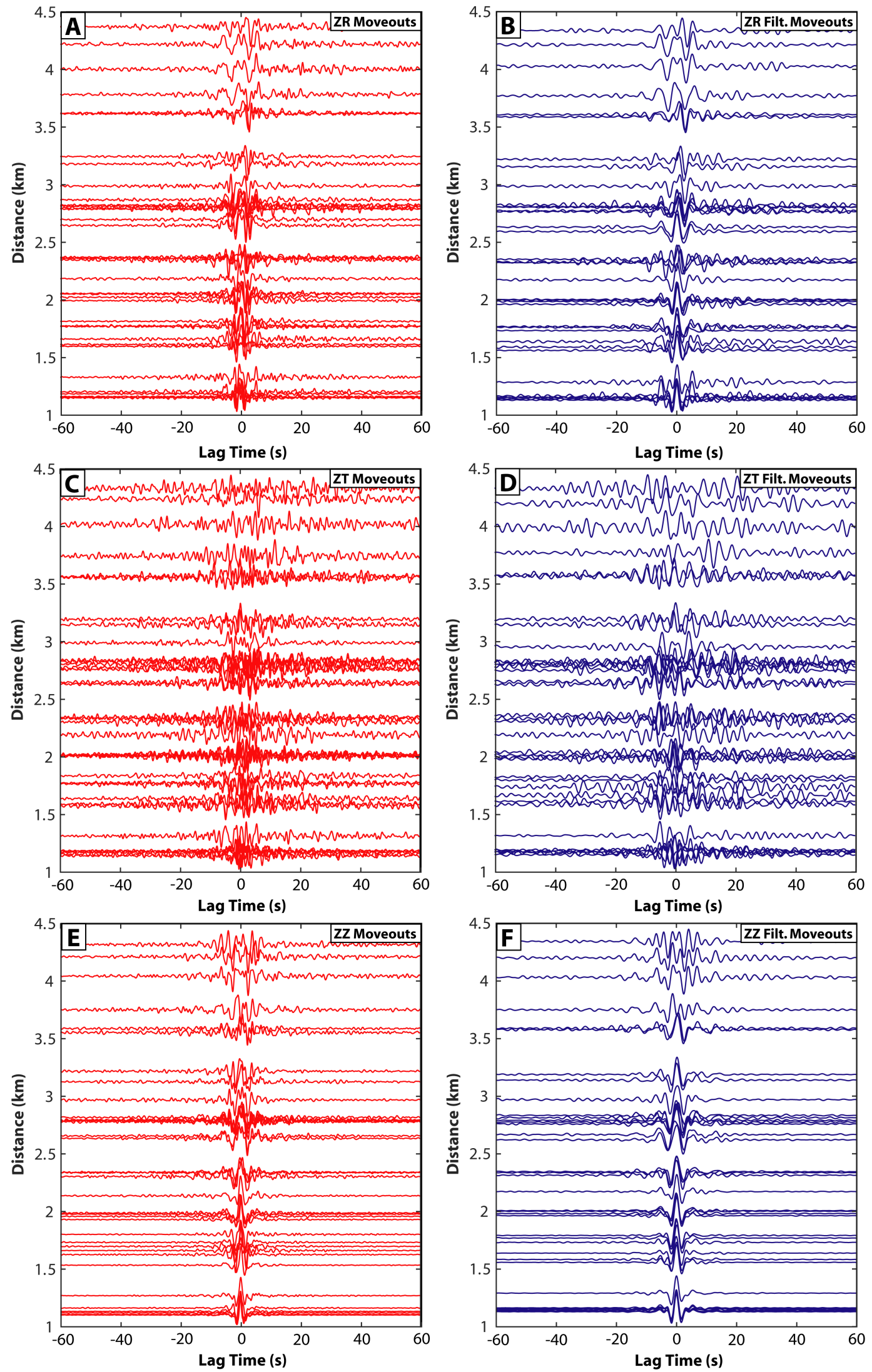

Figure 5.23: Rotokawa 2009-2010 vertical cross-correlation moveouts. 


\subsubsection{Rotokawa stability}

We will now expand our discussion on time and frequency-domain stability first introduced in Sections 5.3.2 and 5.3.3. As previously stated, all of the results shown in this chapter will be using a reference stack between January 1st through October 1st 2009. Additional results using a reference stack between July 1st through October 1st are located in Appendix B.3. Figure 5.24 displays the reference stack, cross-correlation image and magnitude squared coherence for station-pair RT05-RT08 for the RR component. Only the areas in which the MWCS is computed are displayed in the cross-correlation image to facilitate the observation of scatterers [Figure 5.24B]. Overall, we notice both clear scatterers throughout the cross-correlation image as well as in the magnitudesquared coherence.

However, we found that station-pairs may not be consistent in quality across all components. Figures 5.25, 5.26, and 5.27 display both cross-correlation images and magnitude-squared coherence for all nine components. For this example, we found that the RZ, TZ, and ZZ components had significantly worse stability than the rest of the components. Additionally, the cross-components were not as stable as the RR and TT components, which is a phenomenon that we observed throughout our networks. Additionally, we noted that the ZZ component was inconsistent, and could either be as stable as the RR and TT components or of poorer quality than the cross-components. 

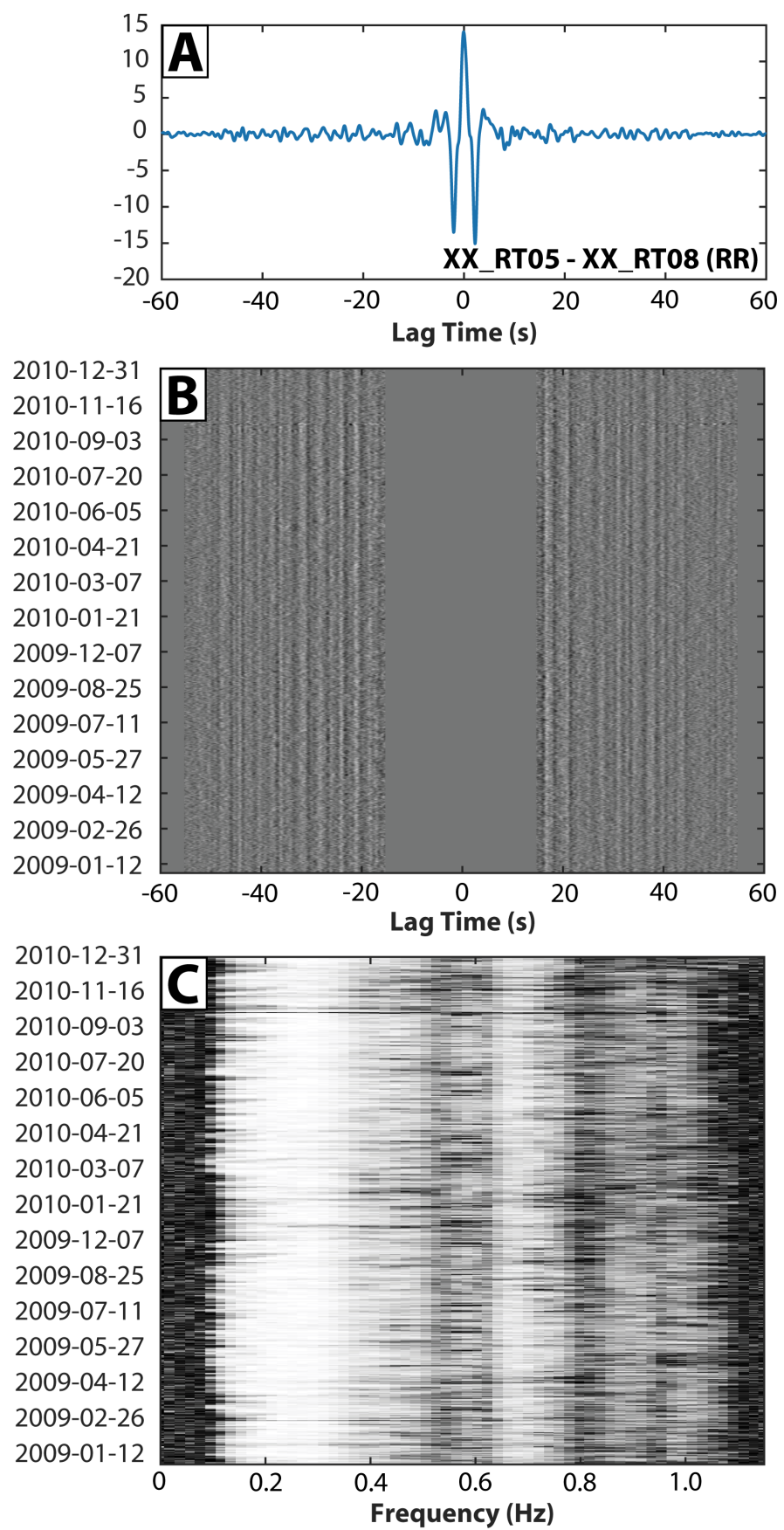

Figure 5.24: [A] Reference stack for Rotokawa RT05-RT08 station pair (2009-01-01 to 2009-10-01). [B] Time-domain stability representation of RT05-RT08 cross-correlations in time. [C] Magnitude squared coherence between the reference stack and the daily stacks for station pair RT05-RT08. 

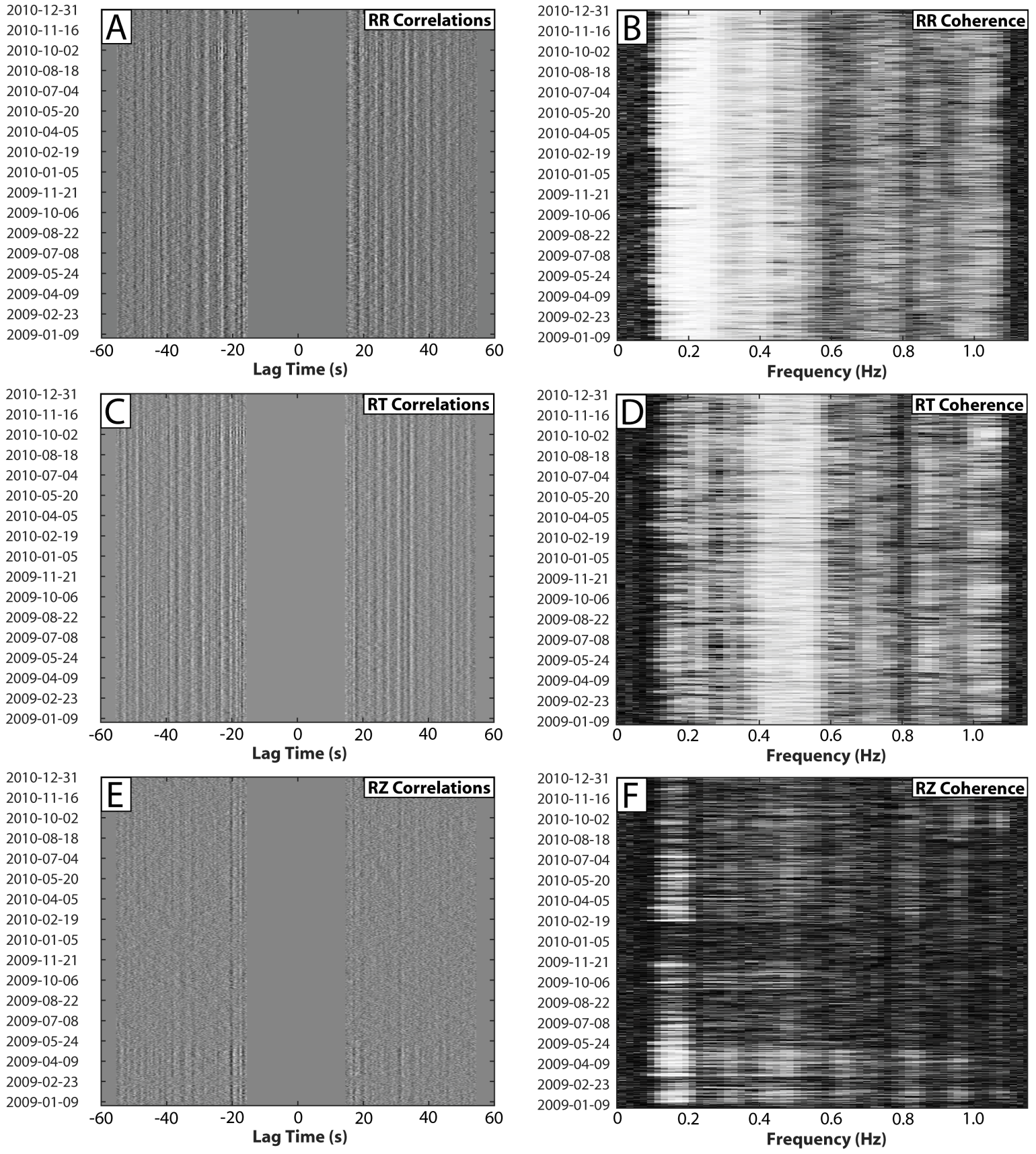

Figure 5.25: Cross-correlation images and magnitude squared coherence for station pair RT05-RT08 for RR [A,B], RT [C,D], and RZ [EF] components. 

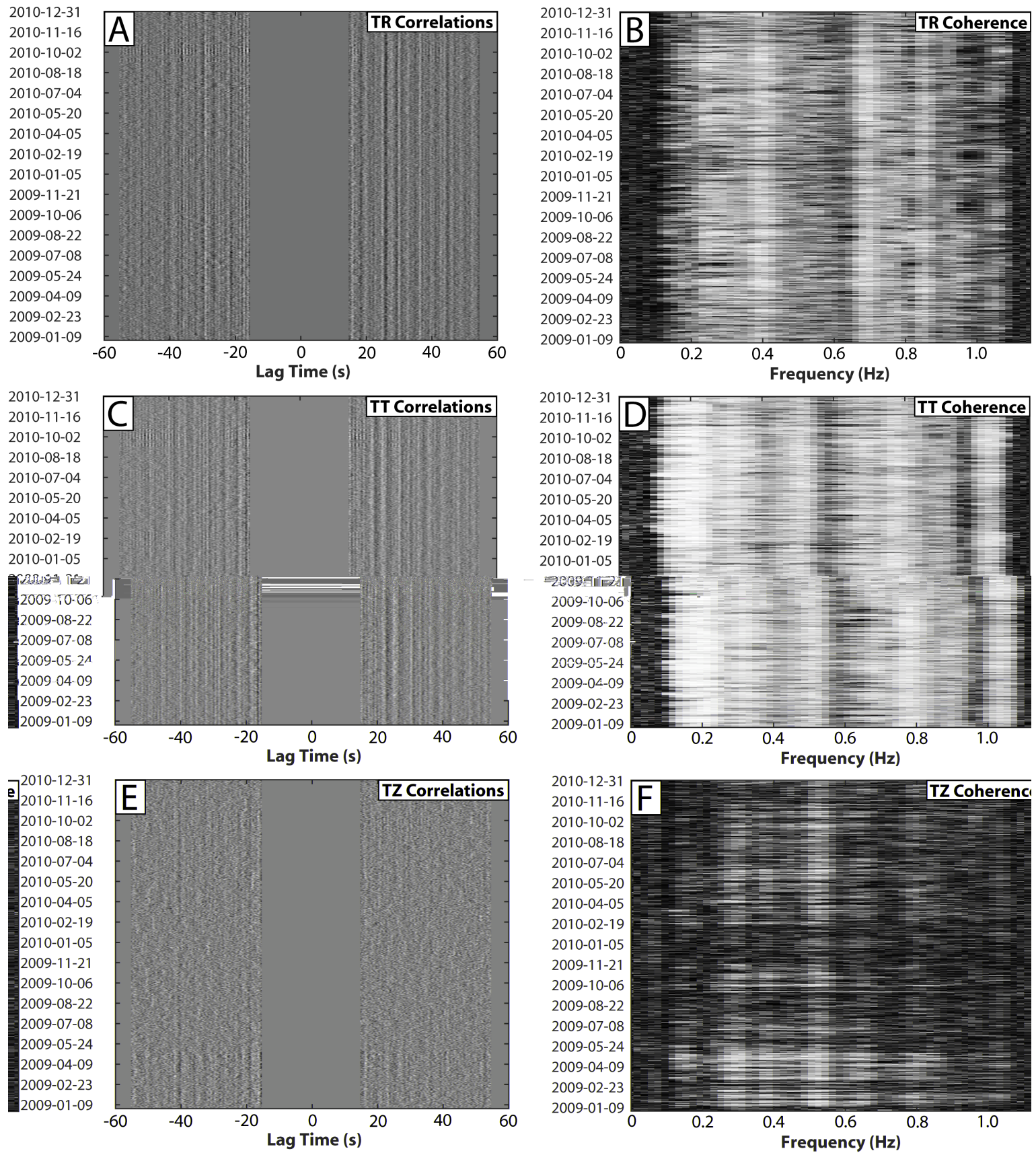

Figure 5.26: Cross-correlation images and magnitude squared coherence for station pair RT05-RT08 for TR [A,B], TT [C,D], and TZ [EF] components. 

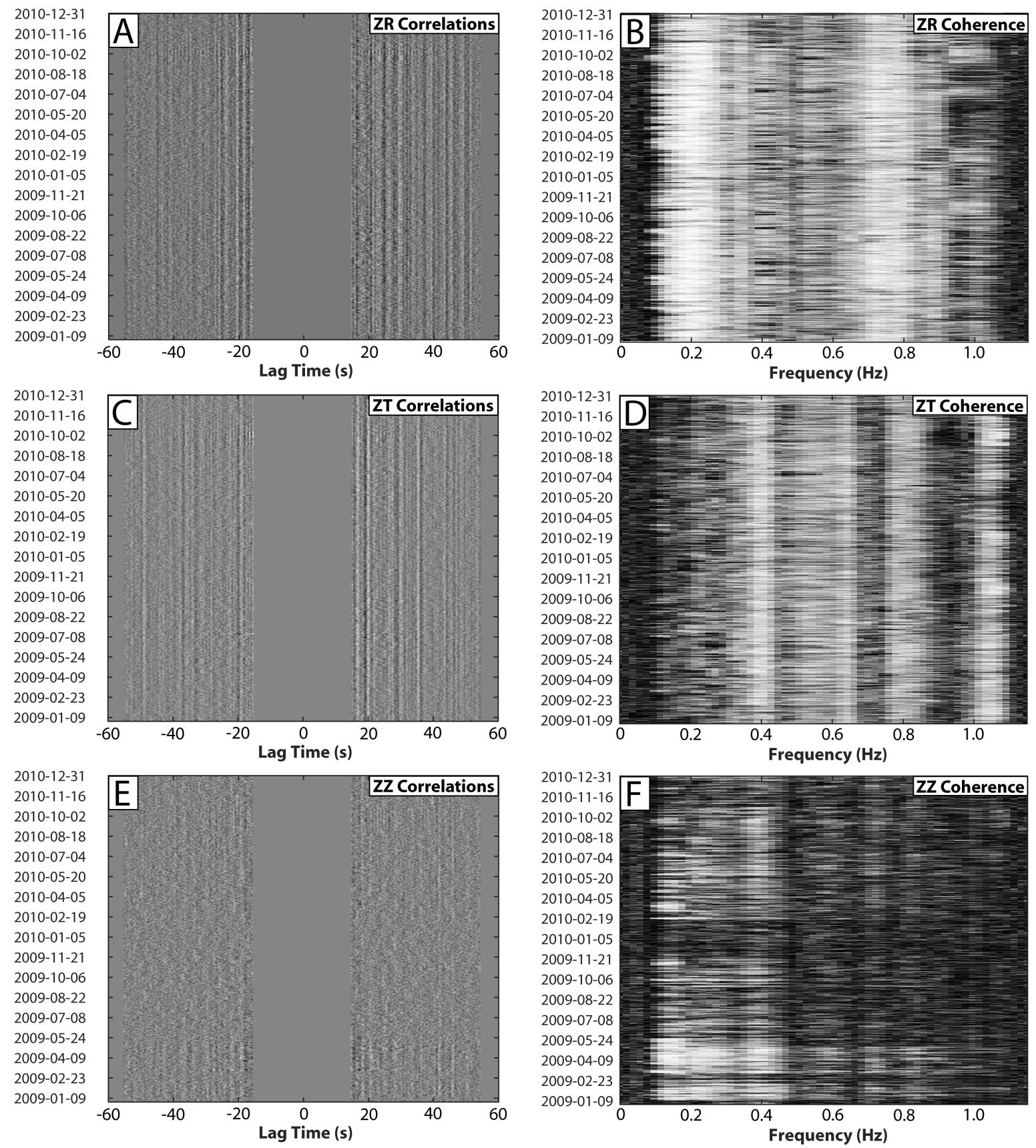

Figure 5.27: Cross-correlation images and magnitude squared coherence for station pair RT05-RT08 for ZR [A,B], ZT [C,D], and ZZ [EF] components. 
Due to the relatively small number of station-pairs in our network, the selection of pairs to be included in the analysis was determined by visual inspection for each of the nine components. In order to prevent bias on the final result, we used a "common sense" approach and only removed data that was clearly not coherent. Figure 5.29 displays one of the station-pairs removed by visual inspection. For this pair, the scatterers in the cross-correlation image are difficult to isolate and are not consistent [Figure 5.29A], the magnitude-squared coherence is low and is zero for certain time periods [Figure 5.29B], and the resulting velocity change is extremely erratic [Figure 5.29C]. The station-pairs removed for each component are listed in Appendix B.1.
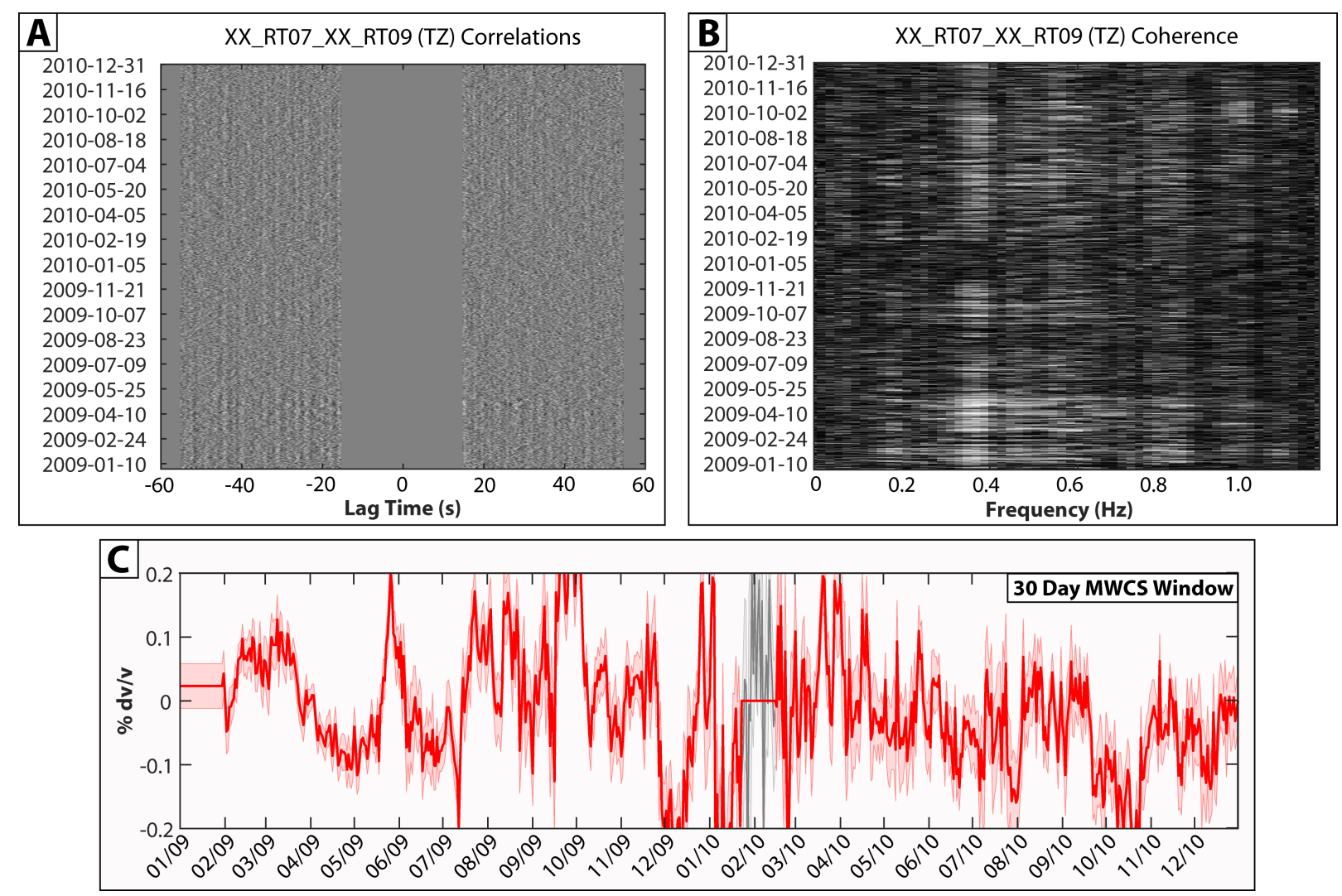

Figure 5.28: Example of bad pair identified by visual inspection. [A] cross-correlation image, [B] magnitude squared coherence, $[\mathrm{C}]$ and resulting 30-day velocity change for station pair RT07-RT09. 


\subsubsection{Sensor orientation issues at Rotokawa}

While visually inspecting the cross-correlation stability and magnitude-squared coherence for each station-pair component, we discovered inconsistencies between the horizontal and vertical components of certain pairs. Specifically, there are clear changes in the cross-correlation images and coherence at specific points in time in the horizontal components but not in the verticals for pairs that include stations RT02, RT10, and RT11. Station information reveals that stations RT10 and RT11 had instruments replaced in June 22nd 2009 and station RT02 had instruments replaced on the 13th of August 2009. The sensors were replaced with instruments of the same type, but no other information regarding the swap is known at this time. In Figure 5.29, we display the differences between the RR and ZZ components of station-pair RT01-RT10 in the time domain $[A, C]$ and the frequency domain $[B, D]$. Clear shifts can be observed in cross-correlation image and coherence of the RR component that correspond exactly with the 22nd of June [Figure 5.29A, B]. As this shift is only observed in the horizontals, and no changes in the ambient noise can be observed sensors that were not swapped, we hypothesize that the orientations before and after the change are not the same. Unfortunately, we cannot know from the cross-correlation images or the coherence whether or not the orientations before or after the change were correct, only that they are not congruent.

These observations call into question what effect the orientation changes could have on the velocity change computations. We consider that the effect is minimal provided that scatterers are still observed in the reference stack and that the time segment prior to the instrument change is not included in the averages of the velocity change computation. The reason for this is that velocity changes are computed relative to the reference stack of each station pair, so as long as the signals are coherent in time, regardless of how the reference stack changes, the relative velocity change should be valid. In the example station-pair RT01-RT10 for the RR component, we can observe through the coherence that the cross-correlations are stable after the sensor change [Figure 5.29B]. This stability was also observed for stations RT02 and RT09. However, we wanted to provide an additional check that the observed velocity change responses were not simply due to the chosen reference stack and the sensor change, and computed the velocity changes produced from July 1st through October 1st 2009 [Figure 5.16, blue rectangle]. These results are displayed in Appendix B.3 and briefly discussed in Section 5.5.4. 

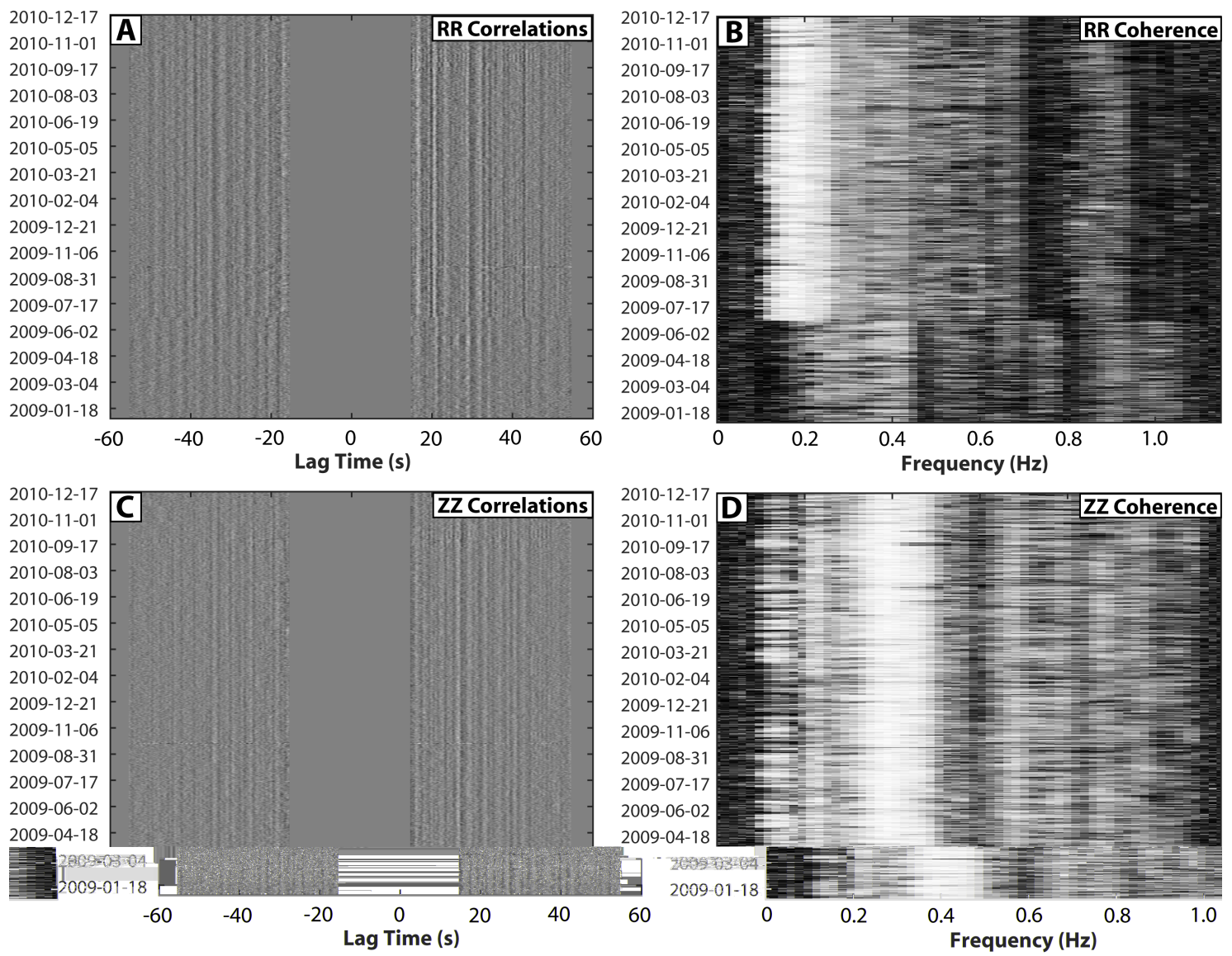

Figure 5.29: Figure illustrating the orientation issues in station-pair RT01-RT10. The sensor is replaced on June 22nd, 2009. [A] Cross-correlation image for radial-radial (RR) component. [B] Magnitude-squared coherence for RR component.[C] Crosscorrelation image for vertical-vertical (ZZ) component. [D] Magnitude-squared coherence for $\mathrm{ZZ}$ component. 


\subsubsection{Rotokawa velocity changes}

As previously discussed, there are two methods of computing the phase slope from the time-delay measurements: forcing the linear regression to go through the origin at a lag-time of zero or unrestricted [Clarke et al., 2011]. We found that for most pairs, both measurements gave the approximately the same values in long-term measurements. However, a few pairs produced anomalous high or low values of velocity that could be observed even when averaged among all pairs. One such example is displayed in Figure 5.30, where a large value of velocity in a station-pair in the unrestricted regression results in a significant deviation in the average [Figure 5.30C, D] but not in the origin-forced regression [Figure 5.30A, B]. Due to this, we chose to use the velocity changes measurements produced by the origin-forced regression.

We computed average velocity-changes for 10, 20, 30, 40, and 50-day MWCS windows using all components and diagonal components (RR, TT, and ZZ) [Figures 5.31 and 5.32]. Additionally, we computed averages for two subsets of pairs corresponding to the injection and production locations [Figures 5.33-5.37]. The number of paths for each grouping is listed in Table 5.5.

\begin{tabular}{||l||l||}
\hline Grouping & Number of paths \\
\hline All field all components & 279 \\
\hline All field diagonal components & 94 \\
\hline Injection subset all components & 60 \\
\hline Injection subset diagonal components & 19 \\
\hline Production subset all components & 89 \\
\hline Production subset diagonal components & 30 \\
\hline
\end{tabular}

Table 5.5: The number of paths for each velocity-change grouping at Rotokawa. 

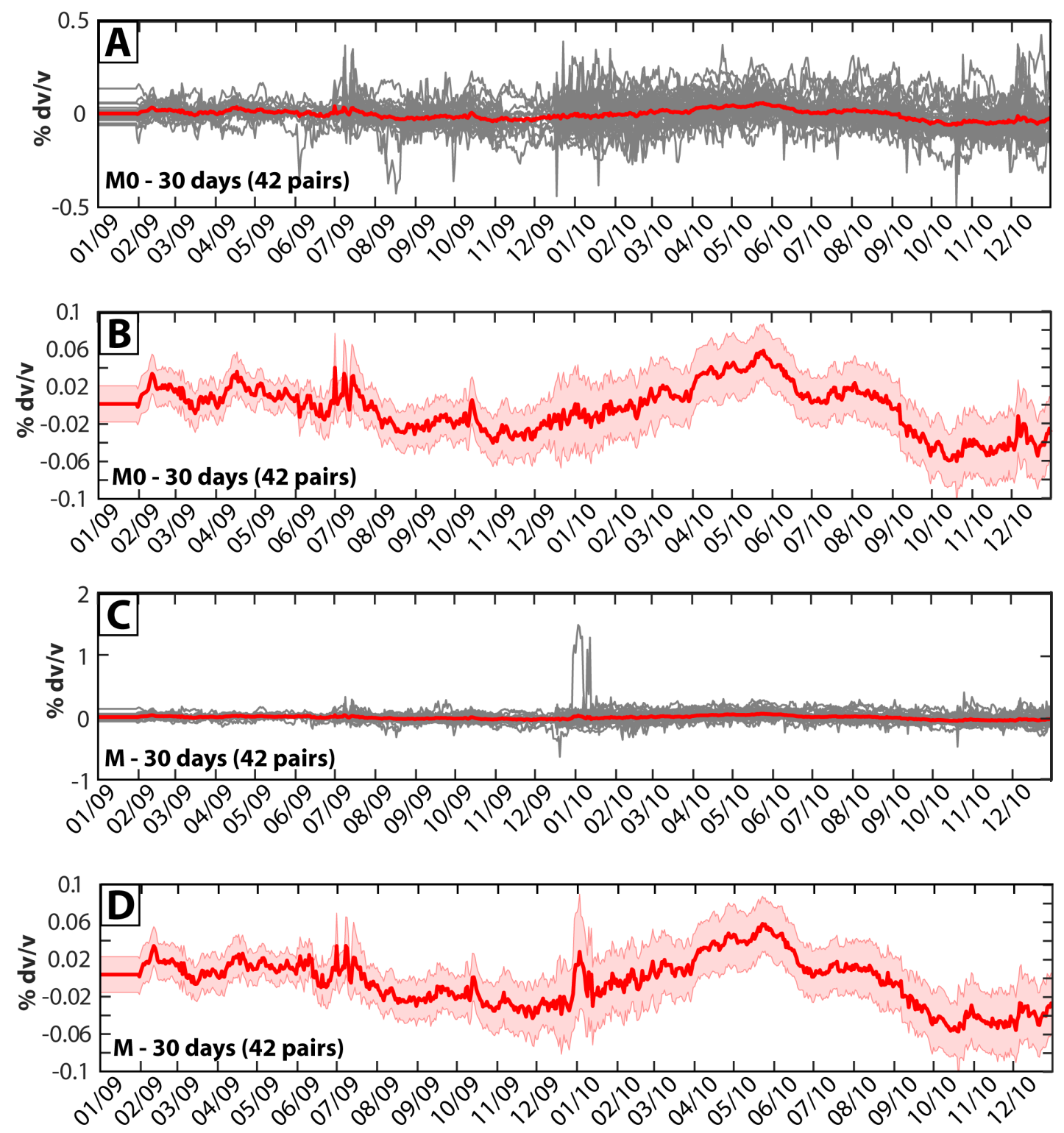

Figure 5.30: Average velocity change for all Rotokawa stations using a 30-day MWCS window using a phase slope forced through the origin (M0) [A, B] and an unrestricted phase slope (M) [C, D]. Gray lines in Figures [A] and [C] represent each individual pair while the red lines are their average (The red lines in [B] and [D] are the same as those in $[A]$ and $[C]$, just scaled between $+-0.1 \%$ velocity change). The root mean squared is displayed as a pink shaded area [Equation 5.7]. 

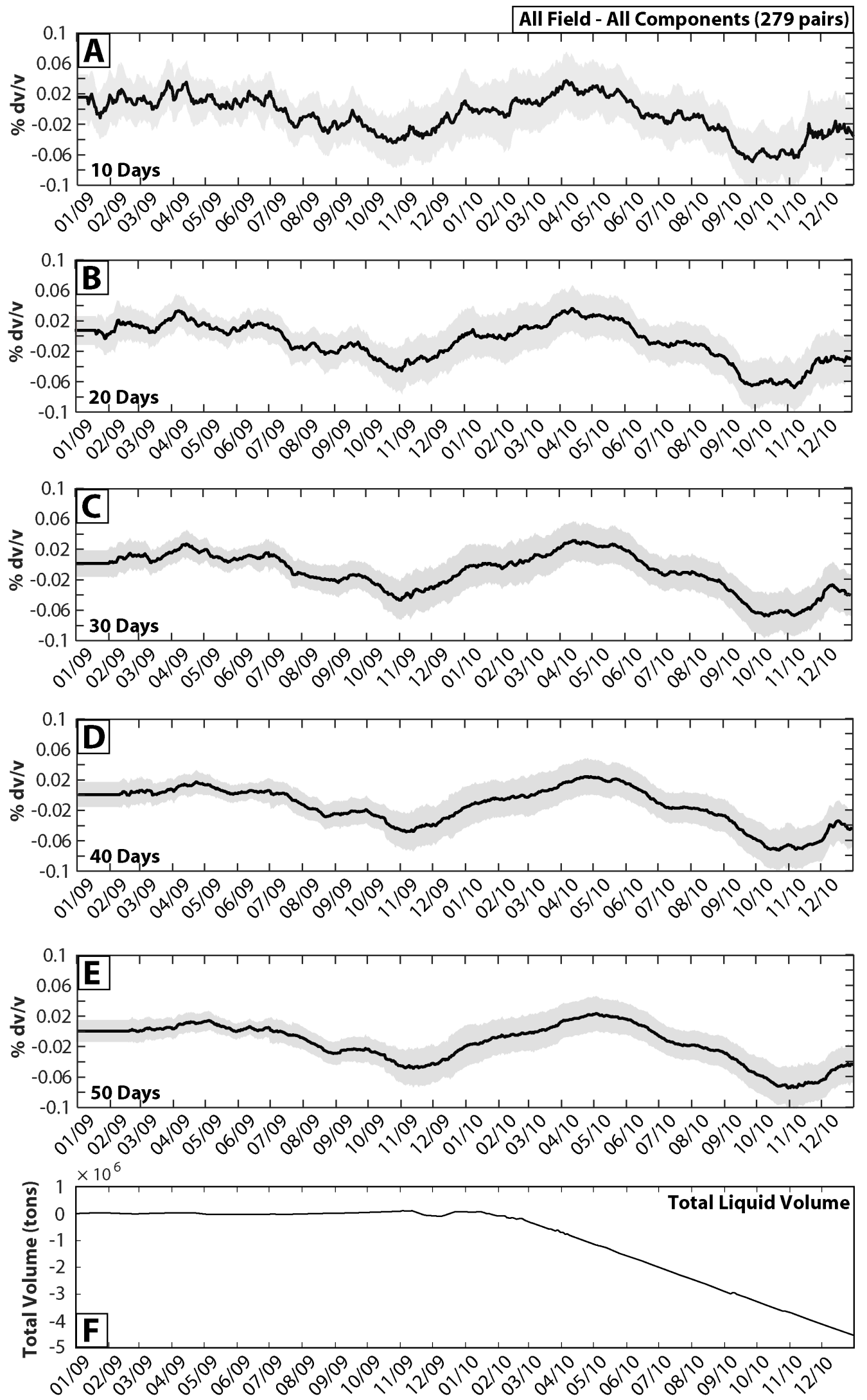

Figure 5.31: Velocity changes using 10-50 day MWCS windows [A-E] and Rotokawa relative liquid volume $[\mathrm{F}]$ using all components of the entire network. 

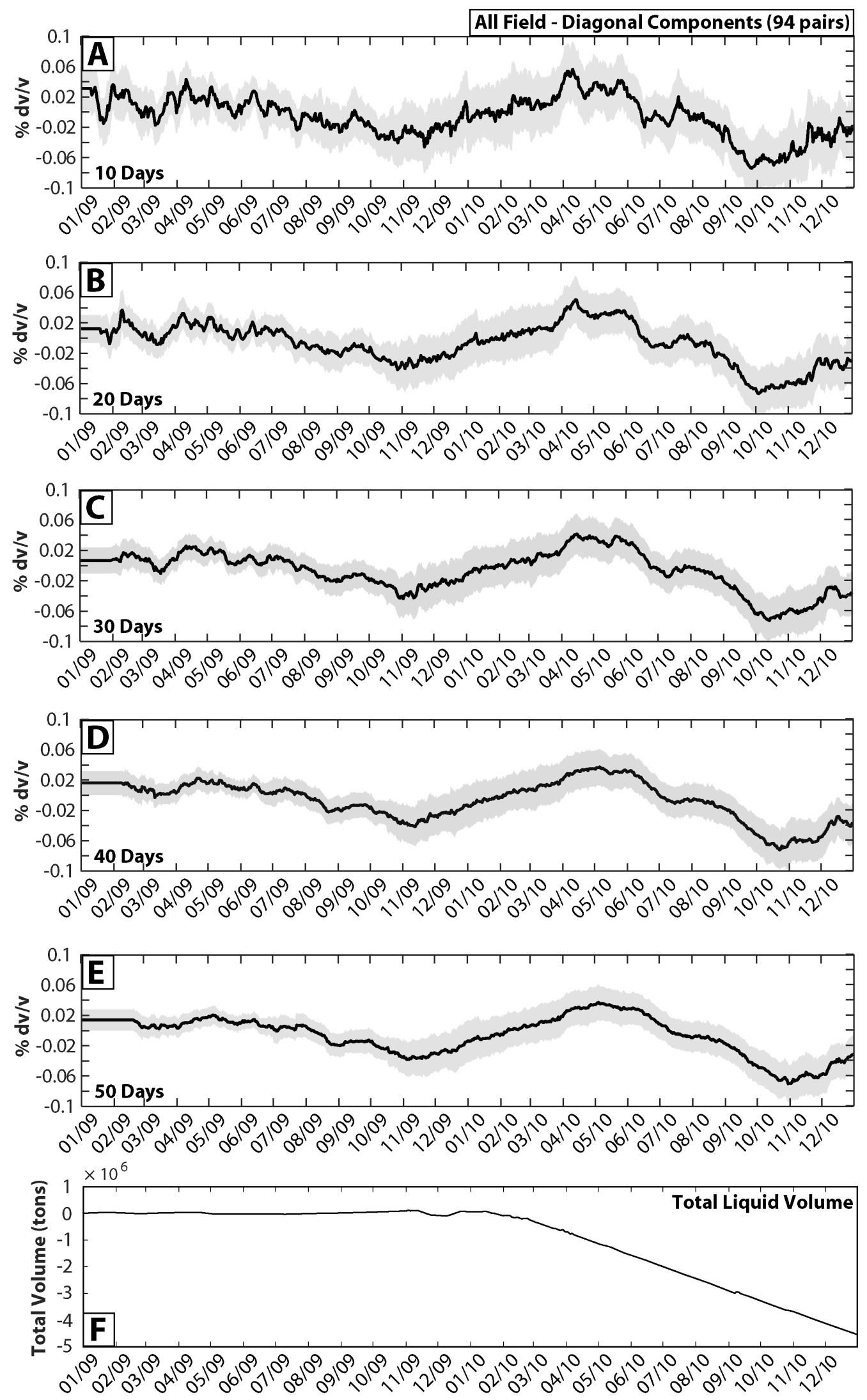

Figure 5.32: Velocity changes using 10-50 day MWCS windows [A-E] and Rotokawa relative liquid volume $[\mathrm{F}]$ using diagonal components (RR, TT, and $\mathrm{ZZ}$ ) of the entire network. 

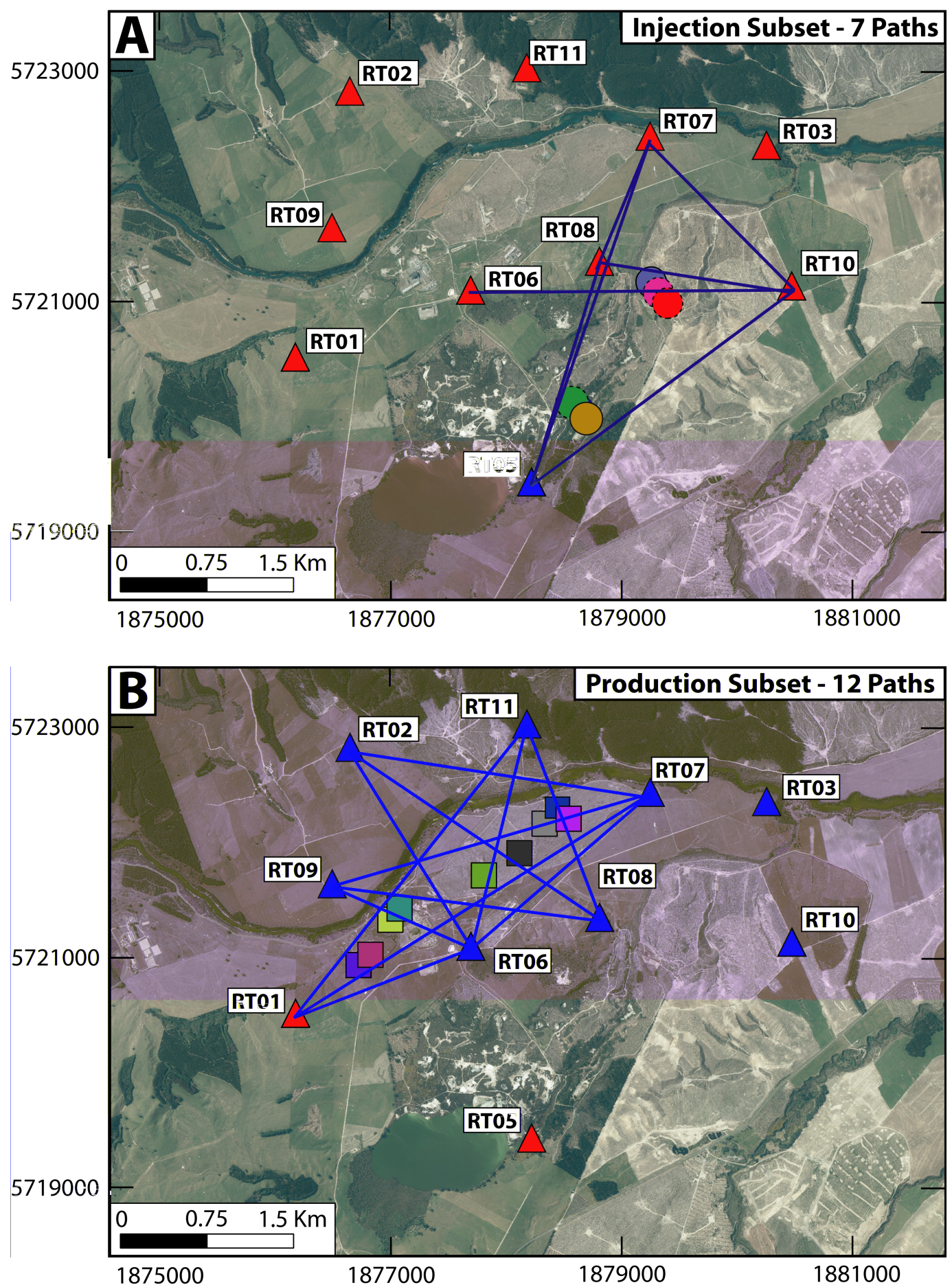

Figure 5.33: Displays the station pair paths used for the injection [A] and production [B] subsets. 

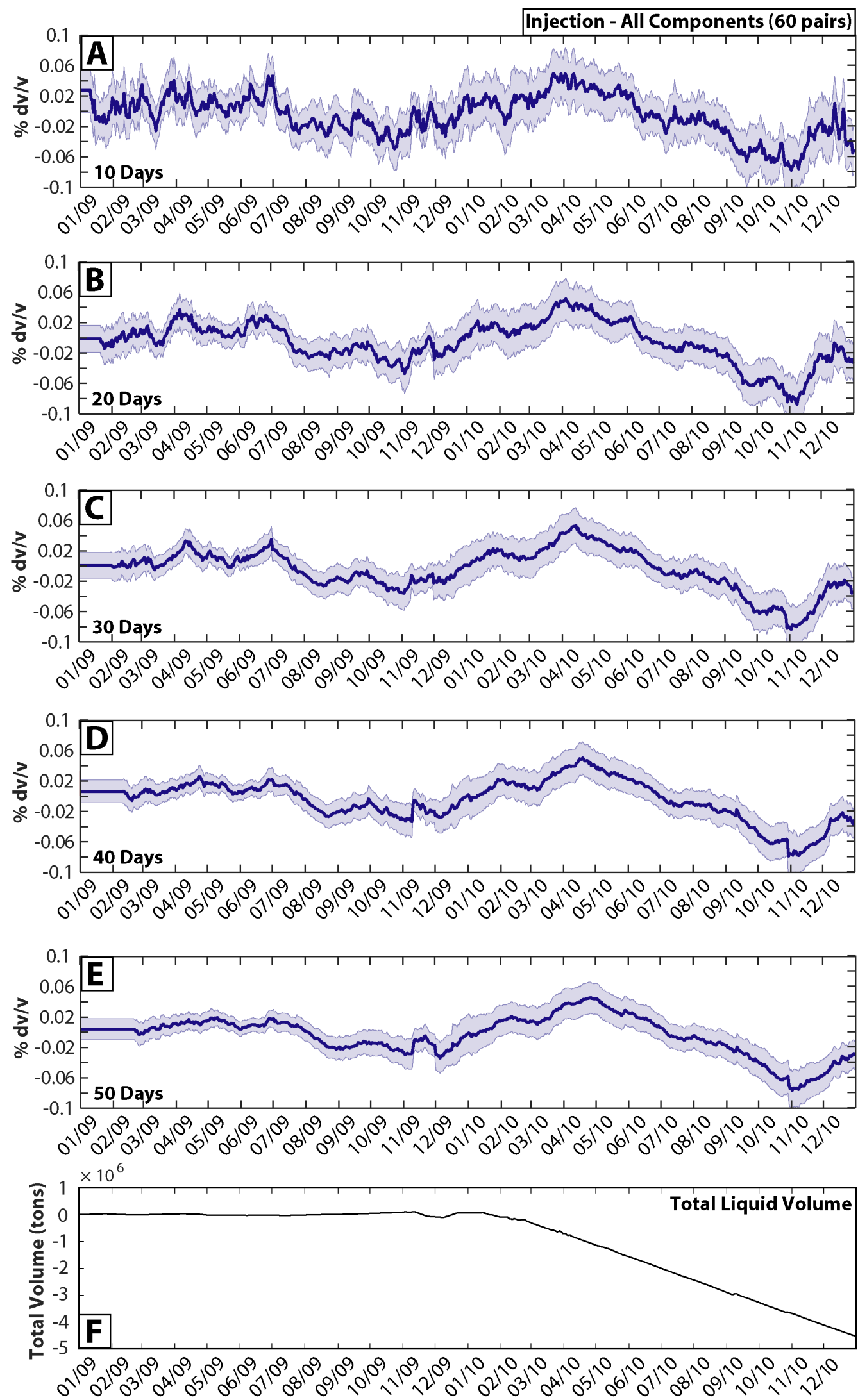

Figure 5.34: Velocity changes using 10-50 day MWCS windows [A-E] and Rotokawa relative liquid volume $[\mathrm{F}]$ using all components of the injection subset. The positive step seen in November of 2009 is due to missing data amongst numerous pairs in this subset. 

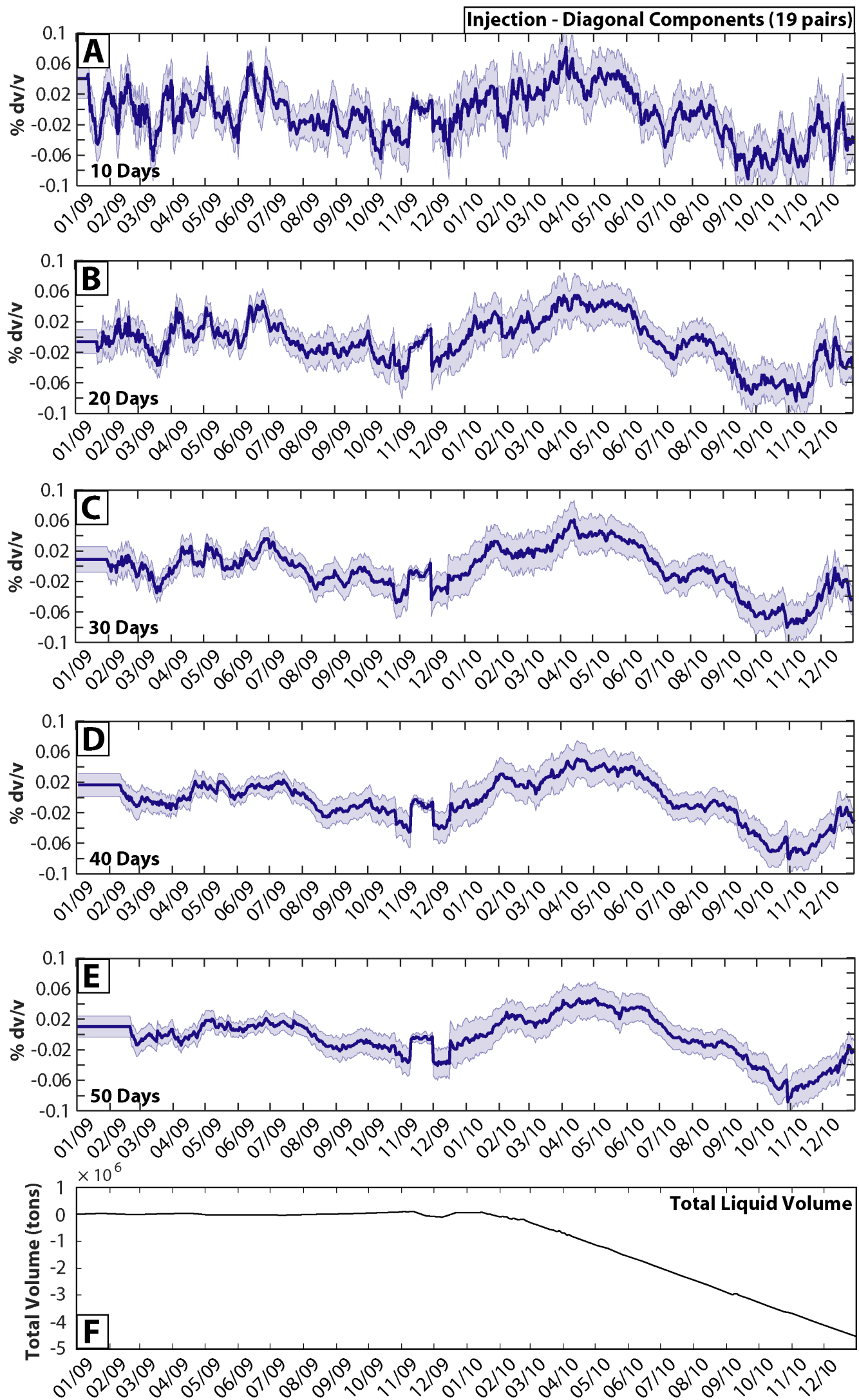

Figure 5.35: Velocity changes using 10-50 day MWCS windows [A-E] and Rotokawa relative liquid volume $[\mathrm{F}]$ using the diagonal components (RR, TT, and $\mathrm{ZZ}$ ) of the injection subset. The positive step seen in November of 2009 is due to missing data amongst numerous pairs in this subset. 

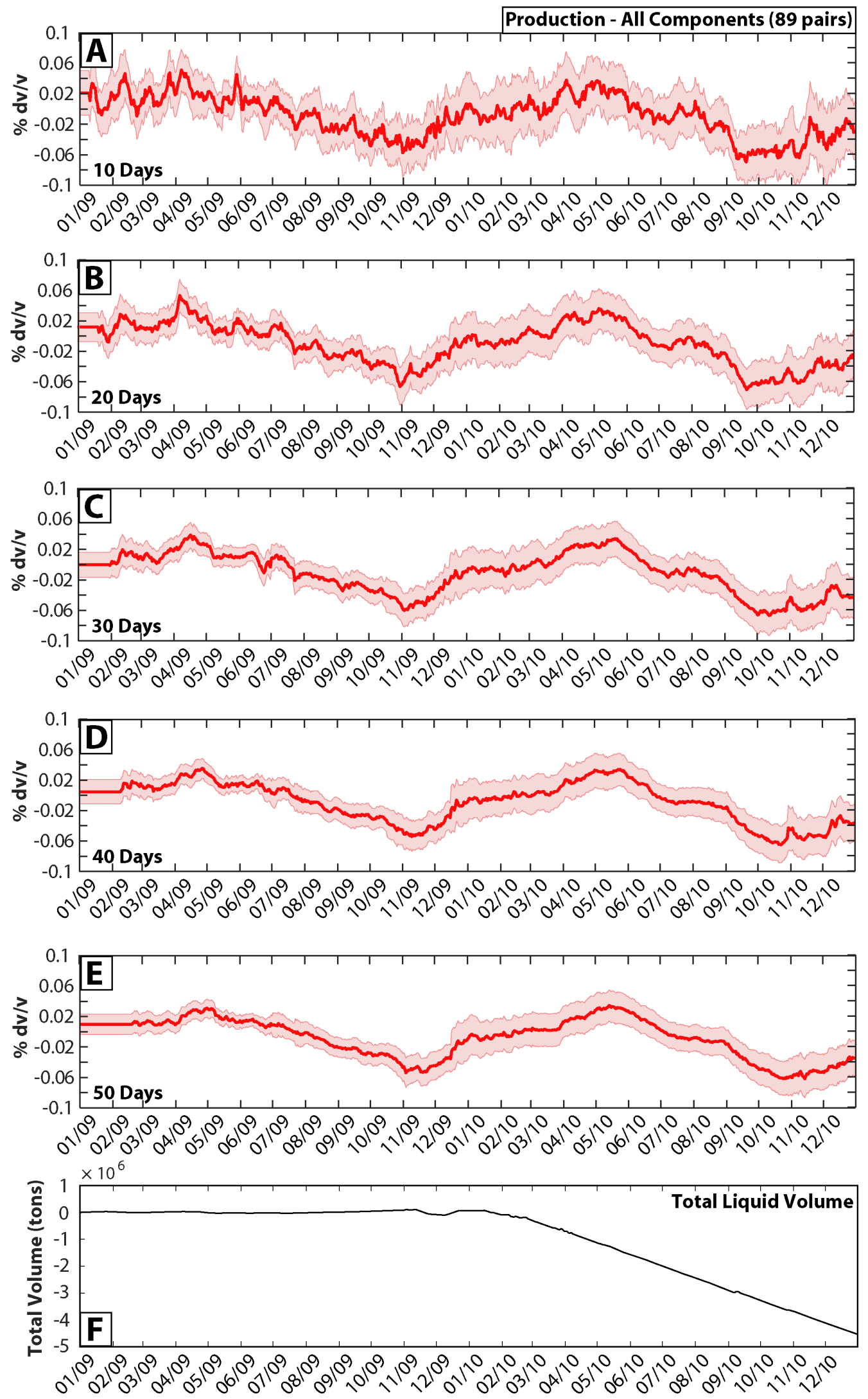

Figure 5.36: Velocity changes using 10-50 day MWCS windows [A-E] and Rotokawa relative liquid volume $[\mathrm{F}]$ using all components of the production subset. 

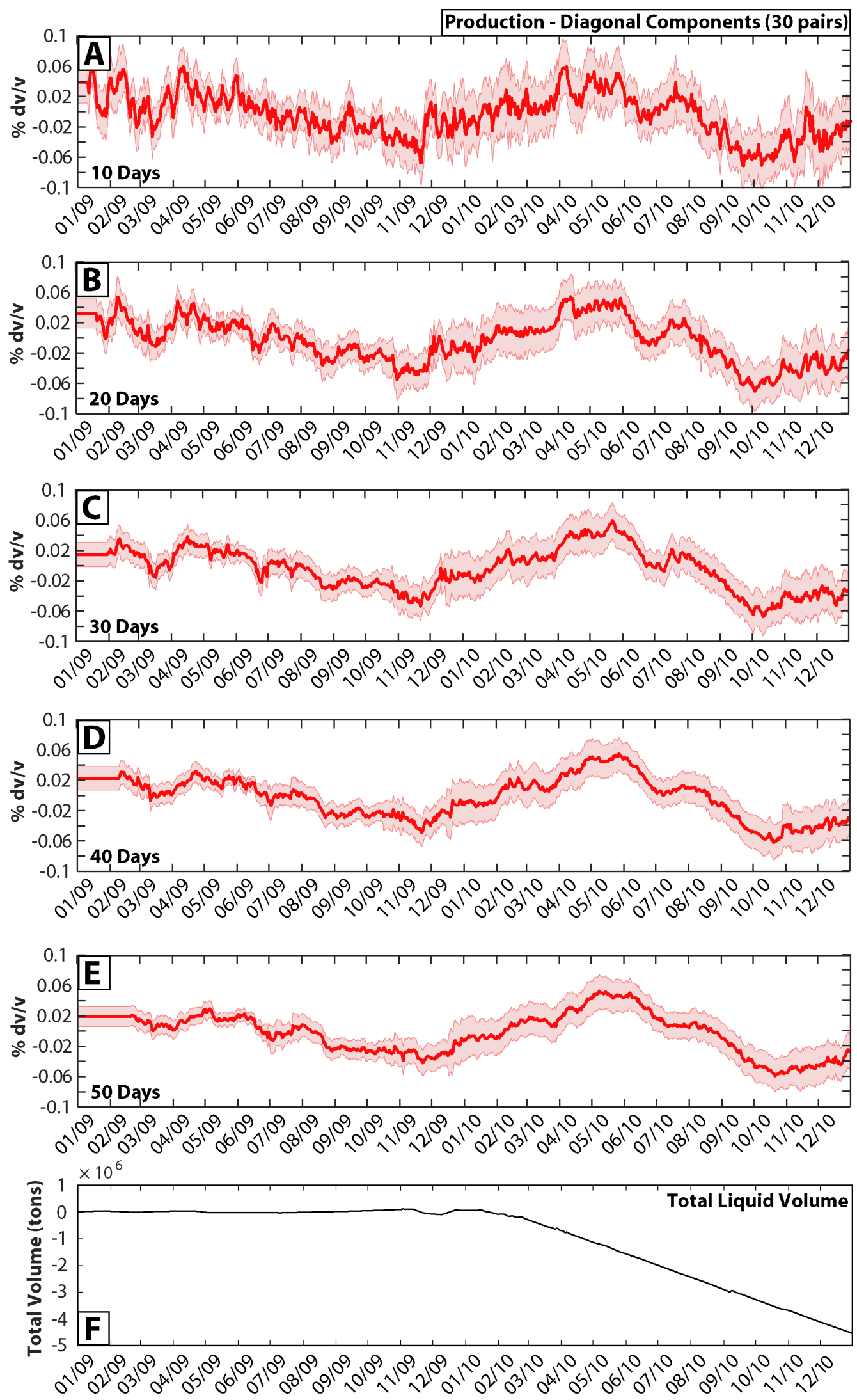

Figure 5.37: Velocity changes using 10-50 day MWCS windows [A-E] and Rotokawa relative liquid volume [F] using the diagonal components (RR, TT, and ZZ) of the production subset. 
Two main trends were found in all of the velocity changes results: a positive velocity change increasing after December 2009 and peaking at approximately April 2010 and a subsequent negative velocity change with a minimum value in the month of October 2010. These measurements are observed throughout the entire field, so we will refer to these as "full-field" responses.

Tables 5.6 and 5.7 outline the maximum positive and negative changes compared to the average velocity of the reference stack for each sensor grouping. We found that the maximum and minimum positive changes were $0.083 \%$ (injection subset diagonal components 10 -day) and $0.025 \%$ (all field all components 50 -day), with a mean value of $0.043 \%$. The maximum and minimum negative changes were $-0.093 \%$ (injection subset diagonal components 50 -day) and $-0.065 \%$ (production subset diagonal components 50 -day), with a mean value of $-0.077 \%$. Additionally, the 10 largest measurements of negative velocity $(-0.093 \%$ through $-0.081 \%)$, and the three largest measurements of positive velocity changes $(0.083 \%$ through $0.056 \%)$, are all from injection groupings [Tables 5.6 and 5.7].

\begin{tabular}{||l||l||l||l||l||l||}
\hline Grouping & 10 Days & 20 Days & 30 Days & 40 Days & 50 Days \\
\hline All field all components & $0.034 \%$ & $0.032 \%$ & $0.029 \%$ & $0.026 \%$ & $0.025 \%$ \\
\hline All field diagonal components & $0.051 \%$ & $0.046 \%$ & $0.038 \%$ & $0.032 \%$ & $0.032 \%$ \\
\hline Injection all components & $0.048 \%$ & $0.051 \%$ & $0.052 \%$ & $0.048 \%$ & $0.043 \%$ \\
\hline Injection diagonal components & $0.083 \%$ & $0.056 \%$ & $0.06 \%$ & $0.048 \%$ & $0.045 \%$ \\
\hline Production all components & $0.032 \%$ & $0.031 \%$ & $0.032 \%$ & $0.029 \%$ & $0.028 \%$ \\
\hline $\begin{array}{l}\text { Production diagonal compo- } \\
\text { nents }\end{array}$ & $0.052 \%$ & $0.047 \%$ & $0.054 \%$ & $0.04 \%$ & $0.047 \%$ \\
\hline \hline
\end{tabular}

Table 5.6: The maximum positive velocity change for each grouping at Rotokawa for each MWCS window size.

\begin{tabular}{||l||l||l||l||l||l||}
\hline Grouping & 10 Days & 20 Days & 30 Days & 40 Days & 50 Days \\
\hline All field all components & $-0.073 \%$ & $-0.073 \%$ & $-0.07 \%$ & $-0.07 \%$ & $-0.07 \%$ \\
\hline All field diagonal components & $-0.08 \%$ & $-0.078 \%$ & $-0.076 \%$ & $-0.077 \%$ & $-0.075 \%$ \\
\hline Injection all components & $-0.082 \%$ & $-0.09 \%$ & $-0.087 \%$ & $-0.083 \%$ & $-0.081 \%$ \\
\hline Injection diagonal components & $-0.092 \%$ & $-0.085 \%$ & $-0.083 \%$ & $-0.085 \%$ & $-0.093 \%$ \\
\hline Production all components & $-0.075 \%$ & $-0.075 \%$ & $-0.069 \%$ & $-0.07 \%$ & $-0.068 \%$ \\
\hline $\begin{array}{l}\text { Production diagonal compo- } \\
\text { nents }\end{array}$ & $-0.079 \%$ & $-0.078 \%$ & $-0.072 \%$ & $-0.069 \%$ & $-0.065 \%$ \\
\hline \hline
\end{tabular}

Table 5.7: The maximum negative velocity change for each grouping at Rotokawa for each MWCS window size. 
There is a step-like increase in velocities in the injection subset of Rotokawa in November of 2009 that does not follow the long-term trend [Figures 5.34 and 5.35]. This step is not observed in the production subsets [Figures 5.36 and 5.37] and is averaged out in the all field velocity change [Figures 5.31 and 5.32]. This is a result of a poorly-averaged measurement due to missing data across several paths of the subset [Figures 5.16 and 5.14] and should not be interpreted as a response due to changes in the medium [Figure 5.38].

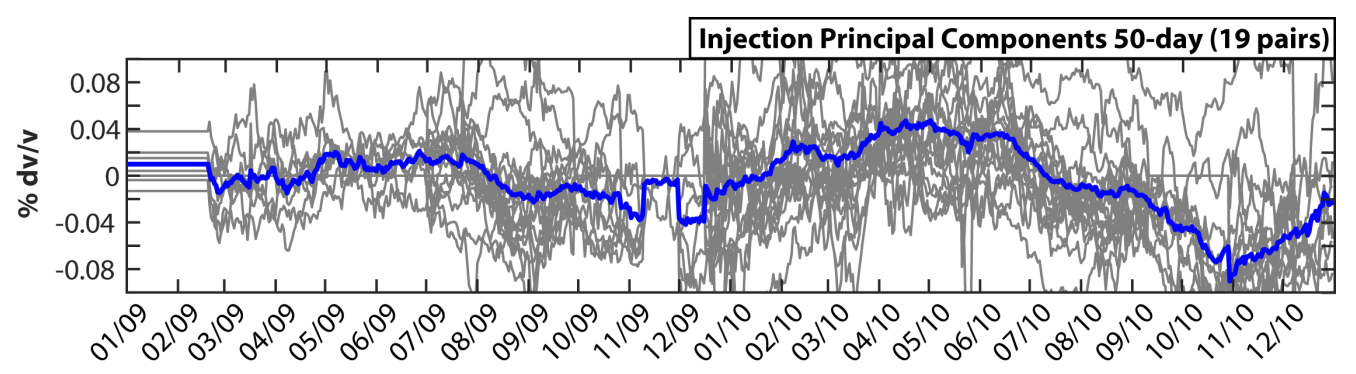

Figure 5.38: Displays the average velocity change (blue) and individual station-pair velocity change (gray) for the Rotokawa diagonal components of the injection subset.

The results using reference stack 2 [Figure 5.16, blue rectangle] are displayed in Appendix B.3 in Tables B.1 and B.2 and Figures B.1-B.6. We observe on all subsets a maximum positive velocity change near April 2010 and a subsequent decrease just like the first reference stack. Using the mean value of the new reference stack for comparison, we found that the maximum and minimum velocity changes were $0.083 \%$ (injection subset diagonal components 20-day) and $0.039 \%$ (all field all components 50-day) for the positive change and $-0.098 \%$ (injection subset diagonal components 10-day) and $-0.052 \%$ (production subset all components 10-day) for the negative change. The mean values of the positive and negative velocity changes were $0.056 \%$ and $-0.066 \%$ respectively. Just like in the first reference stack, we found that the ten largest negative decreases $(-0.098 \%$ through $-0.071 \%)$ and the three largest positive changes $(0.083 \%$ through $0.073 \%$ ) were in the injection subsets. However, the velocity changes in the new reference stack were less stable than those in the first (i.e., Figures 5.34 and B.3). We hypothesize that this may be due to the short amount of data used for the reference stack (3 months instead of 9 months). 


\subsection{Ngatamariki site information}

Ngatamariki is a geothermal field owned and operated by Mercury Energy located only a few kilometers north of Rotokawa. It began electricity production in mid-2013 after a few months of intermittent well stimulation [Clearwater et al., 2015] [Table 5.8]. In this section, we apply the moving-window cross spectral technique (MWCS) to the available seismic data from 2012-2013 at Ngatmariki and compare the computed velocity changes to injection and production information. This dataset represents an opportunity to observe any changes associated with the onset of geothermal production.

\begin{tabular}{||l||l|l||}
\hline Event & Date & $\begin{array}{l}\text { Approximate } \\
\text { Flow (t/h) }\end{array}$ \\
\hline NM8 Stimulation & 2012-06-02 to 2012-07-10 & 170 \\
\hline NM10 Stimulation & 2012-08-29 to 2012-09-24 & 200 \\
\hline NM9 Stimulation & 2012-12-12 to 2013-01-05 & 150 \\
\hline Phase 1 Production & 2013-03-01 to 2013-05-01 & 500 \\
\hline Phase 2 Production & 2013-05-01 to 2013-08-01 & 1100 \\
\hline Phase 3 Production & 2013-08-01 to 2013-12-31 & 2000 \\
\hline
\end{tabular}

Table 5.8: Injection flow in tons/hour for well stimulation and electricity production at Ngatamariki [Clearwater et al., 2015].

\subsubsection{Ngatamariki geology}

The Ngatamariki geothermal field is located only a few kilometers north of the Rotokawa geothermal field, so its geology is similar to that of Rotokawa [Section 5.4.1, Figure 5.1]. However, although the surface geology is composed of pumice alluvium and rhyolitic domes like Rotokawa, Ngatamariki only has a few minor thermal manifestations [Mongillo and Clelland, 1984].

Most of the subsurface geologic information from Ngatamariki was obtained from drilling of wells, first during an initial exploration phase in the 1980's (NM1-NM4) which discovered the permeable high-temperature resource [Wood, 1985, 1986a], then in 2004 (NM5-NM7) to assess its potential, and lastly in 2011 (NM8-NM11) when the power station was being constructed [Chambefort et al., 2015]. Similar to Rotokawa, there are three main aquifers at Ngatamariki: a shallow meteoric groundwater aquifer, an aquifer at intermediate depth composed of chloride-bicarbonate waters, and a deep chloride geothermal reservoir (below $1 \mathrm{~km}$ depth) [Bignall, 2009, Chambefort et al., 2015].

As may be expected, the basement rocks at Ngatamariki are the same Greywacke observed at Rotokawa [Adams et al., 2009]. The basement is overlain by andesitic 
lavas and breccias, the Tahorakuri Formation volcaniclastic and sedimentary successions, and rhyolite lavas and breccias, all of which are included in a set called the Reporoa Group [Gravly et al., 2006, Chambefort et al., 2014] [Figures 5.39 and 5.40]. The minimum thickness of the Reporoa Group at Ngatamariki is $2.1 \mathrm{~km}$, determined by the drilling of NM6 [Chambefort et al., 2014]. The Tahorakuri Formation, the shallowest member of the Reporoa Group, is overlain by ignimbrite up to a thickness of 500 meters, recognized as part of the Whakamaru group [Wilson et al., 1986, Chambefort et al., 2014]. Above the Whakamaru group ignimbrite lies the the volcanic and sedimentary succession of the Waiora Formation [Rosenberg et al., 2009], the lake sediments of the Huka Falls Formation [Grindley, 1965], and the Oruanui ignimbrite of the Oruanui Formation [Leonard et al., 2010, Chambefort et al., 2014]. In addition to the aforementioned geologic layers, certain wells at Ngatamariki are intersected by plutonic rocks including: quartz-bearing diorite, microdiorites, quartz-phyric tonalite, and mafic dikes [Wood, 1986b, Browne et al., 1992, Chambefort et al., 2014]. The surface geology of Ngatamariki, along with a cross-section describing the aforementioned stratigraphy, is displayed in Figures 5.39 and 5.40, adapted from Chambefort et al. [2014]. 


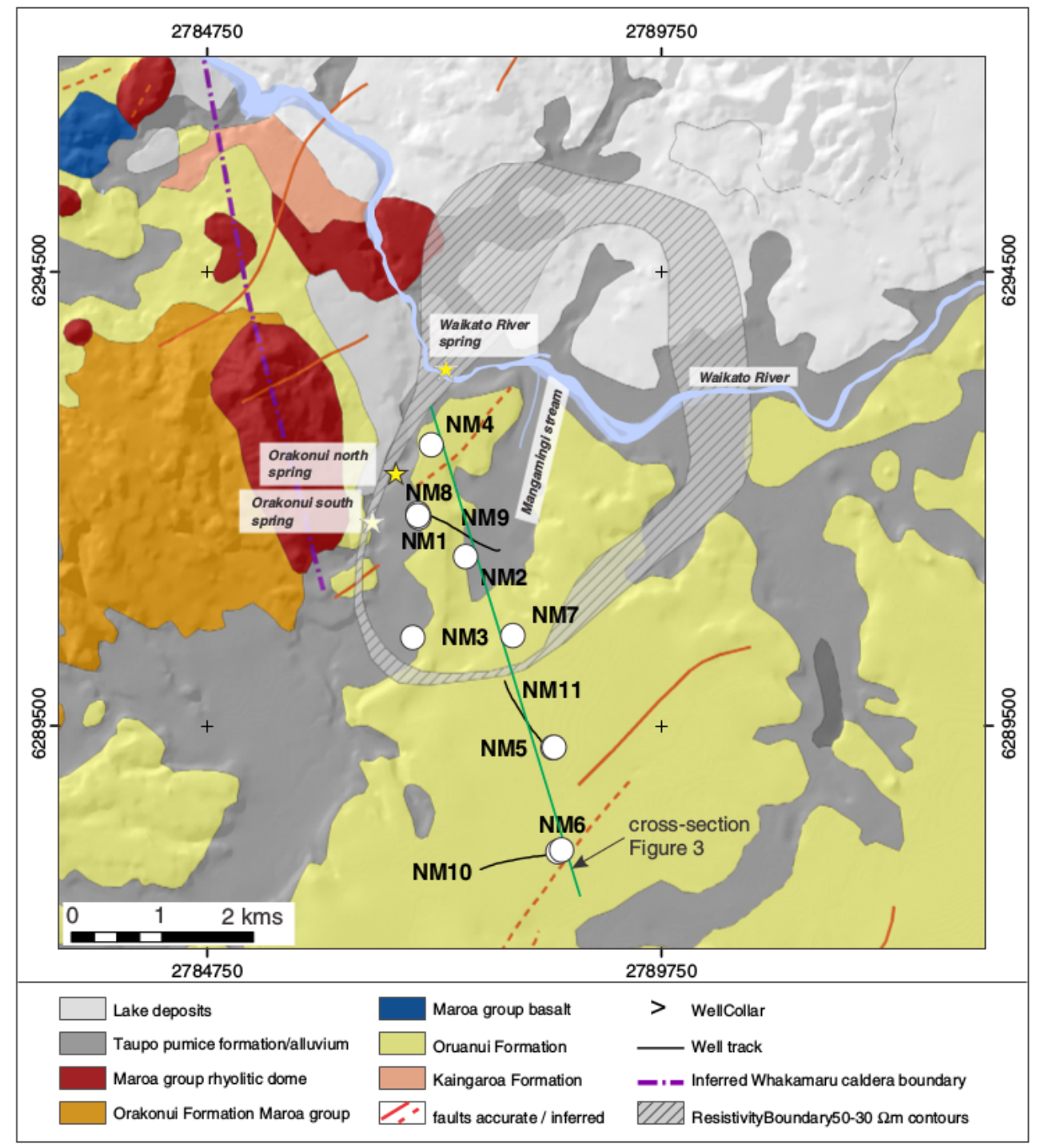

Figure 5.39: Displays the location of the geologic cross section of Figure 5.40 and the surface geology of Ngatamariki. Adapted from Chambefort et al. [2014]. 


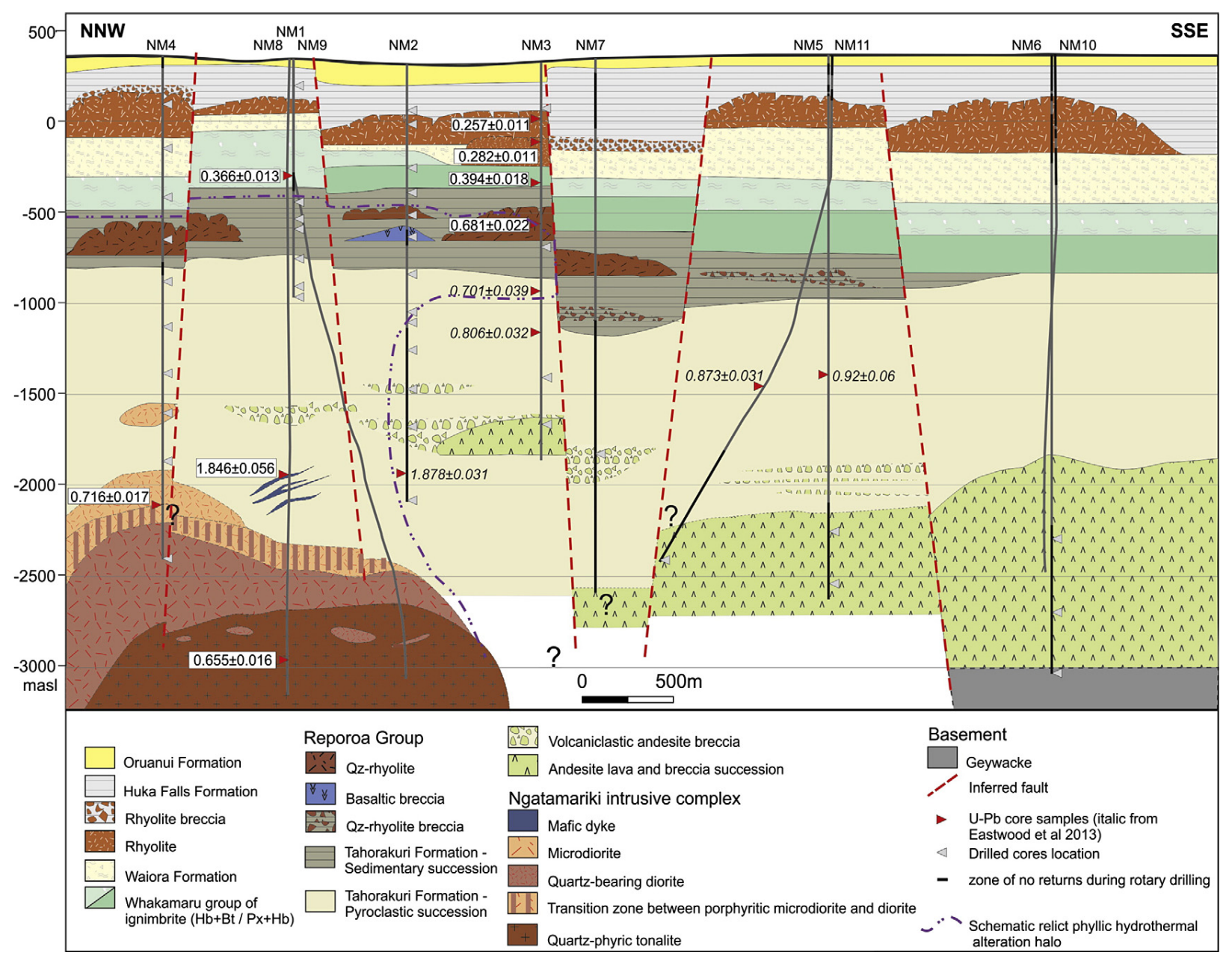

Figure 5.40: Ngatamariki geologic cross section adapted from Chambefort et al. [2014]. The location of the profile is displayed in Figure 5.39. 


\subsubsection{Ngatamariki instrumentation}

During 2012-2013, the time period analyzed in this manuscript for the onset of production, there were five injection wells and three production wells active at Ngatamariki within the deep aquifer [Chambefort et al., 2014] [Table 5.9]. Injection occurred at wells NM04, NM06, NM08, NM09, and NM10 located in the north and south of the geothermal field and production occured at NM05, NM07, and NM11 in the center of the field [Figure 5.41]. During this time, the Ngatamariki seismic network consisted of eleven Geospace 4.5 Hz GS-11D seismometers (NS01-NS11) [Figure 5.41]. Additional borehole instruments (NS12-NS14) were added in 2013, but are not included in this analysis as they were not active prior to the beginning of production.

\begin{tabular}{||l||l||l||}
\hline Well & $\begin{array}{l}\text { Downhole } \\
\text { Depth } \mathbf{( m )}\end{array}$ & Geology \\
\hline NM04 (Injection) & 2742 & Diorite intrusive \\
\hline NM06 (Injection) & 3388 & Greywacke basement \\
\hline NM08 (Injection) & 3492 & Tonalite intrusive \\
\hline NM09 (Injection) & 3543 & Tonalite intrusive \\
\hline NM10 (Injection) & 3027 & Andesite Lava \\
\hline NM05 (Production) & 2987 & Andesite Lava \\
\hline NM07 (Production) & 2953 & Andesite Lava \\
\hline NM11 (Production) & 3097 & Andesite Lava \\
\hline \hline
\end{tabular}

Table 5.9: Injection and production wells at Ngatamariki 2012-2013.

Wells NM08, NM09, and NM10 were stimulated prior to the start of electricity production to assess their injectivity [Clearwater et al., 2015]. Duration and approximate flow rates for the stimulations and the beginning of electricity production are listed in Table 5.8. The data availability for the Ngatamariki seismic network is displayed in Figure 5.42, with red bars showing days with at least some data. Unlike Rotokawa [Figure 5.16], the Ngamatariki seismic network has no gaps throughout its operation after the first initial swap of stations at the end of August 2012 [Figure 5.42]. However, there isn't much data prior to the well stimulation that can be used for a MWCS reference stack. Due to this and our fluctuating velocity change results when using the three-month Rotokawa second refstack [Appendix B.3], we chose to use a reference stack from the beginning of each record (mid-July or September 2012 depending on the station) to the first of February 2013 [Figure 5.42, black rectangle]. In any case, the well stimulation injection rate is approximately an order of magnitude smaller than the production, so we expect the responses to be much smaller than what is observed after the start of production [Table 5.8]. 


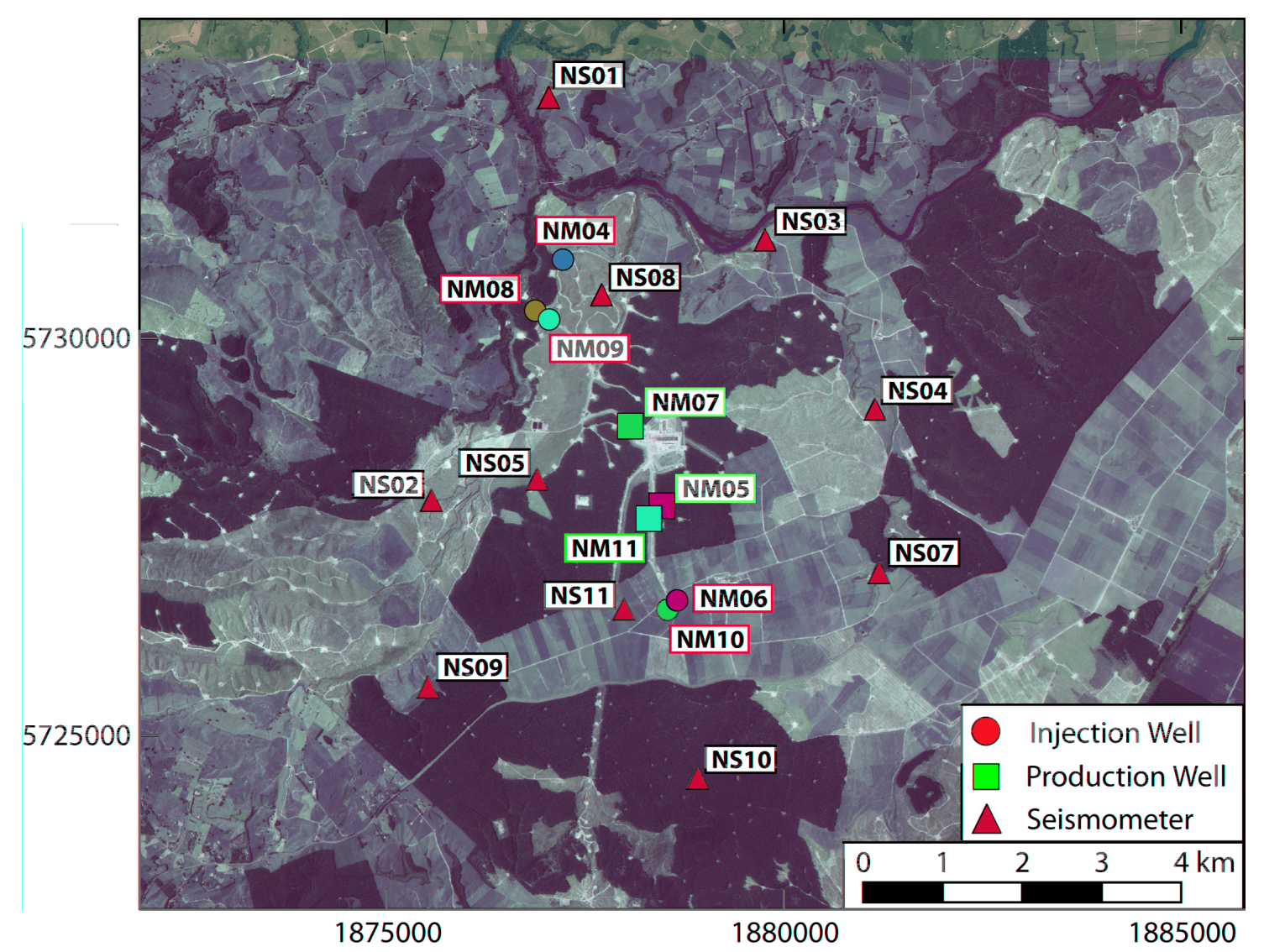

Figure 5.41: Ngatamariki station map for 2012-2013. Seismometers are displayed as blue triangles, injections wells as circles, and production wells as rectangles. The color of the injection and production wells match the color of the flow lines in Figures 5.43 and 5.44 . 


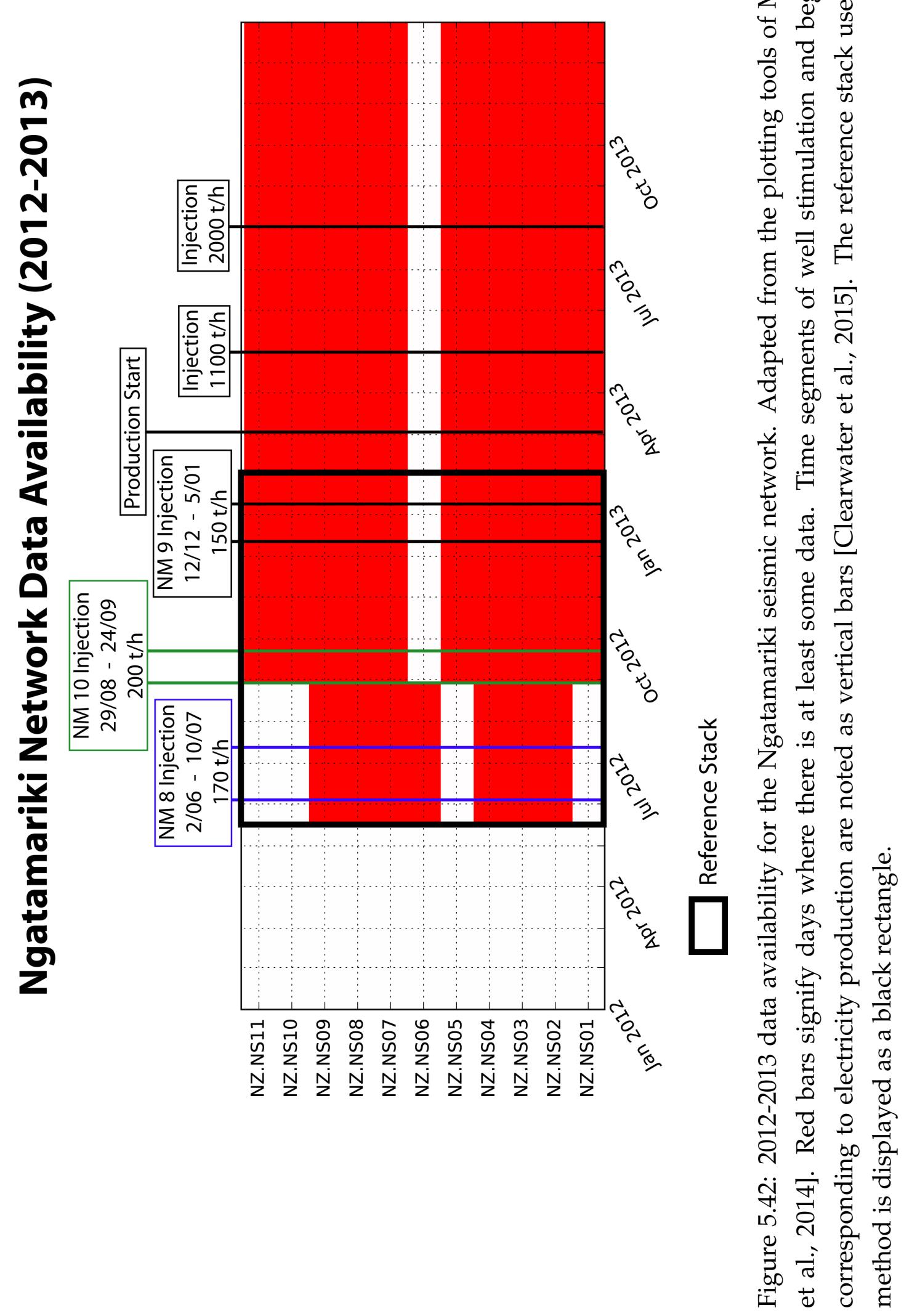


The flow for the Ngatamariki injection and production from March 2013 is displayed in Figures 5.43 and 5.44. The colors for each of the flow rates matches the injection and production well colors in Figure 5.41. Unfortunately, we do not have injection and production rates for the well stimulation prior to production, but we know the approximate flow rate amount and duration from literature [Clearwater et al., 2015]. Figure 5.45 displays the total injection and production at Ngatamariki [A], the total liquid volume computed relative to the start of production (March 2013) [B], the injection-production ratio, and time periods of stimulation for wells NM08, NM10, and NM09. It is important to state that unlike our previous example of Rotokawa [Figure 5.20], Ngatamariki did not have constant injection prior to the start of production [Figure 5.45B].

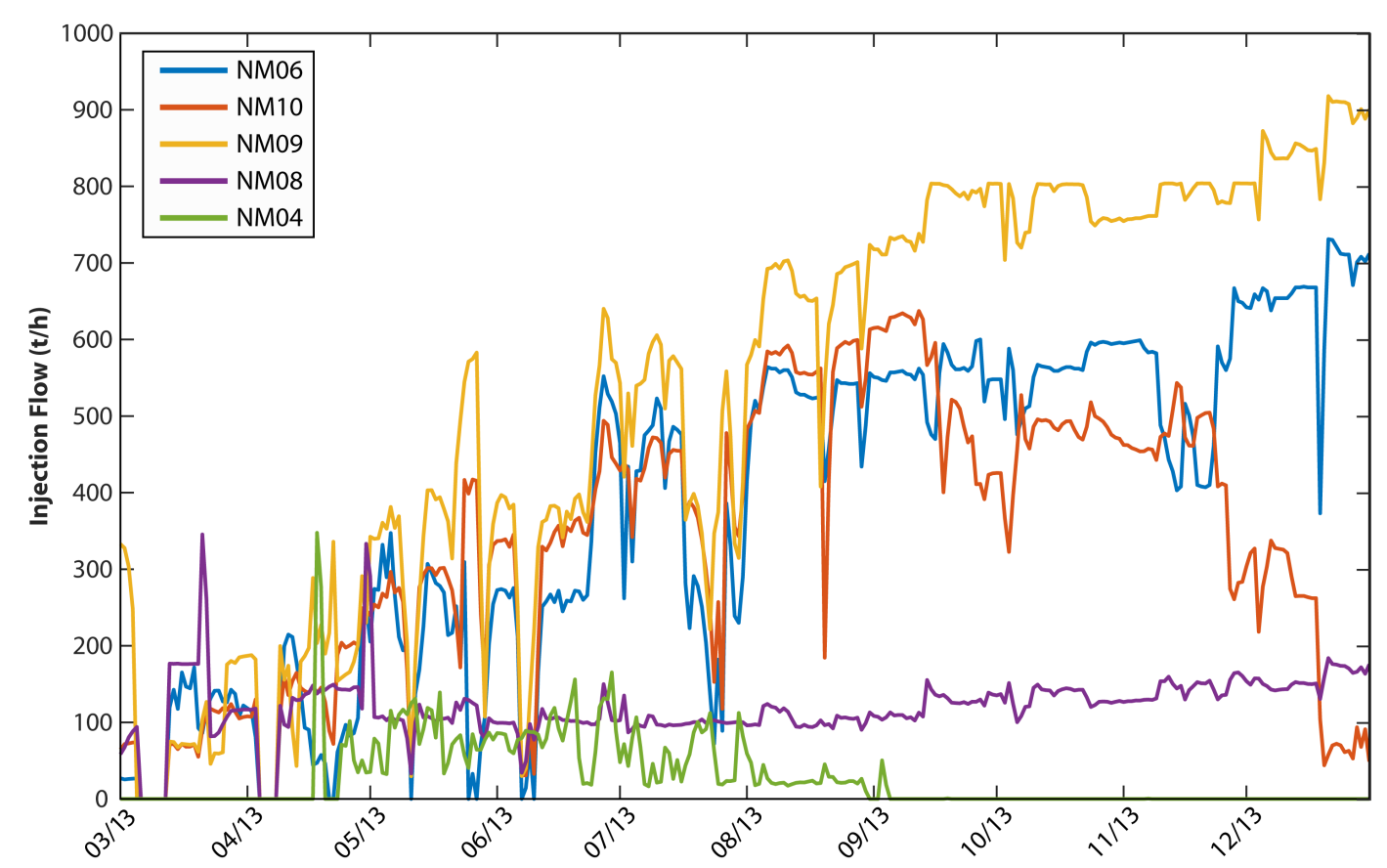

Figure 5.43: Ngatamariki injection flow for 2012-2013. Line color corresponds to the injection well color in Figure 5.41. 


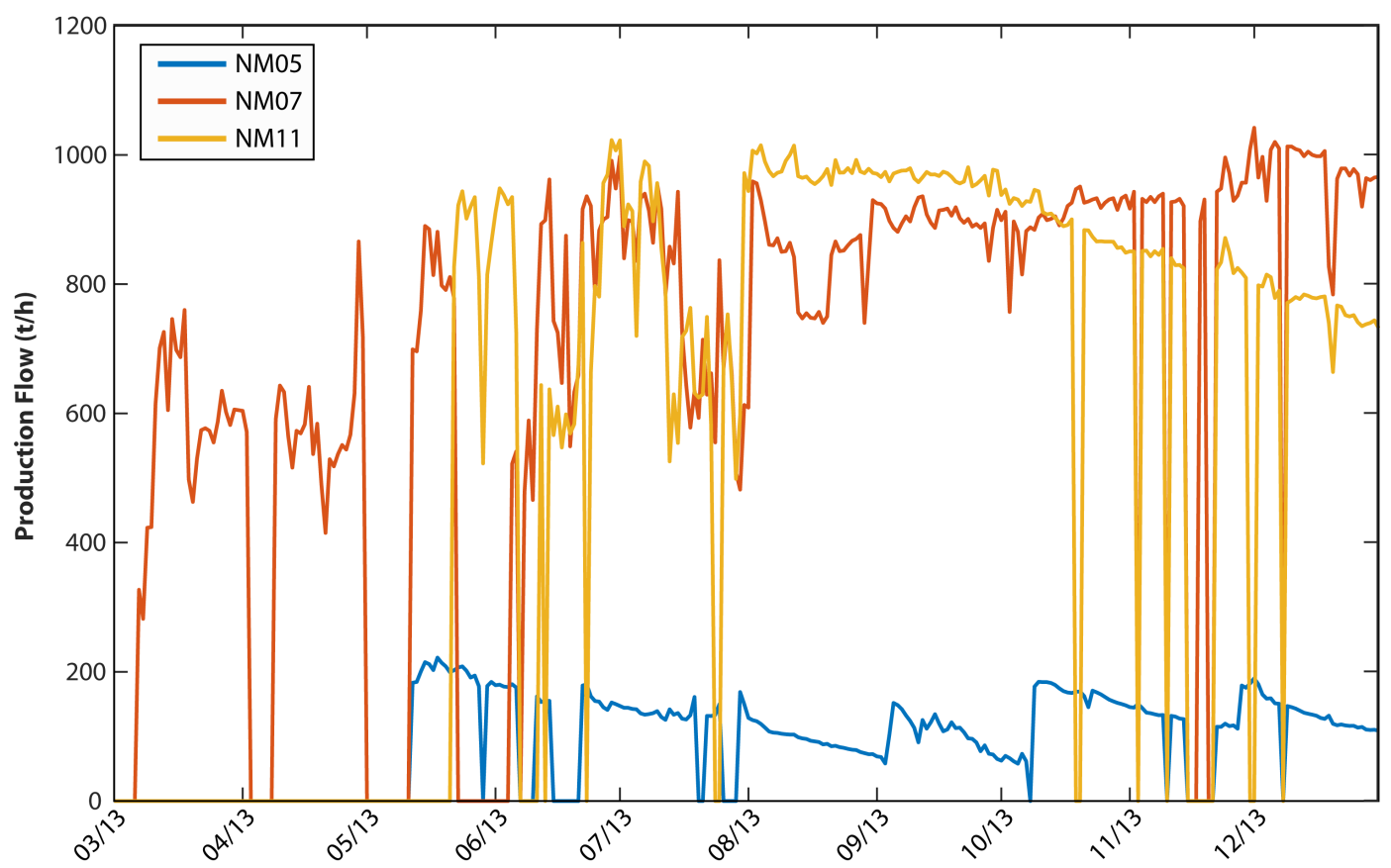

Figure 5.44: Ngatamariki production flow for 2012-2013. Line color corresponds to the production well color in Figure 5.41. 

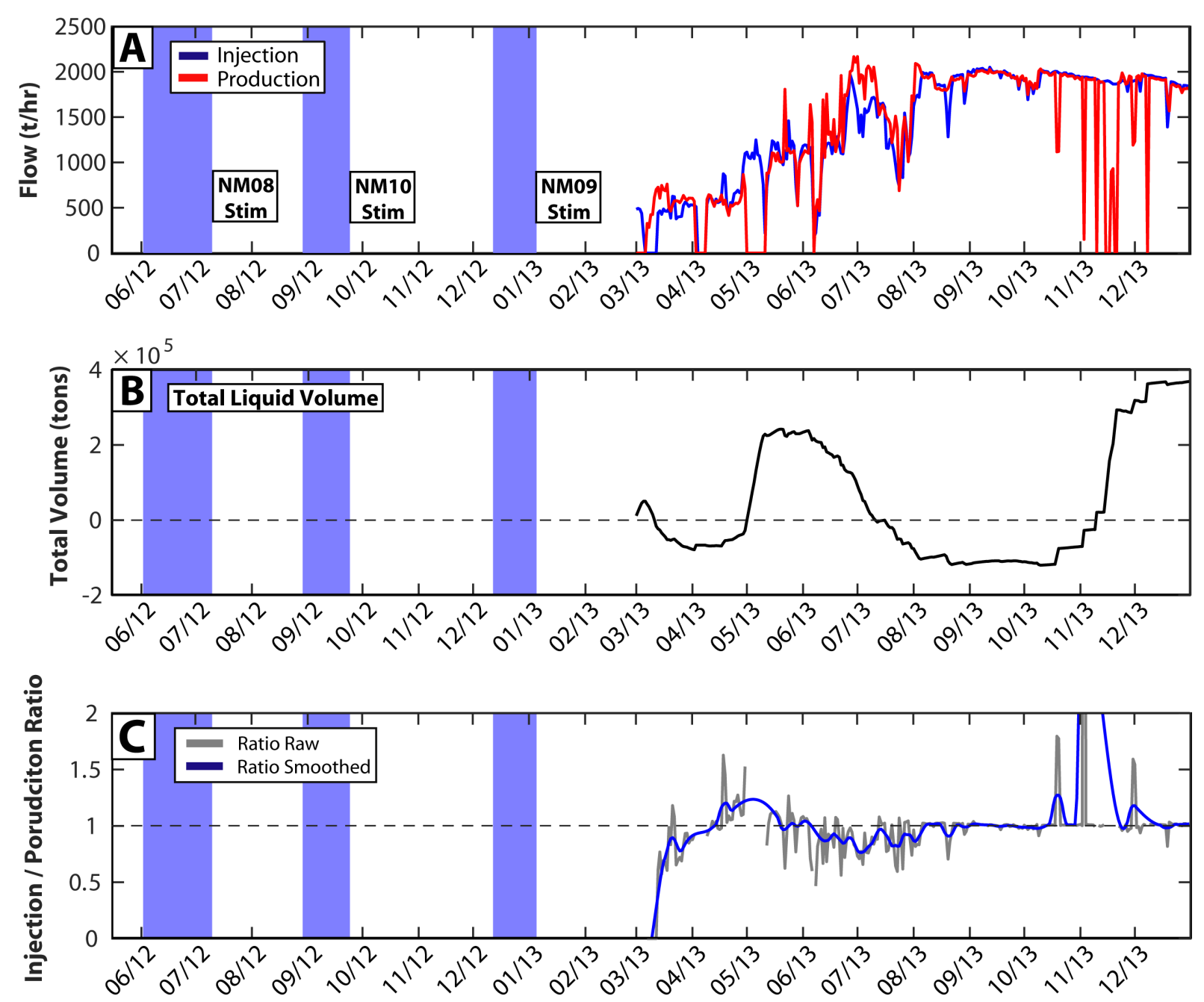

Figure 5.45: [A] Ngatamariki total injection and production flow in tons per hour. [B] Total liquid volume calculated relative to the start of production (1st of March 2013). [C] Smoothed and raw injection-production ratios. Times of well stimulation [Table 5.8] are displayed as blue shaded areas. 


\subsection{Ngatamariki Results}

\subsubsection{Ngatamariki cross-correlations and moveouts}

The cross-correlation moveouts for 2012-2013 Ngatamariki are displayed in Figures 5.46, 5.47 , and 5.48. These cross-correlations were not as clean in terms of ballistic waves or scatterers as their Rotokawa counterparts [Figures 5.21, 5.22, and 5.23]. However, scatterers can still be seen in the coda of the cross-correlations, especially in the diagonal components.

\subsubsection{Ngatamariki stability}

Visual inspection of the stability of the cross-correlation images and the magnitude squared coherence was conducted for the Ngatamariki station pairs for each component using the criteria previously described in Section 5.5.2. The station-pairs that were excluded from analysis are listed in Appendix B.2.

Although we have no gaps in the data, there are two instrument changes occurring during this time period: NS03 on August 29th 2012 and NS09 on March 26th 2013. There appears to be no change in the coherence or cross-correlation stability in NS03 due to the instrument change. The instrument change at NS09 occurred to replace a dead East-component channel. This can be observed in the comparison between horizontal and vertical coherence and cross-correlations for station pairs using that station [Figure 5.49]. Due to this, the velocity changes utilizing the horizontal components of NS09 were excluded entirely. 

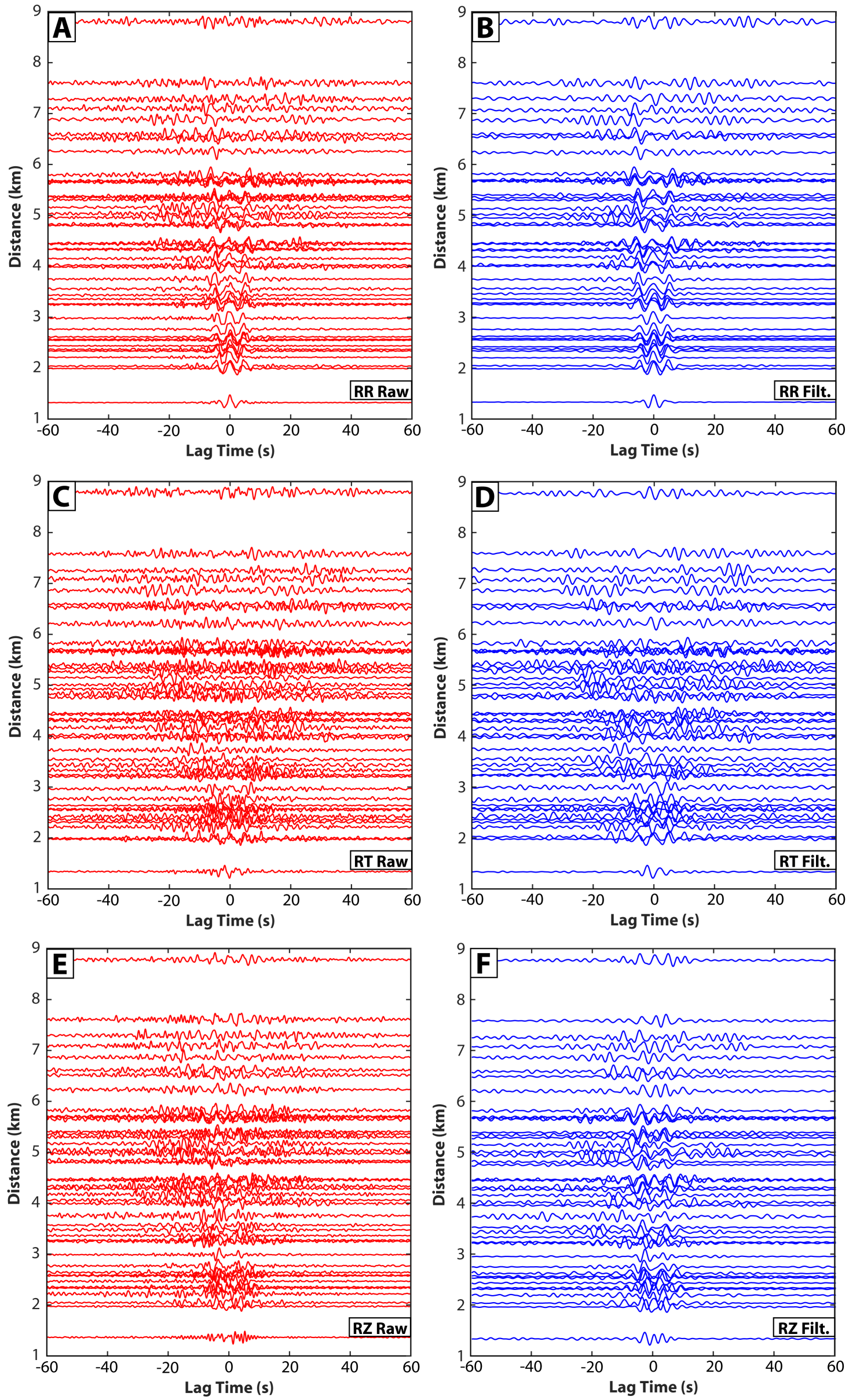

Figure 5.46: Ngatamariki 2012-2013 radial moveouts. The blue data is filtered between 0.1 to $0.4 \mathrm{~Hz}$. 

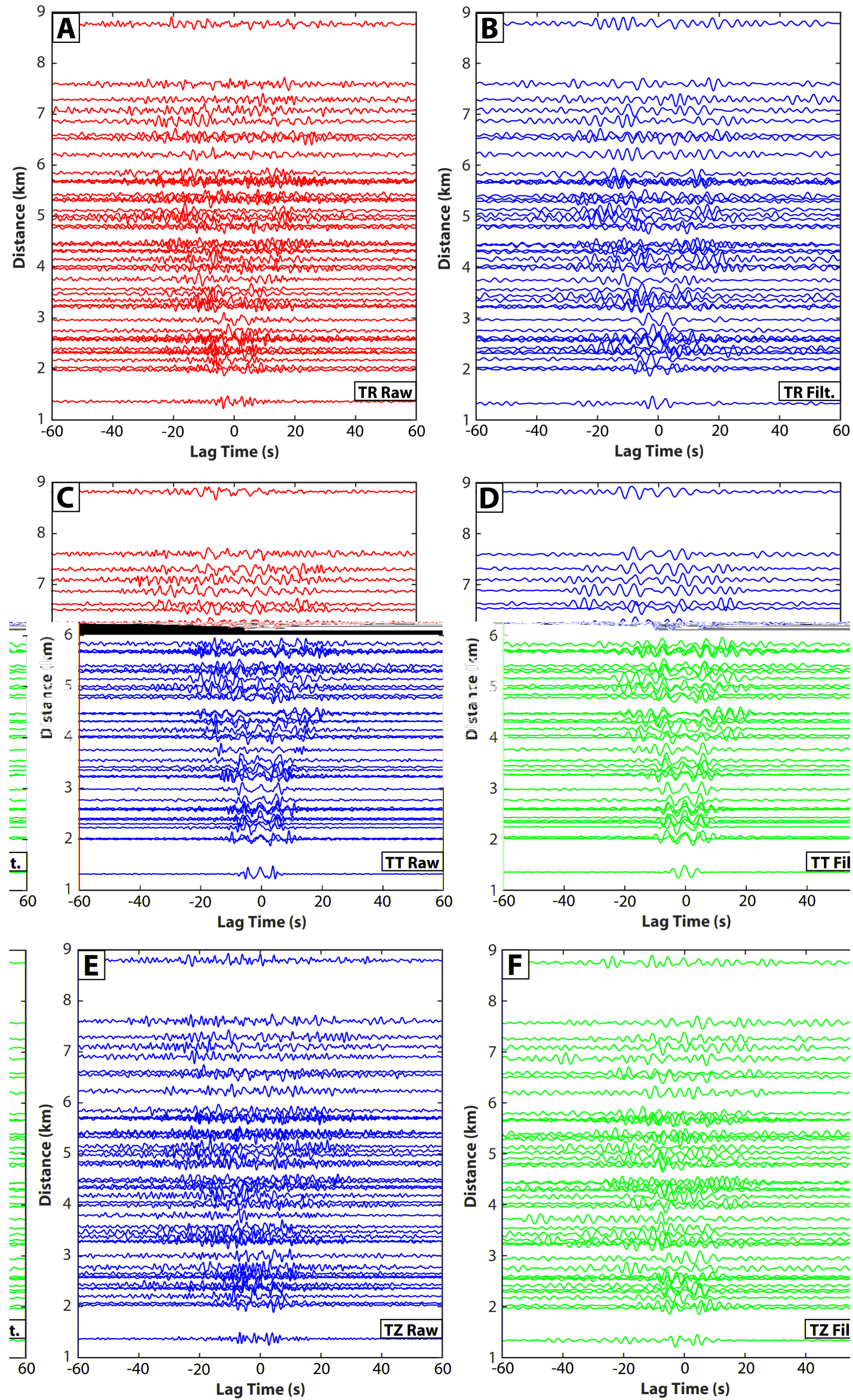

Figure 5.47: Ngatamariki 2012-2013 transverse moveouts. 

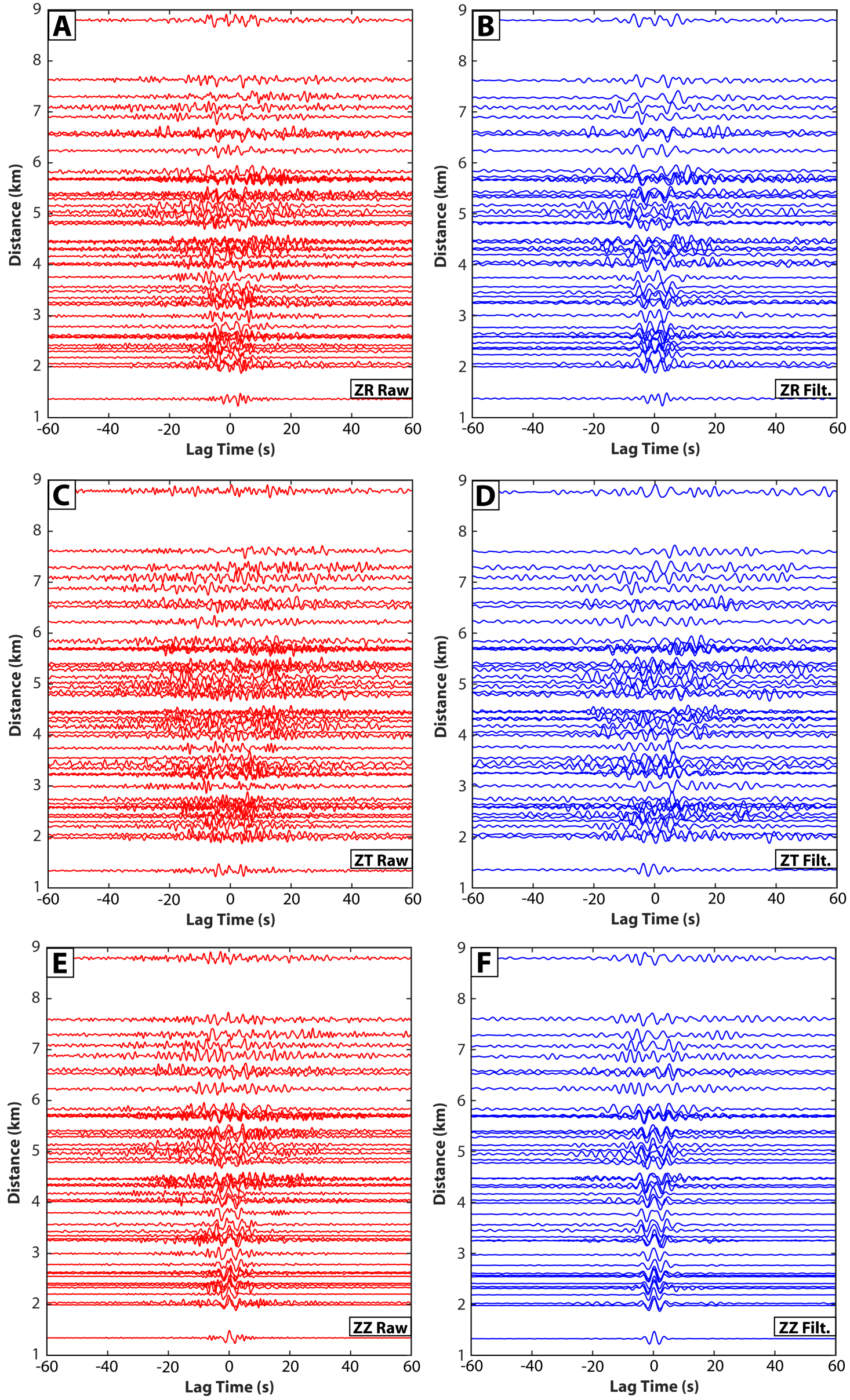

Figure 5.48: Ngatamariki 2012-2013 vertical moveouts. 

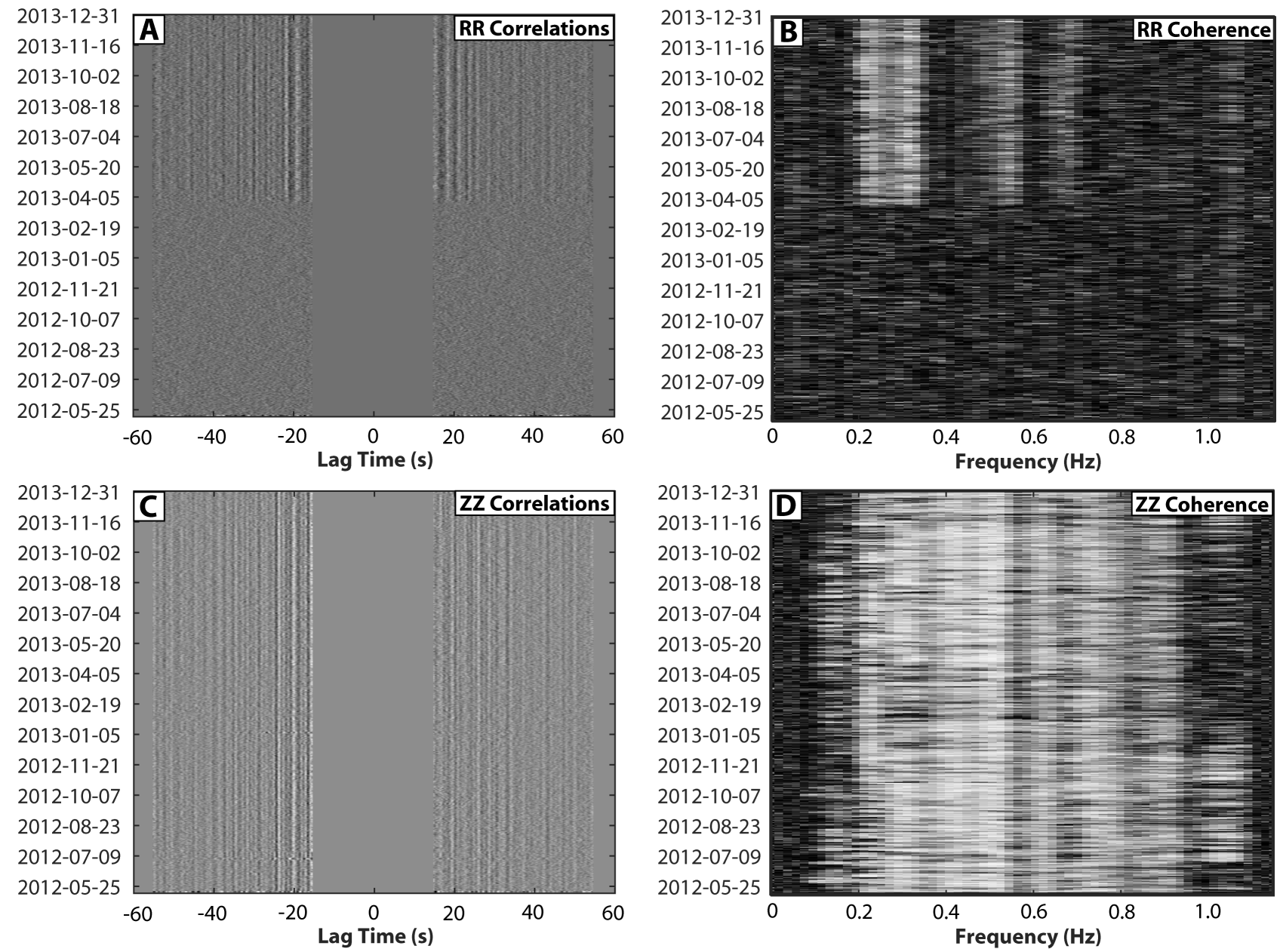

Figure 5.49: Figure illustrating the effects of the dead EHE channel at NS09 (using station-pair NS04-NS09). [A] Cross-correlation image for radial-radial (RR) component. [B] Magnitude-squared coherence for RR component. [C] Cross-correlation image for vertical-vertical (ZZ) component. [D] Magnitude-squared coherence for ZZ component. 


\subsubsection{Ngatamariki velocity changes}

We computed velocity changes across the entire Ngatamariki field [Figures 5.50 and 5.51] and two subsets: Ngatamariki north (6 paths) and south (14 paths) [Figures 5.525.56]. Due to seismic station coverage in relation to the wells, we could not group the field into production and injection subsets. Each velocity change example is compared to the total liquid volume, as previously discussed for the Rotokawa results [Section 5.5.4]. The number of pairs used for each grouping is listed in Table 5.10.

\begin{tabular}{||l||l||}
\hline Grouping & Number of paths \\
\hline All field all components & 261 \\
\hline All field diagonal components & 84 \\
\hline North subset all components & 39 \\
\hline North subset diagonal components & 11 \\
\hline South subset all components & 108 \\
\hline South subset diagonal components & 38 \\
\hline
\end{tabular}

Table 5.10: The number of paths for each velocity change grouping at Ngatamariki. 

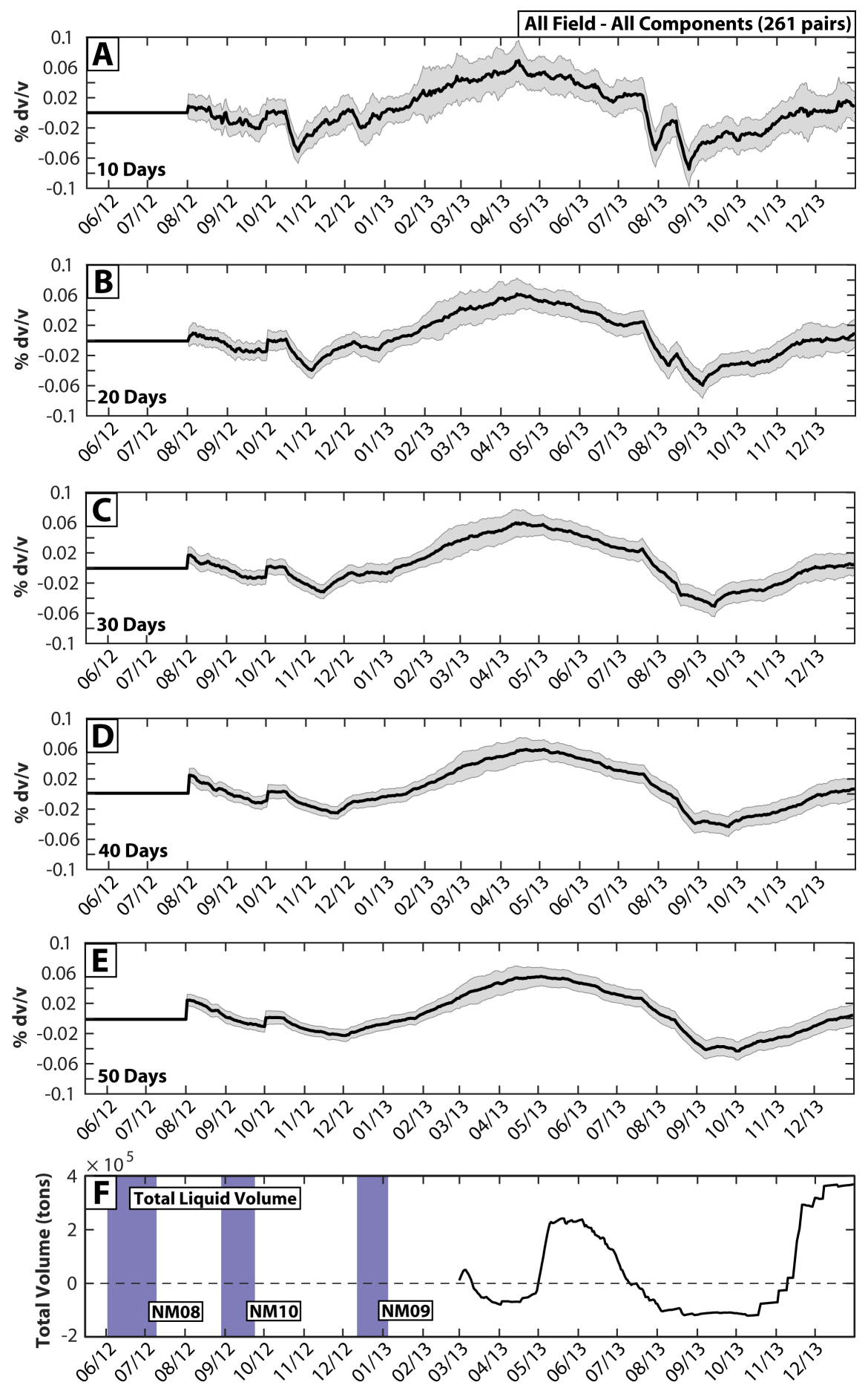

Figure 5.50: Velocity changes using 10-50 day MWCS windows [A-E] and Ngatamariki flow $[\mathrm{F}]$ and relative total liquid volume [G] using all components of the entire field. 

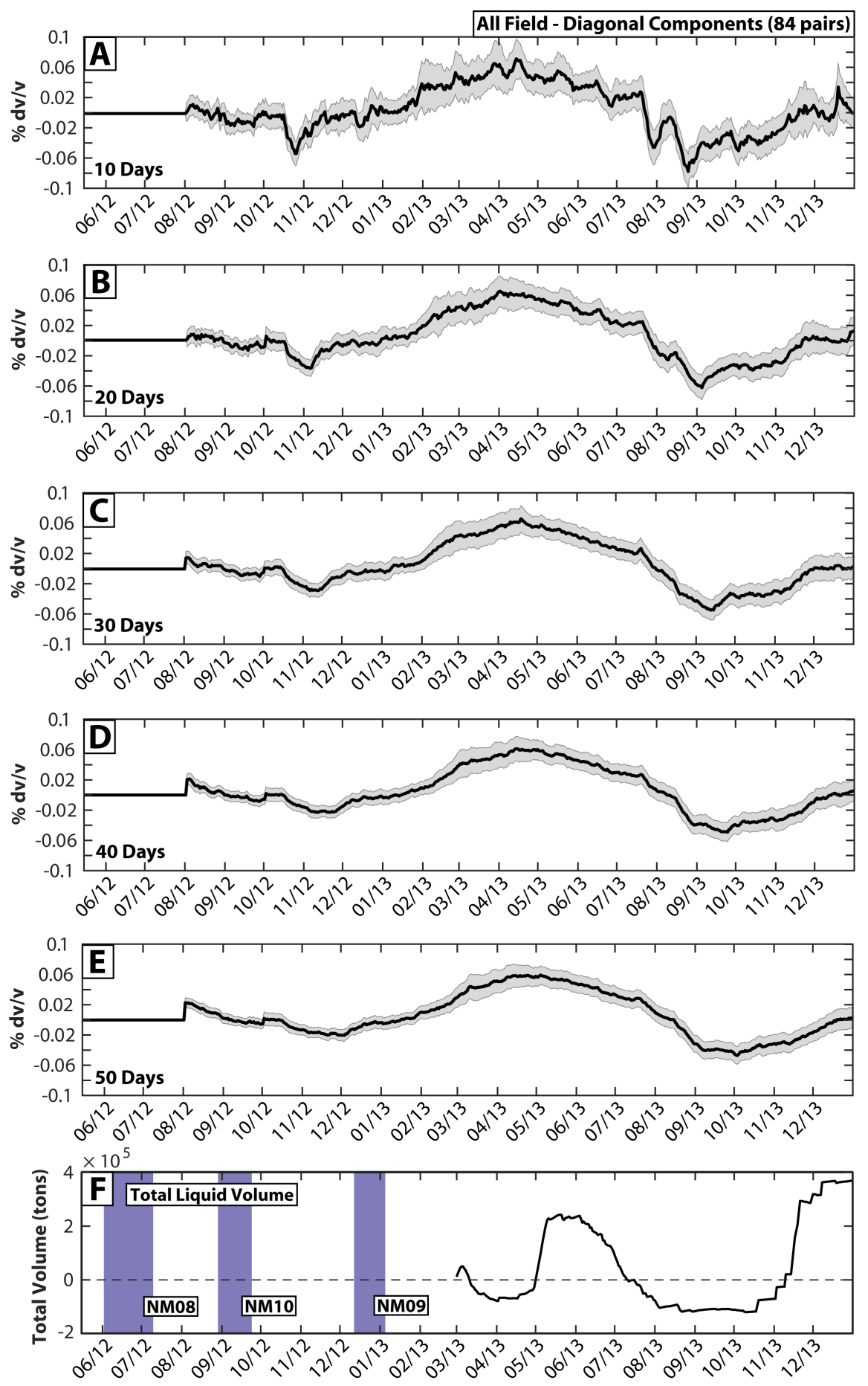

Figure 5.51: Velocity changes using 10-50 day MWCS windows [A-E] and Ngatamariki flow $[\mathrm{F}]$ and relative total liquid volume [G] using the diagonal components (RR, TT, and ZZ) of the entire field. 


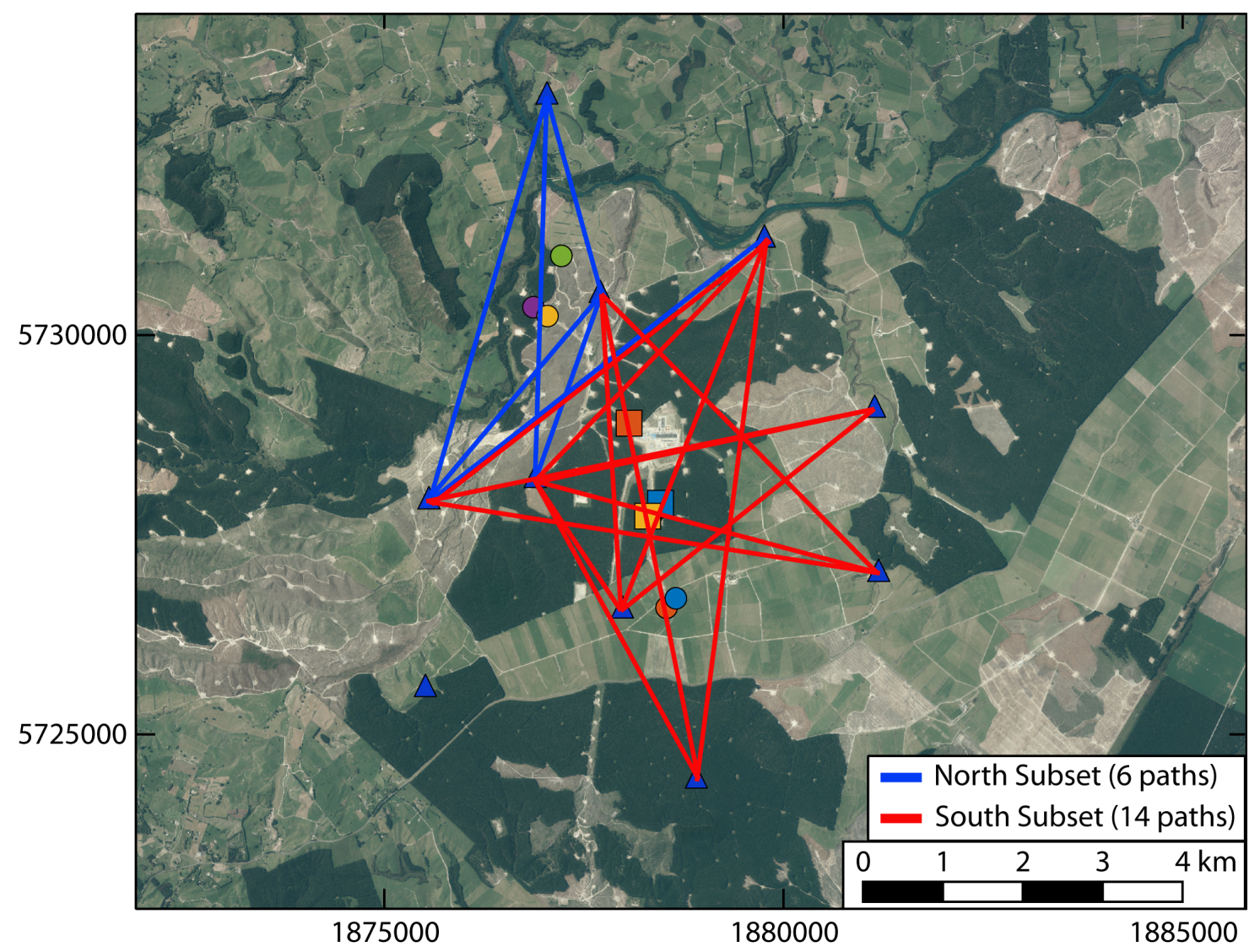

Figure 5.52: Ngatamariki north (blue) and south (red) station-pair subsets. 

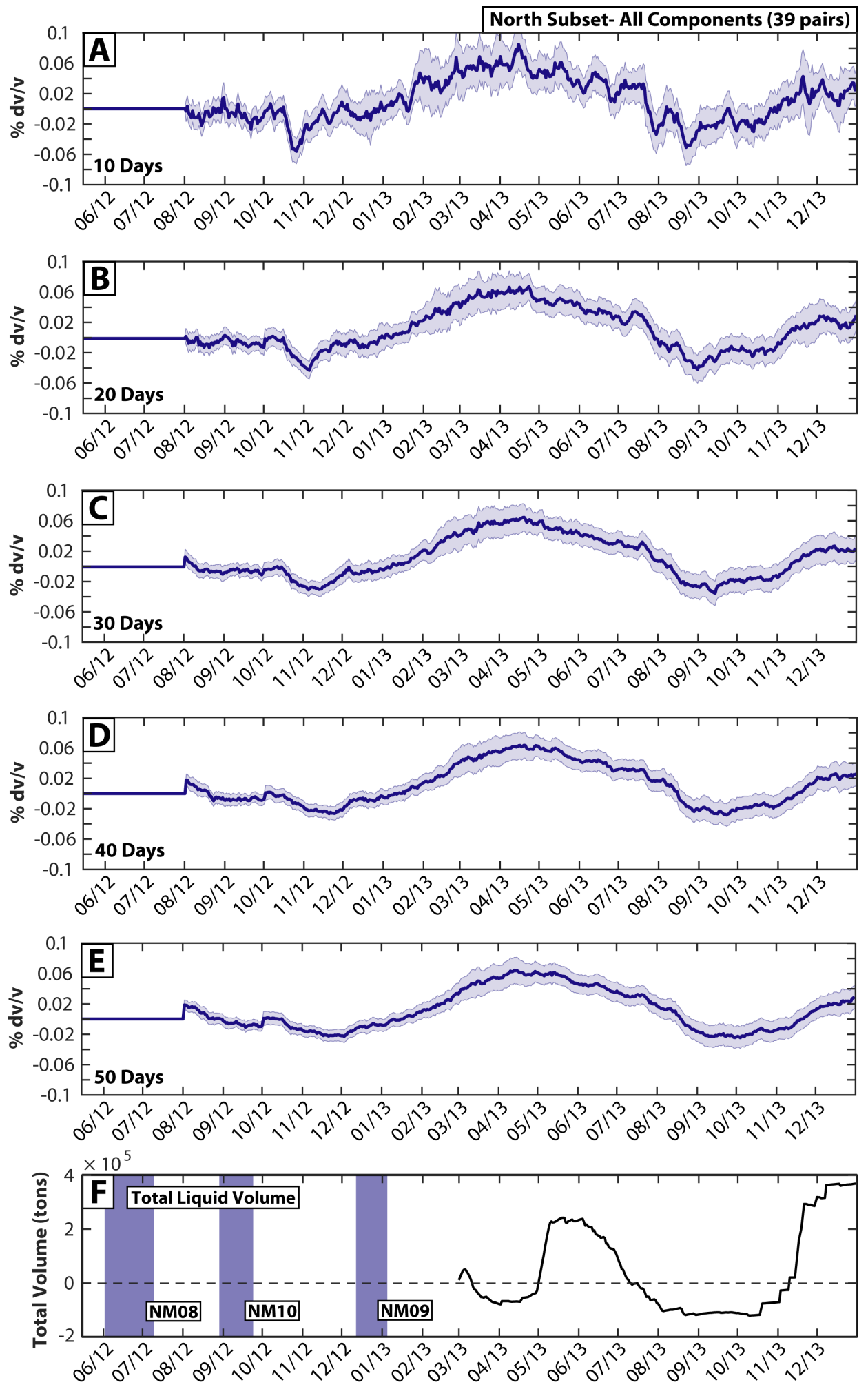

Figure 5.53: Velocity changes using 10-50 day MWCS windows [A-E] and Ngatamariki flow [F] and relative total liquid volume [G] using all components of the north subset. 

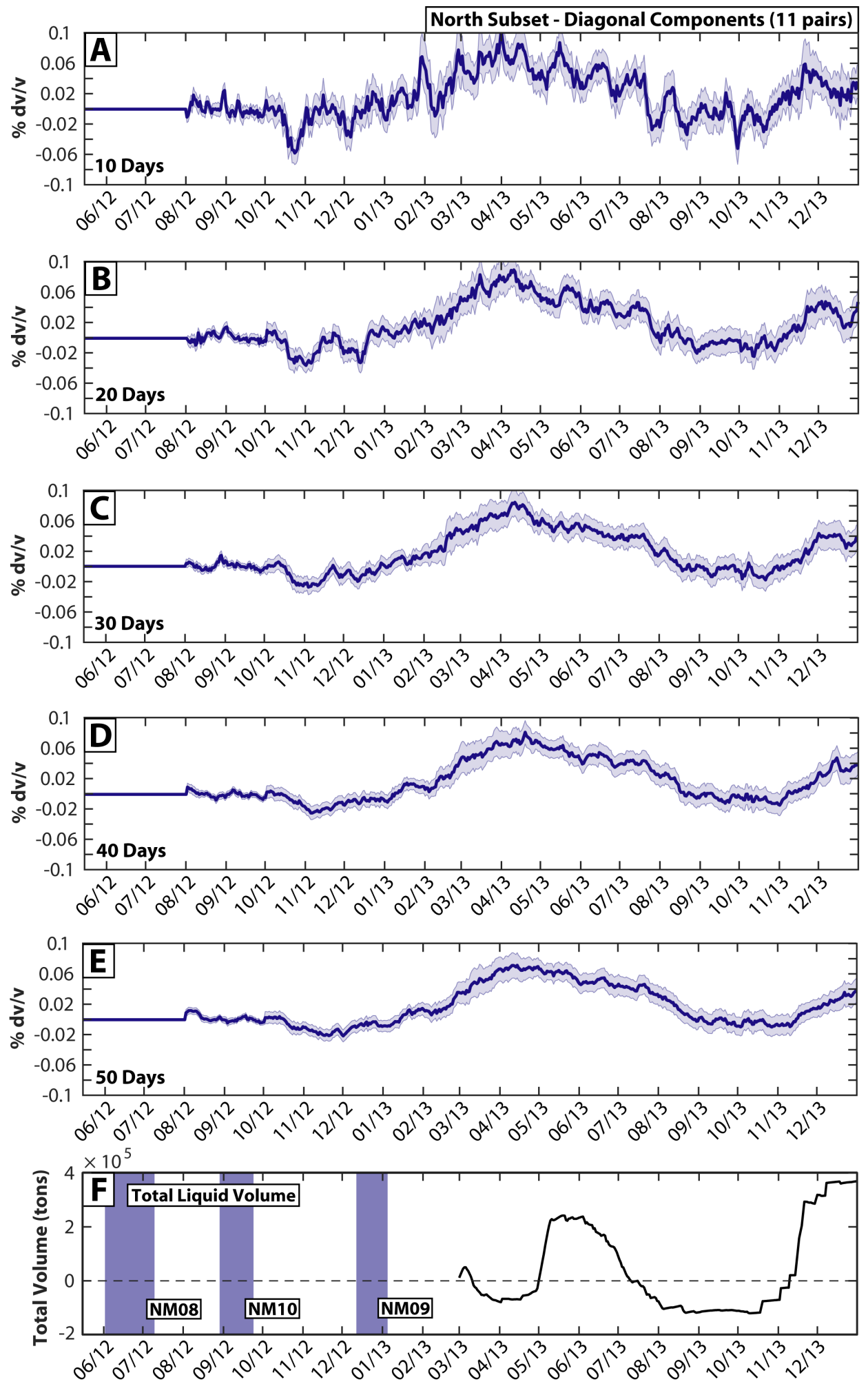

Figure 5.54: Velocity changes using 10-50 day MWCS windows [A-E] and Ngatamariki flow $[\mathrm{F}]$ and relative total liquid volume [G] using the diagonal components (RR, TT, and ZZ) of the north subset. 

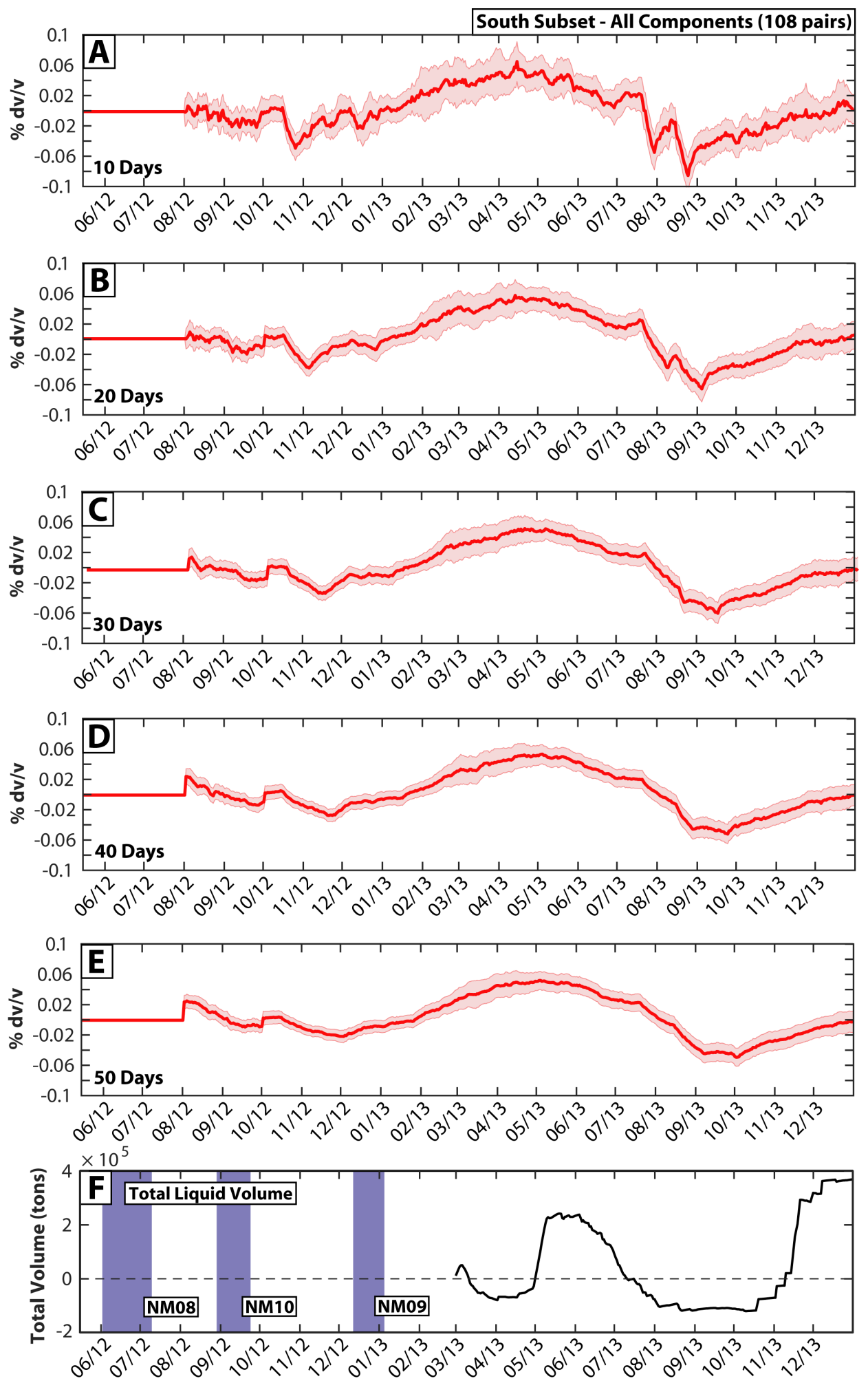

Figure 5.55: Velocity changes using 10-50 day MWCS windows [A-E] and Ngatamariki flow [F] and relative total liquid volume [G] using all components of the south subset. 

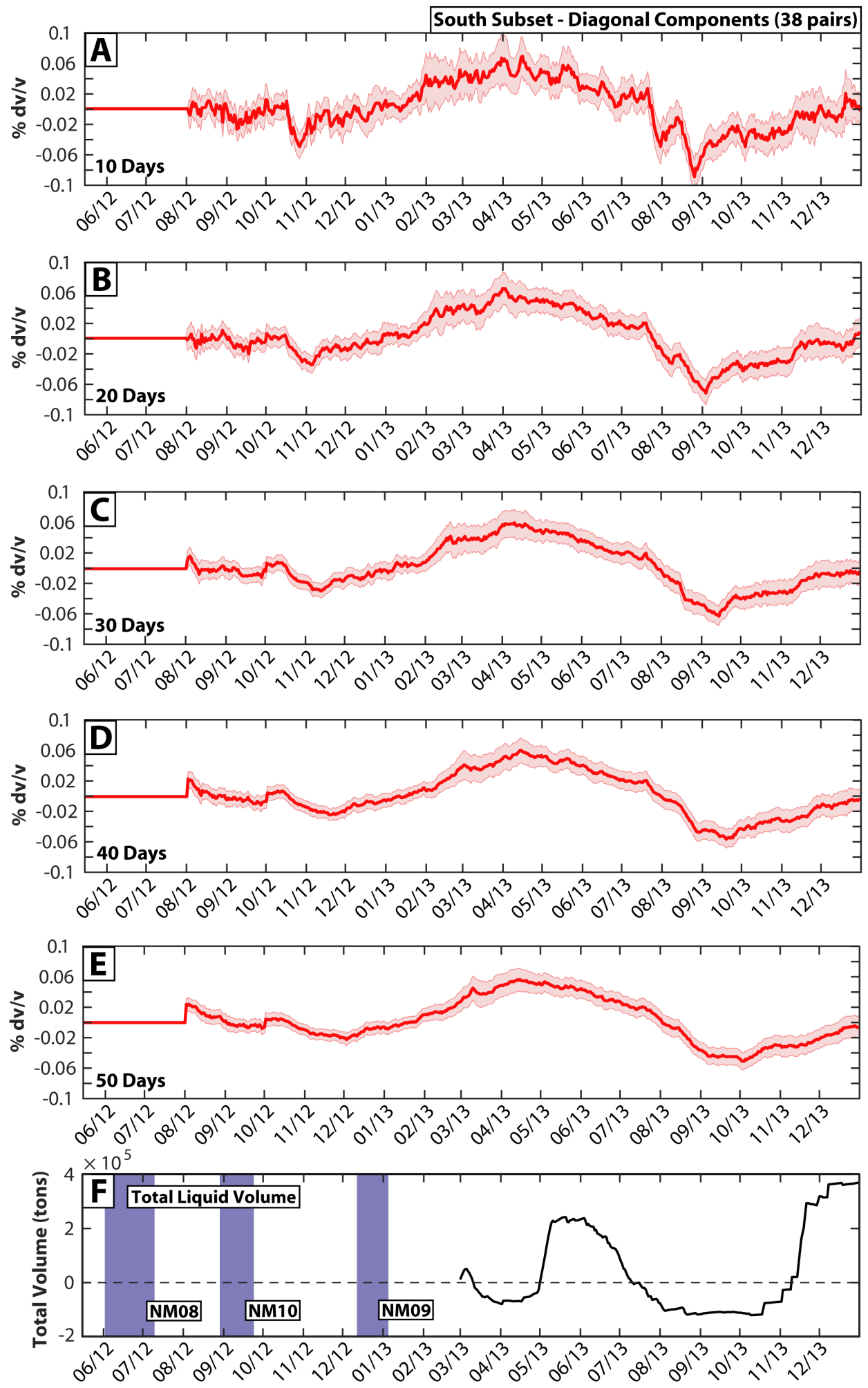

Figure 5.56: Velocity changes using 10-50 day MWCS windows [A-E] and Ngatamariki flow $[\mathrm{F}]$ and relative total liquid volume [G] using the diagonal components (RR, TT, and ZZ) of the south subset. 
We found both a positive and negative full-field change in velocity for all groupings that correlated to changes in the injection and production rates and the total liquid volume. These measurements are tabulated in Tables 5.11 and 5.12. The maximum and minimum positive velocity changes relative to the mean value of the reference stack were $0.105 \%$ (north subset diagonal components 10-day [Figure 5.54A]) and 0.055\% (south subset all components 50-day [Figure 5.55E]) respectively. The maximum negative velocity change was $-0.086 \%$ for the south subset diagonal components 10 -day [Figure 5.56A] and the minimum negative velocity change was $-0.007 \%$ for the north subset diagonal components 50-day [Figure 5.54E]. The mean positive change across all subsets and day-windows was $0.07 \%$ and the mean negative change was $-0.046 \%$.

Additionally, two pulses of increased velocity were detected at the beginning of August and October 2012 on most groupings that appear to follow the stimulation of wells NM08 and NM10. The first velocity increase has a maximum of $0.028 \%$ (south subset all components 50-days [Figure 5.55E]) and has a mean of $0.019 \%$ across all components. The maximum of the second velocity increase is $0.02 \%$ (north subset diagonal components 10-days [Figure 5.54A]) and a mean value of $0.009 \%$ across all components.

\begin{tabular}{|c|c|c|c|c|c|}
\hline Grouping & 10 Days & 20 Days & 30 Days & 40 Days & 50 Days \\
\hline All field all components & $0.074 \%$ & $0.067 \%$ & $0.064 \%$ & $0.062 \%$ & $0.059 \%$ \\
\hline All field diagonal components & $0.076 \%$ & $0.068 \%$ & $0.069 \%$ & $0.064 \%$ & $0.062 \%$ \\
\hline North subset all components & $0.089 \%$ & $0.073 \%$ & $0.07 \%$ & $0.068 \%$ & $0.068 \%$ \\
\hline $\begin{array}{l}\text { North subset diagonal compo- } \\
\text { nents }\end{array}$ & $0.105 \%$ & $0.092 \%$ & $0.087 \%$ & $0.085 \%$ & $0.074 \%$ \\
\hline South subset all components & $0.071 \%$ & $0.062 \%$ & $0.059 \%$ & $0.058 \%$ & $0.055 \%$ \\
\hline $\begin{array}{l}\text { South subset diagonal compo- } \\
\text { nents }\end{array}$ & $0.072 \%$ & $0.07 \%$ & $0.064 \%$ & $0.064 \%$ & $0.059 \%$ \\
\hline
\end{tabular}

Table 5.11: The maximum positive velocity change measurement for each grouping at Ngatamariki for each MWCS window size. 


\begin{tabular}{||l||l||l||l||l||l||}
\hline Grouping & 10 Days & 20 Days & 30 Days & 40 Days & 50 Days \\
\hline All field all components & $-0.07 \%$ & $-0.054 \%$ & $-0.046 \%$ & $-0.041 \%$ & $-0.04 \%$ \\
\hline All field diagonal components & $-0.073 \%$ & $-0.059 \%$ & $-0.051 \%$ & $-0.046 \%$ & $-0.045 \%$ \\
\hline North subset all components & $-0.047 \%$ & $-0.036 \%$ & $-0.03 \%$ & $-0.024 \%$ & $-0.021 \%$ \\
\hline $\begin{array}{l}\text { North subset diagonal compo- } \\
\text { nents }\end{array}$ & $-0.051 \%$ & $-0.022 \%$ & $-0.015 \%$ & $-0.011 \%$ & $-0.007 \%$ \\
\hline South subset all components & $-0.079 \%$ & $-0.062 \%$ & $-0.053 \%$ & $-0.048 \%$ & $-0.046 \%$ \\
\hline $\begin{array}{l}\text { South subset diagonal compo- } \\
\text { nents }\end{array}$ & $-0.086 \%$ & $-0.068 \%$ & $-0.059 \%$ & $-0.053 \%$ & $-0.049 \%$ \\
\hline \hline
\end{tabular}

Table 5.12: The maximum negative velocity change measurement for each grouping at Ngatamariki for each MWCS window size.

\subsection{Discussion}

\subsubsection{Cross-correlation stability, MWCS, and velocity changes}

Velocity changes were computed from ambient seismic noise using the Moving Window Cross-Spectral (MWCS) technique for two geothermal fields at different stages of production: a three-fold increase of production and injection at Rotokawa due to the start-up of a new plant after almost two decades of consistent production, and the beginning of injection and production at Ngatamariki. For both of these datasets, we found velocity changes exhibited responses that were qualitatively correlated with flow rate, i.e. velocity increases with increased fluid amount and decreases when it is removed.

Velocity changes detected via ambient noise are generally between $0.01 \%$ to $1 \%$ [Hillers et al., 2015], so great care must be taken in pre-processing, station outage mitigation, and station-pair selection, as just a few incorrect delay-times can result in misleading velocity change signals [Figures 5.17 and 5.30]. Due to high-frequency noise present at both sites, computed-cross-correlations needed to be whitened prior to onebit normalization to prevent the cultural noise from masking the natural ambient noise signal [Section 2.3]. We found that the cross-components of the Green's function tensors had spurious signals within the data for certain pairs and did not contain clear surface wave move out as predictably as their diagonal-component counterparts [Figures 5.21-5.23, 5.46-5.46]. However, most cross-component pairs were stable in both time and frequency domain, although the quality of that stability was sometimes inferior to the diagonal components, especially the RR and TT.

The cross-correlation image and coherence stability checks were the most important tools for determining the reference stacks in both geothermal fields and the MWCS parameters in Table 5.2. We found that the stability for station-pairs was not consistent 
across all components [Figures 5.25-5.27], and that the quality of the ZZ component was generally inferior to the RR and TT components [Appendix B.1 and B.2]. This is expected, because the vertical component of the noise field for both Rotokawa and Ngatamariki generally had decreased noise amplitude compared to the horizontals [Appendix B.4].

Time and frequency domain stability tools helped reveal instrument orientation errors at Rotokawa [Section 5.5.3] and a dead channel at Ngatamariki [Section 5.7.2]. The instrument changes in Rotokawa occurred half-way through the reference stack and most of the pairs with those stations were stable.

It is important to note that both of these stability checks were computed prior to the MWCS calculation in MSNoise through quick-running scripts. Computing velocity changes is an extremely time-consuming process even on a computer cluster, and the trial-and-error associated with determining the right parameters was greatly diminished by focusing on time and frequency domain stability of the cross-correlations via visual inspection prior to that computation. This was especially true in regards to observing the effect of various reference stacks on the coherence.

We had nine months prior to the onset of production that could be used as a reference stack at Rotokawa. We used two different reference stacks: one from the start of the data through until production commenced ( 9 months) and another much shorter closer to the start of production but after the instrument orientation change ( 3 months) [Figure 5.16]. Although they showed similar results, the second reference stack was significantly less stable [Appendix B.3], which we attribute to the smaller amount of data. Additionally, it is important to note that the first reference stack included both winter and summer seasons, while the second only included winter, which could add additional variations to the velocity changes.

Significant care had to be taken at Rotokawa to minimize the effect of missing data, as we observed that apparent velocity changes increased or dropped dramatically before and after gaps [Figure 5.17]. MSNoise does not accurately remove these oscillations, so a code was written to remove these spurious signals. Although Ngatamariki had virtually no gaps in data after an initial swap at the end of August 2012, we did not have a long time period to use as a reference stack that was free from production and injection [Figure 5.42]. Due to the increased variability observed in Rotokawa using the second reference stack [Appendix B.3], we chose to use April 2012 through January 2013 as the reference stack for Ngatmariki, although this time period is also when wells NM08, NM10, and NM09 were stimulated. As the injection rates during stimulation were approximately an order of magnitude smaller than those during the beginning of electricity production [Table 5.8], we hypothesized that we would still be able to observe velocity changes despite including the stimulation time period in the reference stack. 
Velocity changes were then computed for both Rotokawa and Ngatamariki using all station pairs and spatial subsets for all Green's function components and diagonal components. The ratio of injection-production rates and the total volume of water were computed for both of the geothermal fields [Figures 5.20 and 5.45]. The pairs of the spatial subsets were selected based on whether or not they crossed the areas of production or injection. We found that across both fields, the velocity change was generally consistent between all groupings and was most similar to the total volume of water injected into the aquifer. At Rotokawa, a positive velocity change was detected after April 2010 and was followed by a steady decrease in velocities. These changes were greater in the injection subsets than in the entire field or in the production subset [Tables 5.6 and 5.7], suggesting that the velocity change is dominated by the deformation associated with the injection processes. 


\subsubsection{Time-dependent spatial relationships}

The velocity change results obtained in Sections 5.5.4 and 5.7.3 showed larger positive velocity changes in the injection subsets than in the overall field or in the production subsets [Tables 5.6 and 5.11]. Additionally, we noticed that the velocity trends could be observed on individual station-pair velocity changes and not just their averages (for example, Figure 5.38). This is probably a result of a very consistent natural ambient noise signal. Due to the stability of each station-pair velocity change measurement, we decided further explore the spatial relationships at the geothermal fields in time. Our objective was to try to find additional information regarding the velocity changes beyond the full-field responses computed by the subgroup averages.

We wrote a code to calculate straight-ray pseudo-tomography using the velocity change values for each station pair. Both of the geothermal fields were discretized into a grid, and each station-path assigned its velocity change value to each crossed grid cell. Due to the complexity of determining attenuation for a signal produced by scattering, we approximated that the velocity change value is constant across the ray and that grid cells with overlapping rays average the velocity-changes. Due to their differences in station paths and inter-station distances, 0.0025 and 0.005 decimal degree grid-spacing was chosen for Rotokawa and Ngatamariki, respectively. The pseudotomography was run for each day of velocity change for each component and for diagonal and all-component averages. Each figure of the resulting tomography was then combined using the Linux routine ffmpeg. Due to the large number of repeated operations, the code is parallelized.

We will now discuss an example from Rotokawa and Ngatamariki. Specifically, we use the 30-day MWCS window and the diagonal components average. We found these intervals to be optimal for path smoothing while still retaining features such as well stimulation, which occurred on the scale of a few weeks.

Several spatial relationships, or "localized" responses, can be observed at Rotokawa based on injection and production rates. First, high velocity change values can be observed near the north injection wells for most of the 2009 [Figure 5.57A]. During this time period, only injection well RK20 was operational [Figures 5.14 and 5.18]. As stimulation of well RK22 begins, high velocities can be observed near the south injection wells [Figures 5.18 and 5.57B]. Note that this particular example uses the 30 day MWCS window, which means that the values observed at a specific day contain contributions from the previous twenty-nine days. After the onset of increased injection and production rates, higher velocities are observed across the entire field [Figure 5.57C], which coincided with the all-field average observations of Figure 5.32. After several months of higher production than injection, velocity change values decrease over the entire field, although higher values relative to the average can be observed near the northern 
injection wells [Figure 5.57D].

Three latitude-longitude points corresponding to locations near injection wells, production wells, and midfield were chosen at Rotokawa and observed as time series [Figures 5.57A and 5.58]. As may be expected, the latitude-longitude point time series display similar responses observed in the allfield, injection, and production averages [Figures 5.31-5.37]. 

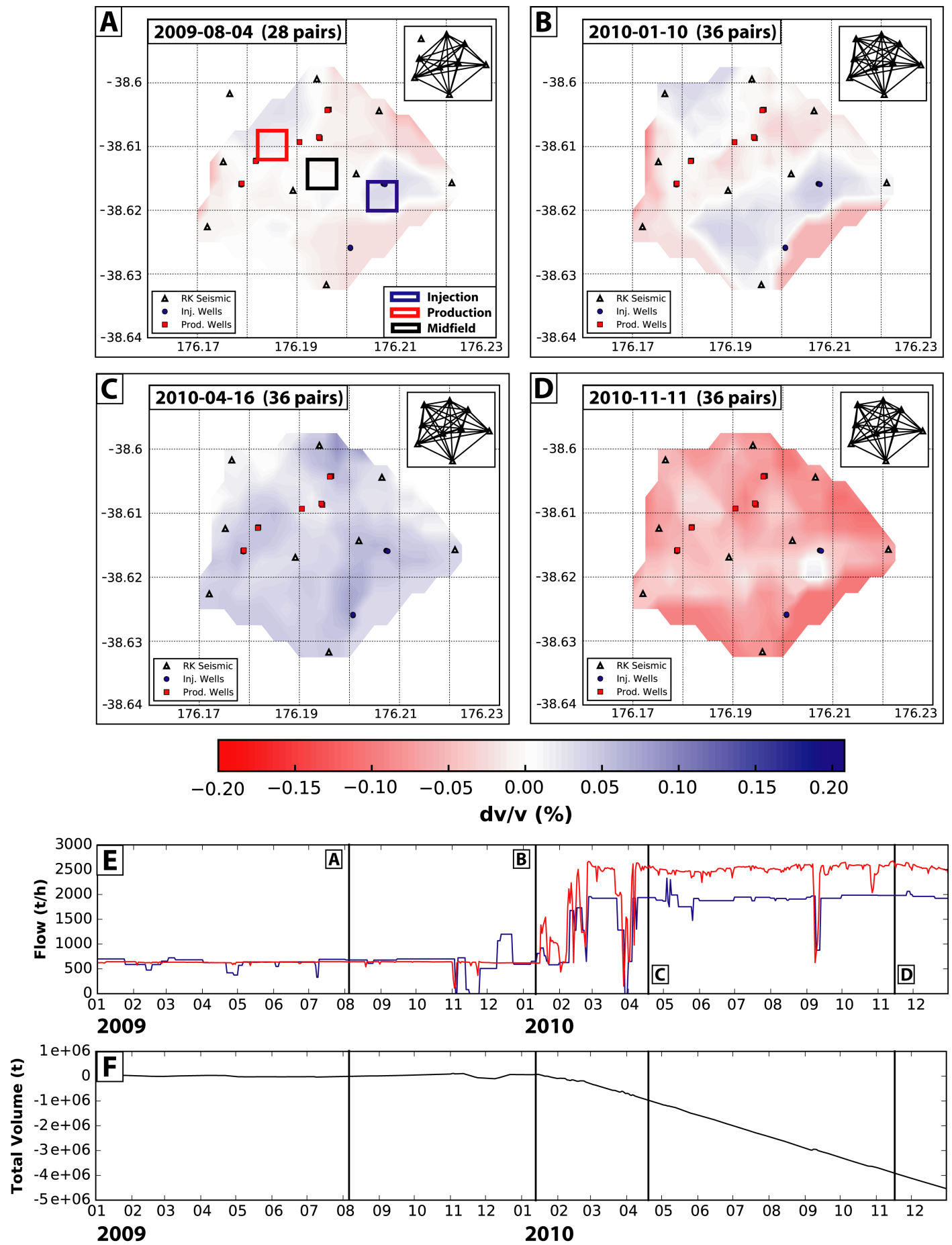

Figure 5.57: Shear-velocity changes across Rotokawa for 2009-08-04 [A], 2010-01-10 [B], 2010-04-16 [C], and 2010-11-11 [D]. The seismic stations, injection wells, and production wells are displayed as white, blue, and red symbols. The blue, red, and black squares show the areas used for the injection, production, and midfield measurements of Figure 5.58. The station-paths used for that day are displayed as an inset on the top-right of the frame. [E] Total injection (blue) and production (red) flow at Rotokawa during the time periods used in A-D. [F] Total liquid volume at Rotokawa during the time periods used in A-D. 

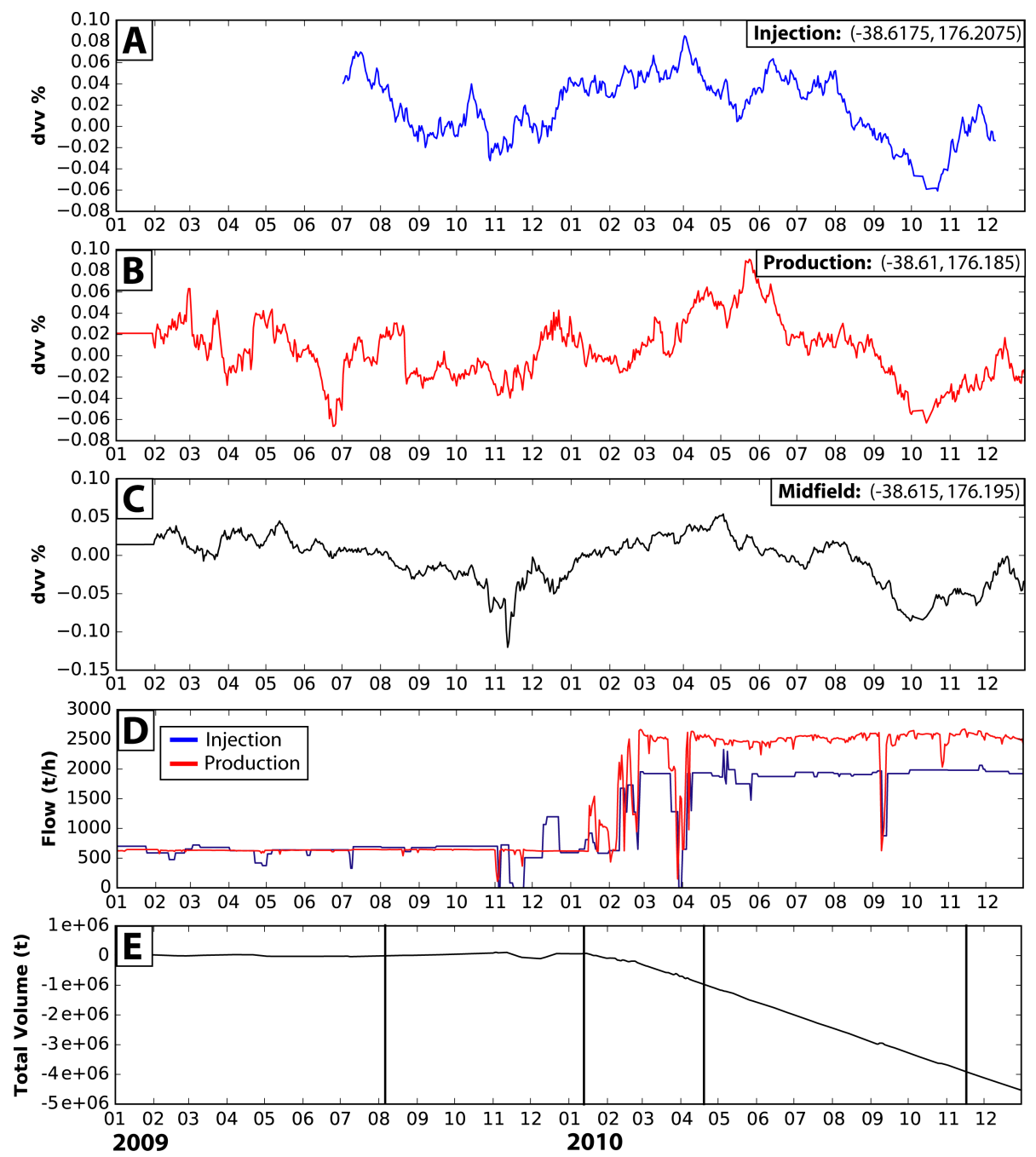

Figure 5.58: Velocity changes over 2009-2010 Rotokawa for injection point ($38.6175,176.2075)[\mathrm{A}]$, production point $(-38.61,176.185)[\mathrm{B}]$, and midfield point ($38.615,176.195)$. [D] Total injection (blue) and production (red) flow at Rotokawa. [E] Total liquid volume at Rotokawa. 
Similar localized responses between the injection wells and velocity were found at Ngatamariki, although the geometry of the injection and production wells is different from Rotokawa. The rate of injection and production are mostly equal [Figure 5.59E], but brief time periods of production stoppage produce large negative and positive total volume values [Figure 5.59F]. Unfortunately, precise stimulation data for injection wells NM08, NM10, and NM09 is not available at time of this writing, although average rates are known from literature [Clearwater et al., 2015] [Table 5.8]. Additionally, the large positive value of injection at the first data point on March 1st, 2013, suggests that the injection had already started. Due to these factors, it's not surprising that high values of velocity changes are observed around the injection wells prior to March 1st [Figure 5.59A]. As production increases relative to production, high values of positive velocity changes are observed across the field, whose timing coincides with a peak in the total volume [Figure 5.59B, F]. This similar phenomenon is also observed when the the total volume becomes negative [Figure 5.59C, F] and when it increases to positive values after mid-October 2013 [Figure 5.59D, F]. Additionally, we observe high velocity measurements in the field near the injection wells and low velocity measurements near the production wells during this last time period [Figure 5.59D]. This feature is observed mostly due to the station geometry in relation to the injection and production well locations.

Similar to Rotokawa, latitude-longitude points were chosen at Ngatmariki near the injection and production wells and in the midfield [Figures 5.59A and 5.60]. Similar responses can be observed throughout the various points, although both the production and injection south points appear to increase slightly during the NM10 stimulation process. As previously observed in Figure 5.59D, velocity changes near the injection wells have a positive increase starting in November 2013 which coincides with increasing values of total volume [Figure 5.60F]. 

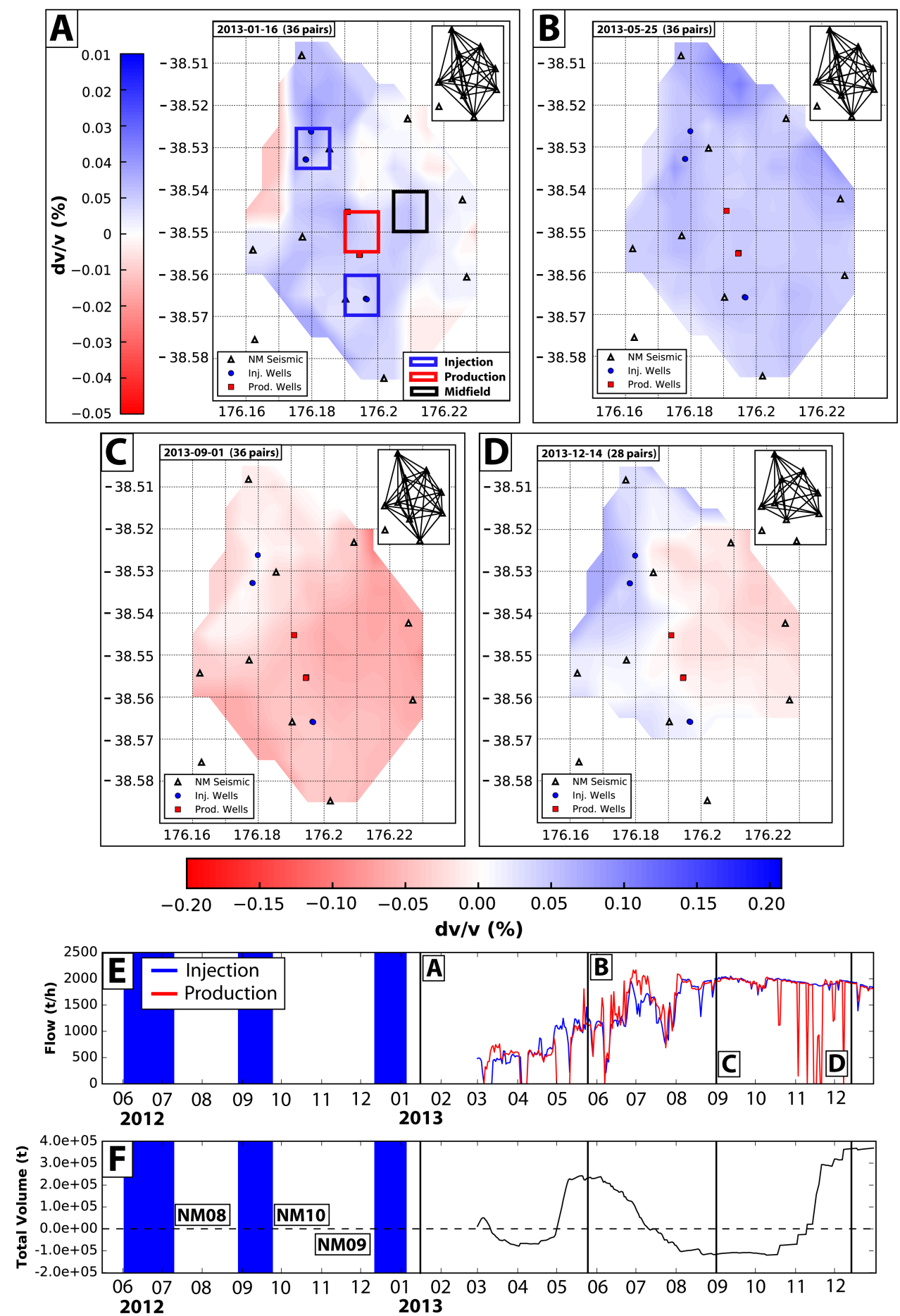

Figure 5.59: Shear-velocity changes across Ngatamariki for 2013-01-16 [A] (Note: This subplot has a darker scale to show contrast on the printed version of this manuscript), 2013-05-25 [B], 2013-09-01 [C], and 2013-12-14 [D]. The seismic stations, injection wells, and production wells are displayed as white, blue, and red symbols. The blue, red, and black squares show the areas used for the injection, production, and midfield measurements of Figure 5.60. The station-paths used for that day are displayed as an inset on the top-right of the frame. [E] Total injection (blue) and production (red) flow at Ngatamariki during the time periods used in A-D. [F] Total liquid volume at Ngatamariki during the time periods used in A-D. 

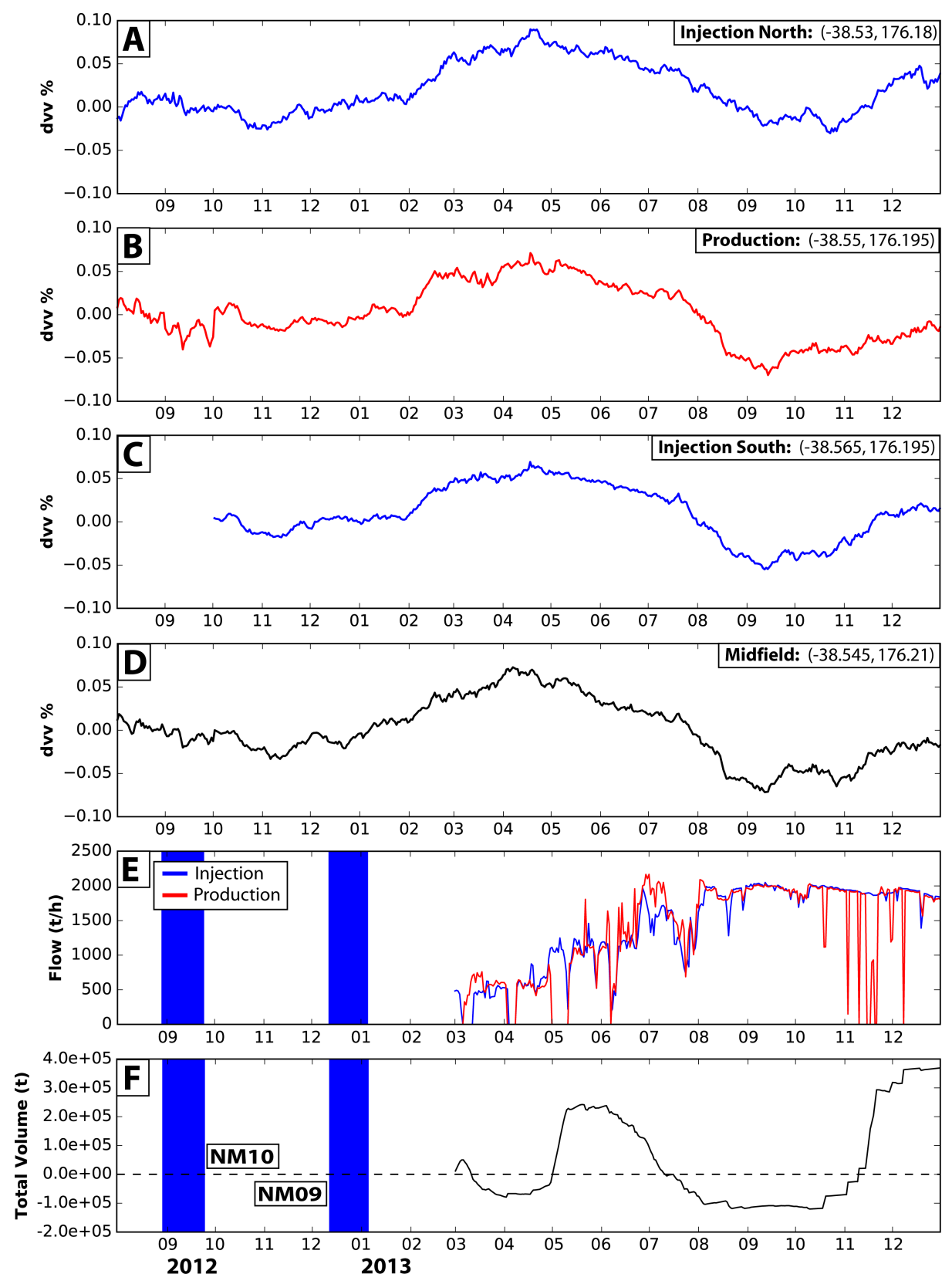

Figure 5.60: Velocity changes over 2012-2013 Ngatamariki for northern injection point $(-38.53,176.18)[A]$, production point $(-38.55,176.195)[B]$, southern injection point ($38.565,176.195)$, and midfield point $(-38.545,176.21)$ [D]. Total injection (blue) and production (red) flow at Ngatamariki. [E] Total liquid volume at Ngatamariki. 


\subsubsection{Mechanism for velocity changes}

A careful analysis of the data, methods, geology, and geothermal production processes is required to adequately determine the mechanisms driving the observed velocity changes. Here, we expand on the examples provided in the theory section [Section 5.2] and compare our results with those obtained in literature. An important detail to remember when analyzing computed velocity changes is that the results display the most dominant mechanism [Wegler et al., 2009], but can also include the contribution of other processes.

A common feature frequently observed in year-long studies of ambient noise is seasonal variability due to changes in groundwater level due to rainfall, which can have variations on the order of $0.1 \%$ [Sens-Schönfelder and Wegler, 2006, Meier et al., 2010]. Higher rainfall during winter increases the groundwater level, which tends to decrease the shear velocity through the saturation of fractures [Sens-Schönfelder and Wegler, 2006, Hillers et al., 2014]. Wegler et al. [2009] addressed the possibility that rainfall could affect the drop of velocities observed after the $M_{w} 6.6$ mid-Niigata earthquake, but used multiple frequency bands to demonstrate the velocity change was not linked to the shallow surface.

The groundwater level $\left(G W L\left(t_{i}\right)\right)$ at a certain time $t_{i}$ can be approximated through a convolution of the precipitation rates with a drainage exponential function [Akasaka and Nakanishi, 2000, Sens-Schönfelder and Wegler, 2006]:

$$
G W L\left(t_{i}\right)=G W L_{0}-\sum_{n=0}^{i} \frac{p\left(t_{n}\right)}{\phi} e^{-a\left(t_{i}-t_{n}\right)}
$$

where $\phi$ is porosity, $a$ is a drainage decay parameter, and $p\left(t_{n}\right)$ is the is the precipitation for day $t_{n}$. We know that the groundwater level at Rotokawa and Ngatamariki is approximately 30 meters [Sewell, 2017, personal comm.] and that the porosity of the Greywacke basement has been determined by laboratory tests as 0.05 [McNamara et al., 2014]. The decay parameter $a$ is frequently approximated to 0.008 [Akasaka and Nakanishi, 2000, Sens-Schönfelder and Wegler, 2006]. We obtained rainfall data $\left(p\left(t_{n}\right)\right)$ during our time of study from the nearby Taupo Aws weather station using NIWA's National Climate Database system and compared it to the velocity changes recorded at Rotokawa and Ngatamariki [Figures 5.61 and 5.62]. 

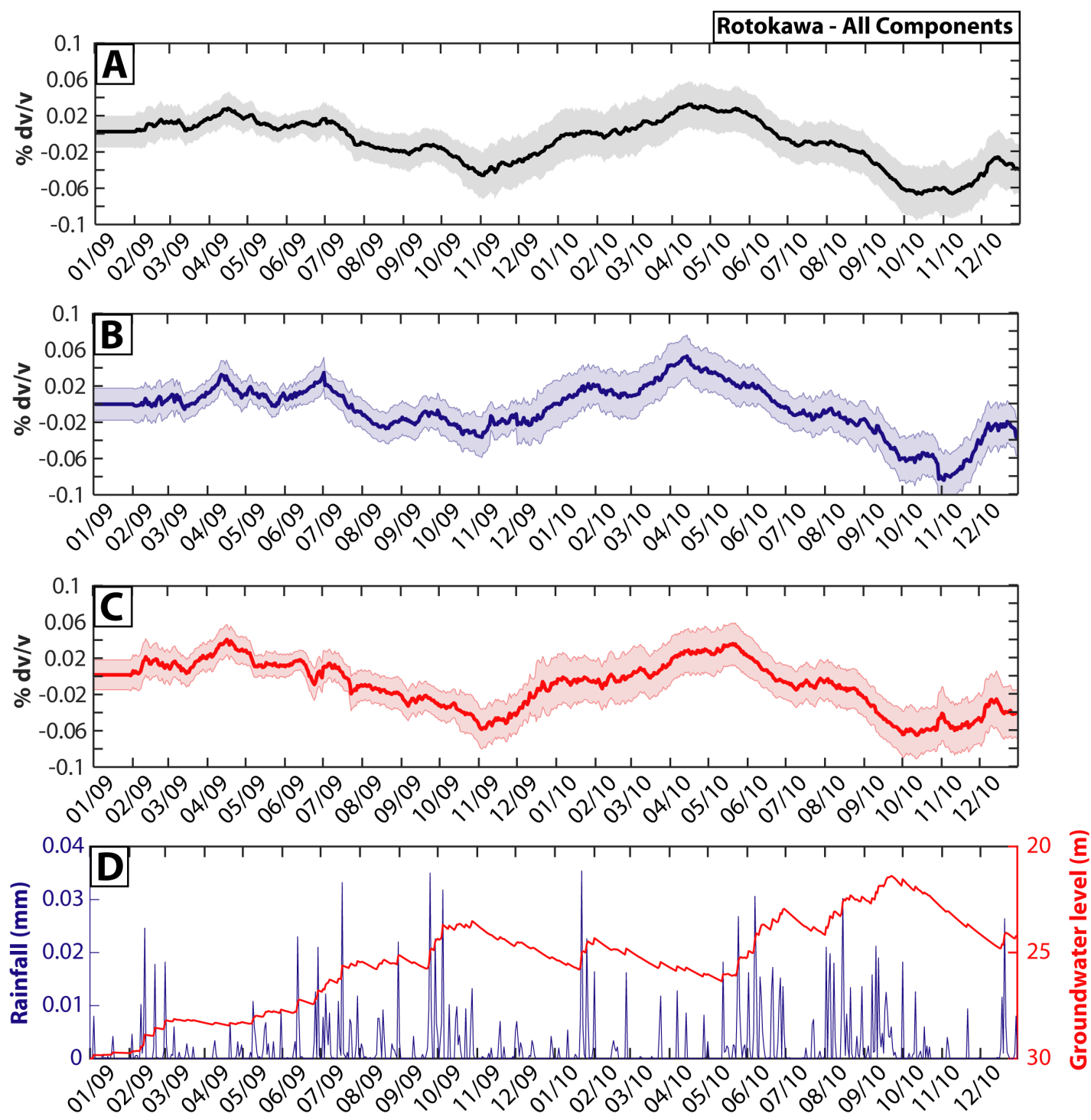

Figure 5.61: Rainfall comparison for Rotokawa velocity changes. Velocity changes for a 30-day MWCS window for [A] all stations, [B] the injection subset, and [C] the production subset. [D] Daily rainfall recorded at weather station Taupo Aws [blue] and predicted water table depth [red]. 

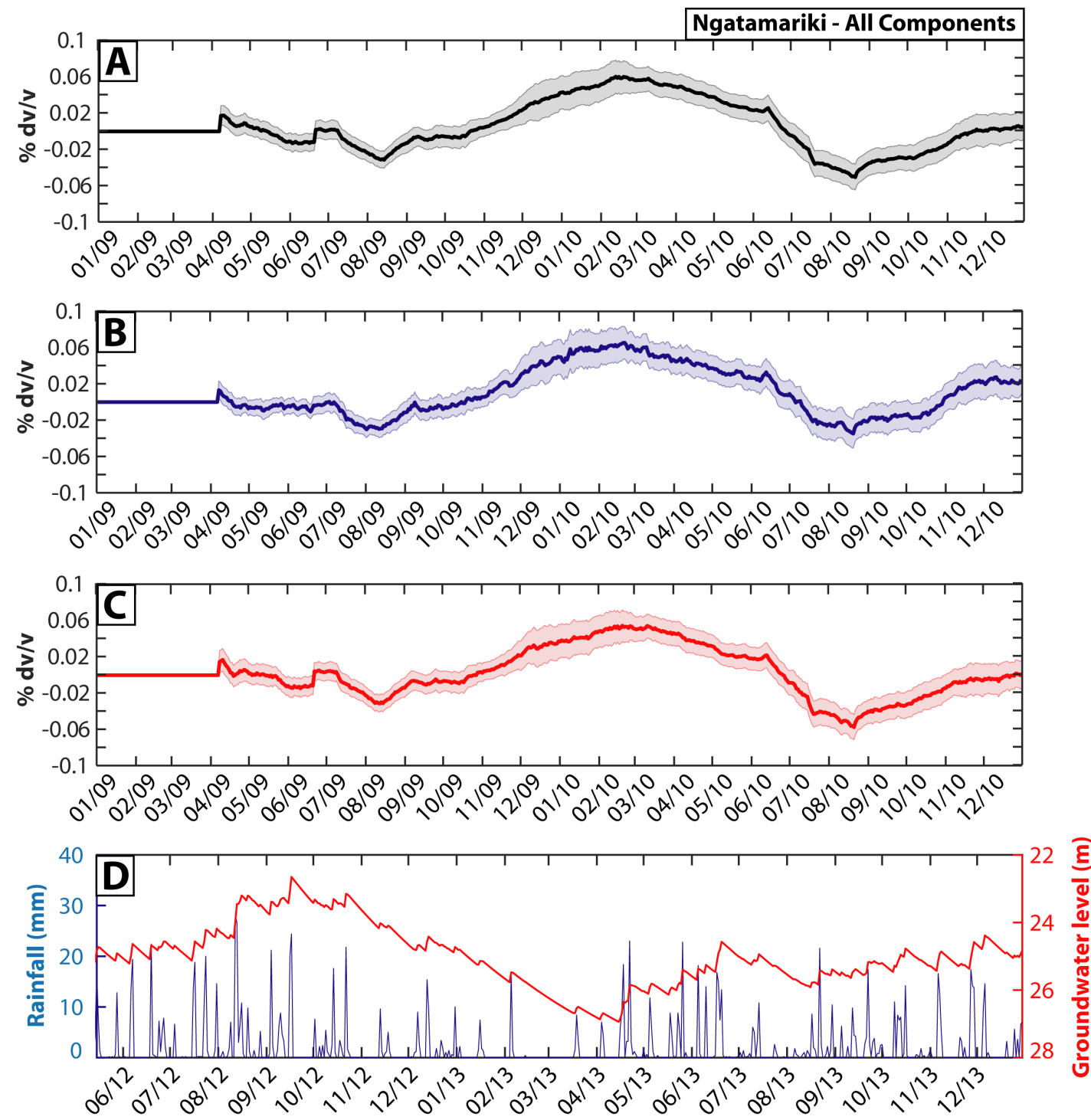

Figure 5.62: Rainfall comparison for Ngatamariki velocity changes. Velocity changes for a 30-day MWCS window for [A] all stations, [B] the north subset, and [C] the south subset. [D] Daily rainfall recorded at weather station Taupo Aws [blue] and predicted water table depth [red]. 
There is a visual correlation between the height of the groundwater level and the velocity change results, specifically at June 2010 (6/10) and October 2010 (10/10) Rotokawa and May 2013 (5/13) Ngatamariki. This suggests that some of the full-field velocity could be in part be due to the groundwater level.

Thermal variations could also potentially explain some of the observed positive velocity changes, especially those observed near the injection wells [Figures 5.57A, B and 5.59A, D], as rocks cool and fracture when interacting with the cold water from the injection [Boden, 2016]. However, the difference between the shear-velocity change from temperature changes as opposed to the change that would be induced by fracturing at a geothermal setting is difficult to quantify. Studies on hydrocarbon rocks have found that $V_{s}$ decreases with increasing temperatures by as much as $6-10 \%$ for a $100 \mathrm{C}^{\circ}$ increase in certain sandstone, although one of the proposed mechanisms, a decrease of the rigidity and bulk modulus due to a reduction viscosity of oil and interfacial force with a rise in temperature, wouldn't apply for our geothermal system [Wang, 1988, Wang and Nur, 1990]. Additionally, it is unclear how a temperature mechanism could explain the large drops in velocity observed at both fields during the startup of production [Figures 5.57D and 5.59C], or the changes between low and high velocities across Ngatamariki when injection remains consistent [Figure 5.59C, D].

The most commonly quantified mechanism for ambient noise velocity changes is a structural change. Initially, variations in the cross-correlation coda were linked to the scattering caused by small-scale inhomogeneities in the shallow structure of Mount St. Helens during a 2004 eruptive period [Sabra et al., 2006]. These observations were quantified as velocity changes for the Piton de la Fournaise volcano on Rèunion island, where a $0.05-0.1 \%$ decrease in velocity prior to an eruption was linked to the dilatation of the volcanic edifice due to magma pressurization [Brenguier et al., 2008b]. Brenguier et al. [2008b] used empirical laws and typical medium constants to quantify the expected pressure and porosity changes resulting from the calculated shear-velocity change, and the results were consistent with models of dike migrations [Pride, 2005]. Obermann et al. [2013] also recorded pre-eruption velocity decreases at Piton de la Fournaise and attributed them to an increase in magma volume.

Negative velocity changes have also been observed following earthquakes, and have been linked to a decrease in crustal stress, non-linear site response due to shaking, and structural weakening due to the creation of new fractures [Wegler and SensSchöndelfer, 2007, Brenguier et al., 2008a, Wegler et al., 2009]. Positive velocity changes were detected during the 2006 M7.5 slow-slip event in Mexico, and were found to related more to the strain rate rather than the strain itself, suggesting that the deformation of the overlying crust has a nonlinear elastic behavior [Rivet et al., 2011].

Two studies at enhanced geothermal fields in France and Switzerland found velocity responses of opposite polarity during well stimulation: negative velocity changes 
were detected as a result of injection at the Soultz-sous-Fôrets field (from 4D seismic tomography of the production-induced micro-earthquakes) [Calò and Dorbath, 2013], and positive velocity changes were found during injection of the Basil geothermal field (from ambient noise) [Hillers et al., 2015]. Hillers et al. [2015] differentiate their response from the Soultz-sous-Fôrets geothermal field due to the presence of an upward migrating deformation transient, which reaches its maximum value in the sedimentary layer above the stimulated formation. They propose a model where the injected layer characterized by slower velocity yields, causing the overlying layers to sag in an upward-migrating order, leading to compaction of the injection layer, increased rigidity, and a velocity increase [Hillers et al., 2015]. In the Hillers et al. [2015] results, velocity values return in pre-injection values after approximately ten days after peak velocity change. Subsidence has been recorded at Rotokawa since 2004, and is primarily caused by production in the deep aquifer, but its rate did not change during the increased injection and production at the start of NAP production, and the rate of measurements (one every few years), cannot be easily compared with our dataset [Sewell, 2016].

In a conference abstract, Jousset et al. [2010] reported a positive velocity change of several percent at the Bouillante geothermal system in the French Antilles coinciding with stoppage of production, and described the possible mechanisms as a phase change in the fluid, a change in porosity, or fracture closure. The described response seems to be similar to what we observe at our geothermal systems when injection exceeds production, but unfortunately there is no information in the abstract regarding the injection rate before and after the production stoppage. We know from the empirical relationships of Brenguier et al. [2008b] that shear-velocity is inversely linked with porosity [Pride, 2005], so a stoppage of both injection and production could potentially decrease porosity and close fractures and produce the observed velocity change, but further detail regarding the geothermal system and injection rates is needed to investigate that assessment.

\subsection{Conclusion}

In this chapter, we utilized the coda of cross-correlation functions to determine shearwave velocity changes at Rotokawa and Ngatamariki, two producing geothermal fields located in the central North Island, New Zealand. Both of the time periods chosen for this study (2009-2010 Rotokawa and 2012-2013 Ngatamariki) coincided with greatly increased production and injection. Cross-correlation and coherence images were used to visually remove station-pairs from analysis. Using coherence, we were able to detect orientation changes for three stations at Rotokawa that underwent instrument swaps. Velocity change averages were conducted across the entire field and for various sub- 
sets using moving window stacks of 10 to 50 days. We detected increases in velocity of approximately $0.05 \%$ to $0.1 \%$ for both geothermal fields at points when the injection rate was greater than production, and a similar magnitude decrease in velocity when production exceeded injection. We refer to this response as the "full-field" velocity change. A straight-ray pseudo-tomography code was used to map the velocity changes obtained at the geothermal fields and determined that positive changes in shear-velocity compared to the average of the field are continuously co-located with injection wells. We distinguish this response from the full-field measurement as "localized" velocity changes.

We propose that there are three possible mechanisms contributing to the velocity changes observed at Rotokawa and Ngatmariki [Figures 5.57 and 5.59]. The first is rainfall, which was visually correlated with the full-field shear-velocity change at certain time periods of the Rotokawa and Ngatamariki datasets. The measured changes have been observed in other datasets at similar magnitudes [Sens-Schönfelder and Wegler, 2006, Meier et al., 2010].

The second is the Hillers et al. [2015] model of compaction and subsequent settling of the injection layer. The NM10 and NM09 well stimulations at Ngatamariki [Figure 5.53] occur during a time period where there is no production, and demonstrate similar responses to the Hillers et al. [2015] study. After large-scale injection begins at Ngatamariki, the dependencies of the velocity changes, correlating primarily to the differences in injection and production, could represent the compaction of the injection layer. At Rotokawa, compaction of the injection layer could occur when injection exceeds production at the beginning of 2010 [Hernandez et al., 2015]. This mechanism does not explain why negative velocity changes are observed when production exceeds injection, and we would expect a higher amount of subsidence to be observed at Rotokawa, which is not the case for this time period [Sewell, 2016]. However, this method could explain the localized positive velocity changes observed near the injection wells in the pseudo-tomography analysis.

The last mechanism we will discuss is one primarily driven by the depth sensitivity of scattered signals recorded at Rotokawa and Ngatamariki. Using the empirical relationships of Brenguier et al. [2008b], we can expect that deformation changes due to injection would reduce the shear-velocity in the injection layer. Expansion in the injection layer would compress the above layers, and would result in a positive velocity change if the depth resolution of the scatterers did not reach the injection layer. This mechanism could explain both the increases and decreases in shear-velocity across the geothermal field.

Finding the various contributions of each mechanism requires depth-dependent measurements of shear-velocity changes, a thorough knowledge of the velocity structure of the geothermal field for the computation of surface wave sensitivity kernels, 
and the calculation of decorrelation for numerous frequency bands. Decorellation is the measure of how cross-correlations change amplitude in time, and has been used in ambient noise studies to isolate time periods and depths where the scattering structure has been altered [Obermann et al., 2013, Hillers et al., 2015]. Hillers et al. [2015] used this technique alongside specific frequency-band filtering and sensitivity kernels to locate upward migrating deformation, which was the basis of his settling mechanism claim. This method could also be used alongside depth-dependent measurements of velocity changes to investigate the depth resolution of the scatterers and assess the likelyhood of each mechanisms driving the full-field and localized velocity changes. 


\section{Chapter 6}

\section{Shear velocity structure inferred using Refraction Microtremor in the upper 200 meters at the Ngatamariki geothermal field, central North Island, New Zealand}

We determine subsurface structure using the Refraction Microtremor method at the Ngatamariki geothermal field, central North Island, New Zealand. The local geology is such that refraction and reflection studies are hindered by energy scattering and attenuation in the near-surface layers. The Refraction Microtremor method uses surface waves from ambient noise and active sources to determine shear-wave velocities in the shallow subsurface. We deployed two lines of 72-channel, $10 \mathrm{~Hz}$ vertical geophones with 10 meter spacing, and were able to model near-surface shear-wave velocity to depths of 57-93 meters for 2D profiles and as much as 165 meters for 1D profiles. Shear-velocity anomalies were detected on one line that are spatially correlated with a fault. The location of the fault was previously inferred from stratigraphic offset in the geothermal wells, suggesting that the Refraction Microtremor method can provide important constraints on near-surface geology in noisy geothermal settings.

Most of the contents of this chapter are taken from a published journal article resulting from this work [Civilini et al., 2016] ${ }^{1}$, but sections regarding the theory [Section 6.3.3] and noise sources [Section 6.3.2] have been expanded with supplementary information to increase clarity.

\footnotetext{
${ }^{1}$ Civilini, F., Pancha, A.,Savage, M.K., Sewell, S., and Townend, J., (2016). Shallow structure from cultural ambient noise at the Ngatamariki geothermal field using Refraction Microtremor (ReMi). SEG Interpretation, (4) 3, SJ87-SJ101, doi: 10.1190/INT-2015-0204.1
} 


\subsection{Introduction}

Seismic exploration in general, and reflection surveying in particular, is challenging in geothermal and volcanic areas due to energy scattering and the highly attenuating properties of near-surface layers [Bannister and Melhuish, 1997, Stern and Benson, 2011]. Surface-wave methods, which are not dependent on energy being reflected or refracted from interfaces, could provide a solution for these challenges. We present here a pilot study of the application of the Refraction Microtremor (ReMi) method, an imaging technique employing surface waves created by cultural ambient noise, to a producing geothermal area to assess the effectiveness of the method as an inexpensive tool for imaging shallow subsurface structures.

The ReMi technique was first devised as a means of efficiently and cheaply constraining shallow shear-velocity structure without the need for intrusive energy sources [Louie, 2001]. The term "Refraction Microtremor" stems from the fact that Louie [2001] used recordings of microtremor recorded by refraction equipment to estimate shearwave velocity structure. It combines the techniques of the Spectral Analysis of Surface Waves (SAWS) [Nazarian and Stokoe, 1984] and Multichannel Analysis of Surface Waves (MASW) [Park et al., 1999] methods with naturally occurring background microtremor. The depth resolution and quality of the velocity model is dependent on the frequencies of the noise source, data sampling rate, record length, geophone spacing, geophone frequency, and array length [Pancha et al., 2008].

The SASW method uses the cross spectrum to determine phase velocities of two receivers near an active source. Unfortunately, this method loses accuracy in noisy environments. SASW assumes that the automatically selected energetic arrivals correspond to fundamental mode surface waves, while in reality they could contain contributions from higher mode surface waves and body waves [Louie, 2001]. The MASW method uses multiple channels to identify and remove noise frequencies and provide a statistical redundancy to the phase velocities [Park et al., 1999]. The depth resolution and accuracy of both of these methods is almost entirely dependent on the frequency content of the active source used. Unless a vibrosize truck is available for the experiment, the active sources used at SASW and MASW will generally have a high frequency content, and as such will only be able to image the shallow subsurface (generally less than 50 meters).

The true advantage of ReMi over SASW and MASW is its ability to utilize the energy produced by background microseisms. Although hammer shots are also frequently used as a ReMi source to obtain resolution for the shallow subsurface, the low frequencies of the background signals can be used to image depths of hundreds of meters. Recent studies have been able to expand the ReMi range of imaging up to a depth of $1 \mathrm{~km}$ [Pancha and Pullammanappallil, 2012]. The frequency content and 
corresponding imaging depth of the source signals used in this experiment will be discussed at length in a later section of this chapter [Section 6.3.4].

The ReMi method has been used to determine shallow velocity structure at various locations worldwide, including the United States [Scott et al., 2004, Thelen et al., 2006], Italy [Castellaro and Mulargia, 2009], Spain [Pérez-Santisteban et al., 2011], Mexico [Chávez-García et al., 2007], Germany [Richwalski et al., 2007], and New Zealand [Louie, 2001, Kaiser and Smith, 2005]. Common applications include the detection of geohazards such as faulting and landslide slip planes [Cashman et al., 2013], characterization of subsurface profiles [Rucker, 2003, Pancha et al., 2008], and liquefaction analysis [Kaiser and Smith, 2005]. Recent studies have been able to expand the ReMi method range of imaging to depths of one kilometer [Pancha and Pullammanappallil, 2012, Louie et al., 2013, 2014]. Results have been verified using synthetics and borehole instruments [Stephenson et al., 2005, Heath et al., 2006, Thelen et al., 2006], as well as other surface-array techniques [Anderson et al., 2007, Chávez-García et al., 2007, Pérez-Santisteban et al., 2011].

Geologic structure in geothermal fields is commonly interpreted from aerial photos, topography, field mapping, microearthquake patterns, and stratigraphic offsets determined from well data [Sugiaman et al., 2004]. However, structures of interest often lack obvious near-surface expressions which results in their locations being based mostly on well stratigraphy [Stimac et al., 2008]. Often, these wells are hundreds of meters apart [Chambefort et al., 2014, McNamara et al., 2016]. The ReMi method has been suggested as a potential tool for geothermal exploration in the Pacific Northwest region of the United States, producing more accurate locations of faults within the top 100 meters than either resistivity or magnetic techniques [Cashman et al., 2013]. Seismic velocity imaging can provide constraints on near-surface geology, thereby identifying possible faults in geothermal fields, which are of interest due to their possible controls on fluid-flow. Seismic velocity is itself essential for determining accurate microseismicity locations, particularly the depths of events, which are often used to interpret injection fluid movements in geothermal reservoirs [Sherburn et al., 2015]. Surface-wave methods can also be used to characterize subsidence at geothermal fields. Subsidence is a common and costly problem associated with geothermal production [White et al., 2005, Allis et al., 2009, Mackenzie, 2012], sometimes requiring the drilling of shallow wells for investigation [Bromley et al., 2009]. Subsidence at geothermal fields has been linked to layers with anomalously high clay content [Bromley et al., 2009], which can be detected using Refraction Microtremor [Rucker, 2003]. 


\subsection{Site and deployment information}

\subsubsection{Geology}

The Ngatamariki geothermal field lies in the central-east portion of the Taupo Volcanic Zone (TVZ) [Figure 6.1], a region of active rhyolitic and andesitic volcanism which began forming approximately $2 \mathrm{Ma}$ as a result of the subduction of the Pacific plate underneath the North Island [Wilson et al., 1995] and subsequent backarc extension [Rowland and Sibson, 2001]. As a result, tectonic studies of the region have relied on refraction surveys using explosive sources and large array transects [Bannister and Melhuish, 1997, Henrys et al., 2003, Stern et al., 2005].

Borehole information at the Ngatamariki geothermal field reveals a greywacke basement underlying andesitic breccia successions on the south side of the field, with an intrusion composed of tonalite and diorite in the northernmost wells, and rhyolitic and sedimentary successions at depths shallower than $1 \mathrm{~km}$ [Chambefort et al., 2014]. Electricity production at Ngatamariki uses an $82 \mathrm{MW}$ binary type power station manufactured by Ormat Technologies, which began operations in mid-2013 and is owned and operated by Mercury [Chambefort et al., 2014].

Within the top 200 meters depth sampled by this analysis, the geology consists of volcanoclastic rocks of the Huka Falls Formation [Chambefort et al., 2014], overlain by the Oruanui Formation ignimbrite [Wilson, 2001, Manville and Wilson, 2004], a layer of loess and gravels [Vucetich and Pullar, 1969], and ignimbrite from the CE 232 Taupo volcanic eruption [Wilson, 1985, Hogg et al., 2012]. Mercury, as part of this study, has provided geology logs interpreted from drill cuttings for the Ngatamariki production and injection wells [Figure 6.1]. These logs generally reveal the contact between the Oruanui and Huka Falls formations, but lack the resolution to determine shallower formations due mostly to poor drill cuttings returns [Hedenquist, 1980, Ramirez and Rae, 2009, Rae et al., 2009, Lewis et al., 2012, 2013a,b].

\subsubsection{Experimental setup}

Two transects were conducted at the Ngatamariki geothermal field on the 17 th and 18th of February 2015. Line A was deployed on the first day trending NW-SE between injection wells NM8, NM9, and NM11 and parallel to an above-ground pipe [Figure 6.1]. Line B was deployed on the second day trending North-South, parallel to a road between the Ngatamariki power station and production wells NM7, NM5, and NM11. The deployment locations were chosen for their proximity to cultural noise sources, the availability of geologic information from well records, and vehicle access. The two-lane road parallel to Line B is an access road to the geothermal power plant, and was mostly devoid of traffic except for the field vehicles used as noise sources for 
this experiment. Chambefort et al. [2014] inferred a fault between NM7 and NM5 from borehole logs, but could not constrain its location and approximated the fault at halfway between wells NM7 and NM5. This location is marked in Figure 6.1 as a black dashed line.

The ReMi method setup used for this experiment was a 72-instrument configuration with 10 meter spacing, consisting of vertical geophones recording at 500 samples per second and with a corner frequency of $10 \mathrm{~Hz}$ [Figure 6.2]. Data were recorded in thirty-second segments in SEG2 format, but converted into SEGY for processing. Recording lengths of 20-30 seconds are common for studies using the ReMi method [Heath et al., 2006, Chávez-García et al., 2007, Anderson et al., 2007]. A total of 43 thirty-second records were captured for Line A, including hammer shots, vehicle signals, and background noise, and 86 thirty-second records were captured for Line B. 


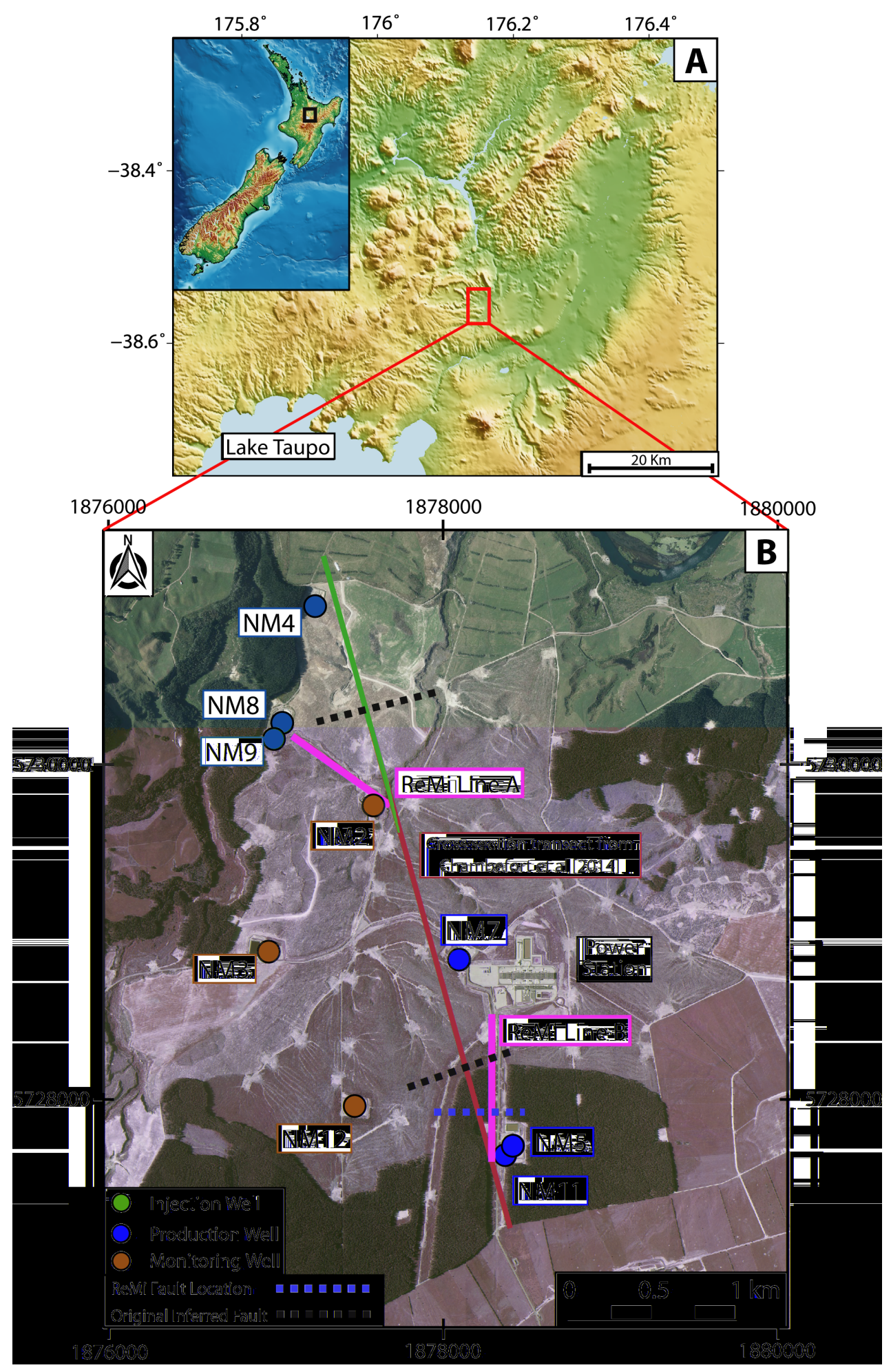

Figure 6.1: [A] The Ngatamariki geothermal field, located approximately $20 \mathrm{~km}$ NorthEast of Taupo in the North Island of New Zealand. [B] Displays a map showing the locations of the deployed ReMi arrays (yellow lines) compared to nearby wells and the original structural cross section presented by Chambefort et al. [2014] (green line). The Chambefort et al. [2014] analysis presented inferred faults (black dashed lines) interpreted from borehole well logs. The inferred fault that transects Line B has been relocated based on the results of this study (red dashed line). 


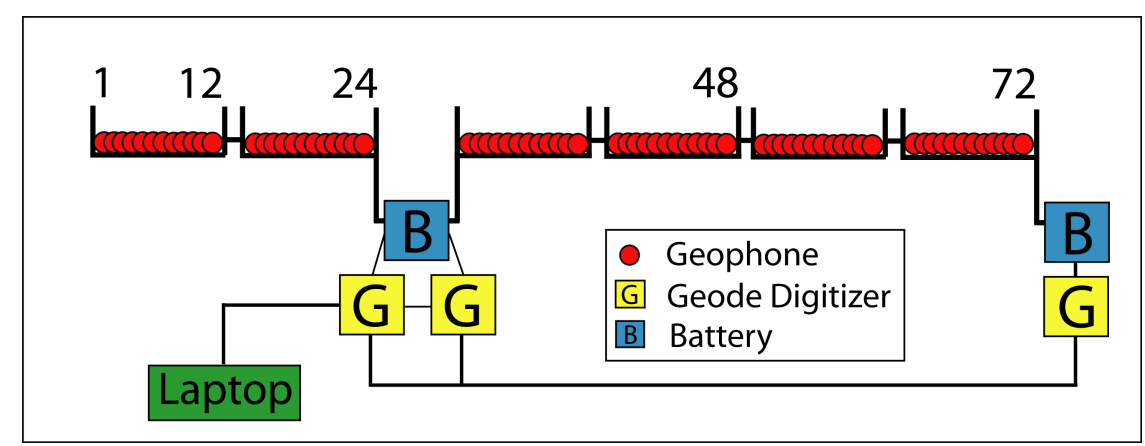

Figure 6.2: Schematic of the 72-channel ReMi deployment used for both Line A and B in this experiment. The sensors used were single component $10 \mathrm{~Hz}$ vertical geophones with an interstation spacing of 10 meters. 


\subsection{ReMi method workflow and processing}

Once data have been collected, analysis using the ReMi method involves three main steps: [1] data processing using the slowness-frequency transform $(p-f)$ [Thorson and Claerbout, 1985], [2] dispersion curve picking using specific slowness and frequency boundaries, and [3] inversion of the dispersion curves. The slowness-frequency $(p-f)$ transformation and manual modeling for this experiment were performed using the software SeisOpt ReMi (C) Optim, 2006), and follow the methodology of Louie [2001], as detailed in the sections below.

\subsubsection{Signal types}

The robustness of the inferred subsurface models depends strongly on the accuracy of the dispersion curve computed from the slowness-frequency transformation of the recorded data. It is important to obtain a dispersion curve that is well resolved across the widest frequency range possible, as high and low frequencies provide information on shallow and deep layers respectively. Three source types were used in this experiment: the background natural and cultural ambient noise of the geothermal field; vehicles moving parallel to the array; and hammer shots [Figure 6.3]. The purpose of using these three sources was to maximize energy levels in as many parts of the frequency spectrum as possible.

Likely background noise sources at Ngatamariki include: the power station, injection and production pipes, vehicles on the service roads of the geothermal field, the Waikato river approximately $2.5 \mathrm{~km}$ to the north, and State Highway 5 approximately $3 \mathrm{~km}$ to the south. Due to the scattering characteristics of the TVZ [Bannister and Melhuish, 1997, Stern and Benson, 2011], we assume that the background noise is a uniform source. The same approximation has been made by other ReMi method experiments employing similar noise sources [Pancha et al., 2008]. In Figure 6.3A, instruments 50-60 appear to record higher amplitudes than their neighbors, and we interpret the amplitudes to be caused by nearby production wells NM5 and NM11 [Figure 6.1].

As the vehicles were traveling parallel to the array, the energy obtained from the vehicle source is most likely a mix of both scattered and in-line energy. Hammer shots were conducted across the array between every second geophone in order to capture high-frequency energy along the entire array length. In the example record of Figure 6.3C, a total of 12 hammer strikes were done at the center of the array. The hammer shots occurred directly in line with the array, and the energy recorded may not be affected by scattering over the distance of the array. However, Line A has a significant amount of elevation change, and is slightly curved, which might promote scattering even for the hammer shots. The implications of the degree of scattering of the noise sources, and how it pertains to the observed dispersion curves, are discussed in the 

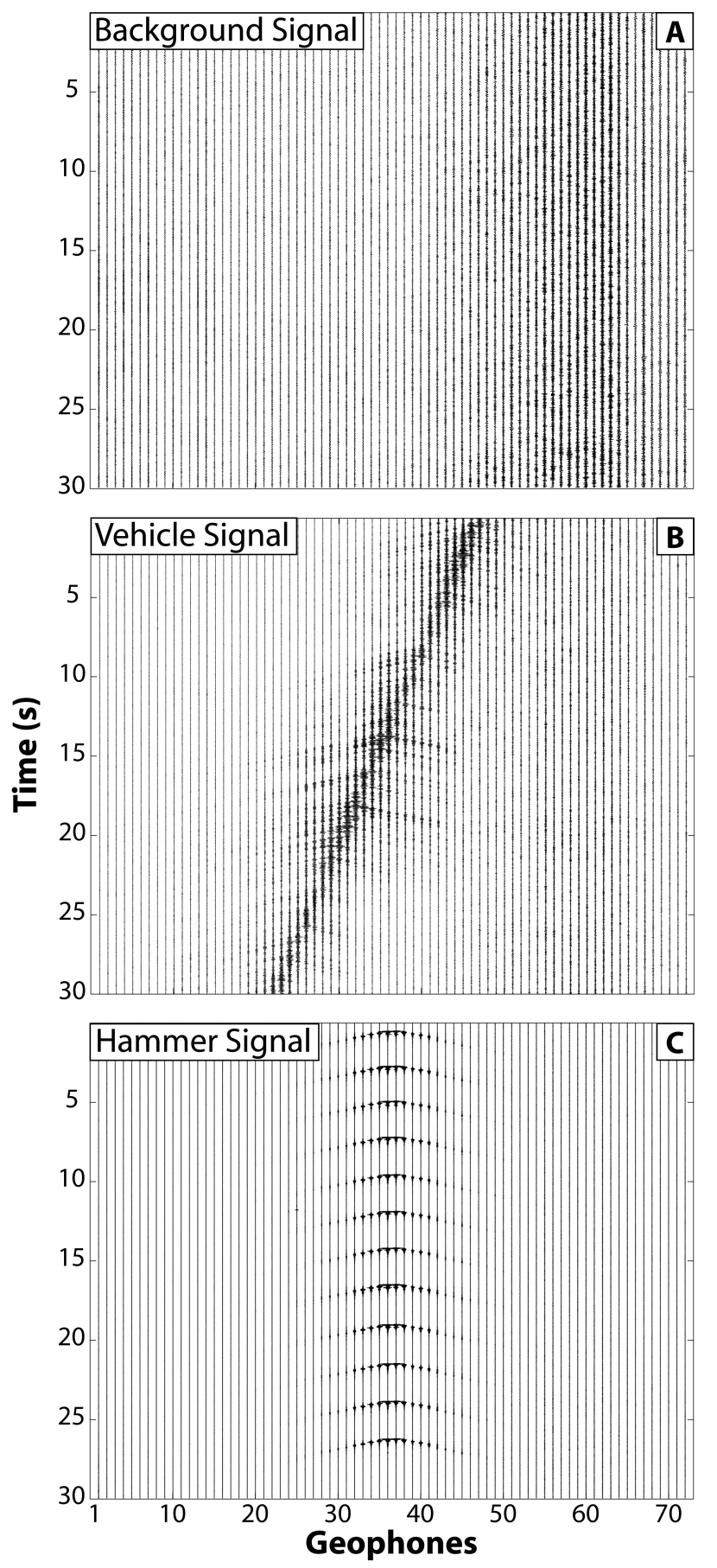

Figure 6.3: An example thirty-second record of each source type at Line B: [A] background, [B] vehicle, and [C] hammer shot noise sources.

"Picking the dispersion curve" section.

Figure 6.4 displays individual instrument and average Fourier transforms for the records presented in Figure 6.3. The background ambient noise [Figure 6.4A] had high energy at 5-22 Hz, while the greatest energy for the vehicle signal was at approximately $17 \mathrm{~Hz}$ [Figure 6.4B]. The hammer source [Figure 6.4C] has high energy for the geophones located closest to the hammer source [Figure 6.3C], and decays across a 
band from 15 to $200 \mathrm{~Hz}$.
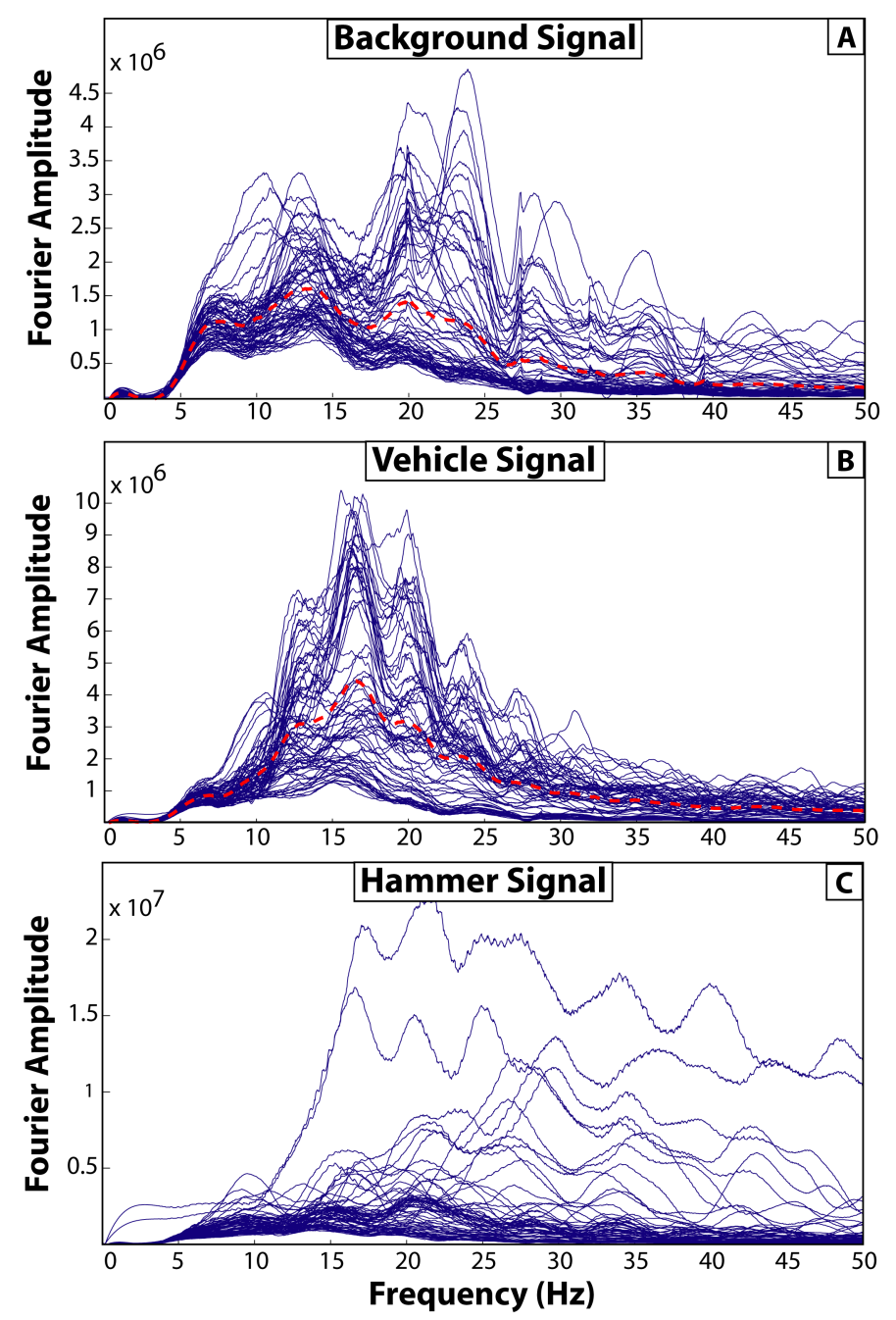

Figure 6.4: Fourier transforms of the example records used in figure 6.3. Each blue line represents the Fourier transformation of an individual geophone of the thirty-second record. In A and B, the mean Fourier transform is displayed as a dashed red line. [A] Fourier transforms for Figure 6.3A (background source): Fourier amplitudes have high energy between 5-22 Hz, [B] Fourier transforms for Figure 6.3B (vehicle source): Energy peaks at approximately $17 \mathrm{~Hz},[\mathrm{C}]$ Fourier transforms for Figure 6.3C (hammer shots): Energy peaks at 15-22 Hz and decays until approximately $200 \mathrm{~Hz}$ (not shown). Note: Sharp decrease below $7 \mathrm{~Hz}$ is due to the $10 \mathrm{~Hz}$ corner frequency of the instruments.

\subsubsection{Characterizing noise sources of the geothermal field}

A deployment of temporary stations at the Rotokawa geothermal field, located approximately $10 \mathrm{~km}$ south of Ngatamariki, was conducted during the same month of the ReMi experiment. The deployment occurred in two stages: Seven $4.5 \mathrm{~Hz}$ Geospace 
11D instruments were deployed from February 4th through February 20th (Deployment A), and then redeployed from February 20th to March 24th in varying locations (Deployment B) [Figure 6.5]. The primary objective of the deployment was to characterize the cultural noise sources in the field. The instruments were placed near the Nga Awa Purua power station, wells, roads, and production pipes. Due to the similarity of the noise sources at Rotokawa and its close proximity to Ngatamariki, we can use these data to infer the likely contribution of the noise sources in the background ReMi recordings [Figures 6.1, 6.4A].

Figure 6.6 displays the probability density functions (PDF) weighted noise means of the stations closest to the each noise source. The weighted noise means were computed using the software PQLX [McNamara and Boaz, 2011] and the methodology is described in Section 2.2. With the exception of DB07, the sensor along Nga Awa Purua road, we found that the weighted means are within 10 decibel of one another for all three components. The production well sensor (DB06) has a flat spectrum in the short period. The power station sensor (DA01) has a sharp peak at approximately 0.04 seconds $(24 \mathrm{~Hz})$ on all components, suggesting a source unique to that location. We hypothesize that this value most likely corresponds to $25 \mathrm{~Hz}$, a multiple of the $50 \mathrm{~Hz}$ electricity oscillation in the power lines extending from the power station. Lastly, the production pipes (DB06 and DA05) have a secondary peak at 0.2 seconds $(5 \mathrm{~Hz})$. This secondary peak is clearly observed on all channels of DB06 and on the EHE channel of DA05.

We can then compare the PDF results from the temporary deployment with the ReMi instrument FFTs for Lines A and B [Figure 6.7]. Based on the locations of Lines $A$ and $B$ [Figure 6.1], we expect the main sources of noise be the production pipe for Line $\mathrm{A}$ and the power station and production wells towards the northern and southern ends respectively of Line $B$.

The FFTs of Line A [Figure 6.7A] have two main peaks: one between 5--10 Hz and a smaller one at approximately $17 \mathrm{~Hz}$. We suggest that the $5--10 \mathrm{~Hz}$ peak corresponds to the production pipe that runs parallel to the line, matching the frequencies we observed in the PDF plot [Figure 6.6, green line]. A similar case could be made for the smaller $17 \mathrm{~Hz}$ frequency peak, which could correspond to the small increase in weighted noise mean observed at 0.6 seconds period in the vertical component of production pipe station DA06 [Figure 6.6C, orange line], but similar increases in production pipe station DB06 or other components are not observed.

The Fourier spectrum of a background recording of Line B has different characteristics than what was observed in Line A [Figure 6.7]. Unlike Line A, where the array was laid out parallel to the production pipe noise source, the beginning of the array (the northern end) is close the Nga Awa Purua power station and the southern end of the array is near two production wells [Figure 6.1]. As may be expected due to the two 

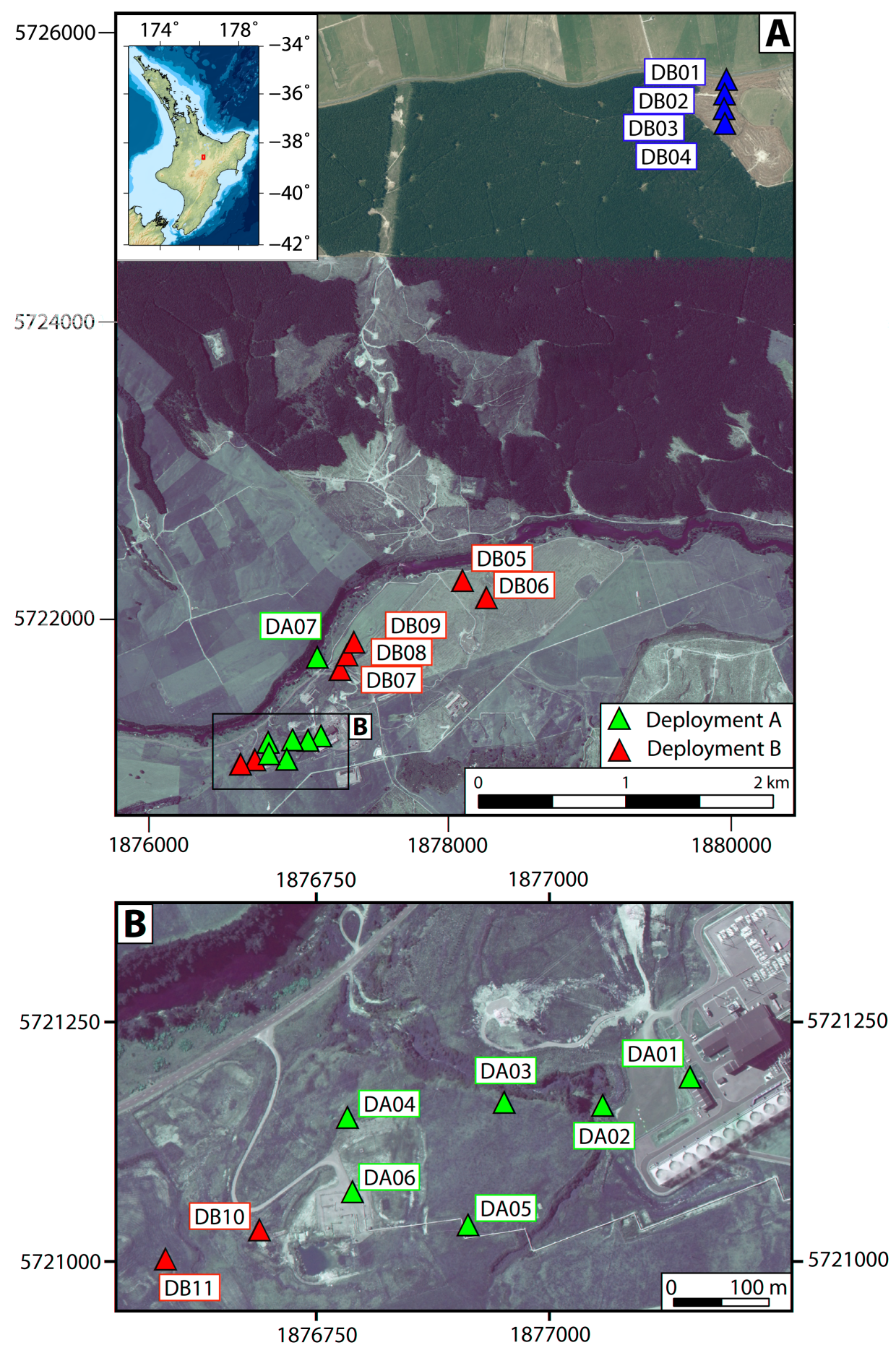

Figure 6.5: Map of the temporary deployment used to measure the amplitude and attenuation of the noise sources at the Rotokawa geothermal field. Map coordinates are in New Zealand Transverse Mercator 2000 (NZGD2000).

noise sources, we found that the Fourier spectrum changed throughout the line from three speaks at 7, 14, and $25 \mathrm{~Hz}$ in the northern end to four peaks at 17, 20, 24, and 28 $\mathrm{Hz}$ in the south [Figure 6.7B]. The northern-most sensor is displayed in Figure 6.7B as a purple line. The $25 \mathrm{~Hz}$ signal detected in the power station noise PDF [Figure 6.6, blue line] is observed in the northern instruments [Figure 6.7B, purple line]. The production 

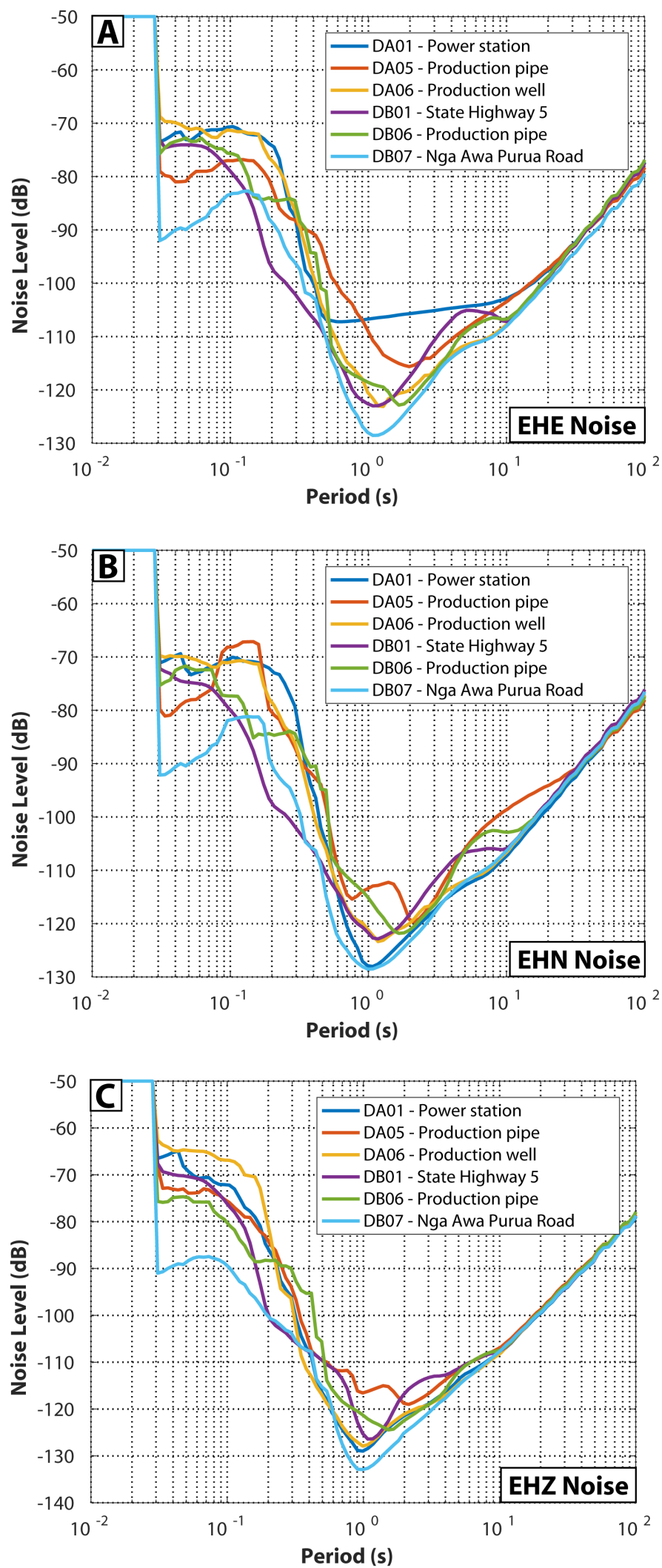

Figure 6.6: Weighted mean noise for instruments closest to noise sources around Rotokawa for [A] EHE, [B] EHN, and [C] EHZ channels.

well noise was computed to have a flat spectrum above $5 \mathrm{~Hz}$ ( 0.2 seconds) and a higher 
noise level than the power station [Figure 6.6C, yellow line], which could explain the higher Fourier amplitudes towards the southern end of the line.

The largest source of error of this exploration into the characteristics of the noise sources is that our analysis doesn't take into account the distance between the sensor and the noise source. Preliminary work to quantify the seismic attenuation of the cultural noise sources and their relationship to the signal-to-noise ratio of microearthquakes was carried out using this temporary deployment, but it is outside the scope of this ReMi project and is discussed in Appendix C.
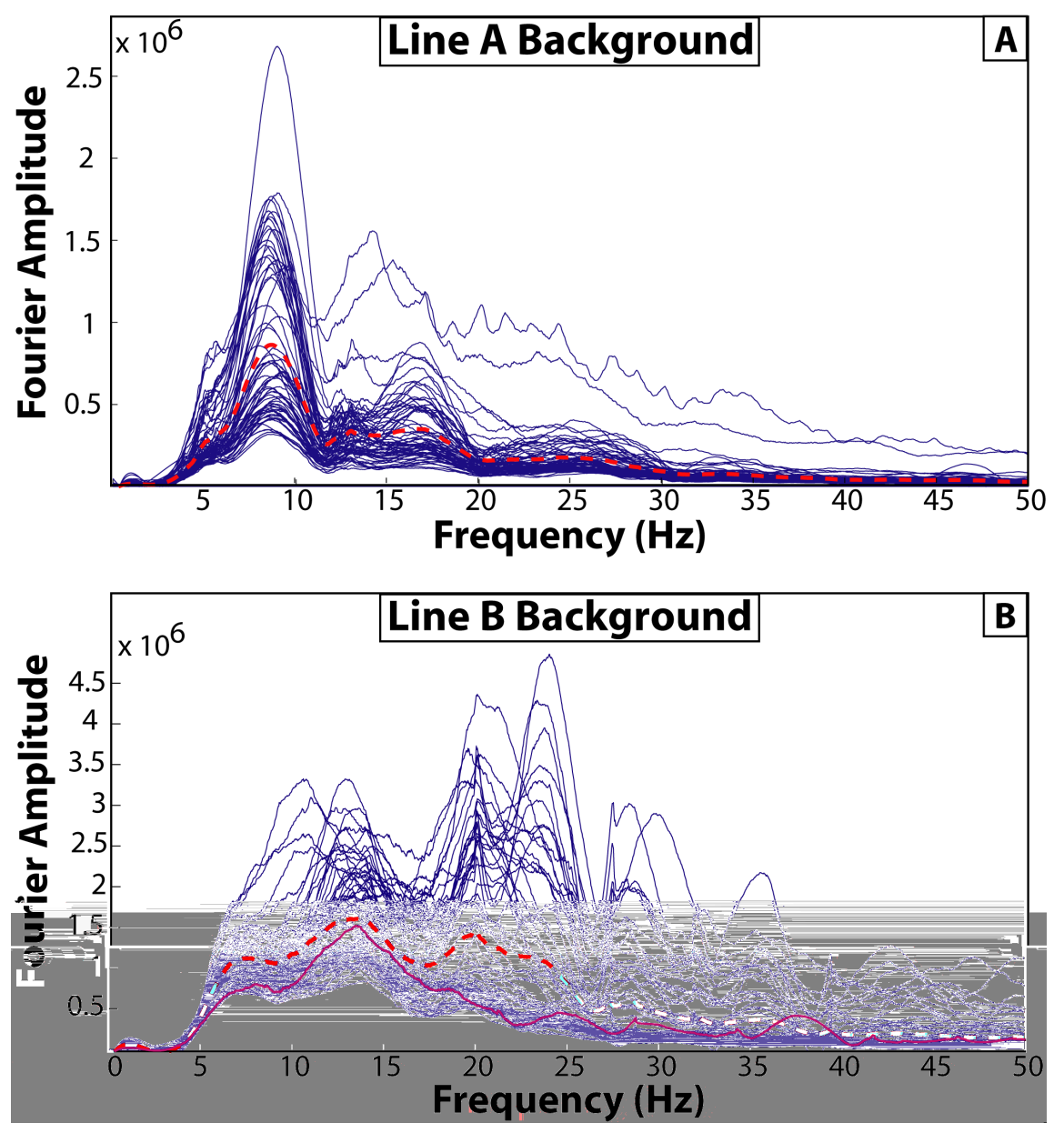

Figure 6.7: [A] ReMi sensor FFTs for a background record in Line A. [B] ReMi sensor FFTs for a background record in Line B. The FFT of the sensor closest to the power station is displayed in purple. The mean is displayed as a red dashed line.

\subsubsection{The slowness-frequency transform}

For each recorded time series, a slowness-frequency $(p-f)$ transformation [Thorson and Claerbout, 1985] was applied to isolate fundamental-mode Rayleigh waves. The $p-f$ transformation occurs in two steps: the signals are first summed as a line integral across the array to represent the data as a function of slowness $(p)$ and ray intercept 
time $(\tau)$, after which the stacks are transformed into the frequency $(f)$ domain using a Fourier transform [Louie, 2001].

From a seismic record $A(x, t)$, positioned a distance $x$ away and at a time $t$, the $p-\tau$ transformation is defined as:

$$
A(p, \tau)=\int_{x} A(x, t=\tau+p x) d x
$$

where $x$ represents the length of the geophone array, in which geophone intervals are at a spacing $d x$ (10 meters in our experiment). The slowness of a surface wave recorded traveling across this array can be defined in terms of $d x$ and $d t$, correlating to the inverse of the apparent velocity $V_{a}$ :

$$
p=\frac{d t}{d x}=\frac{1}{V_{a}}
$$

Note that the apparent velocity $V_{a}$ is different from the true surface wave speed $v$. We can discritize the continuous function [6.1]:

$$
\begin{gathered}
\{x=j d x: j=1,2,3, \ldots\} \\
\{t=i d t: i=1,2,3, \ldots\} \\
\left\{p=p_{0}+l d p: l=1,2,3, \ldots\right\} \\
\{\tau=k d t: i=1,2,3, \ldots\} \\
A\left(p=p_{0}+l d p, \tau=k d t\right)=\sum_{j=0}^{n x-1} A(x=j d x, t=i d t=\tau+p x)
\end{gathered}
$$

where $d t$ is the sample-rate (0.002 seconds for our experiment), $p_{0}$ is the inverse of the minimum velocity (user defined), and $d p$ is the slowness interval at which amplitudes are calculated (automatic). Amplitudes for times outside of $t=\tau+p x$ are estimated by linear interpolation, so a thorough knowledge of the frequencies of the input signals must be known in order to optimize the resolution of the result. The continuous $p-\tau$ function [6.1] is then taken into the frequency domain via a Fourier transform:

$$
F_{A}(p, f)=\int_{\tau} A(p, \tau) e^{-i 2 \pi f \tau} d \tau
$$

and discretized into:

$$
\begin{gathered}
\{f=m d f: m=1,2,3, \ldots\} \\
F_{A}(p, f=m d f)=\sum_{k=0}^{n t-1} A(p, \tau=k d t) e^{-i 2 \pi m d f k d t}
\end{gathered}
$$


The power spectrum can then be calculated by multiplying the discrete Fourier transform with its complex conjugate:

$$
S_{A}(p, f)=F_{A}^{*}(p, f) F_{A}(p, f)
$$

This signal can then be summed together on the same slowness axis:

$$
S_{A}(|p|, f)=\left[S_{A}(p, f)\right]_{p \geq 0}+\left[S_{A}(-p, f)\right]_{p<0}
$$

Depending on the type of signals obtained, it is sometimes useful to add together multiple records:

$$
S_{\text {total }}(|p|, f)=\sum_{n} S_{A n}(|p|, f)
$$

The line integral, or slant stack, sums the time series data along lines of constant slope $p=d t / d x$ (slowness) and intercept $\tau$ (arrival time) [Diebold and Stoffa, 1981, Stein and Wysession, 2003]. The slant stack can be applied from either end of the line, resulting in different concentrations of energy in the $p-f$ plot. We refer to the direction of the slant stack in our analysis as forward or reverse. Once the $p-\tau$ image is transformed into the frequency domain $(p-f)$, a dispersion curve can usually be observed across the spectrum [white boxes, Figure 6.8]. High-energy arcs oriented perpendicular to the dispersion curves are recognized to be aliasing artifacts, produced by truncation and transformation of other phases during the slant stack [Louie, 2001, Optim, 2006].

Within the boundaries of the $p-f$ image, spectral power is normalized for each frequency by a spectral ratio method [Optim, 2006], requiring that the slowness and frequency boundaries of the $p-f$ image be adjusted according to the frequency distribution of the energy observed. In other words, imposing wide frequency and slowness limits during the $p-f$ transformation will identify the energy over a wide range of the dispersion curve, while a transformation using a narrower frequency and slowness limit will more accurately resolve a particular segment of the dispersion curve. Although Fourier transforms of individual records provide an indication of what frequencies should be observed [Figure 6.4], the maximum frequency $\left(F_{\max }\right)$ and minimum velocity $\left(V_{\min }\right)$ boundaries of the $p-f$ transformation are typically selected by trial and error based on the clearest energy contrast in the resulting $p-f$ plot. For example, the background noise contains low-frequency energy that is vital for imaging deeper structure, whereas in order to accurately image the low-frequency end of the dispersion curve, a lower $F_{\max }$ and higher $V_{\min }$ value compared to hammer shot and vehicle sources is required [Figure 6.8].

The selection of the velocity values below the regions of energy is based on energy distribution. In general, noise sources displaced from (not in-line) with the linear array result in energy traveling obliquely to the array and at higher apparent velocities. The apparent velocity $V_{a}$ of a recorded signal is described by: 

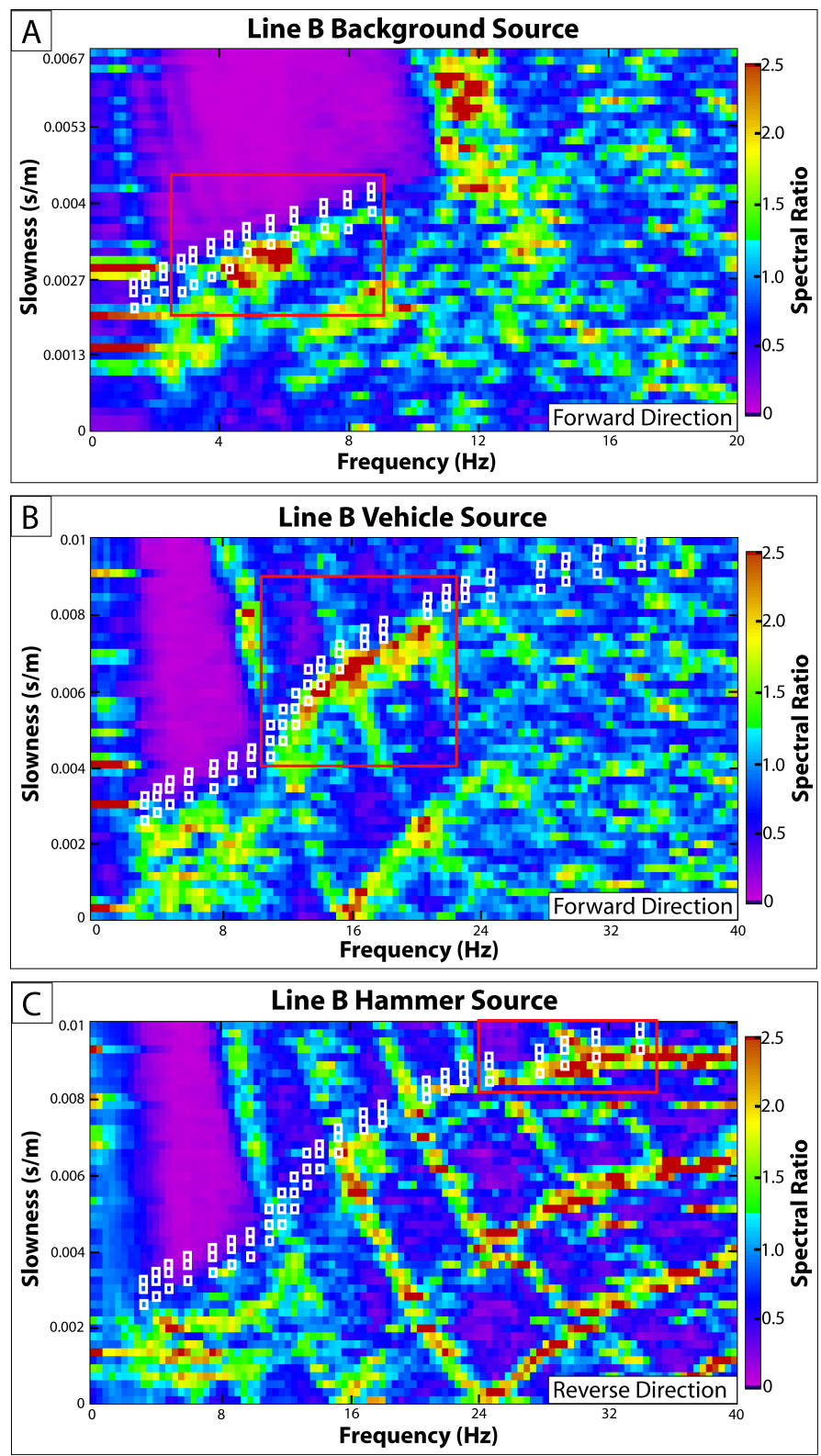

Figure 6.8: Slowness-frequency plots of the [A] background, [B] vehicle, and [C] hammer source records presented in Figures 6.3 and 6.4. The red box corresponds to the picks determined from this particular $p-f$ image. A total of three picks were done for each frequency representing the steepest gradient pick (center) and the high and low limits. The $p-f$ transform of background source records [A] had clearer low-frequency energy than the vehicle and hammer sources, so a new set of picks using different $p-f$ transformation limits was developed for those records.

$$
V_{a}=\frac{v}{\cos (a)}=\frac{1}{p}
$$

Where $v, a$, and $p$ correspond to the in-line phase velocity (or true velocity), angle of incidence, and horizontal slowness respectively. It is important to note that slowness $p$ used in this notation is more appropriately described as an apparent slowness. It is 
helpful to visualize this as an energy distribution from the source recorded at the ReMi array [Figure 6.9]. The energy that travels at a vector perpendicular to the array will reach the array first, while energy traveling parallel to the array will record the greatest slowness. The non-linearity between $p$ and $a$ in Equation 6.14 is described in Table 6.1.

\begin{tabular}{||c|c|c||}
\hline Proportion of $\boldsymbol{v p ( \% )}$ & Propagation Angle $\left.\boldsymbol{a} \mathbf{(}^{\circ}\right)$ & Coverage of $\mathbf{3 6 0} \mathbf{}^{\circ}$ Energy (\%) \\
\hline \hline $0-10 \%$ & $90.0^{\circ}-84.2^{\circ}$ & $6.3 \%$ \\
\hline $10-20 \%$ & $84.2^{\circ}-78.4^{\circ}$ & $6.4 \%$ \\
\hline $20-30 \%$ & $78.4^{\circ}-72.5^{\circ}$ & $6.6 \%$ \\
\hline $30-40 \%$ & $72.5^{\circ}-66.4^{\circ}$ & $6.8 \%$ \\
\hline $40-50 \%$ & $66.4^{\circ}-60.0^{\circ}$ & $7.1 \%$ \\
\hline $50-60 \%$ & $60.0^{\circ}-53.1^{\circ}$ & $7.6 \%$ \\
\hline $60-70 \%$ & $53.1^{\circ}-45.5^{\circ}$ & $8.4 \%$ \\
\hline $70-80 \%$ & $45.5^{\circ}-36.8^{\circ}$ & $9.7 \%$ \\
\hline $80-90 \%$ & $36.8^{\circ}-25.8^{\circ}$ & $12.2 \%$ \\
\hline $90-100 \%$ & $25.8^{\circ}-0^{\circ}$ & $28.7 \%$ \\
\hline
\end{tabular}

Table 6.1: Angular coverage of slowness intervals, adapted from Louie [2001]

The first column $(v p)$, can be interpreted as the accuracy of the calculated slowness value $p$ in comparison with a theoretical true slowness value $p_{\text {true }}$ based on the propagation angle $a$ of column two. The third column is a percentage representation of the coverage of the angles given in column two. This table correlates the geometry of the system to energy, which is observed through the $p-f$ plots, assuming that the horizontally propagating energy comes in equally from all directions [Louie, 2001]. The key point is that $40.9 \%$ of the energy (or $36.8^{\circ}$ of $90^{\circ}$ full coverage) will lie within 80 $100 \%$ accuracy, while the rest will be evenly distributed among the rest of the angles. In other words, most of the energy observed in the $p-f$ output will cluster around $80 \%$ to $100 \%$ of the true value [Figure 6.9]. This can also be seen in the rate of convergence of the cosine function to a true value of slowness [Figure 6.10].

\subsubsection{Picking the dispersion curve}

The next stage of analysis is to select slowness-frequency points to describe the dispersion curve. These slowness-frequency "picks", as we refer to them for the remainder of the manuscript, are the data points used in the inversion. In general, noise sources not in line with the array result in energy traveling obliquely and at higher apparent velocities. The fundamental-mode phase-velocity Rayleigh wave dispersion curve is therefore picked along the steepest gradient in the region of maximum slow- 


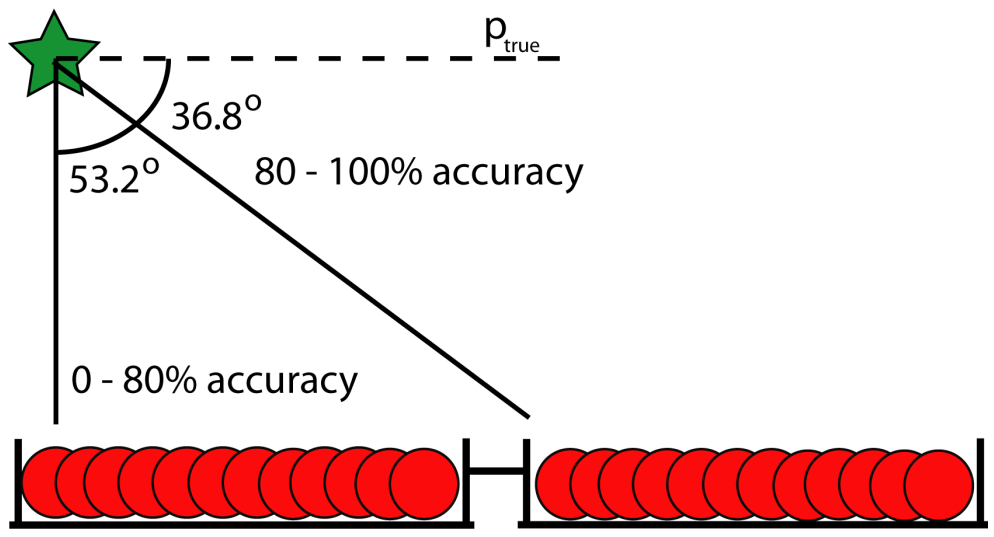

Figure 6.9: Schematic showing the accuracy of slowness measurements for ReMi records based on angular incidence from a source (green star) not in-line with the array [Table 6.1]. The hypothetical $p_{\text {true }}$ value propagates parallel to the array.

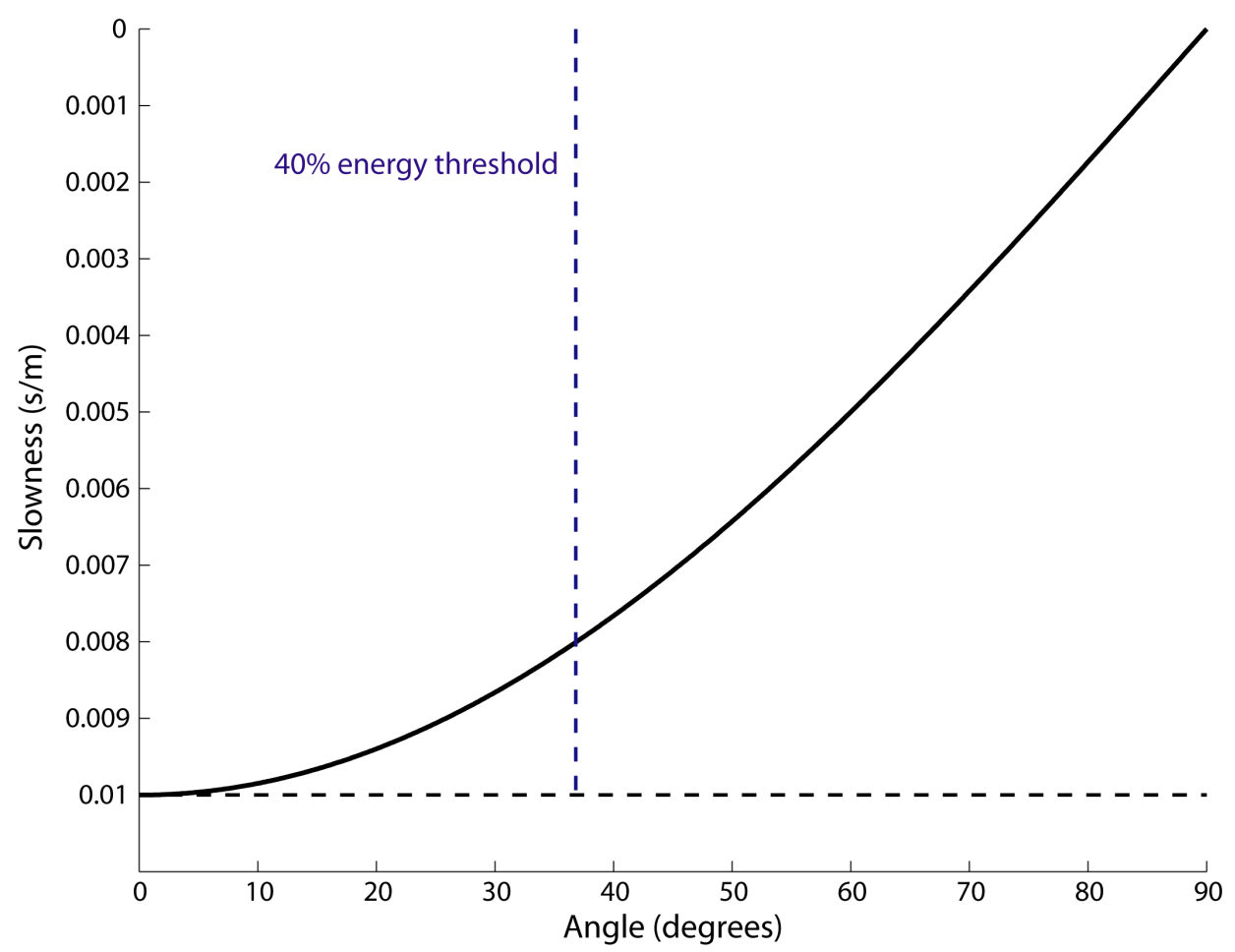

Figure 6.10: Rate of convergence of the cosine function [Equation 6.14] to a true slowness value of $0.01 \mathrm{~s} / \mathrm{m}$.

ness (minimum-velocity) of the energy within the slowness-frequency image to capture waves traveling parallel to the array [Louie, 2001] [Figure 6.8].

As previously mentioned, the energy of the three noise sources used in this experiment is not necessarily isotropic. Pancha et al. [2008] found that the slowness at the steepest gradient of spectral ratio energy of a slowness-frequency plot was consistent for scattered and direct inline noise sources, while the slowness corresponding to the spectral ratio maximum varied. This result was obtained by computing power- 
slowness profiles for different types of noise sources at numerous frequencies. The noise sources used in that experiment included background cultural noise, highway noise from all azimuths, hammer shots, rainfall, a train traveling obliquely and parallel to the array, and a mixture thereof [Pancha et al., 2008]. Therefore, we consider that the methodology of picking the dispersion curve energy at the steepest gradient of the minimum envelope, as is done for isotropic energy, is valid.

In addition to this pick location, which will be referred to as the "steepest gradient" pick location, two additional picks were made to compute error bars, at the maximum and minimum spectral energy locations of each frequency corresponding to the upper and lower limits of the dispersion curve. Figure 6.8 displays the resulting $p-f$ transformation for the thirty-second records from Line B of Figures 6.3 and 6.4 and the corresponding steepest gradient, lower limit, and upper limit picks.

Each $p-f$ image is a transformation of a thirty-second recording of background, vehicle, hammer, or combination of these energy sources, and thus the frequency bounds of highest energy will vary from record to record [Figure 6.4]. Due to this, the final dispersion curve is formulated from analysis of numerous records, as few individual records will have observable energy across the entire band to fully characterize the dispersion curve. Figure 6.8 displays all of our determined picks for Line B as white squares. The example $p-f$ records do not have energy for the entirety of our picked dispersion. The final picks for each line segment presented in this study were computed from an analysis of many dozens of high-energy $p-f$ images. The $p-f$ images of Figure 6.8 were used to determine the dispersion picks within the red squares, while the remainder of the picks were determined from other $p-f$ records.

The $p-f$ transformation can be applied across the entire line or across a subset of instruments. Thus, a $2 \mathrm{D}$ profile can be constructed by analyzing subsets of instruments incrementally across the array at the expense of depth resolution, as the different data subsets will have different frequency content and hence image different depths. Each 2D segment, as well as every $1 \mathrm{D}$ profile using different $F_{\max }$ and $V_{\min }$ boundaries, requires its own set of picks.

Picks are also used to calculate the theoretical maximum depth resolution $\left(Z_{\max }\right)$ of an array segment. This value can be approximated as half the wavelength of the longest-period surface waves, using the phase velocity at the longest-period $\left(C_{1}\right)$ and its corresponding frequency $\left(f_{1}\right)$ for each picked point of the dispersion curve [Park et al., 1999]:

$$
Z_{\max }=\frac{C_{1}}{2 f_{1}}
$$

The $p-f$ transformation boundaries and maximum depths corresponding to steepest gradient, upper limit, and lower limit are tabulated for all modeled segments in Table 6.2. 


\begin{tabular}{||l||l|l|l||}
\hline Segment & $\begin{array}{l}F_{\max } / V_{\min } \\
\mathbf{( H z} / \mathbf{m} / \mathbf{s})\end{array}$ & $\begin{array}{l}\text { Maximum } \\
\text { Pick Depth } \\
(\mathbf{m})\end{array}$ & $\begin{array}{l}\text { Model RMS } \\
\mathbf{( m / s )}\end{array}$ \\
\hline Line A 2D 1-24 & $30 / 85$ & $\underline{93}(77-133)$ & $\underline{2.8} / 5.5 / 3.3$ \\
\hline Line A 2D 49-72 & $30 / 85$ & $\underline{82}(72-120)$ & $\underline{2.9} / 4.0 / 2.4$ \\
\hline Line A 1D & $\begin{array}{l}30 / 85, \\
20 / 150\end{array}$ & $\underline{109}(100-143)$ & $\underline{4.6} / 8.4 / 6.6$ \\
\hline Line B 2D 1-24 & $30 / 85$ & $\underline{57}(50-76)$ & $\underline{4.7} / 3.5 / 4.4$ \\
\hline Line B 2D 61-72 & $30 / 85$ & $\underline{82}(71-103)$ & $\underline{5.4} / 8.6 / 4.7$ \\
\hline Line B 1D & $\begin{array}{l}40 / 100, \\
20 / 150\end{array}$ & $\underline{165}(154-185)$ & $\underline{2.9} / 5.8 / 4.1$ \\
\hline
\end{tabular}

Table 6.2: $p-f$ plot and pick boundaries, maximum theoretical depth for picks [steepest gradient (lower-upper bound)] [Equation 6.15], and RMS values between picks and manually inverted model dispersion curve (steepest gradient/upper/lower picks). Both 1D models required two sets of $p-f$ parameter boundaries to effectively model the high and low frequency dispersion energy.

\subsubsection{D and 2D modeling}

The SeisOpt ReMi Disper (C) Optim, 2006) software contains a 1D manual inverse modeling module developed in C by Yuehua Zeng [Louie, 2001] based on FORTRAN codes of Saito [1988]. The algorithm uses a numerical integration method to iterate over phase velocity for each frequency and can model velocity reversals [Louie, 2001, Pei, 2007]. Parameters that can be modified in this modeling routine include the number of layers, layer shear-velocity, and layer densities.

1D manual inverse models, using all 72 instruments, and 2D manual inverse models, using overlapping instrument segments, were determined using steepest gradient, upper limit, and lower limit dispersion picks determined from $p-f$ plots for each line [Figures 6.11-6.14]. 2D profiles illustrate lateral changes in the velocity structure along the line, resulting from differences in the picked dispersion curves, at the expense of the depth resolution obtainable in the 1D model [Figures 6.11A, 6.13A]. At each end segment of the 2D profiles, corresponding to instruments 1-24 and 49-72 for Line A [Figure 6.11B,C] and 1-24 and 61-72 for Line B [Figure 6.13B,C], we constrained layer boundaries using borehole information from nearby wells. We applied $10 \%$ smoothing, carried out within the Disper program, to interpolate each 1D model segment into a $2 \mathrm{D}$ profile. Interface depths and layer velocities for the upper limit and lower limit manual inverse models are listed in Tables 6.3 and 6.4, while the interface depths and velocities for the steepest gradient set of picks are displayed directly on Figures 6.116.14. Root mean square error (RMS) between the picks and the modeled dispersion 
curve was calculated for each manual inverse model [Table 6.2] and used to drive the number of layers used in the inversion. For the manual inverse models, we used the minimum number of layers necessary to bring the RMS to a value of 5 or lower.

\begin{tabular}{|c|c|c|c|c|c|}
\hline \multicolumn{6}{|c|}{ Manual Inverse Model Interface Depths for Picks (m) } \\
\hline Segment & Int. 1 & Int. 2 & Int. 3 & Int. 4 & Int. 5 \\
\hline Line A 2D 1-24 & $\underline{2}(2 / 2)$ & $\underline{4}(4 / 4)$ & $\frac{20}{(21 / 23)}$ & $\underline{77}(55 / 75)$ & \\
\hline Line A 2D 49-72 & $\underline{2}(2 / 2)$ & $\underline{5}(5 / 5)$ & $\begin{array}{l}\frac{14}{(14 / 14)} \\
\text { (14) }\end{array}$ & $\underline{53}(53 / 53)$ & $\underline{117}(98 / 90)$ \\
\hline Line A 1D & $\underline{3}(3 / 3)$ & $\underline{8}(8 / 8)$ & $\frac{13}{(14 / 13)}$ & $\underline{77}(79 / 73)$ & \\
\hline Line B 2D 1-24 & $\underline{4}(4 / 4)$ & $\begin{array}{l}\frac{64}{(38 / 64)} \\
\text { (38) }\end{array}$ & & & \\
\hline Line B 2D 61-72 & $\underline{2}(2 / 2)$ & $\underline{5}(5 / 5)$ & $\frac{13}{(23 / 13)}$ & $\underline{42}(44 / 36)$ & \\
\hline Line B 1D & $\underline{2}(1 / 2)$ & $\underline{5}(4 / 5)$ & $\frac{43}{(47 / 43)}$ & $\frac{106}{(90 / 106)}$ & \\
\hline
\end{tabular}

Table 6.3: Manual inverse model interface depths for picks [steepest gradient (upper limit / lower limit)] for each 1D and 2D model presented.

Automatic inverse models were computed using the Dinver inversion package to provide a comparison to the manual inverse models [Wathelet, 2008]. Each inversion provided approximately 5000 models which minimized the misfit for each model. The automatic inverse models were bounded by the same geological constraints as their manual inverse model counterparts unless otherwise stated. Interface depths and layer velocities for each best-fit automatic inverse model of minimum misfit are listed in Tables 6.5 and 6.6. Please refer to Section 4.2 for additional information on the Dinver package. 


\begin{tabular}{|c|c|c|c|c|c|c|}
\hline \multicolumn{7}{|c|}{ Manual Inverse Model Layer Velocities for Picks (m/s) } \\
\hline Segment & Layer 1 & Layer 2 & Layer 3 & Layer 4 & Layer 5 & Layer 6 \\
\hline Line A 2D 1-24 & $\begin{array}{l}\frac{160}{(150-} \\
160)\end{array}$ & $\begin{array}{l}\frac{120}{(110-} \\
120)\end{array}$ & $\begin{array}{l}\frac{300}{(280-} \\
340)\end{array}$ & $\begin{array}{l}\frac{420}{(370-} \\
470)\end{array}$ & $\begin{array}{l}\frac{690}{(550-} \\
800)\end{array}$ & \\
\hline $\begin{array}{l}\text { Line A 2D 49- } \\
72\end{array}$ & $\frac{70}{90)}$ & $\begin{array}{l}\frac{240}{(210-} \\
260)\end{array}$ & $\begin{array}{l}\frac{170}{(170-} \\
180)\end{array}$ & $\begin{array}{l}\frac{310}{(280-} \\
390)\end{array}$ & $\begin{array}{l}\frac{430}{(380-} \\
520)\end{array}$ & $\begin{array}{l}\frac{690}{(460-} \\
780)\end{array}$ \\
\hline Line A 1D & $\begin{array}{l}\frac{120}{(120-} \\
130)\end{array}$ & $\begin{array}{l}\frac{210}{(230-} \\
250)\end{array}$ & $\begin{array}{l}\frac{160}{(150-} \\
180)\end{array}$ & $\begin{array}{l}\frac{360}{(340-} \\
450)\end{array}$ & $\begin{array}{l}\frac{740}{(660-} \\
780)\end{array}$ & \\
\hline Line B 2D 1-24 & $\begin{array}{l}\frac{130}{(130-} \\
140)\end{array}$ & $\begin{array}{l}\frac{340}{(310-} \\
380)\end{array}$ & $\begin{array}{l}\frac{450}{(330-} \\
530)\end{array}$ & & & \\
\hline Line B 2D 61-72 & $\begin{array}{l}\frac{160}{(140-} \\
160)\end{array}$ & $\begin{array}{l}\frac{120}{(120-} \\
120)\end{array}$ & $\begin{array}{l}\frac{430}{(420-} \\
500)\end{array}$ & $\begin{array}{l}\frac{330}{(270-} \\
330)\end{array}$ & $\begin{array}{l}\frac{450}{(390-} \\
580)\end{array}$ & \\
\hline Line B 1D & $\begin{array}{l}\frac{110}{(110-} \\
100)\end{array}$ & $\begin{array}{l}\frac{150}{(140-} \\
150)\end{array}$ & $\begin{array}{l}\frac{360}{(340-} \\
410)\end{array}$ & $\begin{array}{l}\frac{460}{(450-} \\
510)\end{array}$ & $\begin{array}{l}\frac{530}{(490-} \\
610)\end{array}$ & \\
\hline
\end{tabular}

Table 6.4: Manual inverse model layer velocities for picks [steepest gradient (lower limit - upper limit)] for each 1D and 2D model presented.

\begin{tabular}{||l||l|l|l|l|l||}
\hline \multicolumn{5}{||c||}{ Best-Fit Automatic Inverse Models Interface Depths (m) } \\
\hline Segment & Int. 1 & Int. 2 & Int. 3 & Int. 4 & Int. 5 \\
\hline Line A 2D 1-24 & 2 & 4 & 15 & $77(C)$ & \\
\hline Line A 2D 49-72 & 1 & 3 & 13 & 50 & $117(C)$ \\
\hline Line A 1D & 4 & 14 & 33 & 72 & \\
\hline Line B 2D 1-24 & 5 & 59 & & & \\
\hline Line B 2D 61-72 & 6 & 8 & 12 & 45 & \\
\hline Line B 1D & 3 & 4 & 9 & 52 & \\
\hline
\end{tabular}

Table 6.5: Layer interface depths for the best-fit automatic inverse models calculated using the Dinver. Symbol C represents a modeling constraint in the manual and automatic inverse models. 


\begin{tabular}{||l||l|l|l|l|l|l||}
\hline \multicolumn{7}{||c||}{ Best-Fit Automatic Inverse Models Layer Velocities (m/s) } \\
\hline Segment & Layer 1 & Layer 2 & Layer 3 & Layer 4 & Layer 5 & Layer 6 \\
\hline Line A 2D 1-24 & 130 & 120 & 260 & 400 & 680 & \\
\hline $\begin{array}{l}\text { Line A 2D 49- } \\
72\end{array}$ & 100 & 130 & 170 & 300 & 410 & 700 \\
\hline Line A 1D & 142 & 180 & 270 & 340 & 490 & \\
\hline Line B 2D 1-24 & 150 & 350 & 490 & & & \\
\hline Line B 2D 61-72 & 140 & 300 & 760 & 320 & 420 & \\
\hline Line B 1D & 110 & 180 & 220 & 330 & 450 & \\
\hline
\end{tabular}

Table 6.6: Best-fit automatic inverse model layer velocity for each 1D and 2D model presented. 


\subsection{Results Line A}

Line A was deployed NW-SE between injection wells NM8, NM9, and NM2 [Figure 6.1]. There is a $39 \mathrm{~m}$ elevation difference between the ends of the array. A $2 \mathrm{D}$ profile was constructed using manual inverse models of 24-instrument segments with 6-instrument overlap and constrained at each end using borehole information [Figure 6.11]. Higher and lower limit manual inverse models created from upper and lower limit picks are also displayed for instruments 1-24 and 49-72 [Figure 6.11B, C]. The 2D profile displayed [Figure 6.11A] was created using the manual inverse models from the steepest gradient set of picks.

We used the depth of the Huka Falls formation top interface, approximately 72-77 meters for instruments 1-24 [Lewis et al., 2012, 2013a] and 117 meters for instruments 49-72 [Hedenquist, 1980] [Figure 6.11C], to constrain our deepest modeled layer. The 117 meter depth of the Huka Falls formation for segment 49-72 had very little effect on the model as it exceeds our imaging depth [Figure 6.11C, Table 6.2]. Due to this, the models for the upper and lower limit picks are not constrained using this depth.

The Huka Falls formation is approximately 100-400 meters thick in this region [Chambefort et al., 2014]. Our lowest frequency steepest gradient 1D and 2D picks have frequencies and velocities of $1.8 \mathrm{~Hz}$ and $400 \mathrm{~m} / \mathrm{s}, 2.2 \mathrm{~Hz}$ and $408 \mathrm{~m} / \mathrm{s}$, respectively, giving theoretical resolution depths of 109 and 93 meters respectively [Equation 6.15, Table 6.2]. Therefore, we infer that the Huka Falls formation, even at its thinnest, would be the deepest formation sampled. Once a model for each end of the array was determined and constrained, we developed manual inverse models for the middle segments, keeping the number of layers constant whenever possible. To reduce the degrees of freedom in the model, we fixed the shear-wave velocity of the deepest layer throughout the profile to $690 \mathrm{~m} / \mathrm{s}$, the velocity which produced the lowest RMS value in the steepest gradient model for the 1-24 instrument segment [Figure 6.11B].

The 1D manual inverse model based on steepest gradient picks is composed of 5 layers and contains a low-velocity layer approximately 4 meters thick with a velocity of $130 \mathrm{~m} / \mathrm{s}$ [Figure 6.12]. Two sets of picks were determined for each of the three 1D models: one using $F_{\max }=30 \mathrm{~Hz}, V_{\min }=85 \mathrm{~m} / \mathrm{s}$ and the other with $F_{\max }=20 \mathrm{~Hz}$, $V_{\min }=150 \mathrm{~m} / \mathrm{s}$ [Table 6.2]. The latter set of parameters was necessary to isolate the low-frequency picks used to model the deepest layer.

Automatic inverse models constructed using the Dinver package were calculated for the 2D 1-24 [Figure 6.11B], 2D 49-72 [Figure 6.11C], and 1D picks [Figure 6.12B, Tables 6.5, 6.6]. The number of layers in each automatic inverse model was set to be the same number as the corresponding manual inverse model, and low-velocity layers were allowed. The automatic inverse models for 1-24 and 49-72 were constrained using the Oruanui and Huka Falls formations interface depths from the borehole informa- 
tion. The 1-24 automatic inverse model with minimum misfit contained a low-velocity layer at 2 meters depth with approximately the same thickness and velocity as that of the manual inverse model [Figure 6.11B, Tables 6.5 and 6.6], but the automatic inverse 49-72 model did not reproduce a low-velocity layer. 

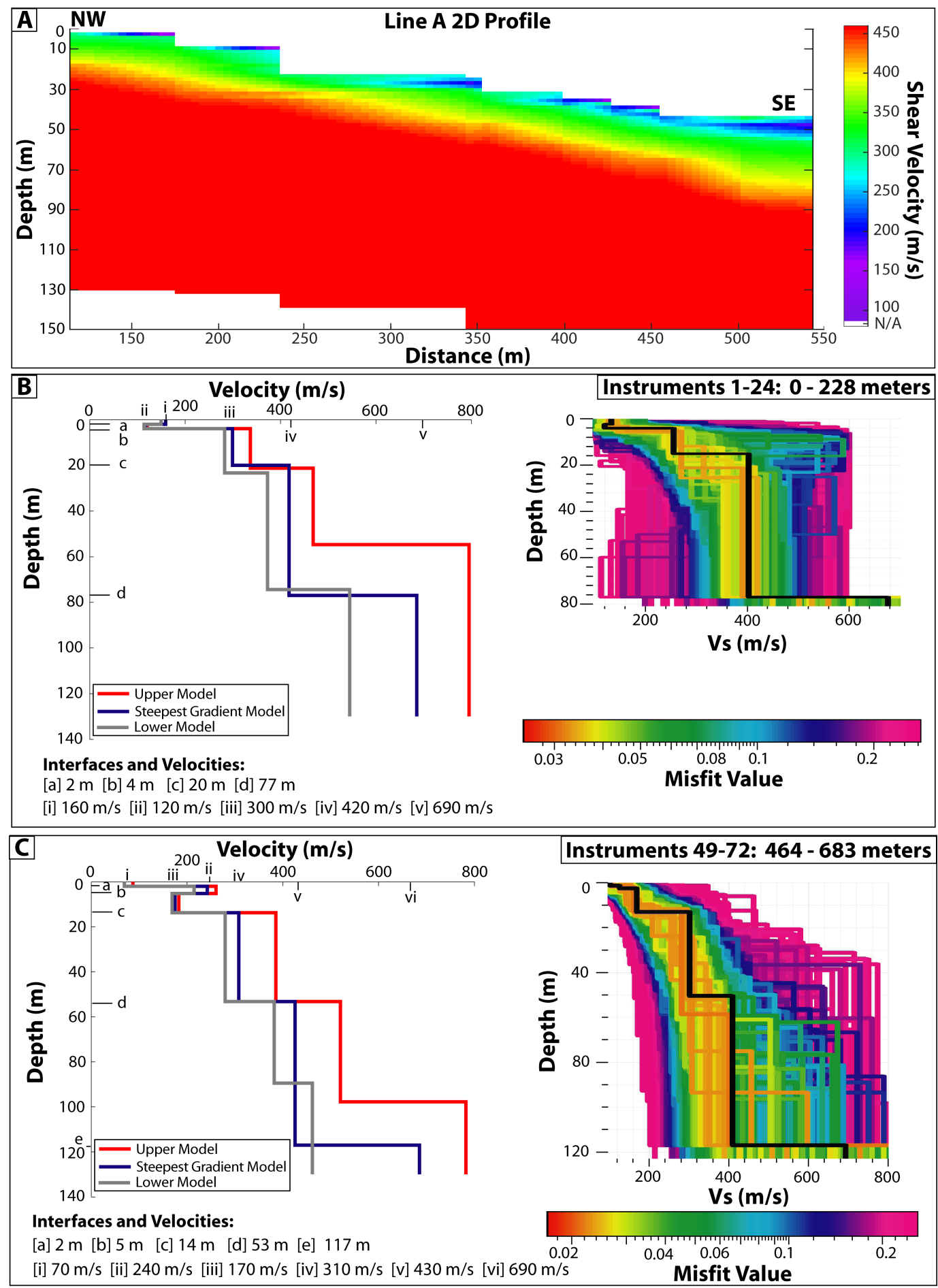

Figure 6.11: [A] 2D shear-velocity profile using 24-instrument segments with 6instrument overlap for Line A. Depth of 0 corresponds to the midpoint of sensors 1-24 (distance 114 meters along array, elevation 374 meters). [B] [Left] Manual inverse models for steepest gradient (blue), upper (red) and lower (gray) dispersion and [Right] automatic inverse models for instruments 1-24 (0-228 meters). [C] [Left] manual inverse models for steepest gradient (blue), upper (red) and lower (gray) limit picks and [Right] automatic inverse models for instruments 49-72 (464-683 meters). The best-fit automatic inverse model is displayed in black in the right hand panel. 

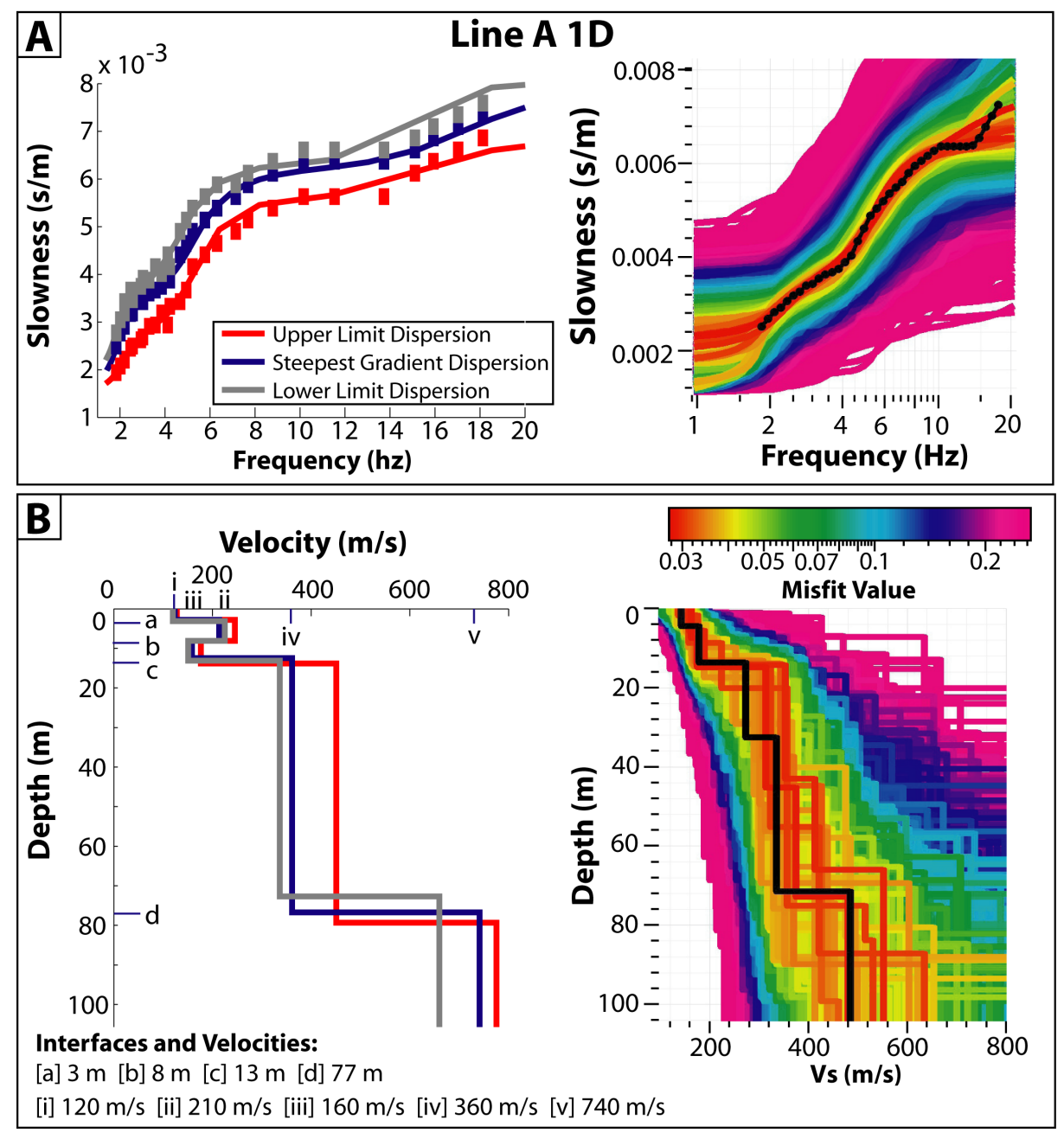

Figure 6.12: Dispersion picks and manual and automatic inverse models for Line A 1D. [A] [Left] Picks and manual inverse model dispersion for steepest gradient (blue), upper limit (red), and lower limit (gray) picks ; [Right] Dispersion from automatic inverse models using the steepest gradient pick set. [B] [Left] Corresponding manual inverse models for each of the dispersion lines and [Right] automatic inverse models obtained from the steepest gradient dispersion picks. The best-fit automatic inverse model is displayed in black in the right hand panel. 


\subsection{Results Line B}

Line B trends North-South away from production well NM7 and the Ngatamariki power station and towards production wells NM5 and NM11, and has an elevation change of $3.9 \mathrm{~m}$ [Figure 6.1]. To model this line, 24-instrument segments with 12instrument overlap were chosen initially to describe the 2D profile, but the 24-instrument segments were found to give large RMS values near the southern end. Where significant changes in the $1 \mathrm{D}$ velocity profiles are noted, additional sub-arrays are required to adequately characterize structure changes along the length of the line. Therefore, the 24-instrument segments were changed to 12-instrument segments with one instrument overlap starting with the band 57-68 until the end of the line.

The depth to the top of the Huka Falls formation is marked as $92 \mathrm{~m}$ for NM7 [Rae et al., 2009], but no cuttings were collected for wells NM5 and NM11 at those depths [Ramirez and Rae, 2009, Lewis et al., 2013b]. The lowest-frequency pick for the 124 instrument segment of the 2D line was $2.7 \mathrm{~Hz}$ with a velocity of $314 \mathrm{~m} / \mathrm{s}$, which gives a theoretical maximum sampling depth of $57 \mathrm{~m}$ [Table 6.2]. Therefore, the 92 $\mathrm{m}$ interface of the Huka Falls formation was too deep to constrain the North end in the profile. Based on our modeling results of Line A [Figure 6.11], and the interface depth we could observe with our picks [Table 6.2], we decided to start the 2D profile using a three-layer model [Figure 6.13B]. In order to reduce the degrees of freedom of the model, the velocity of the lowest layer in the 1D 1-24 model (450 m/s) was kept consistent while manually modeling the 2D profile.

We detected a lateral discontinuity in the profile approximately 560 meters along the line using the overlapping 12-instrument sub-arrays [Figure 6.13A]. Although the beginning of the line could be modeled with a three-layer model [Figure 6.13B], a highvelocity layer approximately 8 to 10 meters in thickness below a depth of approximately $5 \mathrm{~m}$ was necessary to fit the picks of the 12-instrument segment starting from that point [Figures 6.13A, 6.15]. We determined that a five-layer model was optimal to adequately describe the change. The number of layers required to fit the dispersion curve in the manual inverse method was controlled by the RMS: we used the minimum amount of layers necessary to obtain an RMS equal to or less than 5 [Figure 6.15]. Similarly, a five-layer model for the beginning of the array is not necessary, as a three layer model can fit the data to an acceptable RMS value [Table 6.2]. In the 61-72 segment, the shallow low-velocity layer previously modeled in Line A [Figure 6.11, Figure 6.12] is also resolved.

The manual inverse model produced using upper limit picks placed the depth of the deepest interface for segment 1-24 approximately 26 meters shallower than that corresponding to lower and steepest gradient picks [Figure 6.13B]. For segment 6172 , both the upper and lower-limit manual inverse models required a shallow high- 
velocity layer starting at the same depth as the steepest gradient model [Figure 6.13C].

A 1D manual inverse model was developed using all 72 channels with an RMS of $2.9 \mathrm{~m} / \mathrm{s}$ [Figure 6.14]. The main layer interfaces were determined to be at 2, 5, 43, and 106 meters. The lowest frequency pick observed for this $1 \mathrm{D}$ line was $1.3 \mathrm{~Hz}$ with a slowness of slowness of $0.00236 \mathrm{~s} / \mathrm{m}$, which corresponds to a depth resolution of 165 meters [Table 6.2]. The depth of the interface for the top of the Huka Falls formation is known to be approximately 92 meters for NM7 [Rae et al., 2009], but is not known for NM 5 or NM 11 [Ramirez and Rae, 2009, Lewis et al., 2013b]. Due to this, the depth of the deepest interface was not constrained during the modeling for the 1D profile. The resulting depth interface for the highest velocity layer was 106 meters.

Automatic inverse models were run using the steepest gradient picks from the 2D and 1D segments utilizing the same number of layers as the manual inverse models and allowing low-velocity layers. The best-fit automatic model contains a highvelocity layer like that determined manually in the 61-72 segment, although it is approximately $330 \mathrm{~m} / \mathrm{s}$ faster than its manual counterpart [Figure 6.13C, Table 6.6]. 

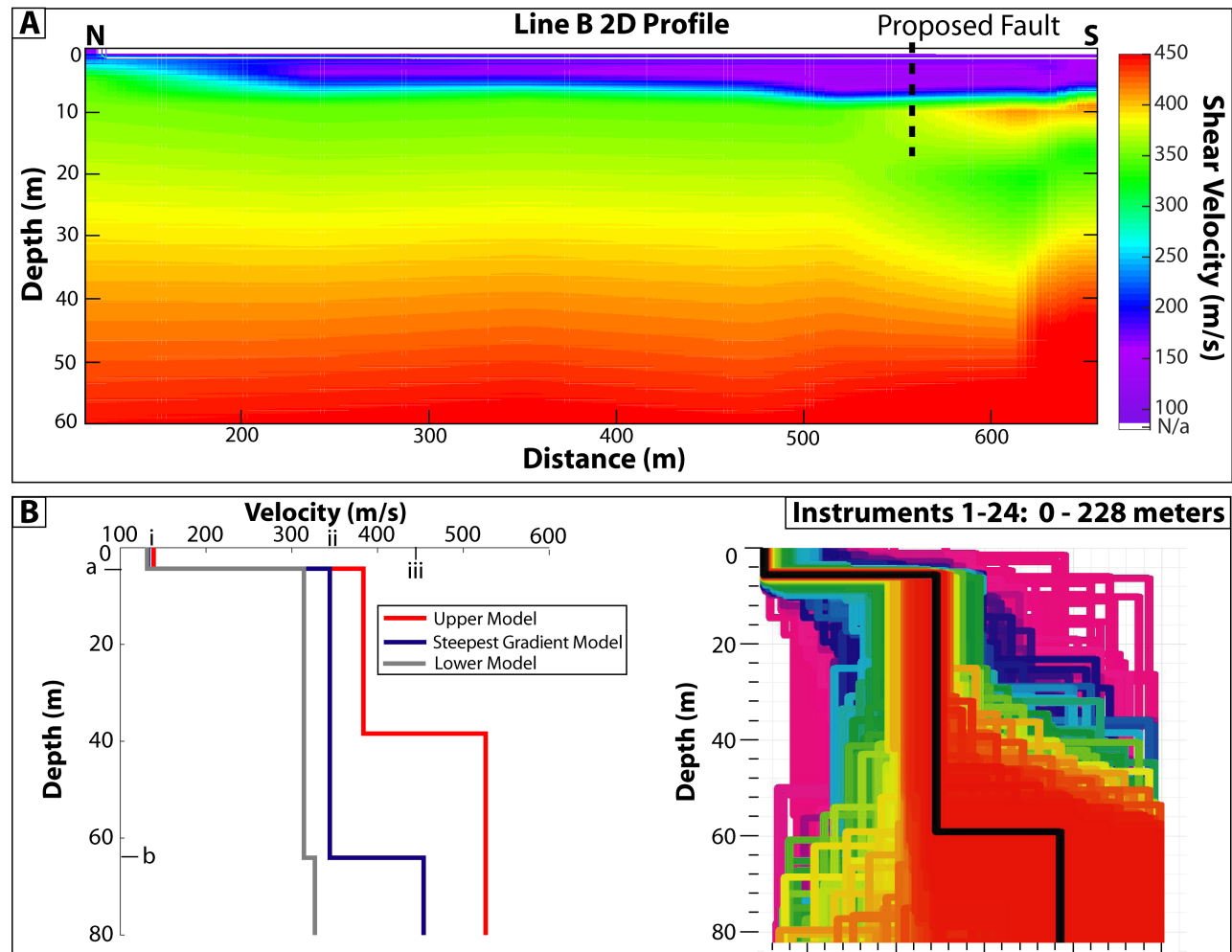

Interfaces and Velocities:

[a] $4 \mathrm{~m}$ [b] $64 \mathrm{~m}$

[i] $130 \mathrm{~m} / \mathrm{s}$ [ii] $340 \mathrm{~m} / \mathrm{s}$ [ii] $450 \mathrm{~m} / \mathrm{s}$
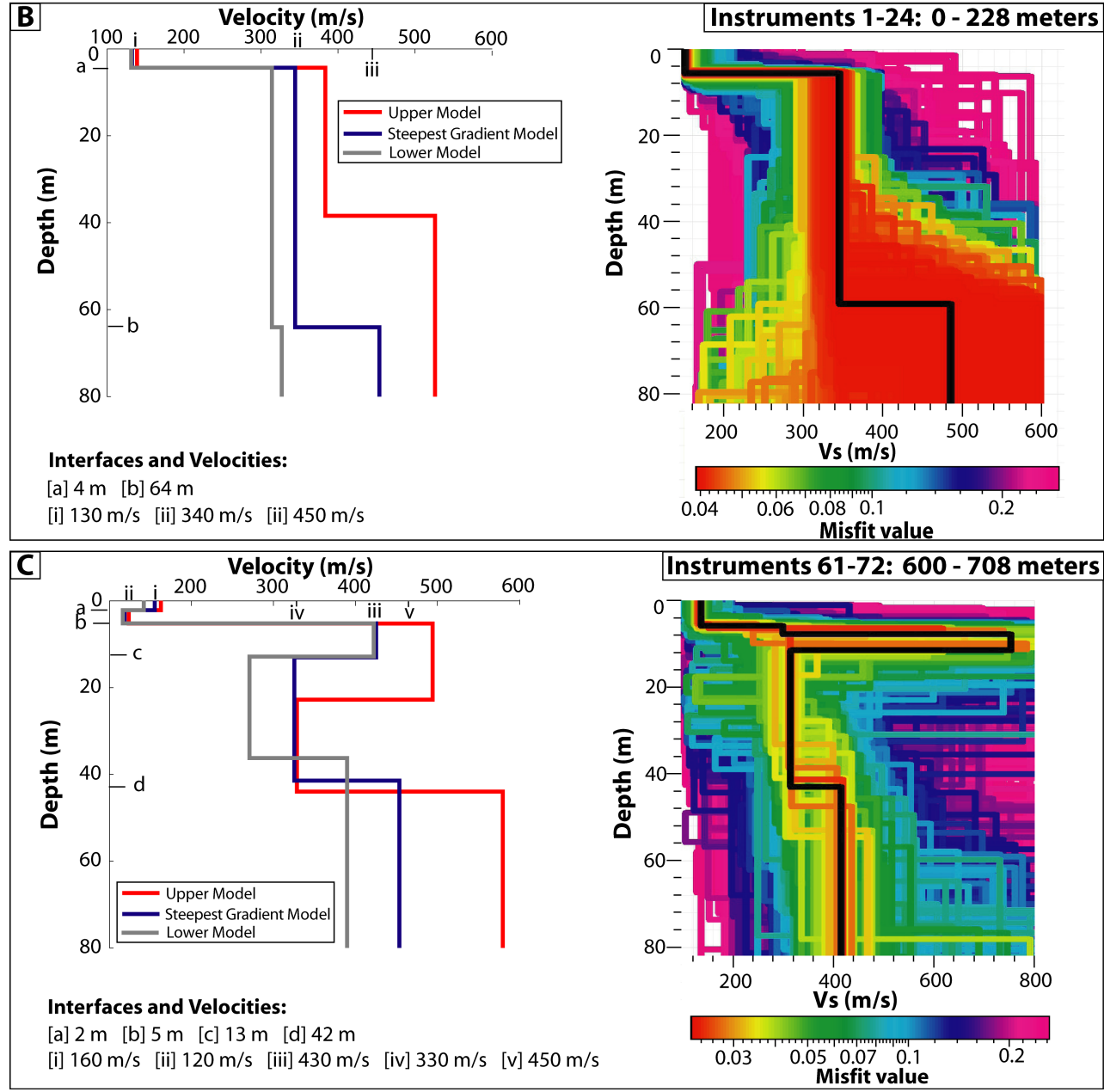

Figure 6.13: [A] 2D shear-velocity profile for Line B. 24-instrument segments with 12-instrument overlap were used until segment 57-68, where it was switched to 12instrument segments with 1 instrument overlap. Depth of 0 corresponds to the midpoint of sensors 1-24 (distance 115 meters along array, elevation 385 meters). [B] [Left] Manually inverted models for steepest gradient (blue), upper (red) and lower (gray) dispersion and [Right] automatic inverse models for instruments 1-24 (0-230 meters). [C] [Left] Manually inverted models for steepest gradient (blue), upper (red) and lower (gray) dispersion and [Right] automatic inverse models for instruments 61-72 (600-708 meters). The best-fit automatic inverse model is displayed in black in the right hand panel. 

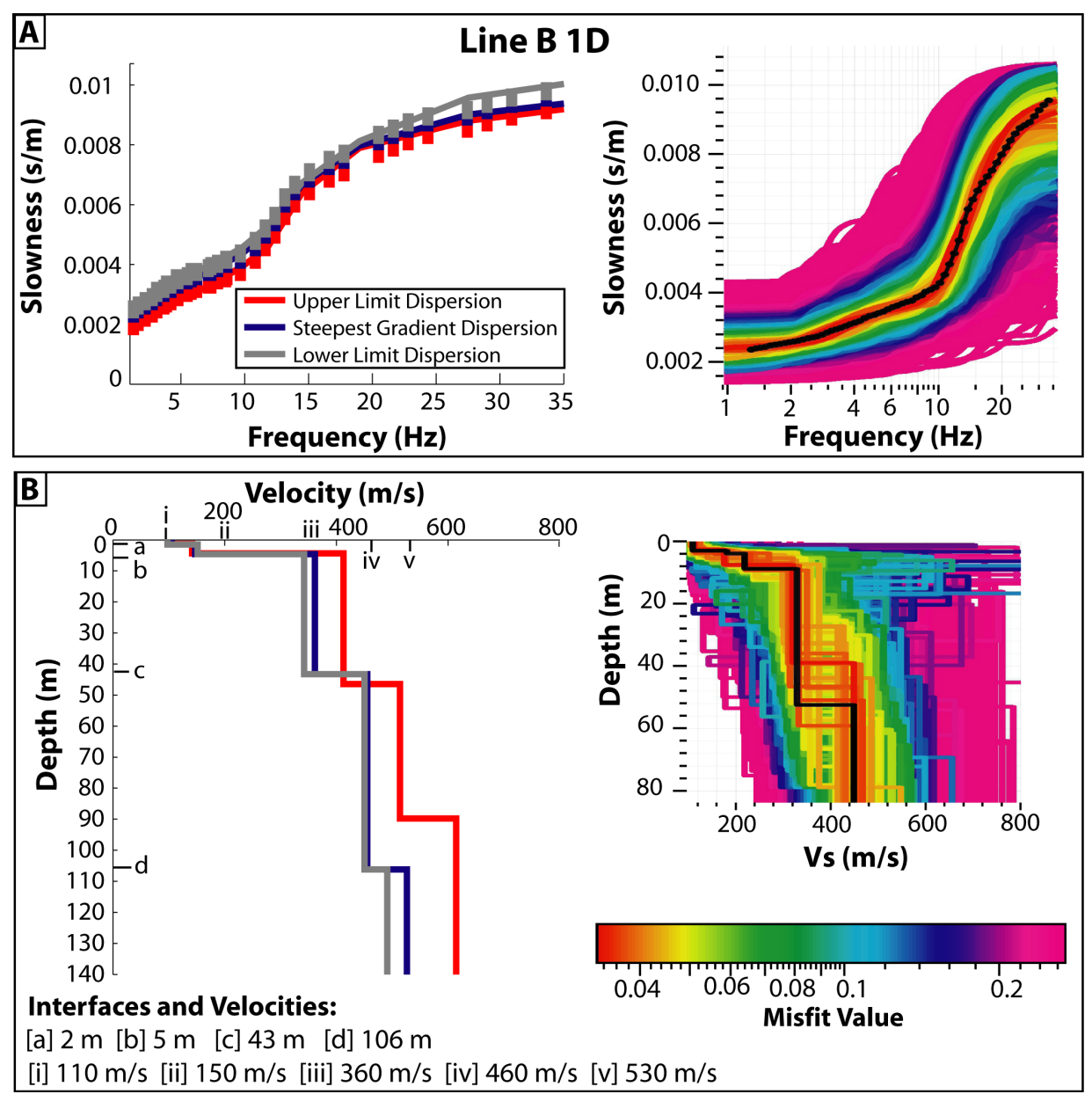

Figure 6.14: Dispersion picks and manual and automatic inverse models for Line B 1D. [A] [Left] Picks and manual inverse model dispersion for steepest gradient (blue), upper limit (red), and lower limit (gray) picks ; [Right] Dispersion from automatic models using the steepest gradient pick set. [B][Left] Corresponding manual inverse models for each of the dispersion lines and [Right] automatic inverse models obtained from the steepest gradient dispersion picks. The best-fit automatic inverse model is displayed in black in the right hand panel. 

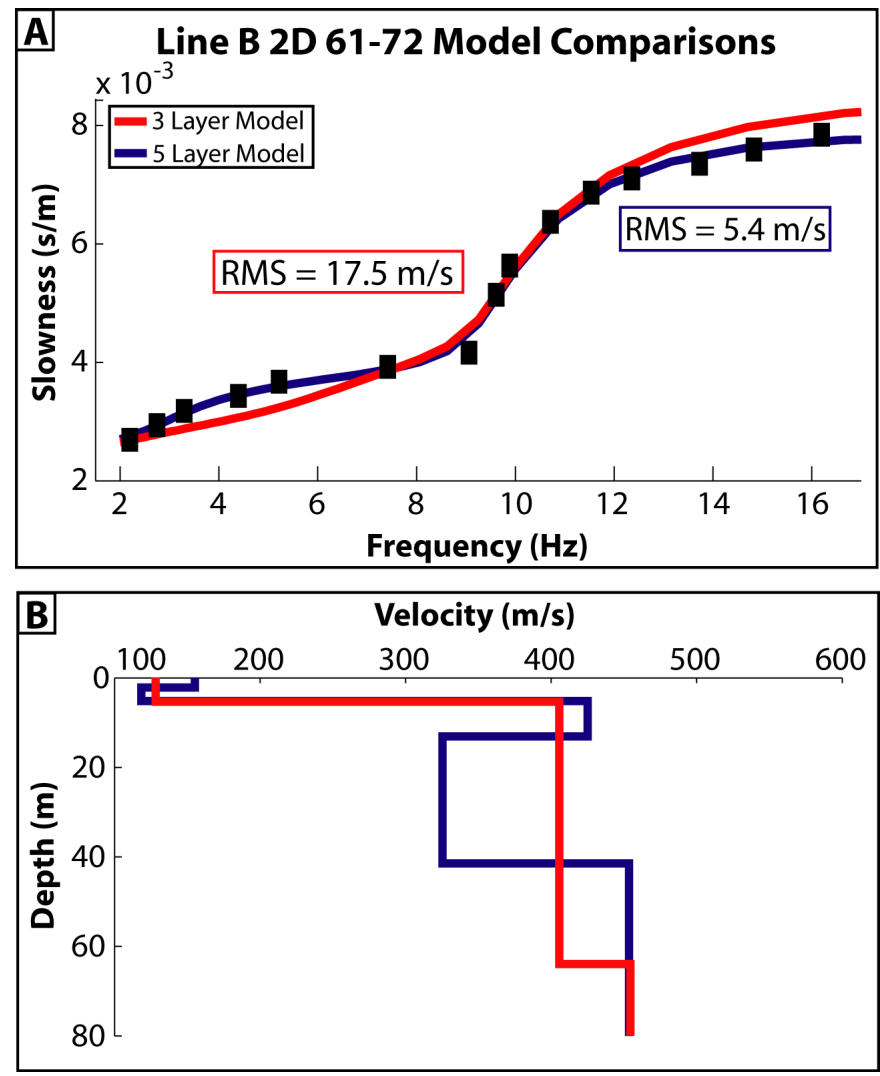

Figure 6.15: Comparisons of a three-layer model and the five-layer model used in Figure 6.13C for the Line B 2D segment 61-72. [A] Manually modeled dispersion curves with picks. [B] Corresponding 1D models for each of the dispersion curves. 


\subsection{Discussion}

Each of the manual inverse models presented was optimized for a minimum number of layers and constrained by available geological information. The interface depth between the Oruanui and Huka Falls formations for the 2D analysis of Line A could be constrained by borehole logs of NM2, NM9, and NM8. We interpret Layers 3 and 4 in the 1-24 segment profile, Layers 4 and 5 in the 49-72 segment profile, and Layer 4 in the 1D profile to correspond to the Oronui formation ignimbrite due to their positioning above the Huka Falls formation. These layers have velocities ranging of $300-430 \mathrm{~m} / \mathrm{s}$ and thicknesses of 64 meters (Line A 1D), 71 meters (Line A 2D 1-24), and 103 meters (Line A 2D 49-72). The thickness of the Oruanui formation in the Line A 2D 49-72 model is an estimate, as we cannot resolve the top depth of the Huka Falls formation at 117 meters [Table 6.2]. The Oruanui ignimbrite was formed from an eruption containing 10 fall units [Wilson, 2001], and its current thickness in the geothermal field is unclear due to post-eruptive flooding and erosion [Manville and Wilson, 2004, Chambefort et al., 2014].

For each of our models for the Line A 2D representation, we determined a lowvelocity layer starting from a depth of approximately $2 \mathrm{~m}$ with a shear-velocity of 120 to $170 \mathrm{~m} / \mathrm{s}$ and a thickness of 2 and 9 meters [Figures 6.11,6.12]. We hypothesize that this feature corresponds to the layer of loess and gravels deposited prior to the $232 \mathrm{CE}$ Taupo eruption [Vucetich and Pullar, 1969, Chambefort et al., 2014], although the geology logs do not have the resolution to verify this at Ngatamariki. For the Line A 2D 49-72 instrument segment and the 1D model, we observe a layer of higher velocity at a depth of 2 meters with shear-velocity and thickness values of $240 \mathrm{~m} / \mathrm{s}$ and 3 meters and $210 \mathrm{~m} / \mathrm{s}$ and 4 meters respectively [Figure 6.11]. We hypothesize that this observed high-velocity layer is the Taupo ignimbrite, deposited during the CE 232 eruption [Wilson, 1985]. These deposits can range in thickness between 10 and 20 meters but are heavily eroded [Chambefort et al., 2014]. The absence of this layer in the 1-24 segment could be because it is an area where the ignimbrite was particularly eroded or otherwise too thin to be detected. Overall, the 1D and 2D profiles are consistent with one another and match well the known geology. Chambefort et al. [2014] inferred a fault approximately halfway between NM4 and NM2 [Figure 6.1], but no velocity discontinuities were observed along our Line A 2D profile. This suggests that the fault zone was too oblique and distant to the line or no displacement could be discerned at the depths we sampled.

Most of the Line B 2D profile could be modeled with a three layer model [Figure 6.13B]. We hypothesize that the top layer for the Line B 1-24 segment is composed of surface deposits due to similar velocities in the top layer of the Line A 2D and 1D models (10-60 m/s of difference). Additionally, the shallow high-velocity and low- 
velocity layers, attributed in Line A to the Taupo ignimbrite and loess and gravel layer, are not detected. Layers 2 and 3 at 4 and 64 meters depth are interpreted to be part of the Oruanui formation, as they are within $30 \mathrm{~m} / \mathrm{s}$ of the $300-430 \mathrm{~m} / \mathrm{s}$ velocity range of the Oruanui formation detected in Line A, and geology logs at NM7 locate the boundary with the Huka Falls formation at 92 meters depth [Rae et al., 2009].

A high-velocity layer, with thickness between 8 and 11 meters and velocity between 410 and $430 \mathrm{~m} / \mathrm{s}$, was detected starting at approximately 560 meters along the profile of Line B [Figure 6.13]. We compared the steepest gradient five-layer model for the 61-72 segment [Figure 6.13C] using the previously established three layer model [Figure 6.13B], but were unable to modify the velocities and depths of the upper two layers to successfully model the picks [Figure 6.15]. A layer of high-velocity was also revealed by automatic inverse models [Figure 6.13C]. The steepest gradient best fit automatic inverse model had a velocity approximately $330 \mathrm{~m} / \mathrm{s}$ greater than our manual model but a layer thickness of 4 meters, which is approximately half the 8 meter thickness of our steepest gradient manual model [Tables 6.5 and 6.6, Figure 6.13C]. This high-velocity layer is also detected in both the lower and upper limit pick models [Figure 6.13C].

We hypothesize that this high velocity layer is caused by a fault crossing the line starting at approximately 560 meters. Chambefort et al. [2014] inferred the presence of a fault between NM7 and NM5 from well logs but could not constrain its location. When projected on our line, Chambefort et al. [2014]'s current inferred location would cross between instruments 5 to 12 in the north end of Line A, corresponding to approximately 50-120 meters along the line [Figure 6.1], which we would have observed in our first 1-24 segment band if this were the correct location. This fault was recognized by the uplift of formations at NM5 and NM11 relative to NM7 [Rae et al., 2009, Ramirez and Rae, 2009, Lewis et al., 2013b, Chambefort et al., 2014], which could explain the higher observed velocities and the step observed for the bottom layer at approximately 610 meters down the profile [Figure 6.13A].

The shallow low-velocity layer and high-velocity discontinuity were not detected in the 1D profile of Line B [Figure 6.14]. This is not surprising, as the 1D profile is an average across the entire line and most of the $2 \mathrm{D}$ profile did not observe these features. Based on the NM7 borehole log, we interpret the deepest manually modeled layer, with an interface depth of 106 meters to be the Huka Falls formation. Layers 3 and 4, which have a combined thickness of 102 meters [Figure 6.14B], are interpreted to be the Oruanui formation, although the modeled velocity and interface depth should be taken as approximate, given that the line has contributions from the high-velocity layer observed in the 2D profile.

The five-layer best-fit automatic inverse model for 1D Line B did not accurately constrain the deepest layer interface, although its velocity of $450 \mathrm{~m} / \mathrm{s}$ was within $80 \mathrm{~m} / \mathrm{s}$ of our manual inverse modeled value. Instead, the most likely model fit an additional 
layer within the top 10 meters. This is because each pick in the automatic inversion routine is weighed the same, and there are more picks in the high frequencies than in the low.

Due to their low-frequency content, the background ambient noise records contained the most important information for determining deep shear velocities at Ngatamariki. The $p-f$ transformation scales the intensity based on a spectral ratio between the observed energy and the maximum energy of the plot parameters [Louie, 2001]. Because of this, the background noise of the geothermal field, although weaker in intensity than the vehicle signal or the hammer shots [Figure 6.4], provided superior dispersion curve picks for lower frequencies [Figure 6.8A], which were essential for determining the deep layer interfaces and velocities of the models. The geophones used in this study have a corner frequency of $10 \mathrm{~Hz}$, so the observed amplitude decreases for frequencies lower than $10 \mathrm{~Hz}$ [Figure 6.4]. Due to the large amplitude at $7 \mathrm{~Hz}$ recorded for the background noise [Figure 6.4A], it is likely that the geothermal field has significant energy at frequencies lower than observed in this study. This suggests that a similar analysis conducted with instruments of lower corner frequencies and with longer lines would yield resolution to significantly deeper depths.

These results suggest that the ReMi method could be applied to detect areas of subsidence within geothermal fields. Although subsidence is not observed at Ngatamariki, areas of anomalously high subsidence (up to 2-3 meters) often form within fields, which can cause damage to the built environment. At the Tauhara geothermal field in New Zealand, this was shown through shallow core drilling to be due to anomalous, mechanically weak, rock within the top 400 meters of the field [Lynne et al., 2013]. As it provides shear-wave velocities in the upper hundreds of meters, the ReMi method may be an effective way of mapping these zones of anomalously soft material, offering a much cheaper alternative to drilling.

\subsection{Conclusions}

We deployed two Refraction Microtremor lines at the Ngatamariki geothermal field and assessed the utility of the method for determining subsurface shear velocities. To our knowledge, this is the first application of the ReMi method at a producing geothermal field. The arrays were comprised of 72-channel, $10 \mathrm{~Hz}$ vertical geophones with 10 meter spacing, and recorded background, vehicle, and hammer shot noise sources in thirty-second records. Manual inverse models were produced for each line using steepest gradient, upper limit, and lower limit dispersion measurements obtained from the $p-f$ transform of the time series records, and were compared to best-fit automatic inverse models obtained using the Dinver software package. Modeled depths using steepest gradient picks ranged between 57 to 93 meters for the 2D profiles and up to 
165 meters for 1D profiles using all 72 channels. A velocity discontinuity was detected starting at approximately 560 meters distance along Line B, and is spatially correlated with an inferred fault. The low-frequency background signal of the geothermal field, most likely produced by the injection pipes along Line A and near the Ngatamariki Power Station and production and injection pipelines in Line B, was superior to the driving and hammer signals for modeling the deepest layers.

The low-frequency background noise of the geothermal field was ideal for imaging deep layers, and we expect that deeper depths, perhaps between 500 meters and 1 kilometer, can be reached by this method if longer lines and instruments with a lower corner frequency are used. 


\section{Chapter 7}

\section{Summary of main results and opportunities for further research}

In this manuscript, we present results from four project chapters in which we use ambient noise to determine shear velocities. We have made the following contributions:

Chapter 3 - Noise analysis and dispersion of the Harrat Al-Madinah volcanic field, Saudi Arabia:

1. This project was the first application of the ambient noise method to compute cross-correlations in Saudi Arabia, which expands the potential for surface wave studies in the region.

2. We computed group and phase Rayleigh and Love velocity maps for the volcanic field surrounding the city of Medina. We resolved a low velocity area co-located with Madinah Basalt geologic structure and the 1245 CE Medina eruption location.

Chapter 4 - Shear-wave tomography of the Harrat Al-Madinah volcanic field, Saudi Arabia:

1. We obtained 1D shear-velocity models with depth for RR, TT, and ZZ components using the surface-wave maps produced in Chapter 3. A joint inversion using all three components was also conducted. Average velocity models were calculated for the field, as well as for two subsets corresponding to an area encompassing the 1256 AD eruption and an area west of the city of Medina off the Harrat basalt.

2. We resolved a velocity increase in the $1 \mathrm{D}$ models representative of a layer change at approximately $20 \mathrm{~km}$ depth. We compared the obtained layer velocities with a previous P-wave refraction study [Mooney et al., 1985] using an approximate Pwave to S-wave speed conversion [Stein and Wysession, 2003] and found that the 
velocities are similar [Table 4.3]. With this verification, the method can now be applied to other areas of Saudi Arabia with confidence. Additionally, the depth resolution of these velocity models (approximately $<40 \mathrm{~km}$ ) complements existing work done using teleseismic earthquakes [Yao et al., 2017].

3. Pseudo-3D shear-velocity models were computed for Harrat Al-Madinah by interpolating the 1D models. Two cross-sections across the volcanic field were compared to existing gravity data. Within the cross-sections, areas of low shearvelocity are spatially co-located with the 1256 CE Medina eruption location and low measurements of gravity. We hypothesize that the shear-velocity is being affected by magma intrusion in the crust, which had been previously detected in this area by P-wave tomography [Abdelwahed et al., 2016] and suggested as a possible cause for low gravity measurements [Langenheim et al., 2016].

Chapter 5 - Velocity variations at New Zealand geothermal fields:

1. We computed cross-correlations from natural ambient seismic noise at the Rotokawa and Ngatamariki geothermal fields despite non-ideal instrumentation and site conditions. Signals within the coda of the cross-correlations were used to obtain measurements of shear-velocity time variations.

2. Shear-velocity positive and negative time variations were found across the entire field using station-pair subsets, and velocity increases were located near injection wells using a straight-ray pseudo-tomography code.

3. We compare the full-field and localized changes with injection and production rates and daily rainfall. We hypothesize that the responses are driven by combination of three mechanisms: [1] rainfall raising the groundwater level, [2] compaction and settling of the injection layer, and [3] expansion and contraction of the formations above the injection layer.

Chapter 6 - Refraction Microtremor at Ngatamariki:

1. This project is the first application of the Refraction Microtremor method to determine shear velocities at a producing geothermal field.

2. The background signals produced by geothermal production were more effective than the vehicle and hammer active sources for modeling the deepest layers. The maximum imaging depth was between 57 to 93 meters for 2D profiles and up to 165 meters for 1D profiles.

3. A velocity discontinuity was detected in one of the Refraction Microtremor lines spatially correlated with an inferred fault, suggesting that this method could be used to cheaply constrain near-surface geology in geothermal regions. 
As discussed throughout this manuscript, ambient noise methods are scalable: the obtainable depth resolution is primarily dependent on the wavelength of the source compared to the inter-station distance [Shapiro and Campillo, 2004, Bonnefoy-Claudet et al., 2006, Bensen et al., 2007]. The projects presented in this thesis used different bandwidths of ambient noise frequencies, and as a result obtained shear-velocities at various depth scales. For the Harrat Al-Madinah tomography (Chapters 3 and 4) we used records of ocean wave natural noise ( $<1 \mathrm{~Hz}$ frequency) to obtain shear velocities up to 25 kilometers depth. In the geothermal fields of the Taupo Volcanic Zone, the station spacing was too small to use the full wavelength of the ocean waves as a signal, so we used surface wave scatterers to determine time-dependent shear-velocity changes within approximately $5 \mathrm{~km}$ depth. The Refraction Microtremor deployment used a 720 meter line of geophones at 10 meter spacing, and we used cultural noise in the field ( $>1 \mathrm{~Hz}$ frequency) and active sources to obtain shear velocities up to a depth of 165 meters. Although the differences in scales required contrasting processing routines to extract surface waves, the fundamental theory behind each project is the same.

There are many opportunities for expanding the research presented in this manuscript. The zero-point noise observed in the cross-correlations of the Saudi Arabia dataset suggests the presence of ambient noise body-waves [Gerstoft et al., 2008, Landes̀ et al., 2010, Zhan et al., 2010], which have been hypothesized as a method to obtain a much deeper depth resolution than is currently possible with ambient noise surface waves [Poli et al., 2012]. Additionally, the methodology we used to compute the ambient noise dispersion maps can be used to map out other volcanic fields in Saudi Arabia. We think that the lateral uncertainty measured for Harrat Al-Madinah (approximately 20-30 km) will be reduced for volcanic fields which have arrays with smaller interstation distance. Conversely, longer inter-station distances will allow increased depth imaging, which can be used to further constrain the observed crustal layer interface. Work to expand the ambient noise analysis conducted in Chapters 3 and 4 for the entire Saudi Arabia array is currently underway.

The results obtained at the New Zealand geothermal fields (Chapter 5) demonstrated that consistent results of velocity changes can be determined despite challenges in instrumentation and data quality. However, the results were limited at both fields due to sensor swaps, the corner frequency of the instrument response, and station outages. In particular, the Ngatamariki array was deployed concurrent to the well stimulation, so there isn't a quiet time segment that could be used for reference. The results could be compared with geothermal fields where there are several months of recorded data prior to the onset of production. Furthermore, the work could be expanded by computing MWCS across various filtering bounds, which could be combined with sensitivity kernels to produce a pseudo-measurement of velocity change at depth. This would be particularly useful when combined with the decorrelation 
methods described by Obermann et al. [2013], which could assess whether or not the upward-migrating deformation mechanism observed by Hillers et al. [2015] at the geothermal field in Basel is occurring at Rotokawa and Ngatamariki. This method could also be used to determine the contribution of increased groundwater level on the shear-velocity. Additionally, most literature compares velocity changes according to geothermal injection, but the effect of sustained production on shear velocities is not known [Jousset et al., 2010, Hillers et al., 2015]. Lastly, we think it may be possible to conduct ambient noise tomography across the two geothermal fields. Although the inter-station distance compared to the ambient noise wavelength is quite short, it may be possible to obtain values of shear-velocity for the north-south paths across both fields [Figure 5.1]. The velocity model could then be compared with those obtained using traditional earthquake tomography.

The exploratory Refraction Microtremor experiment at Ngatamariki [Chapter 6] demonstrated that shear velocities can be obtained at geothermal fields using cultural noise sources from geothermal production. This methodology could provide an effective way to locate zones of subsidence at geothermal fields at a much decreased cost compared to drilling. We think that the maximum depth resolution can be improved by using longer arrays and instruments with a lower corner frequency. 


\section{Appendix A}

\section{Harrat Al-Madinah supplementary information}

\section{A.1 Harrat Al-Madinah seismic stations}

\begin{tabular}{||l||l||l||}
\hline Instrument & Latitude & Longitude \\
\hline RHT01 & 24.2734 & 39.8051 \\
\hline RHT02 & 24.4842 & 40.0862 \\
\hline RHT03 & 24.2471 & 40.1718 \\
\hline RHT04 & 23.993 & 39.878 \\
\hline RHT05 & 23.909 & 39.161 \\
\hline RHT06 & 24.3765 & 39.1923 \\
\hline RHT07 & 24.6658 & 39.0403 \\
\hline RHT08 & 24.7095 & 39.5433 \\
\hline RHT09 & 24.7845 & 39.9147 \\
\hline RHT11 & 24.916 & 39.691 \\
\hline RHT13 & 24.208 & 39.365 \\
\hline RHT14 & 24.386 & 39.757 \\
\hline RHT15 & 24.173 & 39.839 \\
\hline
\end{tabular}

Table A.1: Location of Harrat Rahat seismic stations. 


\section{A.2 Harrat Al-Madinah Station Noise}

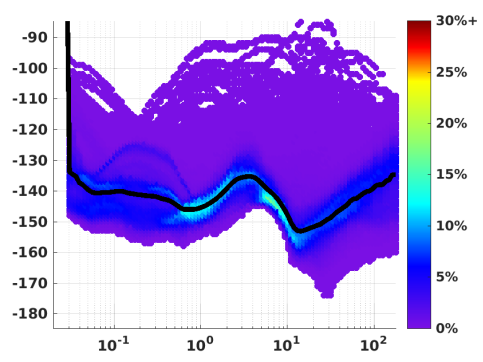

(a) RHT01 HHE

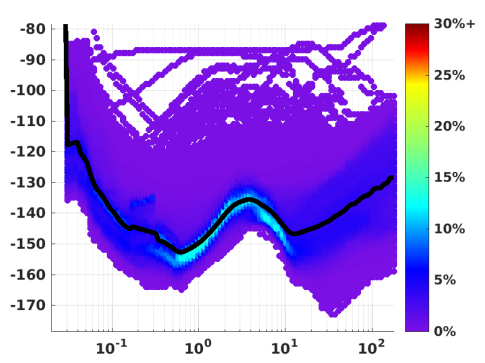

(d) RHT02 HHE

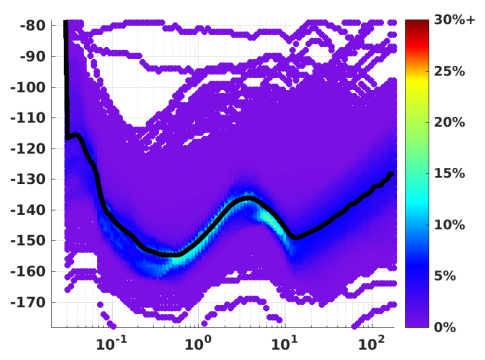

(g) RHT03 HHE

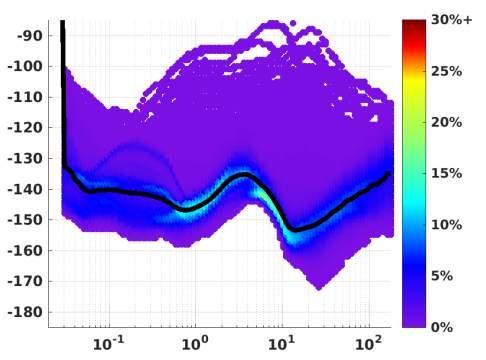

(b) RHT01 HHN

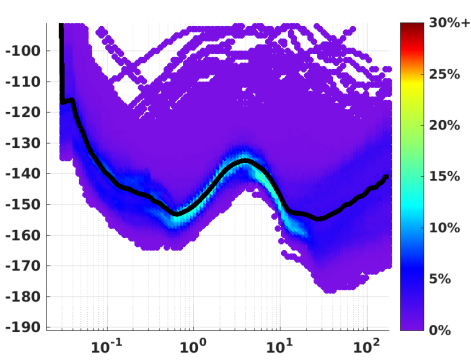

(e) RHT02 HHN

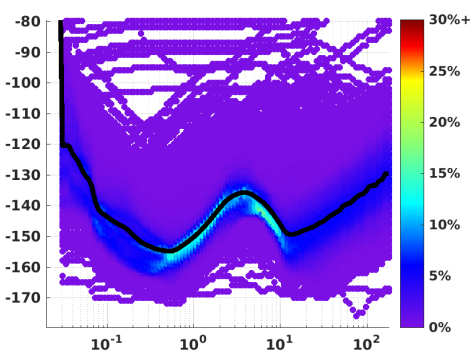

(h) RHT03 HHN

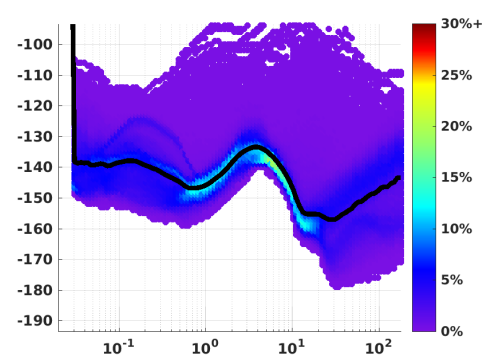

(c) RHT01 HHZ

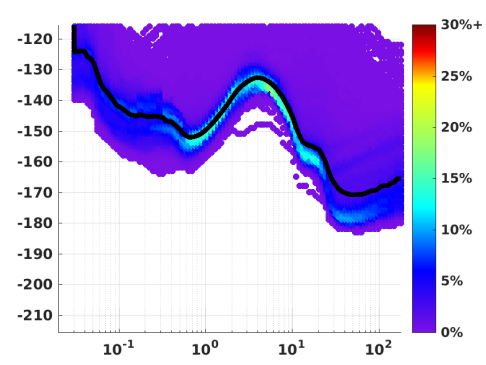

(f) RHT02 HHZ

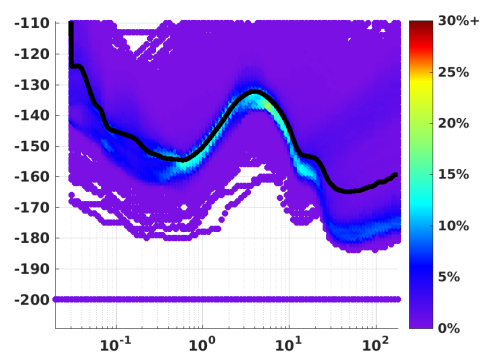

(i) RHT03 HHZ

Figure A.1: Power spectral density for Saudi Arabia stations RHT01--RHT03 for 2014. 


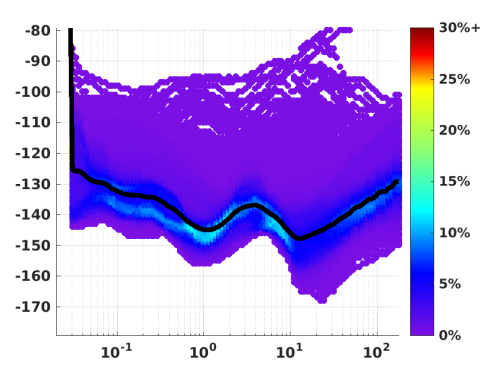

(a) RHT04 HHE

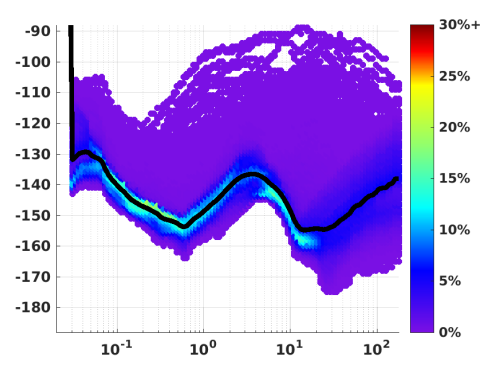

(d) RHT05 HHE

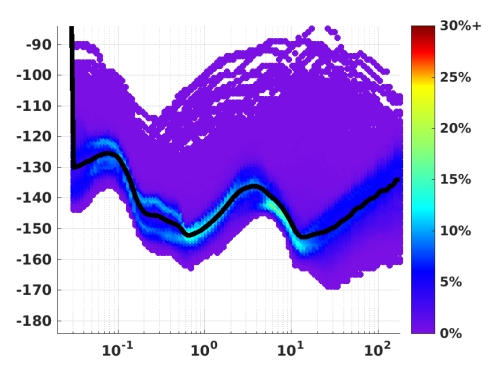

(g) RHT06 HHE

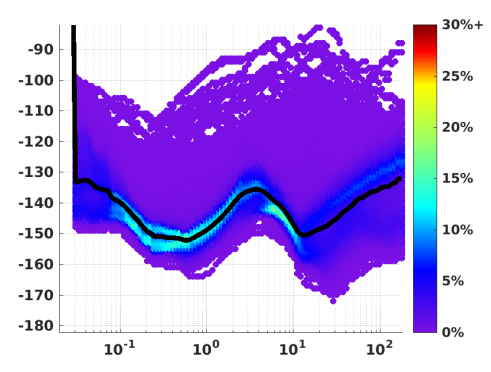

(j) RHT07 HHE

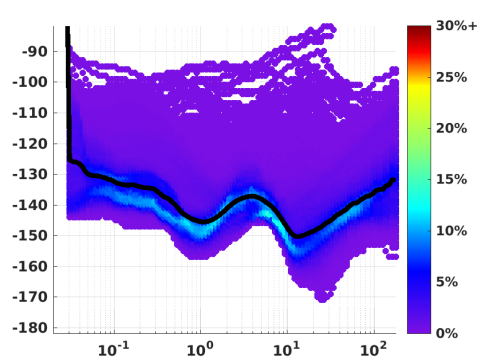

(b) RHT04 HHN

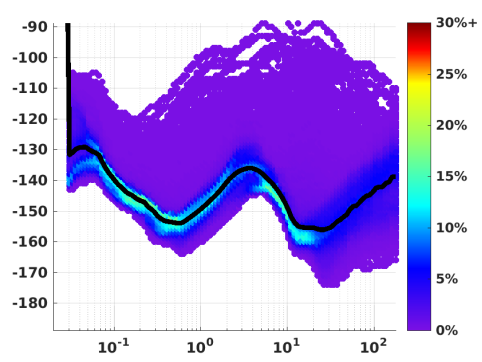

(e) RHT05 HHN

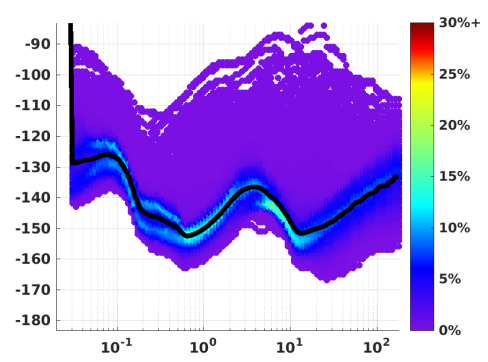

(h) RHT06 HHN

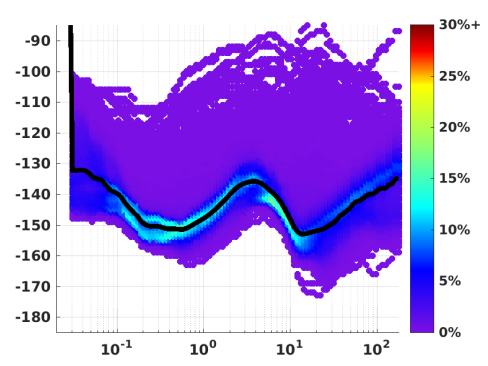

(k) RHT07 HHN

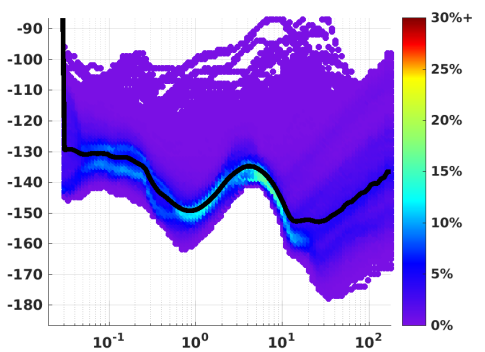

(c) RHT04 HHZ

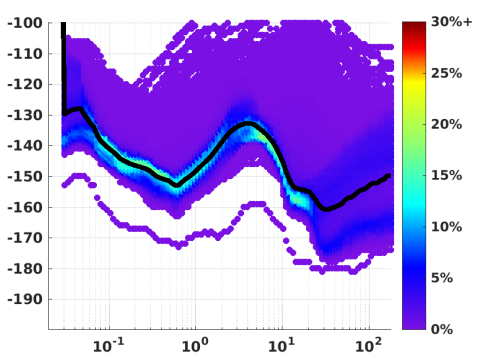

(f) RHT05 HHZ

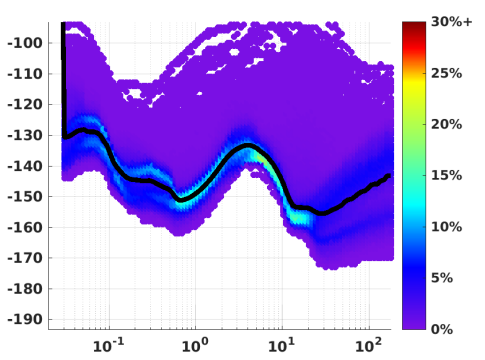

(i) RHT06 HHZ

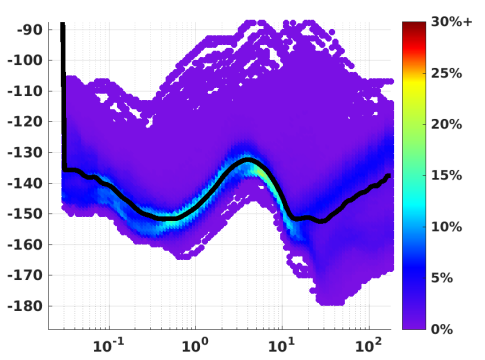

(l) RHT07 HHZ

Figure A.2: Power spectral density for Saudi Arabia stations RHT04--RHT07 for 2014. 


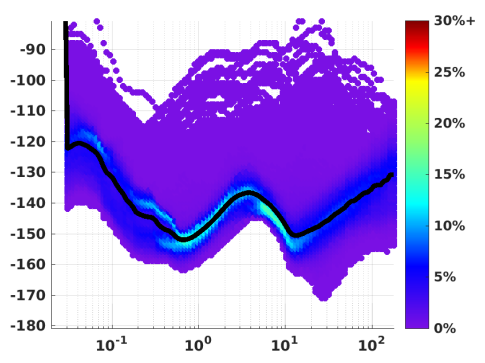

(a) RHT08 HHE

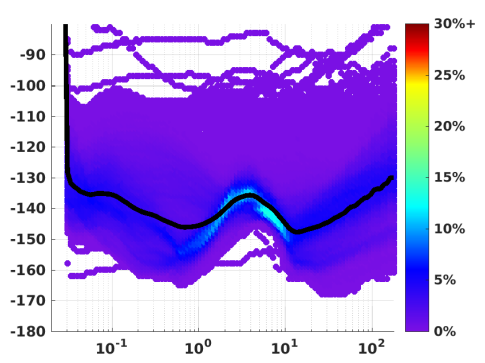

(d) RHT09 HHE

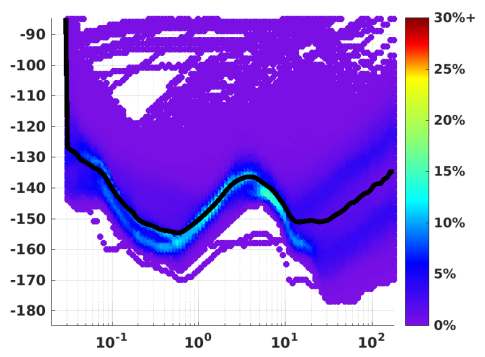

(g) RHT11 HHE

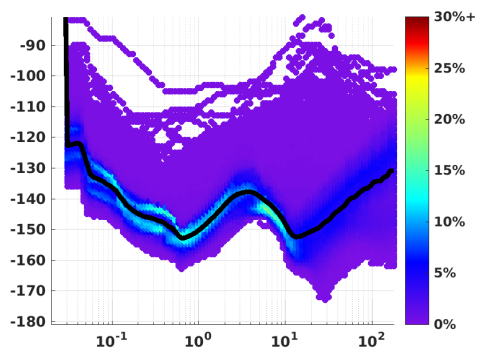

(j) RHT13 HHE

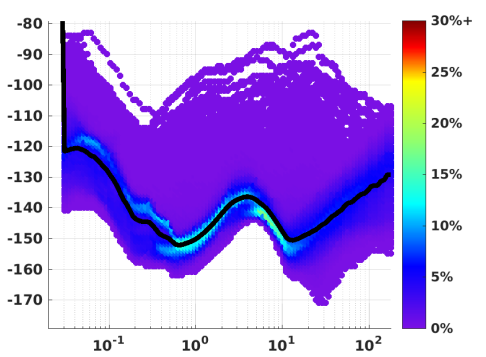

(b) RHT08 HHN

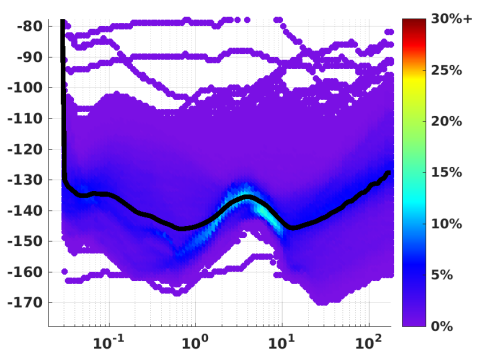

(e) RHT09 HHN

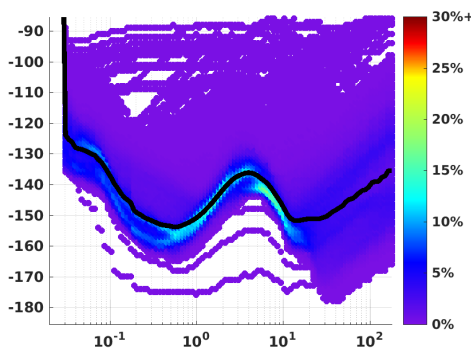

(h) RHT11 HHN

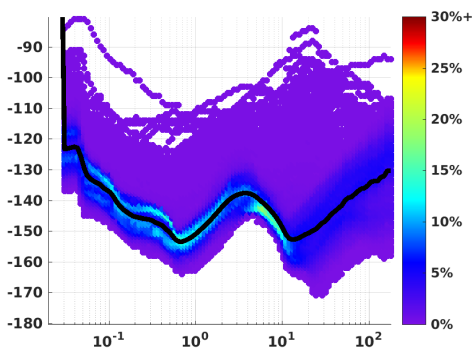

(k) RHT13 HHN

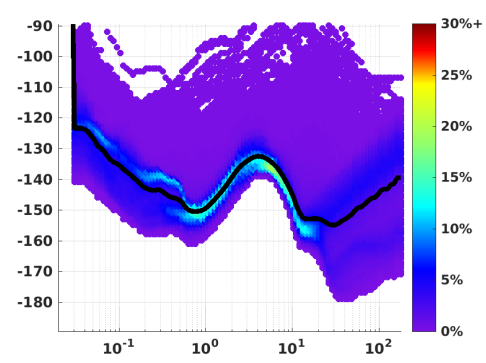

(c) RHT08 HHZ

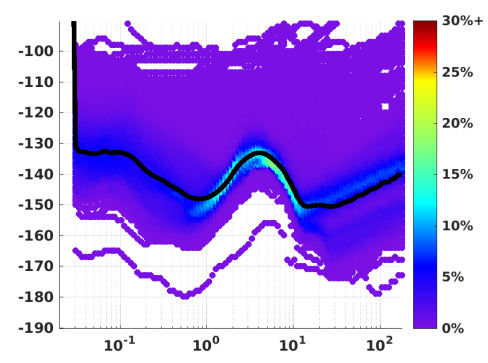

(f) RHT09 HHZ

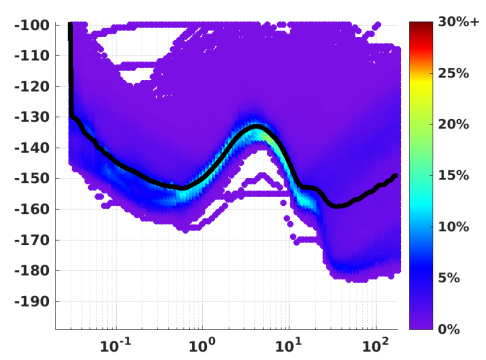

(i) RHT11 HHZ

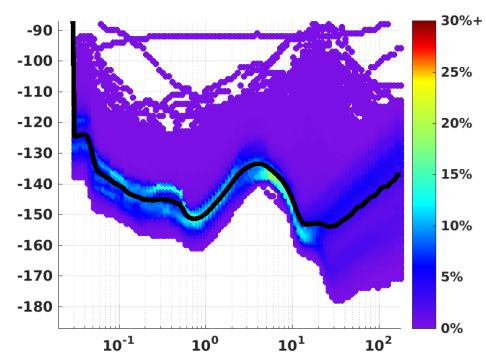

(1) RHT13 HHZ

Figure A.3: Power spectral density for Saudi Arabia stations RHT08--RHT11 and RHT13 for 2014. 


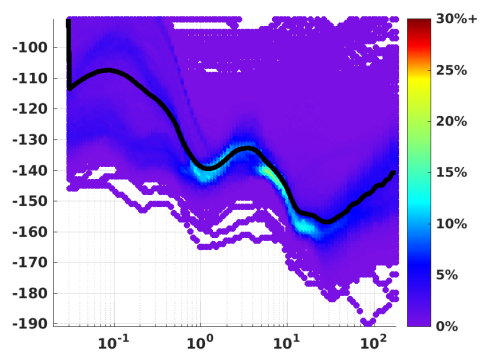

(a) RHT14 HHE

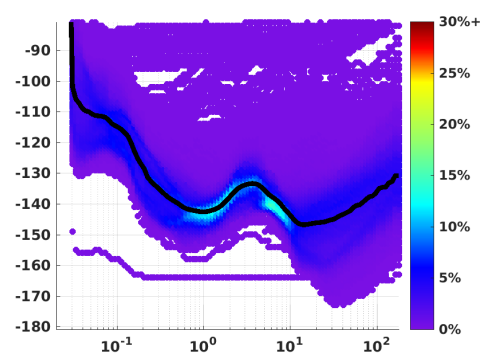

(d) RHT15 HHE

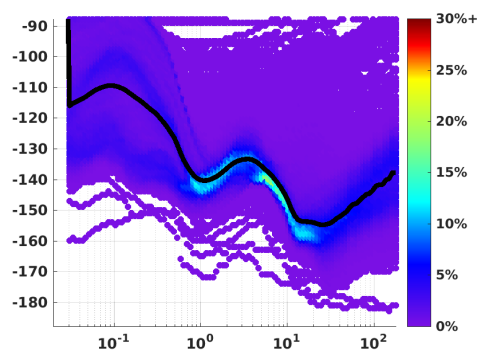

(b) RHT14 HHN

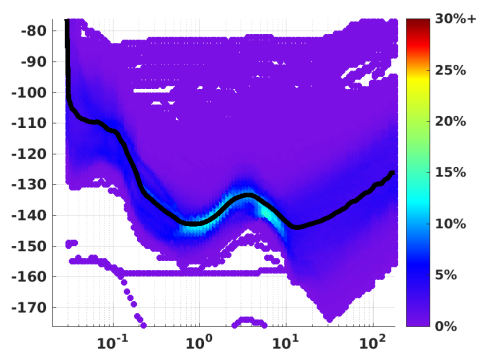

(e) RHT15 HHN

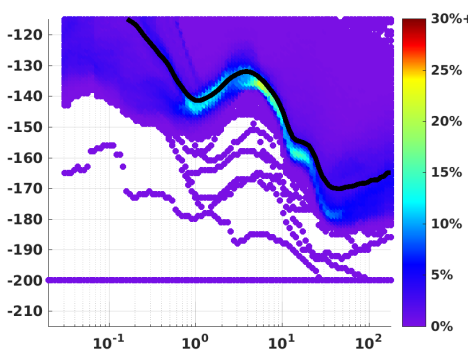

(c) RHT14 HHZ

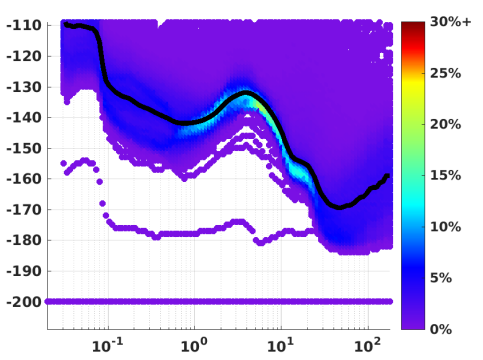

(f) RHT15 HHZ

Figure A.4: Power spectral density for Saudi Arabia stations RHT14 and RHT15 for 2014. 


\section{A.3 Four-month dispersion curves}
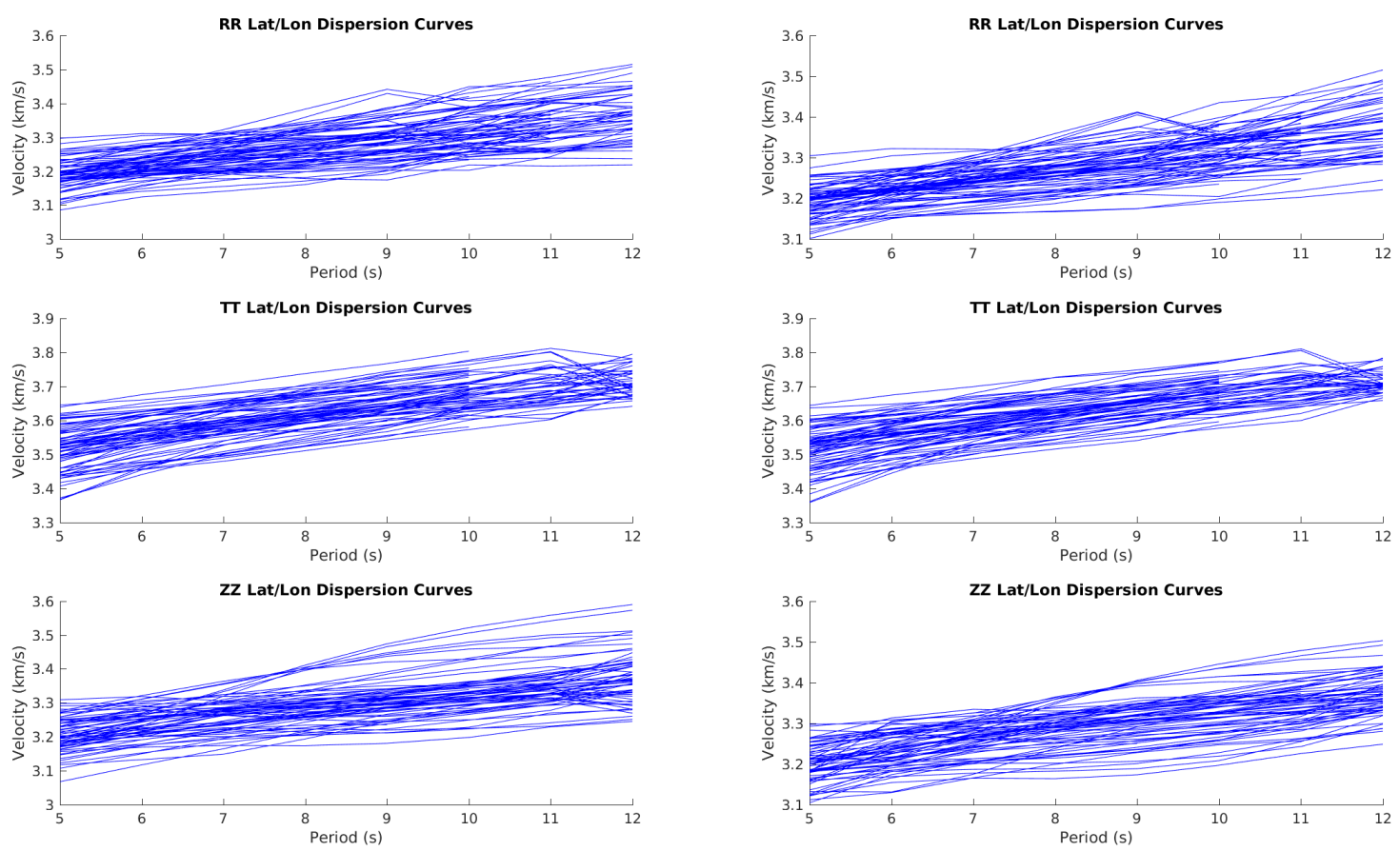

Figure A.5: January--April 2014

Figure A.6: February--May 2014
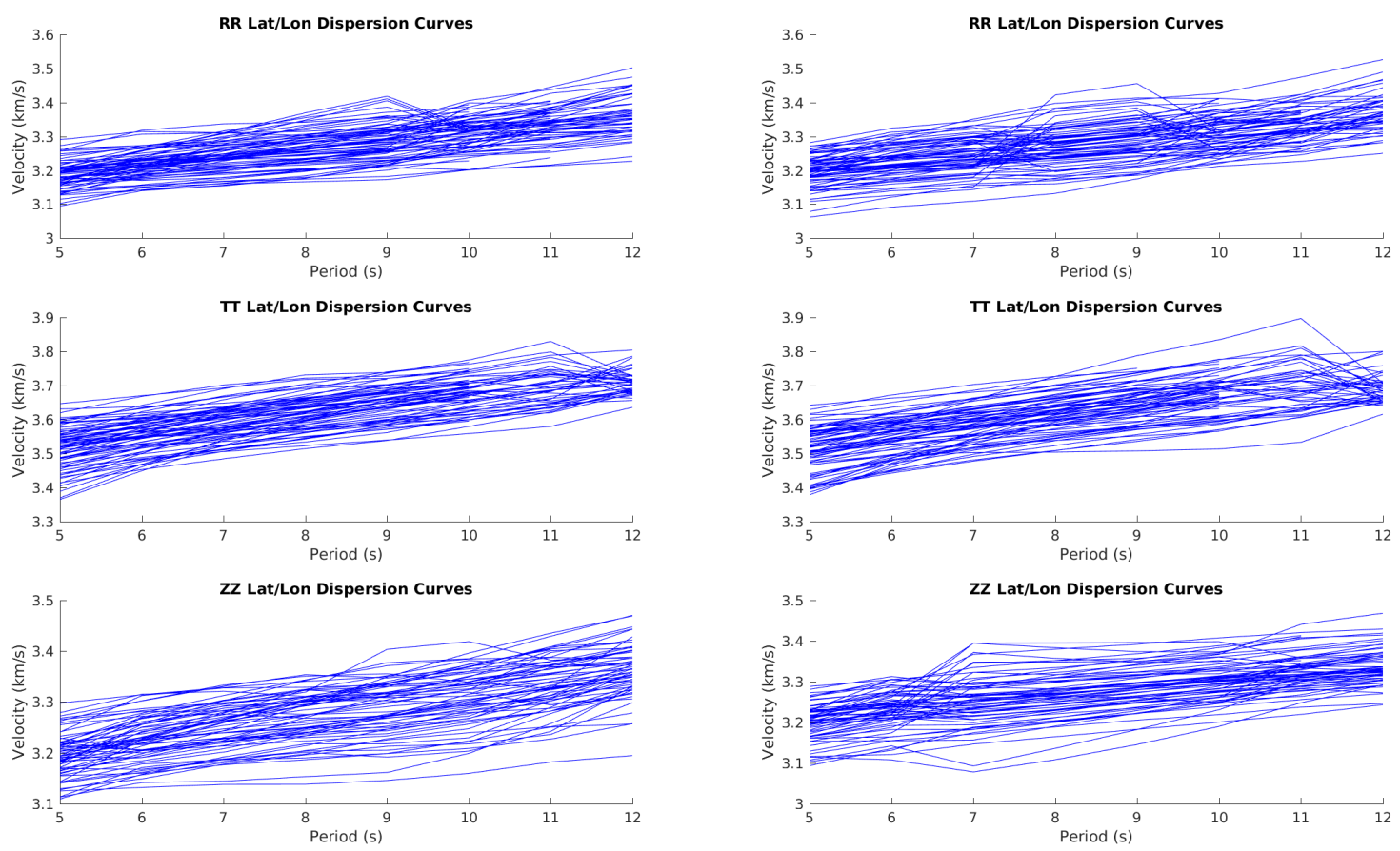

Figure A.7: March--June 2014

Figure A.8: April--July 2014 

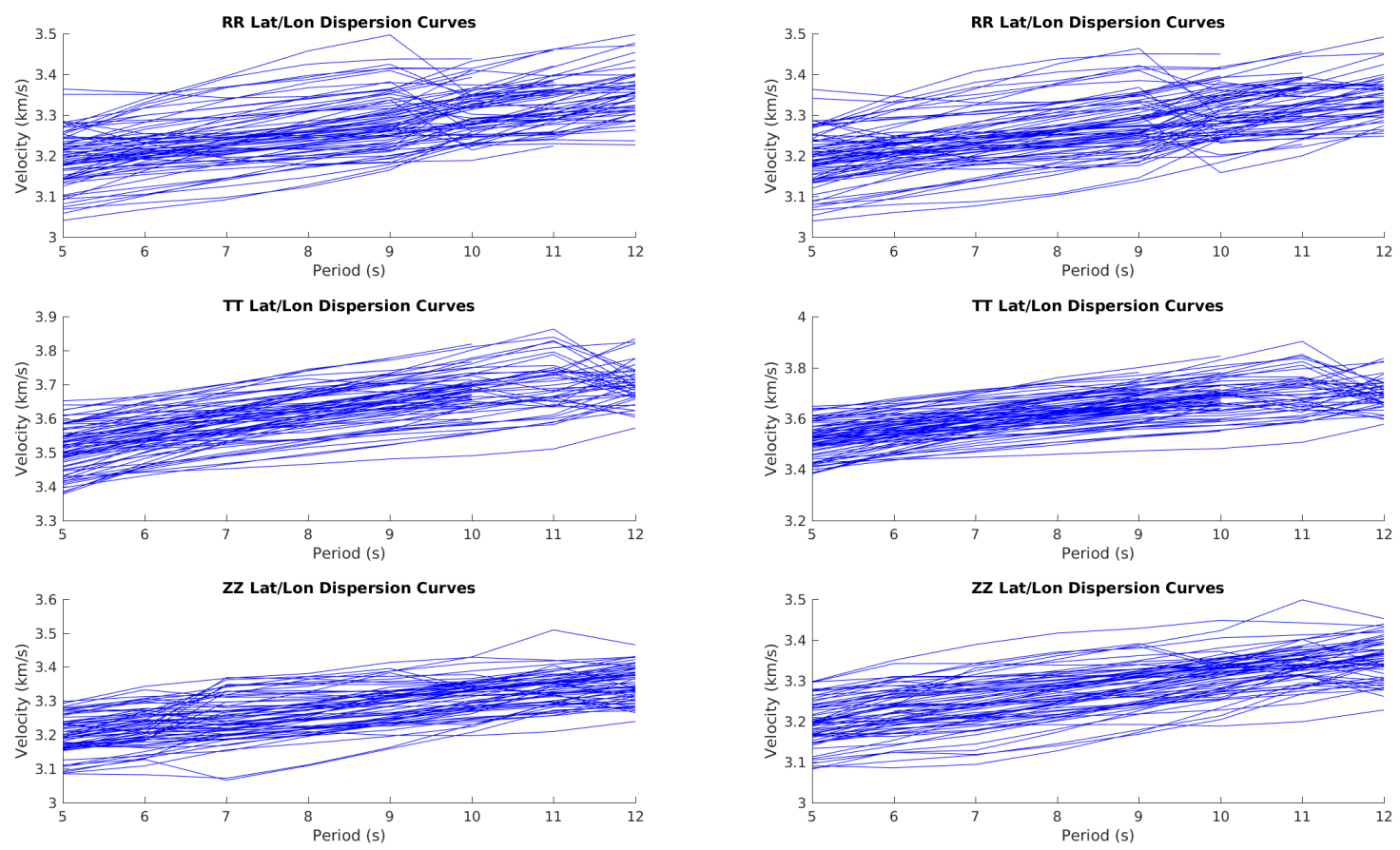

Figure A.9: May--August 2014

Figure A.10: June--September 2014
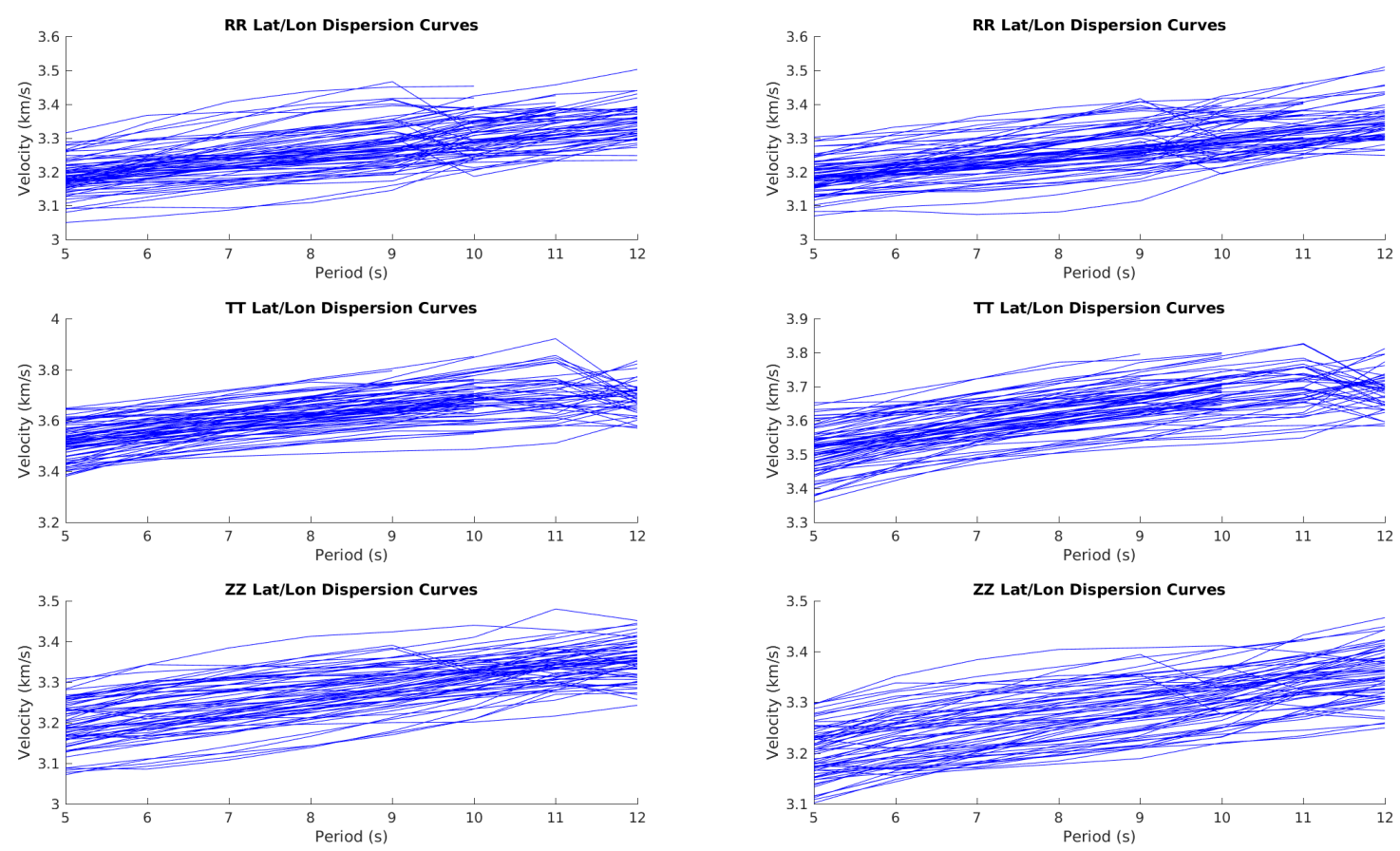

Figure A.11: July--October 2014

Figure A.12: August--November 2014 

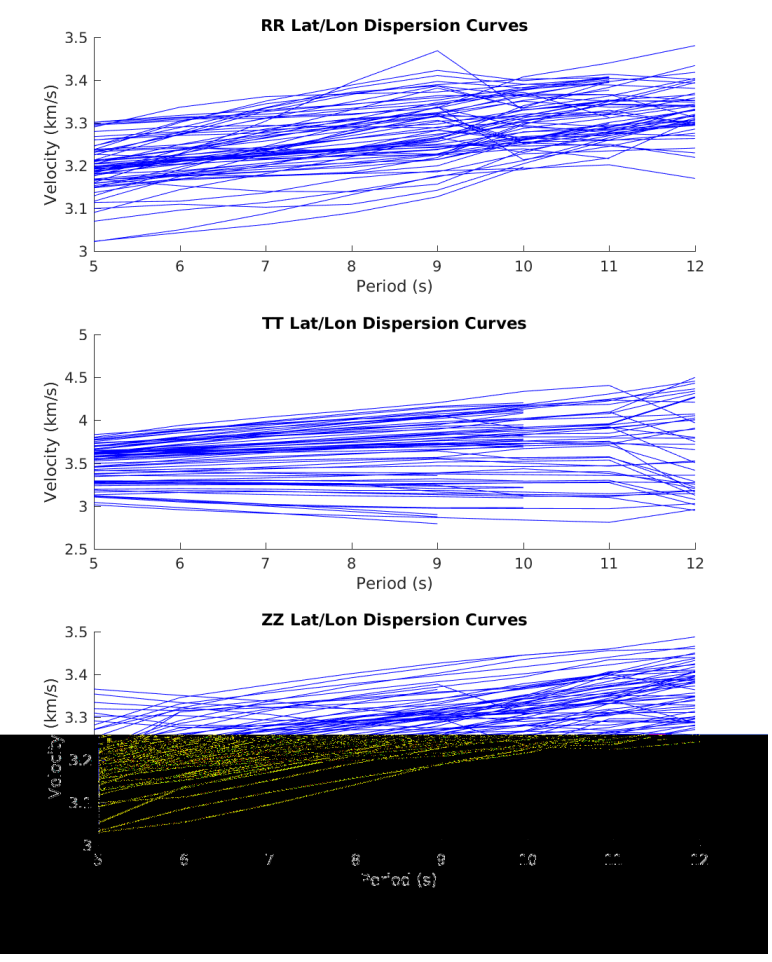

Figure A.13: September--December 2014 


\section{Appendix B}

\section{Velocity changes supplementary information}

\section{B.1 Rotokawa excluded pairs}

- RR: RT02-RT07, RT02-RT08

- RT: RT01-RT02, RT02-RT07, RT02-RT08, RT02-RT10

- RZ: RT01-RT09, RT02-RT07, RT02-RT08, RT02-RT09, RT02-RT11, RT05-RT09, RT06RT09, RT07-RT09, RT08-RT09.

- TR: RT02-RT08

- TT: RT02-RT06, RT02-RT09, RT07-RT10

- TZ: RT01-RT09, RT02-RT09, RT05-RT09, RT05-RT11, RT06-RT08, RT06-RT09, RT07RT09, RT08-RT09, RT08-RT11

- ZR: RT08-RT10, RT08-RT11, RT09-RT10, RT09-RT11

- ZT: RT01-RT02, RT08-RT11, RT09-RT10, RT09-RT11

- ZZ: RT01-RT09, RT02-RT09, RT05-RT09, RT06-RT09, RT07-RT08, RT07-RT09, RT08RT09, RT09-RT10, RT09-RT11

\section{B.2 Ngatamariki excluded pairs}

- RR: NS01-NS04, NS02-NS04, NS02-NS08, NS03-NS08, NS04-NS07

- RT: NS01-NS04, NS02-NS03, NS02-NS05, NS05-NS10

- RZ: NS02-NS08, NS03-NS05, NS03-NS08, NS04-NS08, NS04-NS10 
- TR: NS01-NS04, NS03-NS04, NS04-NS05, NS04-NS08

- TT: NS01-NS04, NS01-NS11, NS02-NS03, NS02-NS10, NS04-NS08

- TZ: NS01-NS03, NS01-NS05, NS01-NS08, NS02-NS04, NS02-NS08, NS02-NS11, NS03-NS05, NS04-NS05, NS04-NS08, NS08-NS10, NS08-NS11

- ZR: NS01-NS02, NS01-NS04, NS01-NS08, NS01-NS10, NS03-NS04, NS05-NS10, NS08-NS10, NS08-NS11

- ZT: NS01-NS04, NS01-NS07, NS01-NS11, NS02-NS04, NS03-NS04, NS05-NS08, NS08-NS11

- ZZ: NS01-NS02, NS01-NS03, NS01-NS05, NS01-NS08, NS01-NS10, NS02-NS05, NS02-NS08, NS02-NS10, NS02-NS11, NS03-NS08, NS05-NS08, NS07-NS10, NS08NS10, NS08-NS11 


\section{B.3 Rotokawa velocity changes reference stack 2}

\begin{tabular}{||l||l||l||l||l||l||}
\hline Grouping & 10 Days & 20 Days & 30 Days & 40 Days & 50 Days \\
\hline All field all components & $0.046 \%$ & $0.043 \%$ & $0.041 \%$ & $0.041 \%$ & $0.039 \%$ \\
\hline All field principal components & $0.058 \%$ & $0.058 \%$ & $0.051 \%$ & $0.049 \%$ & $0.047 \%$ \\
\hline Injection all components & $0.061 \%$ & $0.061 \%$ & $0.059 \%$ & $0.055 \%$ & $0.049 \%$ \\
\hline Injection principal components & $0.083 \%$ & $0.083 \%$ & $0.073 \%$ & $0.066 \%$ & $0.056 \%$ \\
\hline Production all components & $0.057 \%$ & $0.051 \%$ & $0.047 \%$ & $0.046 \%$ & $0.044 \%$ \\
\hline $\begin{array}{l}\text { Production principal compo- } \\
\text { nents }\end{array}$ & $0.068 \%$ & $0.061 \%$ & $0.058 \%$ & $0.059 \%$ & $0.057 \%$ \\
\hline \hline
\end{tabular}

Table B.1: The maximum positive velocity change for each grouping at Rotokawa for each MWCS window size using reference stack 2.

\begin{tabular}{||l||l||l||l||l||l||}
\hline Grouping & 10 Days & 20 Days & 30 Days & 40 Days & 50 Days \\
\hline All field all components & $-0.053 \%$ & $-0.055 \%$ & $-0.056 \%$ & $-0.058 \%$ & $-0.058 \%$ \\
\hline All field principal components & $-0.064 \%$ & $-0.057 \%$ & $-0.057 \%$ & $-0.057 \%$ & $-0.056 \%$ \\
\hline Injection all components & $-0.076 \%$ & $-0.071 \%$ & $-0.079 \%$ & $-0.077 \%$ & $-0.076 \%$ \\
\hline Injection principal components & $-0.098 \%$ & $-0.085 \%$ & $-0.087 \%$ & $-0.085 \%$ & $-0.086 \%$ \\
\hline Production all components & $-0.052 \%$ & $-0.057 \%$ & $-0.055 \%$ & $-0.059 \%$ & $-0.058 \%$ \\
\hline $\begin{array}{l}\text { Production principal compo- } \\
\text { nents }\end{array}$ & $-0.06 \%$ & $-0.061 \%$ & $-0.06 \%$ & $-0.059 \%$ & $-0.057 \%$ \\
\hline \hline
\end{tabular}

Table B.2: The maximum negative velocity change for each grouping at Rotokawa for each MWCS window size using reference stack 2. 

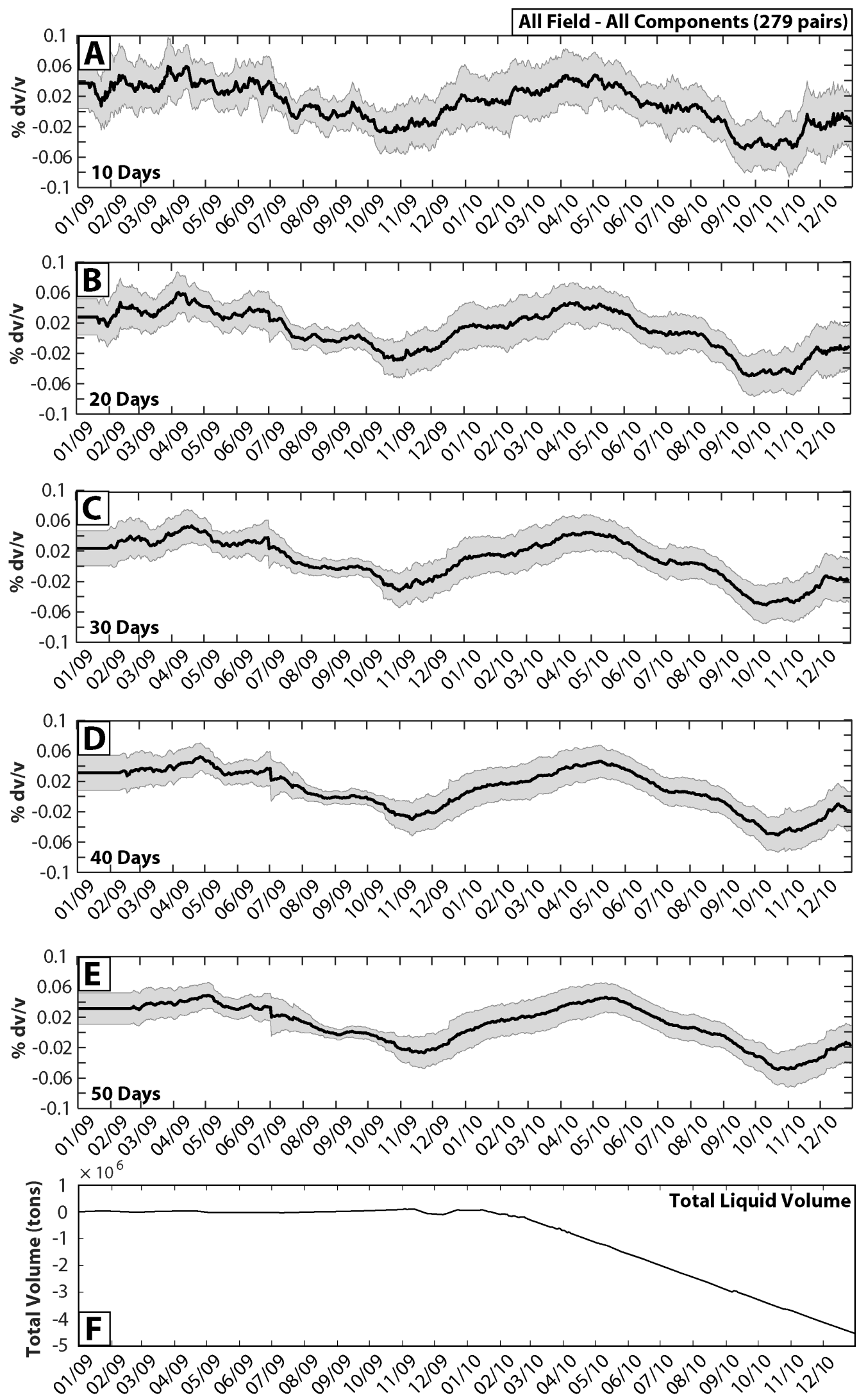

Figure B.1: Velocity changes using 10--50 day MWCS windows [A--E] and Rotokawa relative liquid volume $[\mathrm{F}]$ using all components of the entire network (reference stack 2). 

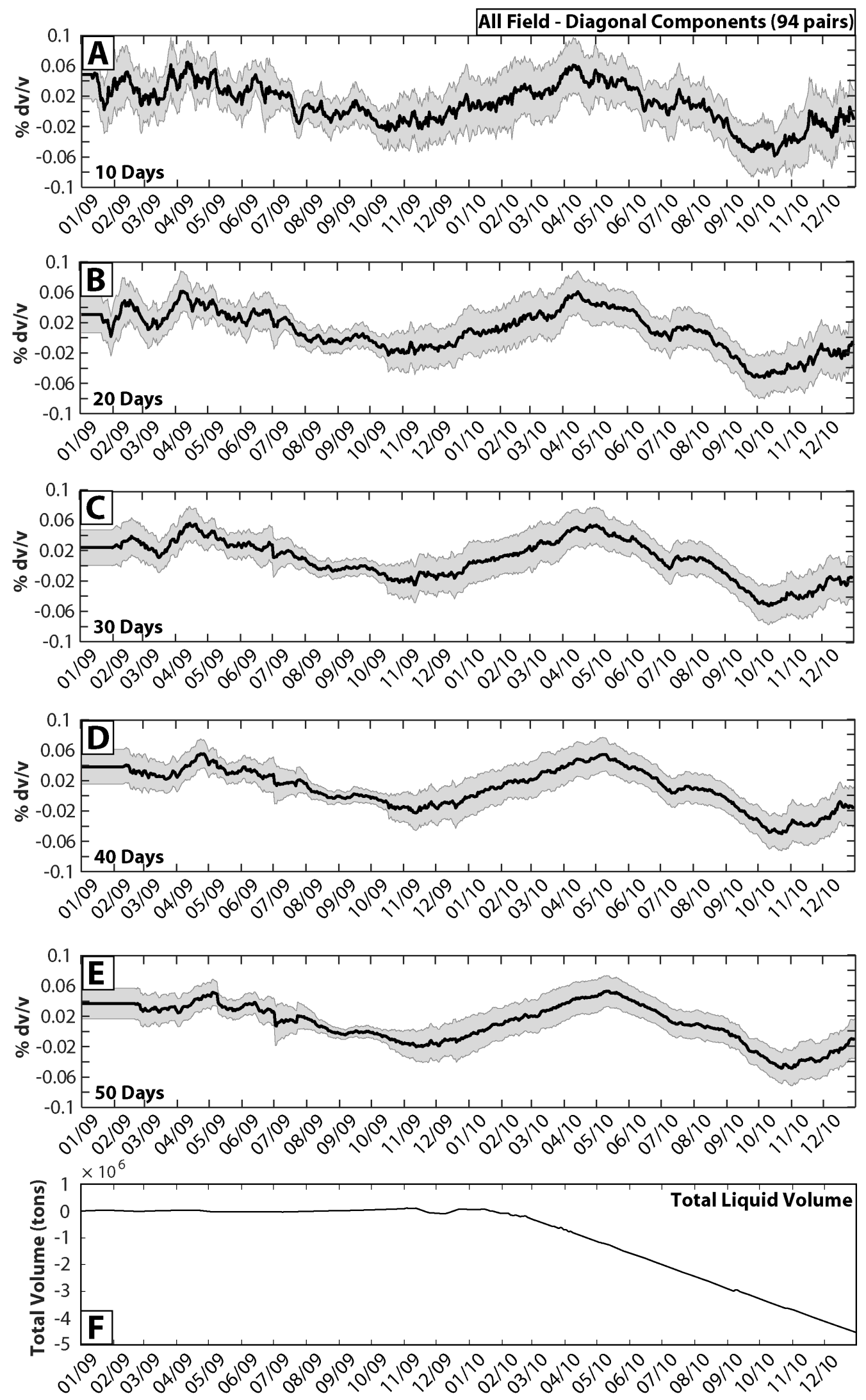

Figure B.2: Velocity changes using 10--50 day MWCS windows [A--E] and Rotokawa relative liquid volume $[\mathrm{F}]$ using diagonal components of the entire network (reference stack 2). 

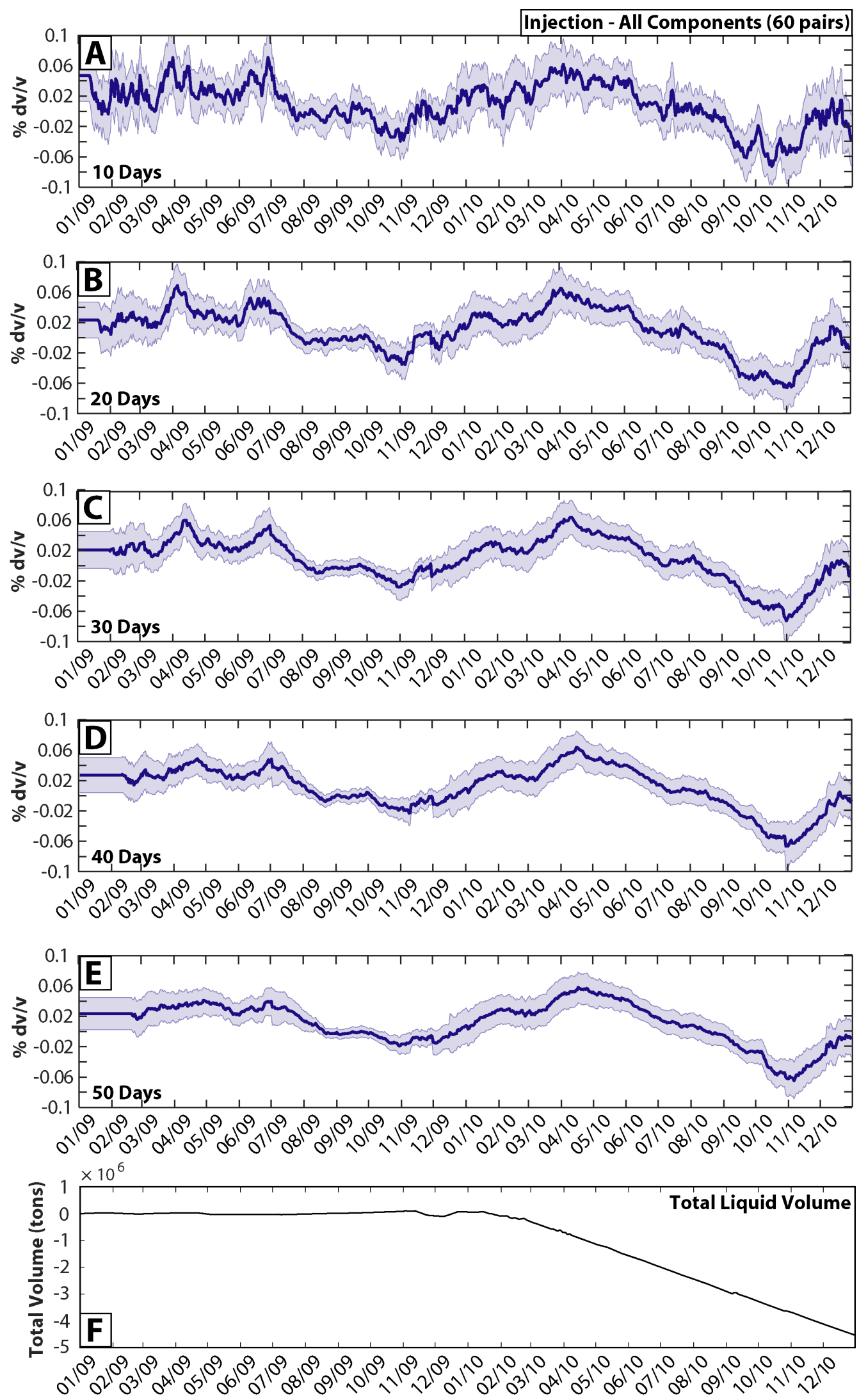

Figure B.3: Velocity changes using 10--50 day MWCS windows [A--E] and Rotokawa relative liquid volume [F] using all components of the injection subset (reference stack 2). 

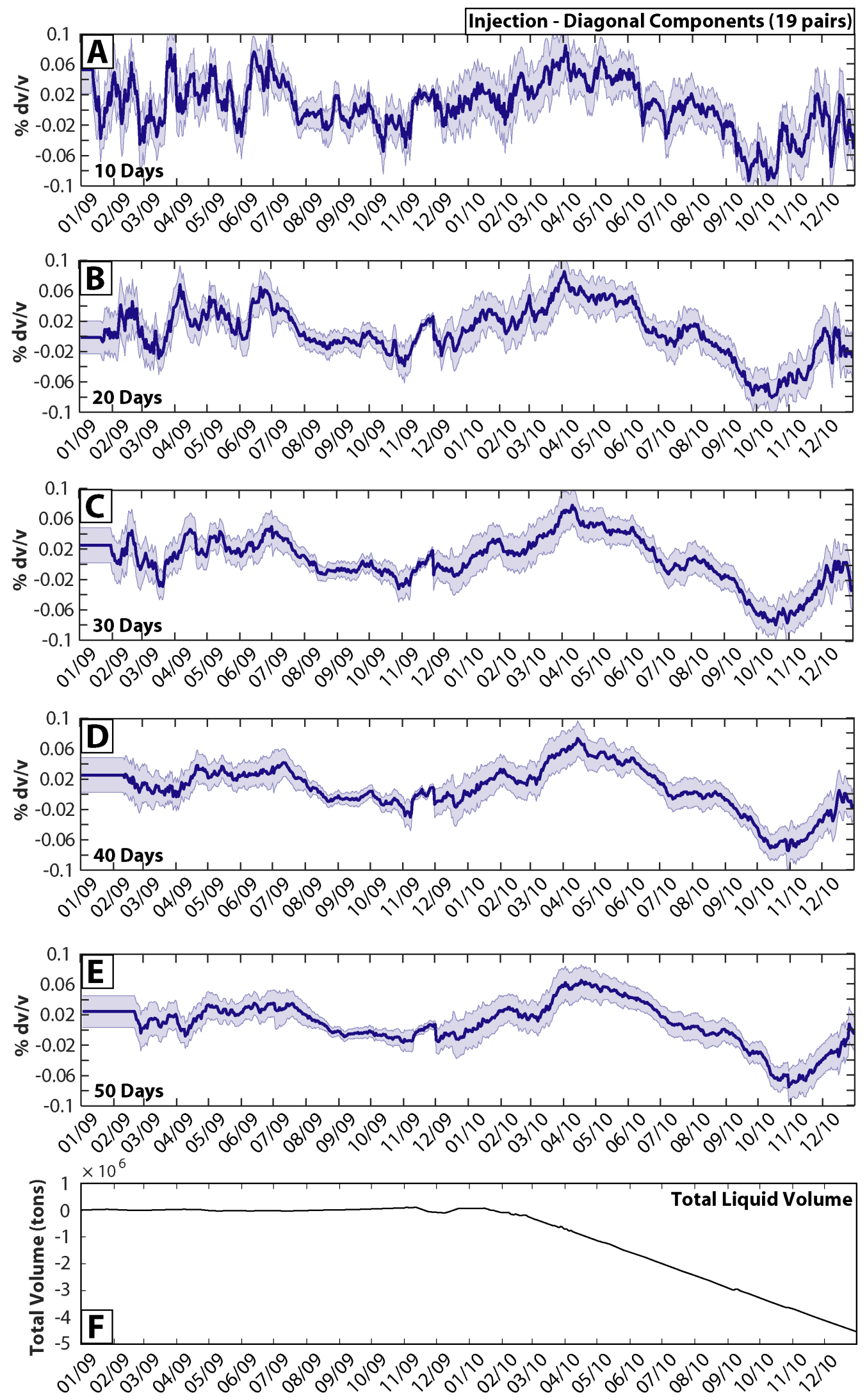

Figure B.4: Velocity changes using 10--50 day MWCS windows [A--E] and Rotokawa relative liquid volume [F] using diagonal components of the injection subset (reference stack 2). 

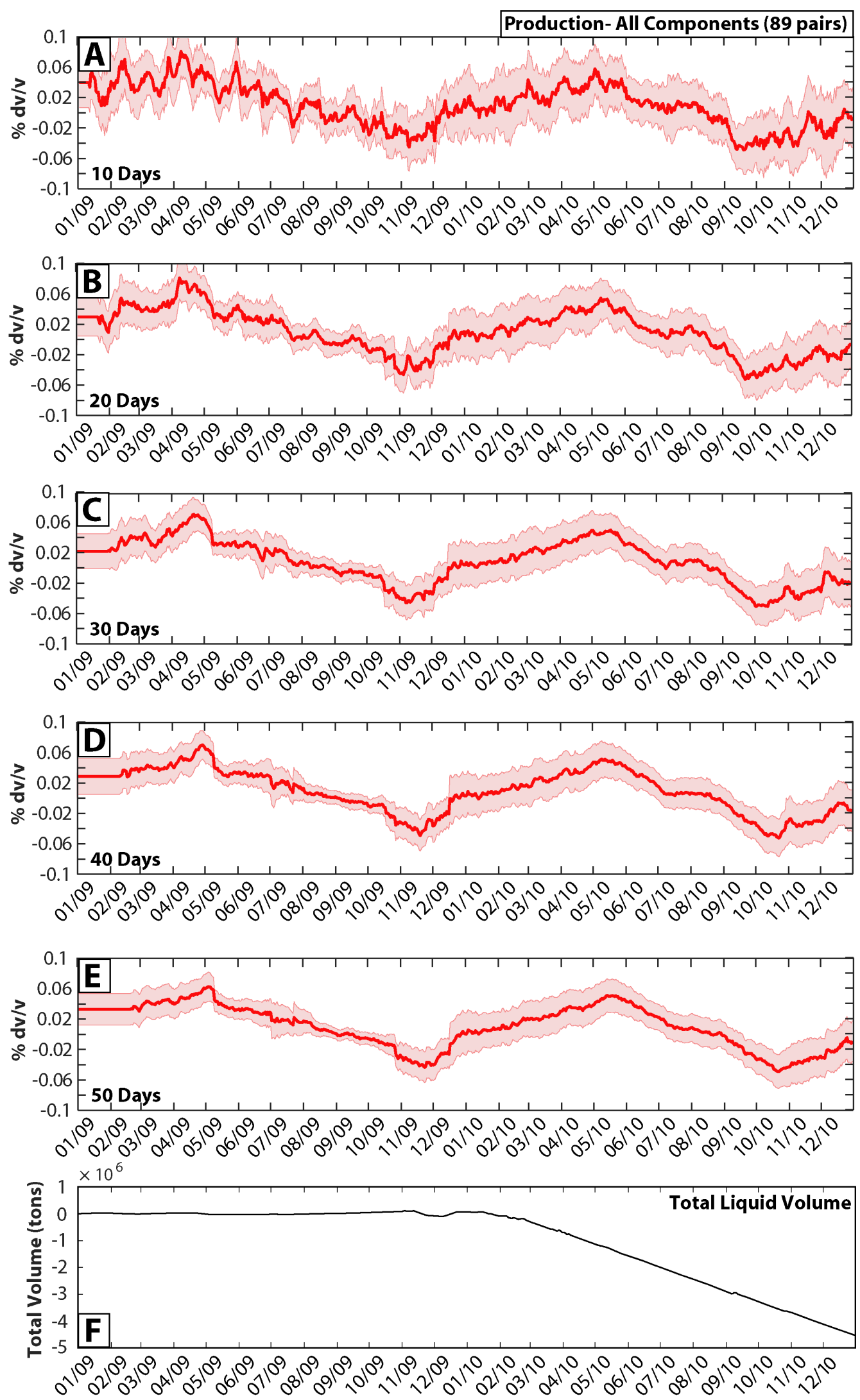

Figure B.5: Velocity changes using 10--50 day MWCS windows [A--E] and Rotokawa relative liquid volume $[\mathrm{F}]$ using all components of the production subset (reference stack 2). 

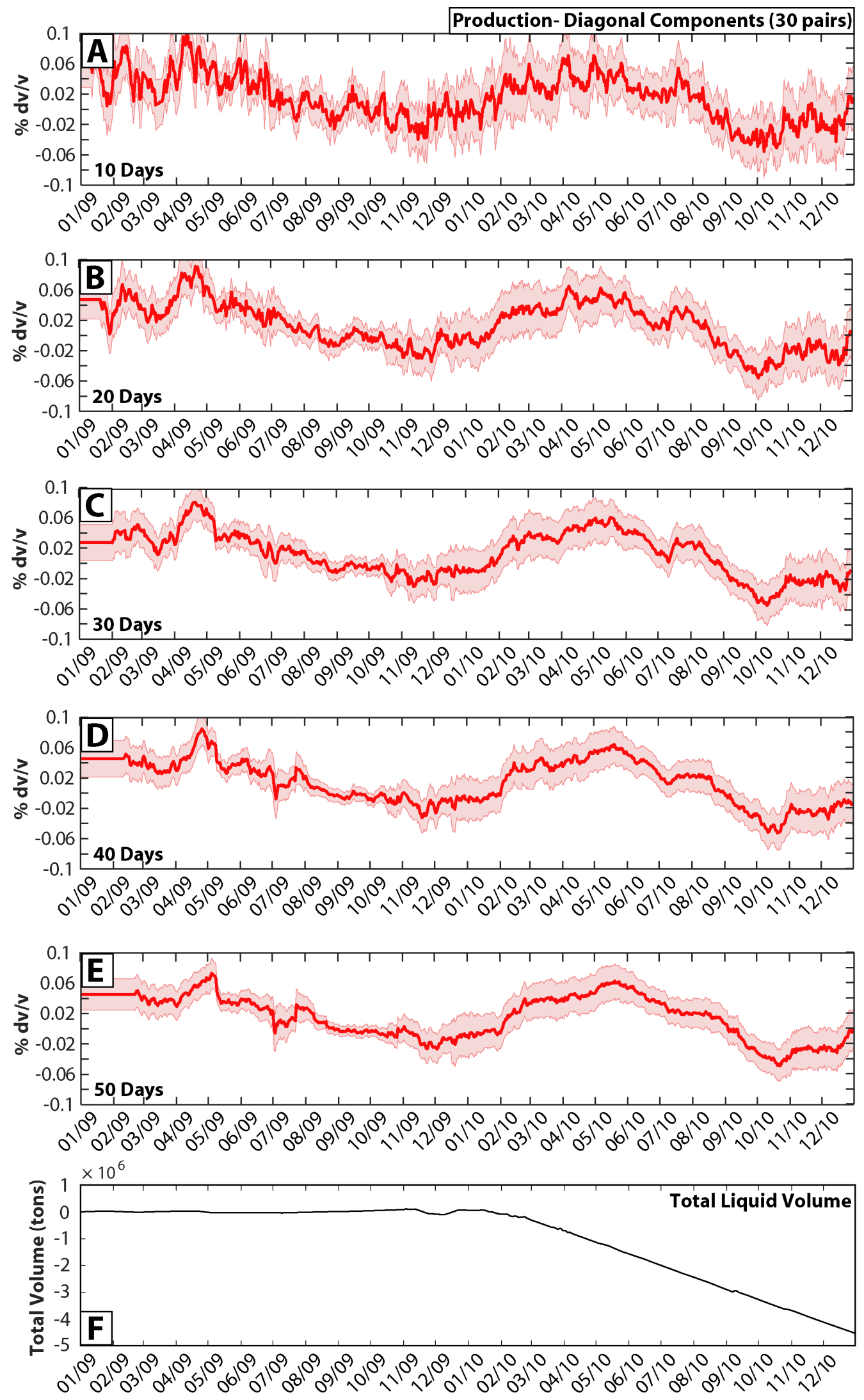

Figure B.6: Velocity changes using 10--50 day MWCS windows [A--E] and Rotokawa relative liquid volume $[\mathrm{F}]$ using diagonal components of the production subset (reference stack 2). 


\section{B.4 Noise at Rotokawa and Ngatamariki}

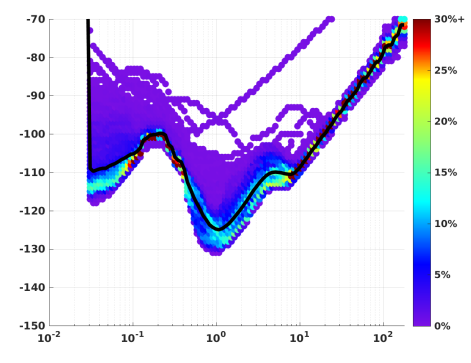

(a) RT01 EHE summer

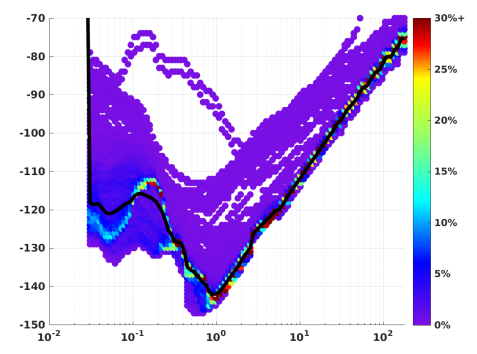

(d) RT02 EHE summer

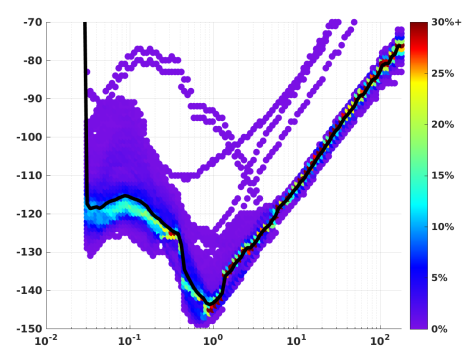

(g) RT03 EHE summer

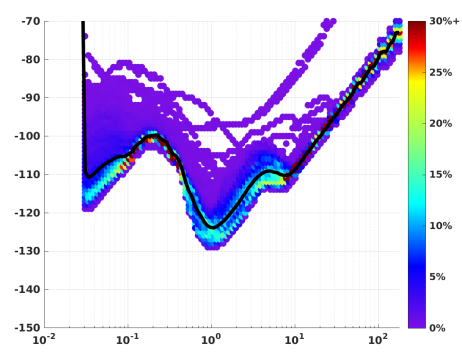

(b) RT01 EHN summer

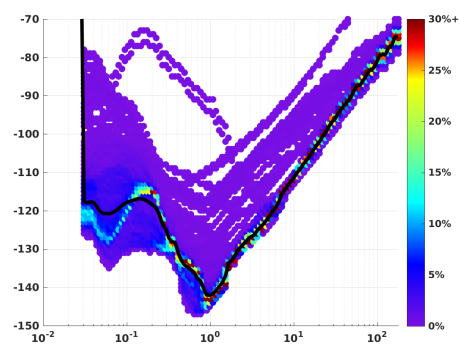

(e) RT02 EHN summer

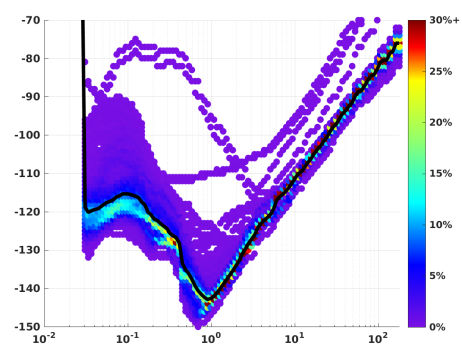

(h) RT03 EHN summer

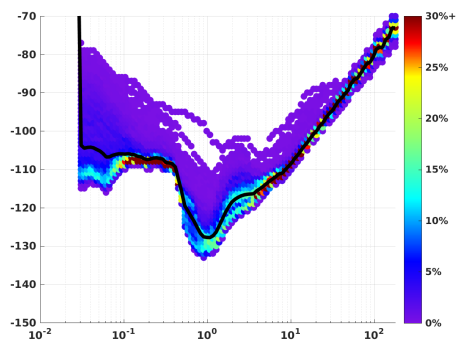

(c) RT01 EHZ summer

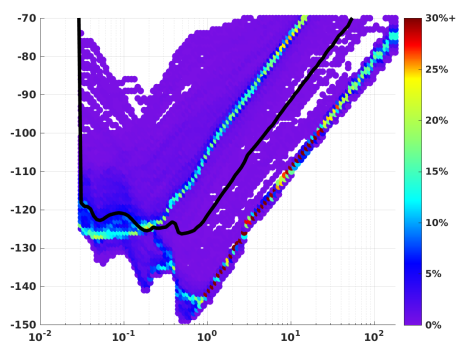

(f) RT02 EHZ summer

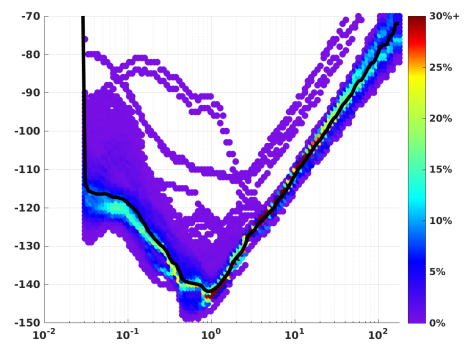

(i) RT03 EHZ summer

Figure B.7: Power spectral density for Rotokawa stations RT01--RT03 for summer. 


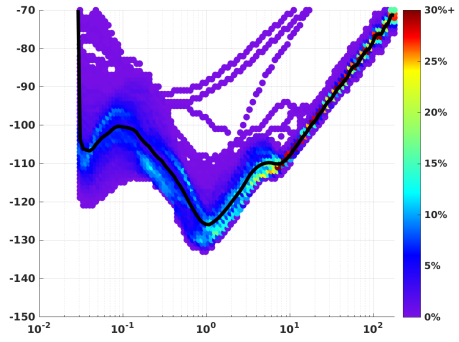

(a) RT05 EHE summer

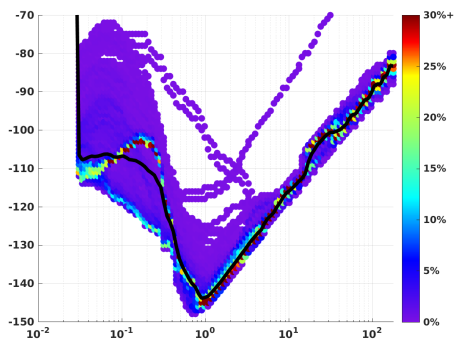

(d) RT06 EHE summer

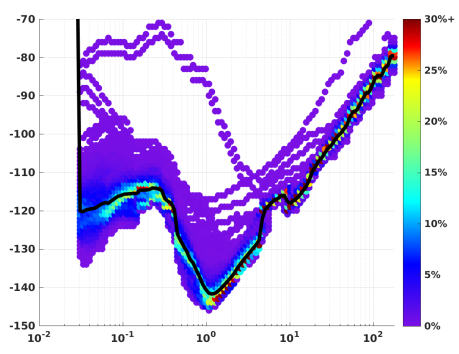

(g) RT07 EHE summer

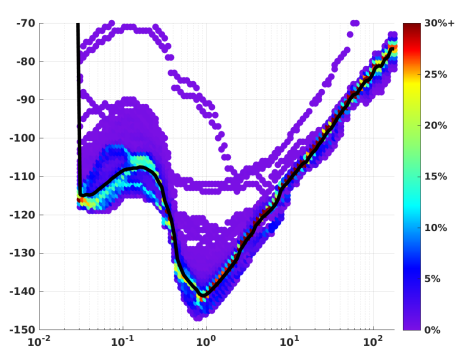

(j) RT08 EHE summer

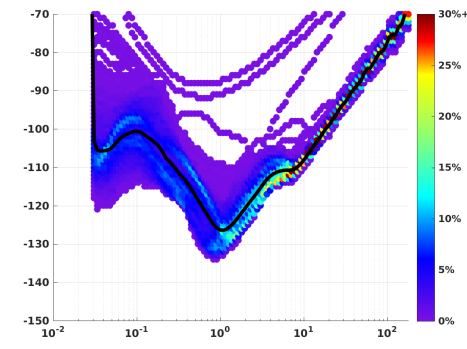

(b) RT05 EHN summer

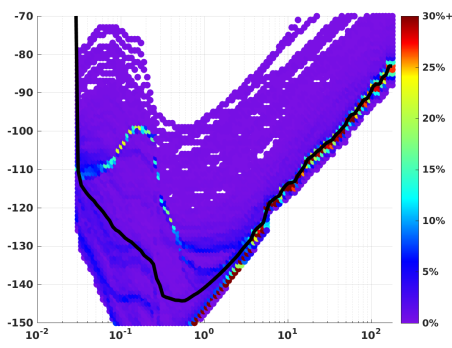

(e) RT06 EHN summer

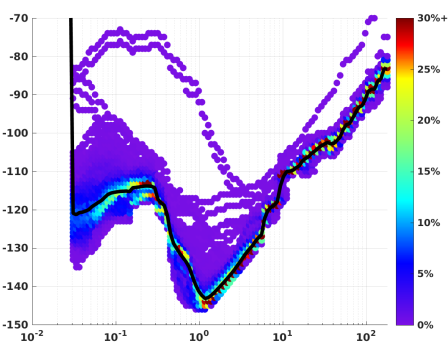

(h) RT07 EHN summer

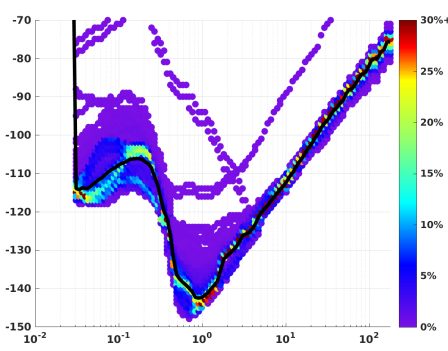

(k) RT08 EHN summer

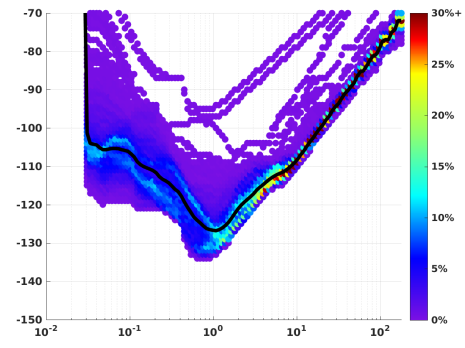

(c) RT05 EHZ summer

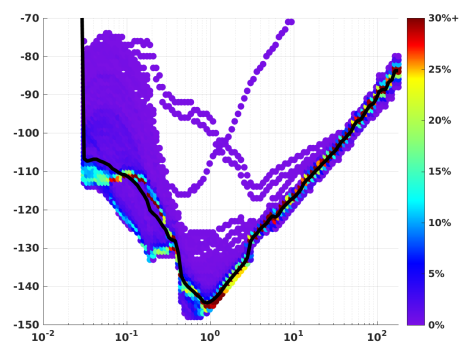

(f) RT06 EHZ summer

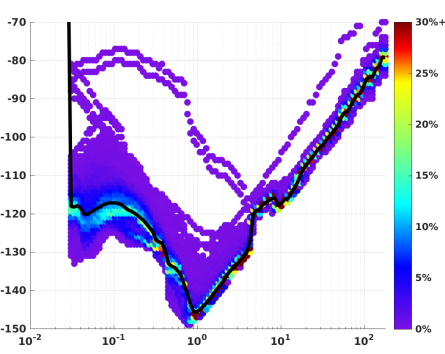

(i) RT07 EHZ summer

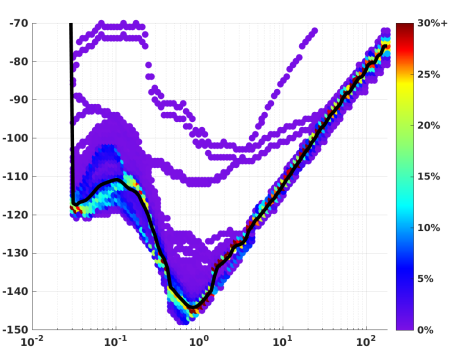

(1) RT08 EHZ summer

Figure B.8: Power spectral density for Rotokawa stations RT05--RT08 for summer. 


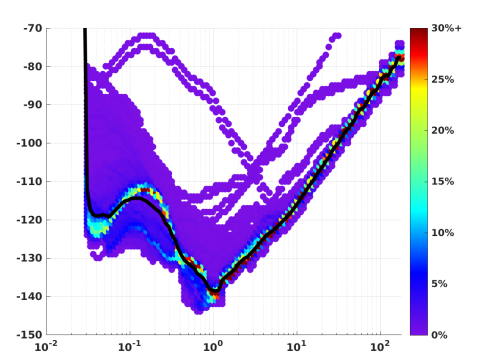

(a) RT09 EHE summer

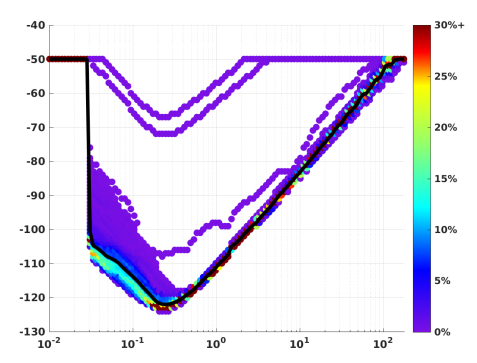

(d) RT10 EHE summer

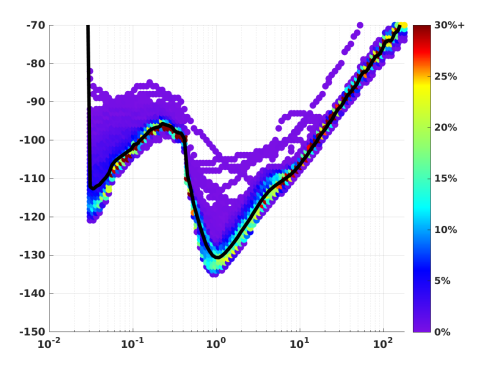

(g) RT11 EHE summer

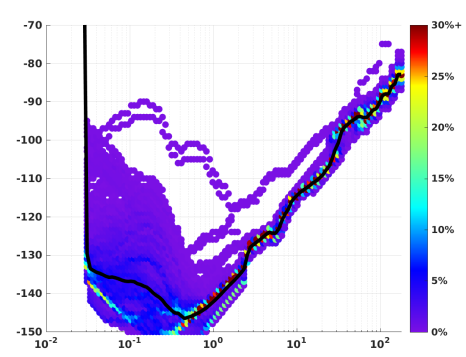

(b) RT09 EHN summer

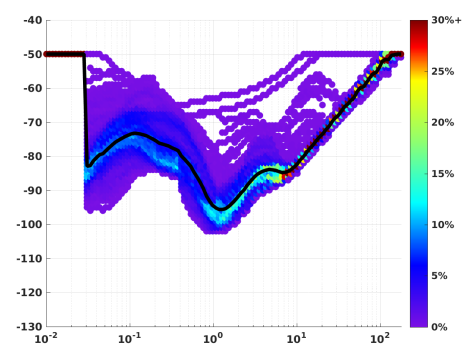

(e) RT10 EHN summer

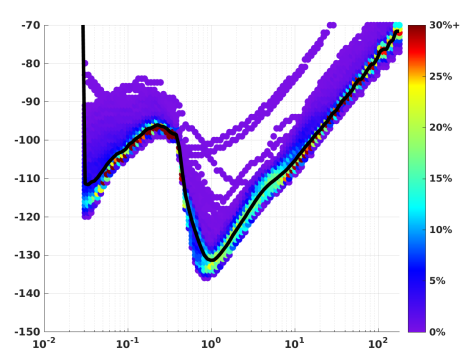

(h) RT11 EHN summer

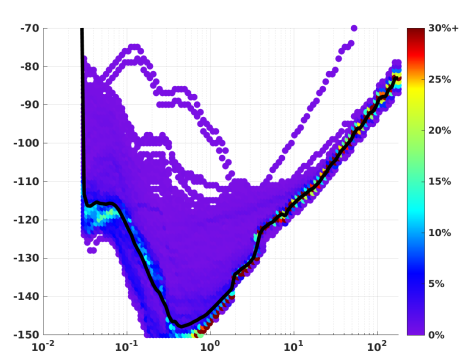

(c) RT09 EHZ summer

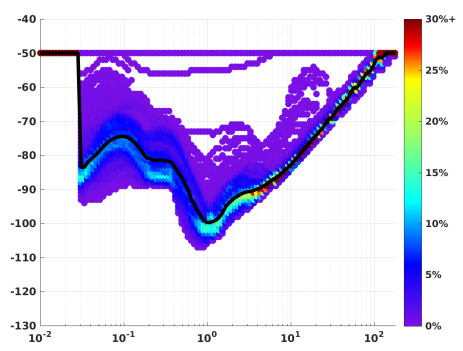

(f) RT10 EHZ summer

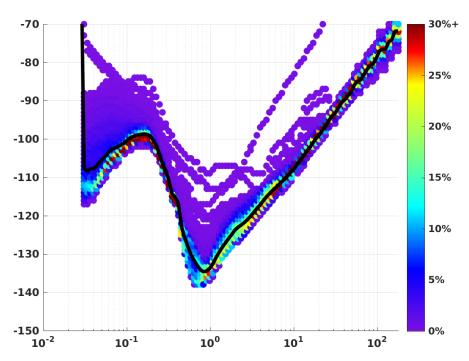

(i) RT11 EHZ summer

Figure B.9: Power spectral density for Rotokawa stations RT09--RT11 for summer. 


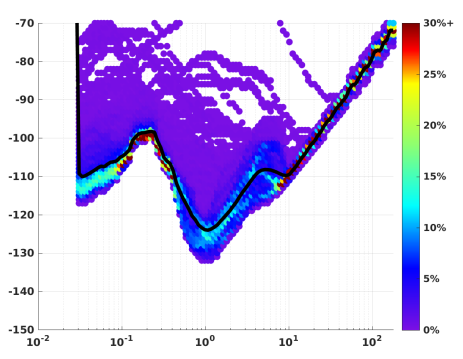

(a) RT01 EHE winter

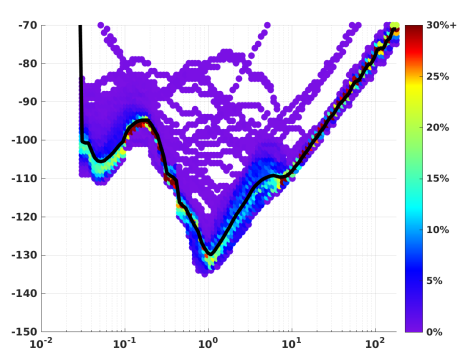

(d) RT02 EHE winter

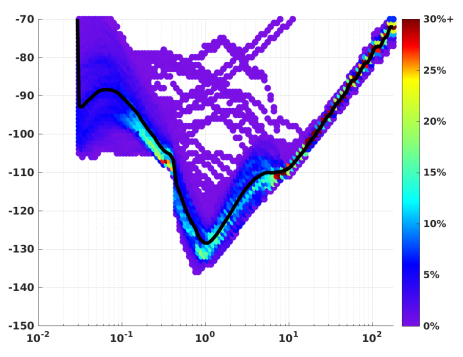

(g) RT03 EHE winter

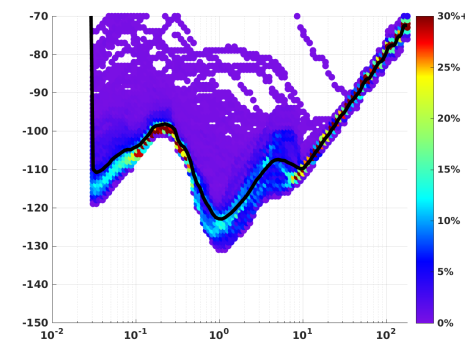

(b) RT01 EHN winter

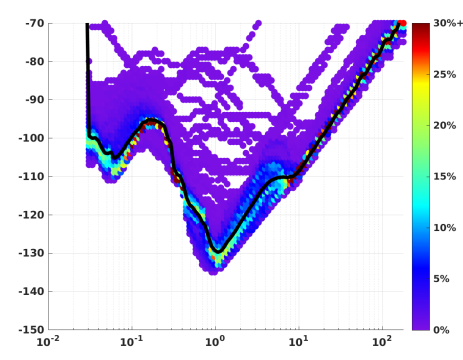

(e) RT02 EHN winter

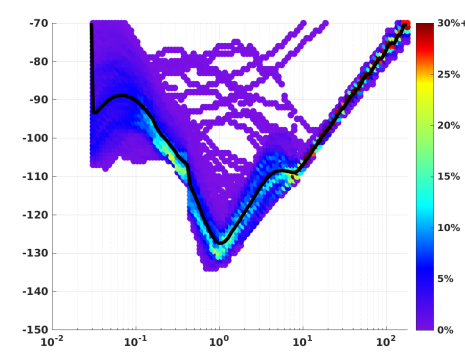

(h) RT03 EHN winter

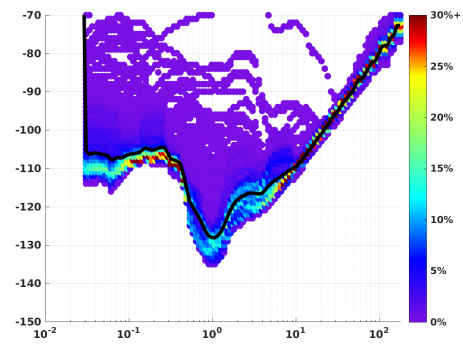

(c) RT01 EHZ winter

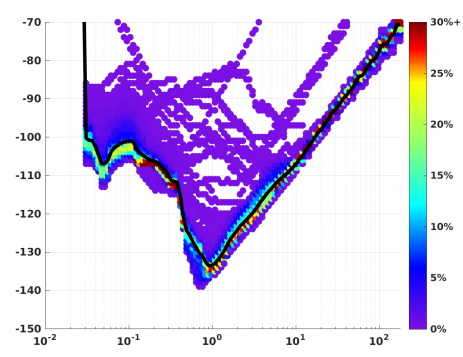

(f) RT02 EHZ winter

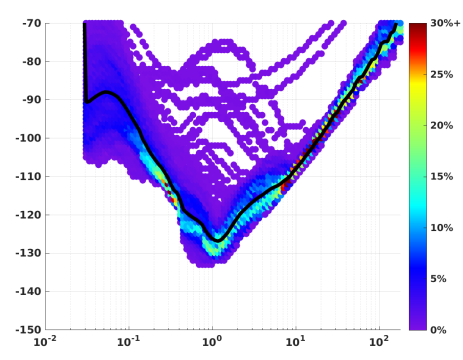

(i) RT03 EHZ winter

Figure B.10: Power spectral density for Rotokawa stations RT01--RT03 for winter. 


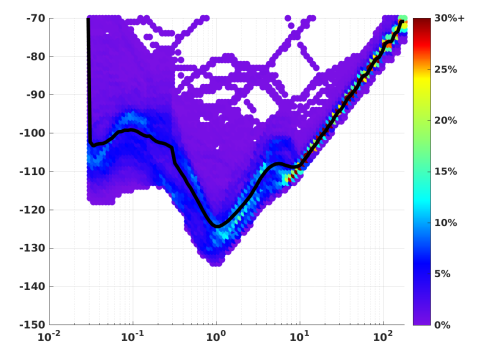

(a) RT05 EHE winter

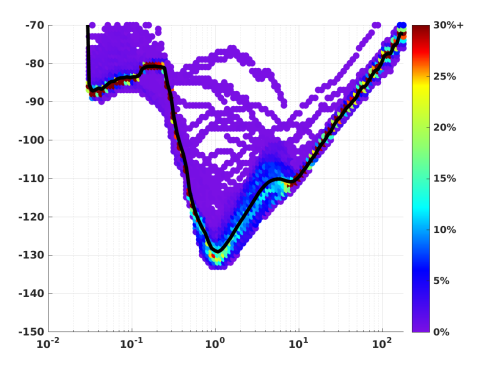

(d) RT06 EHE winter

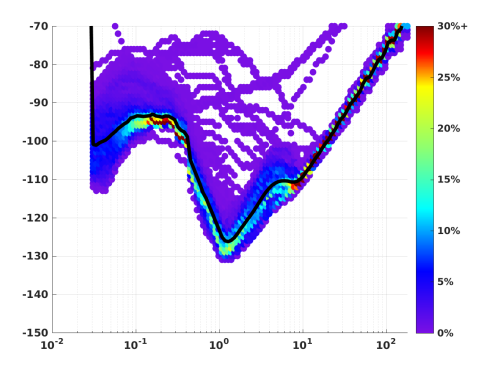

(g) RT07 EHE winter

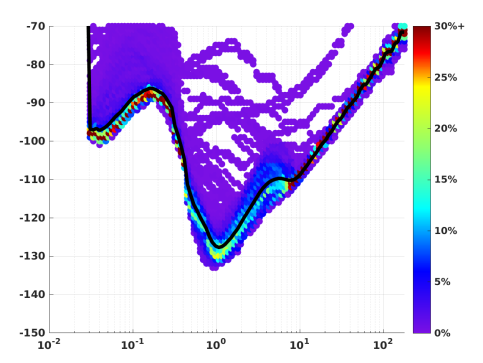

(j) RT08 EHE winter

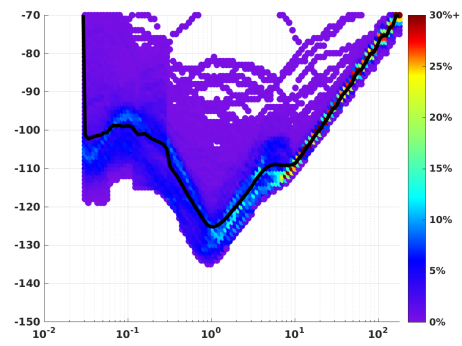

(b) RT05 EHN winter

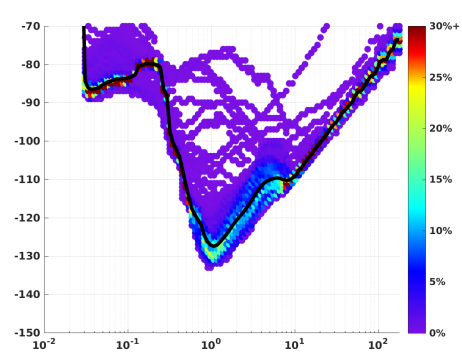

(e) RT06 EHN winter

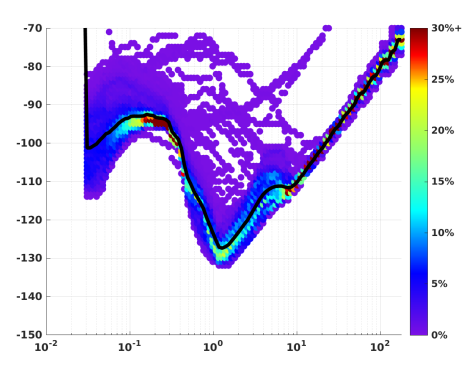

(h) RT07 EHN winter

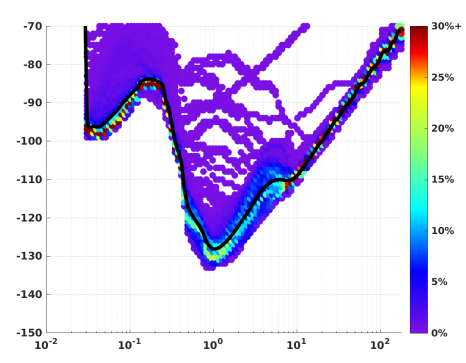

(k) RT08 EHN winter

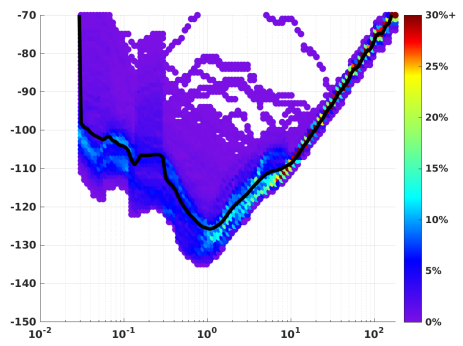

(c) RT05 EHZ winter

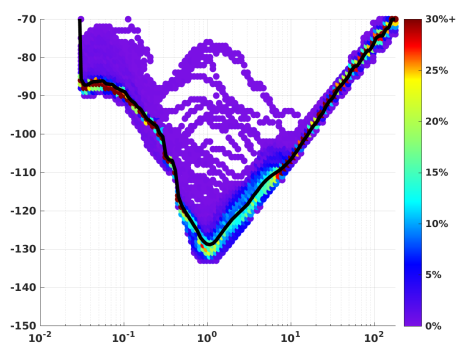

(f) RT06 EHZ winter

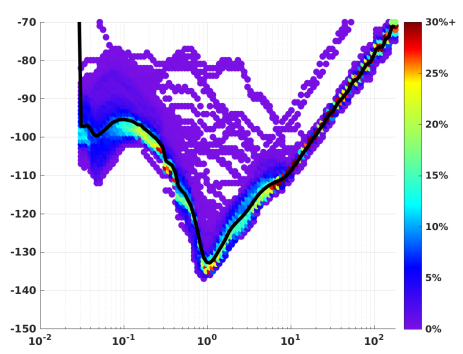

(i) RT07 EHZ winter

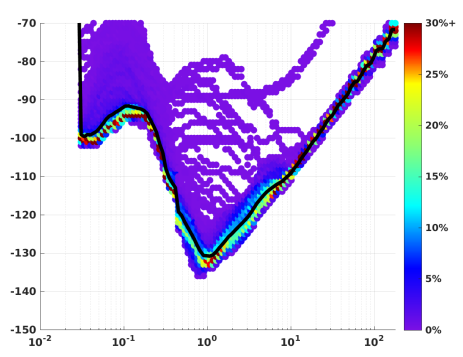

(l) RT08 EHZ winter

Figure B.11: Power spectral density for Rotokawa stations RT05--RT08 for winter. 


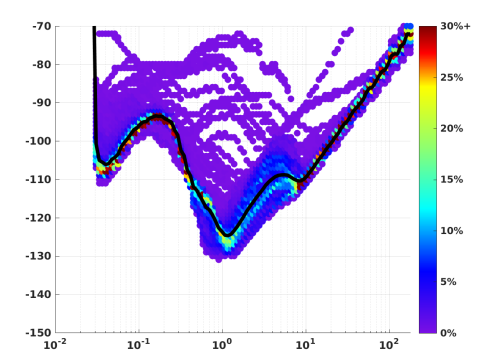

(a) RT09 EHE winter

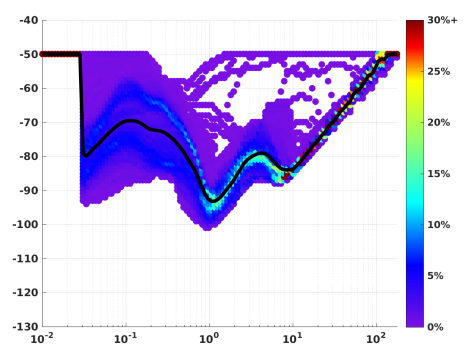

(d) RT10 EHE winter

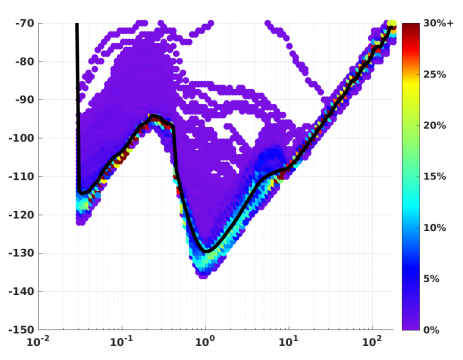

(g) RT11 EHE winter

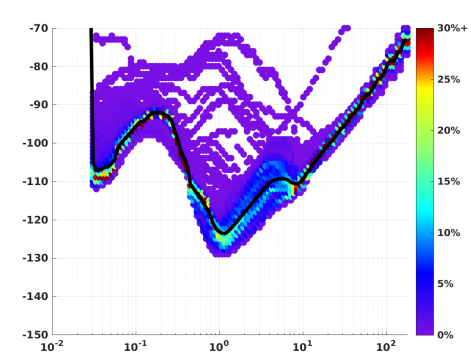

(b) RT09 EHN winter

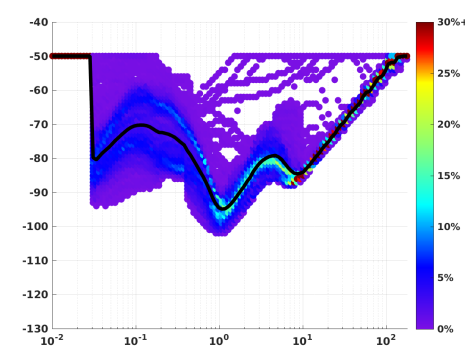

(e) RT10 EHN winter

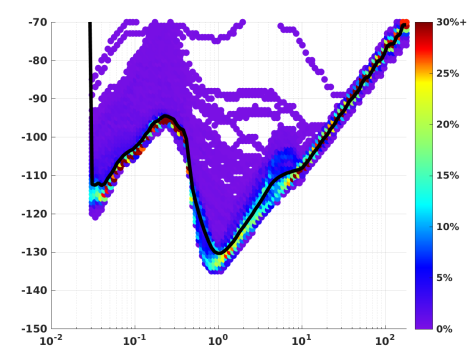

(h) RT11 EHN winter

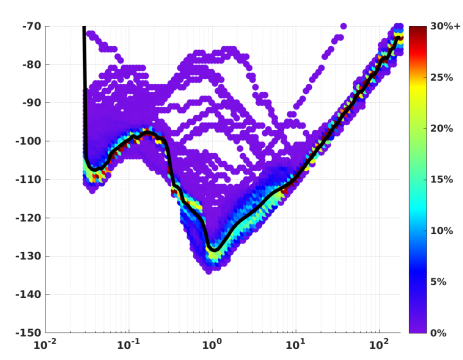

(c) RT09 EHZ winter

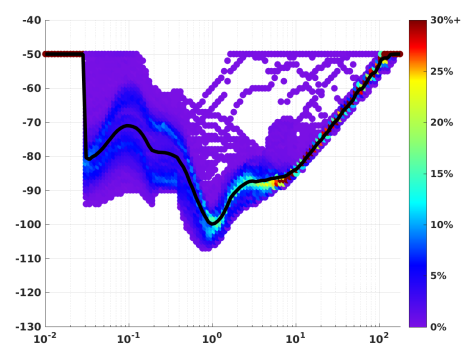

(f) RT10 EHZ winter

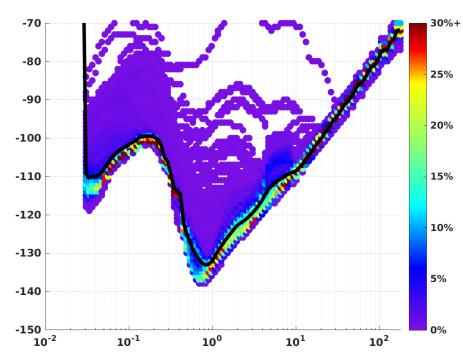

(i) RT11 EHZ winter

Figure B.12: Power spectral density for Rotokawa stations RT09--RT11 for winter. 


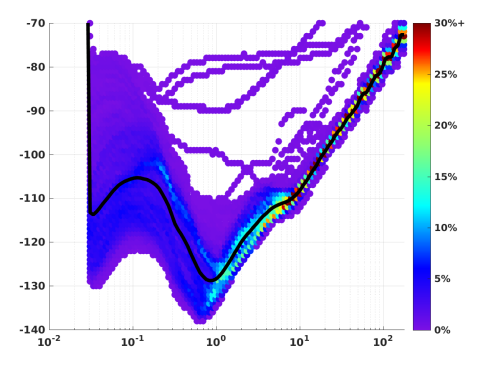

(a) NS01 EHE summer

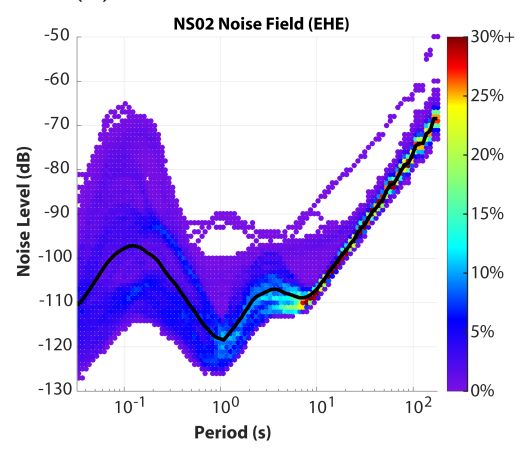

(d) NS02 EHE summer

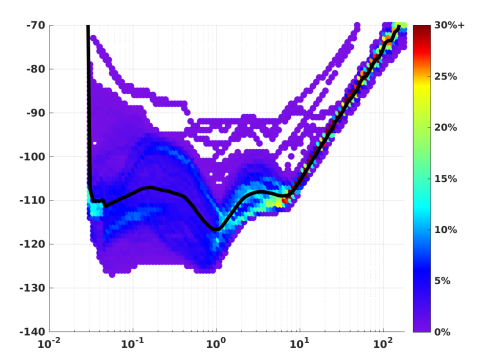

(g) NS03 EHE summer

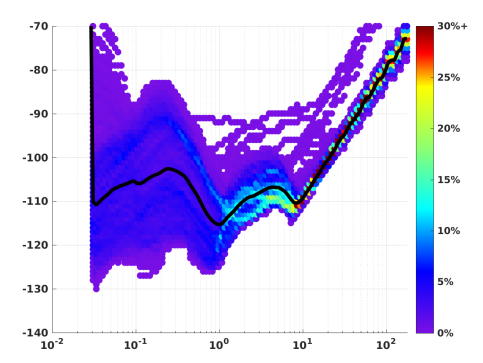

(j) NS04 EHE summer

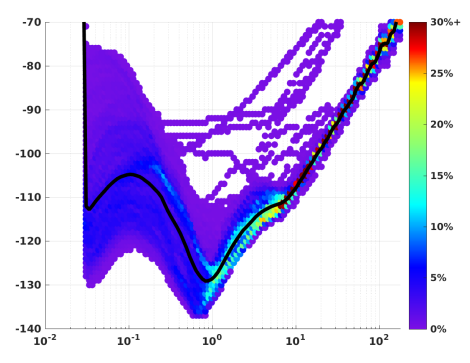

(b) NS01 EHN summer

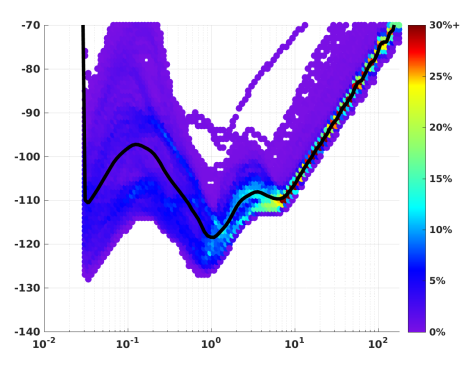

(e) NS02 EHN summer

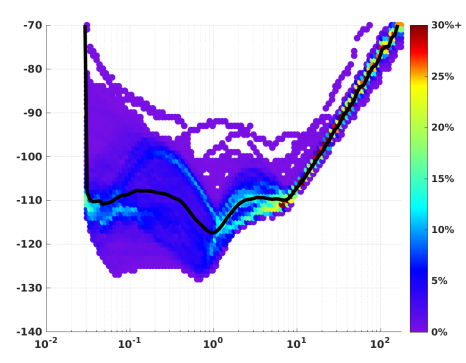

(h) NS03 EHN summer

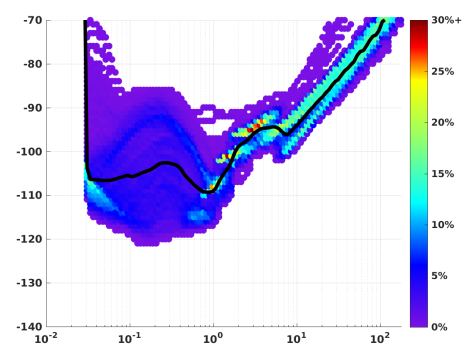

(k) NS04 EHN summer

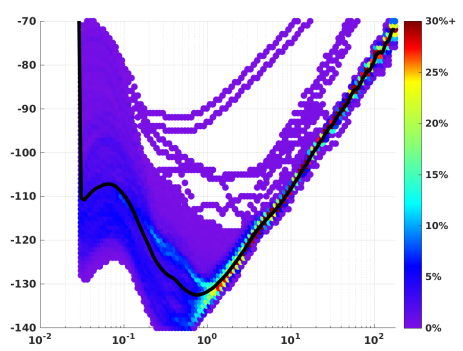

(c) NS01 EHZ summer

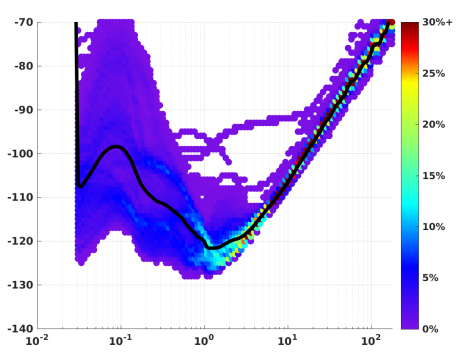

(f) NS02 EHZ summer

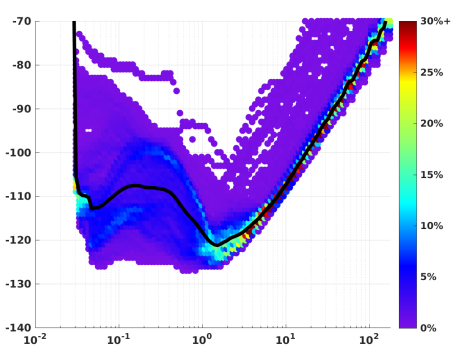

(i) NS03 EHZ summer

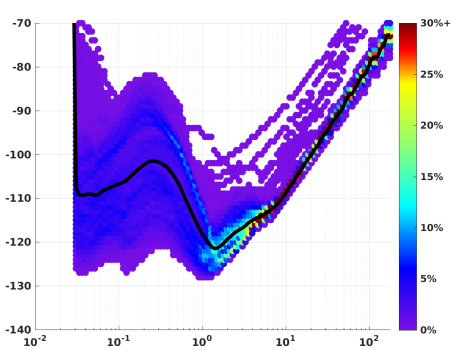

(1) NS04 EHZ summer

Figure B.13: Power spectral density for Ngatamariki stations NM01--NM04 for summer. 


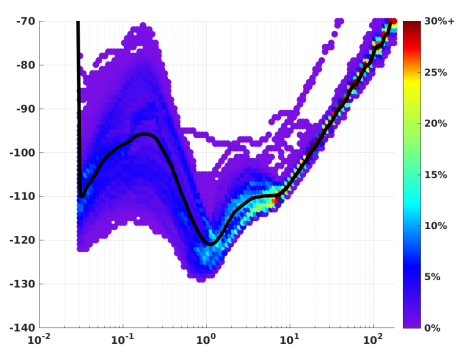

(a) NS05 EHE summer

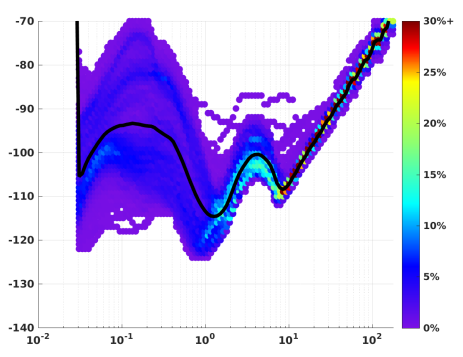

(d) NS07 EHE summer

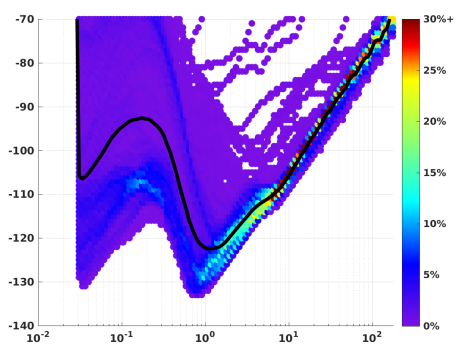

(g) NS08 EHE summer

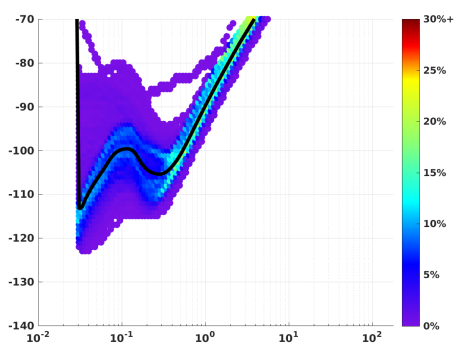

(j) NS09 EHE summer

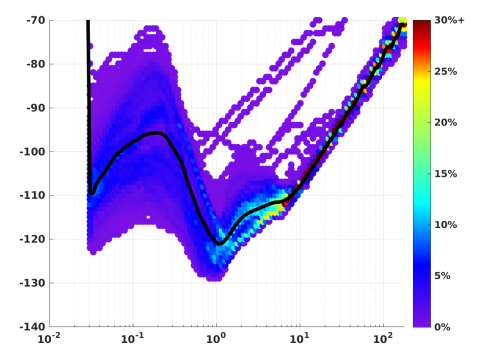

(b) NS05 EHN summer

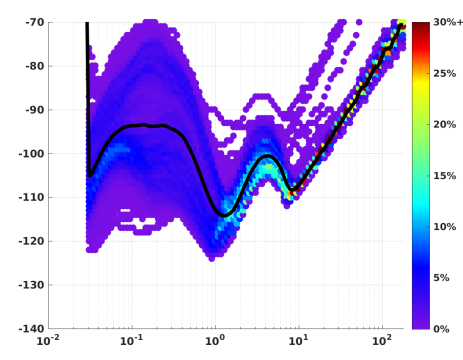

(e) NS07 EHN summer

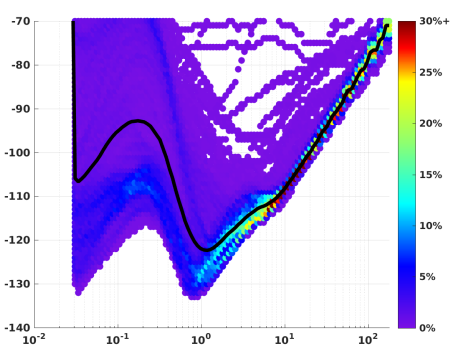

(h) NS08 EHN summer

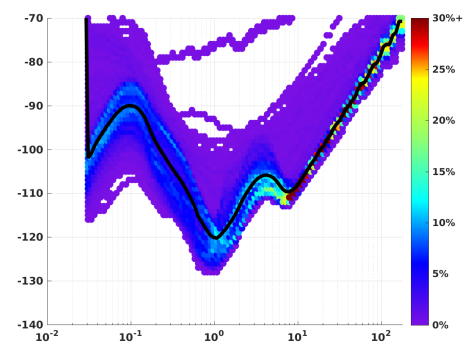

(k) NS09 EHN summer

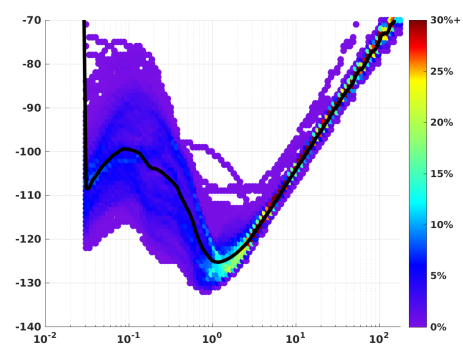

(c) NS05 EHZ summer

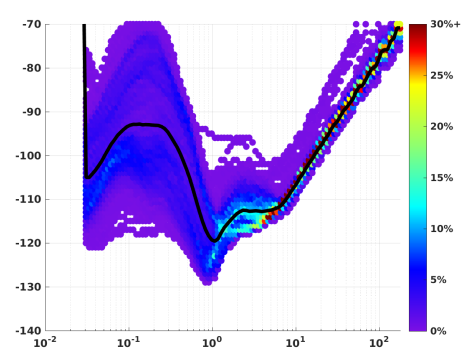

(f) NS07 EHZ summer

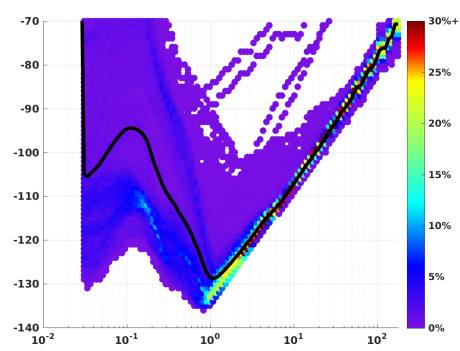

(i) NS08 EHZ summer

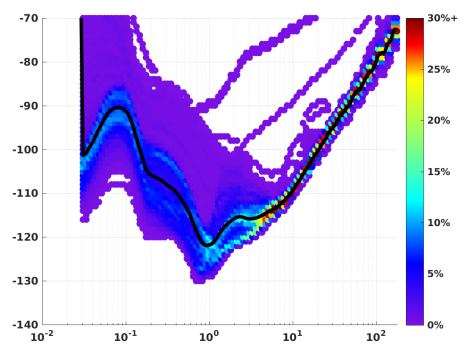

(1) NS09 EHZ summer

Figure B.14: Power spectral density for Ngatamariki stations NM05, NM07--NM09 for summer. 


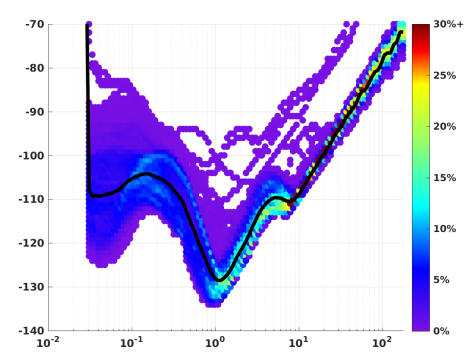

(a) NS10 EHE summer

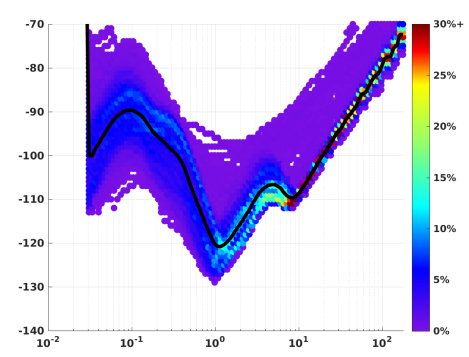

(d) NS11 EHE summer

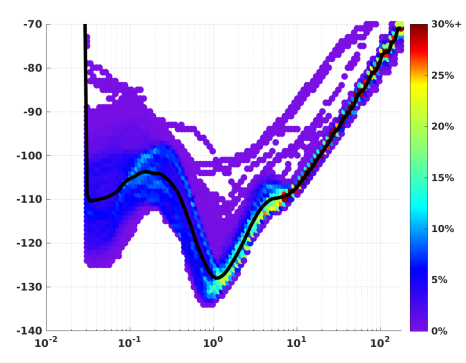

(b) NS10 EHN summer

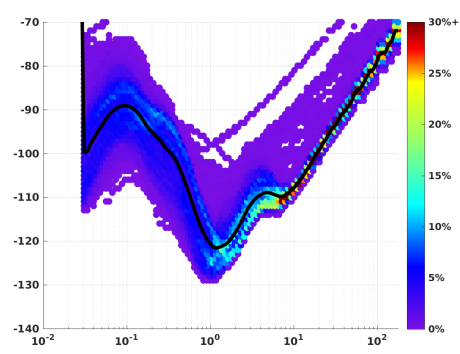

(e) NS11 EHN summer

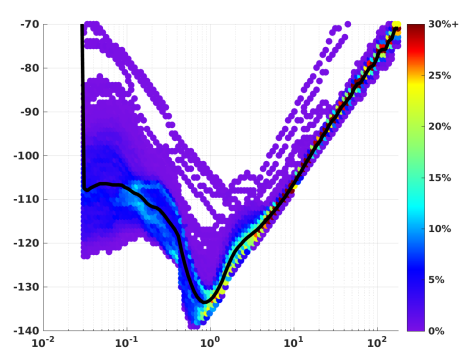

(c) NS10 EHZ summer

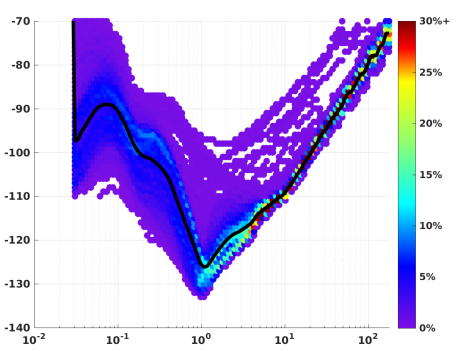

(f) NS11 EHZ summer

Figure B.15: Power spectral density for Ngatamariki stations NM10--NM11 for summer. 


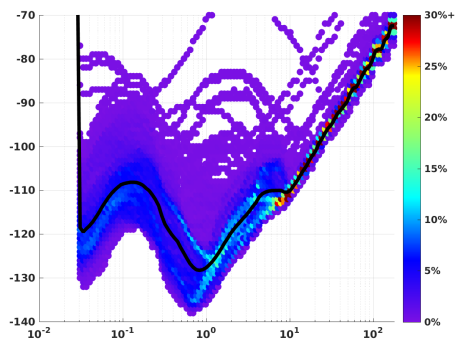

(a) NS01 EHE winter

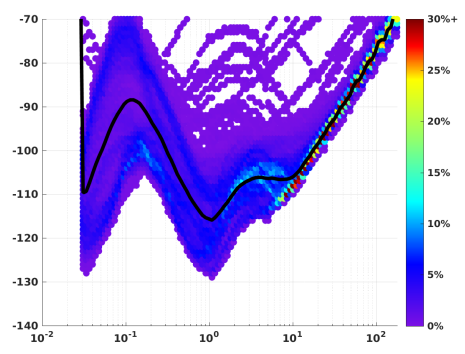

(d) NS02 EHE winter

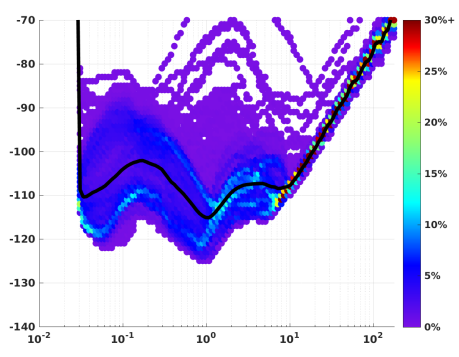

(g) NS03 EHE winter

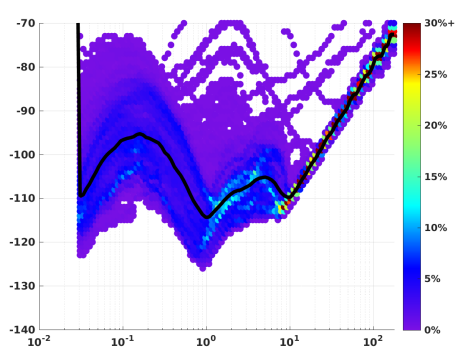

(j) NS04 EHE winter

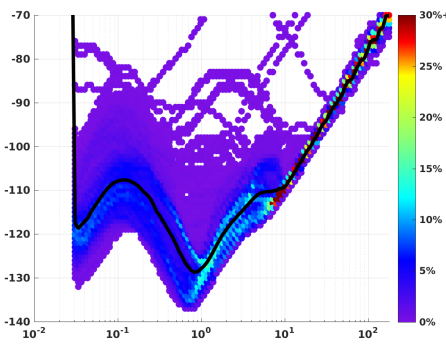

(b) NS01 EHN winter

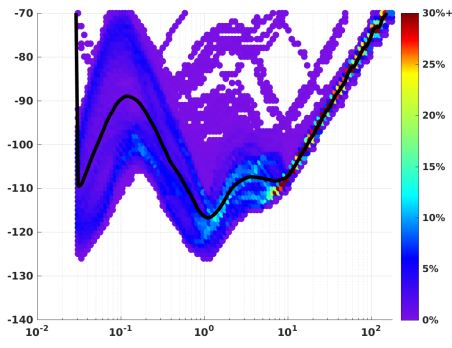

(e) NS02 EHN winter

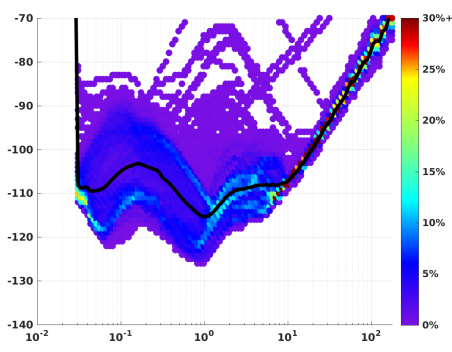

(h) NS03 EHN winter

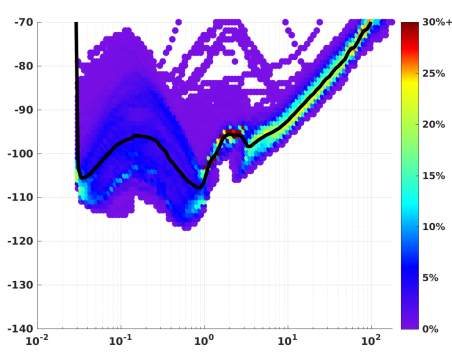

(k) NS04 EHN winter

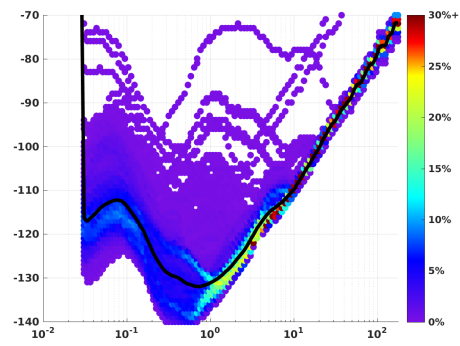

(c) NS01 EHZ winter

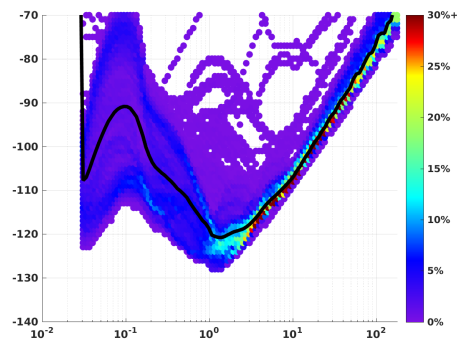

(f) NS02 EHZ winter

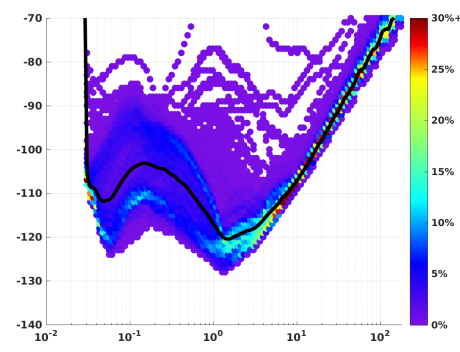

(i) NS03 EHZ winter

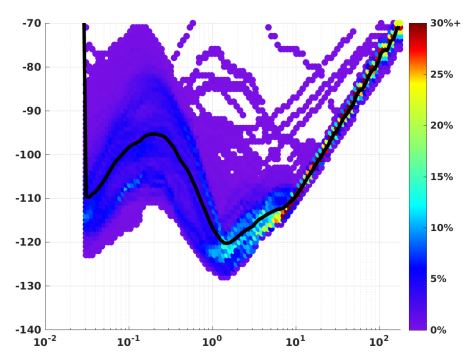

(1) NS04 EHZ winter

Figure B.16: Power spectral density for Ngatamariki stations NM01--NM04 for winter. 


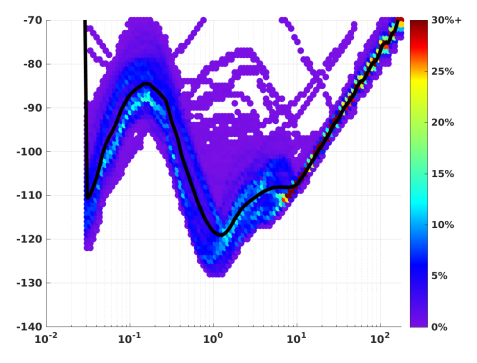

(a) NS05 EHE winter

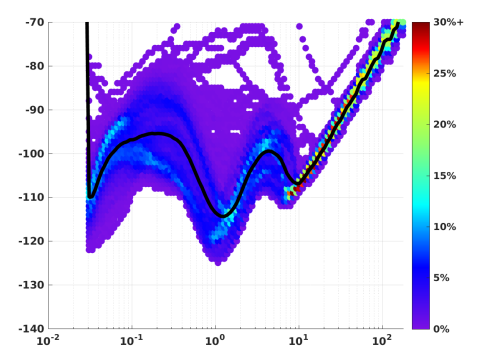

(d) NS07 EHE winter

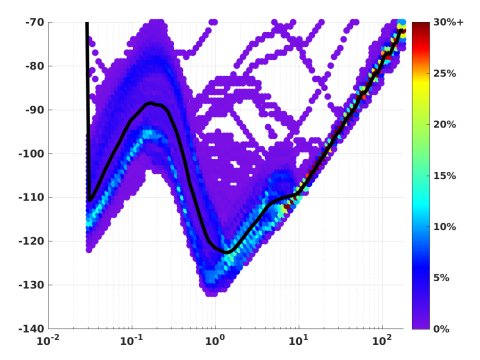

(g) NS08 EHE winter

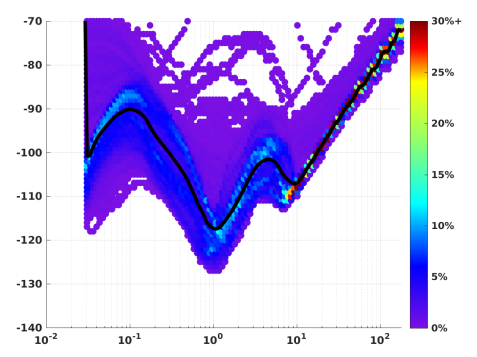

(j) NS09 EHE winter

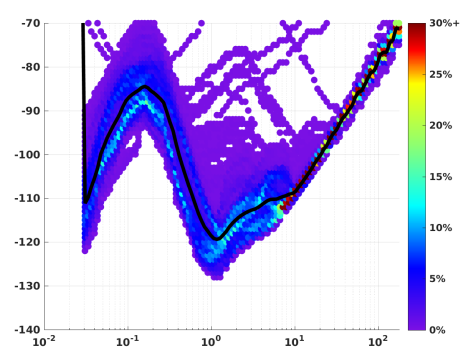

(b) NS05 EHN winter

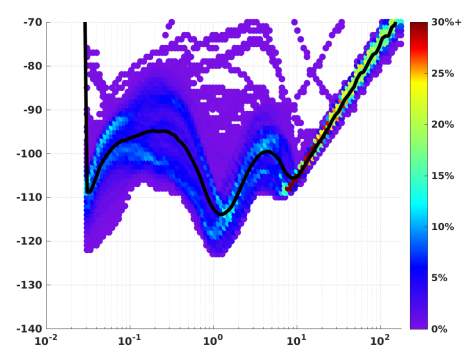

(e) NS07 EHN winter

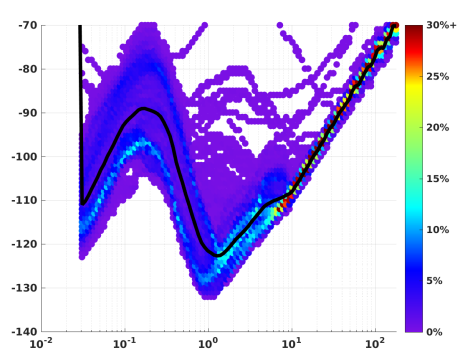

(h) NS08 EHN winter

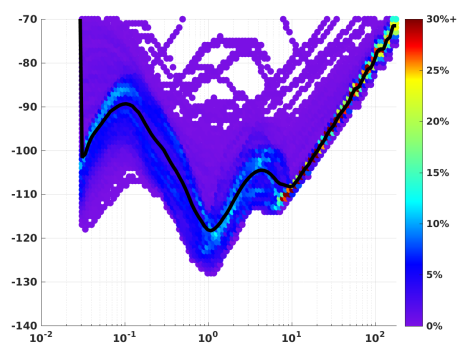

(k) NS09 EHN winter

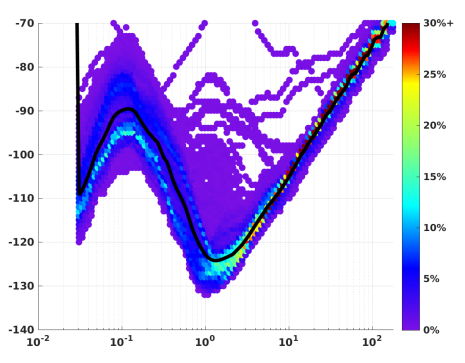

(c) NS05 EHZ winter

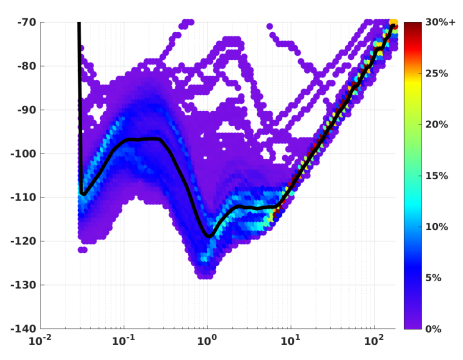

(f) NS07 EHZ winter

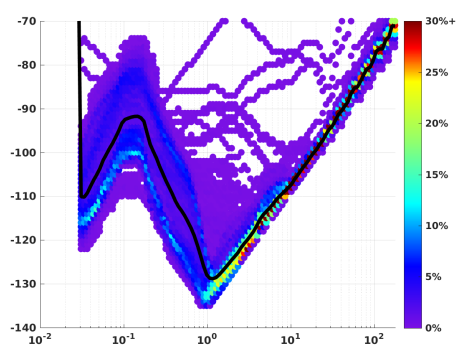

(i) NS08 EHZ winter

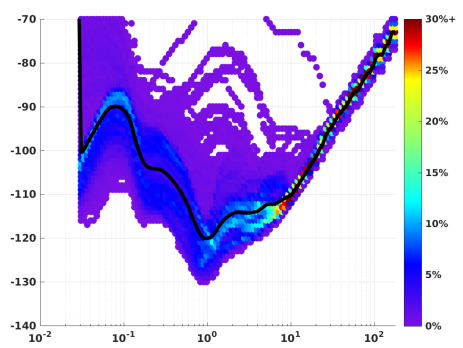

(1) NS09 EHZ winter

Figure B.17: Power spectral density for Ngatamariki stations NM05, NM07--NM09 for winter. 


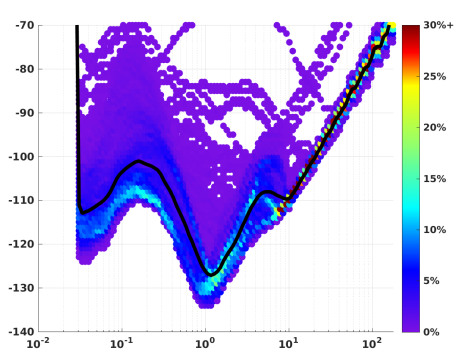

(a) NS10 EHE winter

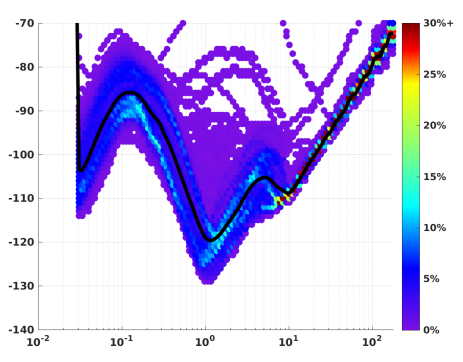

(d) NS11 EHE winter

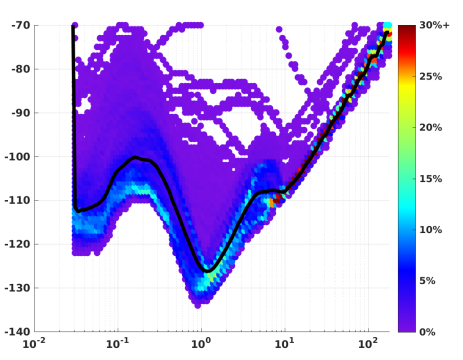

(b) NS10 EHN winter

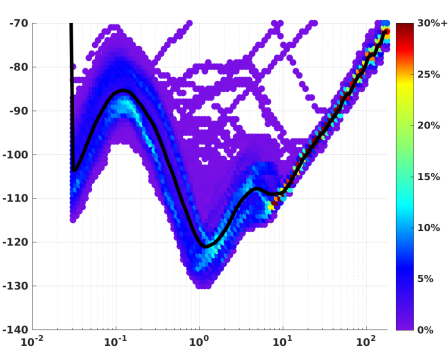

(e) NS11 EHN winter

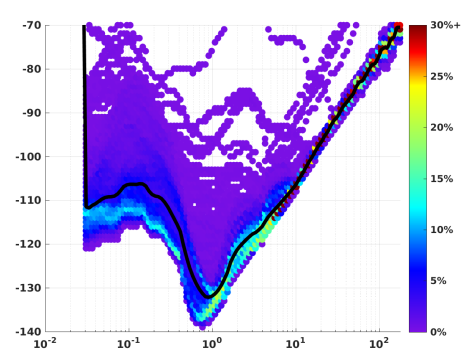

(c) NS10 EHZ winter

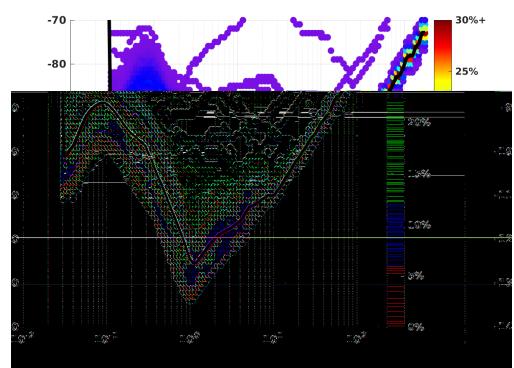

(f) NS11 EHZ winter

Figure B.18: Power spectral density for Ngatamariki stations NM10--NM11 for winter. 


\section{Appendix C}

\section{ReMi cultural noise supplementary information}

\section{C.1 Background}

This Appendix contains supplementary information regarding the sources examined in the ReMi experiment chapter [Section 6.3.2] that is outside the scope of that project. The primary purpose of this section is to assess the seismic attenuation of the cultural noise sources at the Rotokawa geothermal field and explore their effect on microearthquake signal-to-noise ratios (SNRs).

We deployed a temporary set of instruments across Rotokawa in two deployments in early 2015 [Figure C.1]. For more information on the deployment and frequency content of the recorded signals, please refer to Section 6.3.2 in the ReMi experiment chapter. The instrument response curve for the GS-11D instrument is displayed in Figure C.3, and the location of each station in the deployment is detailed in Table C.1.

Probability density functions for each station in the deployment were computed using the software PQLX [McNamara and Boaz, 2011] and are displayed in Appendix Section C.6. As may be expected, the cultural noise value recorded at each station for this deployment was significantly higher than the New High Noise Model, which was determined using natural ambient noise [Figure C.2]. The power spectral densities for all the deployed temporary stations are displayed in Section C.6. The weighted noise means are displayed in Figure 6.6 in Section 6.3.2. 


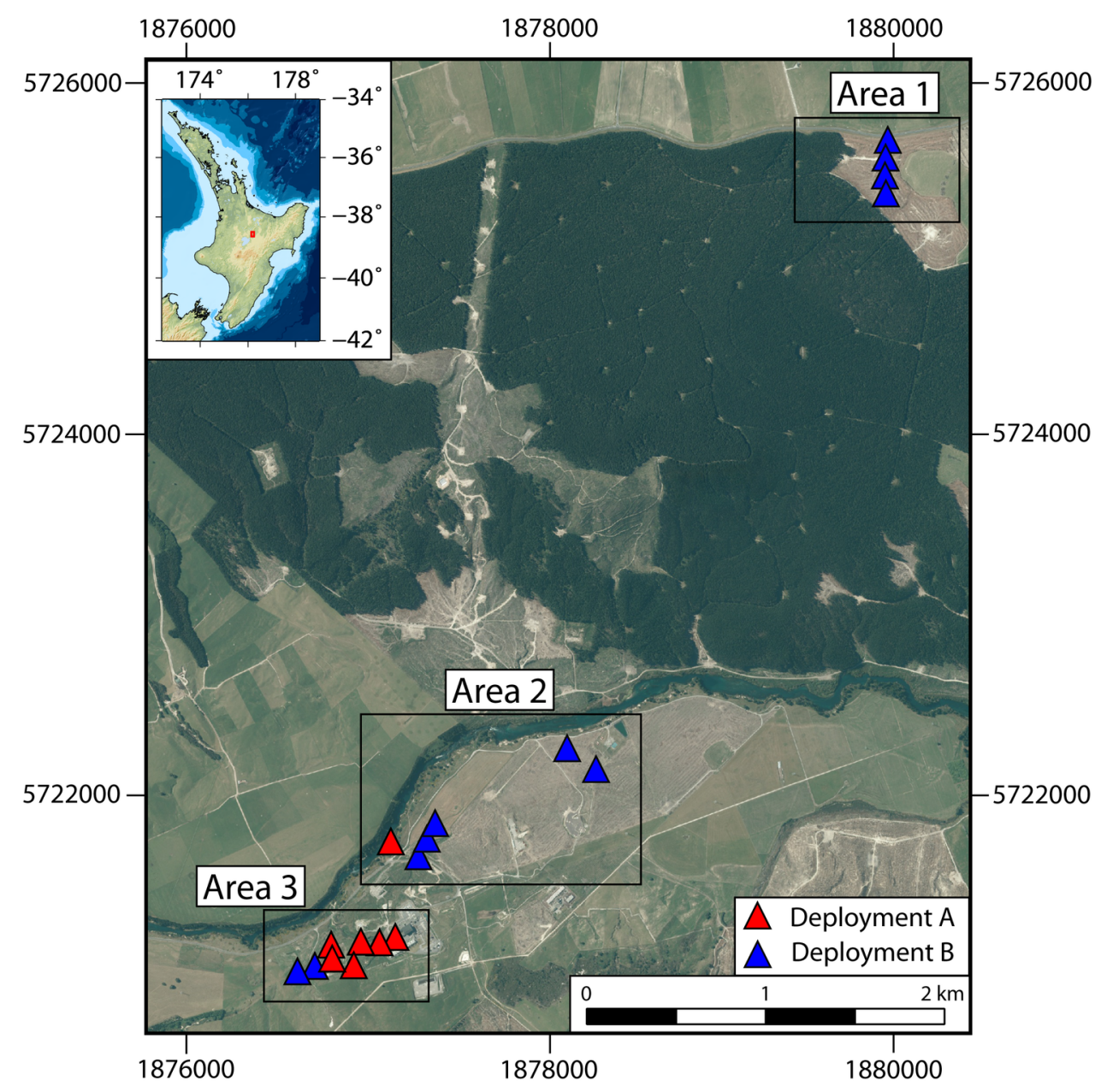

Figure C.1: Map of the temporary deployment used to measure the amplitude and attenuation of the noise sources at the Rotokawa geothermal field. Subsections of each area used for analysis are displayed in boxes. Map coordinates are in New Zealand Transverse Mercator 2000 (NZTM2000). 


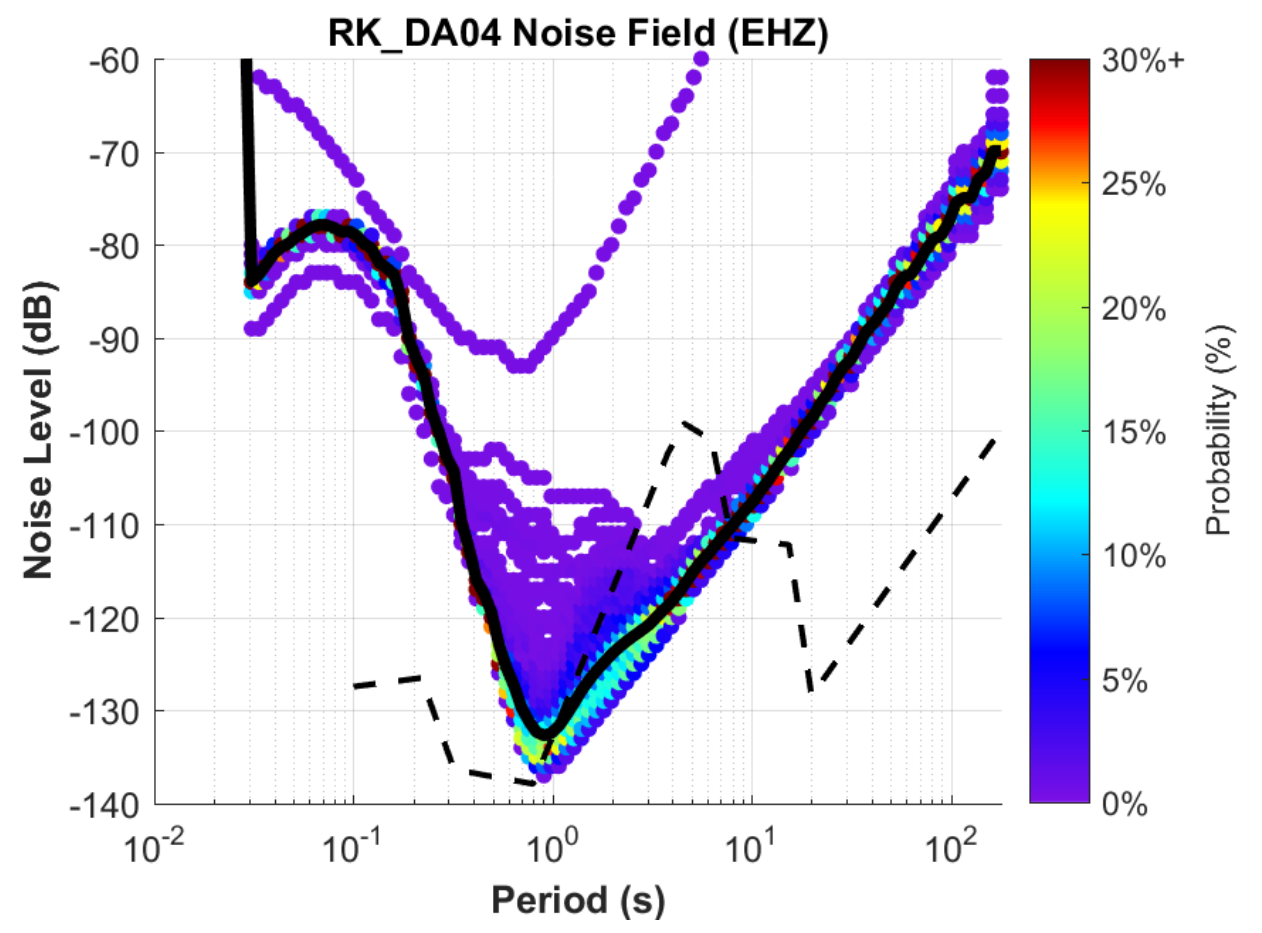

Figure C.2: Noise field for temporary station DA04, its weighted mean (solid line), and the New High Noise Model of Peterson [1993] (dashed line). 


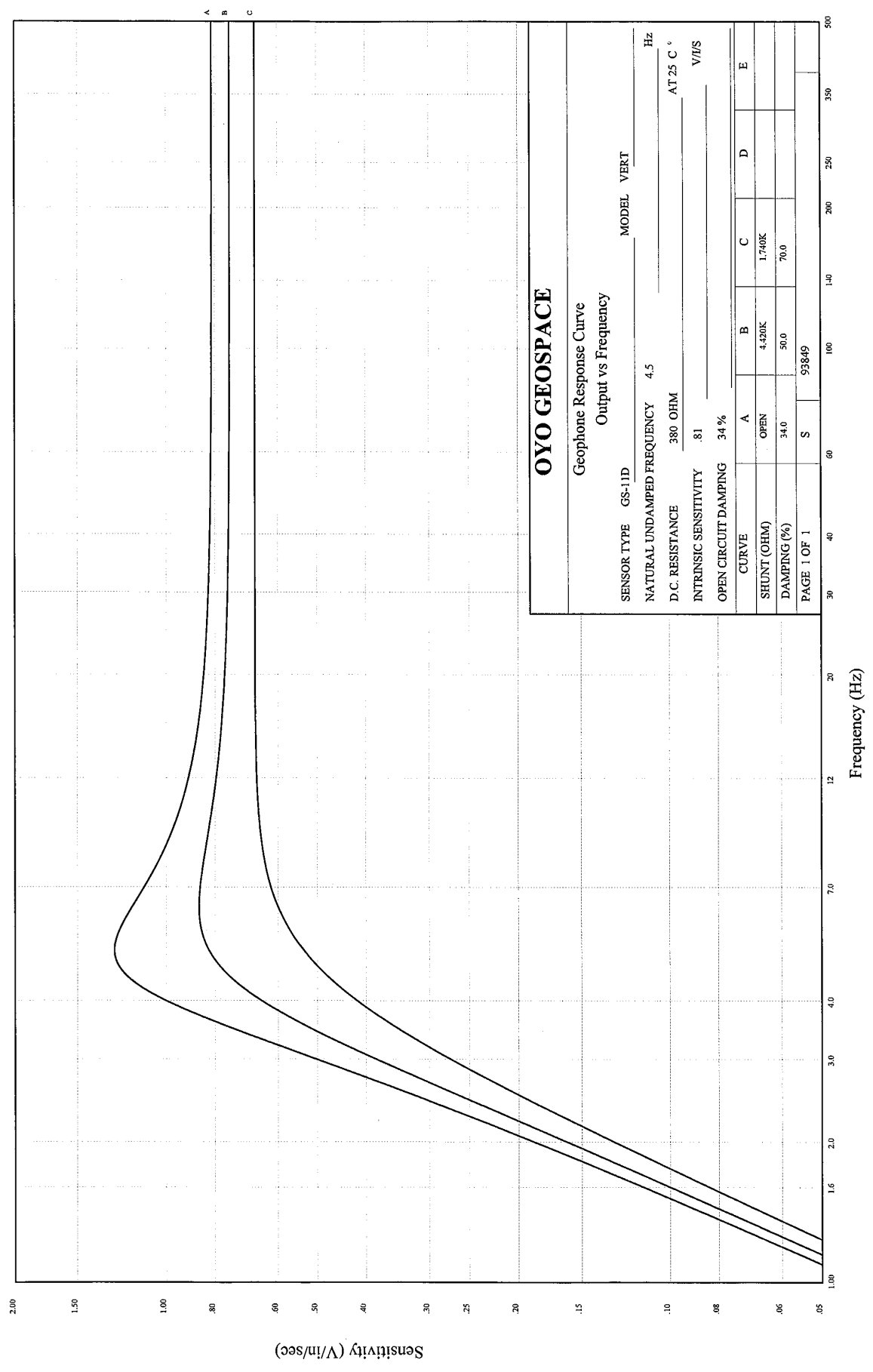




\begin{tabular}{||l||l||l||l||l||}
\hline Station & Installed & Removed & Latitude & Longitude \\
\hline DA01 & $2 / 4 / 2015$ & $2 / 20 / 2015$ & -38.6145 & 176.1830 \\
\hline DA02 & $2 / 4 / 2015$ & $2 / 20 / 2015$ & -38.6148 & 176.1819 \\
\hline DA03 & $2 / 4 / 2015$ & $2 / 20 / 2015$ & -38.6150 & 176.1808 \\
\hline DA04 & $2 / 4 / 2015$ & $2 / 20 / 2015$ & -38.6152 & 176.1797 \\
\hline DA05 & $2 / 4 / 2015$ & $2 / 20 / 2015$ & -38.6160 & 176.1808 \\
\hline DA06 & $2 / 4 / 2015$ & $2 / 20 / 2015$ & -38.6159 & 176.1786 \\
\hline DA07 & $2 / 4 / 2015$ & $2 / 20 / 2015$ & -38.6092 & 176.1833 \\
\hline DB01 & $3 / 13 / 2015$ & $3 / 24 / 2015$ & -38.5735 & 176.2128 \\
\hline DB02 & $3 / 13 / 2015$ & $3 / 24 / 2015$ & -38.5744 & 176.2127 \\
\hline DB03 & $3 / 13 / 2015$ & $3 / 24 / 2015$ & -38.5753 & 176.2127 \\
\hline DB04 & $3 / 12 / 2015$ & $3 / 24 / 2015$ & -38.5762 & 176.2128 \\
\hline DB05 & $3 / 11 / 2015$ & $3 / 24 / 2015$ & -38.6047 & 176.1936 \\
\hline DB06 & $3 / 11 / 2015$ & $3 / 24 / 2015$ & -38.6057 & 176.1955 \\
\hline DB07 & $2 / 20 / 2015$ & $3 / 10 / 2015$ & -38.6104 & 176.1843 \\
\hline DB08 & $2 / 20 / 2015$ & $3 / 11 / 2015$ & -38.6095 & 176.1848 \\
\hline DB09 & $2 / 20 / 2015$ & $3 / 12 / 2015$ & -38.6087 & 176.1853 \\
\hline DB10 & $2 / 20 / 2015$ & $3 / 10 / 2015$ & -38.6161 & 176.1779 \\
\hline DB11 & $2 / 20 / 2015$ & $3 / 10 / 2015$ & -38.6164 & 176.1768 \\
\hline
\end{tabular}

Table C.1: Station information for the cultural noise deployment at the Rotokawa geothermal field. Station coordinates are in WGS84.

\section{C.2 Methodology}

To facilitate the attenuation interpretation, we divided the deployment in three areas and into groups based on noise sources. These groupings are detailed in Figures C.4, C.5, and C.6 and in Table C.2.

The primary tool for determining attenuation are the power spectral densities computed by the program PQLX [McNamara and Boaz, 2011], first described in detail in Section 2.2. We define the decibel scale as a logarithmic measure of power [Peterson, 1993]:

$$
y(\omega)=10 \log _{10} s(\omega)
$$

where $y(\omega)$ and $s(\omega)$ are the values of the power spectral density in units of decibels and $\left(\mathrm{m} / \mathrm{s}^{2}\right)^{2} / \mathrm{Hz}$ respectively. Because the power spectral density is not represented as a ratio of two powers, it is directly correlated with signal amplitude. Therefore, we can 


\begin{tabular}{||l||l||l||}
\hline Grouping & Noise Source & Instruments \\
\hline Area 1 & State Highway 5 & DB01, DB02, DB03, DB04 \\
\hline Area 2 Group 1 & Production pipe & DB05, DB06 \\
\hline Area 2 Group 2 & Nga Awa Purua road & DB07, DB08, DB09 \\
\hline Area 3 Group 1 & Nga Awa Purua power station & DA01, DA02, DA03, DA04 \\
\hline Area 3 Group 2 & Production well & DA04, DA06 \\
\hline Area 3 Group 3 & Production well & DA06, DB10, DB11 \\
\hline
\end{tabular}

Table C.2: Station source information for the Rotokawa cultural noise deployment.

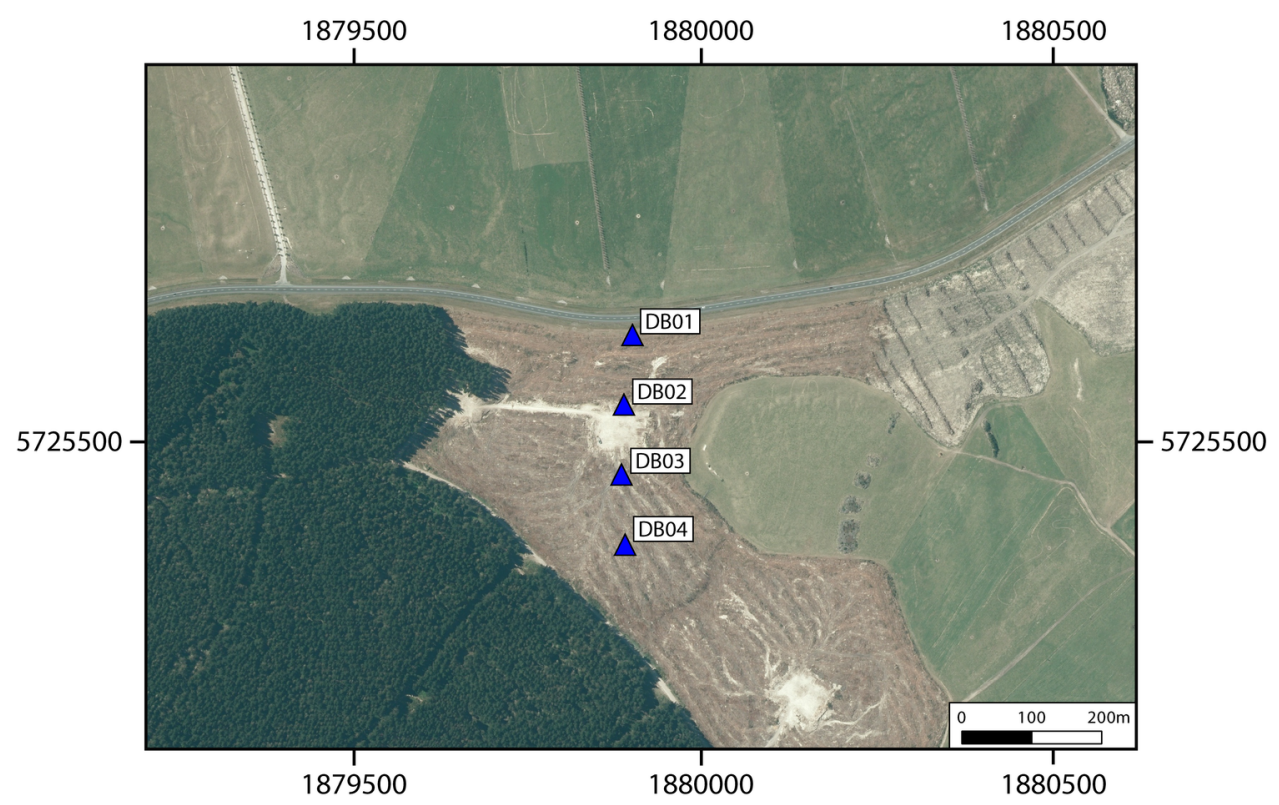

Figure C.4: Area 1 of the cultural noise deployment. Four stations with approximately $100 \mathrm{~m}$ separation extend south of State Highway 5.

switch back and forth between amplitude and decibels using Equation C.1.

We present an example of the process to determine attenuation below, using the sensors of Area 1 EHN components [Figure C.4]. Figure C.7 displays the difference between the decibel level of the plots and the regular amplitude of the signals.

We use the following procedure to determine attenuation: [1] Obtain the maximum noise in the decibel scale between 0.05 and 0.2 seconds (the range of the cultural noise) for a certain grouping of instruments influenced by the same source, [2] convert the distance values of the instruments groupings as a distance away from the instrument closest to the source (referred to as the "source station"), and [3] fit the scatter points with a linear fit [Figure C.8]. 


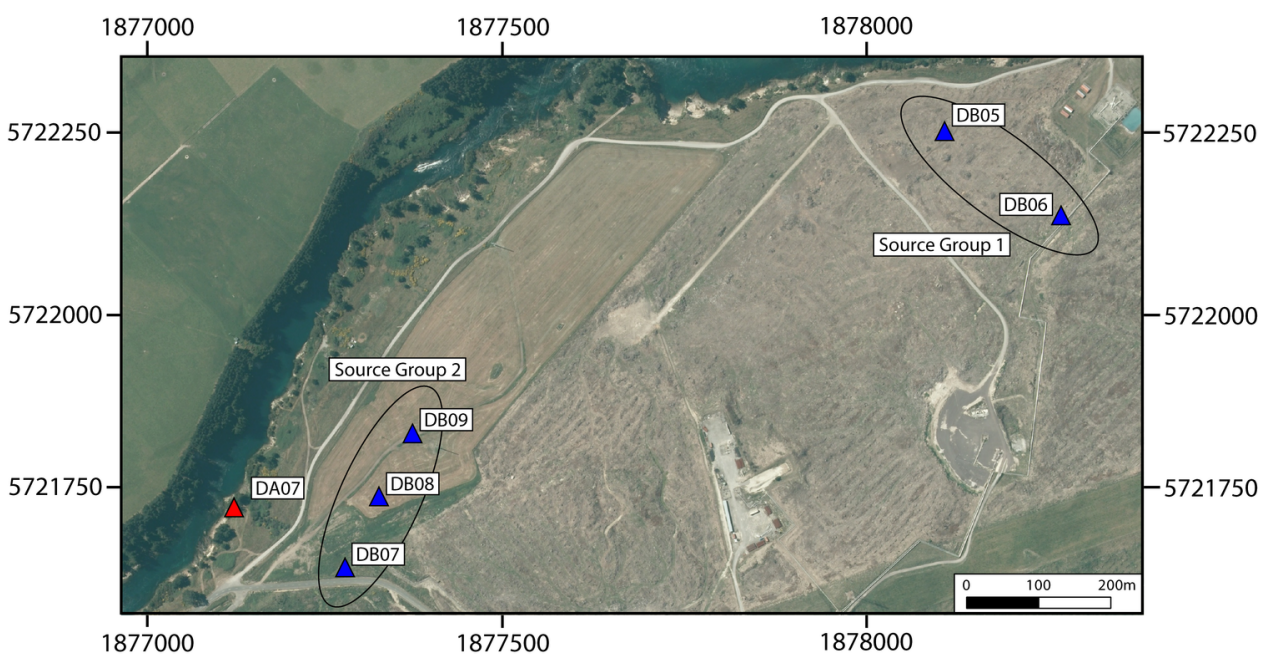

Figure C.5: Area 2 of the cultural noise deployment. Source group 1 lies between two infrequently traveled roads and a production pipe, while source group 2 extends perpendicular from the road used to reach the Nga Awa Purua power station. The battery at DA07 was stolen within a few days of the deployment, so it is not included in a source grouping.

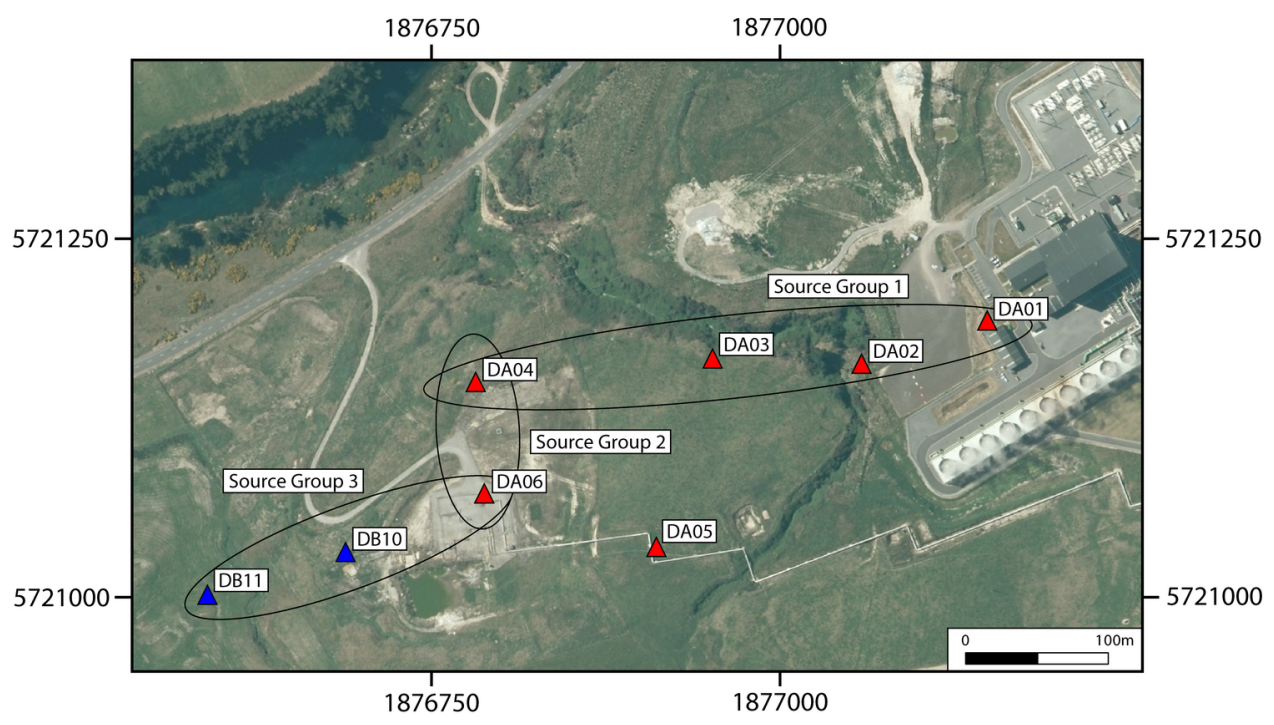

Figure C.6: Area 3 of the cultural noise deployment. Source group 1 was deployed west of the Nga Awa Purua power station. Source group 2 and source group 3 extend in different directions from a production well. 

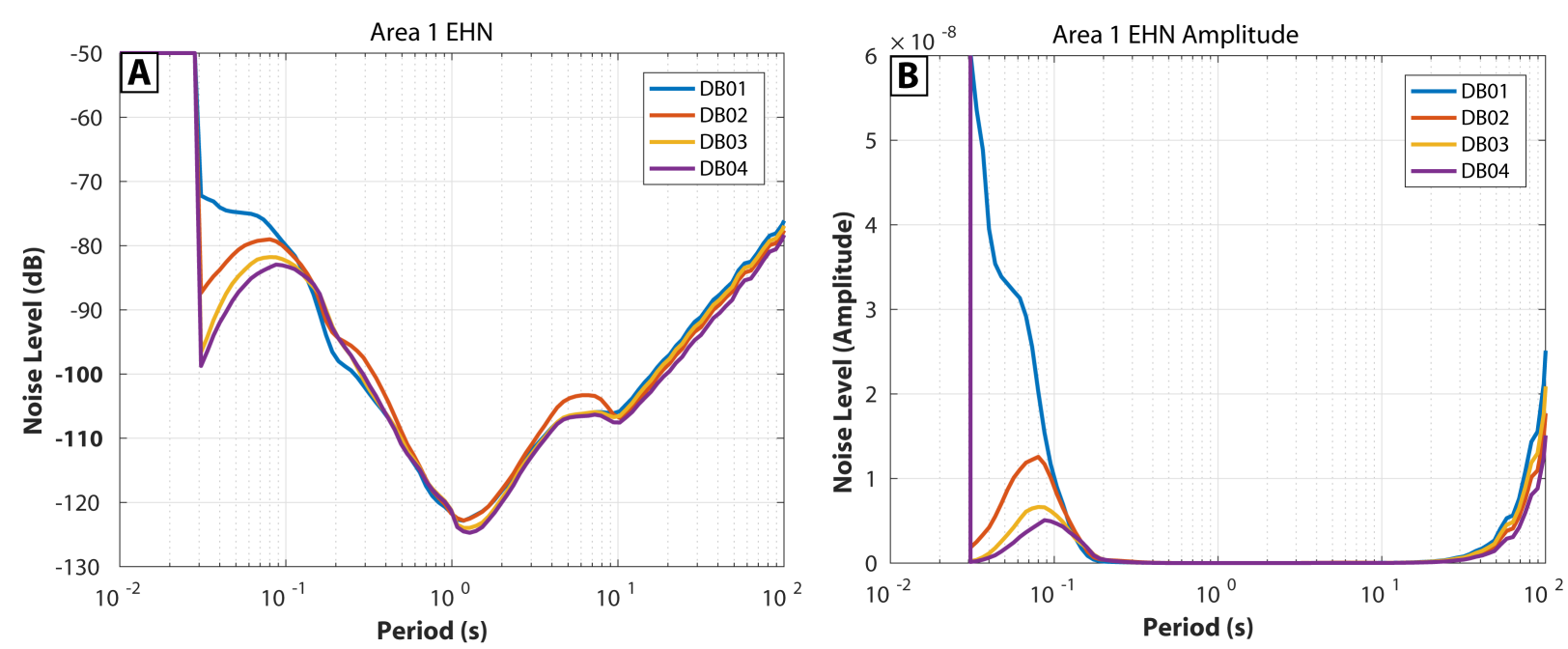

Figure C.7: Power spectral density of EHN component for Area 1 [Figure C.4] sensors using decibels [A] and amplitude [B].

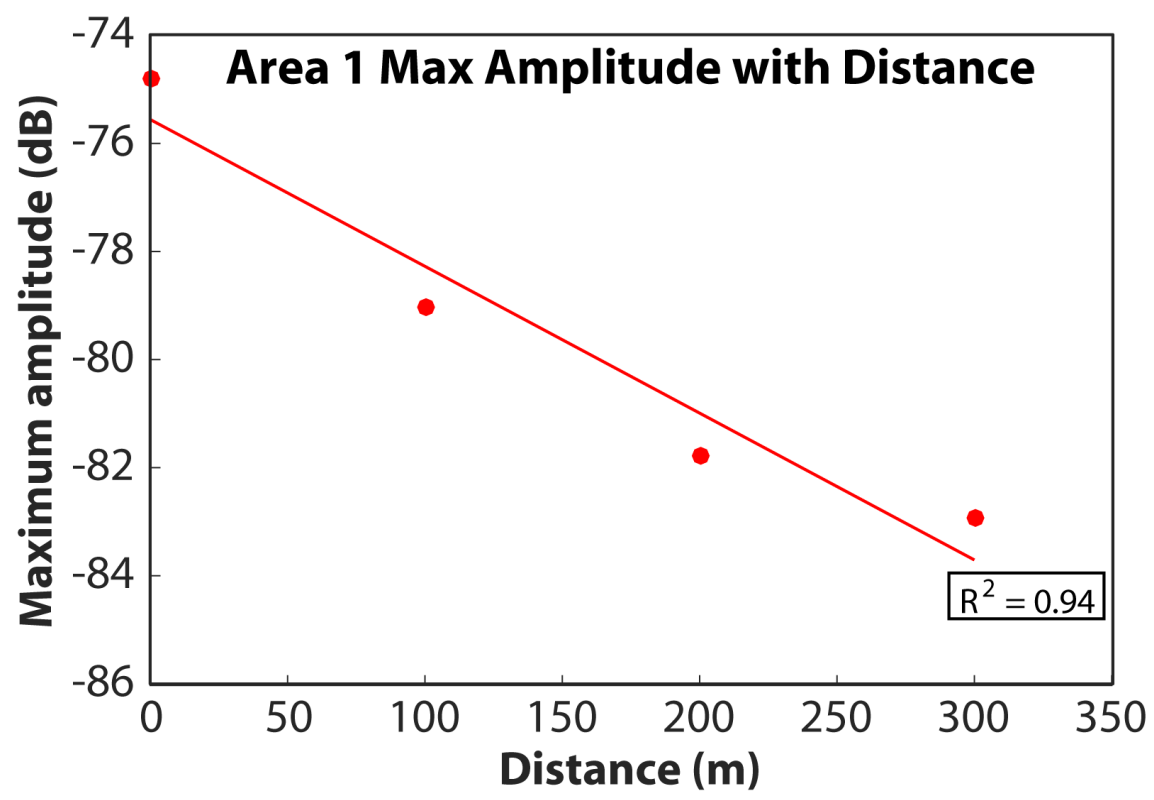

Figure C.8: Attenuation example using Area 1 EHN channels. Scatter plot values are the measured maximum amplitude of the decibel signal between 0.05 -- 0.2 seconds. Displays the linear fit for the data (solid red line) and R squared value. 


\section{C.3 Attenuation results}

The attenuation rate due to a linear fit was computed for all source types [Figure C.9]. The $\mathrm{R}$ squared value was computed for each fit when the instrument number was three or more, and is displayed within each figure in an inset. For Area 3 group 1, DA04 was not included in the modeled decay [Figure C.9D] as it is affected by multiple noise sources. The linear-fit decay displayed in Figure C.9 and the 100 meter decay is tabulated in Table C.3.

\begin{tabular}{||l||l||l||l||l||}
\hline Grouping & Noise Source & $\begin{array}{l}\text { EHE } \\
\text { (dB) }\end{array}$ & $\begin{array}{l}\text { EHN } \\
\text { (dB) }\end{array}$ & $\begin{array}{l}\text { EHZ } \\
(\mathbf{d B})\end{array}$ \\
\hline Area 1 & State Highway 5 & -2 & -3 & -7 \\
\hline Area 2 Group 1 & Production pipe & -7 & -7 & -8 \\
\hline Area 2 Group 2 & Nga Awa Purua road & -5 & -7 & -4 \\
\hline Area 3 Group 1 & Nga Awa Purua power station & -5 & -5 & -7 \\
\hline Area 3 Group 2 & Production well & -4 & -5 & -11 \\
\hline Area 3 Group 3 & Production well & -11 & -10 & -17 \\
\hline
\end{tabular}

Table C.3: Attenuation results in decibels per 100 meters for the linear-fit displayed in Figure C.9. 

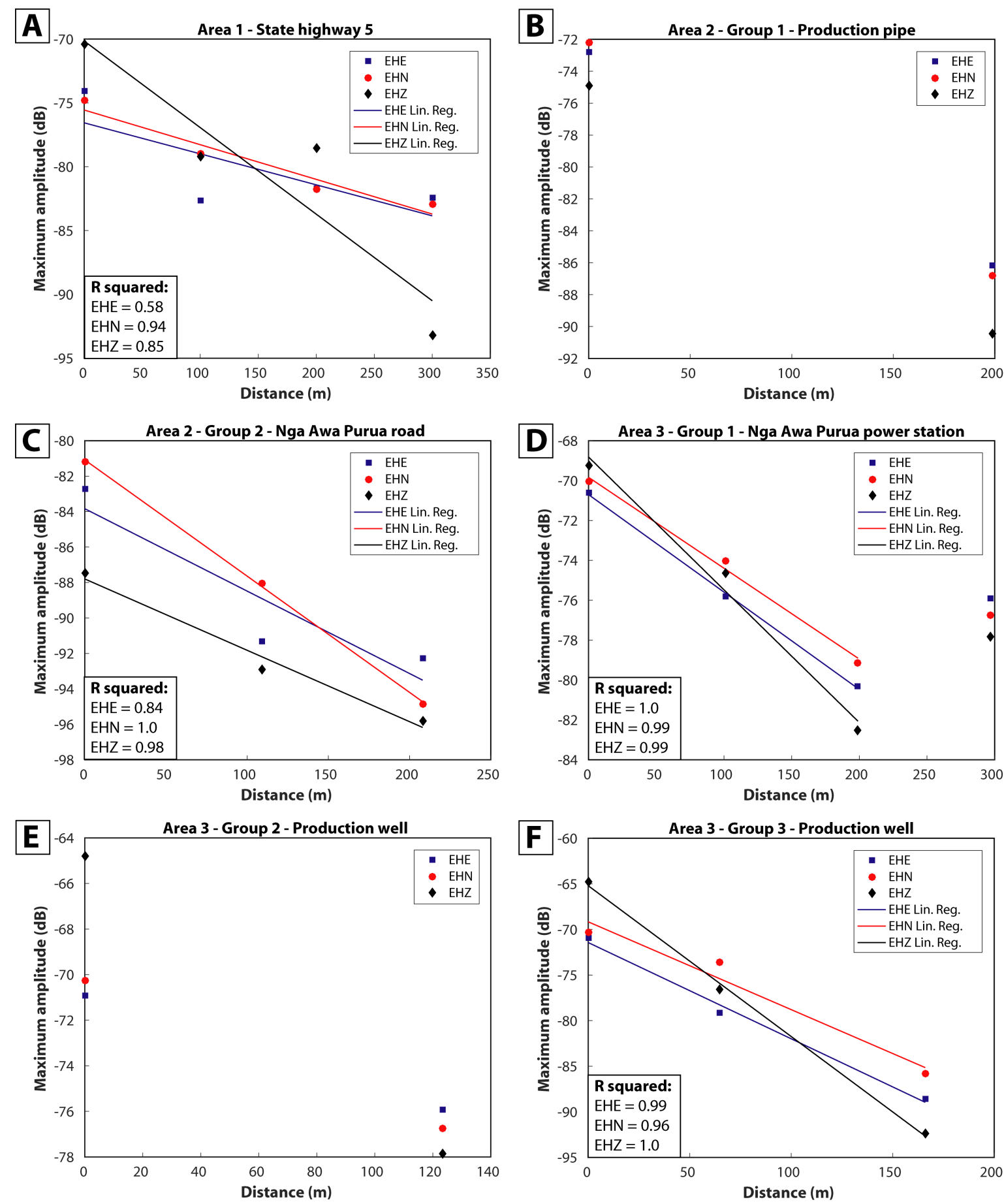

Figure C.9: Noise peak maxima for the Rotokawa geothermal field temporary network and linear fit. Sensor list: [A] Area 1- DB01 (source station), DB02, DB03, and DB04 ; [B] Area 2 group 1- DB06 (source station) and DB05 ; [C] Area 2 group 2- DB07 (source station), DB08, and DB09 ; [D] Area 3 group 1- DA01 (source station), DA02, DA03, and DA04 ; [E] DA06 (source station) and DA04; [F] DA06 (source station), DB10, and DB11. Linear fit and $\mathrm{R}$ squared value are not displayed for grouping with only two stations. 


\section{C.4 Application to microearthquakes}

Sensors should be placed as close as possible to expected microearthquake locations but far enough away from machinery or roads to not be affected by noise as to invalidate wave picks. Throughout this chapter, we have described a Fourier-domain method for determining seismic attenuation utilizing ambient noise power spectral density. In this section, we compare those values to the signal-to-noise ratio of recorded microearthquakes.

Both Rotokawa and Ngatamariki instruments record hundreds of regional, local, and production-induced earthquakes. A Mw 2.0 local earthquake was recorded during our Deployment A time at approximately $4 \mathrm{Km}$ east of the Rotokawa power station (2015-02-08, 10:00:17 UTC, 176.2272 , -38.6078). We computed signal-to-noise ratios for the power station sensors of Area 3 group 1 [Figures C.6 and C.9D] for this event. The power station is a continuously occurring source, so we have a measure of the SNR increase away from the station. The vertical component of the event is displayed in Figure C.10, and the signal-to-noise ratios are listed in Table C.4. The signal-to-noise ratios were calculated by dividing the maximum amplitude of the trace by the standard error the noise prior to the earthquake arrival. With this example, we can compare the attenuation results from the power spectral density with the time-series attenuation using the microearthquake signal-to-noise ratio [Table C.4, Figure C.11].

\begin{tabular}{||l||l||l||l||l||l||l||}
\hline Sensor & $\begin{array}{l}\text { PSD EHE } \\
(\mathbf{d b})\end{array}$ & $\begin{array}{l}\text { PSD EHN } \\
\mathbf{( d b )}\end{array}$ & $\begin{array}{l}\text { PSD EHZ } \\
\mathbf{( d b )}\end{array}$ & $\begin{array}{l}\text { MQ } \\
\text { EHE } \\
(\text { SNR) }\end{array}$ & $\begin{array}{l}\text { MQ } \\
\text { EHN } \\
(\text { SNR) }\end{array}$ & $\begin{array}{l}\text { MQ } \\
\text { EHZ } \\
(\text { SNR) }\end{array}$ \\
\hline DA01 & -70.6 & -70.4 & -69.3 & 25 & 23 & 14 \\
\hline DA02 & -75.8 & -74.0 & -74.6 & 45 & 27 & 37 \\
\hline DA03 & -80.3 & -79.1 & -82.5 & 71 & 60 & 80 \\
\hline
\end{tabular}

Table C.4: Power station attenuation results from sensors DA01, DA02, and DA03 from Area 3 group 1 [Figure C.6]. Units of Power Spectral Density (PSD) are decibel and the micro-earthquake signal-to-noise ratios (SNR) are unitless. 

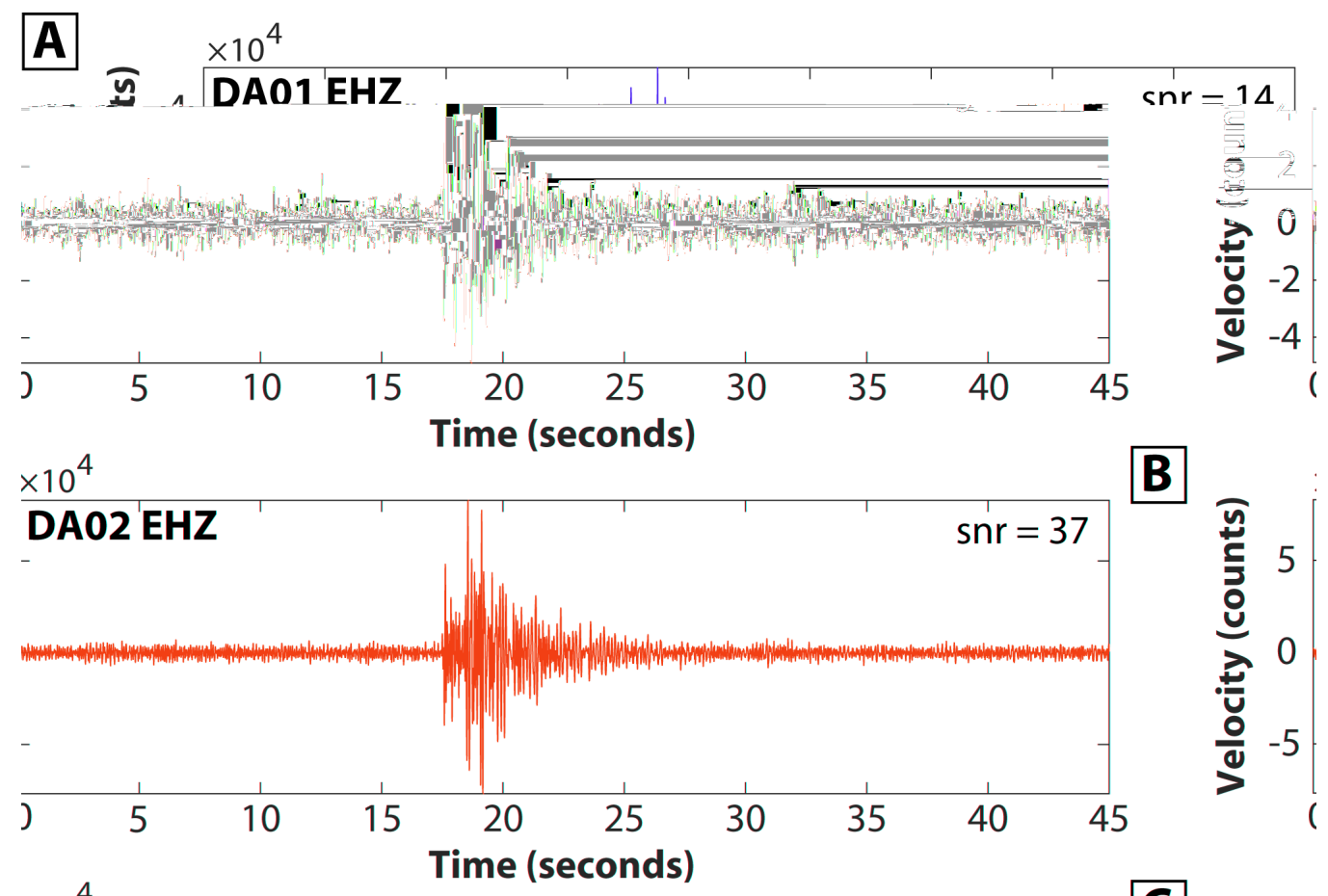

B
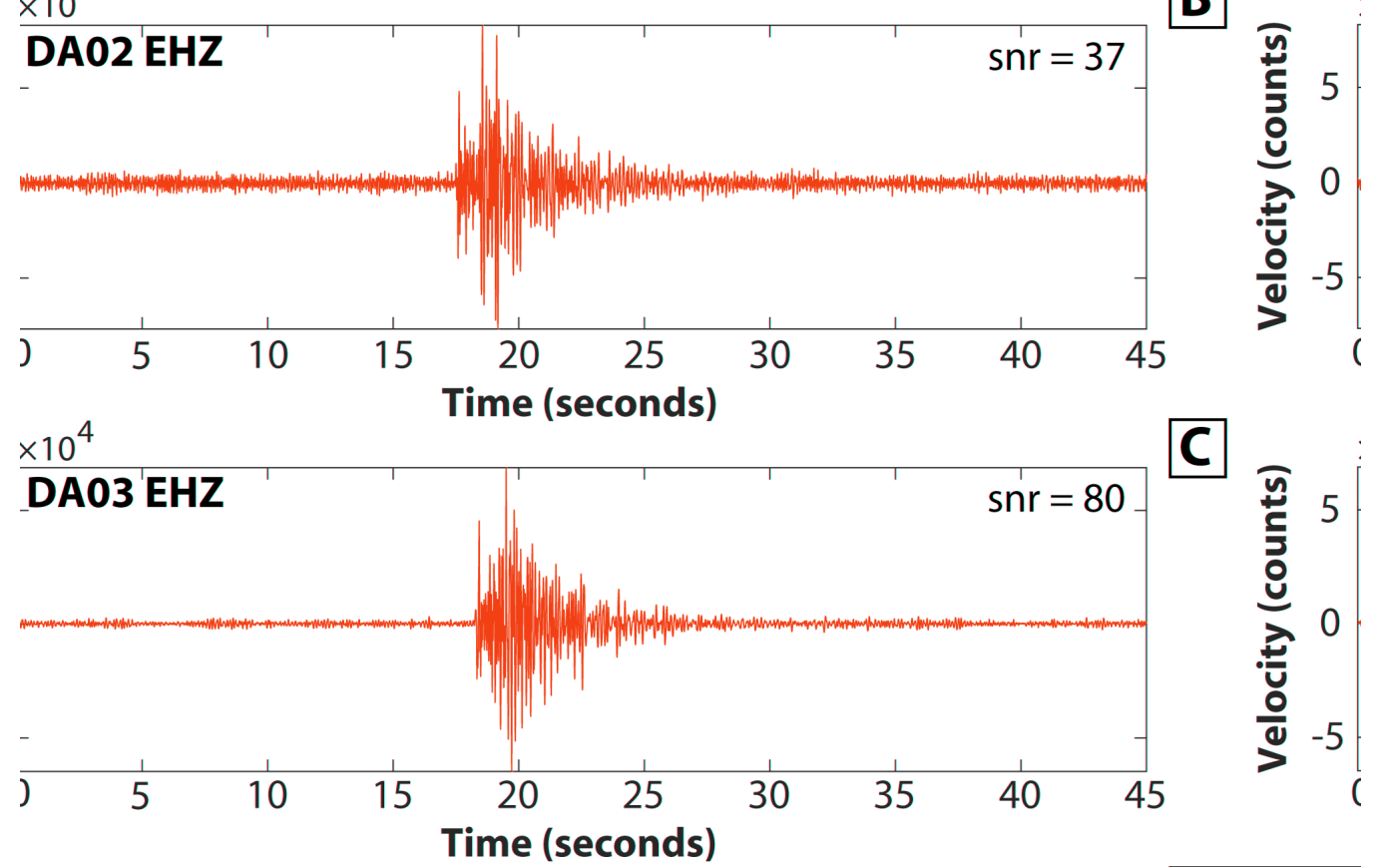

C

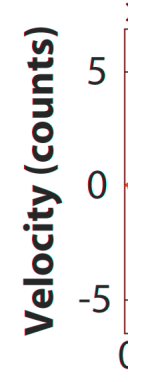

Figure C.10: Velocity time-series for a Mw 2.0 local earthquake recorded during Deployment A at DA01 [A], DA02 [B], and DA03 [C] [Figure C.6]. Each station is at distance of 0,100 , and 200 meters away from the Rotokawa power station. The signalto-noise ratio for each trace is displayed in the top-right inset of each subfigure. 

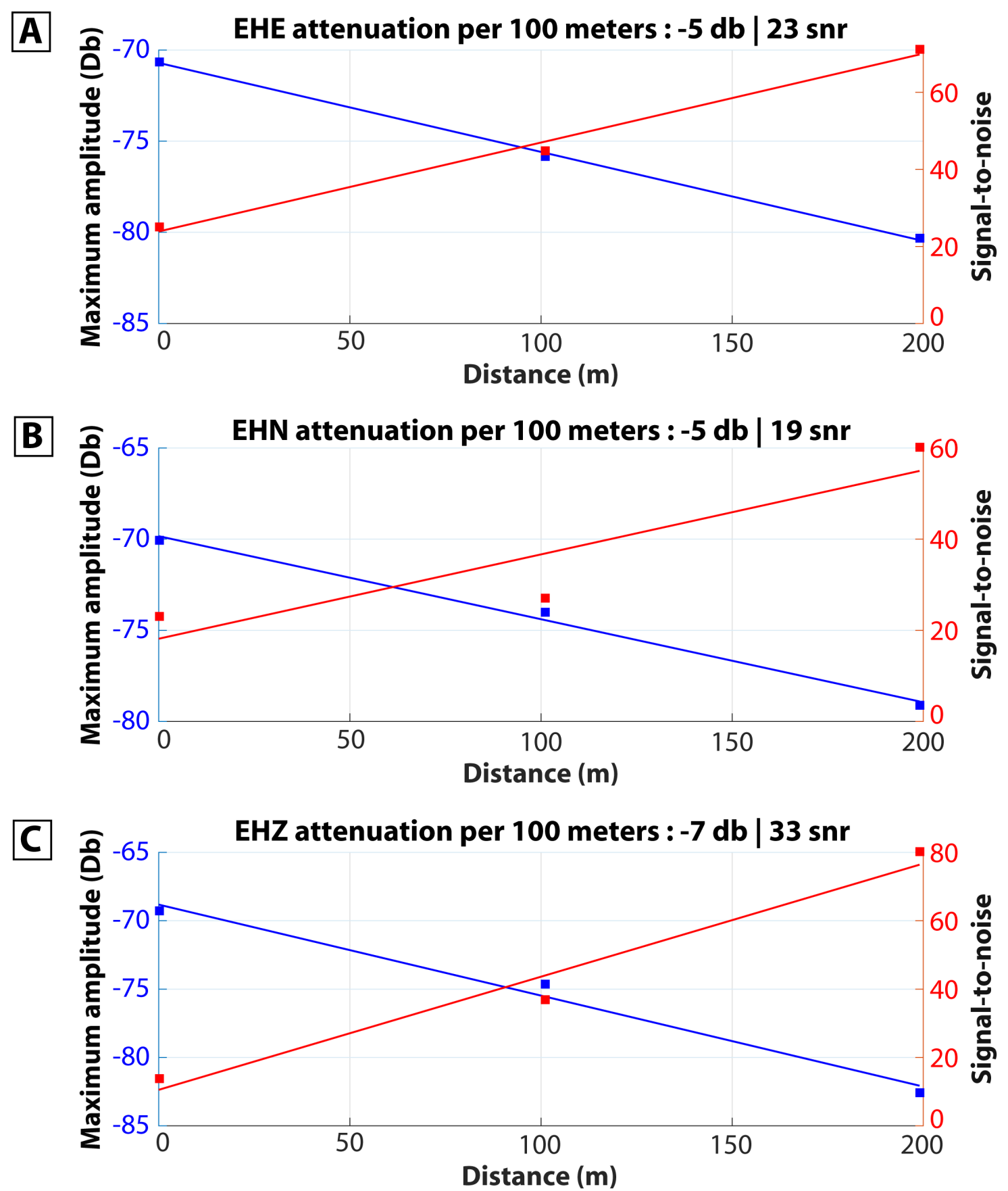

Figure C.11: Amplitude attenuation of a power station source from power spectral density (blue) and microearthquake signal-to-noise ratio (red) for EHE [A], EHN [B], and EHZ [C]. The fitted scatter points represent DA01, DA02, and DA03, which are approximately 0, 100, and 200 meters away from the noise source [Figure C.6]. 


\section{C.5 Discussion and further work}

This is a pilot study on the attenuation rate of cultural ambient noise with distance for typical noise sources at geothermal fields. We found that the noise source with greatest attenuation in all components to be the production well in area 3 group 3, while road noise attenuates the least [Table C.4]. The power spectral density decay per 100 meters for the power station noise, which is a constant cultural noise source in the field, was determined to be $-5,-5$, and -7 decibel for east, north, and vertical components respectively. Using a local earthquake that occurred during the deployment time, we found that this corresponds to a 23,19 , and 33 signal-to-noise ratio increase per 100 meters [Figure C.11]. These measurements provide a correlation between signal-to-noise, the maximum recorded noise amplitude, and the distance from a source.

Several assumptions have been made in the results, including: isotropy of the nearsurface layers, the consistency of the noise level during the deployment, and using signal-to-noise ratio as a proxy for ambient noise contamination. The greatest source of error in our methodology is the selection of power spectral density maximum within the 0.05 -- 0.2 second band. As can be seen from Figure 6.6, certain stations have a definite maximum point (DB05 Figure 6.6A) while others have a steady increase at short periods until the Nyquist frequency is reached (DB01 Figure 6.6B). Due to this, we recommend that only instruments affected by the same source are used in these kinds of analysis despite their similar amplitude. Additionally, measures of error on the weighted nose means [Figure C.9] could be determined by using multiple time segments in the PQLX calculation and determining a standard deviation.

To advance this method, more examples of recorded microearthquakes subjected to varying types of noise fields will need to be studied. Additionally, great care must be taken that the observed signal-to-noise ratio is a reflection of the noise field and not any other mechanism. This method can become a guide for instrumentation deployments, as it quantifies the trade-off between distance from a source and the expected signalto-noise ratio. 


\section{C.6 Station Noise}

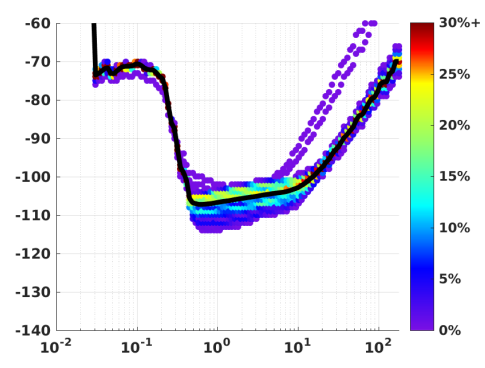

(a) DA01 EHE

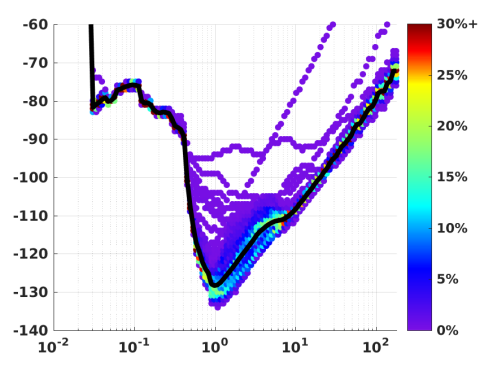

(d) DA02 EHE

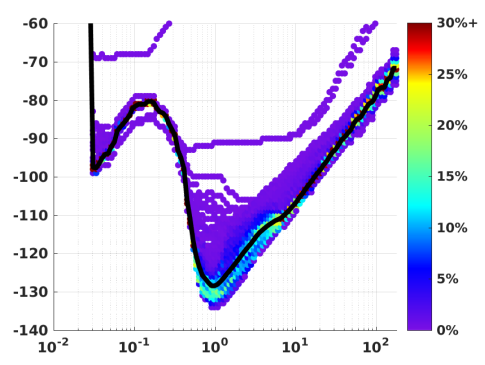

(g) DA03 EHE

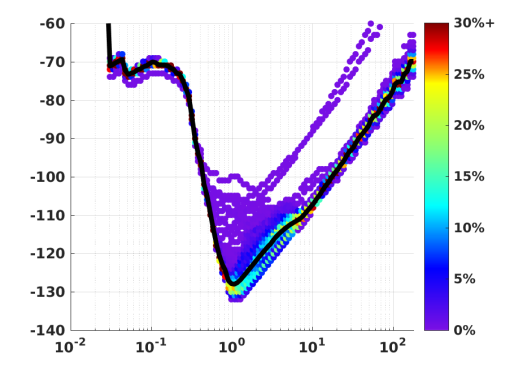

(b) DA01 EHN

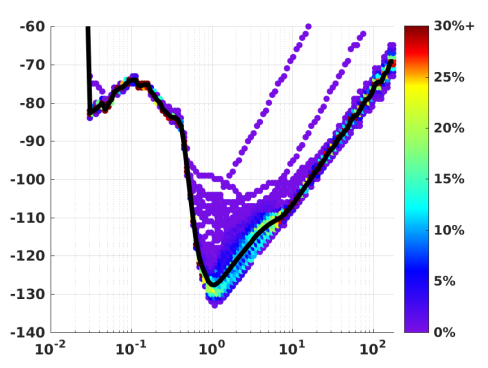

(e) DA02 EHN

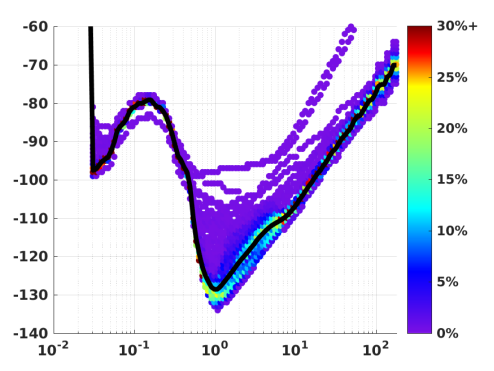

(h) DA03 EHN

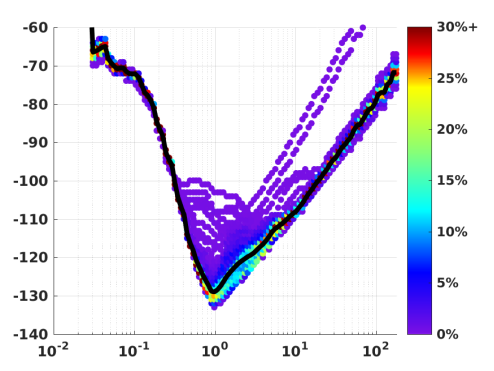

(c) DA01 EHZ

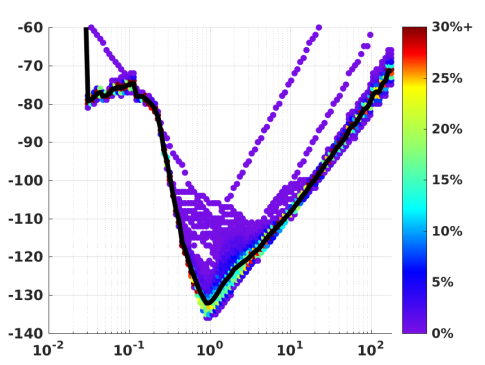

(f) DA02 EHZ

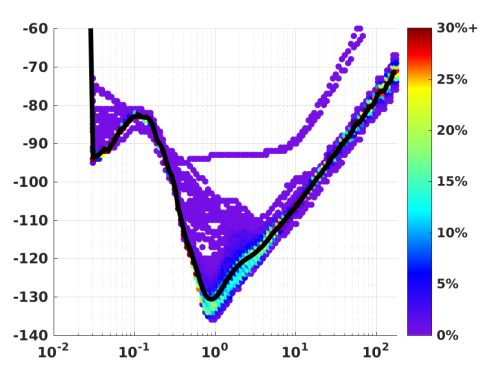

(i) DA03 EHZ

Figure C.12: Power spectral density for Rotokawa temporary stations DA01--DA03. 


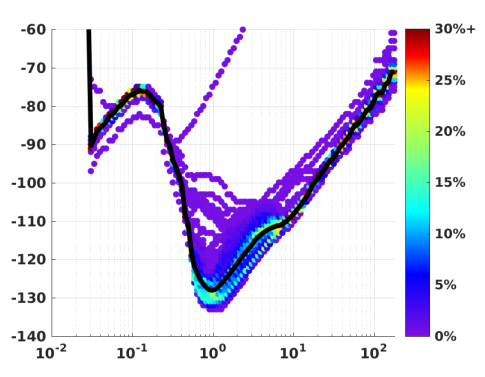

(a) DA04 EHE

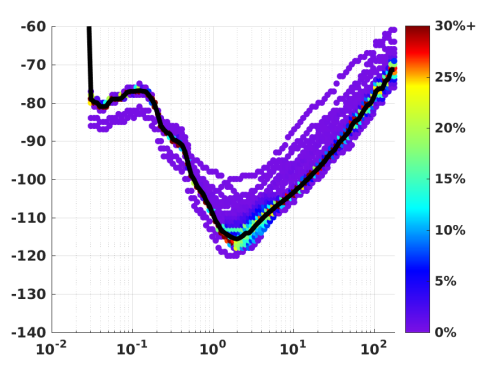

(d) DA05 EHE

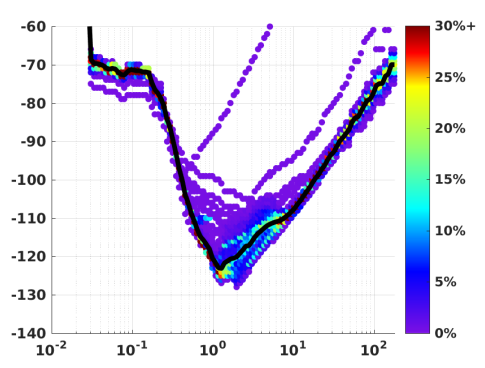

(g) DA06 EHE

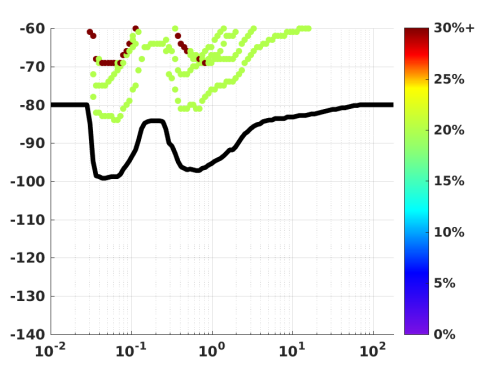

(j) DA07 EHE

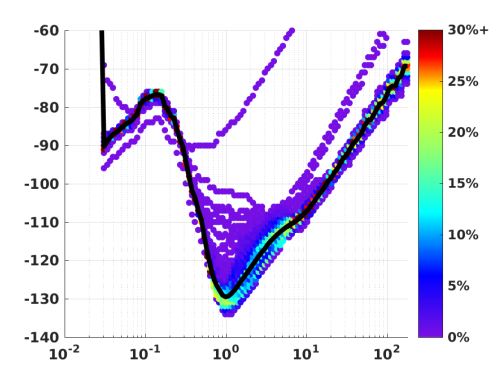

(b) DA04 EHN

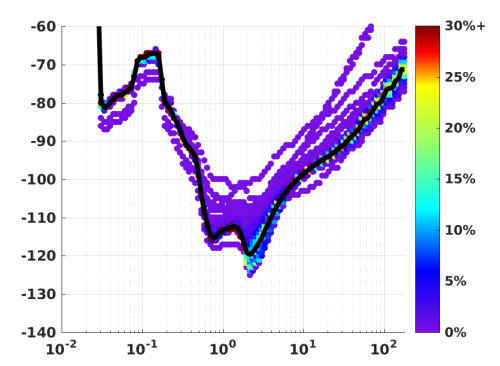

(e) DA05 EHN

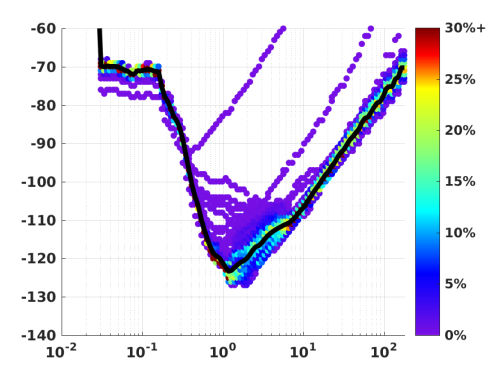

(h) DA06 EHN

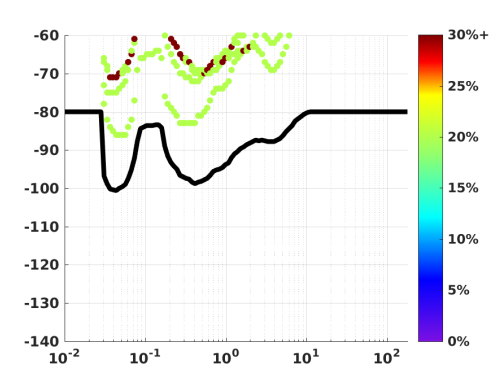

(k) DA07 EHN

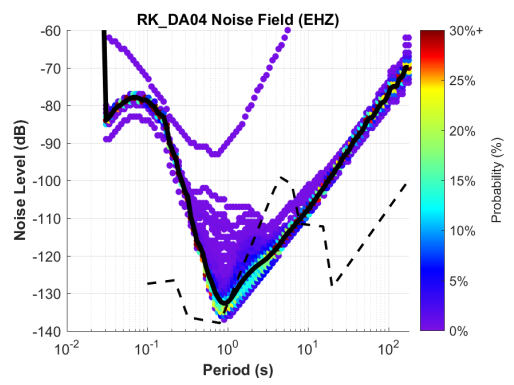

(c) DA04 EHZ

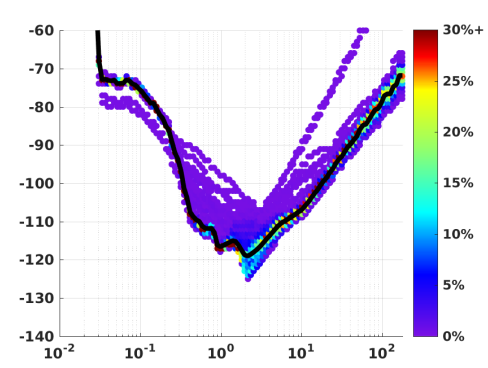

(f) DA05 EHZ

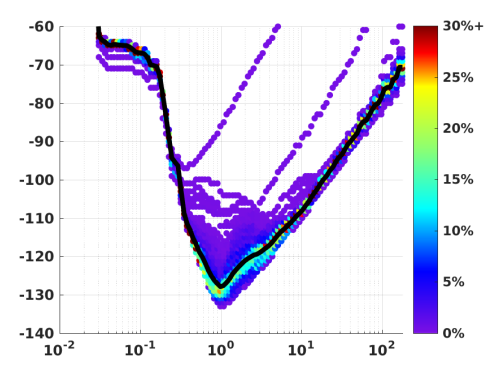

(i) DA06 EHZ

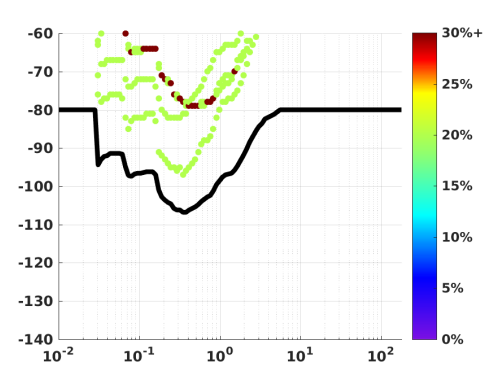

(1) DA07 EHZ

Figure C.13: Power spectral density for Rotokawa temporary stations DA04--DA07. 


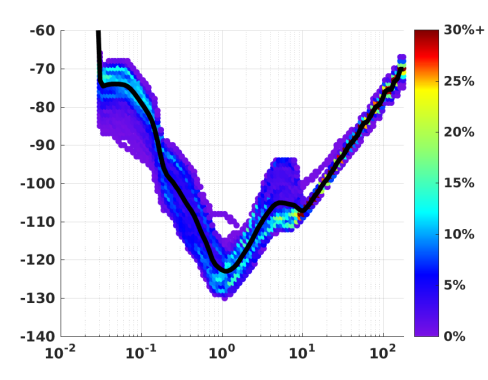

(a) DB01 EHE

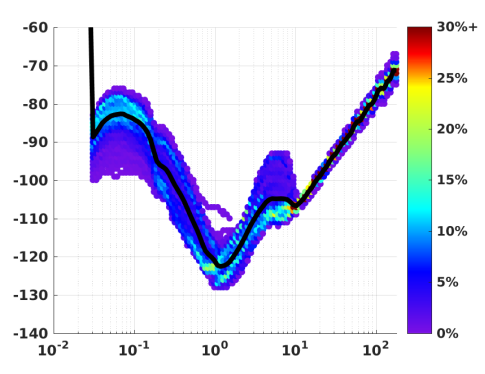

(d) DB02 EHE

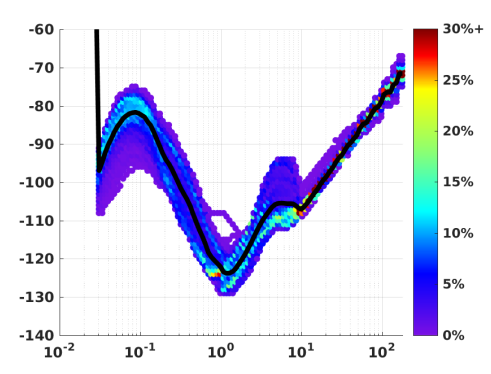

(g) DB03 EHE

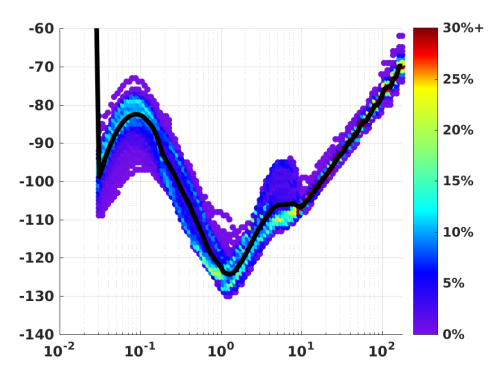

(j) DB04 EHE

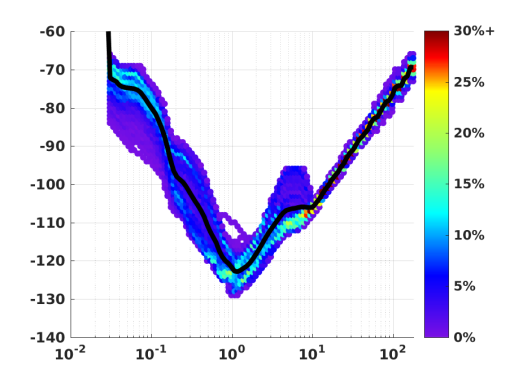

(b) DB01 EHN

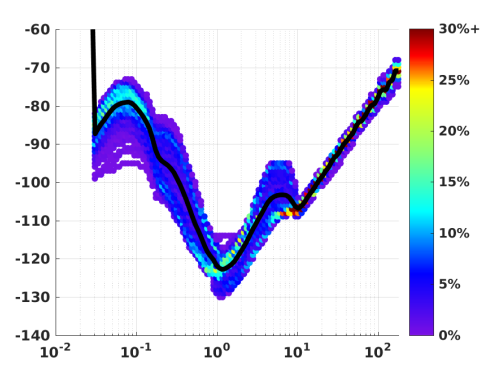

(e) DB02 EHN

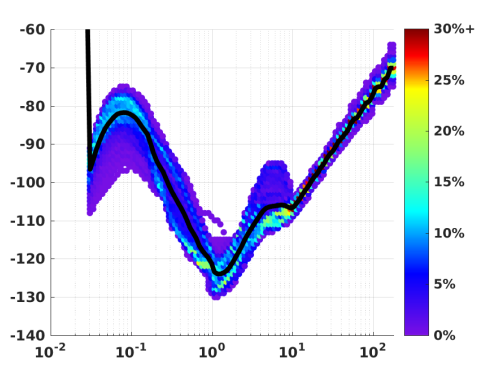

(h) DB03 EHN

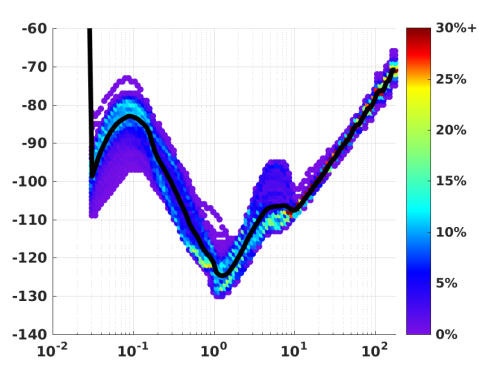

(k) DB04 EHN

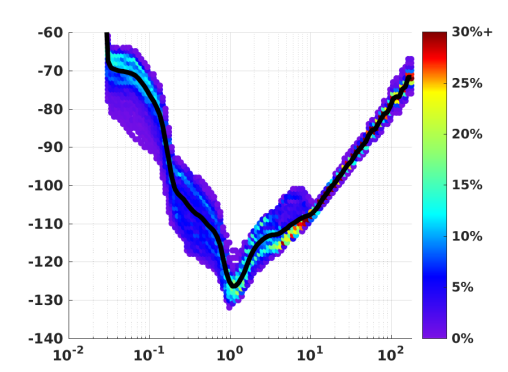

(c) DB01 EHZ

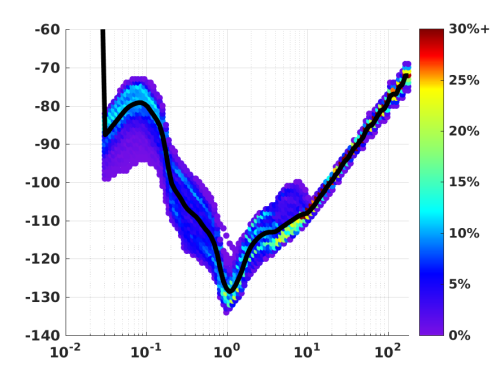

(f) DB02 EHZ

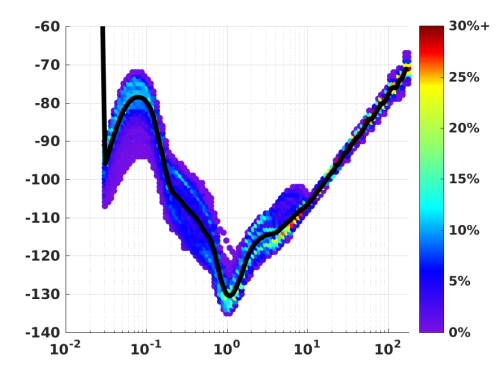

(i) DB03 EHZ

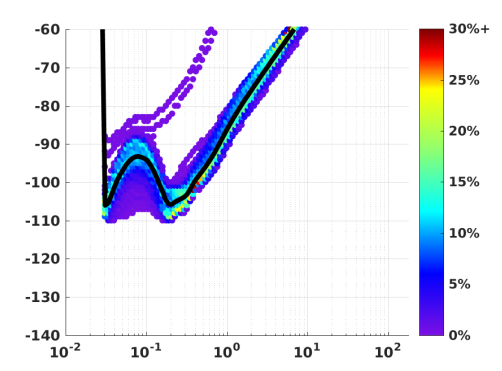

(l) DB04 EHZ

Figure C.14: Power spectral density for Rotokawa temporary stations DB01--DB04. 


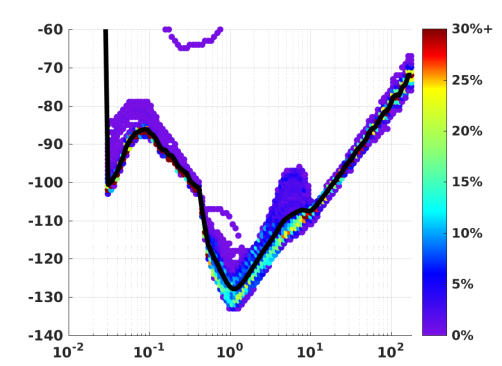

(a) DB05 EHE

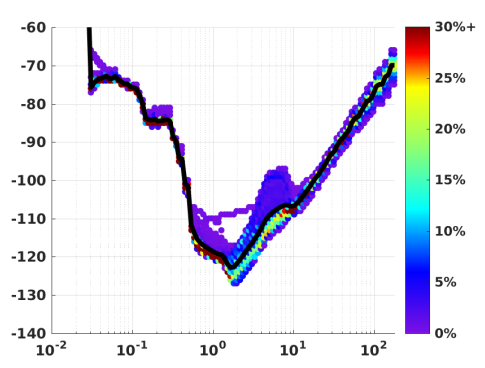

(d) DB06 EHE

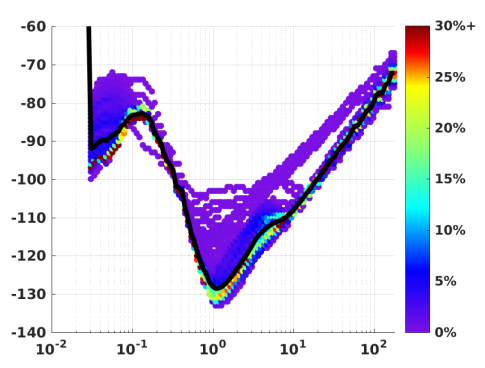

(g) DB07 EHE

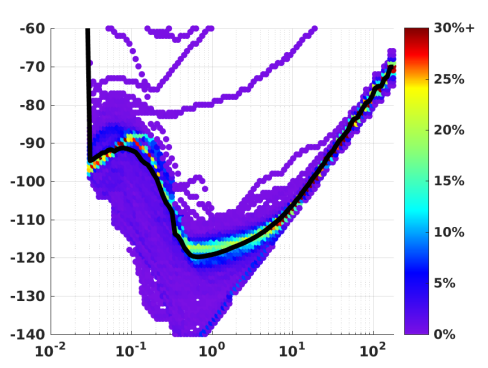

(j) DB08 EHE

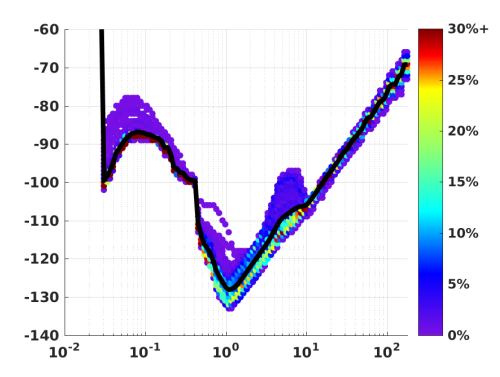

(b) DB05 EHN

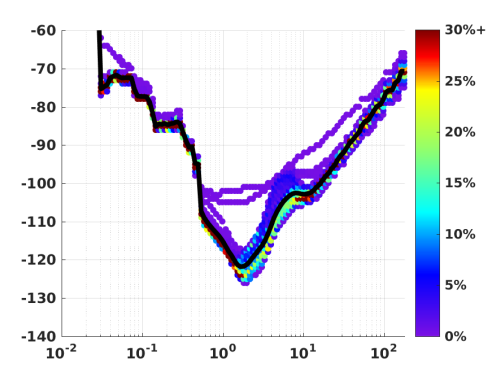

(e) DB06 EHN

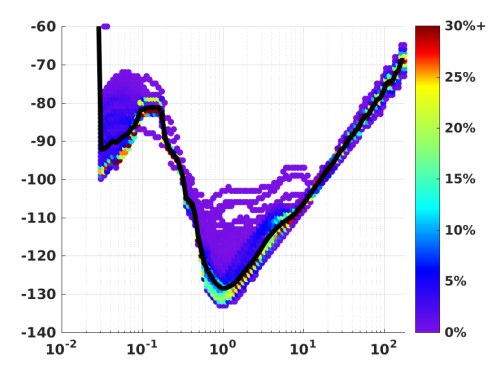

(h) DB07 EHN

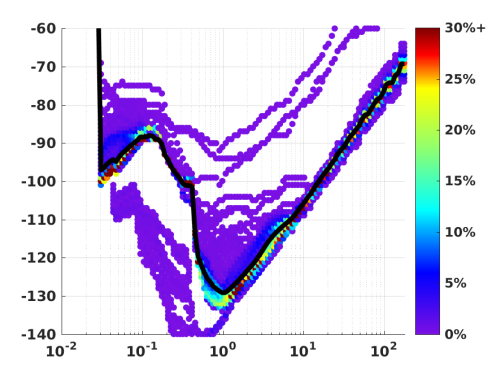

(k) DB08 EHN

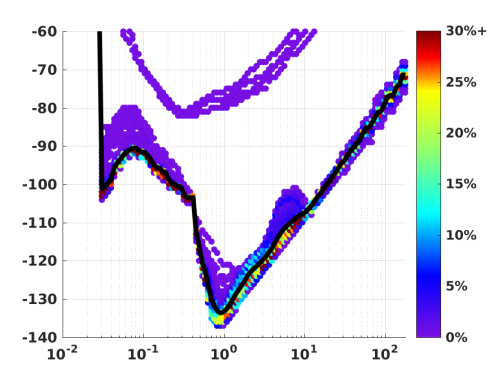

(c) DB05 EHZ

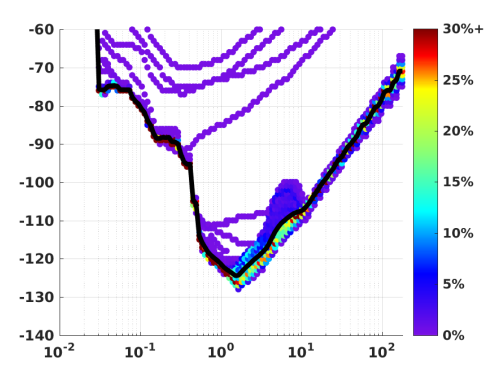

(f) DB06 EHZ

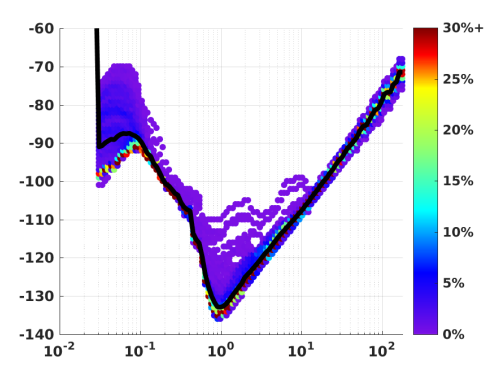

(i) DB07 EHZ

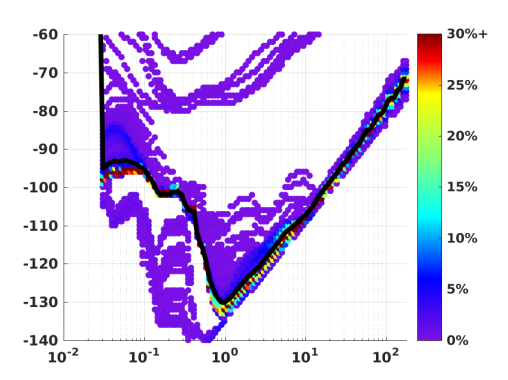

(l) DB08 EHZ

Figure C.15: Power spectral density for Rotokawa temporary stations DB05--DB08. 


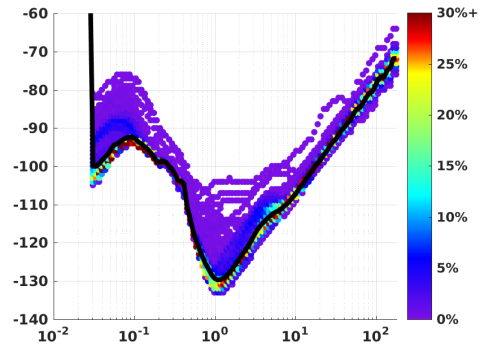

(a) DB09 EHE

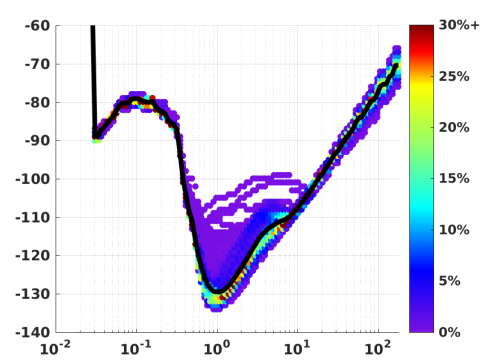

(d) DB10 EHE

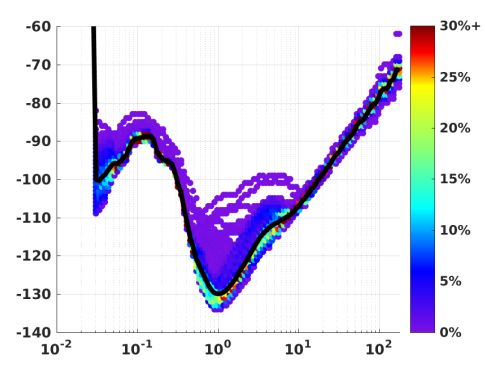

(g) DB11 EHE

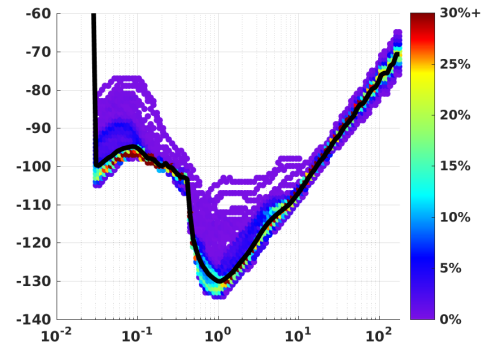

(b) DB09 EHN

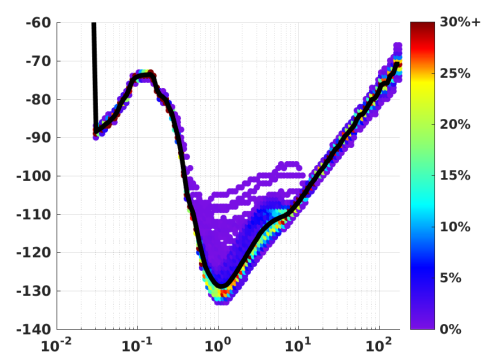

(e) DB10 EHN

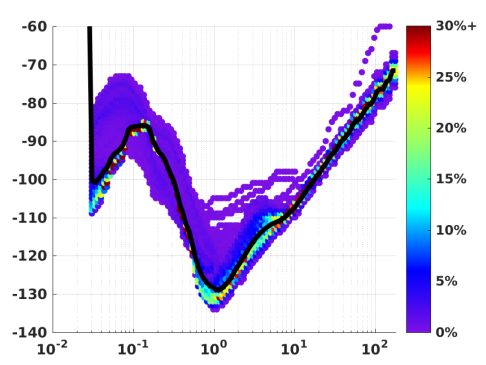

(h) DB11 EHN

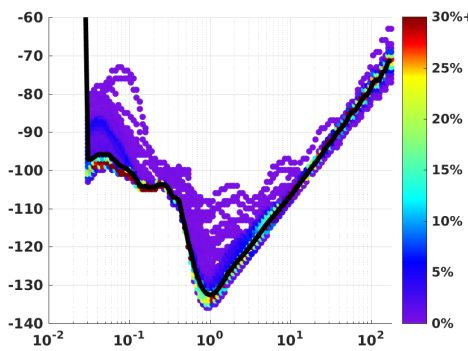

(c) DB09 EHZ

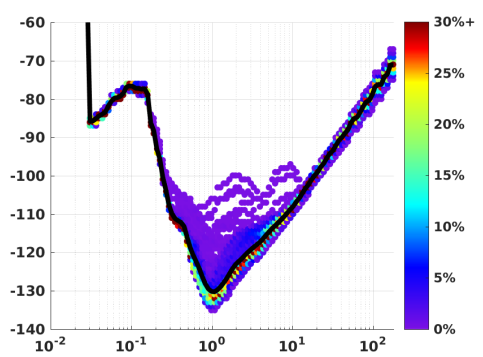

(f) DB10 EHZ

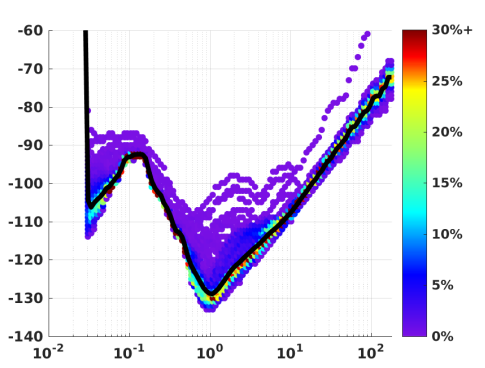

(i) DB11 EHZ

Figure C.16: Power spectral density for Rotokawa temporary stations DB09--DB11. 


\section{Bibliography}

M. F. Abdelwahed, N. El-Masry, M. R. Moufti, C. L. Kenedi, D. Zhao, H. Zahran, and J. Shawali. Imaging of magma intrusions beneath Harrat Al-madinah in Saudi Arabia. Journal of Asian Earth Sciences, 120:17-28, 2016.

C. J. Adams, N. Mortimer, H. J. Campbell, and W. L. Griffin. Age and siotopic characterisation of metasedimentary rocks from the Torlesse Supergroup and Waipapa group in the central North Island, New Zealand. New Zealand Journal of Geology and Geophysics, 52:149-170, 2009.

AFTAN. University of Colorado Boulder Frequency-Time Analysis (AFTAN), http://ciei.colorado.edu/products/, 2013. URL http://ciei.colorado.edu/ Products/.

J. Ahrens, B. Geveci, and C. Law. ParaView: An end-user tool for large-data visualization. The Visualization Handbook, pages 717-731, 2005.

C. Akasaka and S. Nakanishi. Correction of background gravity changes due to precipitation: Oguni geothermal field, Japan. In Proceedings World Geothermal Congress 2000, 2000.

K. Aki. Analysis of the seismic coda of local earthquakes as scattered waves. Journal of Geophysical Research, 1969.

K. Aki and B. Chouet. Origin of coda waves: Source, attenuation, and scattering effects. Journal of Geophysical Research, 80(23):3322-3342, 1975.

R. Allis, C. Bromley, and S. Currie. Update on subsidence at the Wairakei-Tauhara geothermal system, New Zealand. Geothermics, 38:169-180, 2009.

N. Anderson, T. Thitimakorn, A. Ismail, and D. Hoffman. A comparison of four geophysical methods for determining the shear wave velocity of soils. Environmental $\mathcal{E}$ Engineering Geoscience, 13(1):11-23, 2007.

M. W. Asten. On bias and noise in passive seismic data from finite circular array data processed using SPAC methods. Geophysics, 71(6):153-162, 2006. 
G. Baer and Y. Hamiel. Form and growth of an embryonic continental rift: InSAR observations and modelling of the 2009 western Arabia rifting episode. Geophysical Journal International, 182:155-167, 2010.

S. Bannister and A. Melhuish. Seismic scattering and reverberation, Kaingaroa Plateu, Taupo Volcanic Zone, New Zealand. New Zealand Journal of Geology and Geophysics, 40:375-381, 1997.

M. P. Barmin, M. H. Ritzwoller, and A. L. Levshin. A fast and reliable method for surface wave tomography. Pure and Applied Geophysics, 158:1351-1375, 2001.

P. Bedrosian, J. Peacock, M. Dhahry, A. Sharif, and H. Zahran. Magnetotelluric 3-d resistivity modeling: Harrat rahat, 2017.

Y. Behr. Imaging New Zealand's crustal structure using ambient seismic noise recordings from permanent and temporary instruments. PhD thesis, Victoria University of Wellington, 2010.

Y. Behr, J. Townend, S. Bannister, and M. K. Savage. Shear velocity structure of the Northland Peninsula, New Zealand, inferred from ambient noise correlations. Journal of Geophysical Research, 115(B05309), 2010.

G. D. Bensen, M. H. Ritzwoller, M. P. Barmin, A. L. Levshin, F. Lin, M. P. Moschetti, N. M. Shapiro, and Y. Yang. Processing seismic ambient noise data to obtain reliable broad-band surface wave dispersion measurements. Geophysics Journal Intenational, 169:1239-1260, 2007.

M. Beyreuther, R. Barsch, L. Krischer, T. Megies, Y. Behr, and J. Wassermann. ObsPy: A Python toolbox for seismology. Seismological Research Letters, 81(3):530-533, 2010. doi: $10.1785 /$ gssrl.81.3.530.

G. Bignall. Ngatamariki geothermal field geoscience overview. Technical report, GNS Science Consultancy Report, 2009.

P. Bird. An updated digital model of plate boundaries. Geochemistry Geophysics Geosystems, 4(3), 2002.

H. R. Blank and H. S. Sadek. Spectral analysis of the 1976 aeromagnetic survey of Harrat Rahat, Kingdom of Saudi Arabia. Technical report, USGS Numbered Series Report, 1983.

D. R. Boden. Geologic fundamentals of geothermal energy. CRC Press, 2016. 
J. J. Bommer, P. J. Stafford, and S. Akkar. Current empiral ground-motion prediction equations for Europe and their application to Eurocode 8. Bulletin of Earthquake Engineering, 8(5), 2010.

S. Bonnefoy-Claudet, F. Cotton, and P. Bard. The nature of noise wavefield and its application for site effects studies- A literature review. Earth-Science Reviews, 79:205$227,2006$.

W. Bosworth, P. Huchon, and K. McClay. The red sea and gulf of aden basins. Journal of African Earth Sciences, 43(1):334-378, 2005.

D. Bowyer and R. Holt. Case study: development of a numerical model by a multidisciplinary approach, Rotokawa geothermal field, New Zealand. In Proceedings World Geothermal Congress 2010, Bali, Indonesia, 25-29 April 20102010.

F. Brenguier, N. M. Shapiro, M. Campillo, and A. Nercessian. 3-D surface wave tomography of the Piton de la Fournaise volcano using seismic noise correlations. Geophysical Research Letters, 34(L02305), 2007.

F. Brenguier, M. Campillo, C. Hadziioannou, N. M. Shapiro, R. M. Nadeau, and E. Larose. Postseismic relaxation along the San Andreas Fault at Parkfield from continuous seismological observations. Science, 321(5895):1478-1481, 2008a.

F. Brenguier, N. M. Shapiro, M. Campillo, V. Ferrazzini, Z. Duputel, O. Coutant, and A. Nercessian. Towards forecasting volcanic eruptions using seismic noise. Nature Geoscience, 1:126-130, 2008b.

C. Bromley, S. Currie, V. R. Manville, and M. D. Rosenberg. Recent ground subsidence at Crown Road, Tauhara and its probably causes. Geothermics, 38:181-191, 2009.

G. F. Brown. Tectonic map of the arabian peninsula, 1972.

P. R. L. Browne, I. J. Graham, R. J. Parker, and C. P. Wood. Subsurface andesite lavas and plutonic rocks in the Rotokawa and Ngatamariki geothermal systems, Taupo Volcanic Zone, New Zealand. Journal of Volcanology and Geothermal Research, 51:199_ 215, 1992.

M. Calò and C. Dorbath. Different behaviours of the seismic velocity field atSoultzsous-Fôrets revealed by 4-d seismic tomography: case study of GPK3 and GPK2 injection tests. Geophysical Journal International, (194):1119-1137, 2013.

V. E. Camp and M. J. Roobol. The Arabian continental alkali basalt province: Part i. Evolution of Harrat Rahat, Kingdom of Saudi Arabia. Geological Society of America Bulletin, 101:71-95, 1989. 
V. E. Camp and M. J. Roobol. Upwelling asthenosphere beneath western Arabia and its regional implications. Journal of Geophysical Research Solid Earth, 97(B11):15255-15271, 1992.

V. E. Camp, P. R. Hooper, M. J. Roobol, and D. L. White. The Madinah eruption, Saudi Arabia: Magma mixing and simultaneous extrusion of three basaltic chemical types. Bulletin of Volcanology, 49:489-508, 1987.

M. Campillo and A. Paul. Long-range correlations in the diffuse seismic coda. Science, 299:547-549, 2003.

P. H. Cashman, C. D. Peterson, J. N. Louie, S. Pullammanappallil, A. Pancha, C. Sladek, and L. T. West. Testing methods for geothermal exploration in the Pacific Northwest. In 37th Geothermal Resources Council Annual Meeting, Sept. 29 - Oct. 2, Las Vegas, Nevada, volume 37, pages 237-247, 2013.

S. Castellaro and F. Mulargia. Vs30 estimates using constrained H/V measurements. Bulletin of the Seismological Society of America, 99(2A):761-773, April 2009.

S. Castellaro, F. Mulargi, and P. L. Rossi. Vs30: proxy for seismic amplification? Seismological Research Letters, 79(4), 2008.

R. D. Catchings and W. D. Mooney. Crustal structure of the Columbia Plateu: Evidence for continental rifting. Journal of Geophysical Research, 93(B1):459-474, 1988.

I. Chambefort, B. Lewis, C.J.N. Wilson, A.J. Rae, C. Coutts, G. Bignall, and T.R. Ireland. Stratigraphy and structure of the Ngatamariki geothermal system from new zircon $\mathrm{U}-\mathrm{Pb}$ geochronology: Implications for Taupo Volcanic Zone evolution. Journal of Volcanology and Geothermal Research, 274:51-70, 2014.

I. Chambefort, E. Buscarlet, E. C. Wallis, and S. Sewell. Ngatamariki geothermal field, New Zealand: Geology, geophysics, chemistry and conceptual model. Geothermics, 2015.

S. J. Chang, M. Merino, S. V. der Lee, S. Stein, and C. A. Stein. Mantle flow beneath Arabia offset from the opening of the Red Sea. Earth and Planetary Science Research Letters, 38(4):448-454, 2011.

F.J. Chávez-García, T. Domínguez, M. Rodríguez, and F. Pérez. Site effects in a volcanic environment: a comparison between HVSR and array techniques at Colima, Mexico. Bulletin of the Seismological Society of America, 97(2):591-604, 2007.

J. Chiu, S. C. Chiu, and S. G. Kim. The significance of the crustal velocity model in local earthquake locations from a case example of a PANDA experiment in the central United States. Bulletin of the Seismological Society of America, 1997. 
N. I. Christensen. Poisson's ratio and crustal seismology. Journal of Geophysical Research, 101(B2):3139-3156, 1996.

N. I. Christensen and W. D. Mooney. Seismic velocity structure and composition of the continental crust: a global view. Journal of Geophysical Research, 100(B7), 1995.

F. Civilini, A. Pancha, M. K. Savage, S. Sewell, and J. Townend. Inferring shearvelocity structure of the upper $200 \mathrm{~m}$ using cultural ambient noise at the Ngatamariki geothermal field, Central North Island, New Zealand. SEG Interpretation, 4 (3):SJ87-SJ101, 2016. doi: 10.1190/INT-2015-0204.1.

D. Clarke, L. Zaccarelli, N. M. N. M. Shapiro, and F. Brenguier. Assessment of resolution and accuracy of the Moving Window Cross Spectral technique for monitoring crustal temporal variations using ambient seismic noise. Geophysical Journal International, 186:167-882, 2011.

J. Clearwater, L. Azwar, M. Barnes, I. Wallis, and R. Holt. Changes in injection well capacity during testing and plant start-up at Ngatamariki. In Proceedings World Geothermal Congress 2015: Melbourne, Australia, 19-25 April 2015, 2015.

J. R. Cochran and F. Martinez. Evidence from the northern red sea on the transition from continental to oceanic rifting. Tectonophysics, 153:25-53, 1998.

R. G. Coleman. Geologic evolution of the Red Sea. Oxford University Press, 1993.

J. W. Cooley and J. W. Tukey. An algorithm for machine calculation of complex Fourier series. Mathematics of Computation, 19:297-301, 1965.

G. R. Cooper and C. D. McGillen. Probabilitistic methods of signals and system analysis. Holt, Rinehart and Winston, INC, 1971.

B. Delaunay. Sur la sphère vide. Bulletin de l'Académie des Sciences de l'URSS, Classe des sciences mathématiques et naturelles, 6:793-800, 1934.

J. B. Diebold and P. L. Stoffa. The traveltime equation, tau-p mapping, and inversion of common midpoint data. Geophysics, 46(3):238-254, 1981.

D. T. Downs, M. E. Stelten, D. E. Champion, and H. R. Dietterich. Mapping intraplate volcanic fields: A case study. In American Geophysical Union, Fall Meeting 2017, 2017.

C. J. Ebinger and N. H. Sleep. Cenozoic magmatism through east Africa resulting from impact of a single plume. Nature, 395(6704):788-791, 1998.

D. M. Egle. Diffuse wave fields in solid media. Journal of Acoustic Society of America, 1981. 
A. Friedrich, F. Krger, and K. Klinge. Ocean-generated microseismic noise located with the Grfenberg array. Journal of Seismology, 2:47-64, 1998.

K. Galvin, A. Blanchette, W. Mooney, S. L. Klemperer, and H. M. Zahran. Crustal thickness of the middle east and northeast africa. Technical report, U.S. Geological Survey, 2017.

Sensing systems engineering product/customer specification for GS-11D 4/5Hz 380 Ohm vertical. Geospace corporation, Huston, Texas, U.S.A., 1992.

P. Gerstoft, P. M. Shearer, N. Harmon, and J. Zhang. Global P, PP, and PKP wave microseisms observed from distant storms. 35(L23306), 2008.

P. Goudard, P. Roux, and M. Campillo. Small-scale seismic inversion using surface waves extracted from noise cross correlation. The Journal of the Acoustical Society of America, 123((3)):EL26-EL31, 2008.

D. M. Gravly, C. J. N. Wilson, M. D. Rosenberg, and G. S. Leonard. the nature and age of Ohakuri Formattion and Ohakuri Group rocks in surface exposures and geothermal drillhole sequences in the central Taupo Volcanic Zone, New Zealand. New Zealand Journal of Geology and Geophysics, 49, 2006.

G. W. Grindley. Subsurface geology of the Orokei Korako and Te Kopia geothermal fields. Technical report, Geological geothermnal report, New Zealand Geological Survey, 1965.

N. Harmon, D. Forsyth, and S. Webb. Using ambient seismic noise to determine shortperiod phase velocities and shallow shear velocities in young oceanic lithosphere. Bulletin of the Seismological Society of America, 97(6):2009-2023, 2007.

K. Heath, J.N. Louie, G. Biasi, A. Pancha, and S. Pullammanappallil. Blind tests of refraction microtremor analysis against synthetics and borehole data. In Proceedings of the managing risk in earthquake country conference commemorating the 100th anniversary of the 1906 earthquake, April 2006.

J. W. Hedenquist. Stratigraphy and petrology of NM2 - Ngatamariki geothermal field. Technical report, New Zealand Geological Survey Department of Scientific and Industrial Research, 1980.

R. W. Henley and K. I. Middendorf. Geothermometry in the recent exporation wells of Mokai and Rotokawa geothermal fields, new zealand. In Geothermal Resource Council Transcripts, number 9, 1985.

S. Henrys, M. Reyners, and H. Bibby. Exploring the plate boundary structure of the North Island, New Zealand. EOS Transactions AGU, 84(31):289-295, 2003. 
S. Henrys, A. Wech, R. Sutherland, T. Stern, M. Savage, H. Sato, K. Mochizuki, T. Iwasaki, D. Okaya, A. Seward, B. Tozer, J. Townend, E. Kurashimo, T. Iidaka, and T. Ishiyama. SAHKE geophysical transect reveals crustal and subduction zone structure at the southern Hikurangi margin, New Zealand. Geochemistry Geophysics Geosystems, 14(7):2063-2083, 2013.

D. Hernandez, S. Addison, S. Sewell, L. Azwar, and M. Barnes. Rotokawa: Reservoir response of $172 \mathrm{MW}$ geothermal production. In Proceedings 37th New Zealand Geothermal Workshop, 18-20 November 2015, Taupo, New Zealand, 2015.

E. Herrin and T. Goforth. Phase-matched filters: Application to the study of Rayleigh waves. Bulletin of the Seismological Society of America, 67(5):1259-1275, 1977.

G. Hillers, M. Campillo, and K. F. Ma. Seismic velocity variations at TCDP are controlled by MJO driven preciptation pattern and high fluid discharge properties. Earth and Planetary Science Letters, 391:121-127, 2014.

G. Hillers, S. Husen, A. Obermann, T. Planès, E. Larose, and M. Campillo. Noise-based monitoring and imaging of aseismic transient deformation induced by the 2006 Basel reservoir stimulation. Geophysics, 80(4):KS51-KS68, 2015.

A. Hogg, D. Lowe, J. Palmer, G. Boswijk, and C. Ramsey. Definitive high-precision calendar date for the Taupo eruption derived by wiggle-match dating using a New Zealand kauri-derived calibration curve. The Holocene, 4(22):439-449, 2012.

IRIS. SEED reference manual: Standard for the exchange of earthquake data. Incorporated Research Institutions for Seismology, August 2012.

P. R. Johnson and F. H. Kattan. The Geology of the Saudi Arabian Shield. Saudi Geological Survey, 2012.

P. Jousset, A. Bitri, J. Loiseau, and V. Bouchot. Seismic ambient noise study at Bouillante geothermal system, French Antilles. In EGU General Assembly 2010, editor, Geophysical Research Abstracts EGU General Assembly 2010, volume 12, 2010.

A. E. Kaiser and E. G. Smith. Shallow shear-wave velocity from ReMi surface wave dispersion: method and case study. In 2005 New Zealand Society for Earthquake Engineering Conference, 2005.

R. T. Lacoss, E. J. Kelly, and M. N. Toksoz. Estimation of seismic noise structure using arrays. Geophysics, 34(1):21-38, 1969.

M. Landes̀, F. Hubans, N. M. Shapiro, and A. Paul. Origin of deep ocean microseisms by using teleseismic body waves. 115(B05302), 2010. 
V. Langenheim, B. Ritziner, R. Blakely, P. Bedrosian, J. Peacock, A. Shariff, and M. Aldari. Potential fields: Gravity and magnetotellurics. Technical report, U.S. Geological Survey, 2016.

E. Larose. Mesoscopics of ultrasound and seismic waves: Application to passive imaging. Annales de Physique, 31(3):1-126, 2006.

T. Lecocq, C. Caudron, and F. Brenguier. MSNoise, a Python package for monitoring seismic velocity changes using ambient seismic noise. Electronic Seismologist, 85(3): 715-726, May/June 20142014.

G. S. Leonard, J. G. Begg, and C. J. N. Wilson. Geology of the Rotorua area: scale 1:250,000. Technical report, Institute of Geological and Nuclear Sciences, Lower Hutt, New Zealand, 2010.

A. L. Levshin and M. H. Ritzwoller. Automated detection, extraction, and measurement of regional surface waves. Pure and Applied Geophysics, 158:1531-1545, 2001.

A. L. Levshin, V. F. Pisarenko, and G. A. Pogrebinsky. On a frequency-time analysis of oscillations. Annuals of Geophysics, 28:211-218, 1972.

A. L. Levshin, T. B. Yanovskaya, A. V. Lander, B. G. Bukchin, M. P. Barmin, L. I. Ratnikova, and E. N. Its. Seismic surface waves in a laterally inhomogeneous Earth. Kluwer Academic Publishers, 1989.

B. Lewis, I. Chambefort, and A. J. Rae. Geology of injection well NM8-NM8A, Ngatamariki geothermal field. GNS Science Consultancy Report 2012/188, GNS Science, 2012.

B. Lewis, I. Chamefort, A. Rae, and F. Sanders. Geology of well NM9, Ngatamariki geothermal field. GNS Science Consultancy Report 330, 2013a.

B. Lewis, I. Chamefort, A. Rae, F. Sanders, and C. Massiot. Geology of well NM11, Ngatamariki geothermal field. GNS Science Consultancy Report 33, 2013b.

F. Lin, M. P. Moschetti, and M. H. Ritzwoller. Surface wave tomography of the western United States from ambient seismic noise: Rayleigh and Love wave phase velocity maps. Geophysics Journal International, 173:281-298, 2008.

O. I. Lobkis and R. L. Weaver. On the emergence of the Green's function in the correlations of a diffuse field. The Journal of the Acoustical Society of America, 110(6): 3011-3017, 2001.

J. N. Louie. Faster, better: Shear-wave velocity to 100 meters depth from refraction microtremor arrays. Bulletin of the Seismological Society of America, 91:347-64, 2001. 
J. N. Louie, S. K. Pullammanappallil, A. Dorsey, T. West, J. J. Sweeney, and R. J. Mellors. $\mathrm{P}$ - and S-wave imaging of cavity collapse at the site of an underground nuclear test. In American Geophysical Union, Fall Meeting 2013, abstract S33B-2418, 2013.

J. N. Louie, A. Pancha, and S. K. Pullammanappallil. 2D and 3D shear-wave velocity structure to $i 1 \mathrm{Km}$ depth from ambient and active surface waves: Three "deep ReMi" case studies. In American Geophysical Union, Fall Meeeting 2014, abstract S13A-4427, 2014.

B. Y. Lynne, M. Pender, T. Glynn-Morris, and F. Sepulveda. Combining scanning electron microscopy and compressibility measurement to understand subsurface processes leading to subsidence at Tauhara geothermal field, New Zealand. Engineering Geology, 166:26-38, 2013.

H. T. Mackenzie. Determining the origin of localised subsidence features in the Kawerau geothermal field, Bay of Plenty, New Zealand. Master's thesis, University of Canterbury, 2012.

V. Manville and C. J. N. Wilson. The 26.5 ka Oruanui eruption, New Zealand: A review of the reoles of volcanism and climate in the post-eruptive sedimentary response. New Zealand Journal of Geology and Geophysics, 47(3):525-547, 2004.

K. McLoughlin, A. Campbell, and G. Ussher. The Nga Awa Purua geothermal project, Rotokawa, New Zealand. In Proceedings World Geothermal Congress, Bali, Indonesia, 25-29 April 2010, 2010.

D. D. McNamara, D. Faulkner, and E. McCarney. Rock properties of Greywacke basement hosting geothermal reservoirs, New Zealand: Preliminary results. In PROCEEDINGS. Thirty-Eighth Workshop on Geothermal Reservoir Engineering, Stanford Univerisity, Stanford, California, February 24-26, 2014, 2014.

D. D. McNamara, S. Sewell, E. Buscarlet, and I. C. Wallis. A review of the Rotokawa geothermal field, New Zealand. Geothermics, 59:281-293, 2016.

D. E. McNamara and R. I. Boaz. PQLX: A seismic data quality control system description, applications, and users manual. U.S. Geological Survey Open-File Report 2012-1292, 2011.

D. E. McNamara and R. P. Buland. Ambient noise levels in the continental United States. Bulletin of the Seismological Society of America, 94(4):1517-1527, 2004.

U. Meier, N. M. Shapiro, and F. Brenguier. Detecting seasonal variations in seismic velocities within Los Angeles basin from correlations of ambient seismic noise. Geophysical Journal International, 181:985-996, 2010. 
S. D. Milicich and T. Hunt. Thermal features of teh Rotokawa geothermal field. Technical report, GNS Science Consultancy Report, 2007.

M. A. Mongillo and L. Clelland. Concise listing of information on the thermal areas and thermal springs of New Zealand. Technical report, Department of scientific and industrial research, geothermal report 9, Wellington, 1984.

W. D. Mooney, M. C. Andrews, A. Ginzburg, D. A. Peters, and R. M. Hamilton. Crustal structure of the northern Mississippi Embayment and a comparision with other continental rift zones. Tectonophysics, 94:327-348, 1983.

W. D. Mooney, M. E. Gettings, H. R. Blank, and J. H. Healy. Saudi Arabian seismicrefraction profile: A traveltime interpretation of crustal and upper mantle structure. Tectonophysics, 111:173-246, 1985.

M. R. Moufti and K. Németh. Geoheritage of volcanic Harrats in Saudi Arabia, volume XI, chapter 2 - Geological Setting, pages 23-32. Springer, 2016.

M. R. Moufti, M. I. Matsah, M. A. Soliman, and A. M. Moghazi. Arabian plume ddynamic beneath Al-Madinah Al-Munawwarah region and its related geohazards. Technical report, King Abdulaziz City for Science and Technology, Riyadh, Saudi Arabia, 2011.

M .R. Moufti, A. M. Moghazi, and K. A. Ali. 40Ar/39Ar geochronology of the Neogene-Quaternary Harrat Al-Madinah intercontinental volcanic field, Saudi Arabia: Implications for duration and migration of volcanic activity. Journal of Asian Earth Sciences, 62:253-268, 2012a.

M. R. Moufti, A. M. Moghazi, and K. A. Ali. Geochemistry and Sr-Nd-Pb isotropic composition of the Harrat Al-Madinah volcanic field, Saudi Arabia. Gondwana Research, 21:670-689, 2012b.

S. Nazarian and K. H. I. Stokoe. In situ shear wave velocities from Spectral Analysis of Surface Waves. In Prentice-Hall, editor, Proceedings of the 8th World Conference on Earthquake Engineering, volume Vol. III, 1984.

A. Obermann, T. Planès, E. Larose, and M. Campillo. Imaging preeruptive and coeruptive structural and mechanical changes of a volcano with ambient noise. Journal of Geophysical Research: Solid Earth, 118:6285-6294, 2013.

S. Ohmi, K. Hirahara, H. Wada, and K. Ito. Temporal variations of crustal structure in the source region of the 2007 Noto Hanto Earthquake, central Japan, with passive imagy interferometry. Earth Planets Space, 60:1069-1074, 2008. 
Optim. User's manual SeisOpt ReMi version 4.0. Optim software and data solutions, UNR - MS 174, 1664 N. Virginia St., Reno, NV 89557-0141, 2006.

P. P. Overduin, C. Haberland, T. Ryberg, F. Kneier, T. Jacobi, M. N. Grigoriev, and M. Ohrnberger. Submarine permafrost depth from ambient seismic noise. Geophysical Research Letters, 42:7581-7588, 2015.

J. S. Pallister, W. A. McCausland, S. Jonsson, Z. Lu, H. M. Zahran, S. E. Hadidy, A. Aburukbah, I. C. F. Stewart, P. R. Lundgren, R. A. White, and M. R. H. Moufti. Broad accommodation of rift-related extension recorded by dyke intrusion in Saudi Arabia. Nature Geoscience, 2010.

A. Pancha and S. Pullammanappallil. Determination of 3D-velocity structure across the deepest portion of the Reno-area basin. Technical report, USGS NEHRP, 2012.

A. Pancha, J.G. Anderson, J.N. Louie, and S.K. Pullammanappallil. Measurement of shallow shear wave velocities at a rock site using the ReMi technique. Soil Dynamics and Earthquake Engineering, 28:522-535, 2008.

C. B. Park, R. D. Miller, and J. Xia. Multichannel analysis of surface waves. Geophysics, 64(3):800-808, 1999.

D. Pei. Modeling and inversion of dispersion curves of surface waves in shallow site investigations. PhD thesis, University of Nevada, Reno, 2007.

I. Pérez-Santisteban, A. M. Martín J. García-Mayordomo, and A. Carbó. Comparison among SASW, ReMi and PS-logging techniques: Application to a railway embankment. Journal of Applied Geophysics, 73:59-64, 2011.

J. Peterson. Observations and modelling of background seismic noise. Open-file Report 93-322, U. S. Geological Survey, Albuquerque, New Mexico, 1993.

P. Poli, H. A. Pedersen, and M. Campillo. Emergence of body waves from crosscorrelation of short period seismic noise. (188):549-558, 2012.

G. Poupinet, W. L. Ellsworth, and J. Frechet. Monitoring velocity variations in the crust using earthquake doublets: An application to the Calaveras fault, California. Journal of Geophysical Research, 89(B7):5719-5731, 1984.

S. R. Pride. Hydrogeophysics, chapter Chapter 8, pages 253-290. Water Science and Technology Library, Springer, Berlin, 2005.

A. Rae. Rotokawa geology and geophysics. Technical report, GNS Science consultancy Report, 2007. 
A. J. Rae, L. E. Ramirez, and C. Boseley. Geology of exploration well NM7, Ngatamariki geothermal field. GNS Science Consultancy Report 289, 2009.

L. E. Ramirez and A. J. Rae. Geology of injection well NM5, NM5A, Ngatamariki geothermal field. GNS Consultancy Report 14, 2009.

A. Ratdomopurbo and G. Poupinet. Monitoring a temporal change of seismic velocity in a volcano: Application to the 1992 eruption of Mt. Merapi (Indonesia). Geophysical Research Letters, 22(7):775-778, 1995.

Z. Rawlinson. Microseismicity associated with actively exploited geothermal systems: earthquake detection and probabilistic location at Rotokawa and statistical seismic network design at Kawerau. Master's thesis, Victoria University of Wellington, New Zealand, 2011.

S. M. Richwalski, M. Picozzi, S. Parolai, C. Milkereit, F. Baliva, D. Albarello, K. RoyChowdhury, H. van der Meer, and J. Zschau. Rayleigh wave dispersion curves from seismological and engineering-geotechnical methods: A comparison at the Bornheim test site (germany). Journal of Geophysics and Engineering, 4:349-361, 2007.

M. H. Ritzwoller and A. L. Levshin. Eurasian surface wave tomography: Group velocities. Journal of Geophysical Research, 103(B3):4839-4878, 1998.

D. Rivet, M. Campillo, N. M. Shapiro, V. Cruz-Atienza, M. Raiguest, N. Cotte, and V. Kostoglodov. Seismic evidence of nonlinear crustal deformation during a large slow slip event in Mexico. Geophysical Research Letters, 38(L08308):1-5, 2011.

M. D. Rosenberg, G. Bignall, and A. J. Rae. The geological framework of the WairakeiTauhara geothermal system, New Zealand. Geothermics, 38:72-84, 2009.

J. V. Rowland and R. H. Sibson. Extensional fault kinematics within the Taupo Volcanic Zone, New Zealand: Soft-linked segmentation of a continental rift system. New Zealand Journal of Geology and Geophysics, 2001.

M. L. Rucker. Applying the refraction microtremor (ReMi) shear wave technique to geotechnical characterization. In Proceedings of the third international conference on the application of geophysical methodologies and NDT to transportation and infrastructure. Orlando, Florida, December 12-18th, 2003, 2003.

K. G. Sabra, P. Roux, and W. A. Kuperman. Arrival-time structure of the time-averaged ambient noise cross-correlation function in an oceanic waveguide. The Journal of the Acoustical Society of America, 117(1):164-174, 2005. 
K. G. Sabra, P. Roux, P. Gerstoft, W. A. Kuperman, and M. C. Fehler. Extracting coherent coda arrivals from cross-correlations of long period seismic waves during the Mount St. Helens 2004 eruption. Geophysical Research Letters, 33, 2006.

M. Saito. Compound matrix method for the calculation of spheroidal oscillation of the Earth. Seismological Research Letters, 59(29), 1988.

M. Sambridge. Geophysical inversion with a neighbourhood algorithm - I. Searching a parameter space. Geophysical Journal International, (138):479-494, 1999.

E. Sandvol, D. Seber, M. Barazangi, F. Vernon, R. Mellors, and A. Al-Amri. Lithospheric seismic velocity discontinuities beneath the Arabian Shield. Geophysical Research Letters, 1998.

E. Saygin and B. L. N. Kennet. Ambient seismic noise tomography of Australian continent. Tectonophysics, 481(1-4):116-125, 2010.

J.B. Scott, M. Clark, T. Rennie, A. Pancha, H. Park, and J.N. Louie. A shallow shearwave velocity transect across the Reno, Nevada area basin. Bulletin of the Seismological Society of America, 94:2222-2228, 2004.

C. Sens-Schönfelder and E. Larose. Temporal changes in the lunar soil from correlation of diffuse vibrations. Physical Review E, 78(045601):1-4, 2008.

C. Sens-Schönfelder and U. Wegler. Passive image interferometry and seasonal variations of seismic velocities at Merapi volcano, Indonesia. Geophysical Research Letters, 33(L21302):1-5, 2006.

S. Sewell. Rotokawa joint venture limited evidence for applicant. Technical report, Mighty River Power, 2016.

N. M. Shapiro and M. Campillo. Emergence of broadband Rayleigh wave from correlations of the ambient seismic noise. Geophysical Research Letters, 31(7), 2004.

N. M. Shapiro, M. Campillo, L. Margerin, S. K. Singh, V. Kostoglodov, and J. Pacheco. The energy partitioning and the diffusive character of the seismic coda. Bulletin of the Seismological Society of America, pages 655-665, 2000.

N. M. Shapiro, M. Campillo, L. Stehly, and M. H. Ritzwoller. High resolution surface wave tomography from ambient seismic noise. Science, 307:1615-1618, 2005.

P. M. Shearer. Introduction to seismology. Cambridge University Press, 1999.

S. Sherburn, C. Bromley, S. Bannister, S. Sewell, and S. Bourguignon. New Zealand geothermal induced seismicity: An overview. In Proceedings World Geothermal Congress 2015. Melbourne, Australia, 19-25 April 2015, 2015. 
J. O. Smith. Mathematics of the discrete Fourier transform (DFT) with audio applications. W3K Publishing, 2nd edition edition, 2007.

R. Snieder. Extracting the Green's function from the correlation of coda waves: A derivation based on stationary phase. Physical Review E., 69:046610, 2004.

R. Snieder and E. Larose. Extracting Earth's elastic wave response from noise measurements. Annual Review of Earth and Planetary Sciences, 41:183-206, 2013.

R. Snieder, E. Slob, and K. Wapenaar. Lagrangian Green's function extraction, with applications to potential fields, diffusion, and acoustic waves. New Journal of Physics, 12, 2010.

S. Stein and M. Wysession. An introduction to seismology, earthquakes, and Earth structure. Blackwell publishing, 2003.

W. J. Stephenson, J. N. Louie, S. Pullammanappallil, R. A. Williams, and J. K. Odum. Blind shear-wave velocity comparison of ReMi and MASW results with boreholes to $200 \mathrm{~m}$ in Santa Clara valley: Implications for earthquake ground-motion assessment. Bulletin of the Seismological Society of America, 95(6):2506-2516, 2005.

R. J. Stern and P. Johnson. Continental lithosphere of the Arabian Plate: A geologic, petrologic, and geophysical synthesis. Earth-Science Reviews, 101:29-67, 2010. doi: 10.1016/j.earscirev.2010.01.002.

T. Stern and A. Benson. Wide-angle seismic imaging beneath an andesitic arc: Central North Island, New Zealand. Journal of Geophysical Research, 116, 2011.

T. Stern, A. Benson, and W. Stratford. Rocks beneath New Zealand's Central North Island: Mantle or crust? EOS, 86(51), 2005.

J. Stimac, G. Nordquist, A. Suminar, and L. Sirad-Azwar. An overview of the Awibengkok geothermal system, Indonesia. Geothermics, 37:300-331, 2008.

W. Stratford and H. Thybo. Crustal structure and composition of the Oslo Graben, Norway. Earth and Planetary Science Letters, 304:431-442, 2011.

Streckeisen. Portable very-broad-band tri-axial seismometer: STS-2 "Low-Power". G. Streckeisen AG, Messgeraetem CH-8422 Pfungen, Switzerland, 2 edition, May 1995.

F. Sugiaman, E. Sunio, P. Molling, and J. Stimac. Geochemical response to production of the Tiwi geothermal field, Phillippines. Geothermics, 33:57-86, 2004.

Z. Tang, J. Julià, H. Zahran, and P. M. Mai. The lithospheric shear-wave velocity structure of Saudi Arabia: Young volcanism in an old shield. Tectonophysics, 680:8-27, 2016. 
T. Tanimoto. Excitation of normal modes by atmospheric turbolence: source of longperiod seismic noise. Geophysics Journal International, 136:395-402, 1999.

W. A. Thelen, M. Clark, C. T. Lopez, C. Loughner, H. Park, J. B. Scott, S. B. Smith, B. Greschke, and J. N. Louie. A transect of 200 shallow shear velocity profiles across the Los Angeles basin. Bulletin of the Seismological Society of America, 96:1055-1067, 2006.

J. R. Thorson and J. F. Claerbout. Velocity-stack and slant-stack stochastic inversion. Geophysics, 50(12):2727-2741, 1985.

I. M. Tibuleac and M. Eneva. Seismic signature of the geothermal field at Soda Lake, Nevada, from ambient noise analysis. Geothermal Resources Council Transactions, 35 : 1767-1771, 2011.

V. C. Tsai. On establishing the accuracy of noise tomography travel-time measurements in a relalistic medium. Geophysical Journal International, 178:1555-1564, 2009.

V. C. Tsai and M. P. Moschetti. An explicit relationship between time-domain noise correlation and spatial autocorrelation (SPAC) results. Geophysical Journal International, 182:454-460, 2010.

D. L. Turcotte and G. Schubert. Geodynamics. Cambridge University Press, second edition edition, 2002.

L. Uieda, V.C. Oliveira Jr, and V. C. F. Barbosa. Modeling the earth with Fatiando a Terra. In Proceedings of the 12th Python in Science Conference, pages 91-98, 2013.

P. Villamor and K. Berryman. A late Quaternary extension rate in the Taupo Volcanic Zone, New Zealand, derived from fault slip data. New Zealand Journal of Geology and Geophysics, 44:243-269, 2010.

P. Villamor and K. R. Berryman. Evolution of the southern termination of the Taupo Rift, New Zealand. New Zealand Journal of Geology and Geophysics, 49:23-37, 2006.

C. G. Vucetich and W. A. Pullar. Stratigraphy and chronology of late pleistocene volcanic ash beds in Central North Island, New Zealand. New Zealand Journal of Geology and Geophysics, 12(4):784-837, 1969.

I. C. Wallis, C. J. Bardsley, T. S. Powell, J. V. Rowland, and J. M. O'Brien. A structural model for the Rotokawa geothermal field, New Zealand. 35th New Zealand Geothermal Workshop: 2013 Proceedings 17-20 November 2013, 2013.

Z. Wang. Wave velocities in hydrocarbons and hydrocarbon saturated rocks with applications to EOR monitoring. PhD thesis, Stanford University, 1988. 
Z. Wang and A. Nur. Wave velocities in hydrocarbon saturated rocks: experimental results. Geophysics, 55:723-733, 1990.

K. Wapenaar and J. Fokkema. Green's function representation for seismic interferometry. Geophysics, 71(4):SI33-SI46, 2006.

M. Wathelet. Array recordings of ambient vibrations: Surface wave inversion. PhD thesis, Université de Liège, 2005.

M. Wathelet. An improved neighborhood algorithm: Parameter conditions and dynamic scaling. Geophysical Research Letters, 35(L09301), 2008.

U. Wegler and C. Sens-Schöndelfer. Fault zone monitoring with passive image interferometry. Geophysics Journal International, 168:1029-1033, 2007.

U. Wegler, H. Nakahara, C. Sens-Schönfelder, M. Korn, and K. Shiomi. Sudden drop of seismic velocity after the $2004 \mathrm{Mw} 6.6$ mid-Niigata earthquake, Japan, observed with passive image interferometry. Journal of Geophysical Research, 114(B06305):1-11, 2009.

H. Wehr. Dictionary of modern written Arabic (3rd edition). Spoken Language Services Inc, 1976.

T. Wever. The Conrad Discontinuity and the top of the reflective crust - do they coincide? Tectonophysics, 1989.

P. J. White, J. V. Lawless, S. Terzaghi, and W. Okada. Advances in subsidence modelling of exploited geothermal field. In Proceedings World Geothermal Congress 2015. Antalya, Turkey, 24-29 April 2005, 2005.

C. J. N. Wilson. The Taupo eruption, New Zealand ii. The Taupo ignambrite. Philosophical Transactions of the Royal Society of London A, (314):229-310, 1985.

C. J. N. Wilson. The 26.5 ka Oruanui eruption, New Zealand: An introduction and overview. Journal of Volcanology and Geothermal Research, (112):133-174, 2001.

C. J. N. Wilson, B. F. Houghton, and E. F. Lloyd. Volcanic history and evolution of the Maroa-Taupo area, central North Island. Late Cenozoic Volcanism in New Zealand, 23: 194-223, 1986.

C. J. N. Wilson, B. F. Houghton, M. O. McWilliams, M. A. Lanphere, S. D. Weaver, and R. M. Briggs. Volcanic and structural evolution of Taupo Volcanic Zone, New Zealand: A review. Journal of Volcanology and Geothermal Research, 68:1-28, 1995. 
J. Winick, T. Powerll, and E. Mroczek. The natural-state geochemistry of the Rotokawa reservoir. In New Zealand Geothermal Workshop 2009 Proceedings, 16-18 November 2009, Rotorua, New Zealand, 2011.

C. P. Wood. Stratigraphy and petrology of NM2 ngatamariki geothermal field, unpublished report. Technical report, Department of Scientific and Industrial Research, 1985.

C. P. Wood. Stratigraphy and petrology of NM3 ngatamariki geothermal field, unpublished report. Technical report, Department of Scientific and Industrial Research, 1986a.

C. P. Wood. Stratigraphy and petrology of NM4 ngatamariki geothermal field, unpublished report. Technical report, Department of Scientific and Industrial Research, 1986b.

Y. Yang, M. H. Ritzwoller, and C. H. Jones. Crustal structuredetermined from ambient noise tomography near the magmatic centers of the Coso region, southeastern California. Geochemistry Geophysics Geosystems, 12(2), 2011.

Z. Yao, W.D. Mooney, H.M. Zahran, and S. E. Youssef. Upper mantle velocity structure beneath the Arabian shield from Rayleigh surface wave tomography and its implications. Journal of Geophysical Research: Solid Earth, 122, 2017.

Z. Zhan, S. Ni, D. V. Helmberger, and R. W. Clayton. Retrieval of Moho-reflected shearwave arrivals from ambient seismic noise. (102):408-420, 2010.

Z. Zhan, V. C. Tsai, and R. W. Clayton. Spurious velocity changes caused by temporal variations in ambient noise frequency content. Geophysical Journal International, 194: 1574-1581, 2013. 
